\title{
$\frac{10}{4-8}-948 \delta(D$ \\ SANDIA REPORT
}

SAND94-0311 - UC-706

Unlimited Release

Printed March 1994

\section{Triggered Lightning Test Program: Environments Within 20 Meters of the Lightning Channel and Small Area Temporary Protection Concepts}

Richard J. Fisher, George H. Schnetzer

Proparad by

Sandia National Laboratorloe

Albuquorque, Now Mexlco 87186 and Livormore, Calltornla 94550

for the Unitod States Dopartmont of Enorey

under Contract DE-ACO4-94AL85000 
Issued by Sandia National Laboratories, operated for the United States Department of Energy by Sandia Corporation.

NOTICE: This report was prepared as an account of work sponsored by an agency of the United States Government. Neither the United States Government nor any agency thereof, nor any of their employees, nor any of their contractors, subcontractors, or their employees, makes any warranty, express or implied, or assumes any legal liability or responsibility for the accuracy, completeness, or usefulness of any information, apparatus, product, or process disclosed, or represents that its use would not infringe privately owned rights. Reference herein to any specific commercial product, process, or service by trade name, trademark, manufacturer, or otherwise, does not necessarily constitute or imply its endorsement, recommendation, or favoring by the United States Government, any agency thereof or any of their contractors or subcontractors. The views and opinions expressed herein do not necessarily state or reflect those of the United States Government, any agency thereof or any of their contractors.

Printed in the United States of America. This report has been reproduced directly from the best available copy.

Available to DOE and DOE contractors from Office of Scientific and Technical Information PO Box 62

Oak Ridge, TN 37831

Prices available from (615) 576-8401, FTS 626-8401

Available to the public from

National Technical Information Service

US Department of Commerce

5285 Port Royal Rd

Springfield, VA 22161

NTIS price codes

Printed copy: A15

Microfiche copy: A01 
SAND94-0311

Distribution

Unlimited Release

Printed March 1994

Category UC-706

\title{
1993 Triggered Lightning Test Program: Environments Within 20 Meters of the Lightning Channel and Small Area Temporary Protection Concepts
}

\author{
Richard J. Fisher and George H. Schnetzer \\ Electromagnetic Analysis and Testing Department \\ Sandia National Laboratories \\ Albuquerque, New Mexico 87185-0865
}

\begin{abstract}
Vertical electric fields, azimuthal magnetic fields, and earth step potentials at ground level have been measured at 10 and 20 meters from the base of triggered lightning flashes. For incident stroke peak currents in the range of 4.4 to $29 \mathrm{kA}$, vertical electric field change amplitudes as high as $210 \mathrm{kV} / \mathrm{m}$ were observed at $10 \mathrm{~m}$, with rise times of the order of a few microseconds. Magnetic fields were found to follow Ampere's law closely at both 10 and $20 \mathrm{~m}$. Earth step potentials measured over a $0.5-\mathrm{m}$ radial distance at the $10-\mathrm{m}$ and $20-$ $m$ stations were linear with and had the same waveforms as the stroke currents. The step voltages exhibited a $1 / \mathrm{r}$ distance dependence between the two measurement distances. A model that incorporates the presence of a thin surface layer, due to rain water saturation, of much higher conductivity than the bulk of the underlying earth is proposed to explain the observed behavior. Tests were also carried out to evaluate the effectiveness of several concepts for protecting a small exposed object, such as a piece of ordnance at the site of a transportation accident, from either a direct strike or from the indirect effects of electromagnetic fields produced by a nearby lightning flash to ground. Photographs of the occurrence of significant radial filamentary arcing along the surface of the ground from the strike points were acquired. This type of arcing, with a maximum radial extent of at least $20 \mathrm{~m}$, was observed on six of seven of triggered flashes and on all strokes of 15-kA peak amplitude or higher.
\end{abstract}




\section{Acknowledgments}

The authors are indebted to numerous individuals for their significant contributions to the success of the work presented in this report. Among those to whom special thanks are due are P.J. Magnotti, K. Haynes, R. Branstetter, and T. Tran (U.S. Army's Armament Research, Development, and Engineering Center (ARDEC) at Picatinny Arsenal and Ft. McClellan, Alabama) for their technical participation and general on-site support. The excellent and enthusiastic technical support throughout the fielding period provided by J.A. Chael, R. Davis, and G.O. Miller (Dept. 9324, SNL) is very gratefully acknowledged. The numerous discussions of theoretical aspects of this project with L.K. Warne (Dept. 2753, SNL) and his assistance in the interpretation of the experimental data, particularly the earth step potential measurements, is gladly acknowledged, as is his development of the model for earth potentials presented in Appendix C. The extended elucidating discussions of the experimental results with M.A. Uman, V.A. Rakov, and R. Thottapillil of the University of Florida Lightning Research Laboratory were also very constructive. Finally, we owe thanks for the many valuable suggestions received from B.J.C. Burrows (formerly of the Lightning Test and Technology Centre, Culham Laboratory, United Kingdom) during the planning and data review portions of the program. The support and encouragement of M.A. Chiefa and J.E. Grenert (ARDEC), M. Jones (United Kingdorn Atomic Weapons Establishment, Aldermaston), M.E. Morris (Dept. 2753, SNL) and S.D. Spray (Dept. 12331, SNL) were critical to the success of the program and are most gratefully acknowledged. 


\section{Contents}

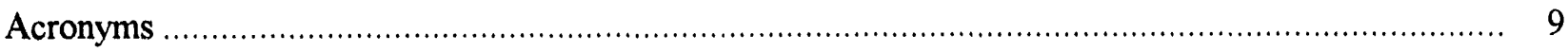

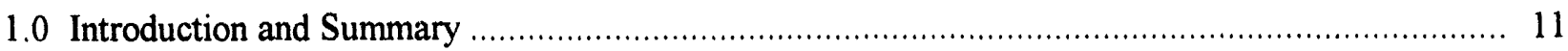

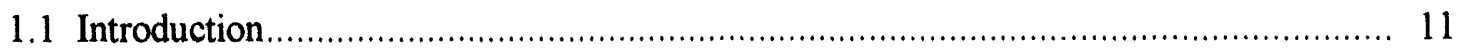

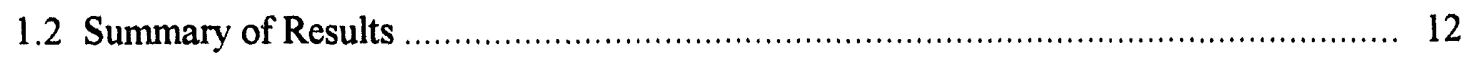

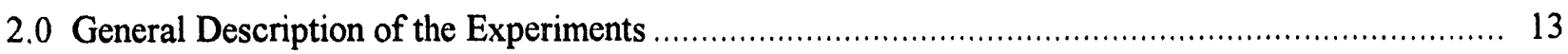

2.1 Characterization of Close-in Lightning Environments ..................................... 13

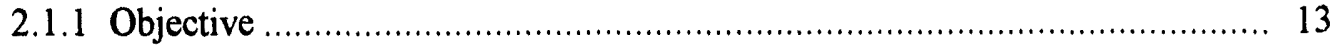

2.1.2 Experimental Approach............................................................. 13

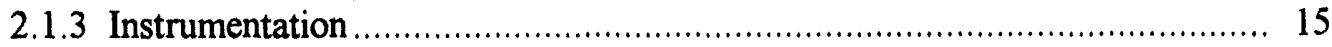

2.1.3.1 Incident Flash Currents ...................................................... 17

2.1.3.2 Vertical Electric and Horizontal Magnetic Fields ....................... 18

2.1.3.3 Earth Step Potentials...................................................... 18

2.1.3.4 Photographic Coverage ....................................................... 21

2.2 Comparison of Temporary Lightning Protection System Concepts .......................... 21

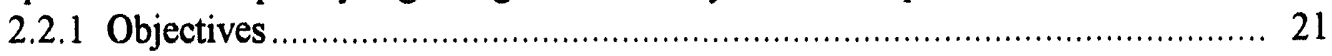

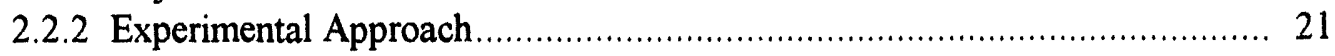

2.2.2.1 Direct-Strike Prevention.................................................. 21

2.2.2.2 Protection from the Nearby Lightning Environment .................... 24

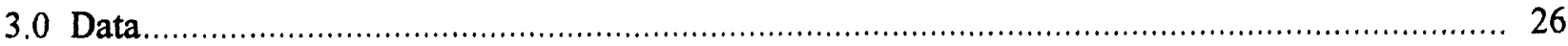

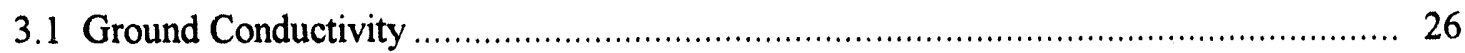

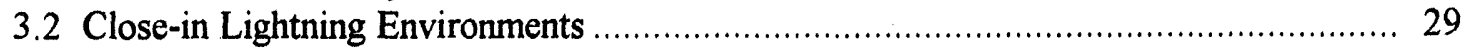

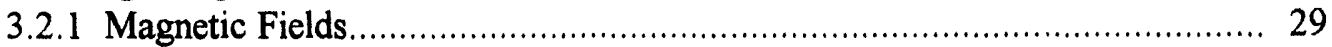

3.2.2 Vertical Electric Fields ............................................................... 33

3.2.3 Radial Earth Step Potentials ........................................................ 38

3.2.4 Incident Flash Currents ................................................................... 45

3.2.5 Surface Arcing Near the Bases of Lightning Channels ........................... 48

3.3 Temporary Lightning Protection Concept Tests ................................................ 56

3.3.1 Direct-Strike Suppression............................................................. 56

3.3.1.1 The Effect of a Grounded Mast ............................................. 56

3.3.1.2 Dielectric Shield .............................................................. 59

3.3.2 Reduction of Nearby Lightning Environments ................................... 60

3.3.2.1 Effects of a Shielding Cage ................................................... 60

3.3.2.2 Effect of a shallowly Buried Ground Ring Electrode ................... 60

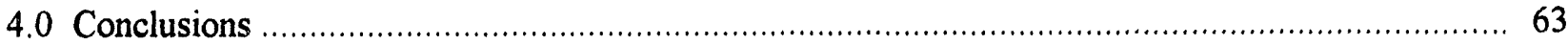

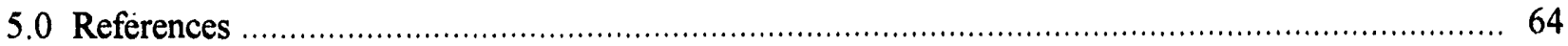

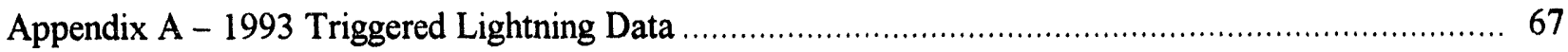

Appendix B - Derivation of the Relationship for Obtaining Earth Conductivity fiom a 4-Probe Measurement ......................................................................... 323

Appendix C - Potential Distribution about an Earth Lightning Strike with Conducting Surface Layer.... 325

Appendix D - Annotated Schematic Diagrams of the Time Histories of 1993 Triggered Flashes........... 343 


\section{Illustrations}

2-1 The SATTLIF deployed at Ft. McClellan, Alabama in readiness for 1993 RTL tests .................14

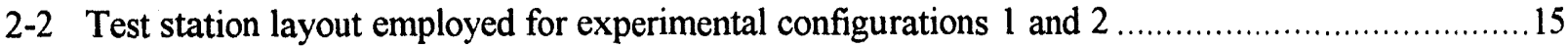

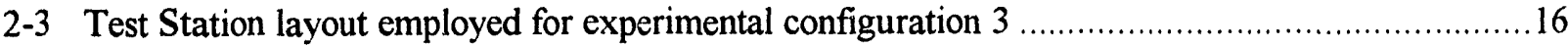

2-4 Basic instrumentation complement deployed at each test station ........................................ 16

2-5 Connection of the bottom of the LTA to the short earthing rod directly below

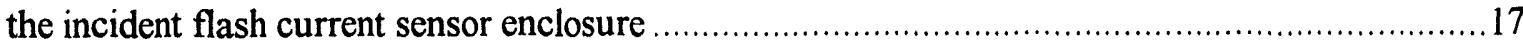

2-6 Schematic of the vertical electric field sensor and its signal conditioning electronics ..................19

2-7 Diagram of the earth step potential measurement instrumentation configuration ......................19

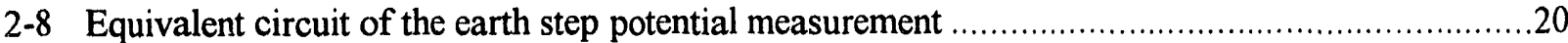

2-9 Assumed geometry leading to the commonly employed model for earth step potentials given by equation $(2-1)$

2-10 Temporary lightning protection mast proposed by AWE for use at weapon transportation accident sites 22

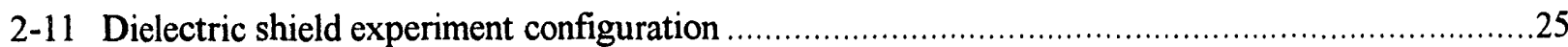

2-12 Grounded partial Faraday cage made from steel wire fencing material installed over a test station sensor complement (cage has been outlined in white to enhance visibility) ........................25

3-1 Overview of the triggered lightning test site at Ft. McClellan, Alabama ..................................27

3-2 Diagram of the measurement technique employed in determining the earth

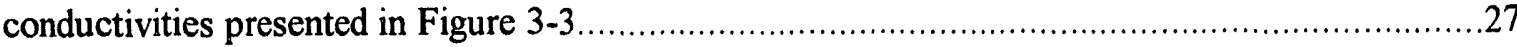

3-3 Earth conductivities derived from daily measurements conducted as shown in Figure 3-2 _..........28

3-4 Lightning channel geometry leading to the model for the azimuthal magnetic field at the ground given in equation (3-1)

3-5 Comparison of peak magnetic fields measured at unperturbed stations at $10 \mathrm{~m}$ and $20 \mathrm{~m}$ from the strike point with Ampere's Law

3-6 Comparison of the waveforms of the magnetic fields recorded at $20.7 \mathrm{~m}$ from the strike point on both strokes of flash 93-12 with those of the associated recorded incident return-stroke currents; (a) full records from stroke 1, (b) same data as (a) on an expanded scale, (c) full records from stroke 2 , and (d) same data as (c) on an expanded time scale

3-7 Schematic of the typical flash current time history produced by the lightning triggering technique employed during the present experiments

3-8 Full flash current records obtained during flashes (a) 93-02 and (b) 93-03; the chosen recording sensitivities are such that saturation of the return-stroke components occurred as shown in each case

3-9 Typical vertical electric field of a return stroke preceded by a dart leader as measured at Station $2(9.3 \mathrm{~m})$ on flash 93-02; (a) full record, and (b) same data on an expanded time scale 


\section{Illustrations (Continued)}

3-10 Two examples of electric fields produced at $9.3 \mathrm{~m}$ from the strike point by return strokes preceded by dart-stepped leaders; (a) last of seven strokes in flash 93-02, and (b) last of the two strokes in flash 93-03.

3-11 The relationship between peak return-stroke current and amplitude of leader field change measured at $9.3 \mathrm{~m}$ from the strike point

3-12 The relationships between peak return-stroke current and vertical electric field changes produced by preceding dart leaders at distances from the strike point of $9.3 \mathrm{~m}$ and $19.3 \mathrm{~m}$.

3-13 The relationship between the electric field change due to the return- stroke portion of the discharge and peak return-stroke current as measured at $9.3 \mathrm{~m}$ and $19.3 \mathrm{~m}$ from the strike point.

3-14 Earth step potential as a function of return-stroke current as measured at $10 \mathrm{~m}$ and $20 \mathrm{~m}$ from the strike point

3-15 Comparison of the waveforms of earth step potentials measured $10 \mathrm{~m}$ from the strike point on both strokes of flash 93-12 with those of their corresponding incident return-stroke currents normalized to the peak amplitude of the step potentials; (a) full data records, stroke 1, (b) expanded time scale of data in (a); (c) full data records, stroke 2, and (d) expanded time scale of data in (c)

3-16 Comparison of the waveforms of the earth step potentials measured $20 \mathrm{~m}$ from the strike point on both strokes of flash 93-12 with those of their corresponding incident return-stroke currents normalized to the peak amplitude of the step potentials; (a) full data records, stroke 1, (b) expanded time scale of data if (a); (c) full data records, stroke 2, and (d) expanded time scale of data in (c)

3-17 Earth step potentials at $10 \mathrm{~m}$ and $20 \mathrm{~m}$ from the strike point as functions of return-stroke amplitude determined for various strokes at points $60 \mu \mathrm{s}$ into the responses

3-18 Two element earth step potential model accounting for the presence of a highly conducting thin surface layer......

3-19 Radial filamentary surface arcing emanating from the base of the strike point of the second stroke in flash 93-12; stroke current was $29.6 \mathrm{kA}$

3-20 Radial surface arcing from the base of flash 93-03; view of the radial extent of the arc beyond the orange cage at station 1 is blocked by the SATTLIF's electrical generator and auxiliary storage container; arcing occurred on the first and fourth return strokes.

3-21 Scorched grass on a golf course green near Tucson, Arizona resulting from a lightning strike to the (dielectric) flagpole at the center of the pattern. (Photograph courtesy of E.P. Krider, Institute of Atmospheric Physics, University of Arizona).

3-22 Percentages of return strokes producing filamentary arcing from the base of the strike point as a function of peak stroke current

3-23 (a) Magnetic field and (b) earth step potential records obtained at Station 1 during stroke 1 of flash 93-03, during which the metal cage was contacted by the ground arc shown in Figure 3-20 


\section{Illustrations (Continued)}

3-24 Installation of current sensor on the grounding braid connecting one corner of the wire shielding cage with its adjacent $0.5-\mathrm{m}$ deep ground rod

3-25 Current recorded on the grounding strap on the corner of the wire cage nearest to the point of arc interception shown in Figure 3-20 on stroke 1 of flash 93-03

3-26 Effect of strike rod height $\mathrm{H}$ on the vertical electric fields measured at $9.3 \mathrm{~m}$ and $19.3 \mathrm{~m}$ from the strike point.

3-27 Location of recording stations with respect to the nominal 45-degree cones of protection of the $4.5-\mathrm{m}$ and $11-\mathrm{m}$ strike poles used during the present tests.

3-28 Vertical electric field reduction factors measured at distances $D$ of $9.3 \mathrm{~m}$ and $19.3 \mathrm{~m}$ from the strike point due to the increase in strike rod height $\mathrm{H}$ from $4.5 \mathrm{~m}$ to $11 \mathrm{~m}$

3-29 The effect of the presence of the wire cage on the magnetic fields measured at Station 1

3-30 The effect of the presence of the wire cage on the earth step potentials measured at Station

3-31 The effect of the wire cage on vertical electric fields measured during the same strokes at $9.3 \mathrm{~m}$ from the strike point; shown are the ratios of electric fields measured at Station 1, with the cage installed, to those measured the same distance from the strike point at Station 2, where no cage was present

3-32 The effects of the presence of ground ring electrode and cage, separately and in combination, on earth step voltages at $10 \mathrm{~m}$ from the strike point

\section{Tables}

2-1 $\mathrm{E}_{\mathrm{V}}$ Measurements at 10 and $20 \mathrm{~m}$ to Determine the Effect of an Increase in Strike Rod Height .....23

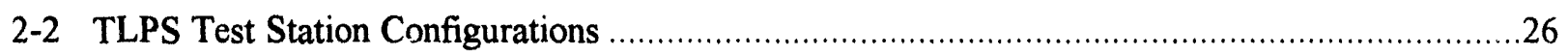

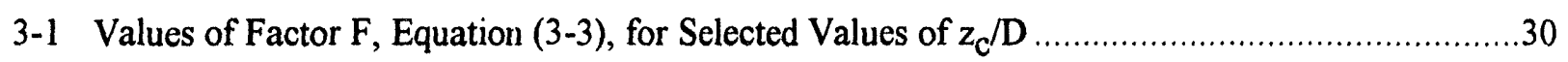

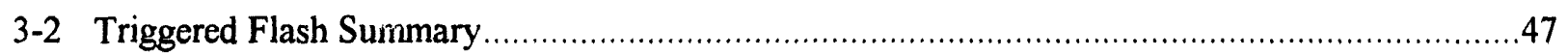

A-1 Summary of Available Data According to Flash Number ...................................................67 


\section{Acronyms}

ARDEC U.S. Army Armament Research, Development, and Engineering Center AWE United Kingdom's Atomic Weapons Establishment

CTG cloud-to-ground (lightning flash)

CVR current viewing resistor

DoD U.S. Department of Defense

FM frequency modulated

FOL fiber optic link

GRE ground ring electrode

ICC initial continuous current

LPS lightning protection system

LTA launch tube assembly

RTL rocket-triggered lightning

SATTLIF Sandia Transportable Triggered Lightning Instrumentation Facility

SNL Sandia National Laboratories

SOTS Security Operations Test Site

TLPS temporary lightning protection system

UK United Kingdom 


\section{Triggered Lightning Test Program: Environments Within 20 Meters of the Lightning Channel and Small Area Temporary Protection Concepts}

\subsection{Introduction and Summary}

\subsection{Introduction}

For the past five years Sandia National Laboratories (SNL) and the U.S. Army's Armament Research, Development and Engineering Center (ARDEC) ${ }^{1}$ have been carrying out a collaborative investigation of the interaction mechanisms of lightning with munitions and their related handling and storage facilities. The fundamental objective of this effort is the development of an improved understanding of the performance of various elements of conventional lightning protection systems (LPS) employed at such facilities. As part of this ongoing effort, a third series of experiments using triggered lightning as the test source took place during the summer of 1993 at the Department of Defense (DoD) Security Operations Test Site (SOTS), Ft. McClellan, Alabama, which is operated by ARDEC. During the period from July 24 through September 26, the Sandia Transportable Triggered Lightning Instrumentation Facility (SATTLIF) [1] was employed to conduct experiments that had two primary objectives. The first was the measurement of the electric and magnetic fields at ground level at radial distances of 10 and 20 meters from the earth attachment point of cloud-to-ground (CTG) lightning, plus the measurement of radial earth step potentials at the same distances. The second general objective was to evaluate, by direct comparison, the effectiveness of alternative conceptual techniques for protecting exposed munitions (at the site of a transportation accident, for example) from the effects of direct-strike and nearby lightning.

During the fielding period, seven flashes were initiated to the designated ground strike point using Sandia's standard rocket-triggered lightning techniques [2,3]. All of the triggered flashes were of the negative CTG type; that is, the type of lightning that effectively lowers negative charge from the cloud to the earth. ${ }^{2}$ The seven triggered flashes contained a total of approximately 50 return strokes. About 250 high resolution digitized measurements were acquired during 31 of the strokes. The data included incident stroke currents at the channel base, vertical electric and azimuthal horizontal magnetic fields, and radial earth step potentials. By inference, the latter also yield radial electric fields and earth current densities near the surface of the ground. Additional data records of the low level, slowly varying channel current components were obtained on magnetic tape. The measurements were supplemented by numerous video records of each flash from various angles; 16-mm high speed movies with 5-ms time resolution of five of the flashes; and various, sometimes spectacular, $35-\mathrm{mm}$ still photographs.

The 1993 effort yielded a wealth of pioneering experimental data, among the most significant of which are

- The first characterization of close-in (10 and $20 \mathrm{~m}) \mathrm{E}$ and $\mathrm{H}$ field environments,

- The first simultaneous multiple-station measurements of ground level vertical electric (E) and horizontal magnetic $(\mathrm{H})$ fields and earth step potentials (allowing the first experimental determination of the spatial uniformity of each of these quantities as functions of radial distance and azimuthal angle about the base of lightning channels),

1 Fire Support Armaments Center (FSAC), U.S. Army Armament Research Development \& Engineering Center; Picatinny Arsenal, New Jersey 07806-5000.

2 Globally, more than 90 percent of all CTG lightning is of negative polarity. 
- The first measurements of earth step potentials caused by the flow of lightning current in the earth outward from the base of a strike to ground (with results that apparently invalidate the use of the most commonly employed model for predicting these voltages),

- The first definitive photographic documentation of and statistics on the existence of radial filamentary surface arcing emanating from ground strike points (even to a ground of relatively high conductivity), and

- The first direct measurements of the electric field reduction produced within the cone of protection of an adjacent vertical lightning protection mast.

In the following sections of this report, each of the various experiments is described in detail. The data are summarized and their most significant features and implications are discussed. Because of the phenomenological significance of the entire body of data and its general applicability to the technology of lightning protection systems, the entire set of high resolution records of the measurements are documented in Appendix A for future reference.

These experimental results provide the basis for considerable future analysis and modeling efforts that will be reported separately on a topical basis as each progresses.

\subsection{Summary of Results}

The results of these experiments now represent the most definitive existing data base on the range of environments that can exist close to the base of a lightning ground strike. In general, the data validate previous existing models for predicting close-in electric and magnetic fields. The measurements of earth step potentials as a function of radial distance from the strike point, however, exhibit a definite $1 / \mathrm{r}$ dependence in distinct variance with expectations based on the conventional model that is commonly used throughout the lightning literature, which predicts a $1 / r^{2}$ range dependence.

To first approximation, the azimuthal distribution of electric and magnetic fields and earth potentials around the base of a lightning channel can be taken to be uniform, although this will not be true in situations in which buried conductors (e.g., cables, pipes, large tree roots) are present. Furthermore, the waveforms of close-in magnetic fields and earth step potentials can also be taken to have the same shape as the incident flash currents.

The presence of a tall $(11-\mathrm{m}$, in the present case), grounded strike rod or mast has been demonstrated experimentally under actual lightning conditions to reduce substantially the vertical electric field within and somewhat beyond its nominal 45-degree "cone of protection." The use of such a pole has been concluded to represent the most effective immediately available measure for dramatically reducing the probability of a direct strike to a low-lying critical item located within its protection zone. Additionally, if the pole is properly oriented with respect to the longitudinal axis of the item to be protected, it provides the collateral benefits of minimizing coupling to the protected item driven by radial electric and azimuthal magnetic fields and radial earth step potentials.

Six out of seven flashes triggered during these experiments produced localized diffuse (fireball) arcing at the ground point of the channel over a radial extent of 1 to $2 \mathrm{~m}$. Furthermore, 100 percent of all component strokes in these flashes that had peak current amplitudes of $15 \mathrm{kA}$ or more produced radial filamentary surface arcing along the ground out to a maximum range of at least $20 \mathrm{~m}$, the limit of the detailed monitoring capability. As inferred from one data point corresponding to the documented interception of a radial arc by one of $10-\mathrm{m}$ measurement stations, the arc delivered an estimated current of at least $1 \mathrm{kA}$ to the station, or approximately 5 percent of the total peak current of the associated stroke. On 
the basis of these results, suppression of the development of such arcing or precluding its contact with any critical nearby item should constitute a priority objective in the design of any local area lightning protection system, second only to the prevention of a direct strike. It is believed that this can be achieved through a properly designed grounding system at the base of an adjacent protection mast.

No significant reduction of the magnetic fields or earth step potentials due to a strike nearby, but not directly to the item to be protected, resulted from the installation of either a grounded metallic wire mesh cage over the object, the installation of a shallowly buried bare metallic ground ring electrode around it, or from the combination of both. No practical alternatives to these concepts for providing temporary, local protection from the intense electromagnetic environments close to the terminus of a strike to ground have as yet been identified. The presence of the cage was effective in reducing the local vertical electric field by factors of 20 or more.

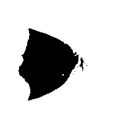

\subsection{General Description of the Experiments}

\subsection{Characterization of Close-in Lightning Environments}

\subsubsection{Objective}

The specific objective of this phase of the program was to provide a set of simultaneous measurements of ground level electromagnetic environments (amplitudes and waveforms of the vertical electric field $E_{Z}$, horizontal azimuthal magnetic field $\mathrm{H}_{\mathrm{a}}$, and radial earth step potential $\mathrm{V}_{\mathrm{s}}$ ) from multiple stations located at radial distances of 10 and 20 meters from the ground strike point of lightning. The measured step potentials permit the inference of earth current densities and radial electric fields within the earth at each of the measurement sites.

\subsubsection{Experimental Approach}

Figure 2-1 shows the SATTLIF deployed at the site of the experiments, which was an area fieshly cleared of pine woods vegetation during the spring of 1993. The composition and conductivity of the soil are discussed in Section 3.1.

Data were obtained for two basic test bed configurations. In the first, an identical complement of three separate sensors, one for each of the desired measurement quantities, was installed at three separate stations. As indicated in Figure 2-2, each station lay on a 10-m radius arc centered around the ground rod below the rocket launch tube assembly, which constituted the designated earth attachment point for each triggered flash. As is discussed in more detail in Section 2.2.2.2, Station 1 was modified by the addition of a steel wire mesh cage positioned over the sensors and grounded at each of its four corners by means of standard 16-mm diameter zinc-plated steel electrical ground rods, each driven $0.5 \mathrm{~m}$ deep into the earth. Station 3 was modified by the installation of a bare, \#2 gauge, copper wire circular ground ring electrode (GRE). The radius of the ring was about $3 \mathrm{~m}$, and the cable was buried at a depth of about $0.15 \mathrm{~m}$. The significance of the modifications at Stations 1 and 3 pertains to the evaluation of local area temporary protection system (TLPS) concepts, which is addressed later in Section 2.2.2.2 and is not germane to the present discussion. Data corresponding to nine return strokes were acquired with this sensor arrangement on August 3, 1993 during flashes 93-02 and 93-03. In this configuration, and throughout all of the other experiments, Station 2 was left unperturbed, both to serve as the primary 10-m recording site for the environments measurements and as the comparative response standard for the TLPS tests. 

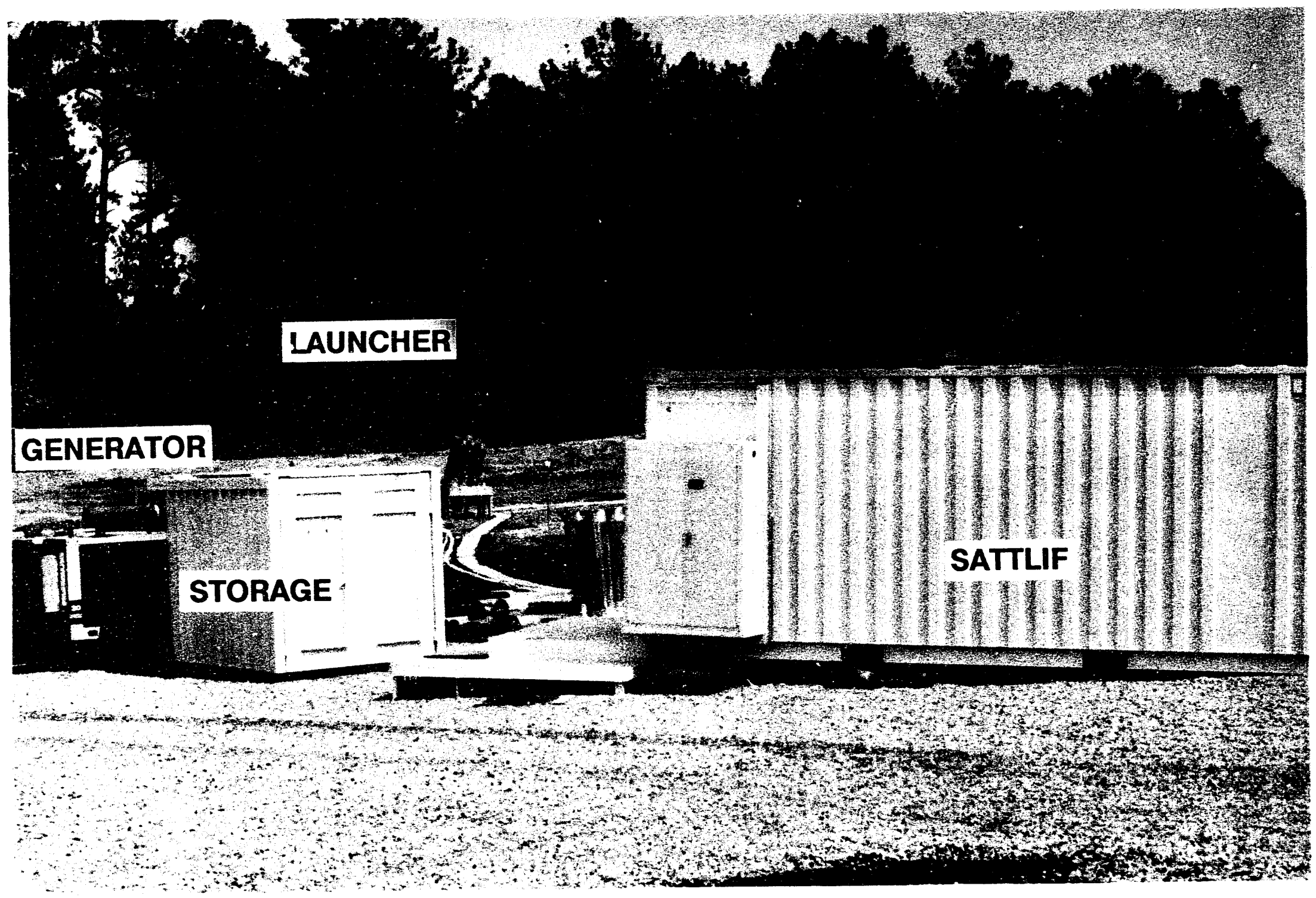

Figure 2-1 The SATTLIF deployed at Ft. McClellan, Alabama in readiness for 1993 RTL tests 


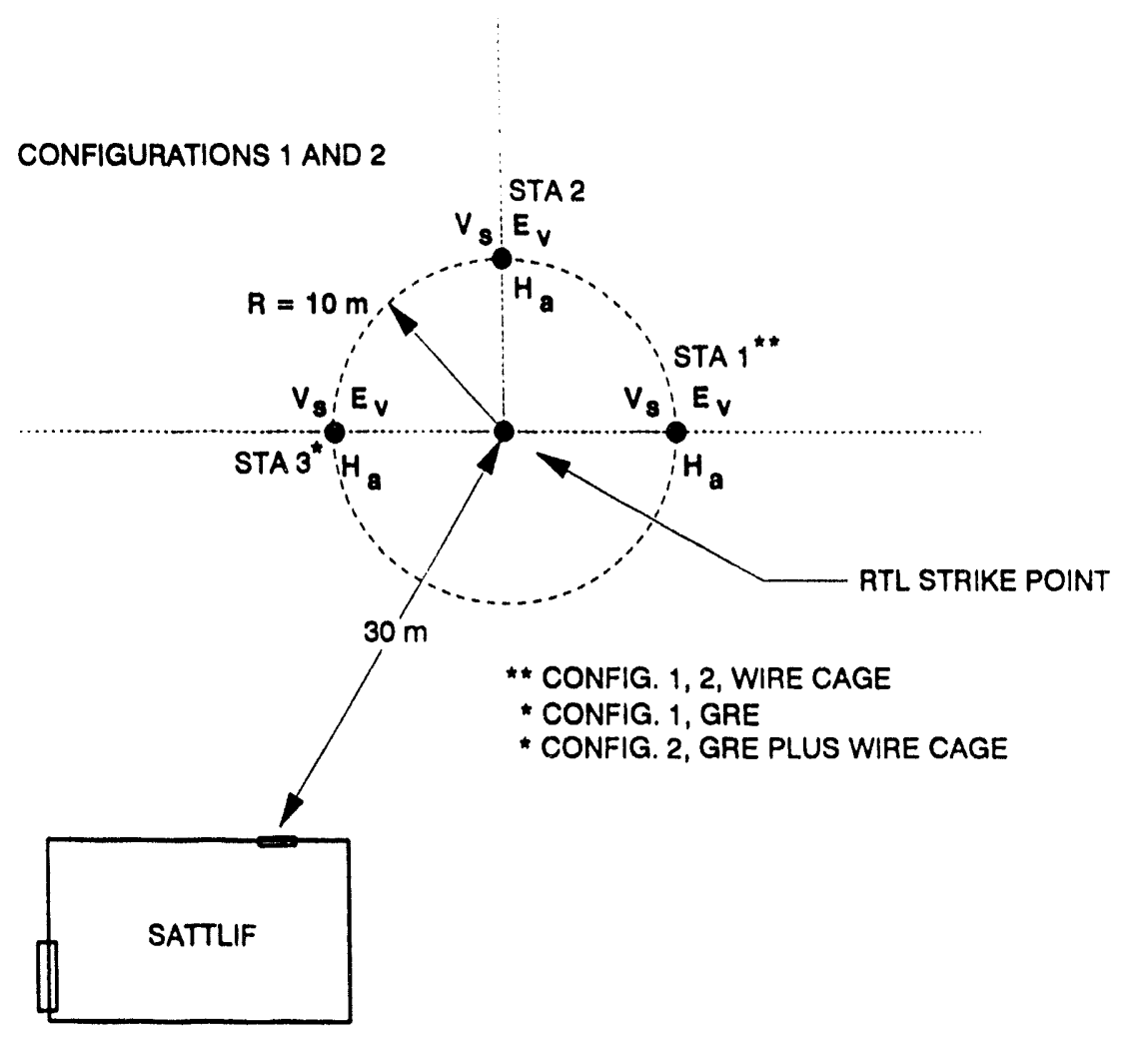

Figure 2-2 Test station layout employed for experimental configurations 1 and 2

Additional modifications were made to Stations 1 and 3 prior to the next storm during which data were acquired. The principal change was the addition of a wire cage at Station 3 similar to the one at Station 1. Data were obtained for a total of 11 return strokes under this configuration on August 18, 1993 during flashes 93-08 and 93-09. Unfortunately, a problem with the common pneumatic system line controlling the actuators of the fiber optic transmitters from several of the sensors led to the loss of data from the affected channels during the second storm.

Following a review of the data from the storm on August 18, a different station layout was implemented as is most conveniently described by means of Figure 2-3. The instrumentation assignments in this configuration, in which none of the TLPS provisions were included, reflects a prioritization of the measurement set driven by the limited number of available data channels. This deployment of sensors was left intact during storms that occurred on September 3 (flashes 93-12 and 93-14, with a total of 3 return strokes), and on September 7 (flash 93-15, yielding data from an additional 8 strokes).

The arrangement of sensors and their associated instrumentation enclosures employed at each station was as shown in Figure 2-4. The results of the experiments are summarized and discussed in Section 3.

\subsubsection{Instrumentation}

With the exception of the step voltage measurement, the sensors, signal conditioning, data transmission links, and recording instrumentation employed during these experiments were substantially the same as were used during the 1991 triggered lightning tests of a munitions storage bunker [3] and are documented in detail in Reference 3. That description is not repeated herein, although relevant overall instrumentation characteristics and operating points specific to the present experiments are summarized. The earth voltage measurement is discussed in Sections 2.1.3.3 and 3.2.3. 


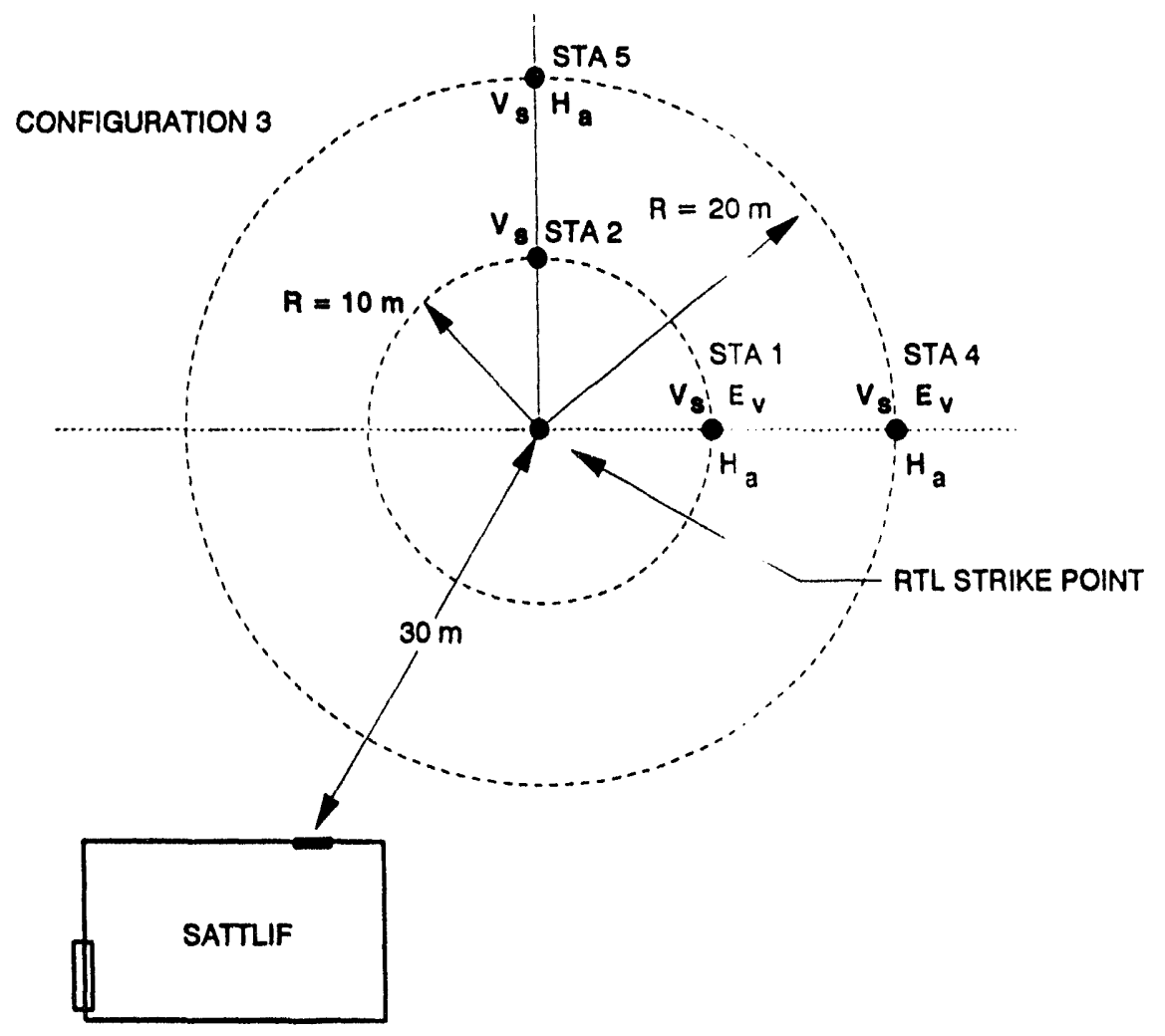

Figure 2-3 Test Station layout employed for experimental configuration 3

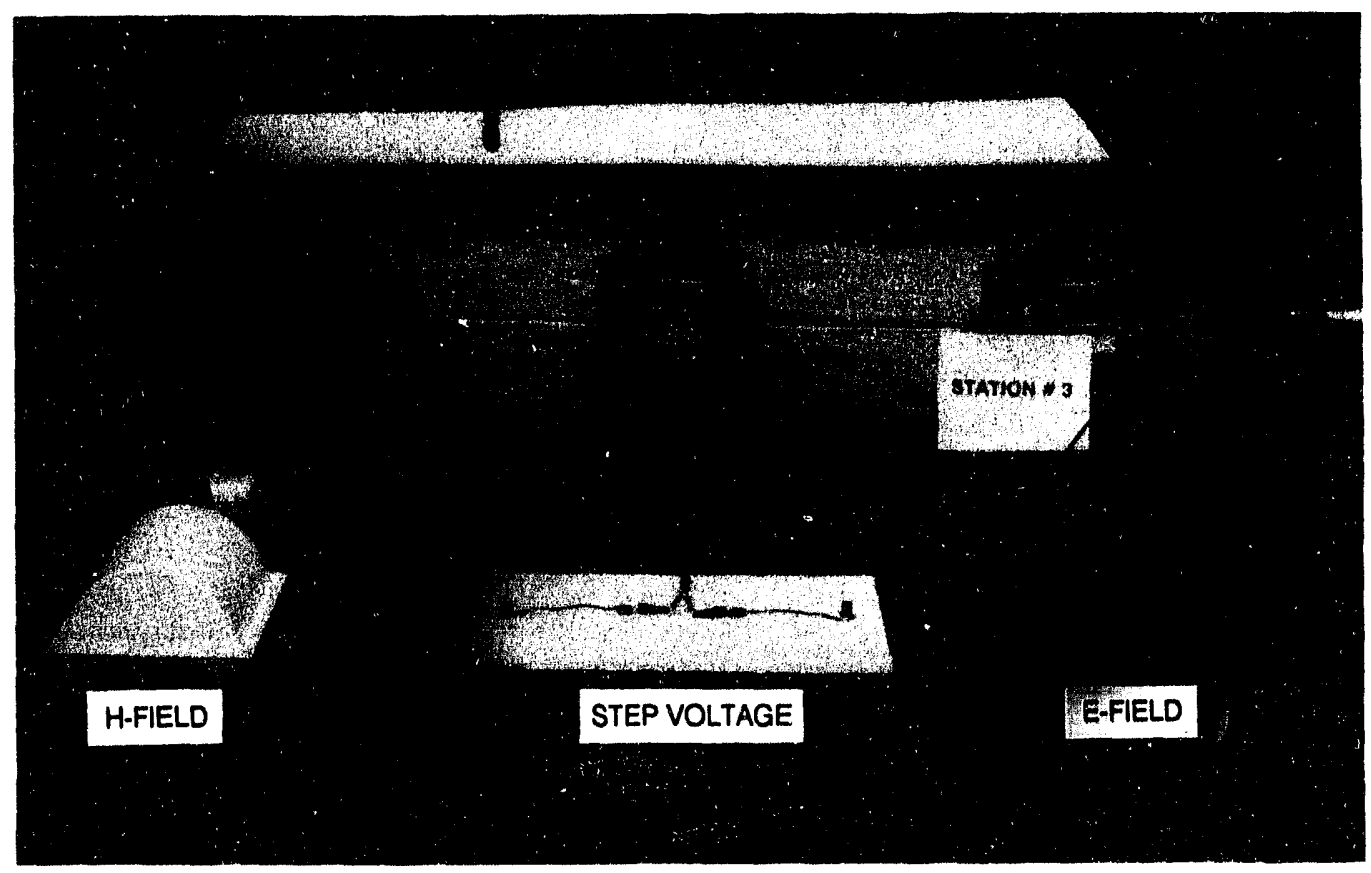

Figure 2-4 Basic instrumentation complement deployed at each test station 
2.1.3.1 Incident Flash Currents. Per our standard practice, the incident flash current flowing onto the launch tube assembly at the base of the lightning channel is sensed directly by means of a T\&M Research Model K-5000-10, 1-m $\Omega$ coaxial current viewing resistor (CVR). The CVR is mounted in an electromagnetically sealed steel enclosure which also contains two separate fiber optic transmitters, their batteries, and pneumatically controlled actuator components. The steel enclosure is inserted physically and electrically between the metallic frame of the LTA and the earthing point directly beneath it (Figure 2-5). The legs of LTA are made of fiberglass, intended to provide high voltage electrical isolation of the strike rod, launch tubes, and aluminum supporting frame from the earth, thereby encouraging all the incident current to flow to earth through the CVR.

During the 1993 experiments, the high amplitude (kiloamperes to tens of kiloamperes, typically), fast transient return-stroke current signals were transmitted, digitized at a $25-\mathrm{MHz}$ sampling rate, and stored with an overall mensurement bandwidth of about $10 \mathrm{~Hz}$ (set by the low frequency response of the particular NanoFast FOL transmitter unit employed) to just under $6 \mathrm{MHz}$, (set by the measured 60-ns 10-to-90 percent rise time of the CVR). Based on the nature of our previous triggered lightning data, a full scale measurement sensitivity of $48 \mathrm{kA}$ was selected. The common trigger level for the ten available LeCroy $9400 \mathrm{~A}$ digitizer channels was the equivalent of $3.8 \mathrm{kA}$.

Low amplitude currents, such as the continuing current components of the flash, were transmitted over a separate, frequency modulated (FM) fiber optic link. These signals were recorded on an FM analog magnetic tape channel, with an overall bandwidth from zero to approximately $400 \mathrm{kHz}$. The upper 3-dB limit was set by the response of the FM fiber optic link. The selected sensitivity for this channel resulted in a saturation level of approximately $2 \mathrm{kA}$ at the upper end and a resolution capability of approximately 20 to $30 \mathrm{~A}$ after post-test data processing using a $125-\mathrm{kHz}$ low pass filter to reduce tape recorder noise.

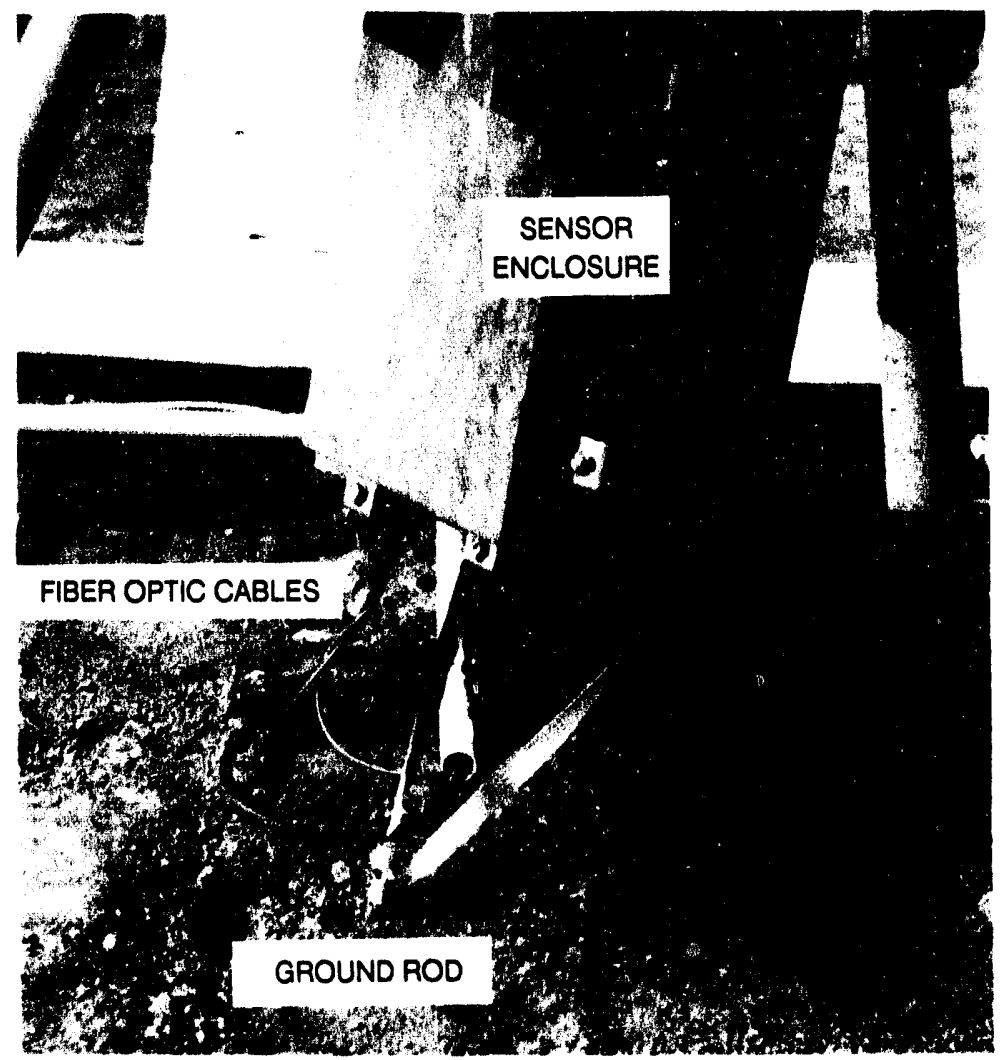

Figure 2-5 Connection of the bottom of the LTA to the short earthing rod directly below the incident flash current sensor enclosure 
2.1.3.2 Vertical Electric and Horizontal Magnetic Fields. The measurements of $E_{z}$ and $H_{a}$ were performed in the same manner as during the previous testing discussed in reference 3 . The sensitivity of the $E_{z}$ channel was set by means of a capacitive voltage divider comprised of $C_{a}$, the antenna capacitance (approximately $5 \mathrm{pF}$ ), and $\mathrm{C}_{1}$, which was physically added at the input of the amplifier circuit shown in Figure 2-6. An initial full scale sensitivity corresponding to $200 \mathrm{kV} / \mathrm{m}$ was selected on the basis of unpublished data provided by the University of Florida from similar measurements performed by them at 30 meters from triggered lightning channels at the Kennedy Space Center during 1991 [4].

The magnetic field measurements were conducted using commercially available EG\&G MGL-4 B-dot sensors, with active integration performed prior to transmission of the signal over the fiber optic link. The sensitivities of the channels were preselected based on Ampere's law applied to a maximum channel current of $48 \mathrm{kA}$.

2.1.3.3 Earth Step Potentials. The sensing of the step potential at each site was achieved using a tworod arrangement as shown in Figure 2-7. Each probe consisted of a 0.4-m length of standard 16-mm diameter zinc-plated soft steel electrical ground rod, driven $0.3 \mathrm{~m}$ into the earth. The separation distance of the probes was in all cases $0.5 \mathrm{~m}$.

The design of the measurement was based on the model indicated in Figure 2-8. The model does not account for any magnetic field coupling to the connecting cables between the two sensor probes. However, the upper limit of this contribution can be readily shown to be of the order of tens of volts, which is negligible compared to the magnitude of the recorded step voltages and is therefore justifiably ignored in the model.

As a starting point in the development of values for the circuit elements in Figure 2-8, consider the standard hemispherical model of a low frequency current distribution in the earth due to the injection of incident lightning current $\mathrm{i}(\mathrm{t})$ as shown in Figure 2-9. We assume a homogeneous earth with a uniform, constant conductivity $\sigma$ throughout. According to this model, the open circuit voltage $V_{\mathrm{e}}$ that would appear across two points at the surface at radial distances $r$ and $r+\Delta r$ is

$$
\begin{aligned}
& \mathrm{V}_{\mathrm{e}}=\mathrm{i}(\mathrm{t}) \rho /(2 \pi)[1 / \mathrm{r}-\mathrm{l} /(\mathrm{r}+\Delta \mathrm{r})], \text { or } \\
& \mathrm{V}_{\mathrm{e}} \approx \mathrm{i}(\mathrm{t}) \rho \Delta \mathrm{r} /\left(2 \pi \mathrm{r}^{2}\right)=\mathrm{i}(\mathrm{t}) \Delta \mathrm{r} /\left(2 \pi \sigma \mathrm{r}^{2}\right)
\end{aligned}
$$

where $\rho$ is the resistivity of the earth, and $\Delta r \ll r$. If two rods are driven into the earth at $r$ and $r+\Delta r$ to detect $V_{e}$, their terminal source resistance is given by

$$
\mathrm{R}_{\mathrm{e}} \approx \cosh ^{-1}(\Delta \mathrm{r} / \mathrm{d}) / \pi \sigma \ell
$$

where $\mathrm{d}$ is the diameter of the each rod, and $\ell$ is the depth of each in the earth [5]. The approximate equality is in recognition of the contribution of fringing when the depth $\ell$ is comparable to $r$. For $(\Delta \mathrm{r} / \mathrm{d})>>1,(2-2)$ reduces to

$$
\mathrm{R}_{\mathrm{e}} \approx \ln (2 \Delta \mathrm{r} / \mathrm{d}) / \pi \sigma \ell
$$

The average conductivity measured in the vicinity of the test site was $1.8 \times 10^{-3} \mathrm{~S} / \mathrm{m}$ (Section 3.1 ). The probe separation $\Delta \mathrm{r}$ was $0.5 \mathrm{~m}$; the depth $\ell$ and diameter $\mathrm{d}$ of each probe were $0.3 \mathrm{~m}$ and $16 \mathrm{~mm}$, respectively. Hence, from (2-3), $\mathrm{R}_{\mathrm{e}} \approx 2400 \Omega$. 


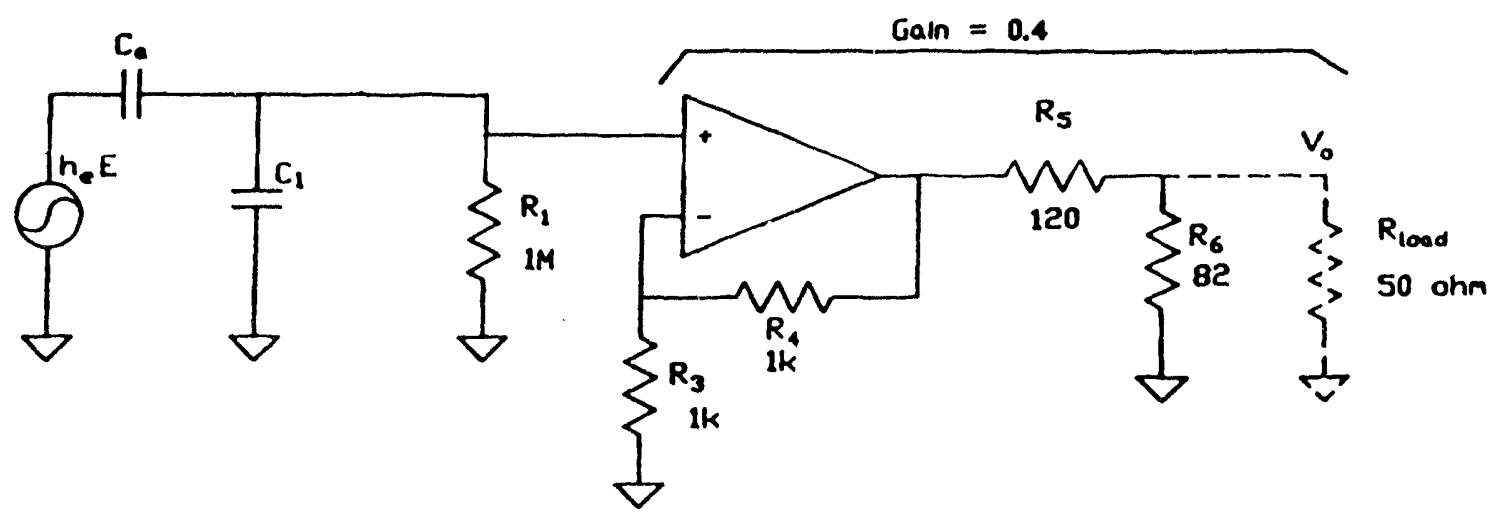

$V_{0}$ \&s $0.4 \frac{C_{e}}{\left(C_{a}+C_{1}\right)} h_{e} E$

C. is the E-field probe capacitance

$C_{\text {in }}$ in the amplifier input capacitance including the cable

$h$. Is the effective height or the probe

$E$ is the vertical electric fleld

Low Freq. Cutoffi

$$
f_{c}=\frac{1}{6.28 R_{1}\left(C_{a}+C_{1}\right)}
$$

Figure 2-6 Schematic of the vertical electric field sensor and its signal conditioning electronics
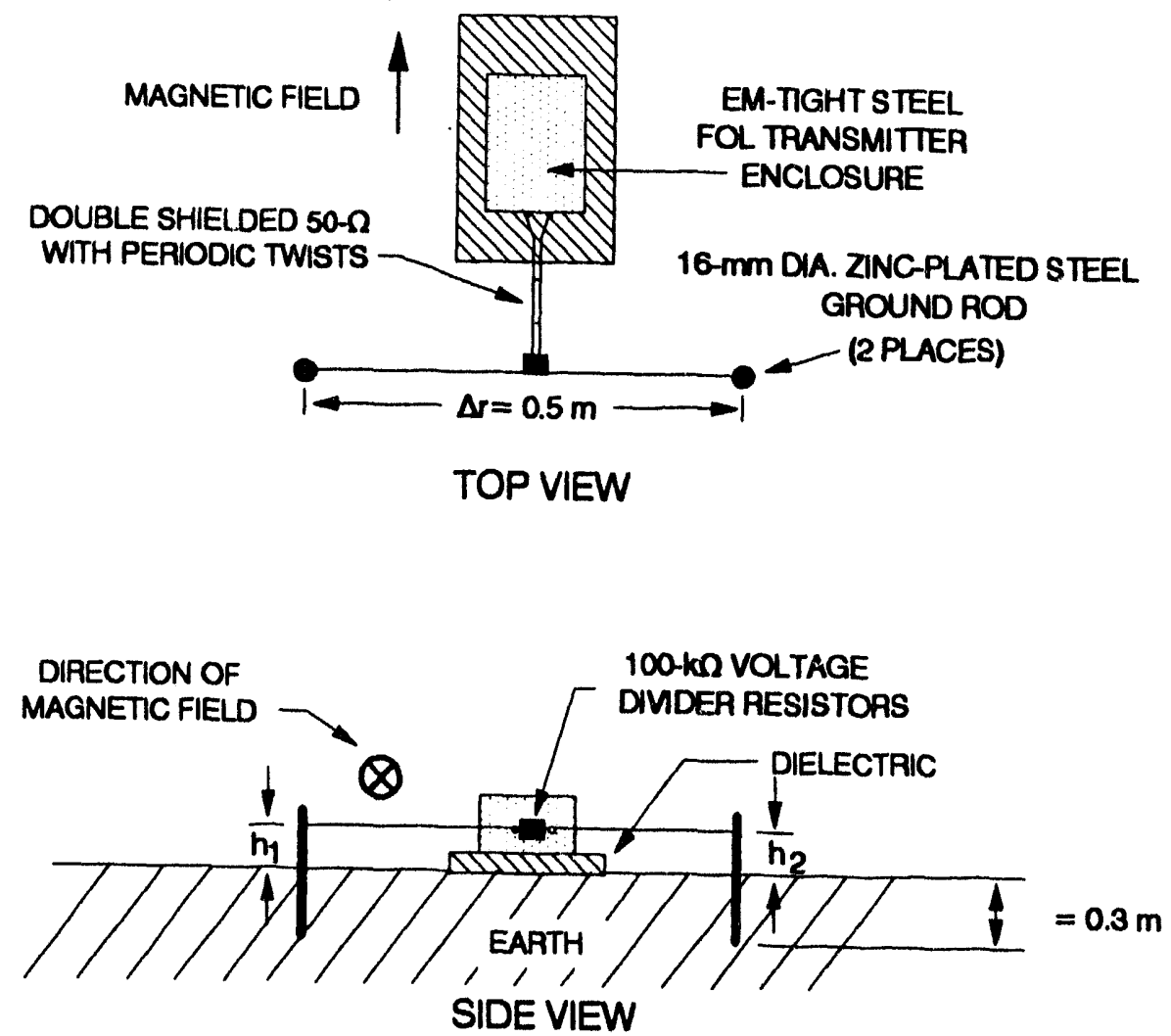

Figure 2-7 Diagram of the earth step potential measurement instrumentation configuration 


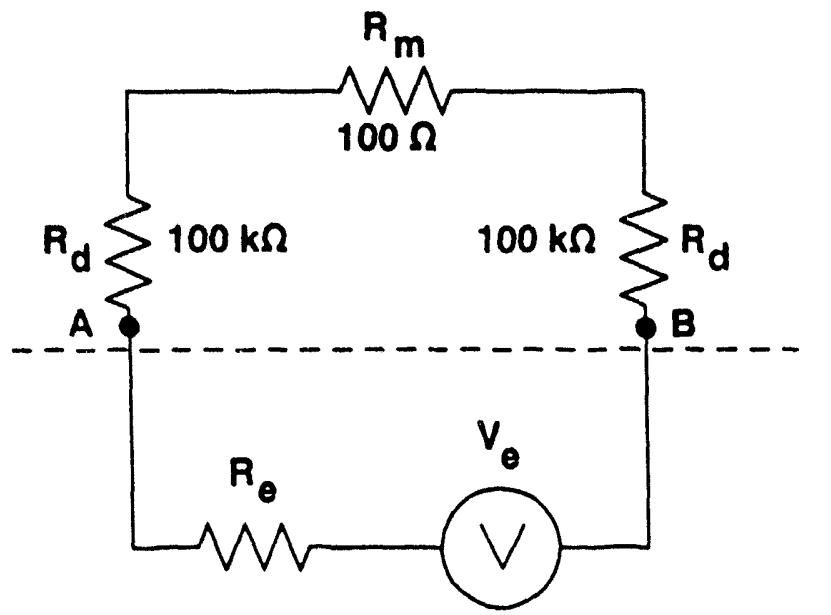

$R_{m}=$ FOL INPUT RESISTANCE

$R_{d}=$ VOLTAGE DIVIDER RESISTANCE

$R_{e}=$ SOURCE RESISTANCE AT MEASUREMENT PROBES

$V_{e}=$ OPEN CIRCUIT SOURCE VOLTAGE AT MEASUREMENT PROBES

$A, B=$ STEP POTENTIAL PROBES

2-8 Equivalent circuit of the earth step potential measurement

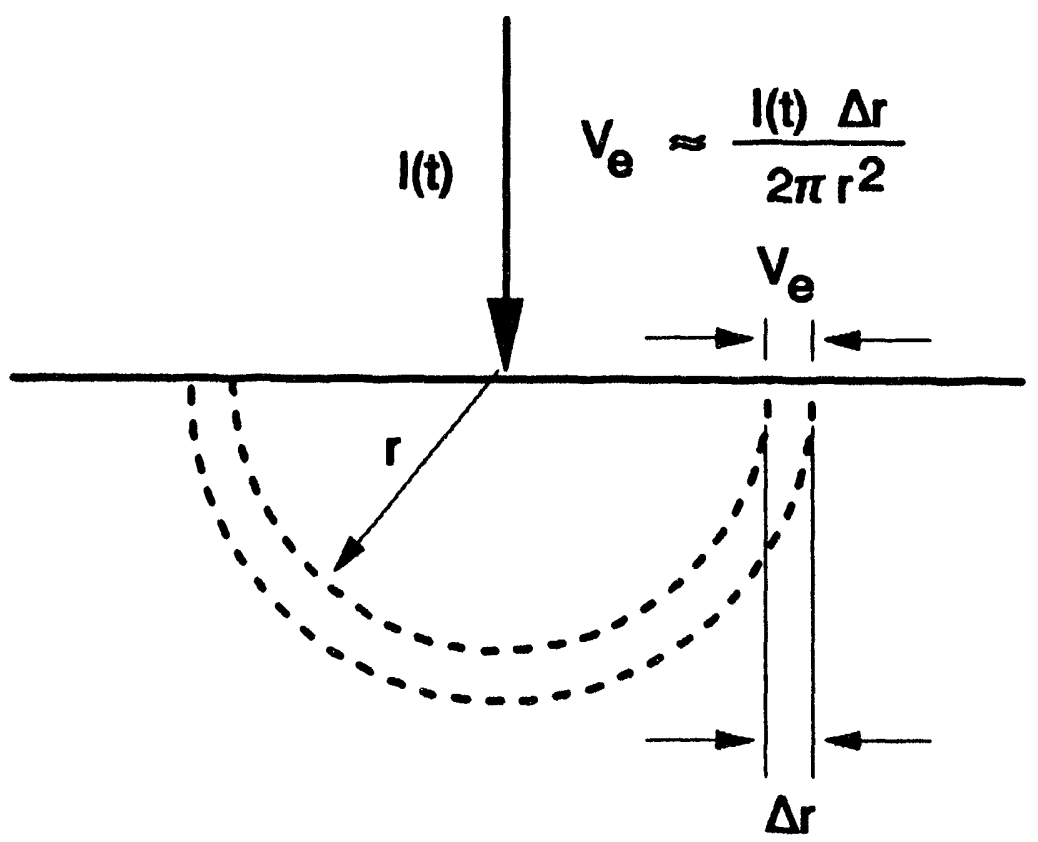

Figure 2-9 Assumed geometry leading to the commonly employed model for earth step potentials given by equation $(2-1)$ 
$V_{e}$ in series with $R_{e}$ constitutes the Thevenin source presented by the earth at the measurement terminals, represented in Figure $2-8$ by points $\mathrm{A}$ and $\mathrm{B}$. The two $100-\mathrm{k} \Omega$ voltage divider resistors and the $100-\Omega$ FOL input impedance form the load across the probes. Because the load impedance is so much larger than the 2.4-k $\Omega$ source impedance, the step voltage is not perturbed by the presence of the measurement circuit.

Initially, consideration was given to the possibility of coupling of the intense vertical electric field into the measurement system due to any minor difference in the heights of the two wires that are connected to the probes. As is discussed in Section 3.2.3, however, there is no indication in the recorded step voltage data of any significant coupling of this type, with possible exceptions occurring in cases in which the voltage response was of relatively low amplitude. A source term corresponding to this mechanism could be added to the basic model shown in Figure 2-8, but, on the basis of the bulk of the step voltage data, it appears to be unnecessary. The nature of the open circuit earth voltage $V_{e}$, which, in fact, was found not to behave as predicted by $(2-1)$, is also addressed in further detail in Section 3.2.3.

2.1.3.4 Photographic Coverage. Photographic coverage of each triggered event was provided in the form of video records from several angles and fields of view, a 16- $\mathrm{mm}$ framing camera movie with 3- to $5-\mathrm{ms}$ time resolution, and various $35-\mathrm{mm}$ still photographs. One specially configured black and white charge coupled device video camera was arranged to view the last few meters of the channel and attachment point on each flash with sufficient neutral density filtering to provide excellent definition of the central channel core. This particular camera was also equipped with a fiber optic isolation link so that its coverage was uninterrupted by the severe electromagnetic fields generated by the test events themselves. This complement of photographic coverage proved to be invaluable during the course of the tests, as will be indicated during discussions of the acquired data of various types that appear in Section 3.

\subsection{Comparison of Temporary Lightning Protection System Concepts}

\subsubsection{Objectives}

The primary objective of this set of experiments was to obtain a direct comparison of the effectiveness of various proposed concepts for protecting munitions or other relatively small exposed objects from the effects of lightning. There are two aspects of the protection requirement: elimination of direct strikes to the exposed object, and protection from the intense electromagnetic environments from a nearby strike.

\subsubsection{Experimental Approach}

2.2.2.1 Direct-Strike Prevention. The intense vertical electric field caused by a downwardpropagating lightning leader initiates upward electrical discharges (streamers) from objects on the ground below the approaching leader tip. These objects might include trees or other vegetation, fences, vehicles, personnel, or, in one scenario of specific interest, for example, an exposed piece of ordnance at the site of a transportation accident. The ultimate strike point of the impending flash is determined by which of the upward reaching streamers makes first contact with the descending leader. Prevention of a direct strike to an object of interest can be achieved either by suppressing streamer emission from it (at least in comparison with other nearby objects), or by providing a much more effective streamer source from a designated safe strike point other than the object to be protected.

One proposal put forward and favored by the United Kingdom's Atomic U. apons Establishment (AWE) for providing temporary protection of an exposed weapon is the erection of a well-grounded metallic mast within $4 \mathrm{~m}$ of the object (Figure 2-10). The proposed height of the mast is about $10 \mathrm{~m}$. The 


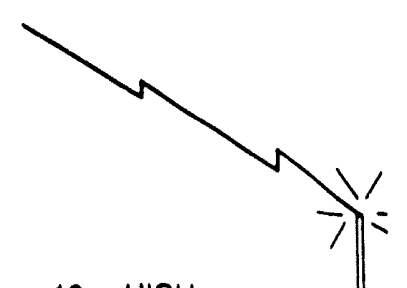

LIGHTNING PROTECTION MAST

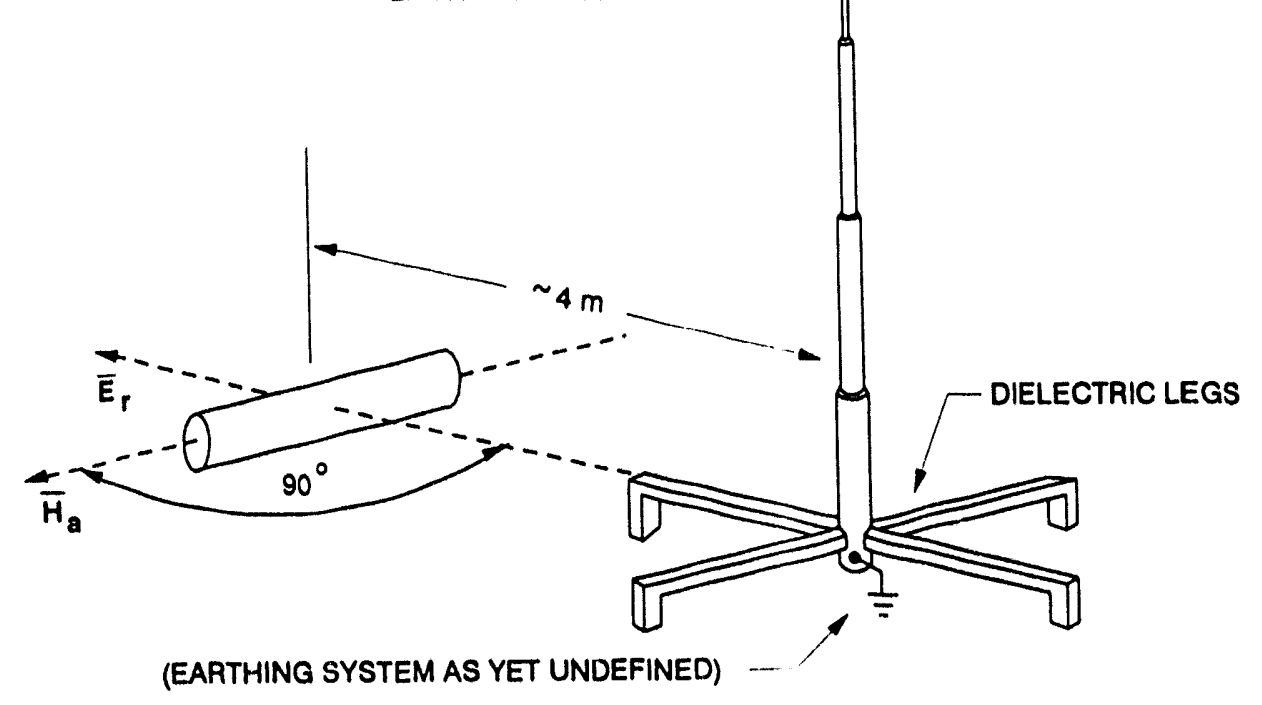

Figure 2-10 Temporary lightning protection mast proposed by AWE for use at weapon transportation accident sites

protection afforded by such a mast derives from a combination of both the mechanisms mentioned above. First, because of its relatively good earthing provisions and its substantial height advantage, it represents, to a considerable degree, the most efficient streamer emitter in the vicinity. Secondly, due to its proximity to the weapon, it also provides electrostatic shielding of the weapon from the vertical field produced by the descending leader. This shielding reduces the field at the site of the weapon and, hence, reduces the intensity of streamers originating from the weapon itself. For sharp points in the absence of significant wind, streamer current has been found to be directly proportional to the square of the applied electric field. Any reduction in local field intensity, therefore, produces a second order reduction of streamer current.

Use of a properly grounded mast would also provide a collateral benefit, since it could be so located with respect to the longitudinal axis of the item to be protected that the radial electric field and the azimuthal magnetic field due to the lightning current flowing on and outwards from the rod would be perpendicular and parallel, respectively, to the long axis of the object. This orientation of fields minimizes coupling into the object. The $10-\mathrm{m}$ strike rod is not high enough to affect the overall probability of a ground flash to the immediate vicinity of concern, but its presence dramatically lowers the probability of a direct strike to an object located within sonie defined zone of protection. This aspect is further discussed in Section 3.3.1.1 in connection with the results of the measurements involving the mast that are described below. The limitations of such an approach are that it might not always be capable of being readily implemented because of terrain features and that emergency response teams may not always be equipped with such a mast.

Experiments were made to measure the effect of the presence of an $11-\mathrm{m}$ pole on the vertical electric field at the 10- and 20-m stations defined in Figure 2-3. An extension pole was added to the usual strike rod mounted atop the LTA, resulting in an overall pole height of $11 \mathrm{~m}$ above the ground. Without the extension, the top of the usual strike rod was $4.5 \mathrm{~m}$ above ground. During flashes 93-12 and 93-14 occurring on 
September 3, the vertical electric field was measured simultaneously at 10 and $20 \mathrm{~m}$ from the strike point with the extension pole in place. The extension was then removed, and on September 7 an additional flash was triggered. This set of measurements is summarized in Table 2-1. The resulting data and their implications are discussed in Section 3.3.1.1.

Table 2-1.

Ev Measurements at 10 and $20 \mathrm{~m}$ to Determine the Effect of an Increase in Strike Rod Height

\begin{tabular}{cccc}
$\begin{array}{c}\text { Flash } \\
\text { ID }\end{array}$ & $\begin{array}{c}\text { Rod Height } \\
(\mathbf{m})\end{array}$ & $\begin{array}{c}\text { No. } \\
\text { Stokes }\end{array}$ & $\begin{array}{c}\text { Amp. Range } \\
(\mathbf{k A})\end{array}$ \\
\hline $93-12$ & 11 & 2 & $14-30$ \\
$93-14$ & 11 & 1 & 6 \\
\hline $93-15$ & 4.5 & 8 & $8-11$ \\
\hline
\end{tabular}

An alternative concept for eliminating a direct strike to a weapon, potentially attractive as a stop-gap measure to be employed by an initially arriving accident response team, is the installation of a dielectric tarpaulin or blanket of some type over the weapon. In principle, an adequately thick layer of dielectric over an object would inhibit the formation and free upward propagation of streamers from the weapon. This would ultimately result in the termination of any nearby approaching lightning leader on some other object providing a stronger, less inhibited upward streamer.

The use of dielectric shielding of electric field enhancing features in high voltage iystems is a well established practice. Furthermore, it has also been shown to be effective under lightning conditions during previous Sandia triggered lightning experiments. In those experiments, the use of a dielectric shell was employed to preclude attachment of the triggered lightning to the edge of a fixture that was being used to hold metallic specimens that were intentionally being exposed to the lightning currents $[2,3]$. With the shield in place, attachment invariably occurred at the centers of the exposed specimens and not on the shielded sharp edges of the fixture. The real question of interest in the present context was whether a practical thickness of pliable dielectric material can reliably hold up under the intense electrical stress due to the field from an approaching lightning leader.

Presuming a favorable resolution of that issue, the dielectric blanket concept is still attended by the practical down-side possibility of material defects due to either manufacturing origin or age and wear. Such defects would degrade the standoff capability of the dielectric and reduce the reliability of the protection afforded by the blanket. Nevertheless, it was decided to investigate the concept since this could be done in a preliminary fashion without interfering with any of the other higher priority aspects of the summer's program.

Accordingly, one attempt was made to test the idea during the storm that occurred on August 18. The end of one of the launch tube extension pieces was covered with 20 layers of 6 -mil $(0.15 \mathrm{~mm})$ thick polyethylene sheeting (dielectric strength of $18 \mathrm{kV} / \mathrm{mm})^{3}$. Of the list of otherwise suitable candidate materials available for use in the experiment, polyethylene had the highest dielectric strength, although a

3 CRC Handbook of Chemistry and Physics, 50th Edition, The Chemical Rubber Company, (1970), p. E-66. 
blanket incorporating one or more layers of polyimide (Kapton ${ }^{4}$ ) or equivalent would provide a dielectric strength of about a factor of ten higher for comparable thicknesses. The polyethylene sheets were secured to the top of the tube by ordinary duct tape. The total thickness of $3 \mathrm{~mm}$ provided a nominal breakdown voltage of about $55 \mathrm{kV}$. As indicated in Figure 2-11, the experiment consisted of mounting the covered extension on top of one of the launch tubes that did not contain a rocket. This placed the covered end of the extra tube at an overall height from the ground of about $4.5 \mathrm{~m}$, and set up a very severe test of the ability of the shield to function as desired. Should it succeed, the test would provide convincing evidence of the viability of the concept. Failure, on the other hand, would not necessarily rule it out, since the test was conservatively severe in several aspects. Failure in this first screening test would, however, dictate the use of much more refined experimentation to assess the utility of such an approach. The results are discussed in Section 3.3.1.2.

2.2.2.2 Protection from the Nearby Lightning Environment. An exposed and possibly physically damaged weapon lying on the ground can conceivably result from an aircraft crash or other transportation accident. In such an event, the weapon needs protection not only from the possibility of a direct lightning strike, but also from the intense electromagnetic environments caused by a nearby flash to ground. In principle, adequate protection can readily be provided by completely enclosing the exposed object in a metallic structure. In practice, however, this may be neither possible nor advisable in view of a number of other overriding considerations. The issue then becomes one of determining the relative effectiveness of various substitute, more practically achievable, approaches.

Electrical coupling is driven by both electric and magnetic fields. Furthermore, close to the base of a lightning strike to ground, the channel current spreads out more or less radially in the earth, and, in flowing through the resistance of the earth, develops potential differences along the surface in some way proportional to the radial distance between points of interest and the amplitude of the incident lightning current. The resulting transient voltages in the ground, which, for average return strokes, can readily reach tens of kilovolts or more close to the strike point, can drive currents through metallic objects lying on the surface.

Several conceptual approaches were tested that have been considered for providing some degree of protection to a weapon exposed to nearby lightning environments. The concepts included the use of a multiply grounded wire mesh cage over the weapon to reduce the $\mathrm{E}$ and $\mathrm{H}$ fields, the installation of a shallowly buried ground ring electrode (GRE) around the immediate vicinity of the weapon, and a combination of both techniques. The effectiveness of each measure was evaluated by comparing the measured $E_{z}, H_{a}$, and $V_{s}$ quantities measured at each of the three stations shown in Figure 2-2, located $10 \mathrm{~m}$ from the triggered lightning strike point. At Station 1, a wire mesh cage was positioned over the three sensors, as shown in Figure 2-12. The $1.2 \times 1.2 \times 2.4-\mathrm{m}$ cage was constructed of galvanized steel wire of about 2-mm diameter, with a mesh size of about $5 \times 10 \mathrm{~cm}$. The cage was variously grounded at either two or four of its corners, depending on the particular test, using $0.5-\mathrm{m}$ deep lengths of standard 16-mm diameter zinc-plated steel electrical ground rods. During flashes 93-02 and 93-03, Station 3 had a GRE installed around it. The GRE consisted of a circular ring of 3-m diameter of \#2 gauge wire buried at a depth of $0.15 \mathrm{~m}$. The ring was further grounded at 4 equi-spaced positions around its circumference using the same type of ground rods as were described above. During flashes 93-08 and 93-09, a steel wire cage identical to the one installed at Station 1 was installed at Station 3 in addition to the GRE. During all of the flashes, Station 2 was left unmodified, and the $\mathrm{E}_{\mathrm{z}}, \mathrm{H}_{\mathrm{a}}$, and $\mathrm{V}_{\mathrm{s}}$ data from that station served as the standard for comparing the effectiveness of the cage and GRE in reducing the lightning environment. Within the limits of the reasonable assumptions that the earth properties can be considered uniform over the 10-m

4 Trade name of the DuPont Company's polyimide insulating material, DuPont Catalogue E-86239 (5M-8186), 1985 


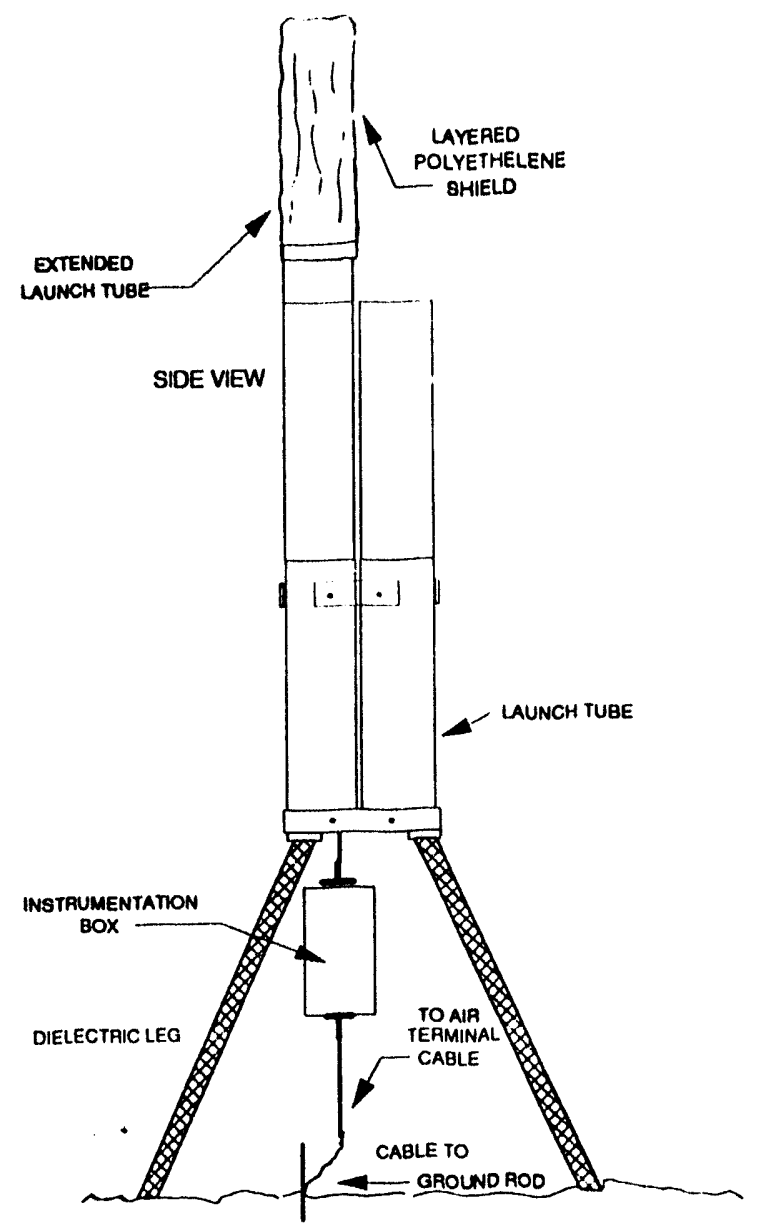

Figure 2-11 Dielectric shield experiment configuration

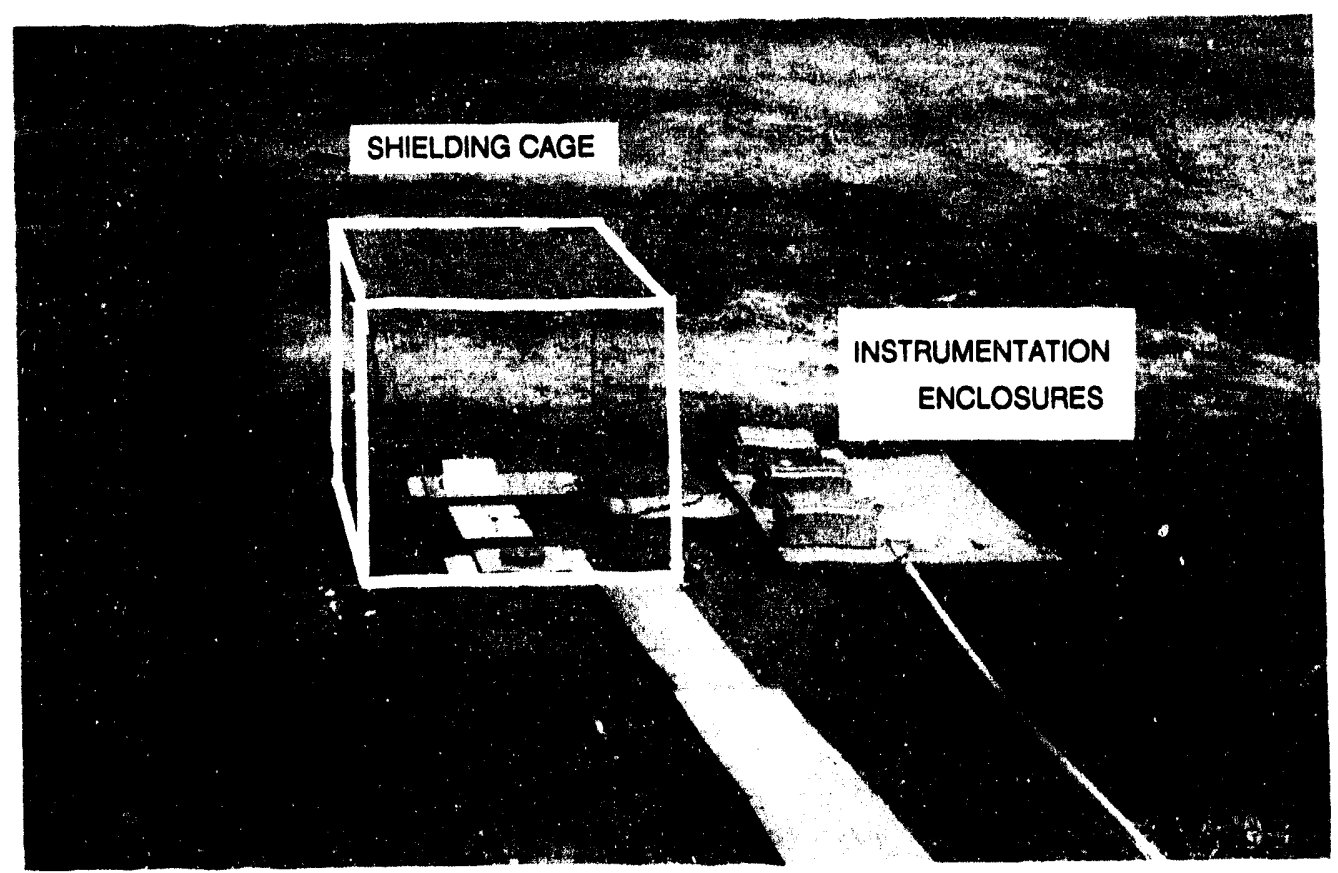

Figure 2-12 Grounded partial Faraday cage made from steol wire fencing material installed over a test station sensor complement (cage has been outlined in white to enhance visibility) 
radius involved in these tests and that the lightning channel is essentially vertical within the last few hundred meters near the ground, the results of these tests represent direct comparisons conducted under identical conditions.

The station configuration variations implemented during different flashes are summarized in Table 2-2, and the test results are discussed in Section 3.3.2.

Table 2-2.

TLPS Test Station Configurations

\begin{tabular}{cccc} 
Flash & Station 1 & Station 2 & Station 3 \\
\hline $\begin{array}{c}93-02 \\
\text { and } \\
93-03\end{array}$ & $\begin{array}{c}\text { Cage; } \\
4 \text { rods, } 0.5 \text { m deep }\end{array}$ & None & $\begin{array}{c}\text { Circular GRE; } \\
4 \text { rods, 0.5 m deep }\end{array}$ \\
\hline $\begin{array}{c}93-08 \\
\text { and } \\
93-09\end{array}$ & $\begin{array}{c}\text { Cage; } \\
2 \text { rods }\end{array}$ & None & $\begin{array}{c}\text { Cage plus GRE; } \\
2 \text { rods on cage }\end{array}$ \\
\hline
\end{tabular}

\subsection{Data}

\subsection{Ground Conductivity}

The test bed for these experiments was located in an area of approximately $20,000 \mathrm{~m}^{2}$ of ground that had been cleared of typical Alabama pine woods vegetation within forty-five days of the start of the testing. The general site layout is shown in Figure 3-1. The soil composition is heavy red clay. Throughout the active test period, there was very little regrowth of vegetation within the cleared and tilled area indicated in the figure. Ground resistivity measurements were conducted nearly daily over a period of fifty days from the commencement of the test readiness period, which occurred in late July. Readings were taken along the radials through each experiment station (1 through 5) defined in Figures 2-2 and 2-3, and in both the north/south and east/west directions at the rocket launcher site.

The measurements were made using a Biddle Model 250241 Megger instrument and the four probe technique illustrated in Figure 3-2, with a probe separation distance a of $5 \mathrm{~m}$ and a corresponding depth $\mathrm{b}$ of $0.25 \mathrm{~m}$, in accordance with the specified procedures in the Megger's operation manual. The probes were made of the same zinc-plated steel ground rod material as was used for the earth step potential measurements discussed in Section 2.1.3.3. In order to make the measurement procedure as repeatable from day to day as possible, four measurement probes were permanently installed at each site for the duration of the fielding period. All that was therefore required to perform the measurement each morning was to connect the Megger drive and pick-up cables to their respective probes at each station and null out the deviation of the indicator needle.

Conductivities are inferred from the measured data according to the relationship specified by the instrument manufacturer as follows:

$$
\sigma=(2 \pi \mathrm{aR})^{-1}
$$


STANDING WOODS

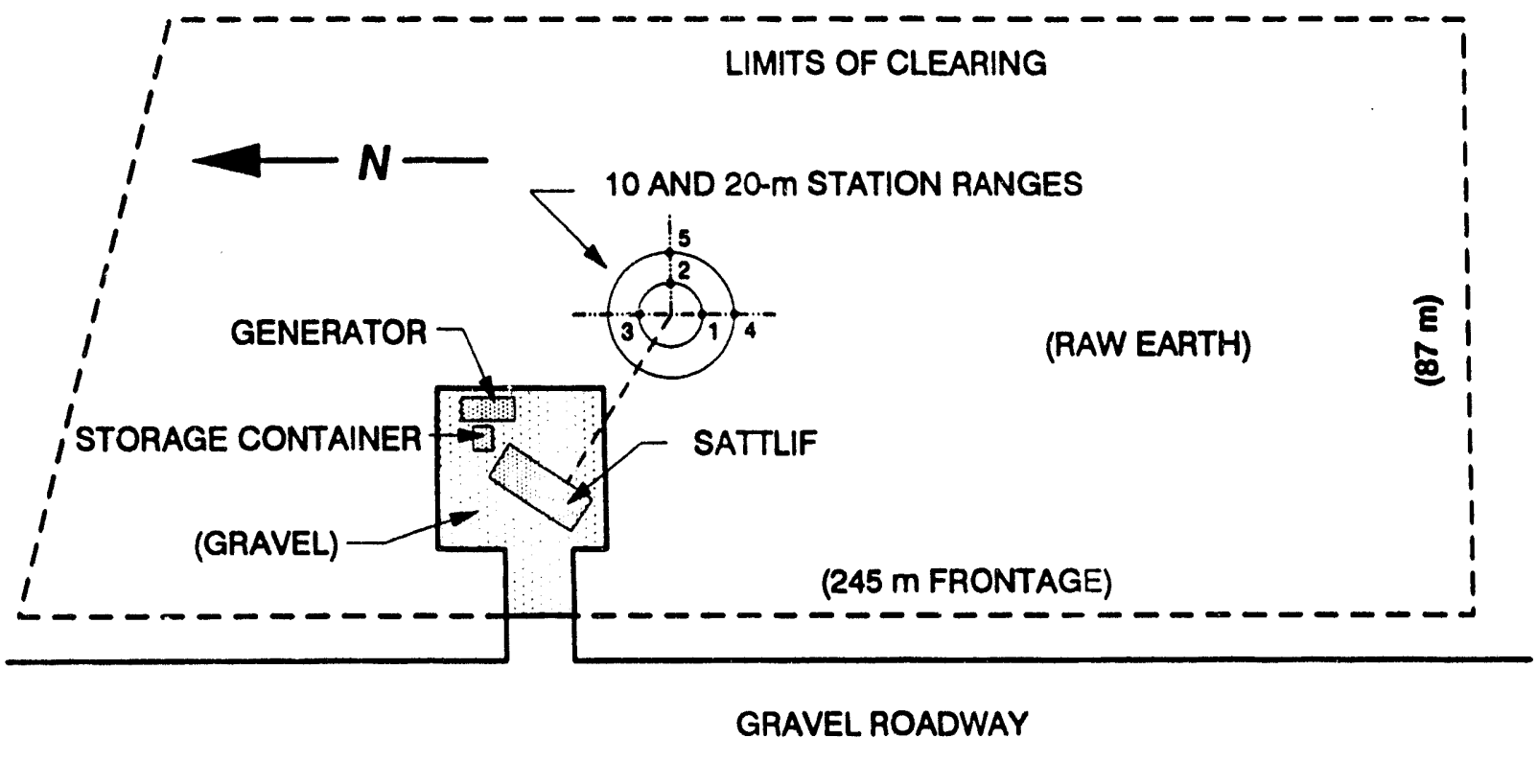

Figure 3-1 Overview of the triggered lightning test site at Ft. McClellan, Alabama

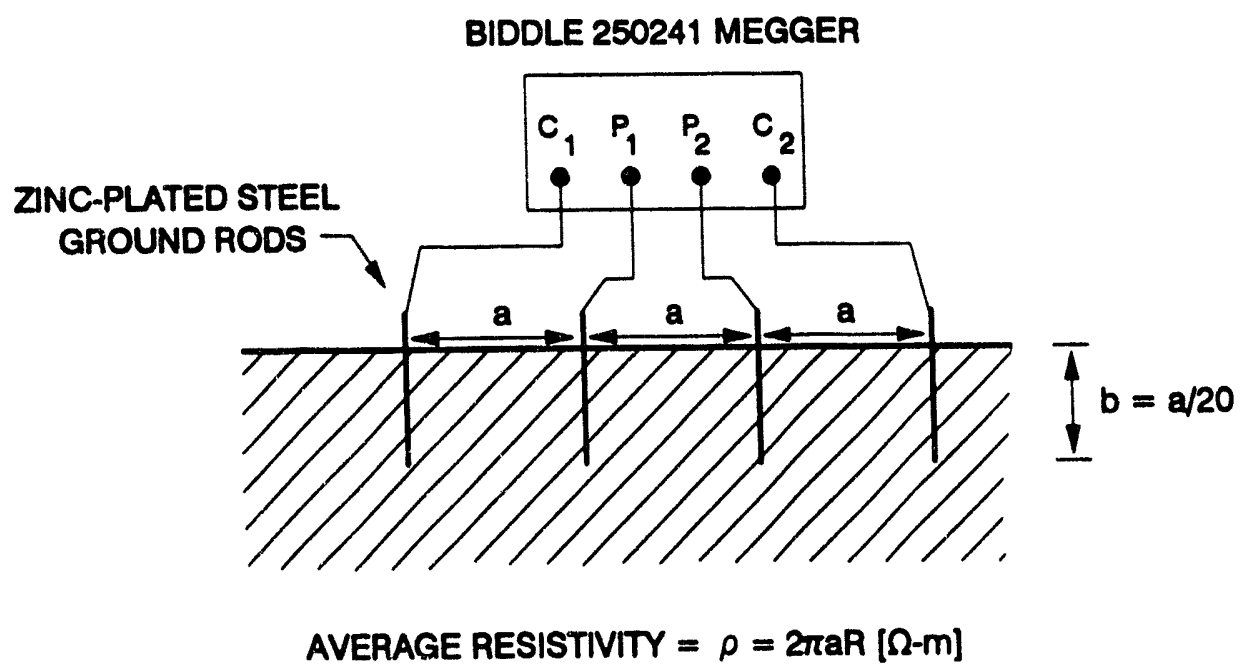

Figure 3-2 Diagram of the measurement technique employed in determining the earth conductivities presented in Figure 3-3 
where $\mathrm{a}$ is the probe separation distance and $\mathrm{R}$ is the resistance value indicated by the instrument when the needle is properly nulled. A derivation of (2-4) is given in Appendix B. Of significance is the fact that conductivities derived from the Megger measurements represent a complicated average of earth conductivity over a depth approximately comparable to the probe separation distance used in the Megger measurements, which in the present case was $5 \mathrm{~m}$. Referring to Figure 2-9, it is seen that the conductivity at a depth corresponding to radius $r$ from the strike point plays a dominant role in determining the step voltage developed over an increment of $\Delta r$ at the surface. Therefore, step potential predictions based on conductivities derived from the Megger measurements conducted with a 5-m probe separation are subject to uncertainties that increase with radial distance beyond $5 \mathrm{~m}$ from the attachment point. This factor is addressed further in Section 3.2.3, in which the measured step voltage data are discussed.

The conductivities derived according to (2-4) from the data at each of the stations are plotted in Figure 3-3. The installation of the GRE at Station 3 took place on the eighth day of the record period. As might be expected, since the Megger drive and sensing probes straddle the 3-m GRE radius, the indicated conductivity at that station exhibits a distinct discontinuity on the day the GRE was installed. Furthermore, a period of very heavy daily rain showers occurred for several days starting on day 18, from which point on significant discontinuities are apparent in the data from Station 3. The erratic behavior of the indicated conductivity at Station 3 after that time appears to be related to changes in contact resistance between the GRE and the surrounding earth in response to changing moisture content in the soil. While an interesting observation in itself, this behavior is not directly indicative of the earth conductivity at that station. The data from that station were, therefore, not included in the determination of the overall average test site conductivity.

The mean value of conductivity, based on the weighted average of the readings from all sites except Station 3, is $1.8 \times 10^{-3} \mathrm{~S} / \mathrm{m}$, corresponding to a resistivity of $556 \Omega-\mathrm{m}$. The average value of conductivity obtained during 1991 at a site several hundred meters away at which the earth had not been disturbed was $2.6 \times 10^{-3} \mathrm{~S} / \mathrm{m}$.

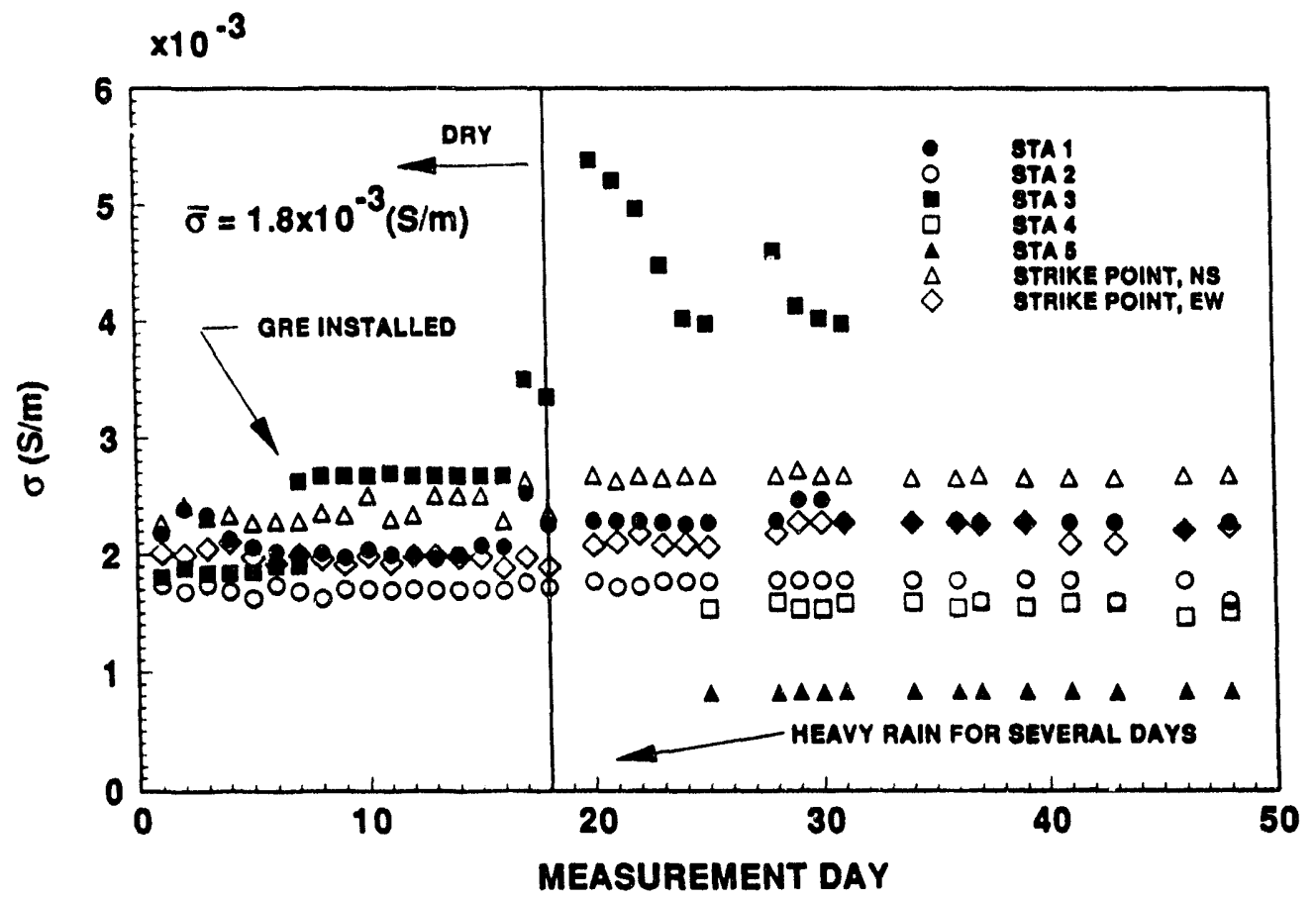

Figure 3-3 Earth conductivities derived from dally measurements conducted as shown in Figure 3-2 


\subsection{Close-in Lightning Environments}

\subsubsection{Magnetic Fields}

The general expression for the azimuthal magnetic field at the ground a radial distance $D$ from the base of an idealized straight, vertical lightning channel carrying current $i(z, t-R / c)$ is $[6]$

$$
H(D, t)=\frac{1}{2 \pi}\left[\int_{0}^{L} \frac{\sin \theta}{R^{2}} i\left(z, t-\frac{R}{c}\right) d z+\int_{0}^{L} \frac{\sin \theta}{c R} \frac{\partial}{\partial t} i\left(z, t-\frac{R}{c}\right) d z\right]
$$

Here $\mathrm{c}$ is the velocity of light, and the remaining geometrical parameters are as defined in Figure 3-4. The ground plane is assumed to be perfectly conducting. The first and second terms in (3-1) are the magnetic induction and the magnetic radiation fields, respectively.

Over some range of $\mathrm{D}$, it can be expected that (3-1) reduces approximately to the Ampere's law relationship

$$
H(D, t)=\frac{i(t)}{2 \pi D}
$$

and the range over which (3-2) holds is of practical interest for purposes of specifying the magnetic field environment and analyzing the electromagnetic response of an object that is exposed to nearby lightning.

When $D$ is small, $\sin \theta$ decreases rapidly with $z$, and current elements at heights up the channel beyond some point $z_{c}$ contribute negligibly to the integrals in (3-1). Furthermore, over the relatively short contributing section of channel below $\mathrm{z}_{\mathrm{c}}$, the current may be taken to be uniform and retardation effects can be neglected. Detailed numerical evaluation of (3-1) has been carried out for a return-stroke current incorporating typical parameter values [7]. The results show that at $10 \mathrm{~m}$ from the base of the channel the

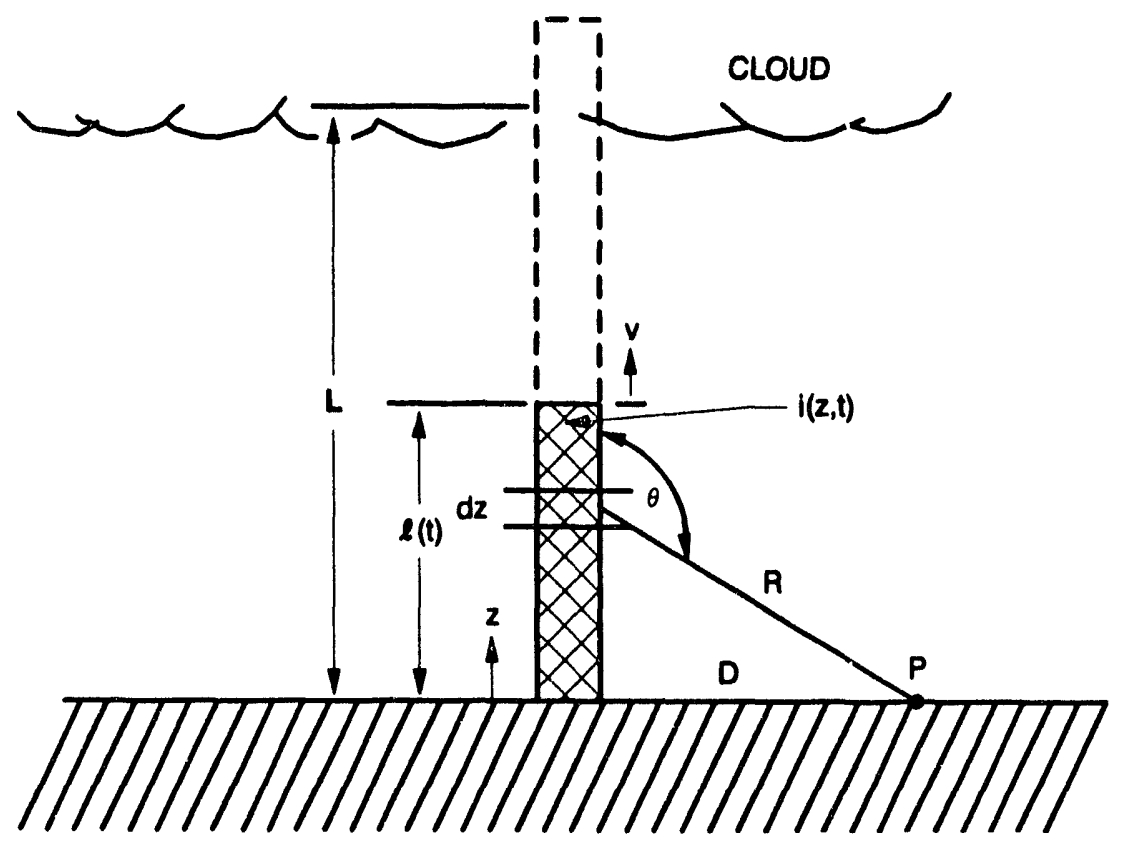

Figure 3-4 Lightning channel geometry leading to the model for the azimuthal magnetic field at the ground given in equation (3-1) 
ratio of the peak of the radiation field to that of the induction field is about 0.2 . Furthermore, the timing of the two field components is such that the radiation field affects the peak of the total field by less than 5 percent. The same analysis also demonstrated that, at a distance of $10 \mathrm{~m}$ from the channel, contributions from the bottom $20 \mathrm{~m}$ of channel current account for about 90 percent of the peak amplitude of the field.

Ignoring the radiation term in (3-1) on the basis of the above analysis, the same results can be obtained more directly by examining the induction field alone.

$$
\begin{aligned}
H(D, t) & =\frac{i(t)}{2 \pi} \int_{0}^{z_{c}} \frac{\sin \theta}{R^{2}} d z=\frac{i(t)}{2 \pi} \int_{0}^{z_{c}} \frac{D}{\left(D^{2}+z^{2}\right)^{3 / 2}} d z \\
& =\frac{i(t)}{2 \pi D}\left[\frac{\left(z_{c} / D\right)}{\sqrt{1+\left(z_{c} / D\right)^{2}}}\right]=\frac{i(t)}{2 \pi D} F
\end{aligned}
$$

Values of $F$ as a function of $z_{c} / D$ are given in Table 3-1, from which it can be seen that for $z_{c} / D=2$, the magnetic field given by (3-3) is within about 10 percent of that given by Ampere's law. This result closely agrees with the outcome of the detailed numerical analysis quoted above. For $z_{c} / D=5$, agreement with Ampere's law is to within 2 percent. However, as D increases, the contributing length of channel needed to maintain the desired $z_{c} / D$ ratio also increases. For example, at $D=20 \mathrm{~m}, 100 \mathrm{~m}$ of channel is required to maintain the $z_{c} / D$ ratio of 5 . For $z_{c}=100 \mathrm{~m}$, the retardation time from that point to $D$ is $R / c=$ $3 \times 10^{-7} \mathrm{~s}$, which is long enough to be important at the higher frequencies of interest that contribute to the rise time of the field. Furthermore, the validity of the assumption of a uniform current between $z_{c}$ and the ground also becomes more tenuous. So, once again, the question becomes one of determining the range out to which (3-3) can be used to estimate the magnitude of $\mathrm{H}$ with adequate accuracy. Also of interest is the degree to which the field predicted by (3-3) might be affected by any local inhomogeneities in earth properties.

Table 3-1.

Values of Factor $F$, Equation (3-3), for Selected Values of $z_{c} / D$

\begin{tabular}{cc}
$\mathbf{z}_{\mathbf{c}} / \mathbf{D}$ & $\mathbf{F}$ \\
\hline 0.5 & 0.45 \\
1.0 & 0.71 \\
2.0 & 0.89 \\
5.0 & 0.98 \\
10.0 & 1.0 \\
\hline
\end{tabular}

Plotted in Figure 3-5 are the peak values of $\mathrm{H}_{\mathrm{a}}$ recorded at the unperturbed stations ${ }^{5}$ located at radial distances of 10.7 and 20.7 meters from the strike point, respectively. The data points are plotted as a function of the peak amplitudes of their associated return-stroke currents, and linear regression lines are displayed for each data set. In addition, the curves corresponding to (3-2) as applied to each of the two

5 That is, stations at which the sensor sites were unperturbed by the presence of any of the TLPS modifications discussed in Section 2.2.2.2. 


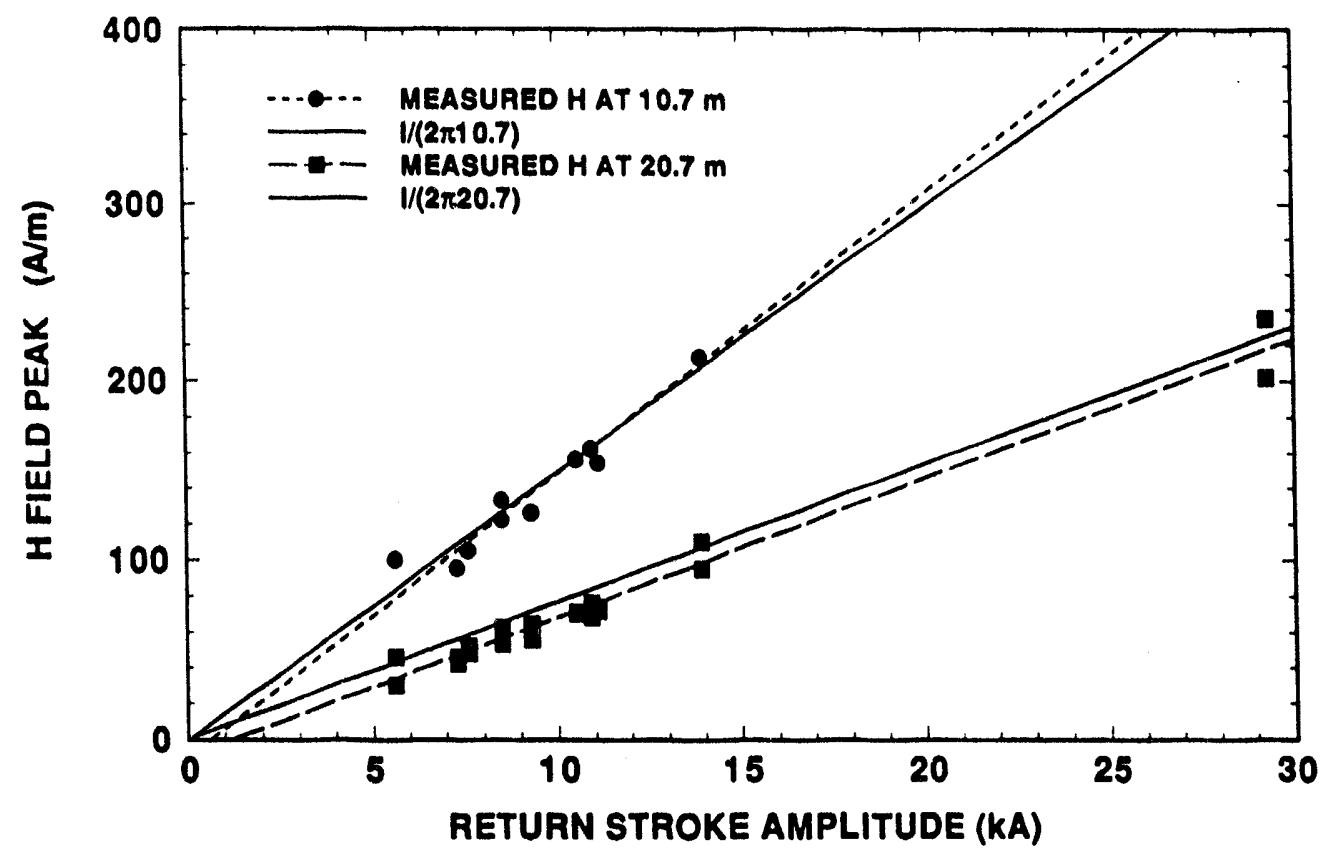

Figure 3-5 Comparison of peak magnetic fields measured at unperturbed stations at $10 \mathrm{~m}$ and $20 \mathrm{~m}$ from the strike point with Ampere's Law

radial distances are plotted for comparison with their respective experimental data sets. There is close agreement between the theoretical and experimental values at both distances, implying that (3-2) holds well out to 20 meters, under the particular conditions of the present experiments.

The correspondence of the magnetic field to the incident current is further illustrated in Figure 3-6, in which the measured field data traces recorded at Stations 4 and $5(D=20.7 \mathrm{~m})$ are given for both strokes of flash 93-12. Overlaid on the same plots are the waveforms of the fields estimated by applying (3-2) to the incident currents recorded at the base of the channel for the same two strokes. In each case, the peaks of the fields estimated from the incident currents and the magnetic field from Station 5 have been normalized to the peak value of the field at Station 4. The data are presented on two time scales for each stroke. The correspondence is so close on stroke 1 that it is very difficult to distinguish the separate traces. On stroke 2, however, while there is excellent agreement at the early times and at the peaks, there are growing discrepancies with increasing time. These discrepancies are thought to be caused by leakage currents through the shield of the cable that connects the MGL-4 sensor with the instrumentation box that houses the integrator and FOL transmitter. Because of the integration process, only a small leakage current is necessary to produce the type of contamination suspected in this example. As can be noted from Figure 3-6c, the field at Station 4 decays more slowly with time than does the incident current; while at Station 5, it apparently drops off faster than the current. If leakage current through the shields of the connecting cables is the explanation for the discrepancies, it follows that the direction of flow of those currents at the two stations was opposite. This might well be possible if the currents were driven by vertical electric field coupling and the small differences in heights between the sensor and instrumentation boxes at each station happened to have been reversed. 

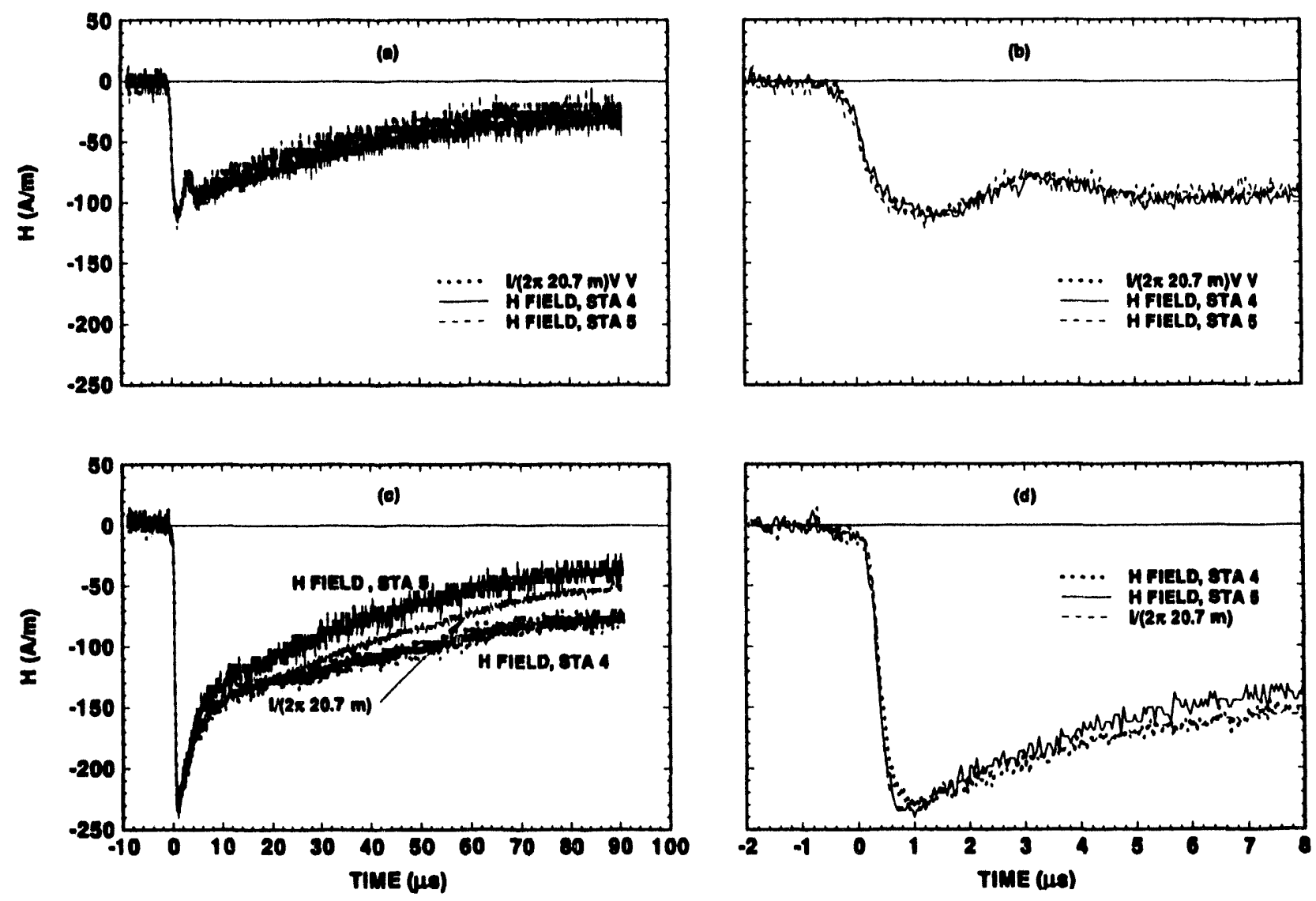

Figure 3-6 Comparison of the waveforms of the magnetic fields recorded at $20.7 \mathrm{~m}$ from the strike point on both strokes of flash 93-12 with those of the associated recorded incident return-stroke currents; (a) full records from stroke 1, (b) same data as (a) on an expanded scale, (c) full records from stroke 2, and (d) same data as (c) on an expanded time scale

Additional evidence of even stronger contamination of the late time portion of some of the magnetic field data acquired towards the end of the fielding period has lead to the conclusion that the plywood sheets on which the instrumentation boxes were sitting had become increasingly saturated with moisture during the course of the summer. This is thought to have resulted in the development of a path for ground loop current flow on the shield of the connecting cable as the dielectric isolation provided by the plywood decreased. Increasing the electrical isolation of the instrumentation boxes from the ground has been identified as an area for improvement during future efforts of this type.

Waveshape agreement to the same degree as that indicated in Figure 3-6 has been noted in all of the data for which similar overlays have been plotted, although this comparison has not been carried out for the entire set of data. Even in those cases in which the late time portion of the magnetic field is obviously contaminated, their wavefronts and peaks exhibit the same kind of agreement with their counterpart stroke currents as is apparent in Figure 3-6.

The evidence typified in Figures 3-5 and 3-6 clearly justifies the usual practice of using the relationship of (3-2) to define magnetic fields from nearby lightning channels in specifying weapon environments. Although the data available from the present study explicitly cover return-stroke incident current amplitudes only up to $30 \mathrm{kA}$, there appears to be no indication that use of (3-2) at higher current amplitudes would result in any significant error of practical concern. 


\subsubsection{Vertical Electric Fields}

Triggered lightning of the type initiated during the present experiments is different from naturally initiated lightning in that the component strokes in triggered lightning are almost always preceded by dart leaders. This is so apparently because the channel prepared by upward streamers from the ascending end of the triggering wire and the initial continuous current (ICC) that flows for hundreds of milliseconds following wire vaporization leave a channel that remains marginally hotter than the surrounding atmosphere. This hotter channel represents the preferential path for a downward propagating leader, and, unless the interval is so long that the residual channel temperature falls below some critical value, the leader progresses continuously, readily re-charging the channel as it travels earthward. In effect, the first stroke of a flash triggered in this way involves the same initiating physics as do subsequent strokes in naturally occurring flashes, which are also generally preceded by dart leaders [e.g., Chapter 12, Ref. 9]. Figure 3-7 illustrates the main features of the type of flash current generally produced by the technique employed during the present testing, and in Figures 3-8a and b are plotted the incident currents recorded during flashes 93-02 and 93-03.

Figure 3-9 is a plot of a typical vertical electric field change recorded at $10 \mathrm{~m}$ from the base of a stroke that was preceded by a dart leader. This particular example, recorded at Station 2, occurred on stroke 1 of flash 93- 02. The $\mathrm{V}$ shape of the overall field change is characteristic of electric field changes out to well beyond $500-\mathrm{m}[4,8,10]$. The wavefront portion preceding the bottom of the $\mathrm{V}$ is associated with the approaching dart leader. The rate of change and peak amplitude of the leader portion of the field change are related to the leader's propagation velocity and linear charge density, respectively. The rapid change following the peak corresponds to the neutralization of the leader charge as the return-stroke wavefront propagates back up the channel from the ground. ${ }^{6}$ In a manner analogous to that described in Section 3.2.1 in connection with magnetic fields, the electric field at the ground close to the base of a lightning channel is predominantly influenced by charges and currents on portions of the leader/return-stroke channel up to only a few tens of meters of height [4].

(NOT TO SCALE)

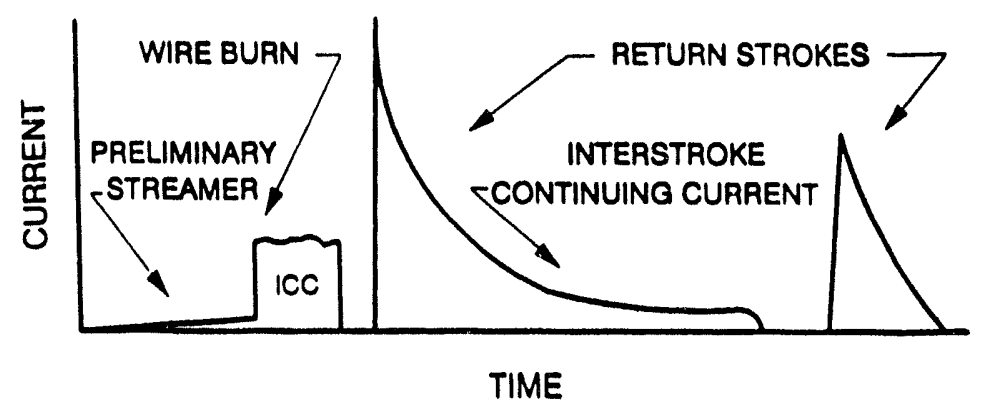

Figure 3-7 Schematic of the typical flash current time history produced by the lightning triggering technique employed during the present experiments

6 More precisely, the return-stroke wavefront originates and propagates upward from the junction between an upward streamer from the strike point and the tip of the downward traveling leader. There is some evidence [e.g., 2 and 11] that, for dart-leader initiated strokes, this junction occurs at from 1 to 10 meters above the source of the streamer. The similar junction point between a stepped leader and its complementary upwardrising streamer occurs much higher above the streamer source, typically at heights of 50 to $100 \mathrm{~m}$, depending on structure height [e.g., Chapter 6, Ref. 9]. 

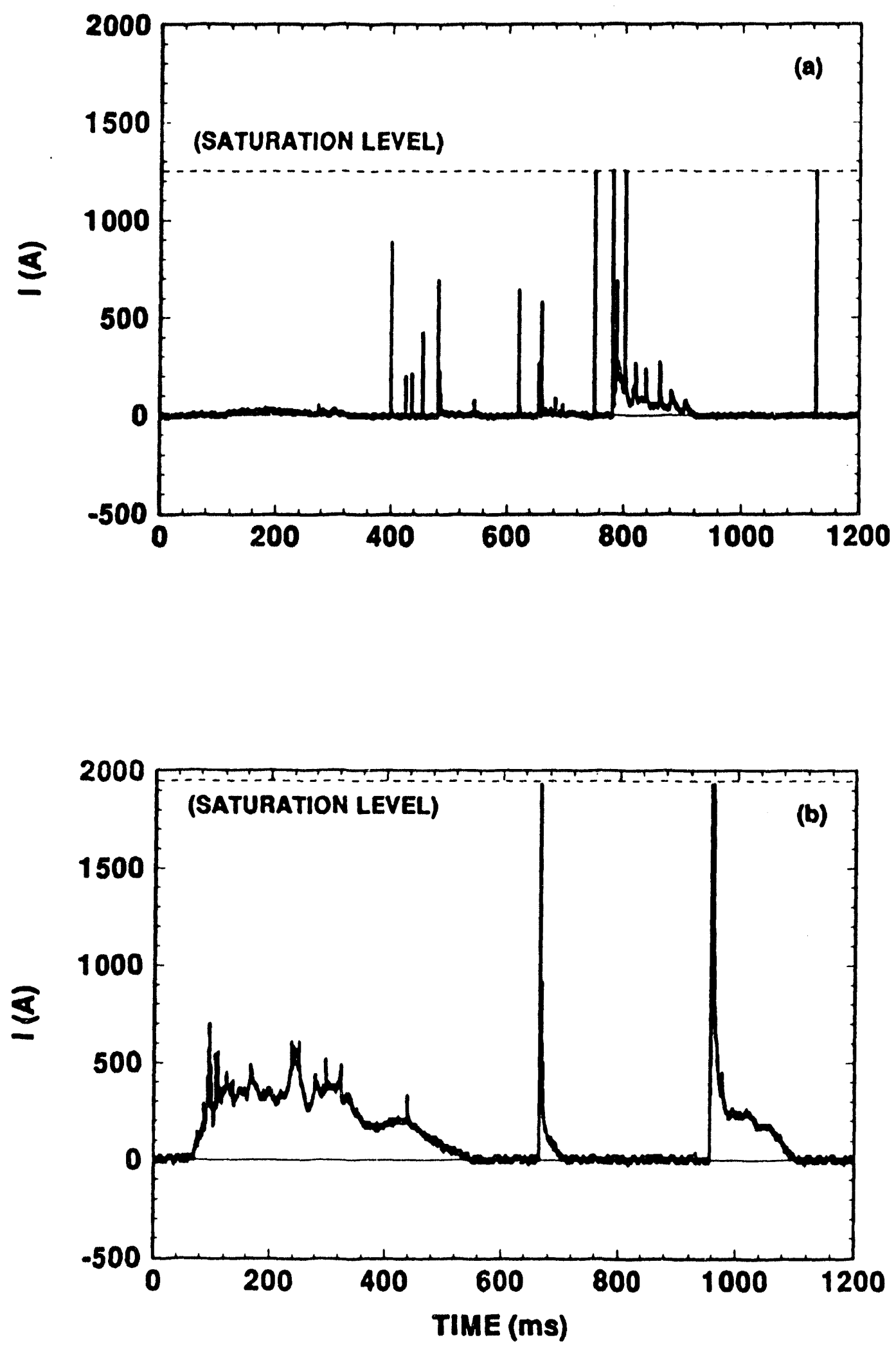

Figure 3-8 Full flash current records obtained during flashes (a) 93-02 and (b) 93-03; the chosen recording sensitivities are such that saturation of the returnstroke components occurred as shown in each case 

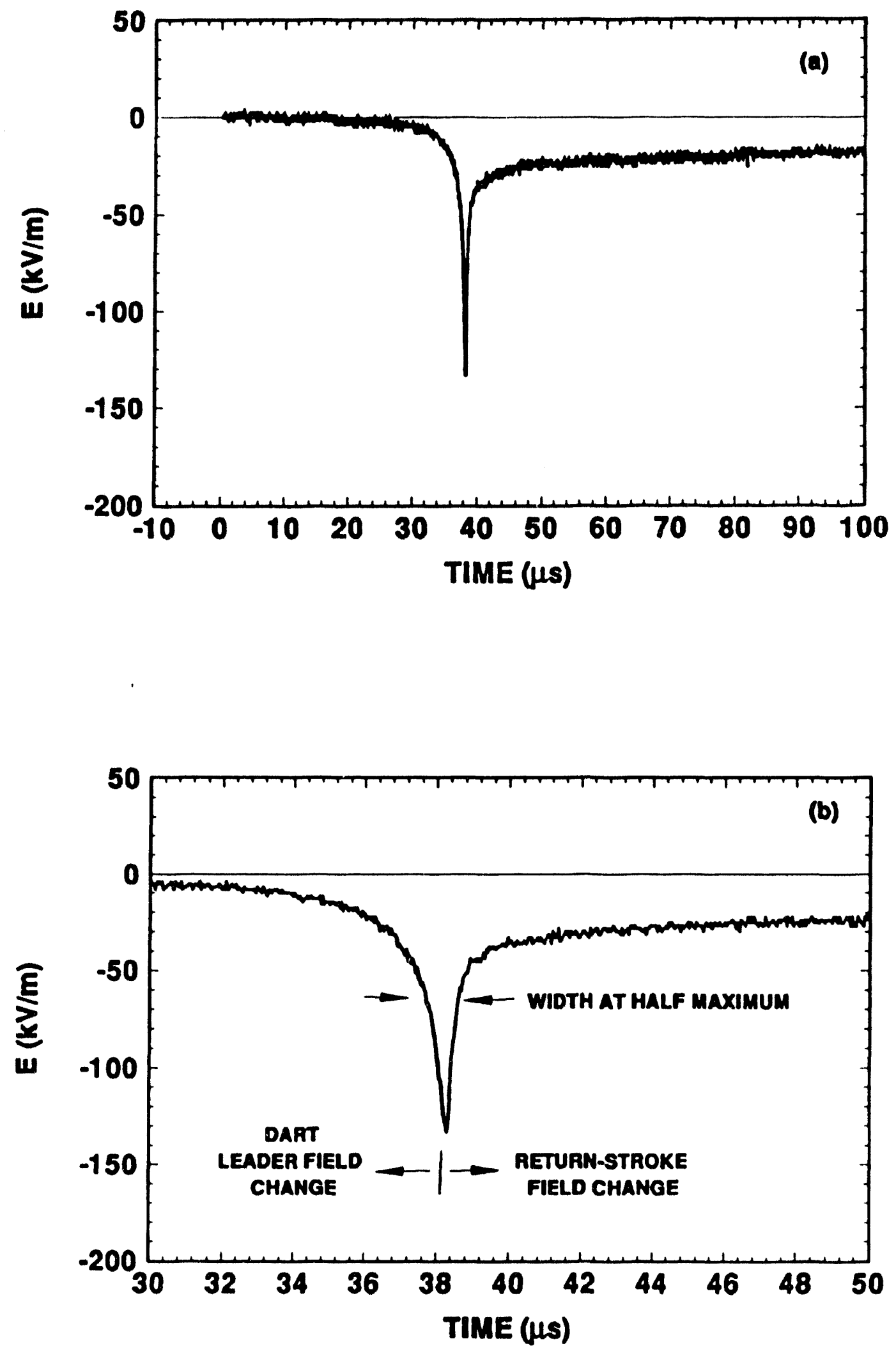

Figure 3-9 Typical vertical electric field of a return stroke preceded by a dart leader as measured at Station 2 (9.3 $\mathrm{m}$ ) on flash 93-02; (a) full record, and (b) same data on an expanded time scale 
On relatively infrequent occasions, and in particular on strokes following long interstroke intervals, the temperature of the existing channel evidently decays to a degree that, below some point on the leader's path to earth, there no longer exists a preferential path. In such a case, the dart leader transitions into a stepping mode similar to that of a stepped leader preceding the first stroke in a naturally initiated flash. This hybrid type of leader is called a dart-stepped leader.

On two occasions during the present experiments, measurements of electric field changes from strokes preceded by dart-stepped leaders were obtained. These are shown in Figures 3-10a and b. The record in Figure 3-10a is from the final stroke in flash 93-02, which, as can be noted from Figure 3-8a, follows its preceding stroke by an interval of about $345 \mathrm{~ms}$. The median interstroke interval for triggered flashes in Florida and Alabama is $48 \mathrm{~ms} \mathrm{[12];} \mathrm{so} 345 \mathrm{~ms}$ represents a very long interval. Furthermore, at least over the last 5 meters or so of the channel visible in our various video and film records, this stroke followed a distinctly different path to its attachment point on the top of the LTA than did the first six strokes of the same flash. Particularly interesting features of this record include the presence of the series of steps on the rising portion of the wavefront and the slower overall rise time of the leader portion of the field change in comparison with that shown in Figure 3-9. The average step interval is about $6.3 \mu \mathrm{s}$. This interval is quite consistent with data presented by Krider et al. for dart-stepped leaders in naturally initiated flashes in Florida and Arizona [13], but it is somewhat shorter than the mean interval of $11.9 \mu \mathrm{s}$ reported by Schonland for 6 strokes occurring in South Africa [14]. The latter data, however, correspond to sections of channel higher above ground than do either the present measurements or those of Krider et al. Available data on stepped leaders preceding first strokes in naturally initiated lightning suggest that stepping distances and intervals decrease near the ground [e.g., Chapter 5 of Reference 9].

The companion record in Figure 3-10b corresponds to the second and final stroke in flash 93-03. The interstroke interval preceding this stroke was about $290 \mathrm{~ms}$ (Figure 3-8b). As was the case with the last stroke of flash 93-02, this one also terminated on the LTA via a different channel segment than did the other stroke in flash 93-03. The mean stepping interval apparent in the record is $5.4 \mu \mathrm{s}$.

In F:gure 3-11, peak return-stroke current amplitude is plotted against dart leader field changes observed at a radial distance of $9.3 \mathrm{~m}$ from the base of the channel. The regression line has a correlation coefficient of 0.85 . The observed linearity is presumably related to the fact that the peak amplitude of a return-stroke current is proportional to the charge per unit length deposited by the leader on the last hundred meters or so of its channel. Assuming a uniform linear charge density, the proportionality constant is the return-stroke wavefront propagation velocity. In Figure 3-11 there appear two different data points at each of the return-stroke current levels for which data are presented. These points correspond to the two separate field change measurements made at Stations 2 and 3, respectively, on the same strokes. ${ }^{7}$ At return-stroke amplitudes of 8.6 and $10.5 \mathrm{kA}$, the two data points overlap. In all other cases, those associated with Station 2 (the solid dots) consistently fall at lower values of leader fields than do the points from Station 3, by an average of 5.6 percent. There is, at the moment, no ready explanation for this systematic pattern in terms of instrumentation effects, since the sensors and associated components at both stations were identically constructed, calibrated, and deployed. Unlike the situation with the magnetic field measurements, cable leakage currents would not substantially contaminate the electric field records, since no integration takes place. It is noted that during flashes 93-02 and 93-03, Station 3 was configured with the GRE installed. Whether its presence can account for the systematic spread in the measurements at the two stations is undetermined at this time.

7 Recall that during flashes 93-02 and 93-03, a wire mesh cage was installed over the sensors at Station 1 . Because of this perturbation, the Station 1 data are not included in the present discussion. The effect of the cage on the electric field at that station is discussed in Section 3.3.2.1. 

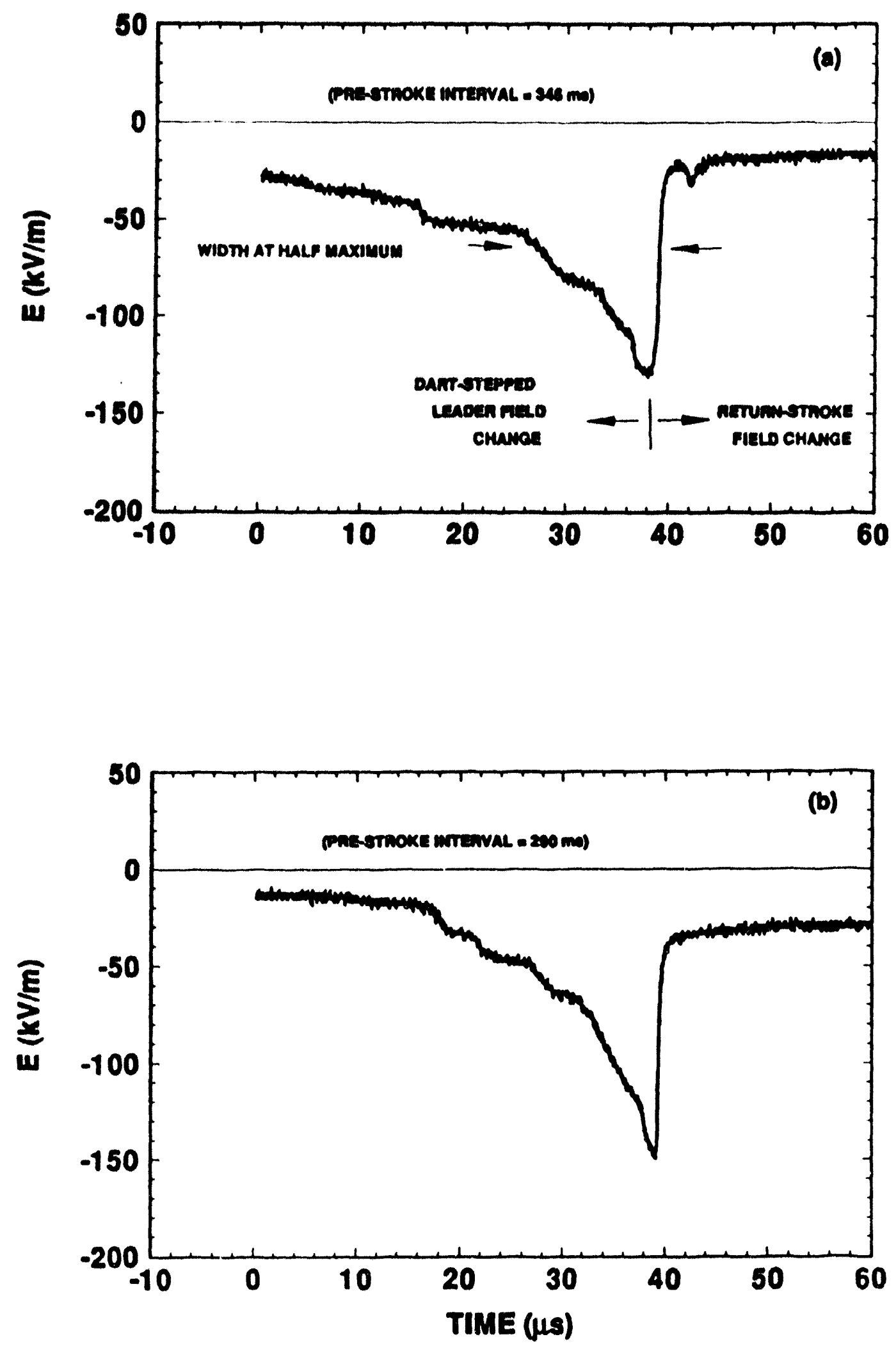

Flgure 3-10 Two examples of electrle filds produced at $8.3 \mathrm{~m}$ from the strike point by return strokes preceded by dart-stopped leaders; (a) last of seven strokes In fiash 93-02, and (b) last of the two strokes $1 /$; flash 93-03 


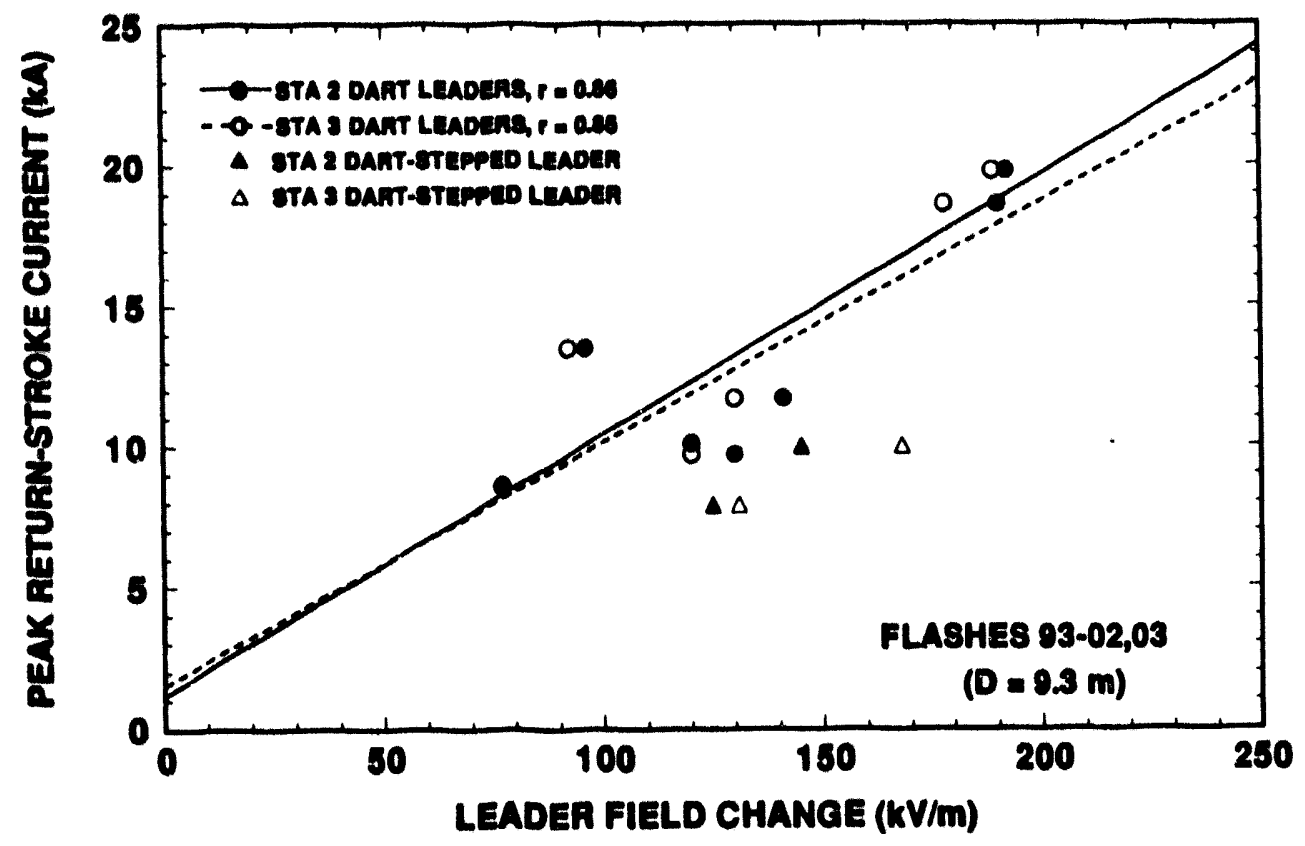

\section{Flgure 3-11 The relationshlp between peak retum-stroke current and amplitude of loader field change measured at $9.3 \mathrm{~m}$ from the strike point}

Also included in Figure 3-11 are the data points that correspond to the strokes that were preceded by dart-stepped leaders. These points were not included in the regression line computations, but are simply shown for comparison with the points that correspond to strokes initiated by dart leaders.

Similar plots are given in Figure 3-12, where peak return-stroke current is plotted against the maximum dart leader field changes recorded at unperturbed stations during all flashes for which the strike rod height was $4.5 \mathrm{~m}$. Data from both the 9.3 and $19.3-\mathrm{m}$ stations are shown.

The maximum field change associated with the return-stroke portions of the waveforms are plotted against the peak return-stroke current in Figure 3-13 for both recording distances from the channel. The definition of what constitutes the return-stroke portion of the field change is somewhat open to interpretation. For present purposes, it was simply defined to be the value of the field at the beginning of the return stroke minus the value at the $100-\mu$ s point in the plotted records. The origin of the relationship of the data at the two distances is obscure and will require detailed modeling to understand.

\subsubsection{Radial Earth Step Potentials}

The technique used to measure earth step potentials at different locations around the lightning strike point is discussed in Section 2.1.3.3. The major results are summarized in Figure 3-14, in which the peak voltages recorded at the unperturbed 10 - and $20-\mathrm{m}$ stations are plotted against return-stroke current. As is evident from the figure, the voltages developed over the $0.5-\mathrm{m}$ probe separation distance at each station are highly linear with respect to the driving currents that produced them. This implies that if volumetric breakdown took place within the soil, it must have occurred only locally near the injection point. At $10 \mathrm{~m}$, the maximum measured step voltage was approximately $7 \mathrm{kV}$, corresponding to an electric field within the earth of about $14 \mathrm{kV} / \mathrm{m}$, about a factor of 100 below the ionization threshold that would be expected for that type of soil [15]; so no volumetric breakdown would have occurred at that range. 


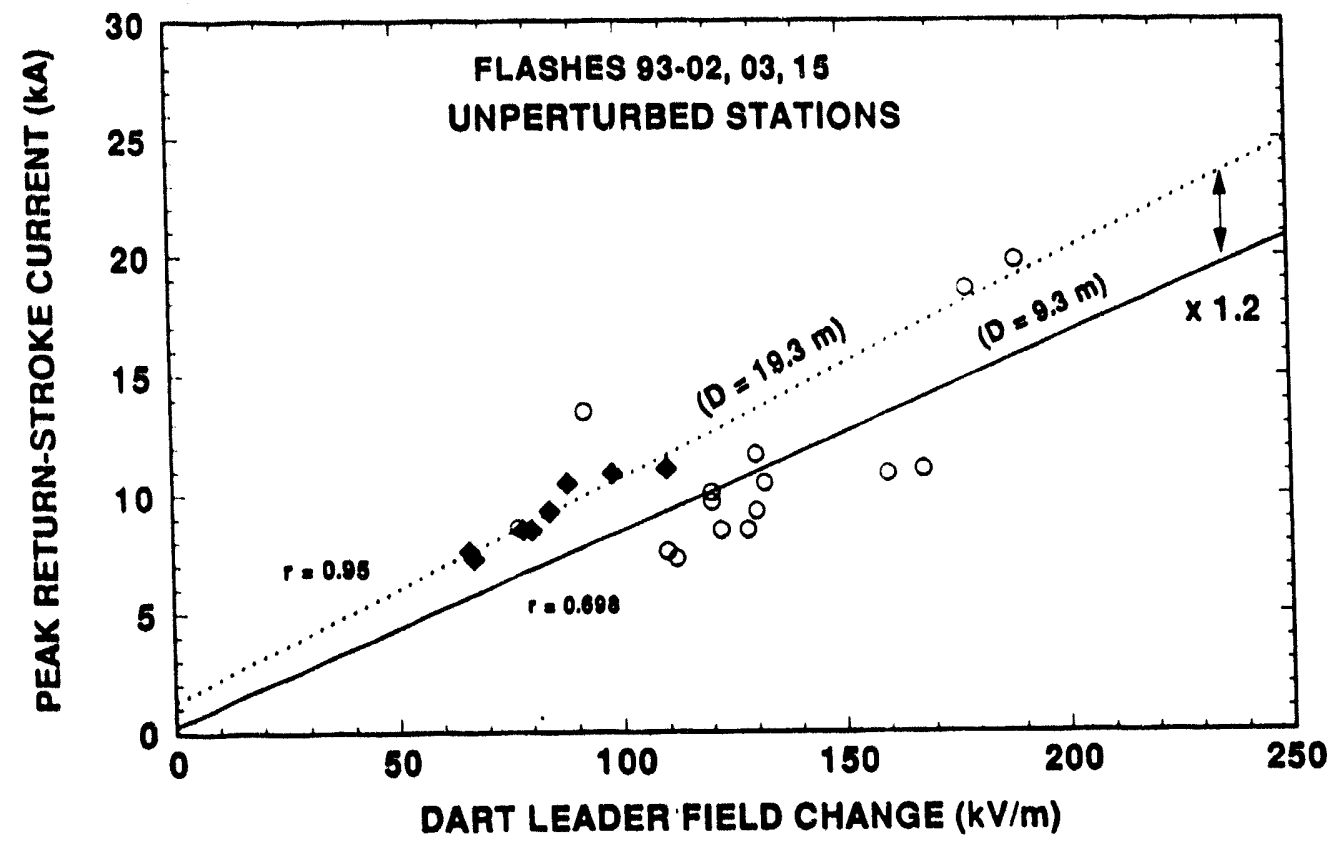

Figure 3-12 The relationships between peak return-stroke current and vertical electric field changes produced by preceding dart leaders at distances from the strike point of $9.3 \mathrm{~m}$ and $19.3 \mathrm{~m}$

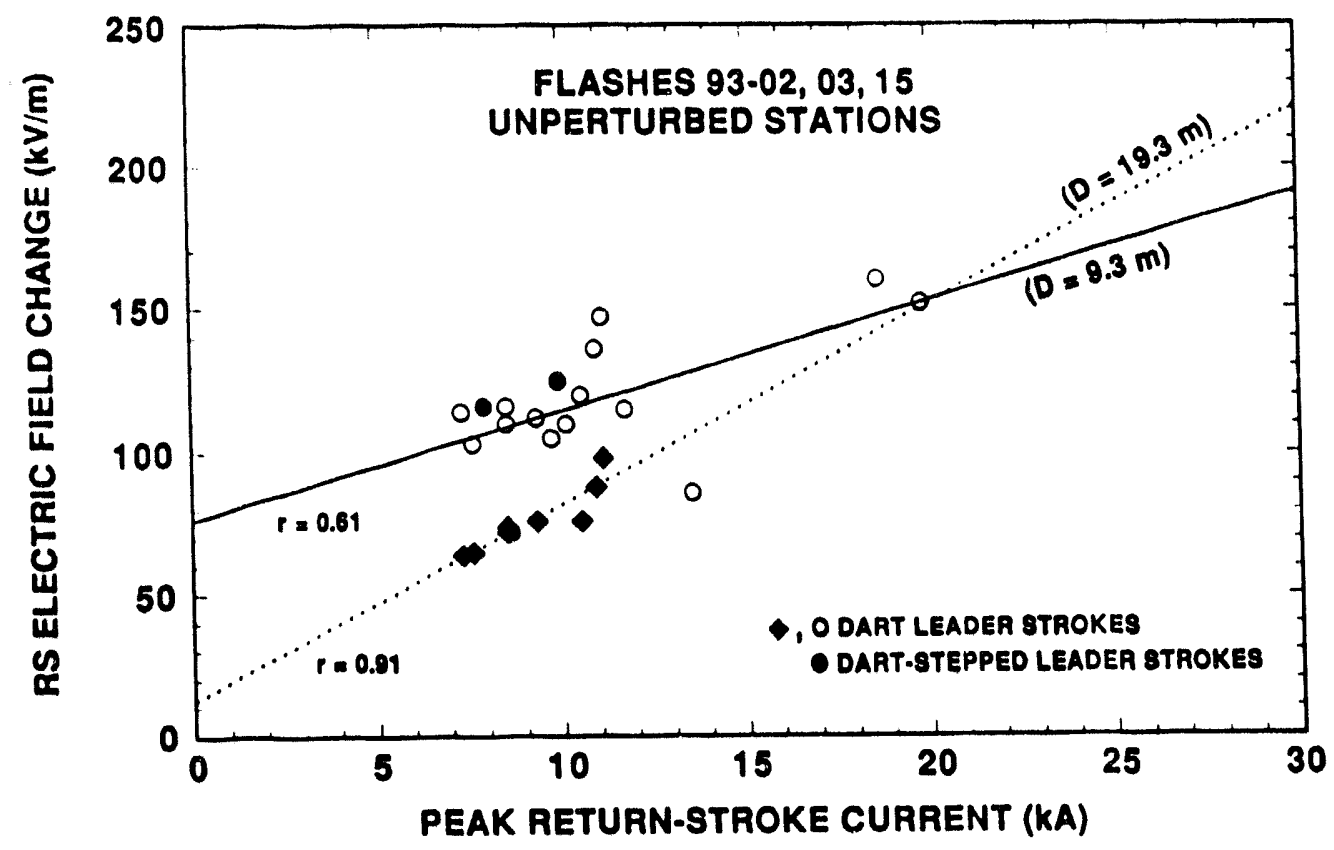

Figure 3-13 The relationship between the electric field change due to the return-stroke portion of the discharge and peak return-stroke current as measured at $9.3 \mathrm{~m}$ and $19.3 \mathrm{~m}$ from the strike point 


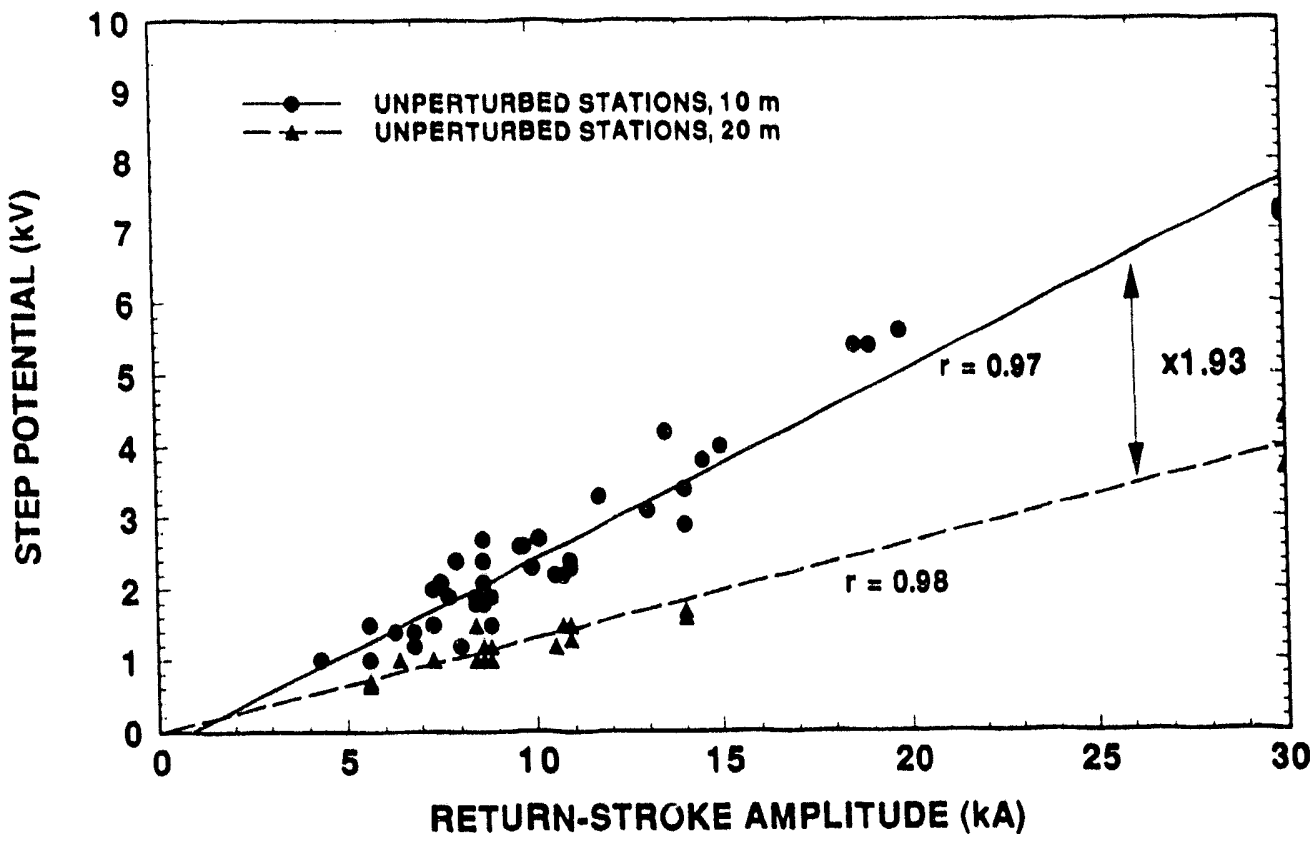

Figure 3-14 Earth step potential as a function of return-stroke current as measured at $10 \mathrm{~m}$ and $20 \mathrm{~m}$ from the strike point

In those cases for which data from multiple stations at the same distance are available, the voltages from the different stations agree quite closely. Therefore, the spatial distribution of current densities flowing outward from the injection point appears to be essentially uniform. Perhaps more precisely stated, there appears to be no significant net effects from any local nonuniformities. This result would, of course, not hold for situations in which buried conductors, such as pipes, cables or major tree roots, are present. The spatial uniformity of the observed voltages is discussed further in Section 3.2 .5 in connection with the presence of significant filamentary arcing along the surface of the ground.

The waveshapes of the recorded voltages are substantially the same as their associated return-stroke currents. By way of example, comparisons of the shapes of the voltages and their corresponding returnstroke currents are given in Figures 3-15 and 3-16 for both component strokes of flash 93-12. In Figure 3-15, the incident current waveforms of strokes 1 and 2, normalized to the peak voltages obtained at Station $2(\mathrm{D}=10 \mathrm{~m})$, are overlaid on the voltage traces. The agreement is virtually complete. A similar comparison is given in Figure 3-16 for the Station 5 data $(D=20 \mathrm{~m})$. In the latter, the agreement at early times is not as good as is the case at Station 2, but it is still substantial. Certainly for engineering applications the waveshapes can justifiably be taken to be the same. From a phenomenological viewpoint, detailed modeling will be required to resolve whether the deviations are real or represent some artifact of the experimental approach. It may be that the discrepancies are simply related to the noisy nature of the data at the $20-\mathrm{m}$ stations. In some of the other data from relatively low amplitude return strokes, notably so in flash 93-15 (see Appendix A), the waveshape agreement is somewhat degraded. For the most part, however, in those cases in which the data are relatively clean, the observed waveshape agreement is similar to that shown in Figures 3-15 and 3-16.

A further significant feature is the relationship between the 10- and 20-m station data revealed in Figure 3-14. The fall-off with distance is very nearly a factor of two, which represents a $1 / \mathrm{r}$ dependence. The hemispherical half-space model of current density due to current injected into the earth at a single point (Figure 2-9), which is commonly employed in the lightning literature for estimating driving voltages in lightning protection system applications, predicts a potential difference over a step distance $\Delta r$ of

$$
V_{S}=(I \rho / 2 \pi)[(1 / r)-1 /(r+\Delta r)]
$$



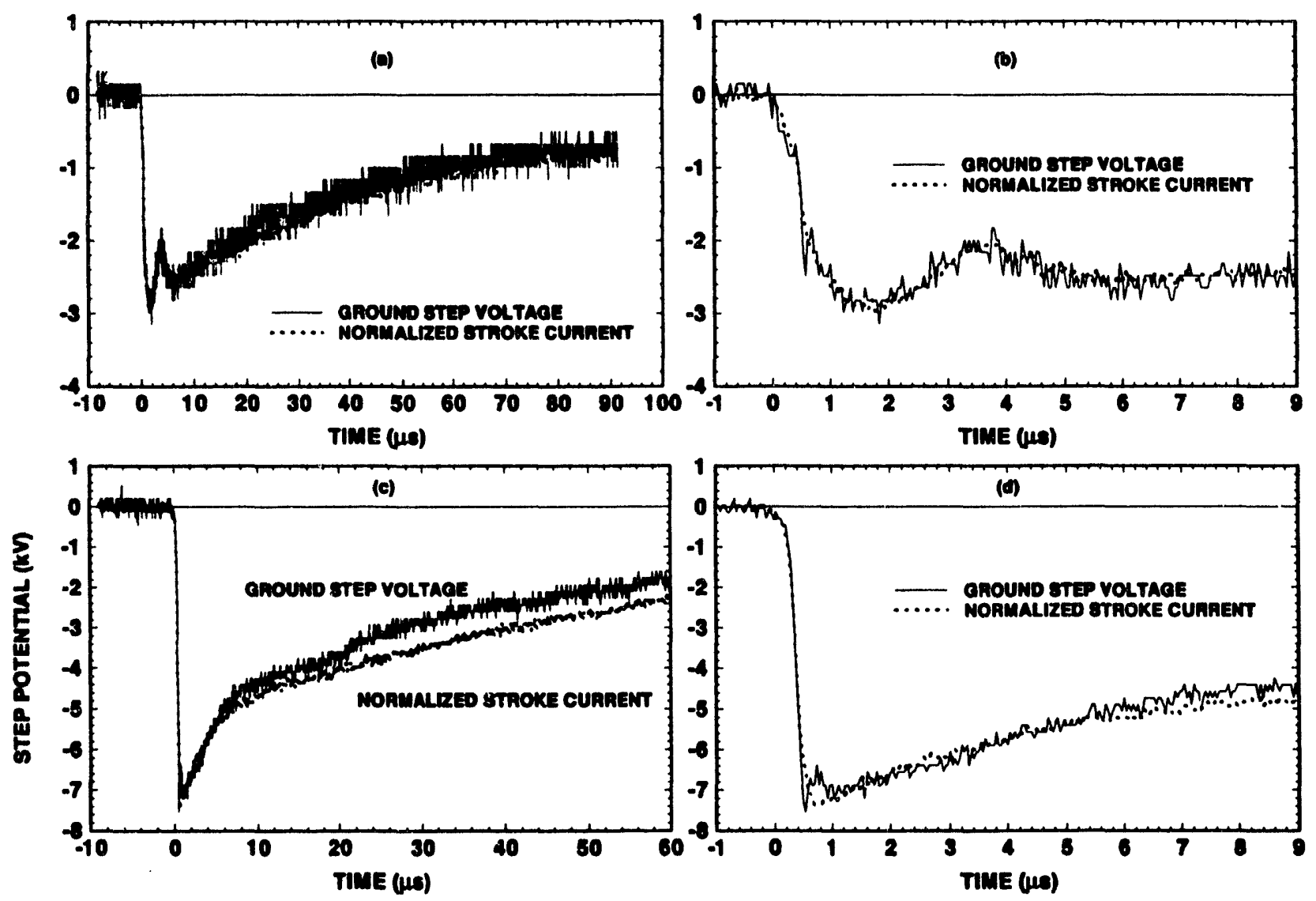

Figure 3-15 Comparison of the waveforms of earth step potentials measured $10 \mathrm{~m}$ from the strike point on both strokes of flash 93-12 with those of their corresponding incident returnstroke currents normalized to the peak amplitude of the step potentials; (a) full data records, stroke 1, (b) expanded time scale of data in (a); (c) full data records, stroke 2, and (d) expanded time scale of data in (c)

which, for $\Delta \mathrm{r}<<\mathrm{r}$, reduces to

$$
\mathrm{V}_{\mathrm{S}} \approx(\mathrm{I} \rho / 2 \pi)\left(\Delta \mathrm{r} / \mathrm{r}^{2}\right)
$$

and indicates a $1 / \mathrm{r}^{2}$ dependence for the step voltage.

Applying (3-4) with the average measured conductivity of $1.8 \times 10^{-3} \mathrm{~S} / \mathrm{m}$ results in values for apparent ground step resistance $R_{g}=V_{S} / I$ of 0.44 and 0.11 at 10 and $20 \mathrm{~m}$, respectively. Neither of these predictions agrees very well with the value determined from the slope of its corresponding data line in Figure 3-14; and, of course, theie is still the matter of the $1 / \mathrm{r}$ decrease with distance to be accounted for.

Skin depth considerations suggest that, at some radial distance from the injection point, the current should, in effect, become concentrated in a sheet of depth $\delta$ along the surface of the ground. This would result, in a $1 / r$, rather than $1 / r^{2}$, dependence of the current density. It can be shown that a current injected into the earth at a single point transitions to a current sheet mode within a radial distance corresponding to approximately one skin depth from the point of injection. Thus, strictly speaking, the hemispherical halfspace model applies only out to a radial distance comparable to the skin depth corresponding to the highest frequency of interest. 

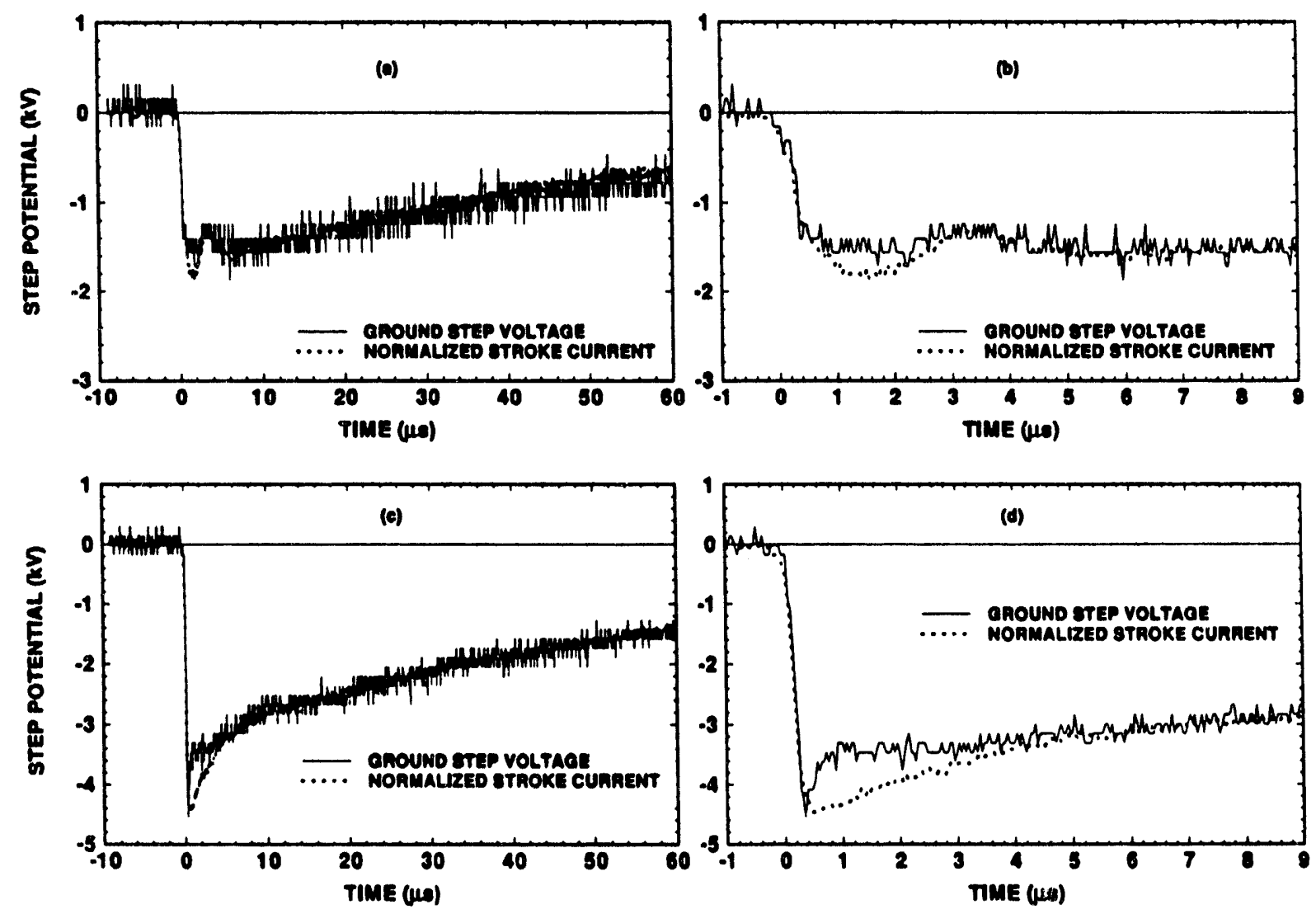

Figure 3-16

Comparison of the waveforms of earth step potentials measured $20 \mathrm{~m}$ from the strike point on both strokes of flash 93-12 with those of their corresponding incident return-stroke currents normalized to the peak amplitude of the step potentials; (a) full data records, stroke 1, (b) expanded time scale of data in (a); (c) full data records, stroke 2, and (d) expanded time scale of data in (c)

Skin depth is defined as

$$
\delta=(\pi f \sigma \mu)^{-1 / 2} \text {. }
$$

For $\mathrm{f}=3.5 \mathrm{MHz}$ (representing a reasonable upper bound frequency of interest in a return stroke with a rise time of about $0.1 \mu \mathrm{s}$ ) and $\sigma=1.8 \times 10^{-3} \mathrm{~S} / \mathrm{m}$ (Section 3.1 ), $\delta=6.3 \mathrm{~m}$; at $3.5 \mathrm{kHz}$, below which more than 80 percent of the return-stroke energy content resides, $\delta=190 \mathrm{~m}$. For frequencies corresponding to times into the voltage waveforms of $\sim 1 \mu$ s (corresponding to the approximate maximum time to peak of the voltages plotted in Figure 3-14), $\delta \approx 20 \mathrm{~m}$, about a factor of two greater than that which would support a skin-depth explanation to account for the observed behavior of the peak voltages.

A comparison of the voltages at 10 and $20 \mathrm{~m}$ is shown in Figure 3-17, which is similar to that shown in Figure 3-14, except that here the comparison point is taken at points $60 \mu$ s into the voltages and siroke currents. At $60 \mu \mathrm{s}$, the dominant frequencies are much lower, implying commensurately greater wavelengths and skin depths. Were the skin depth explanation to apply in the present case, it would be expected that, at later times, the roll-off with distance would be governed by the $1 / \mathrm{r}^{2}$ dependence. Instead, the data of Figure 3-17 indicate that the functionality is closer to the same $1 / \mathrm{r}$ relationship that was exhibited by the early time peak data shown in Figure 3-14. Therefore, while the data of Figures 3-14 and 3-17 clearly indicate that the current was flowing predominantly in a sheet mode at a distances between 10 and 20 meters, factors other than skin depth are evidently involved in producing the current concentration at the surface. 


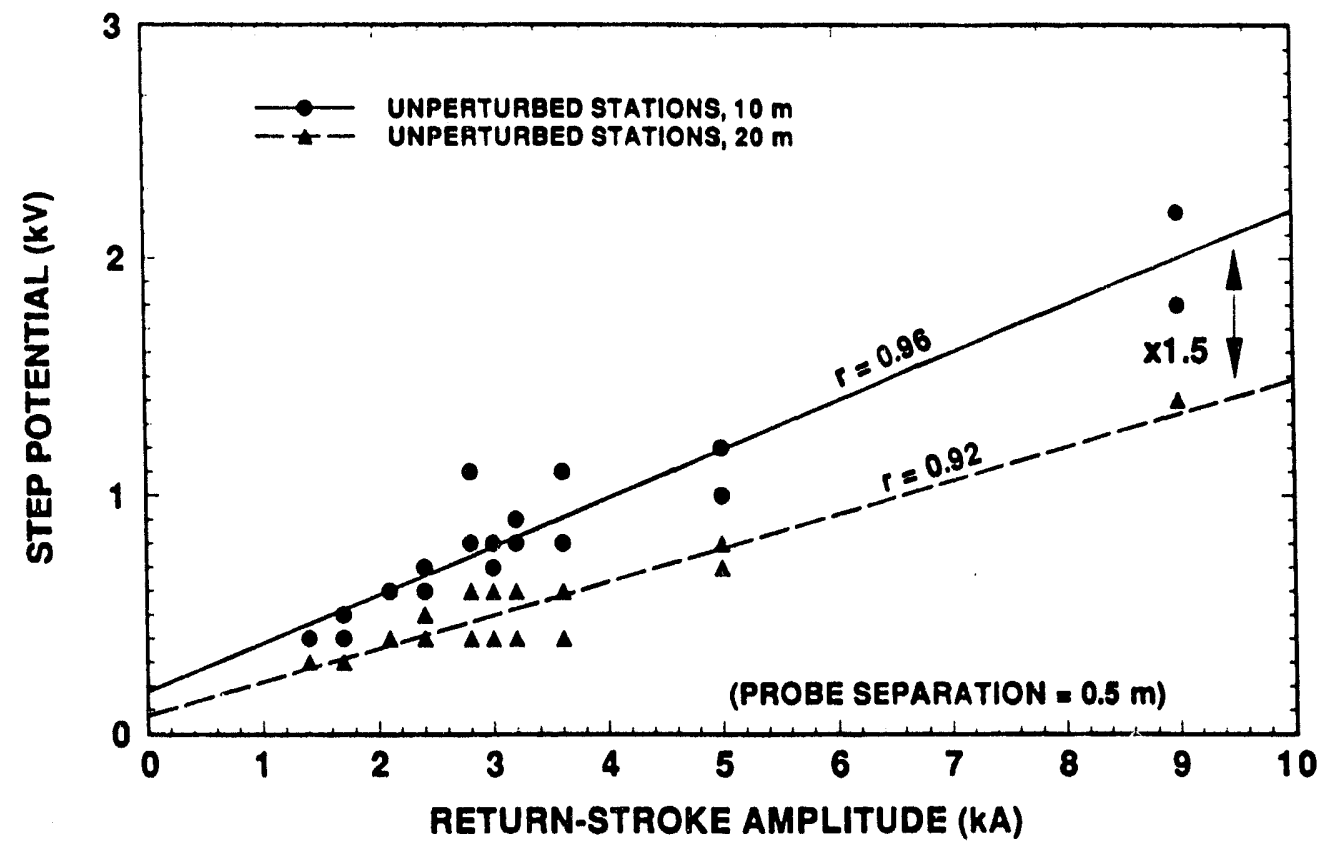

Figure 3-17 Earth step potentials at $10 \mathrm{~m}$ and $20 \mathrm{~m}$ from the strike point as functions of returnstroke amplitude determined for various strokes at points 60 Hs into the responses

One possibility is that, due to the intense rainfall that occurred during each of the storms for which data were acquired, the conductivity along a shallow layer at the surface was significantly higher than in the bulk of the earth below that layer. In this way a large fraction of the current would flow within the surface layer and result more nearly in the observed $1 / \mathrm{r}$ voltage dependence.

This view of the problem is illustrated in Figure 3-18, in which the half- space below the lightning current attachment point is depicted as consisting of a thin surface layer of conductivity $\sigma_{\mathrm{S}}$ situated above the rest of the half space of conductivity $\sigma$, taken to be the average conductivity determined from the Megger measurements. The voltage developed over $\Delta r$ at radial distance $r$ from the strike point on the earth's surface can be considered to result from the flow of the injected current I through the parallel combination of the resistances of the annulus and the half spherical shell below it. The resistance of per unit thickness of the annulus is

$$
\mathbf{R}_{\mathbf{a}}=\mathbf{R} /(2 \pi \mathrm{r})[\Omega / \mathrm{m}]
$$

where $\mathbf{R}$ is the surface resistance, in $\Omega$ /square, of the layer. The corresponding resistance per unit width of the hemispherical shell is

$$
R_{S}=1 /\left(2 \pi r^{2} \sigma\right)[\Omega / m]
$$

A rigorous development of this circuit approximation is presented in Appendix C.

Per the circuit representation in the Figure 3-18, the open circuit voltage per unit step width, that is, the radial electric field at the surface, is given by

$$
E_{r}=(I R / 2 \pi r)(1+o R r)^{-1}[V / m]
$$

and the voltage developed over step width $\Delta \mathrm{r}$ is therefore 


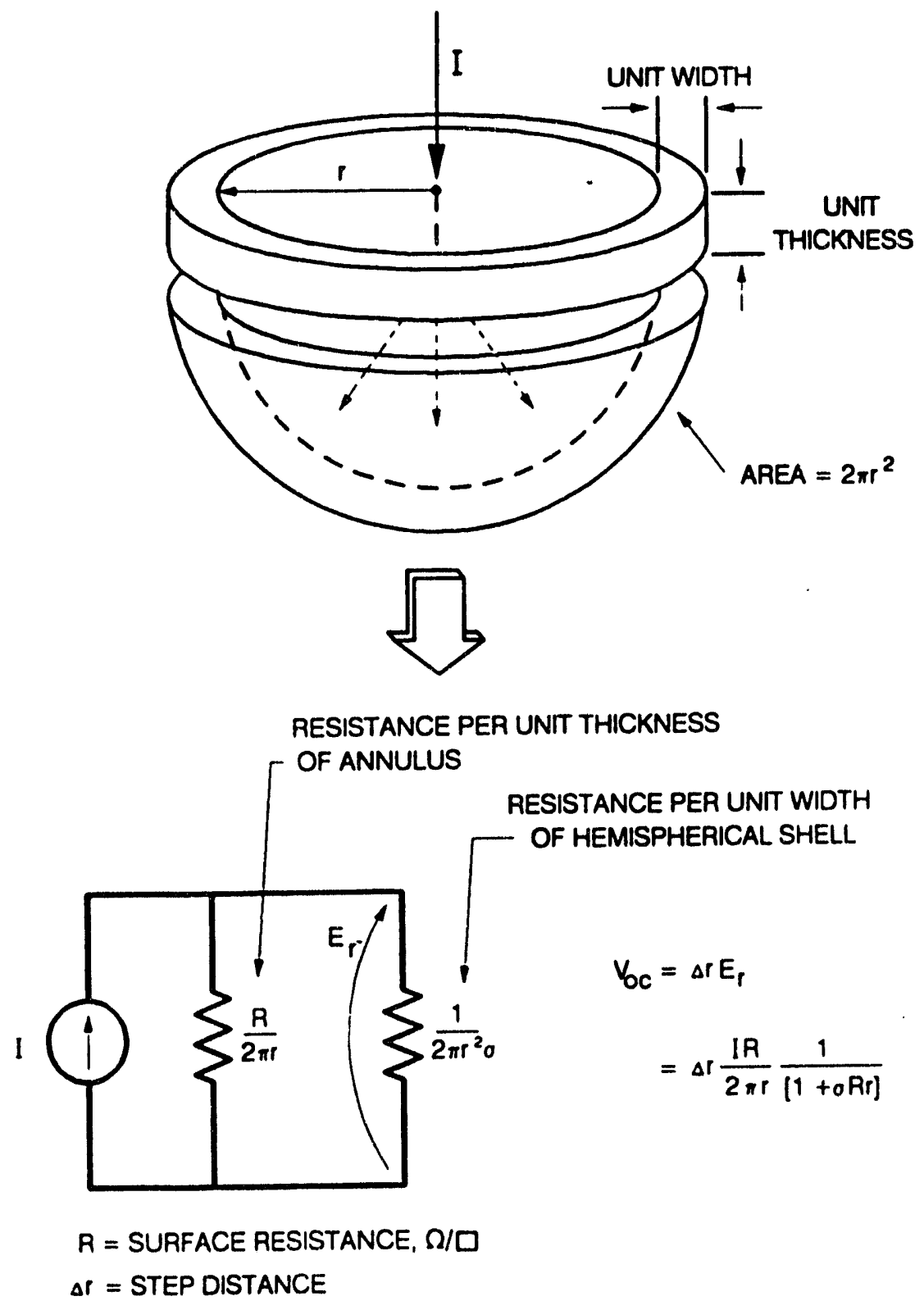

Figure 3-18 Two element earth step potential model accounting for the presence of a highly conducting thin surface layer

$$
\mathrm{V}_{\mathrm{S}} \approx(\mathrm{IR} \Delta \mathrm{r} / 2 \pi \mathrm{r})(1+\sigma \mathrm{Rr})^{-1}[\mathrm{~V}]
$$

for $\Delta r<<r$. From the data of Figure 3-14, effective resistances $R_{g}=V_{S} / I$ of 0.24 and $0.12 \Omega$ are obtained from the slopes of the fitted lines for radial distances of 10 and $20 \mathrm{~m}$, respectively. From (3-5)

$$
R_{g}=V_{S} / I=(R \Delta r / 2 \pi r)(1+\sigma R r)^{-1}(\Omega) .
$$

Solving for $\mathbf{R}$ in terms of $\mathbf{R}_{\mathbf{g}}$ yields

$$
R=2 \pi r /\left(1 / R_{g}-2 \pi r^{2} \sigma\right)(\Omega-m) .
$$


Evaluating (3-7) at $\mathrm{r}=10 \mathrm{~m}$ and $\sigma=1.8 \times 10^{-3} \mathrm{~S} / \mathrm{m}$ yields $\mathrm{R}=66.1 \Omega$. However, substitution of $R=66 \Omega$ and $\sigma=1.8 \times 10^{-3} \mathrm{~S} / \mathrm{m}$ in (3-6) for $\mathrm{r}=20 \mathrm{~m}$ results in $\mathrm{R}_{\mathrm{g}}=0.078$, about 37 percent lower than the observed experimental value of 0.12 . If, however, at $20 \mathrm{~m}$ we employ $\sigma=1 \times 10^{-3}$, which is more nearly what was measured at the 20-m stations (Figure 3-3), then the agreement at both distances improves to within 10 percent. Use of $\sigma=1.0 \times 10^{-3} \mathrm{~S} / \mathrm{m}$ at Stations 4 and 5 is at least reasonable, and doing so leads to quite good agreement between the theoretical and experimental results. Nevertheless, with the presently available experimental data, there is no way of definitively validating that the proposed model adequately describes the mechanism leading to the observed behavior of the measured data. In fact, a combination of factors may be contributing to the observed outcome. Without further experimental data under varying conditions, it may not be possible to validate the proposed, or any other, model the results with certainty.

In spite of the lack of a definitive explanation for the observed results, two significant practical conclusions may be safely drawn from the step potential measurements:

1. Within 20 meters of the strike point, the waveshape of the step voltage can be taken to be the same as that of the incident current. This conclusion supports the validity of applying the existing extensive statistical data base on stroke current parameters (in particular, rise time and rate of current rise) to earth potentials when assessing the interaction of lightning with buried cables, pipes and lightning protection systems.

2. At least under the conditions represented in the present experiments, the magnitudes of earth potentials fall off with distance as $1 / r$, a rate that is slower than the $1 / r^{2}$ factor conventionally employed in the analysis of lightning interactions with facilities and in the design of lightning protection systems. Prudence would suggest employing the more conservative $1 / \mathrm{r}$ dependence in lightning interaction assessments.

\subsubsection{Incident Flash Currents}

The standard technique employed in directly recording incident flash currents is discussed in Section 2.1.3.1. During the course of the present experiments, however, several problems occurred in carrying out these particular measurements. The problems included loss of data due to the occurrence of arcing down the legs of the LTA, which resulted in a by-passing of the current sensor suspended below the LTA frame (Figure 2-5) by some fraction of the total incident current. Also, during several shots, miscellaneous other instrumentation problems occurred that caused the loss of some of the directly measured stroke and/or continuing current data.

The height of the aluminum rocket launcher frame above the ground was initially adjusted to be approximately $0.5 \mathrm{~m}$ at its lowest point, a circumstance resulting from the requirement to cant the launcher somewhat to ensure the impact of the spent rockets within a designated safety zone. The length of the conductor (including the steel enclosure containing the current sensor and fiber optic transmitters) between the bottom of the launch tube frame and the ground was about $1 \mathrm{~m}$. Its inductance was, therefore, about $1 \mu \mathrm{h}$. A nominal return-stroke current of 10-kA peak amplitude and rise time of about $0.3 \mu$ s flowing down this conductor would produce an inductive voltage between the frame and the ground of the order of $10 \mathrm{kV}$, with a short duration corresponding to that of the wavefront of the incident stroke current. At this level the dielectric (fiber glass) legs of the LTA were considered to provide more than adequate voltage standoff between the LTA frame and the ground. However, the additional possible effect of a high ground rod resistance was not initially given sufficient consideration.

By design, the ground rod employed under the LTA was chosen to be short. The intention was to approximate closely ground attachment point conditions that exist when a naturally initiated flash terminates at the earth's surface. Therefore, the length of the rod was initially chosen to be only about 
$0.3 \mathrm{~m}$. The rod itself was of zinc-plated steel of $16-\mathrm{mm}$ diameter. The low frequency resistance of such a rod can be estimated using the Liew-Darveniza model [15], from which

$$
R=1 /(2 \pi \ell \sigma) \ln \left[\left(r_{0}+\ell\right) / r_{0}\right][\Omega],
$$

where $\sigma$ is the conductivity of the earth, and $r_{0}$ and $\ell$ are the radius and length of the rod in the earth, respectively. Using $\sigma=2.2 \times 10^{-3} \mathrm{~S} / \mathrm{m}$, the conductivity measured in the immediate vicinity of the launcher, $R \approx 260 \Omega$. It follows that, under these conditions, a return-stroke current of $10-\mathrm{kA}$ peak amplitude would result in a voltage on the launcher frame with a peak value in excess of $2.5 \mathrm{MV}$ with respect to "infinity" within the half space of earth below the LTA and a duration corresponding to that of the incident stroke current. About one-half of that value of voltage is developed over the distance out to the legs of the launcher.

This resistive voltage was evidently responsible for initiating surface tracking and subsequent arcing down the dielectric LTA legs. The fraction of the total incident current taking this shunt path to earth, of course, would by-pass sensor and cause an error in the recorded data. That this phenomenon had occurred on some individual strokes was discovered during the initial data review following the first storm. It was noted that the magnetic fields recorded at each of the three $10-\mathrm{m}$ recording stations agreed quite closely with each other, but that the channel currents deduced from those fields using Ampere's law deviated substantially from what had been measured at the base of the LTA during some (but not all) strokes. Detailed study of the video records from the first two flashes (93-02 and 03) appeared to corroborate the presence of such arcing, although the evidence was somewhat inconclusive due to image blooming and the obscuring presence of smoke from the rocket plumes.

Prior to the next storm, the $0.3-\mathrm{m}$ ground rod was replaced by a longer one of $1.3-\mathrm{m}$ length, and the LTA frame was raised to the limit permitted by its existing legs. Unfortunately, however, during the second storm, a separate, unrelated instrumentation problem caused the loss once again of the directly measured incident current data. Following that storm, during the process of reconfiguring the launcher with a tall strike rod to support the next scheduled experiment, extensions were added to the legs of the LTA, thereby raising the frame to a height of about $1.3 \mathrm{~m}$ above the ground. From that point on, no further occurrence of arcing down the legs was ever detected, either in the measured data or in any of the photographic or video records. In fact, as is documented in Section 3.2.1, the data from the last two storms show that the peak amplitudes of the magnetic fields recorded at the 10-m stations during flashes triggered from this configuration are related to the directly measured stroke currents by the Ampere's law relationship to within better than 5 percent (e.g., Figure 3-5).

On the basis of this established relationship, peak values of the currents of strokes that occurred during the earlier storms in which the directly measured currents were either known to be in error or were otherwise lost outright are taken to be those deduced by applying Ampere's law to the magnetic fields measured at the unperturbed $10-\mathrm{m}$ monitoring station. Furthermore, in view of the results discussed in Section 3.2.1, it has been decided that, in future operations of this kind, a magnetic field sensor will always be employed as a backup to the usual direct measurement of the incident stroke current.

Table 3-2 provides a summary of information relevant to the seven triggered flashes, including the current peaks obtained on component strokes for which digitized records were obtained. Under the standard assumption that return-stroke peak currents are log-normally distributed, the geometric mean of the present sample of 31 strokes is $10.2 \mathrm{kA}$, although the distribution is slightly truncated because of the 4-kA instrumentation trigger level. The corresponding mean value of triggered strokes obtained by SNL in previous experiments conducted in Florida and Alabama is $12 \mathrm{kA}$, which is also the median value associated with subsequent return strokes in naturally initiated lightning [12]. The maximum stroke current obtained was about $30 \mathrm{kA}$, and the minimum, above the measurement trigger level, was $4.4 \mathrm{kA}$. 
Table 3-2.

Triggered Flash Summary

\begin{tabular}{|c|c|c|c|c|c|}
\hline $\begin{array}{l}\text { Shot } \\
\text { ID }\end{array}$ & Date & $\begin{array}{c}\text { Time } \\
\text { (GMT) }\end{array}$ & $\begin{array}{c}\text { E-Field } 1 \\
(\mathbf{k V} / \mathbf{m})\end{array}$ & $\begin{array}{c}\text { Stroke } \\
\text { No. }{ }^{2}\end{array}$ & $\begin{array}{c}\mathbf{I p}^{3} \\
(\mathbf{k A})\end{array}$ \\
\hline $93-02$ & $8 / 3 / 93$ & $23: 21$ & 5.5 & $\begin{array}{l}1 \\
2 \\
3 \\
4 \\
5 \\
6 \\
7\end{array}$ & $\begin{array}{c}11.7^{*} \\
9.7^{*} \\
19.8^{*} \\
8.6^{*} \\
13.5^{*} \\
10.1^{*} \\
7.9^{2}\end{array}$ \\
\hline 93-03 & $8 / 3 / 93$ & $23: 47$ & 9.0 & $\begin{array}{l}1 \\
2\end{array}$ & $\begin{array}{l}18.6^{*} \\
10.0^{*}\end{array}$ \\
\hline 93-08 & $8 / 13 / 93$ & $19: 46$ & 4.0 & $\begin{array}{l}1 \\
2 \\
3 \\
4 \\
5 \\
6 \\
7 \\
8\end{array}$ & $\begin{array}{c}8.0^{*} \\
4.4^{*} \\
14.5^{*} \\
13.0^{*} \\
7.7^{*} \\
6.3^{*} \\
15.1^{*} \\
9.9^{*}\end{array}$ \\
\hline 93-09 & $8 / 13 / 93$ & $21: 35$ & 6.0 & $\begin{array}{l}1 \\
2 \\
3\end{array}$ & $\begin{array}{c}7.5^{*} \\
18.9^{*} \\
8.6^{*}\end{array}$ \\
\hline $93-12$ & $9 / 3 / 93$ & $20: 05$ & 5.5 & $\begin{array}{l}1 \\
2\end{array}$ & $\begin{array}{l}13.9 \\
29.3\end{array}$ \\
\hline $93-14$ & $9 / 3 / 93$ & $20: 21$ & 5.2 & 1 & 5.6 \\
\hline $93-15$ & 9/7/93 & $19: 46$ & 5.4 & $\begin{array}{l}1 \\
2 \\
3 \\
4 \\
5 \\
6 \\
7 \\
8\end{array}$ & $\begin{array}{c}8.5 \\
9.3 \\
7.6 \\
10.9 \\
8.5 \\
11.1 \\
10.5 \\
7.3\end{array}$ \\
\hline
\end{tabular}

1 Ambient electric field at time of launch; polarity in each case corresponds to negative charge overhead.

2 Listed stroke number corresponds to strokes exceeding digitizer trigger level of $\sim \mathbf{4} \mathbf{k A}$.

3 Starred values as deduced from magnetic field at $10 \mathrm{~m}$. 


\subsubsection{Surface Arcing Near the Bases of Lightning Channels}

Among the more significant results of the present experiments was the documentation of the presence and frequency of occurrence of large scale surface arcing emanating from the ground point of the triggered lightning channels. The primary source data used for this purpose was the collection of various video and high speed 16- $\mathrm{mm}$ framing camera records. In addition, details of the spatial patterns of the arcing were obtained from the two 35-mm still photographs shown in Figures 3-19 and 3-20. These two photographs represent the first available detailed images of this type of arcing while actually in progress, although the existence of such behavior has previously been well established by after-the-fact photographs of its effects [e.g., 17 - 19]. One of the most elucidating records of this kind is shown in Figure 3-21.

In addition to the radial filamentary arcing documented in Figures 3-19 through 3-21, fairly large diffuse arcs, perhaps most effectively described as "fireballs," were noted to occur with regularity at the base of the launcher out to a radial extent of 1 to $2 \mathrm{~m}$. The fireball effect, without discernible accompanying filamentary branching, was observed in some instances even during the ICC phase of the triggered flashes. Given the ground rod resistance of $260 \Omega$ calculated in Section 3.2.4, air breakdown is not surprising around the sharp edges of the top of the ground rod and metal clamps used to attach it to the down conductors from the LTA (Figure 2-5). The ICC component of flash 93-03 shown in Figure 3-8b, for example, has an average amplitude of about $300 \mathrm{~A}$ over much of its duration. That level of current would produce a potential at the ground rod connection of nearly $80 \mathrm{kV}$. Under these conditions, the resulting local electric field enhancement at the sharp edges of the ground rod would surely exceed the nominal atmospheric breakdown threshold of about $30 \mathrm{kV} / \mathrm{cm}$.

This sort of fireball arc accompanied one or more of the return strokes in six out of seven of the flashes. Furthermore, 100 percent of all strokes in these flashes with current peaks in excess of $15 \mathrm{kA}$ produced filamentary arcs. The maximum range of any of the arcs was at least $20 \mathrm{~m}$, which was the limit of our detailed monitoring capability. The frequency of occurrence of the observed branched arcing as a function of return-stroke amplitude is shown in Figure 3-22.

As discussed in Section 2.2.2.2, during flashes 93-02 through 93-09, a wire cage was installed over the sensors located at Station 1. This cage, painted orange for photography purposes, can be seen clearly in Figure 3-20. From this photograph, it appears that one branch of the surface arc terminated onto the cage. This event is corroborated by the data traces shown in Figure 3-23, which correspond to the magnetic field and earth step potential recorded at Station 1 during the first stroke of Flash 93-03. Zero time in the plots coincides with the beginning of the return-stroke current. The large discontinuities that occur in each trace at about $35 \mu \mathrm{s}$, which also occur in the electric field measurement at that station, are attributed to the interception by the cage of the current flowing in the arc that attached to the cage. These abrupt excursions do not appear in the data acquired during that stroke at either of the other two stations that were active during this flash. (See Appendix A.)

All four corners of the cage were earthed by means of $0.5-\mathrm{m}$ deep ground rods. Two of the braided straps that were used to connect the corners of the cage to their respective rods were instrumented as shown in Figure 3-24 to measure the currents they carried. Plotted in Figure 3-25 is the current recorded at the corner of the cage nearest to the apparent point of attachment of the arc. The current exceeded the instrumentation saturation level but appears to have reached a peak value somewhat in excess of $1 \mathrm{kA}$, which represents about 5 percent of the peak incident current of that struke. The corresponding currents measured on events in which there was no observable arcing in the vicinity of the cage had much lower peak amplitudes, typically in the range of a few tens of amperes. 


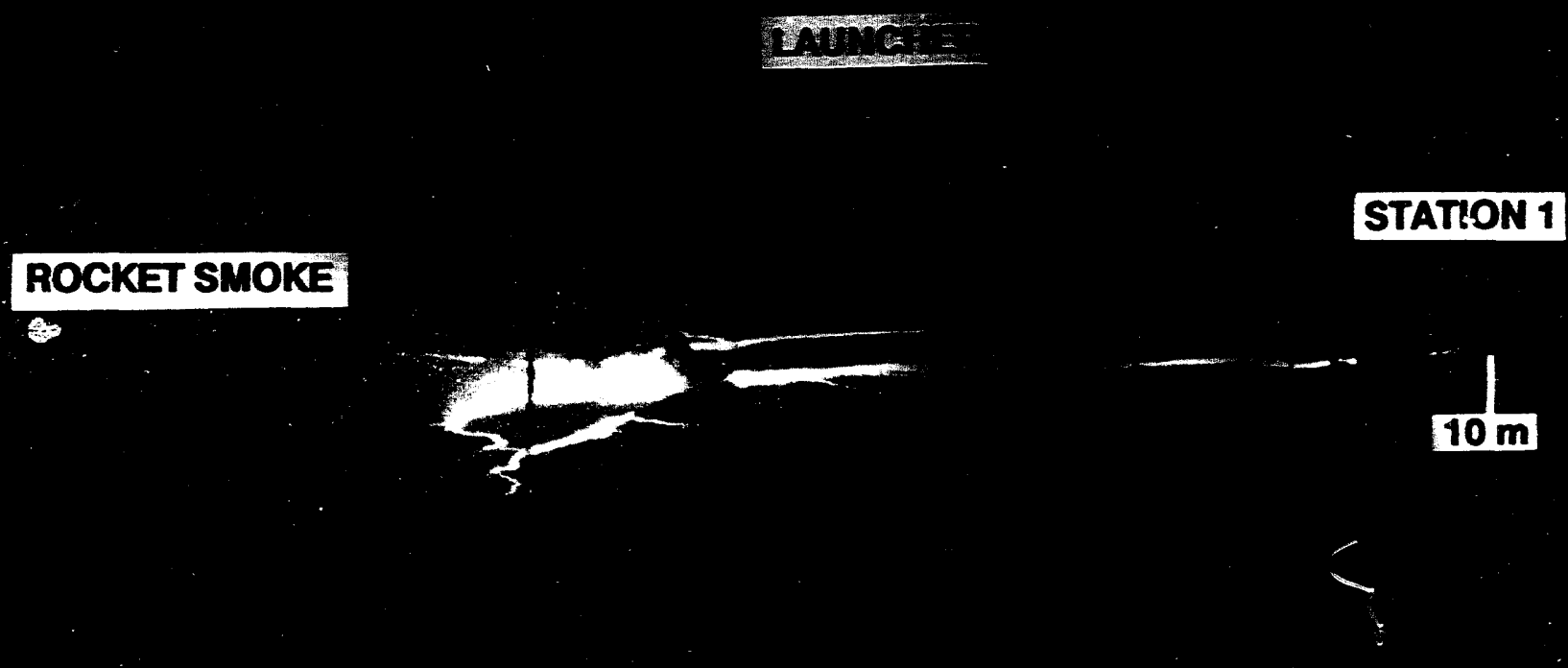

CONDUITS

FIBER OPTIC CABLES

PNEUMATIC LINES

Figure 3-19 Radial filamentary surface arcing emanating from the base of the strike point of the second stroke in flach 93-12; stroke current was $29.6 \mathrm{kA}$ 


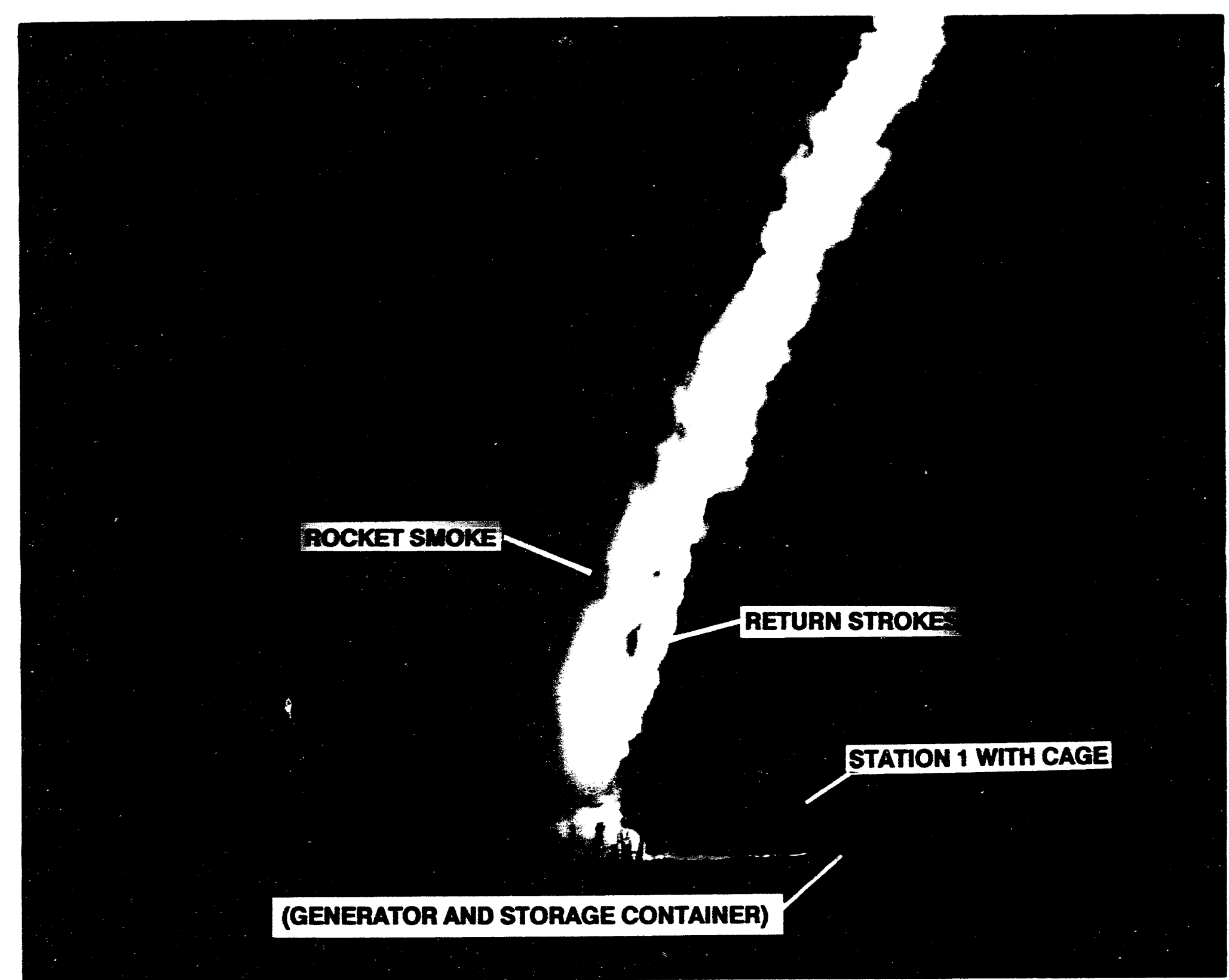
by the SATTLF's electrical generator and auxiliary storage container; arcing occurred on the first and fourth retum strokes 


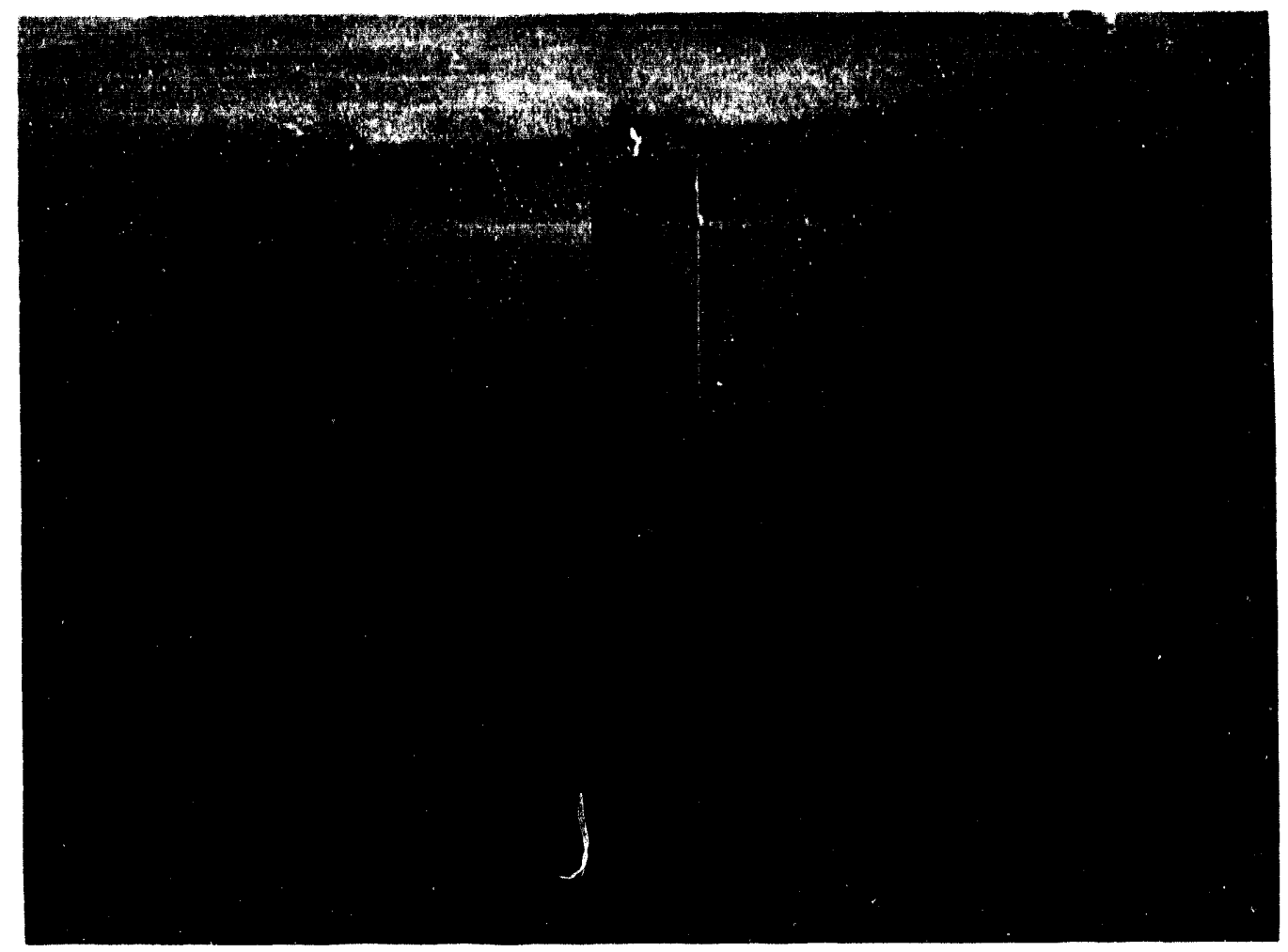

Figure 3-21 Scorched grass on a golf course green near Tucson, Arizona resulting from a lightning strike to the (dielectric) flagpole at the center of the pattern. (Photograph courtesy of E.P. Krider, Institute of Atmospheric Physics, University of Arizona)

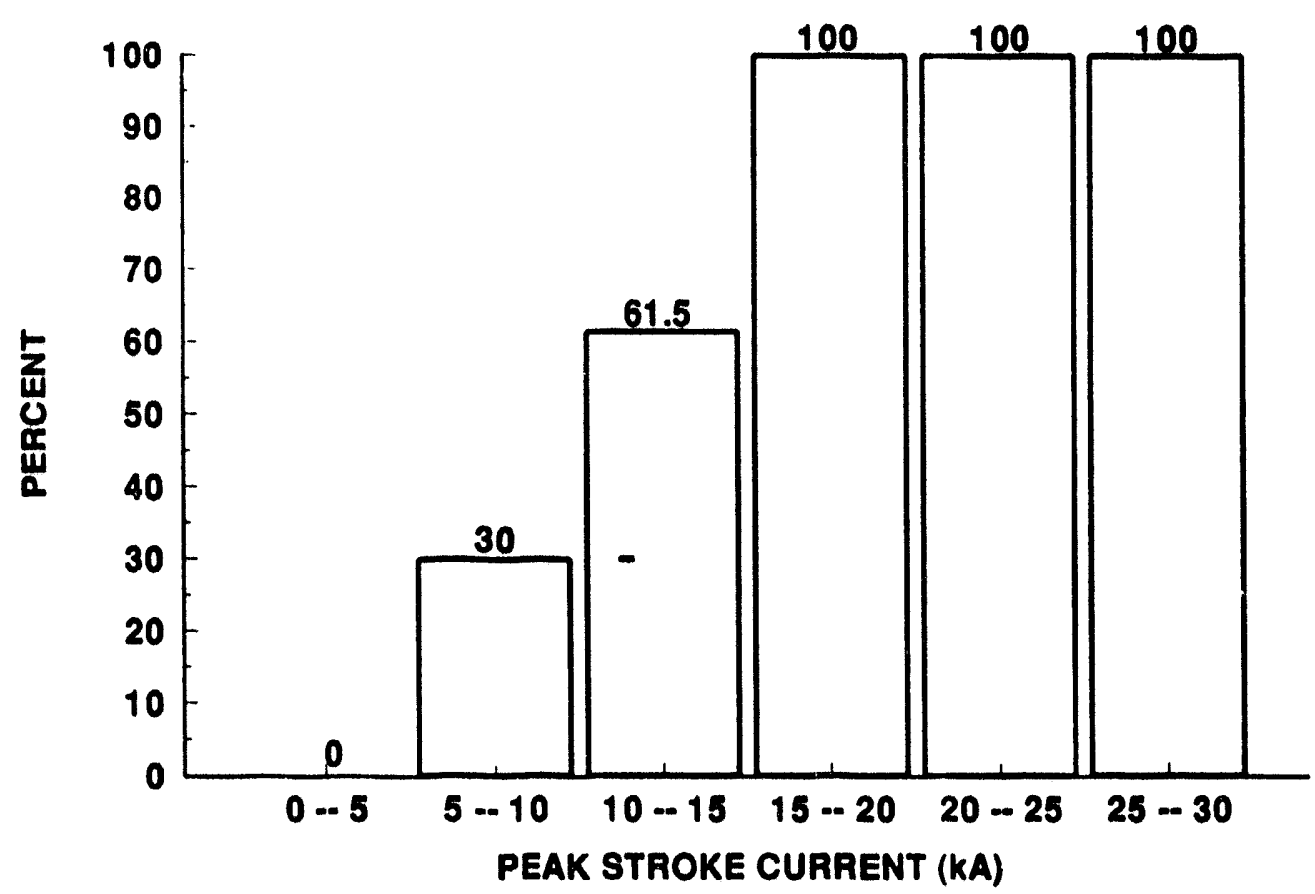

Figure 3-22 Percentages of retum strokes producing detectable filamentary arcing from the base of the strike point as a function of peak stroke current 

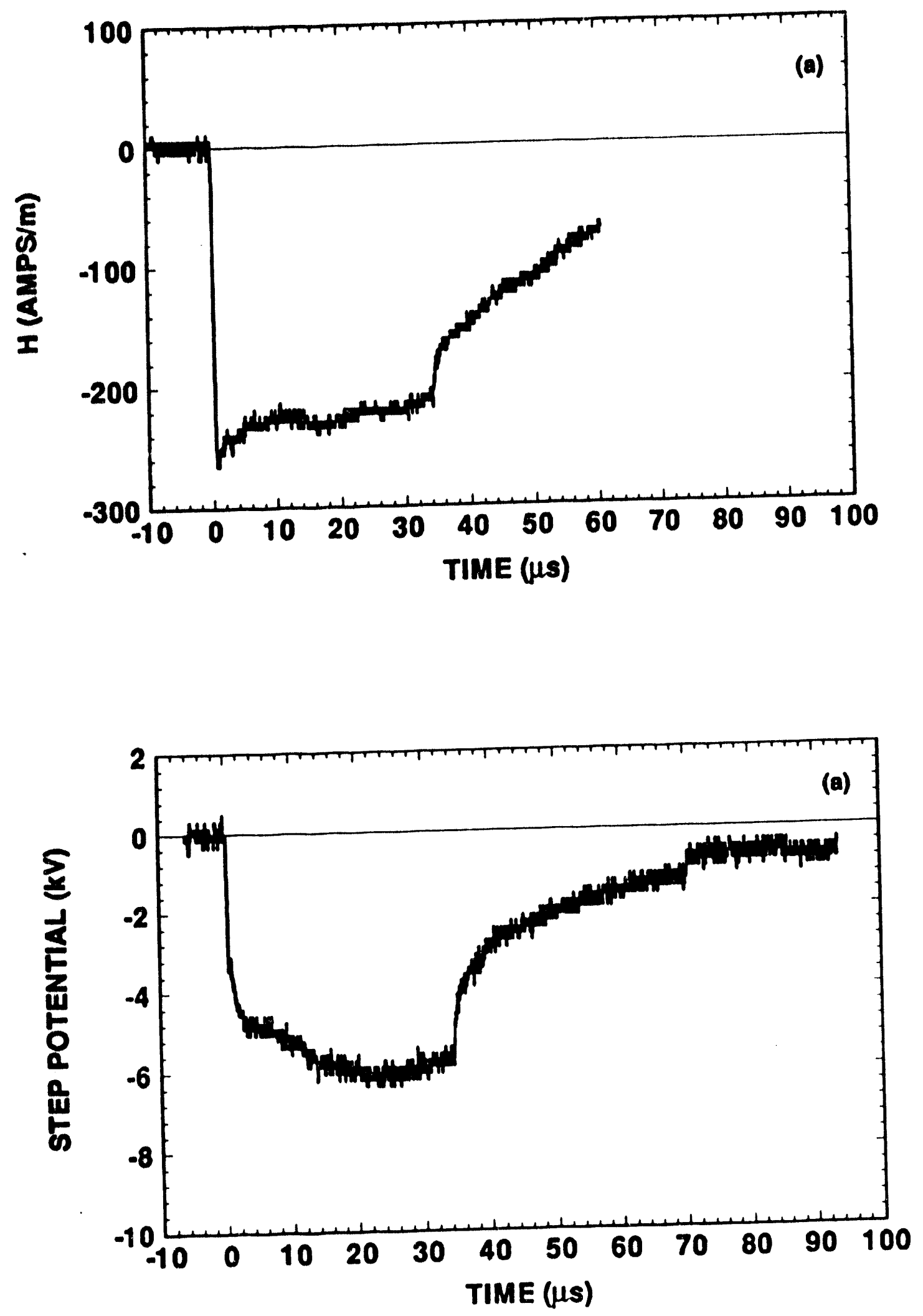

Figure 3-23 (a) Magnetic field and (b) earth step potential records obtained at Station 1 during stroke 1 of flash 93-03, during which the metal cage was contacted by the ground are shown in Figure 3-20 


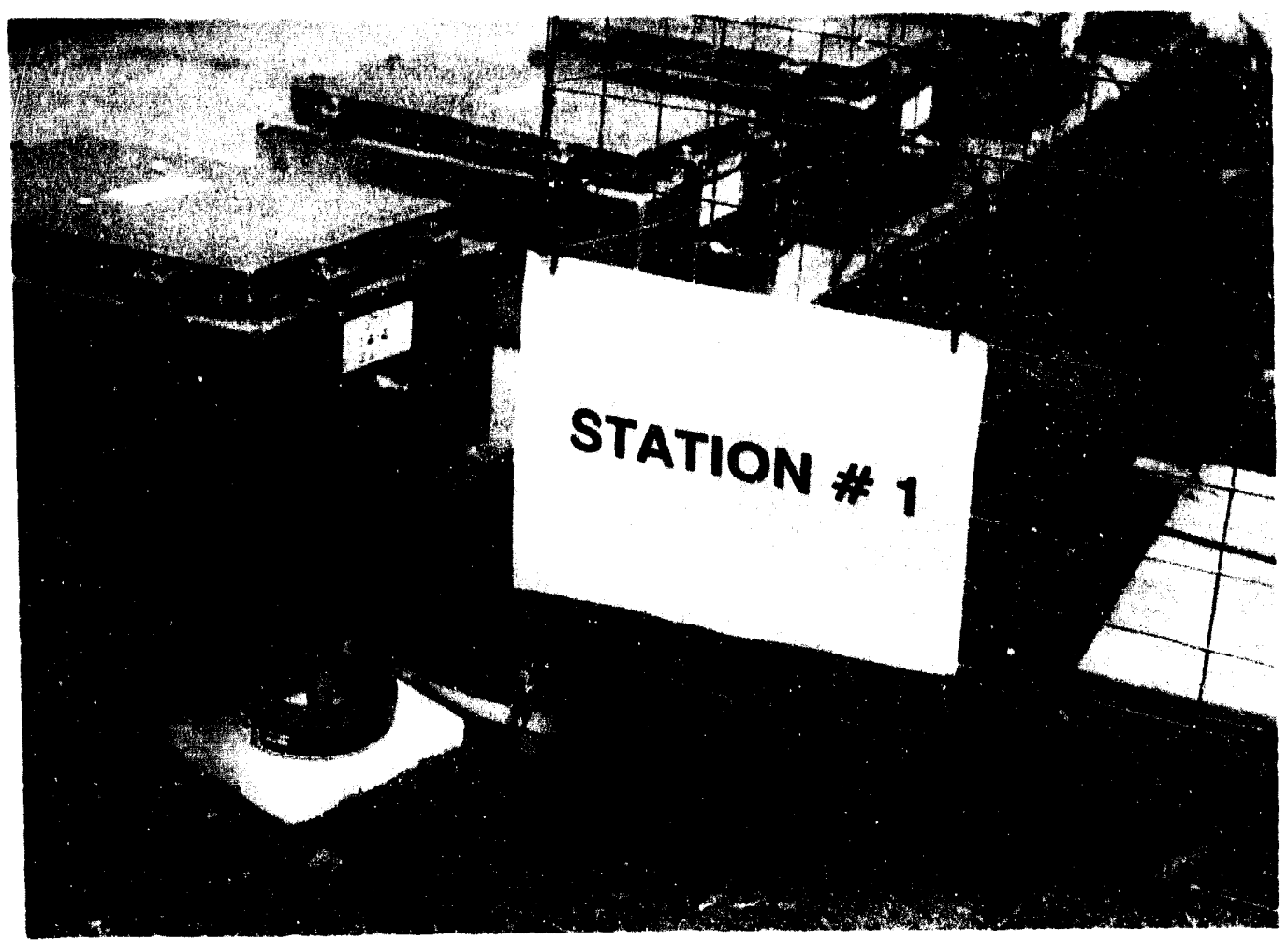

Figure 3-24 Installation of current sensor on the grounding braid connecting one corner of the wire shielding cage with its adjacent $0.5-\mathrm{m}$ deep ground rod

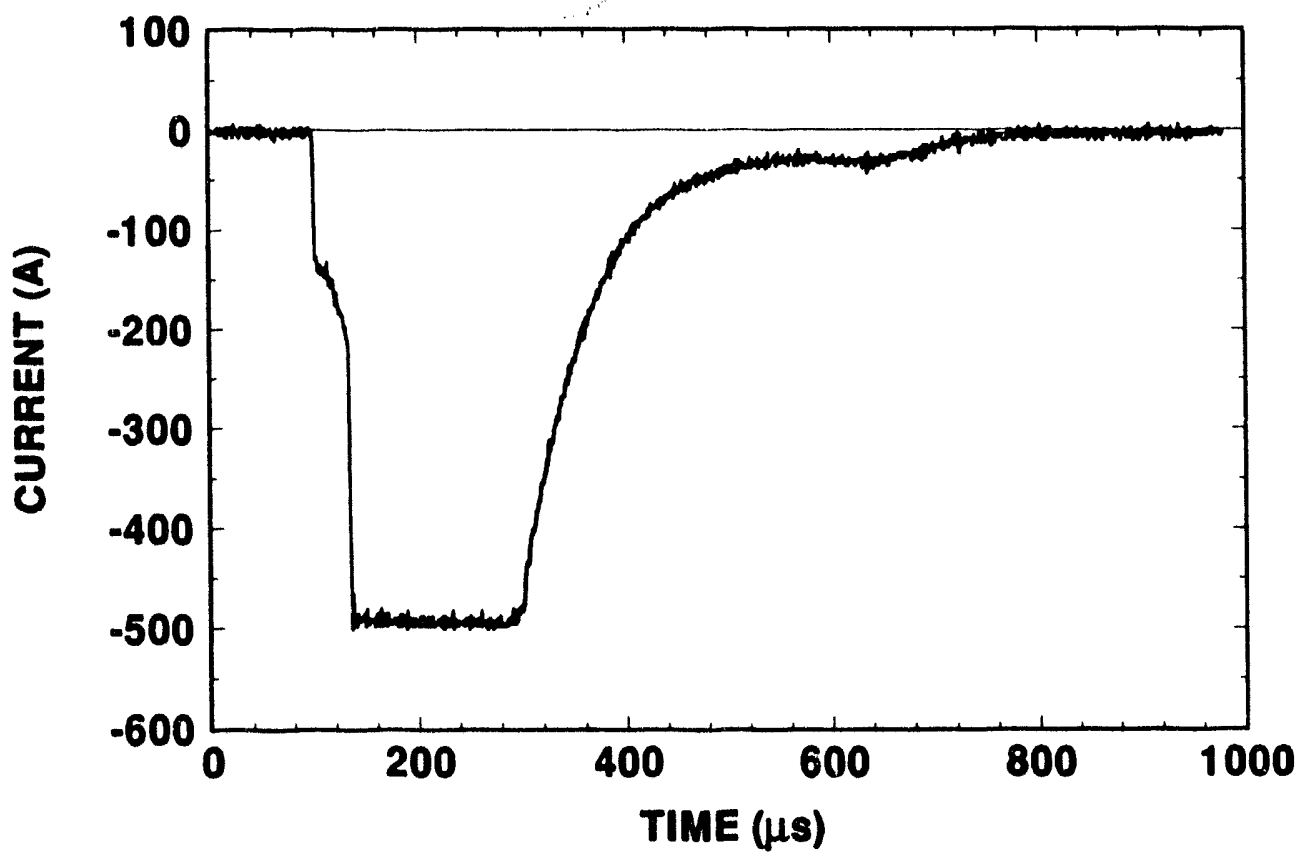

Figure 3-25 Current recorded on the grounding strap on the corner of the wire cage nearest to the point of arc interception shown in Figure 3-20 on stroke 1 of flash 93-03 
Evidence similar to that presented in Figure 3-23 of the interaction of a surface arc with one or more of the deployed sensors was also obtained during flashes 93-02 and 93-12. During the latter flash, which is the one shown in Figure 3-19, excursions appear in the step voltage data of both Stations 1 and 4, which lie on the same outward radial from the strike point, with the effect being more pronounced at the $20-\mathrm{m}$ station. From this and the available photographic evidence, it appears that the arc reached a distance of at least $20 \mathrm{~m}$.

The maximum return-stroke current amplitude obtained during these tests was $29.4 \mathrm{kA}$, and it occurred on stroke 2 of flash 93-12. Assuming that surface arc range is a function of stroke current amplitude, a linear extrapolation would represent the upper bound of the range. Whether the functionality is actually linear is problematic; but, all other factors being equal, the linear assumption would yield an upper bound. As an illustration, if the range of the arc occurring on flash 93-02 (Figure 3-20) is taken to be $25 \mathrm{~m}$, then the limit of the range of an arc that could accompany a 200-kA stroke would be something in excess of $150 \mathrm{~m}$.

Following each storm, the vicinity near the launcher was carefully examined for evidence of fulgurites. None were found, even directly below the ground rod under the LTA. This result is consistent with those of a recent experiment carried out by the New Mexico Institute of Mining and Technology. In that experiment, it was arranged that triggered lightning current passed through layers of various types of soils. Fulgurites were found to have been formed only in those layers composed predominantly of sand [20]. In other recent triggered lightning tests, carried out in an area of sandy soil in the north central region of Florida, the ground attachment points were intentionally positioned over instrumented buried power distribution cables. Fulgurites as long as a meter or more were formed by the lightning current as it arced through the soil before attaching to the buried cables [21].

\subsection{Temporary Lightning Protection Concept Tests}

\subsubsection{Direct-Strike Suppression}

As discussed in Section 2.2.2.1, two approaches to providing temporary protection from a direct lightning strike to a small exposed object were tested. The two techniques were the installation of a tall electrically conductive, grounded mast near the object, and the use of a dielectric blanket to cover the object and thereby suppress the development of upward streamers from its extremities. The rationale behind each concept and descriptions of the tests are provided in Section 2.2.2.1. In the following, the results of each test are presented and discussed.

3.3.1.1 The Effect of a Grounded Mast. The vertical electric fields at 9.3 and $19.3 \mathrm{~m}$ from the base of triggered lightning channels were measured under experimentally similar conditions during two storms (Table 2-1). The only modification made between storms was the replacement of the $11-\mathrm{m}$ high strike rod employed during the first storm with a $4.5-\mathrm{m}$ rod during the second storm. The resulting data are summarized in Figure 3-26.

The locations of Stations $1(9.3 \mathrm{~m})$ and $4(19.3 \mathrm{~m})$ with respect to the nominal 45 -degree cones of protection associated with each of the strike rods are diagrammed in Figure 3-27. In the case of the 4.5-m rod, both stations fell well outside the 45 -degree protection zone; 8 while with the $11-\mathrm{m}$ pole, Station 1 fell

8 Definitions of "zones of protection" are based on varying interpretations of results from numerous theoretical and empirical electrostatic and statistical models that have been developed over the years in attempts to quantify the probabilities of strikes as functions of range from the highest object within a local vicinity. The protection zone concept is best taken as providing general guidance rather than definitive quantitative boundaries of protection. 


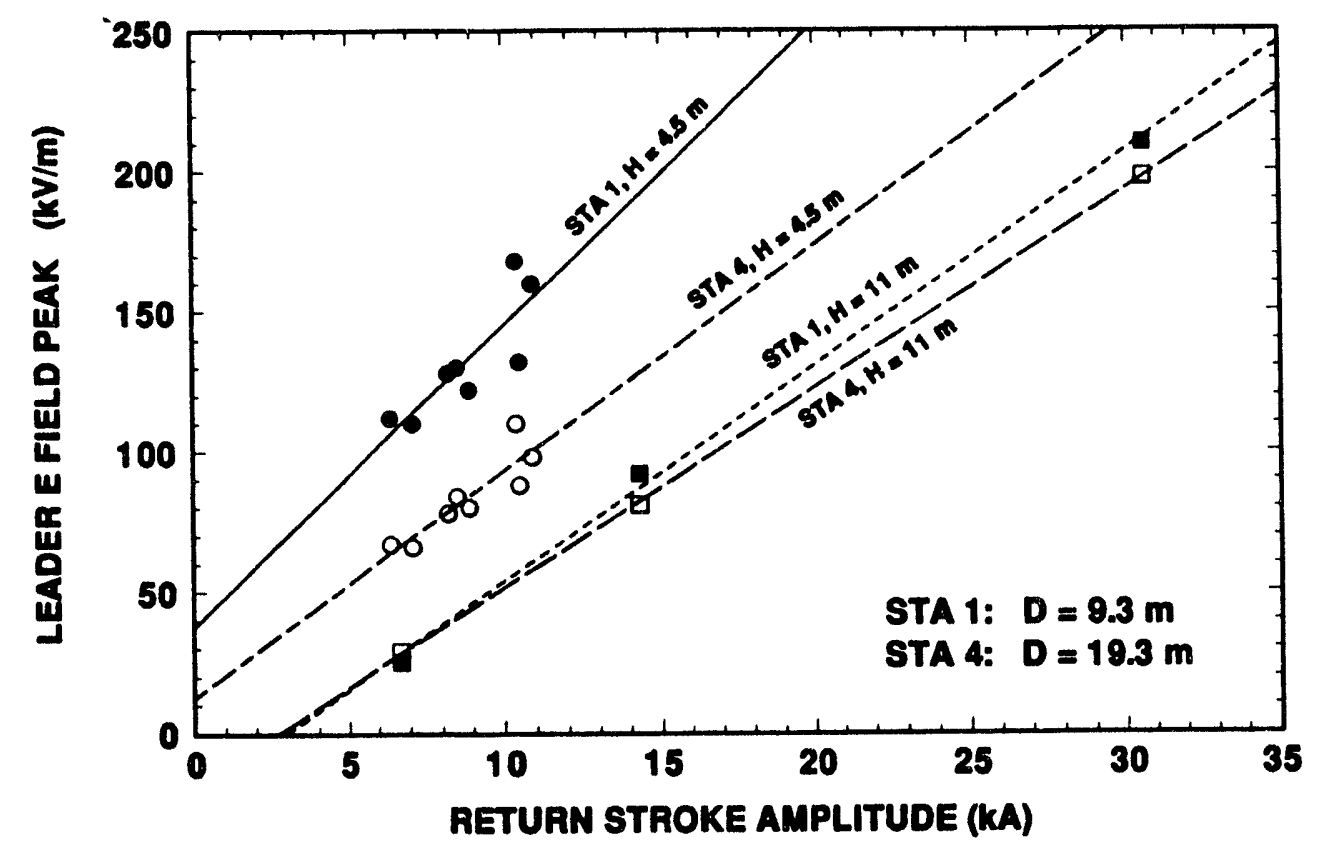

Figure 3-26 Effect of strike rod height $H$ on the vertical electric fields measured at $9.3 \mathrm{~m}$ and $19.3 \mathrm{~m}$ from the strike point

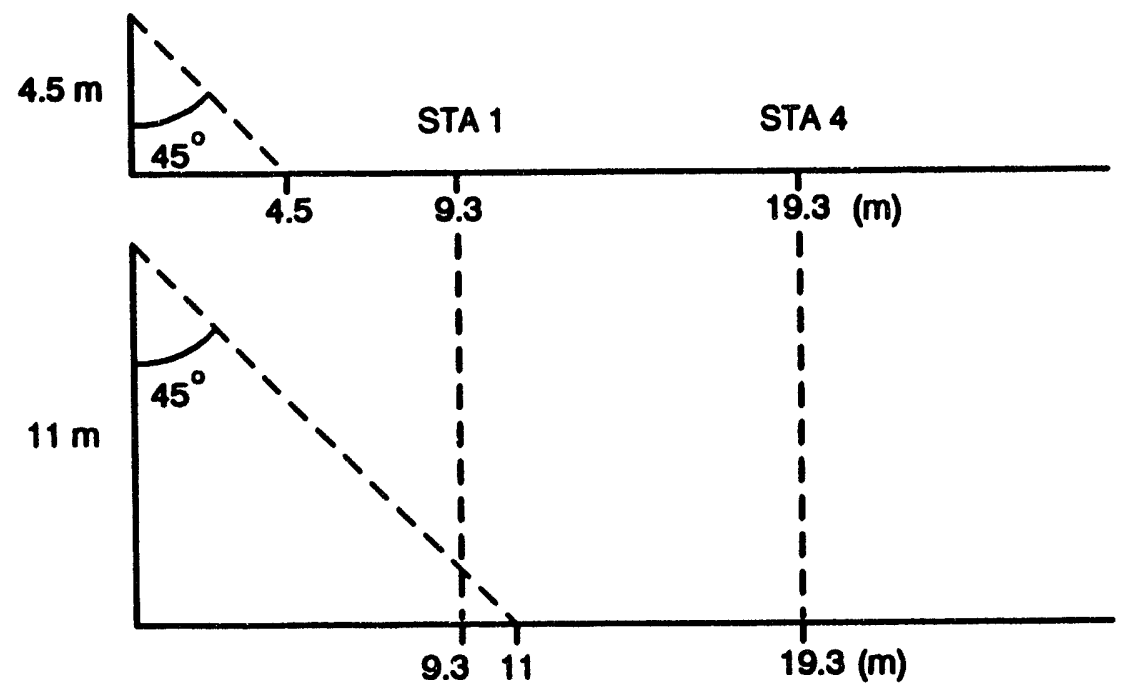

Figure 3-27 Location of recording stations with respect to the nominal 45-degree cones of protection of the 4.5-m and 11-m strike poles used during the present tests 
just within the boundary of the cone, and Station 4 lay at a distance of about a factor of two beyond this imaginary boundary. The question is: To what degree does the increased height of the grounded strike rod reduce the vertical electrostatic field at each station due to an approaching lightning leader?

Consider first the results at Station 1. To be compared are the two lines in Figure 3-26 corresponding to the solid circle $(4.5-\mathrm{m}$ pole) and solid square $(11-\mathrm{m}$ pole) data points. At any given return-stroke amplitude, the higher pole resulted in a vertical electric field intensity that was reduced by a factor of approximately 0.45 . Similarly, as determined from the open circle and open box data lines pertaining to Station 4, which was well outside the zone defined by the cone, the reduction factor was 0.65 -still significant. (This latter observation illustrates the ill-defined nature of the shielding boundary provided by an elevated grounded object.)

In Figure 3-28, these results are further condensed and extrapolated beyond the range covered by the limited data available from the present experiments. The vertical field reduction factor due to an increase of pole height from 4.5 to $11 \mathrm{~m}$ is plotted as a function of the ratio of the radial distance $D$ from the base of the $11-\mathrm{m}$ pole to its height. (In the abscissa of the figure, $\mathrm{H}=11 \mathrm{~m}$.) At distances very large compared to the pole height, the influence of the mast is negligible; that is, the reduction factor approaches unity. At the other extreme very near the base of the mast, ignoring dynamic electromagnetic effects, the vertical field approaches zero.

In applying these results to an analysis of the effectiveness of the pole in protecting an object from a direct strike, it is important to bear in mind that reduction of the vertical field at the ground is only one of several contributing factors. Under low wind conditions, streamer current is roughly proportional to the square of the local ambient electric field [e.g., 16]. Therefore, other factors being equal, an increase in the height of the pole simultaneously produces an increase in the streamer current from its tip and a decrease in the current emitted from the protected object. The changes in the currents from each of the two emitters would be proportional to the square of the incremental changes in applied electric fieid intensities

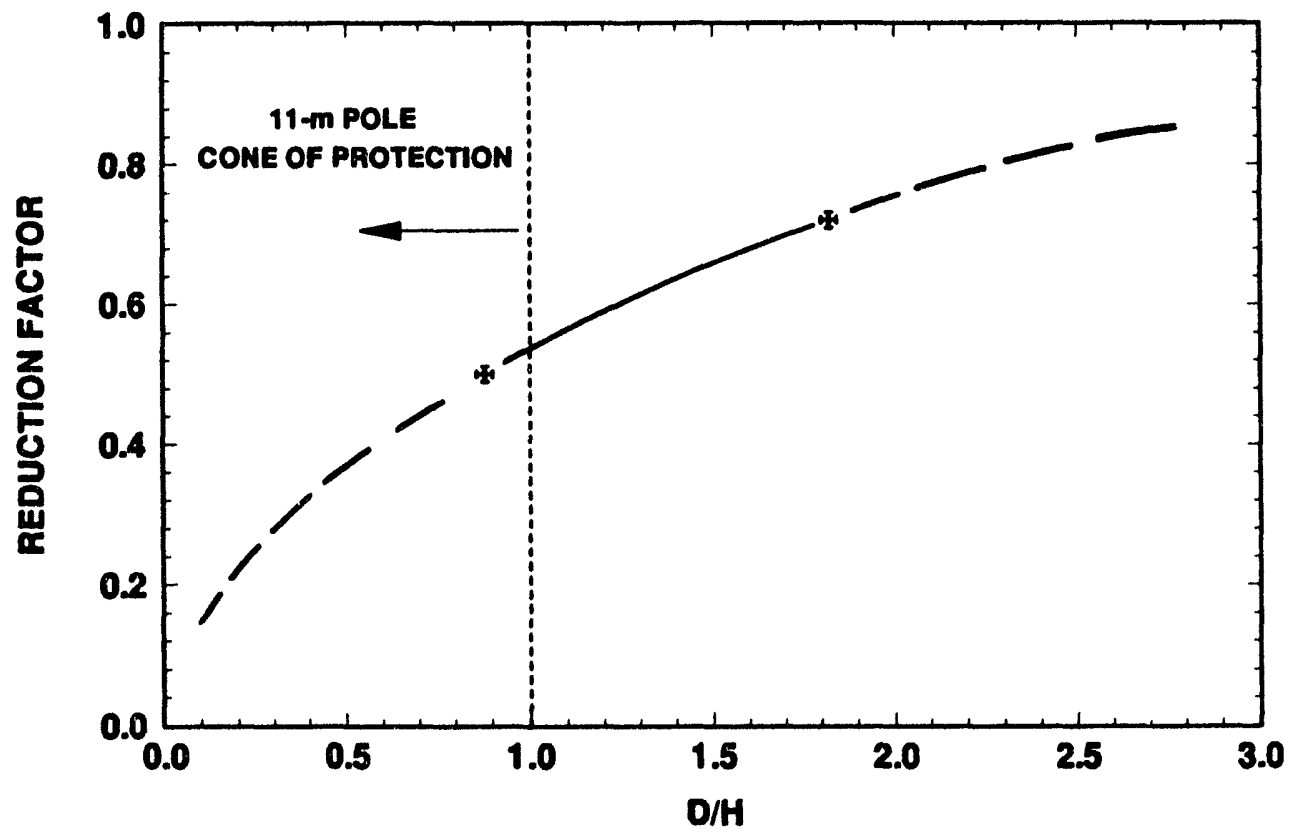

Figure 3-28 Vertical electric field reduction factors measured at distances $D$ of $9.3 \mathrm{~m}$ and $19.3 \mathrm{~m}$ from the strike point due to the increase in strike rod height $\mathrm{H}$ from $4.5 \mathrm{~m}$ to $11 \mathrm{~m}$ 
experienced by each of the points producing the streamers. Finally, there is the advantageous effect associated with the closer physical proximity of the elevated tip of the mast to a vertically downward approaching lightning leader tip. While extremely difficult to quantify reliably, the combined effect of all these factors results in a substantial decrease in the probability of a strike anywhere within the mast's zone of protection, with that probability falling dramatically with decreasing range from the base of the pole.

3.3.1.2 Dielectric Shield. An alternative concept for reducing the probability of a direct strike to an object that was briefly investigated was the use of a shielding layer of dielectric material over the top of the elevated end of a spare rocket launch tube cylinder. Details of this experiment are described in Section 2.2.2.1. In this section, the results of the test are presented and discussed.

The dielectric layer that was installed over the open end of the aluminum launch tube was made up of 20 layers of $0.15-\mathrm{mm}(6-\mathrm{mil})$ thick polyethylene plastic. This tube was installed on the LTA in a manner such that its top was $1.3 \mathrm{~m}$ higher than that of the adjacent tube from which the rocket was launched (Figure 2-11). The test was conducted during flash 93-08. As usual, about 3 seconds after the rocket cleared the tube, the lightning initiation process caused vaporization of the trailing wire from the rocket and an ICC of about 220-ms duration. (Note that the lower end of the trailing wire was connected to the LTA frame at the bottom of the tube from which the rocket was launched and was not directly attached to the "protected" tube extension.) Following a quiescent period of about $10 \mathrm{~ms}$, the usual sequence of return strokes and continuing currents took place. (Refer to Figure 3-7.) In this instance, the flash consisted of a total of 17 return strokes, the first of which had a peak amplitude of $8 \mathrm{kA}$. An annotated schematic diagram of the time history of the flash is given in Appendix D.

As documented by the high speed 16-mm cinematic film record of this flash, the initial return stroke and all others, with one possible exception, attached directly to the top edge of the "protected" tube.

This test of the dielectric shield concept was highly severe in several aspects. In an actual transportation accident scenario in which this protection measure would be applied, the weapon would presumably be lying essentially horizontally on the ground, with its extremity elevated no more than about a meter above the ground. In the present test, because of the lingering presence of the channel conditioned by the wire-burn and ICC phases of the triggered flash, the dart leader initiating the first of the return strokes was artificially directed to seek earth immediately next to elevated tube. This would not be the case in an actual situation. It can, therefore, be appreciated that the electric stress applied across the dielectric during the present test was grossly enhanced beyond what would occur in a natural situation.

The present test was intentionally conducted in this worst-case spirit. The idea was that a demonstrated successful performance by the crude protective cover of polyethylene used herein would surely constitute a convincing demonstration of the viability of the basic idea. This was not the outcome, however, and a much more refined investigation will now be required to establish any ultimate utility of the idea under more realistic conditions. Here the operative word is practicality, since the object of the investigation would really be to define the thickness of the best suited material for such an application that would reliably withstand a reasonable upper bound electrical stress that can be associated with the lightning protection requirement. From that determination, along with consideration of other numerous operational factors and requirements, the practicality of the concept could be judged.

One outcome of the present test that bears due consideration is the fact that the plastic material was noted to be on fire (fully flaming) following the eleventh return stroke, which was accompanied by a robust continuing current of nearly 250 -ms duration. It would appear to be completely unacceptable practice to introduce any inflammable material into close proximity with a damaged weapon. 


\subsubsection{Reduction of Nearby Lightning Environments}

The techniques that were tested for reducing the intensity of electromagnetic environments at a local site very near a lightning strike to ground are described in detail in Section 2.2.2.2. In that section, the test methodology is also discussed. In the following, the results of the comparative tests on the effectiveness of the three environment reduction measures are presented. These include installation of a grounded steel wire mesh over the sensors, installation of a shallowly buried bare copper cable around the sensors, and a combination of both.

3.3.2.1 Effects of a Shielding Cage. A steel wire cage, grounded at all four of its corners, was installed over the sensors at Station 1 (Figure 2-12). Its effect on the peak magnetic field 10-m from the base of two flashes, 93-02 and 93-03, is shown in Figure 3-29 in comparison with the fields recorded at Stations 2 and 3. On the basis of these data, the presence of the cage produces a decrease in the field of only about 12 percent on average. This effect shows up in the magnetic field waveforms primarily as a rounding off of the peaks. (Refer to the magnetic field data for each station on flashes 93-02 and 93-03 in Appendix A.)

A similar comparison of the effect of the cage on measured earth step potentials is given in Figure 3-30. In that figure, the peak values of the step voltages measured during flashes in which the cage was installed are compared with the corresponding voltages obtained during flashes in which it was removed. From these data, it is clear that the cage provides no step voltage reduction of any practical significance.

As would be anticipated, the cage does more significantly affect the vertical electric field within its interior volume. This is demonstrated in Figure 3-31, in which are plotted the ratios of peak values of the electric fields measured at Station 1 with the cage installed to those measured at the unshielded 10-m stations on the same flashes. As indicated in the figure, the wire cage provided reduction of the vertical field by factors of about 25 to 40 . The error bars result from uncertainty in reading the peak values of the noisy low amplitude electric field signals from under the cage.

3.3.2.2 Effect of a Shallowly Buried Ground Ring Electrode. The GRE was installed around the sensors at Station 3 during flashes 93-02 and 93-03. In principle, the main effect is intended to be a shunting of earth currents around the zone within its circumference in order to reduce potential differences developed between different points within the surrounded area.

The solid line in Figure 3-29 relates to the magnetic field data at both Stations 2 and 3; that is, to both the unperturbed station and to the one at which the GRE was installed. As can be seen, there is no significant spread in the combined set of data points; which is to say, there is basically no effect of the presence of the buried cable on magnetic fields at Station 3. By implication, therefore, there is no significant difference in the radial component of the ground current density at the two stations.

This observation is further emphasized by the data shown in Figure 3-32. Here the solid line corresponds to step voltages measured at Station 2 during all flashes. The data points acquired at Station 3 during flashes in which the GRE was installed are shown as solid dots in the figure. It is evident that the GRE had very little effect on those voltages. However, as is indicated by the dashed line associated with the triangular data points, with the addition of a grounded wire cage, the combination did produce a reduction in the observed voltages of about a factor or 1.6. Why this is so is not clear, given the lack of any significant effect due to either the cage or GRE separately. In any event, the factor of 1.6 is not likely to warrant the economic and operational costs associated with adopting their use as standard EOD practice. 


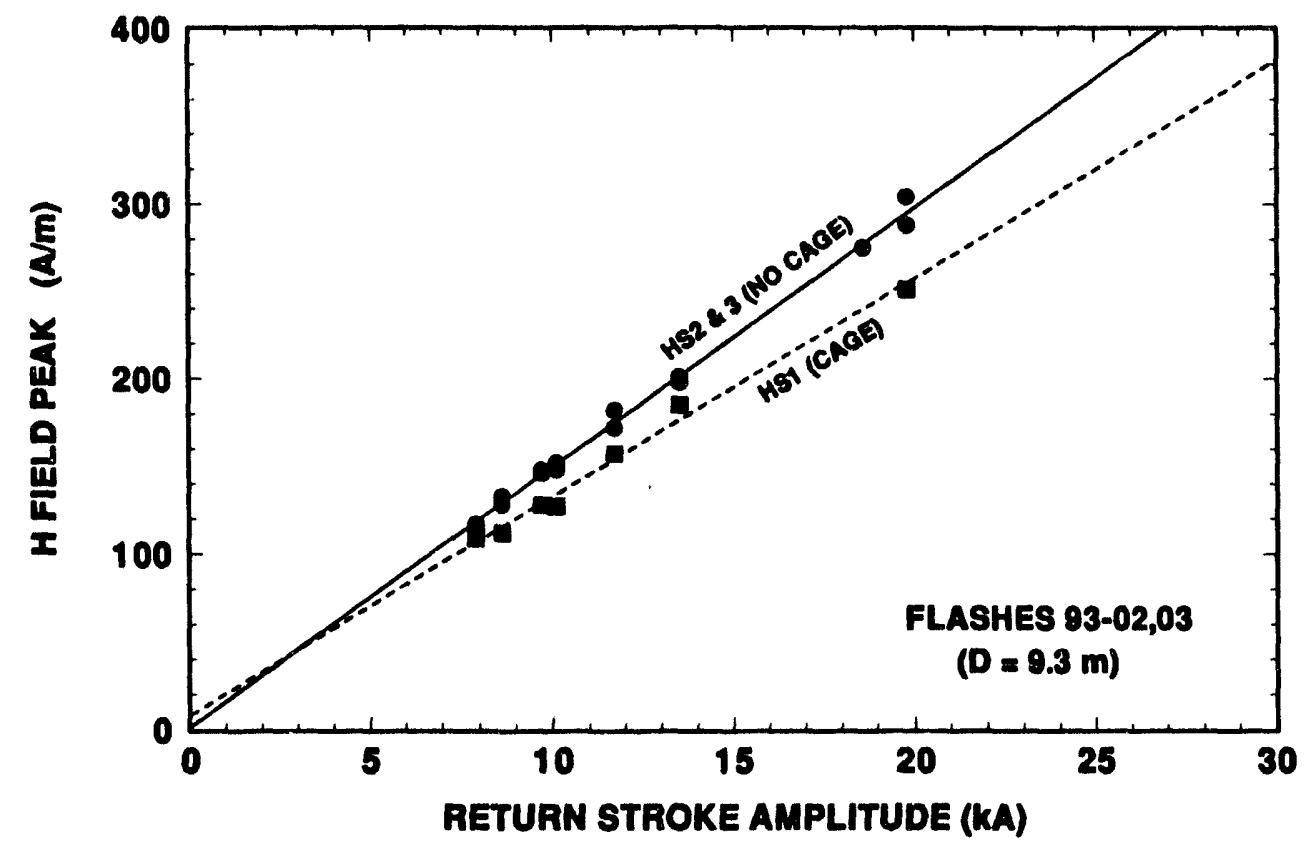

Figure 3-29 The effect of the presence of the wire cage on the magnetic fields measured at Station 1

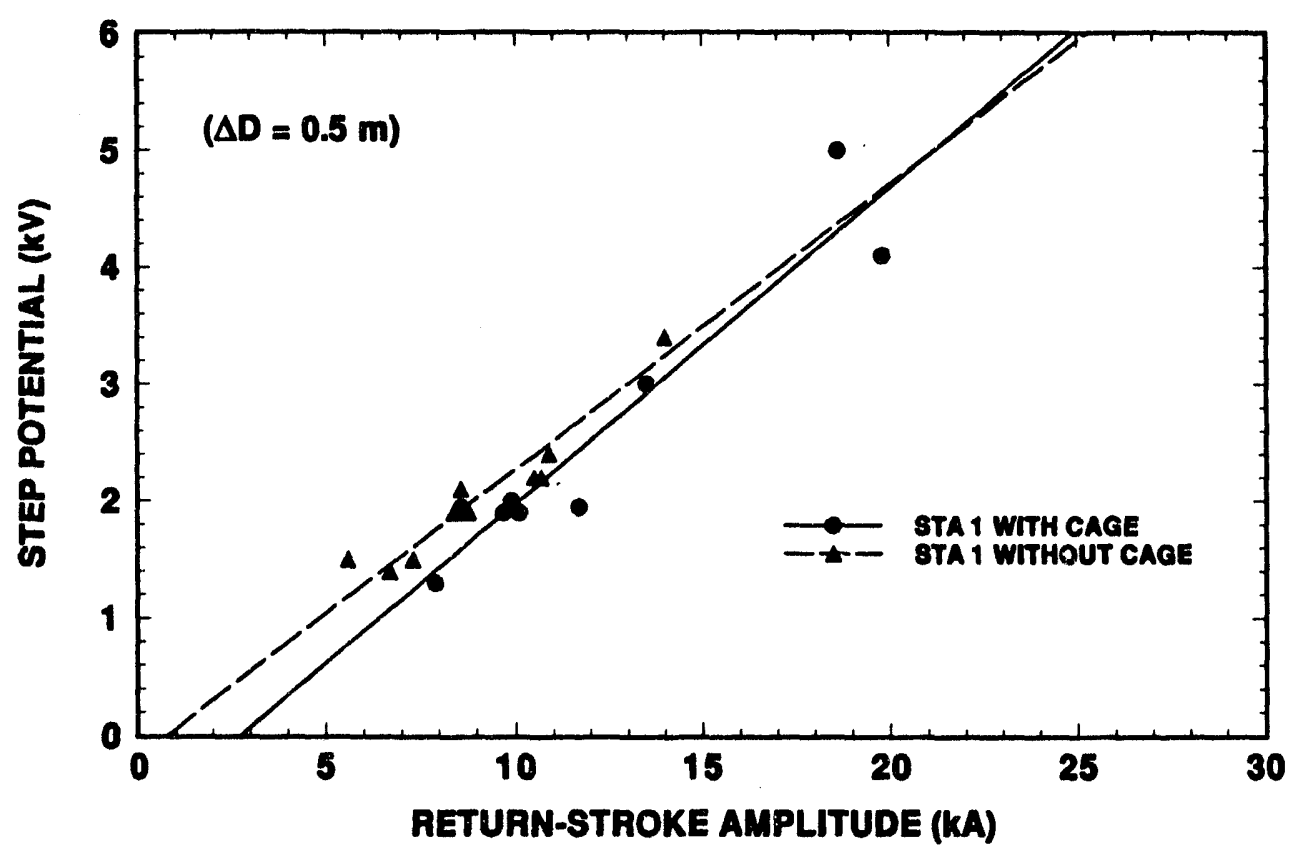

Figure 3-30 The effect of the presence of the wire cage on the earth step potentials measured at Station 1 


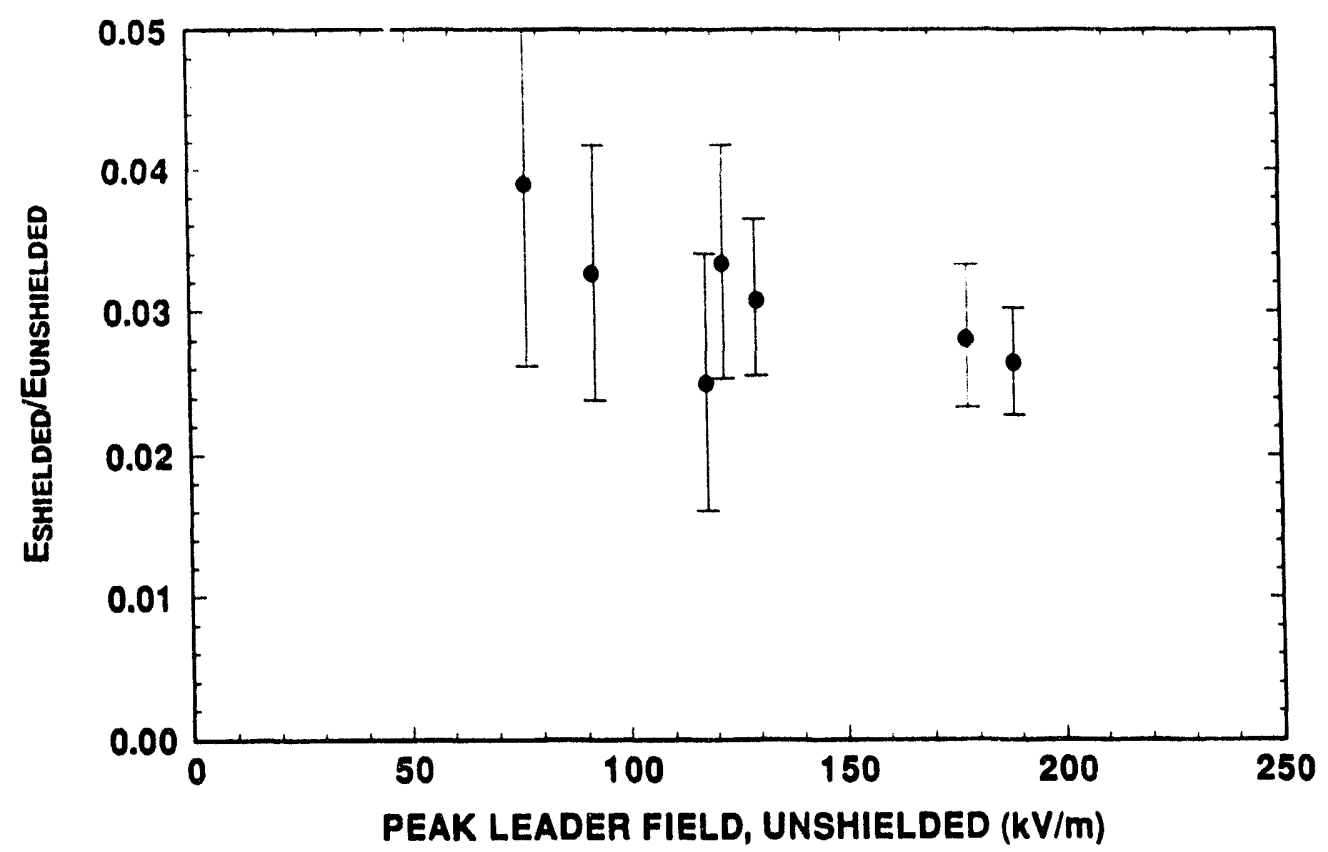

Figure 3-31 The effect of the wire cage on vertical electric fields measured during the same strokes at $9.3 \mathrm{~m}$ from the strike point; shown are the ratios of electric fields measured at Station 1, with the cage installed, to those measured the same distance from the strike point at Station 2, where no cage was present

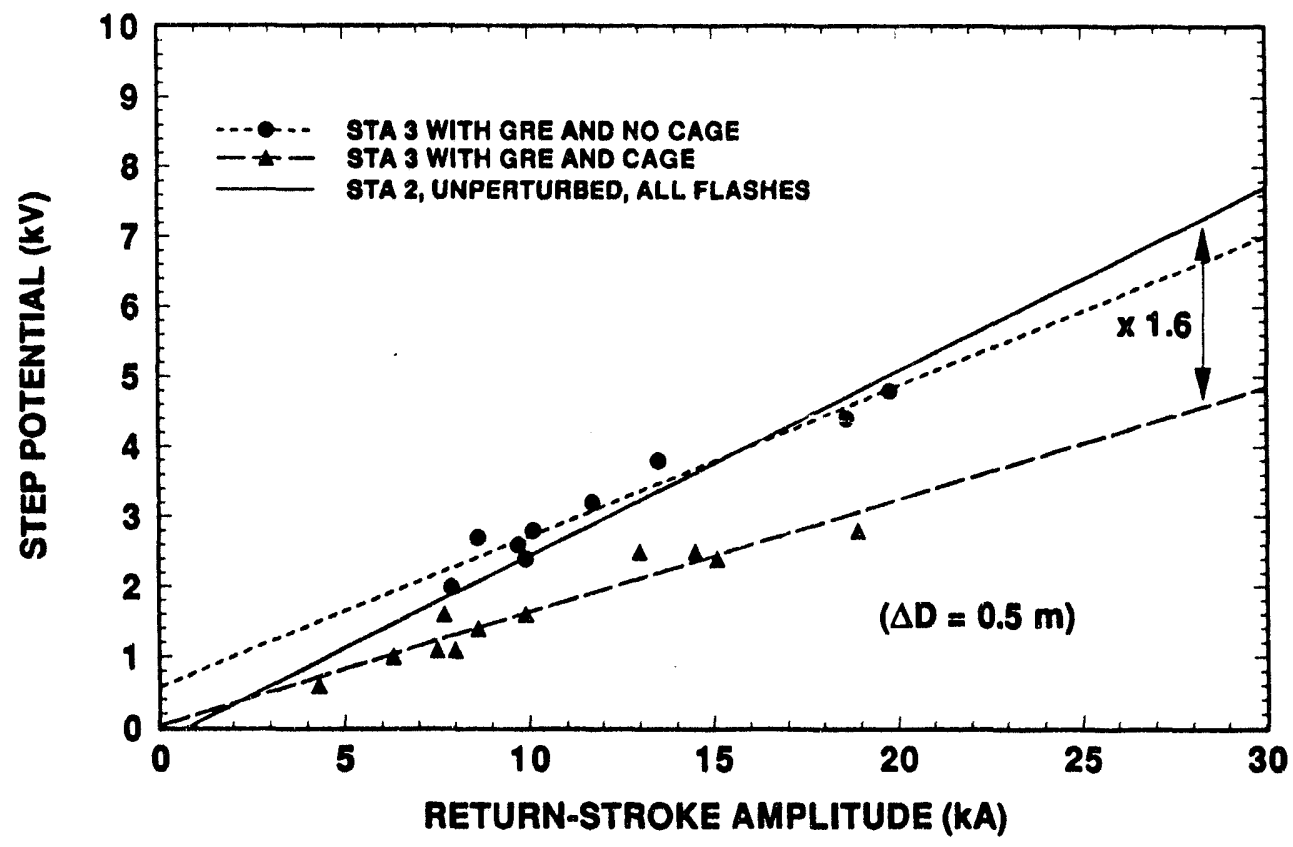

Figure 3-32 The effects of the presence of ground ring electrode and cage, separately and in combination, on earth step voltages at $10 \mathrm{~m}$ from the strike point 


\subsection{Conclusions}

The main conclusions derived to date from analysis of the data from the experiments to characterize the electromagnetic environment close to the base of a lightning channel are given below. These conclusions are strictly valid only over the appropriate ranges covered by the available data base, which, with respect to return-stroke amplitude, is from 4.4 to $30 \mathrm{kA}$. Extrapolation well beyond those limits appears to be reasonable; but, as always, extrapolation is accompanied by an increase in uncertainty.

1. The magnetic field at ground level out to a range of 20 meters from the base of a lightning channel is well described by Ampere's law.

2. Peak return-stroke current amplitudes are linear (with correlation coefficients of 0.65 and 0.95 at $10 \mathrm{~m}$ and $20 \mathrm{~m}$, respectively) with respect to the vertical electric field changes produced by approaching dart leaders.

3. Vertical electric field change amplitudes as high as $300 \mathrm{kV} / \mathrm{m}$, occurring with rise times of the order of a few microseconds, can be experienced at a range of $20 \mathrm{~m}$ from the lightning channel for a return-stroke of $30 \mathrm{kA}$ amplitude. (If the linear relationship of item 2 is assumed to hold, a field change in excess of $2 \mathrm{MV} / \mathrm{m}$ would precede a 200-kA subsequent stroke.)

4. Earth step potentials measured at 10 and $20 \mathrm{~m}$ from the channel base are linear with returnstroke current. Over the range of 10 to $20 \mathrm{~m}$, their magnitudes fall off as $1 / \mathrm{r}$.

5. The likelihood of significant radial filamentary surface arcing out to ranges well in excess of $20 \mathrm{~m}$ from the base of the channel must be considered to be part of the hazardous environment of a nearby lightning flash. Individual branches of the arc can carry significant fractions of the total incident return-stroke current.

The data from the test of a vertical metallic protection mast confirm its effectiveness in significantly reducing the probability of a direct strike to an item located within a protection zone of some radius from its base, probably somewhat in excess of the height of the rod. The strike rods employed during the present tests were intentionally poorly grounded. It is probable that by optimizing the earthing provisions at the base of such a protection mast, the occurrence and severity of arcing at the base of the mast would be substantially reduced or eliminated and the direction of any residual surface arc propagation could be controlled. Without some sort of well grounded preferential strike rod, uncontrolled surface arcing will occur around the strike point to raw earth with a very high probability, even in regions of relatively high earth conductivity.

The concept of using a dielectric blanket of some sort over an object to suppress the development of streamers from its extremities did not prove successful during its initial test. The test, however, was drastically severe, and a more refined investigation will be required to resolve whether the approach offers any ultimate practical utility.

Neither the installation of a partial Faraday cage over a designated monitoring point nor the presence of a circular, shallowly buried ground ring electrode around that point $10 \mathrm{~m}$ from the base of the triggered lightning channels proved to be significantly effective in reducing the lightning environments. While installation of the cage did produce a decrease in vertical electric field of a factor of 20 or more, neither measure appreciably affected either the magnetic field or radial electric field within the earth, which is responsible for producing earth step potentials. 


\subsection{References}

1. Schnetzer, G.H. and R.J. Fisher, "The Sandia Transportable Triggered Lightning Instrumentation Facility," Proc. 1991 International Aerospace and Ground Conference on Lightning and Static Electricity, NASA Conf. Publication 3106, Cocoa Beach, Florida, April 16-19, 1991.

2. Fisher, R.J. and G.H. Schnetzer, 1990 Rocket-Triggered Lightning Field Tests at Kennedy Space Center, Florida, SAND90-2926, March 1991.

3. Schnetzer, G.H. and R.J. Fisher, 1991 Rocket-Triggered Lightning Test of the DoD Security Operations Test Site (SOTS) Munitions Storage Bunker, Ft. McClellan, Alabama, Volume 1, SAND91-2343, February 1992.

4. M. Rubinstein, F. Rachidi, M.A. Uman, R. Thottappillil, C.A. Nucci, E.M. Thompson, P. Medelius, "Characterization of Vertical Electric Fields at $500 \mathrm{M}$ and $30 \mathrm{M}$ from Triggered Lightning," Submitted to J. Geophys. Res., January 1994.

5. Ramo, S., J.R. Whinnery, and T. Van Duzer, Fields and Waves in Communications Electronics, J. Wiley and Sons, New York, (1967) p. 444.

6. Lin, Y.T., M.A. Uman, and R.B. Standler, "Lightning Return Stroke Models," J. Geophys. Res., Vol. 85, No. C3, March 20, 1980.

7. Unpublished data; R. Thottappillil, University of Florida, November 1993.

8. Unpublished data; R. Thottappillil, University of Florida, November 1993.

9. Uman, M.A., The Lightning Discharge, Academic Press, Orlando, (1987).

10. Uman, M.A. et al. "Multiple-Station Measurements of Close Electric and Magnetic Fields Produced by Triggered Lightning," Fall Meeting, Amer. Geophys. Union, San Francisco, December 6-10, 1993.

11. Idone, V.P., "Length Bounds for Connecting Discharges in Triggered Lightning Subsequent Strokes," J. Geophys. Res., Vol. 95, No. D12, November 20, 1990.

12. Fisher, R.J., G.H. Schnetzer, R. Thottappillil, V.A. Rakov, M.A. Uman, and J.D. Goldberg, "Parameters of Triggered Lightning Flashes in Florida and Alabama," J. Geophys. Res., Vol. 98, No. D12, December 20, 1993.

13. Krider, E.P., C.D. Weidman, and R.C. Noggle, "The Electric Fields Produced by Lightning Stepped Leaders," J. Geophys. Res. Vol. 82, 1977.

14. Schonland, B.F.J., "The Lightning Discharge," Handbk. Phys., 22:576-628 (1956), as adapted in Table 8.3 of Ref. 9 above.

15. Liew, A.C. and M. Darveniza, "Dynamic Model of Impulse Characteristics of Concentrated Earths," Proc. IEE, Vol. 121, No. 2, February 1974.

16. Chalmers, J.A., Atmospheric Electricity, 2nd Edition, Pergamon Press (1967). 
17. Colton, F.B., "Lightning in Action," National Geographic, 97-106, 809-28, 1950.

18. Viemeister, P.F., The Lightning Book, Doubleday, New York (1961).

19. Krider, E.P., "On Lightning Damage to a Golf Course Green," Weatherwise, Vol. 30, No. 3 , June 1977.

20. Davis, D.A., W.C. Murray, W.P. Winn, Q. Mo, P.R. Buseck, J. Knell, and B.D. Hibbs, "Fulgurites from Triggered Lightning," Trans. Amer. Geophys. Union, October 1993.

21. Uman, M.A., V.A. Rakov, R. Thottappollil, J. Versaggi, A. Eybert-Berard, L. Berret, P.P. Barker, and S.P Hnat, "Multiple-Station Measurements of Close Electric and Magnetic Fields Produced by Triggered Lightning Discharges," Trans. Amer. Geophys. Union, October 1993. 


\section{Appendix A \\ 1993 Triggered Lightning Data}

Presented in this appendix is a full set of all the measurements obtained during the course of the experiments covered in this report. Where appropriate, each data trace is presented on two time scales, corresponding to the full digitized record and to an expanded version that makes examination of the wavefront possible.

Table A-1 summarizes the set of data that were acquired. This list reflects intentional apportioning of the available instrumentation and the fact that, in some cases, data were lost for one reason or another. The data are grouped by flash number, with the types of measurements being presented in the following order: full flash current, individual return-stroke currents, magnetic fields, vertical electric fields, earth step potentials, and miscellaneous. In each category, the data are given in ascending stroke number order.

Table A-1.

Summary of Avallable Data According to Flash Number

\begin{tabular}{|c|c|c|c|c|c|c|}
\hline \multirow[b]{2}{*}{ Flash } & \multirow[b]{2}{*}{ Measurement } & \multicolumn{5}{|c|}{ Station } \\
\hline & & 1 & 2 & 3 & 4 & 5 \\
\hline $\begin{array}{c}\text { 93-02 } \\
7 \text { Strokes } \\
{\text { (Unreliable } I_{\text {inc }}{ }^{*} \text { ) }}^{\text {Und }}\end{array}$ & $\begin{array}{l}E_{V} \\
H_{a} \\
V_{s}\end{array}$ & $\begin{array}{l}X \\
X \\
X\end{array}$ & $\begin{array}{l}X \\
X \\
X\end{array}$ & $\begin{array}{l}X \\
X \\
X\end{array}$ & $\begin{array}{l}-- \\
-- \\
--\end{array}$ & $\begin{array}{l}- \\
- \\
--\end{array}$ \\
\hline $\begin{array}{c}93-03 \\
2 \text { Strokes } \\
\text { (Unreliable I Inc) }\end{array}$ & $\begin{array}{l}E_{v} \\
H_{a} \\
V_{s}\end{array}$ & $\begin{array}{l}X \\
X \\
X\end{array}$ & $\begin{array}{l}\mathbf{X} \\
\mathbf{X} \\
\mathbf{X}\end{array}$ & $\begin{array}{l}X \\
X \\
X\end{array}$ & $\begin{array}{l}-- \\
-- \\
-\end{array}$ & $\begin{array}{l}-- \\
-- \\
-\end{array}$ \\
\hline $\begin{array}{l}\text { 93-08 } \\
8 \text { Strokes } \\
\text { ( } I_{\text {inc }} \text { lost) }\end{array}$ & $\begin{array}{l}\mathrm{E}_{\mathrm{v}} \\
\mathrm{H}_{\mathrm{a}} \\
\mathrm{V}_{\mathrm{s}}\end{array}$ & $\begin{array}{l}X \\
X \\
--\end{array}$ & $\begin{array}{l}-- \\
\mathbf{X} \\
\mathbf{X}\end{array}$ & $\begin{array}{l}X \\
-- \\
X\end{array}$ & $\begin{array}{l}-- \\
-- \\
--\end{array}$ & $\begin{array}{l}-- \\
-- \\
--\end{array}$ \\
\hline $\begin{array}{c}\text { 93-09 } \\
3 \text { Strokes } \\
\left(I_{\text {inc }} \text { lost }\right)\end{array}$ & $\begin{array}{l}E_{v} \\
H_{a} \\
V_{S}\end{array}$ & $\begin{array}{l}X \\
X \\
--\end{array}$ & $\begin{array}{l}-- \\
X \\
X\end{array}$ & $\begin{array}{l}\mathbf{X} \\
-- \\
\mathbf{X}\end{array}$ & $\begin{array}{l}-- \\
-- \\
-\end{array}$ & $\begin{array}{l}-- \\
-- \\
-\end{array}$ \\
\hline $\begin{array}{c}\text { 93-12 } \\
2 \text { Strokes } \\
\text { (Good I inc) }\end{array}$ & $\begin{array}{l}\mathrm{E}_{\mathrm{v}} \\
\mathrm{H}_{\mathrm{a}} \\
\mathrm{V}_{\mathrm{S}}\end{array}$ & $\begin{array}{l}X \\
X \\
X\end{array}$ & $\bar{x}$ & $\begin{array}{l}-- \\
-- \\
--\end{array}$ & $\begin{array}{l}\mathbf{X} \\
\mathbf{X} \\
\mathbf{X}\end{array}$ & $\begin{array}{l}-- \\
X \\
X\end{array}$ \\
\hline $\begin{array}{c}93-14 \\
1 \text { Stroke } \\
\left(\text { Good } I_{\text {inc }}\right)\end{array}$ & $\begin{array}{l}E_{v} \\
H_{a} \\
V_{s}\end{array}$ & $\begin{array}{l}X \\
X \\
X\end{array}$ & $\begin{array}{l}-- \\
-- \\
X\end{array}$ & $\begin{array}{l}-- \\
-\end{array}$ & $\begin{array}{l}X \\
X \\
X\end{array}$ & $\begin{array}{l}-- \\
X \\
X\end{array}$ \\
\hline $\begin{array}{c}\text { 93-15 } \\
8 \text { Strokes } \\
\left.\text { (Good I } \text { inc }_{\text {) }}\right)\end{array}$ & $\begin{array}{l}\mathrm{E}_{\mathrm{v}} \\
\mathrm{H}_{\mathrm{a}} \\
\mathrm{V}_{\mathrm{S}}\end{array}$ & $\begin{array}{l}X \\
X \\
X\end{array}$ & $\begin{array}{l}-- \\
-- \\
X\end{array}$ & $\begin{array}{l}-- \\
-- \\
--\end{array}$ & $\begin{array}{l}\mathbf{X} \\
\mathbf{X} \\
\mathbf{X}\end{array}$ & $\begin{array}{l}- \\
\mathbf{X} \\
\mathbf{X}\end{array}$ \\
\hline
\end{tabular}

${ }^{*} I_{\text {inc }}=$ directly recorded return-stroke current 


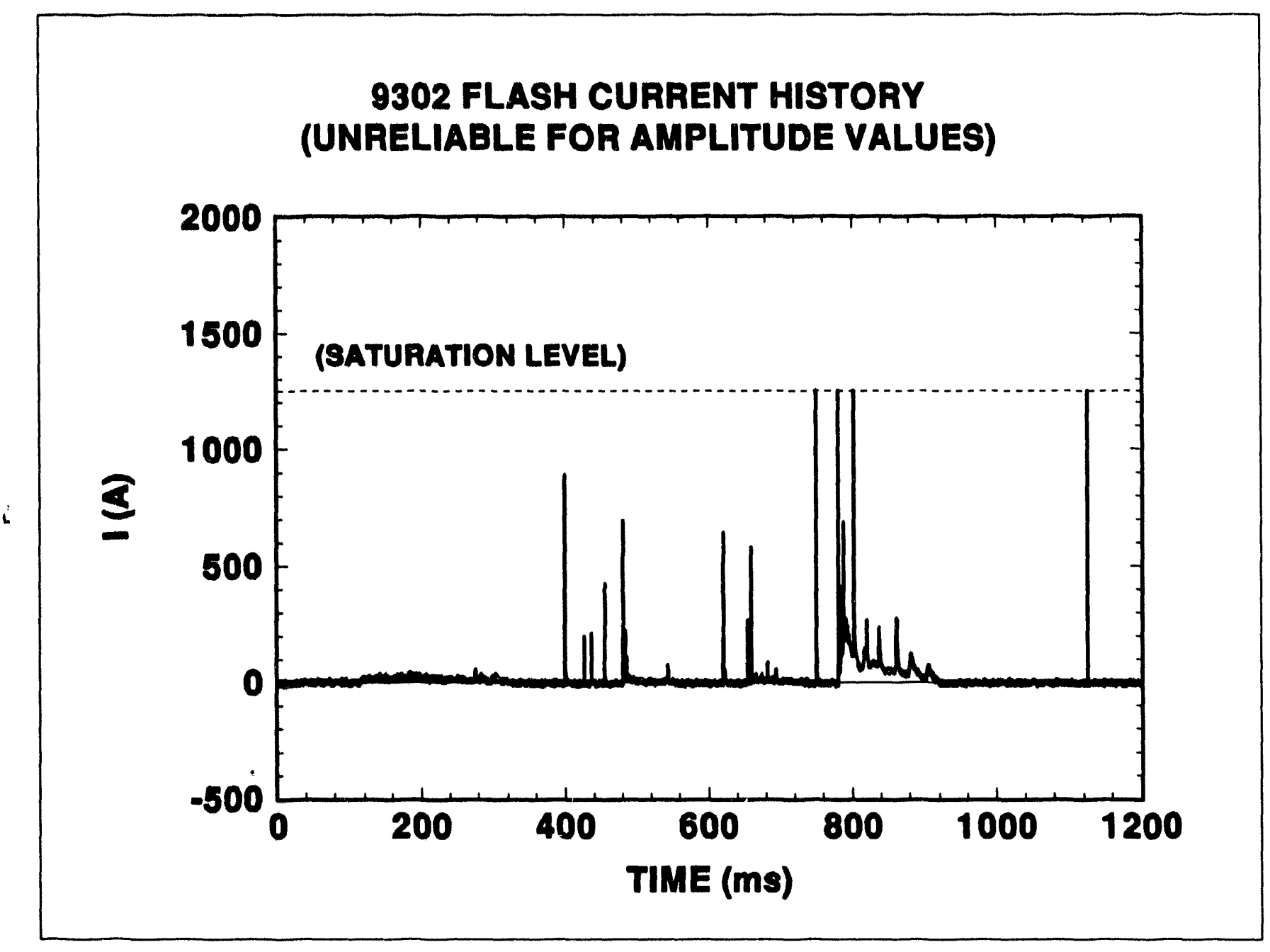


93-02 STROKE 1

HS1
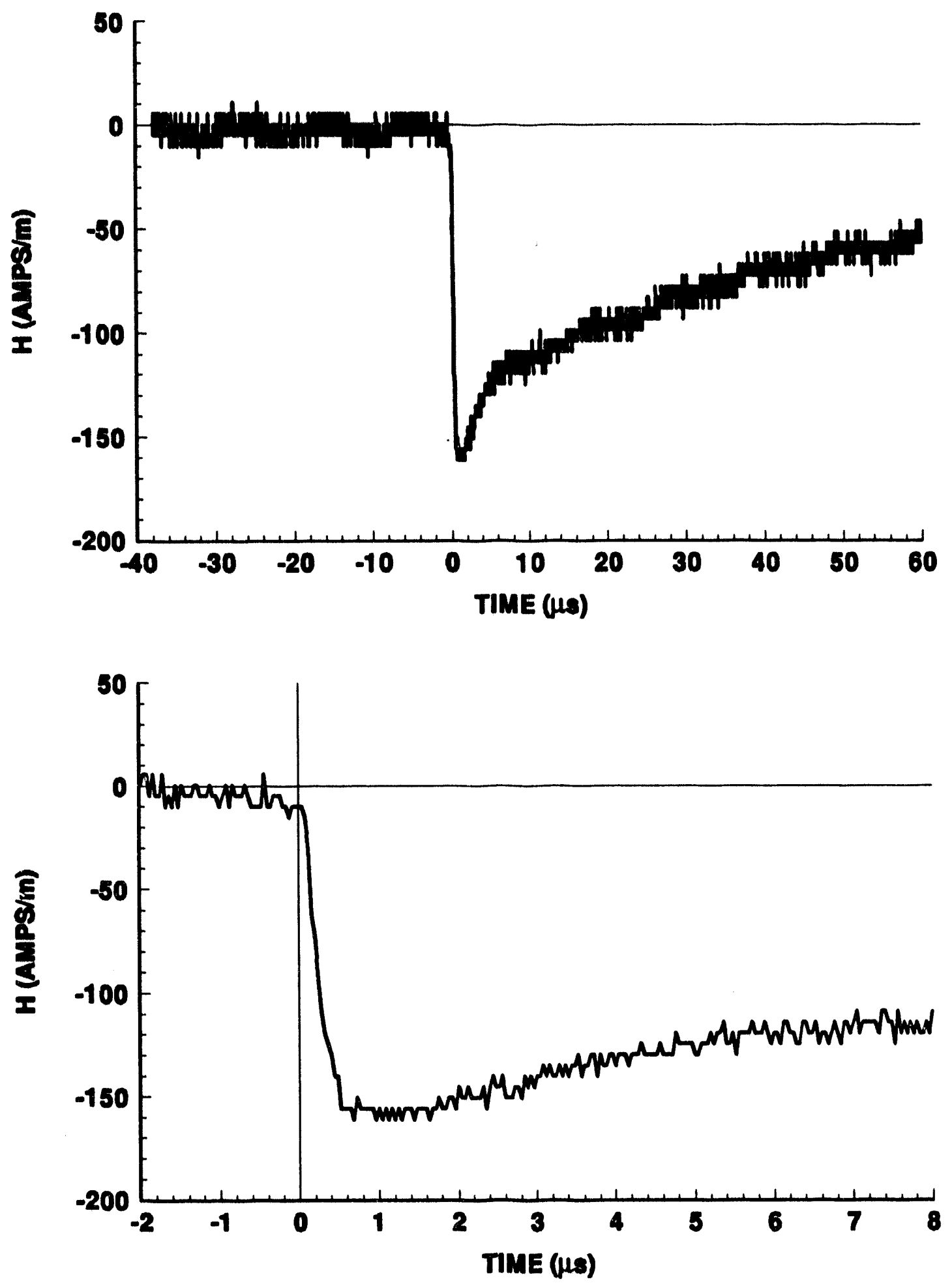


\section{3-02 STROKE 2}

HS1
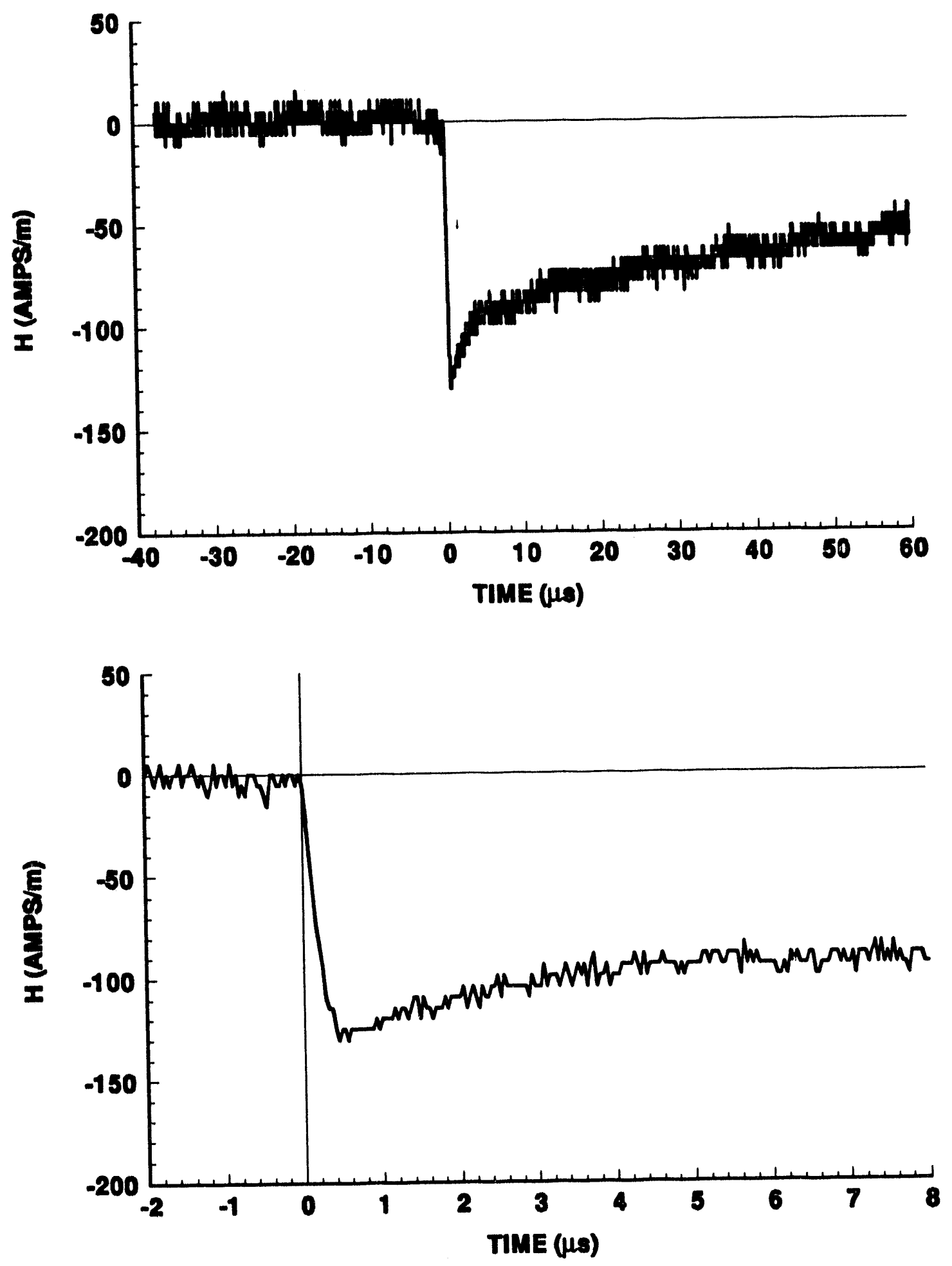


\section{STROKE 3}

\section{HS1}
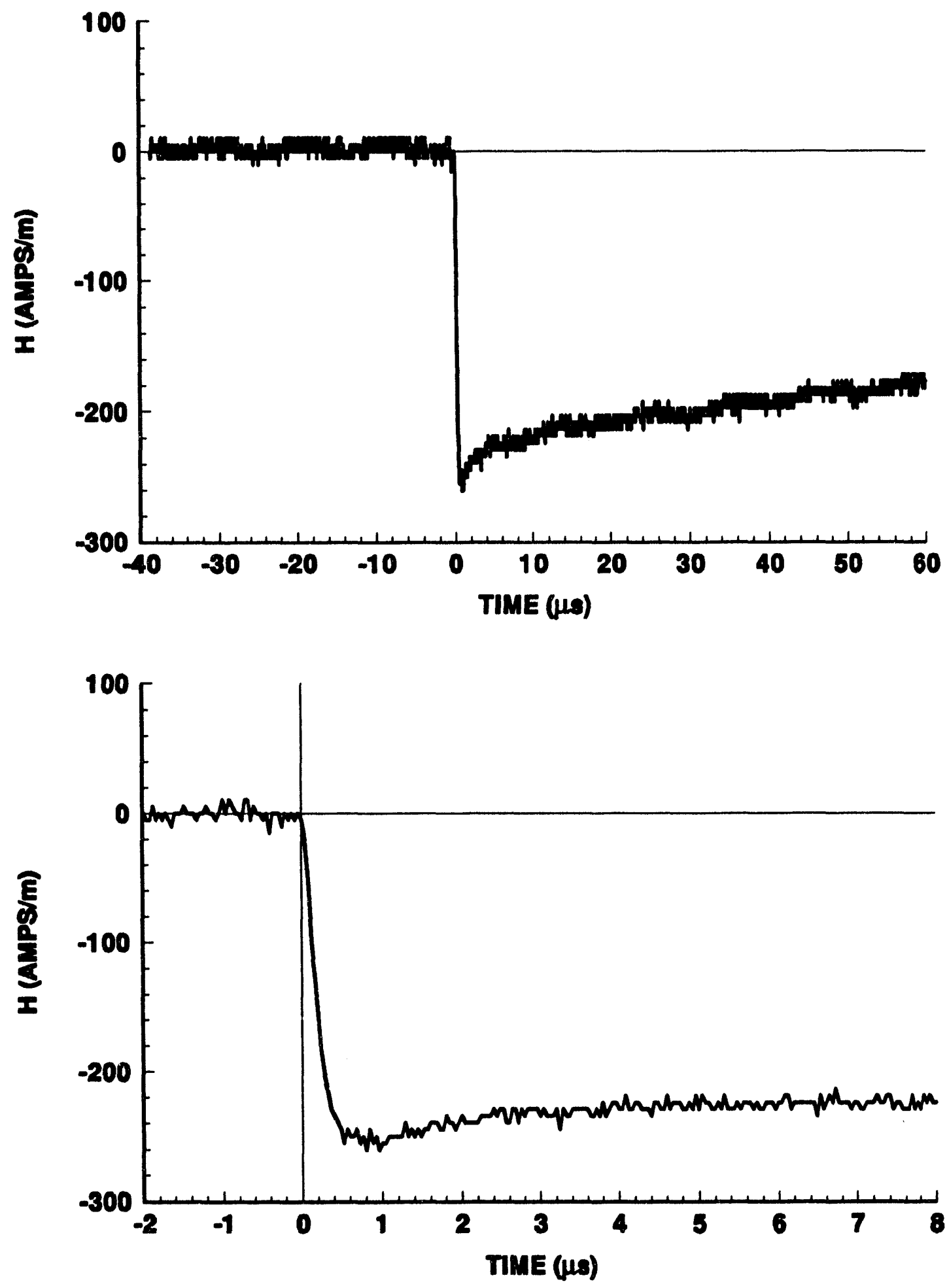


\section{STROKE 4 \\ HS1}
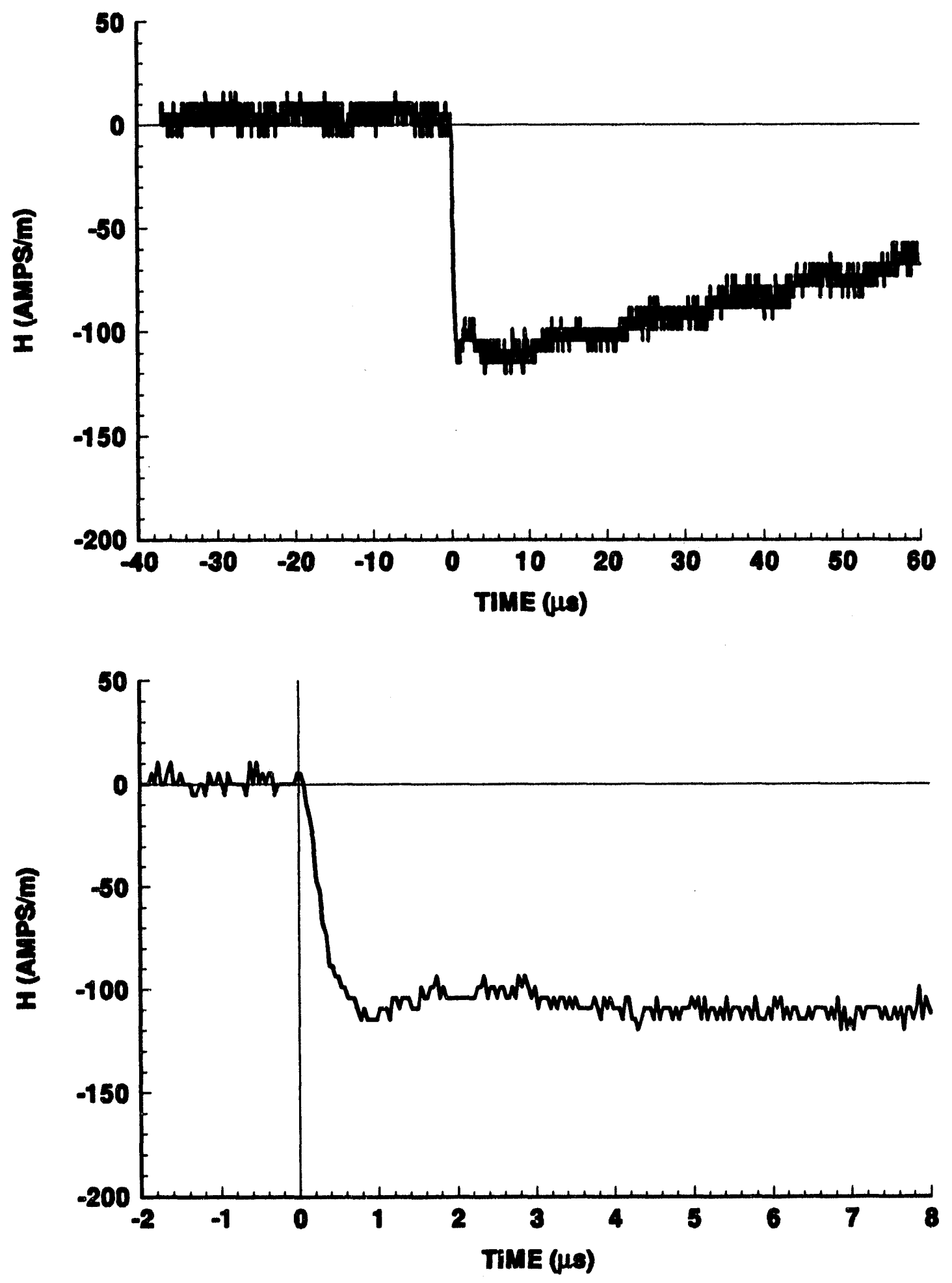


\section{STROKE 5}

HS1
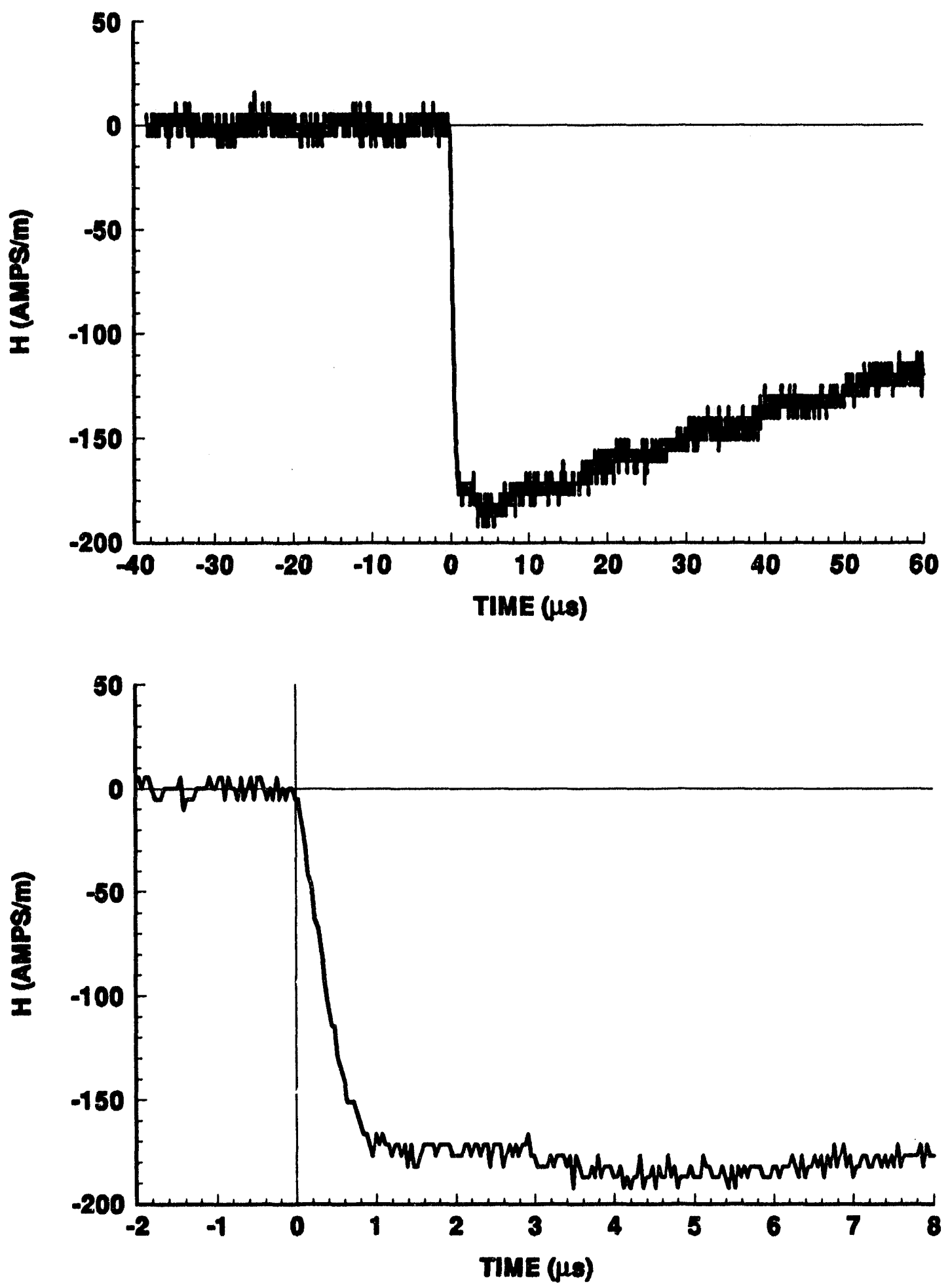


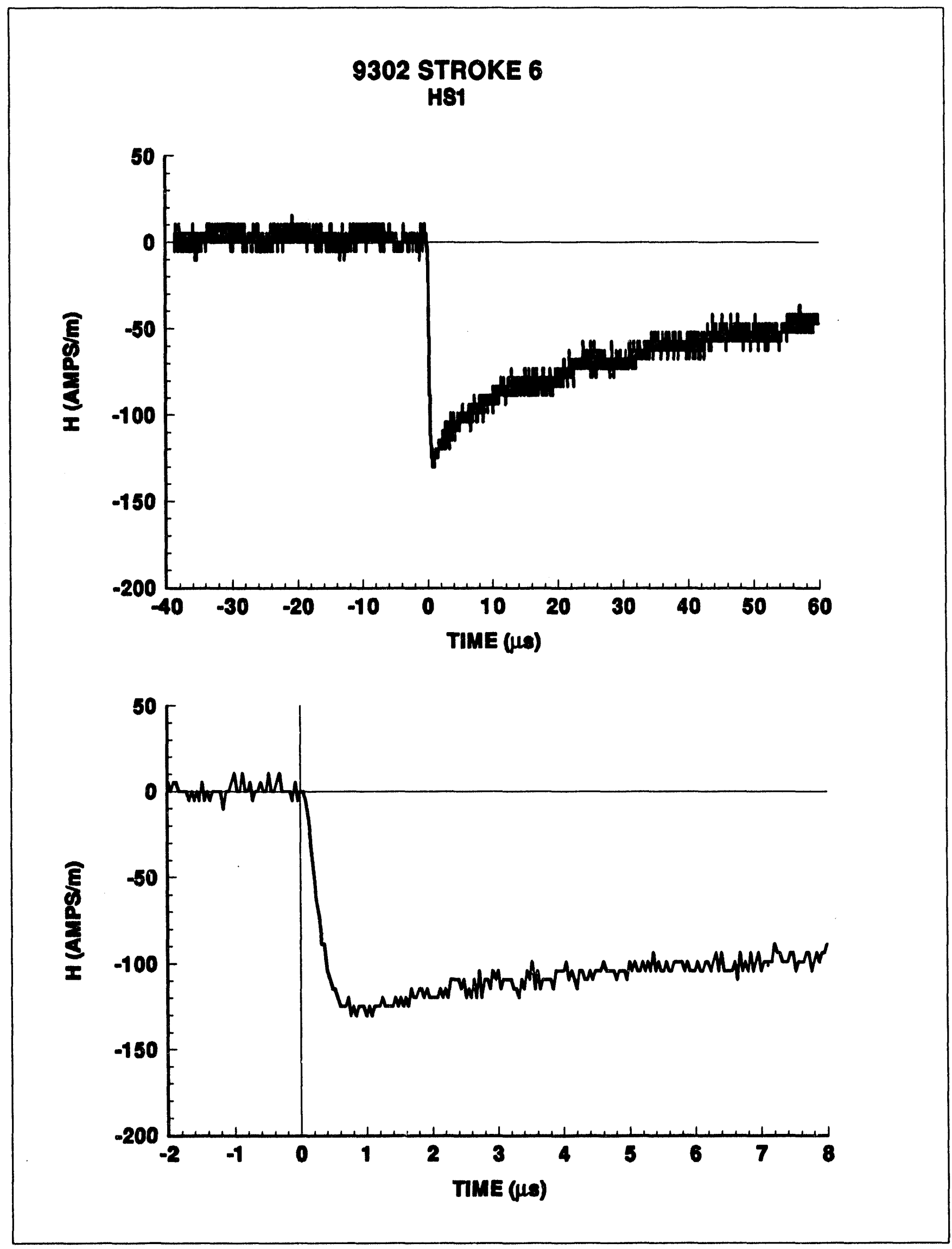




\section{STROKE 7}

HS1
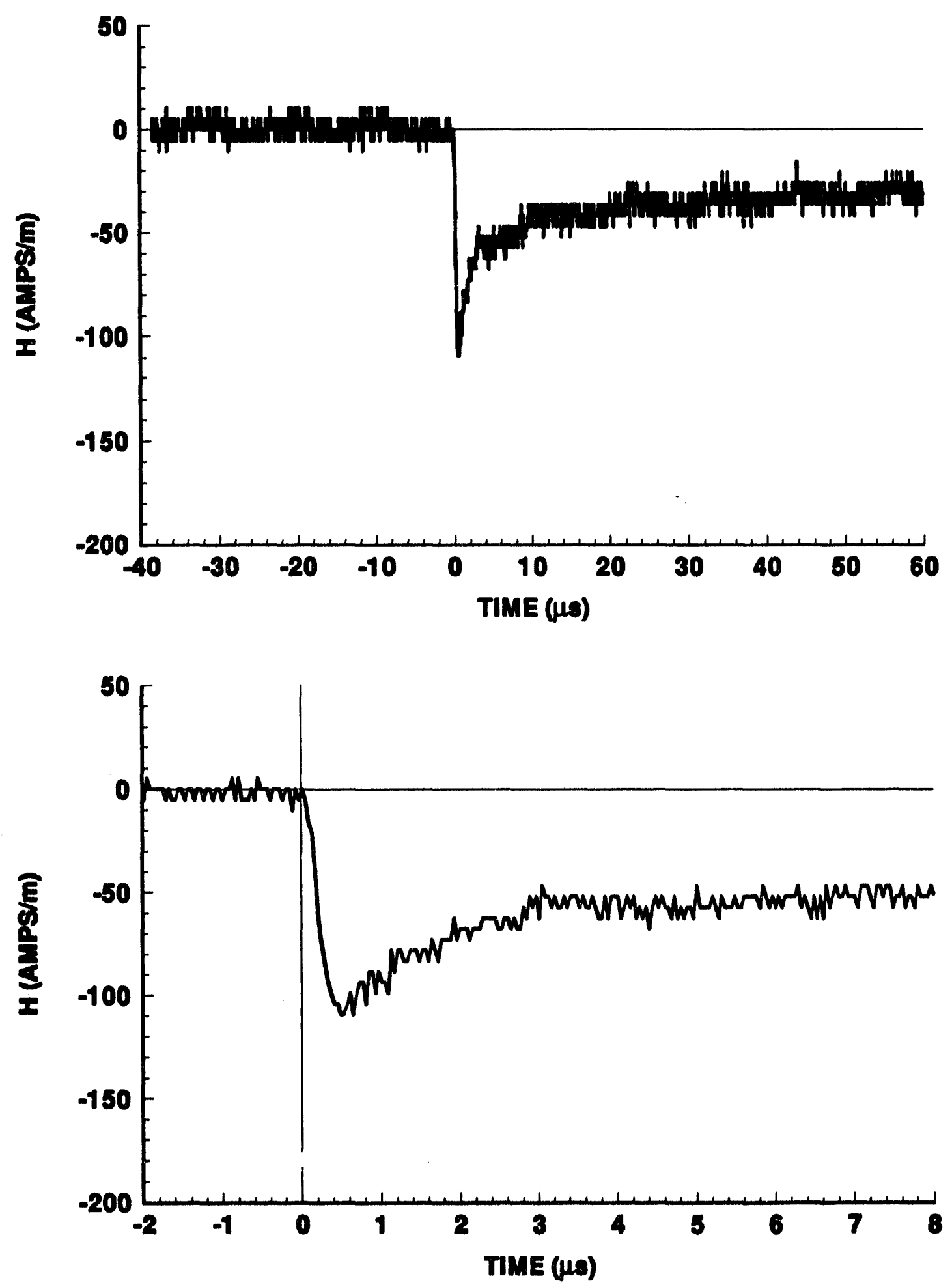


\section{STROKE 1}

HS2
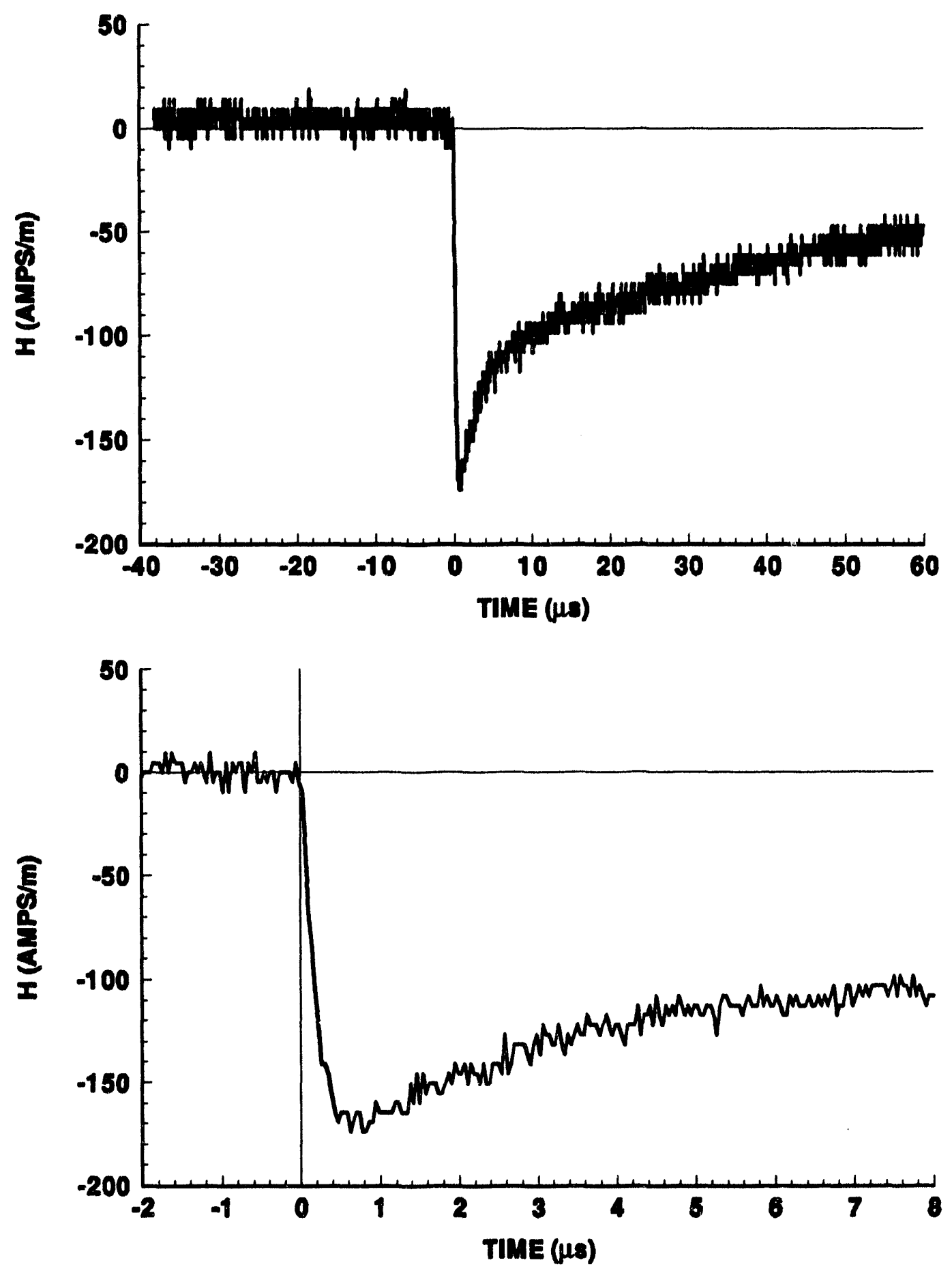


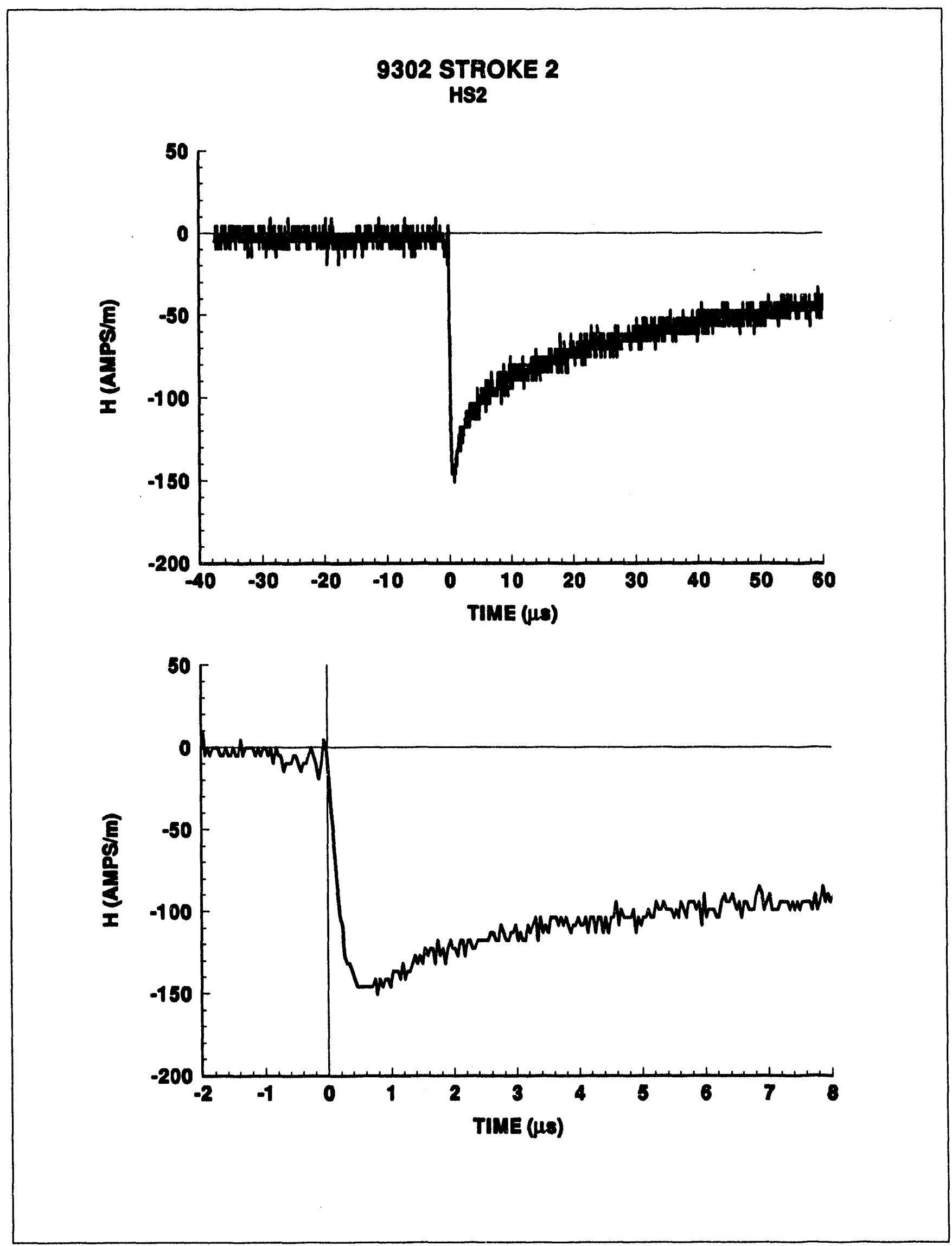




\section{STROKE 3 \\ HS2}
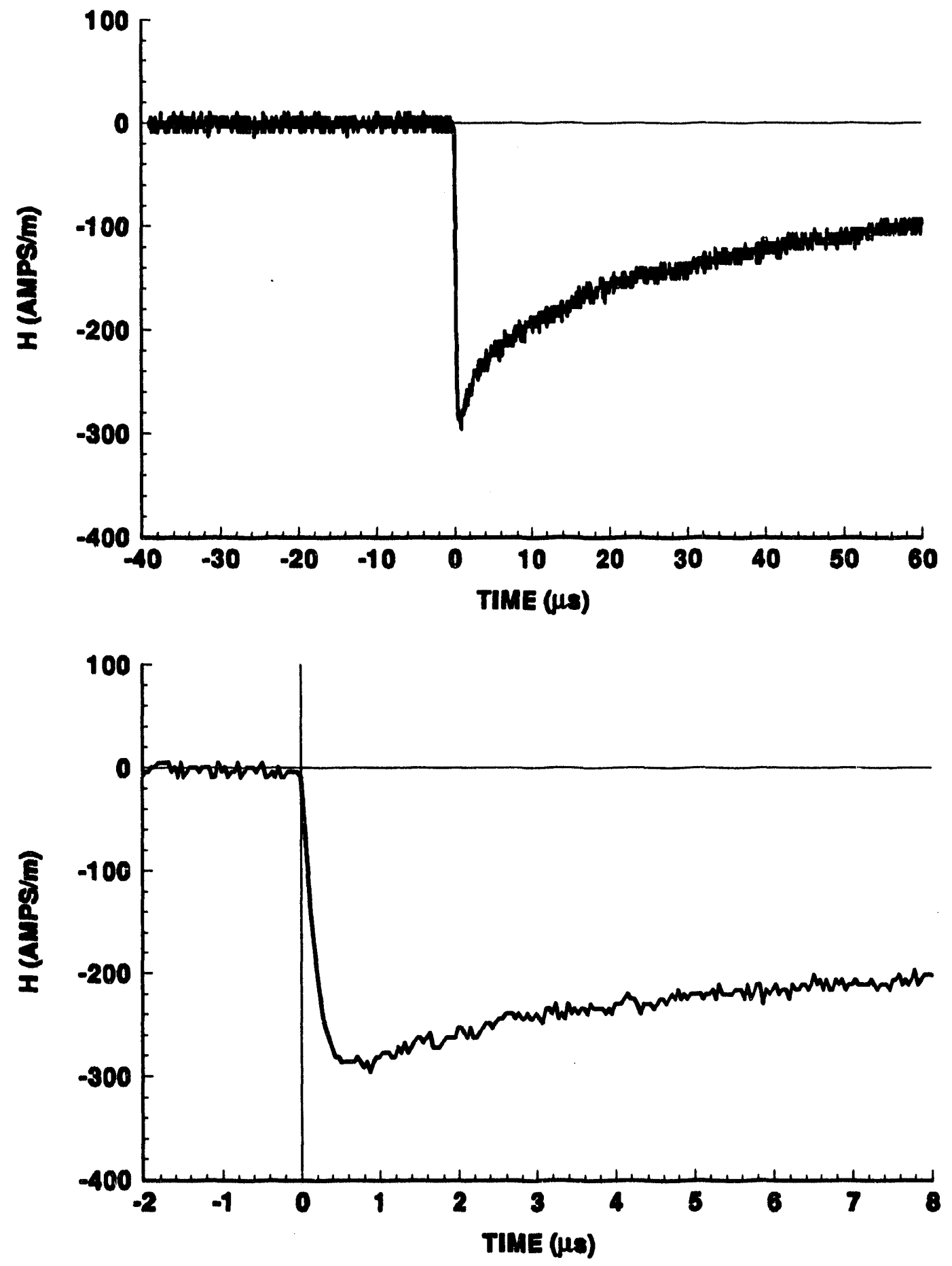


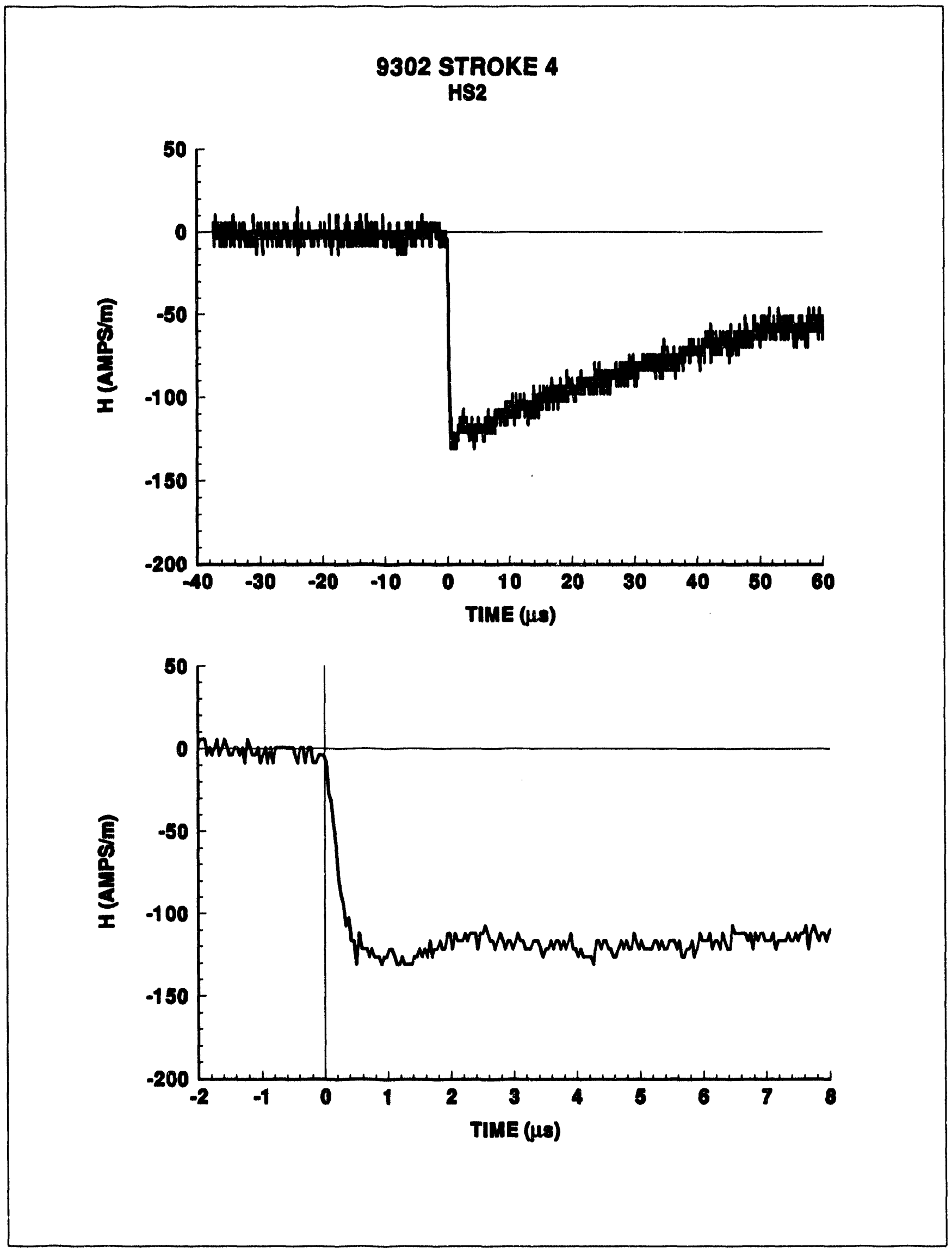




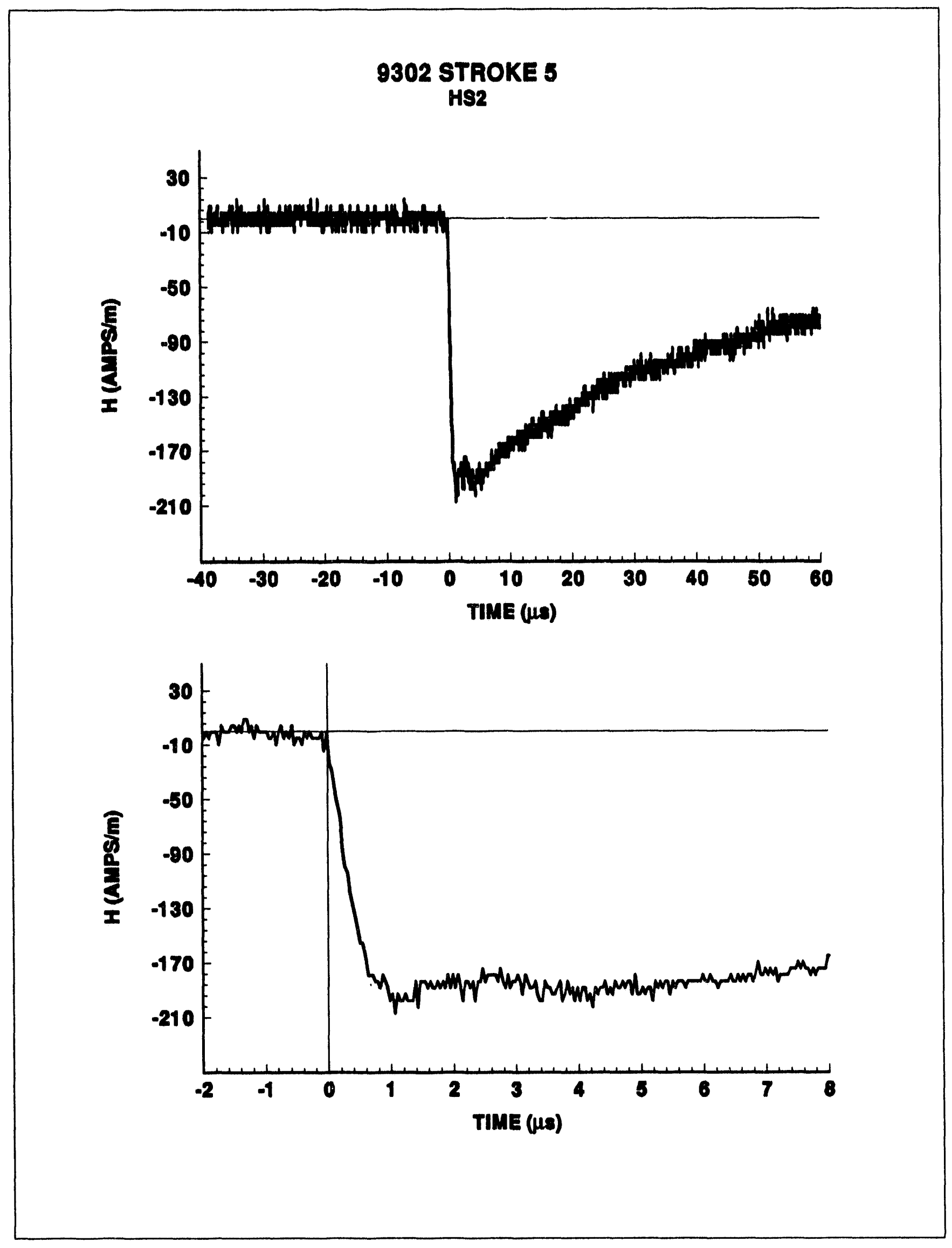




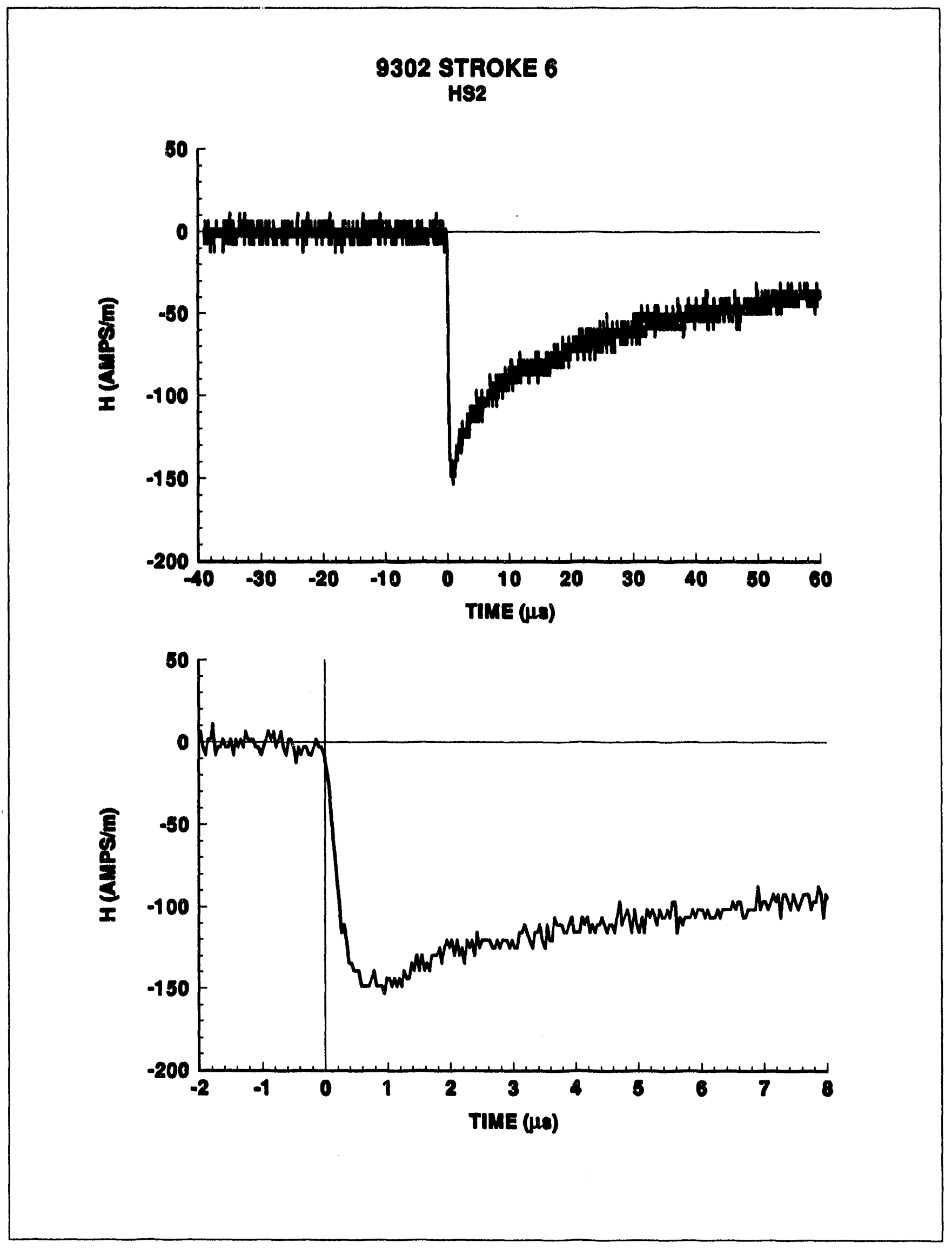




\section{STROKE 7}

HS2
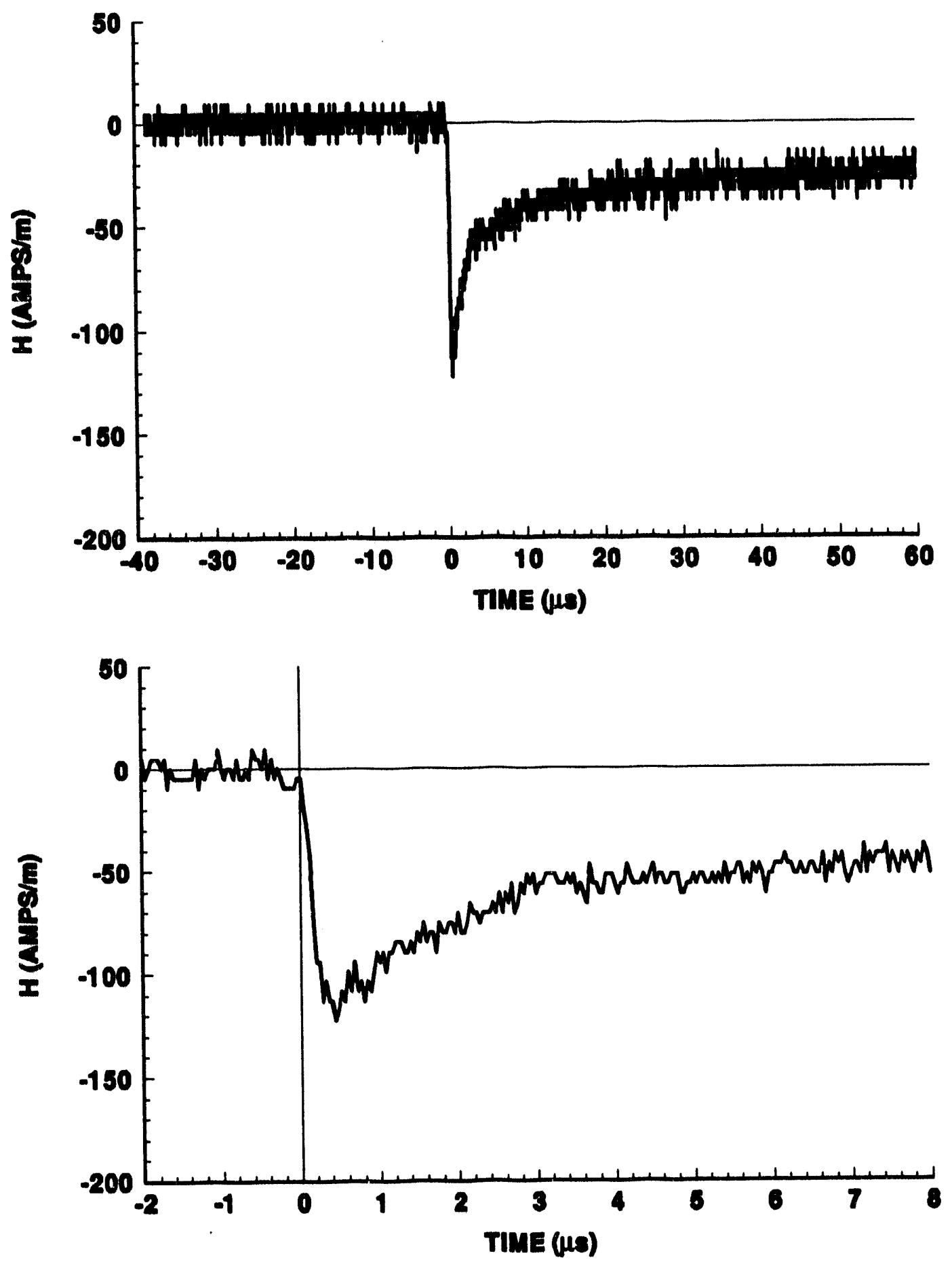

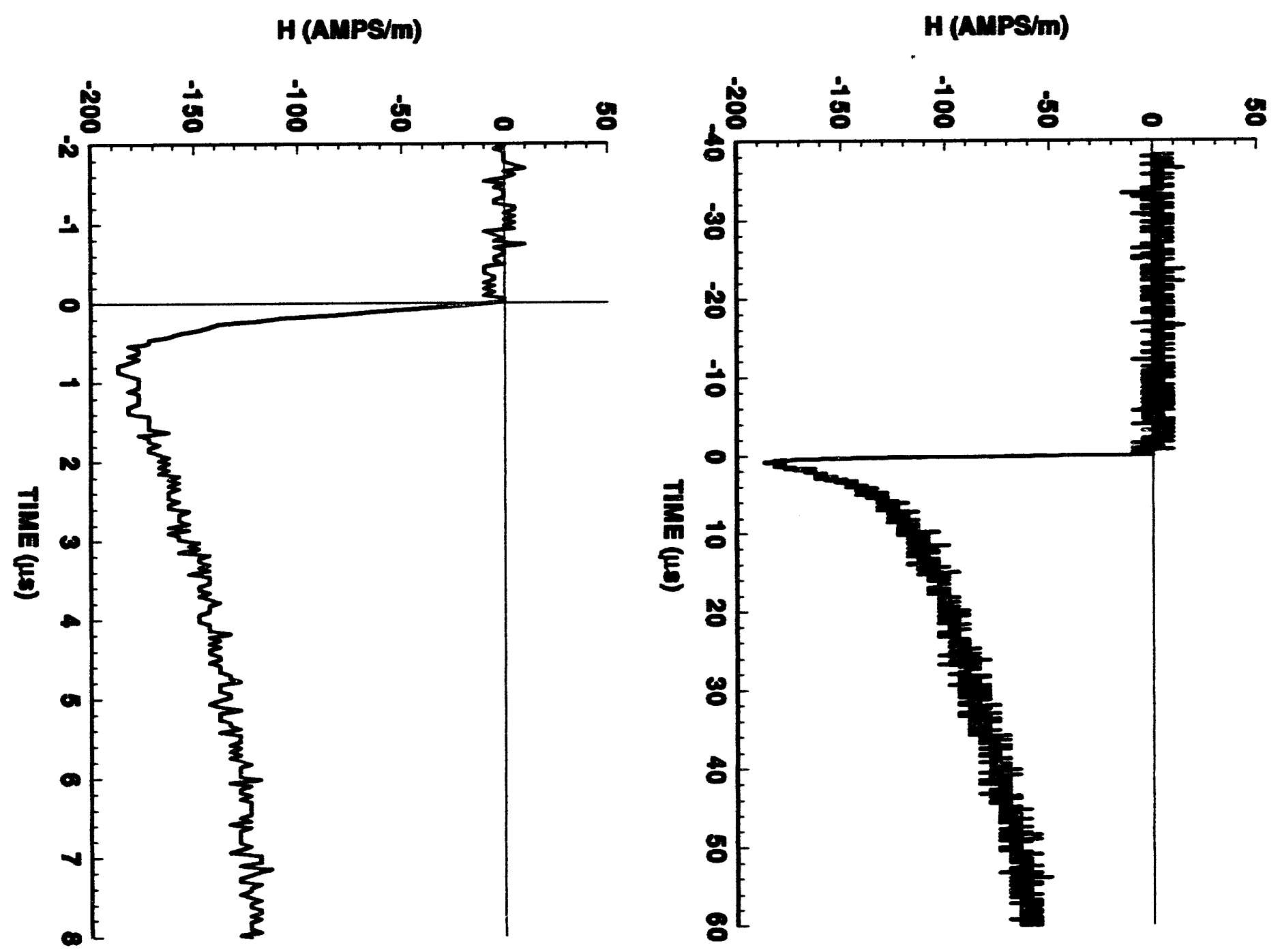

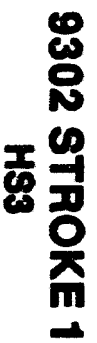




\section{STROKE 2}

HS3
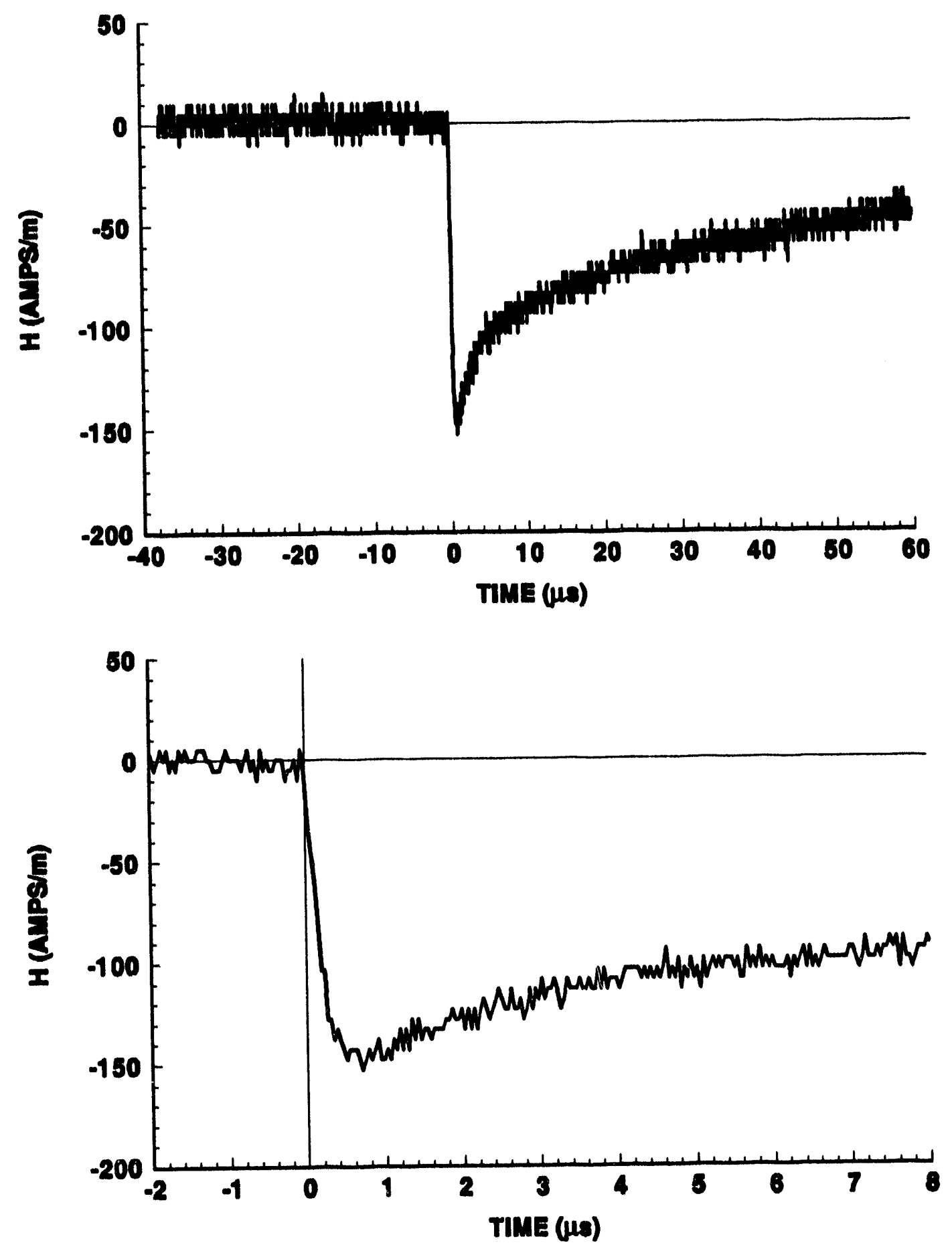


\section{STROKE 3}

HS3
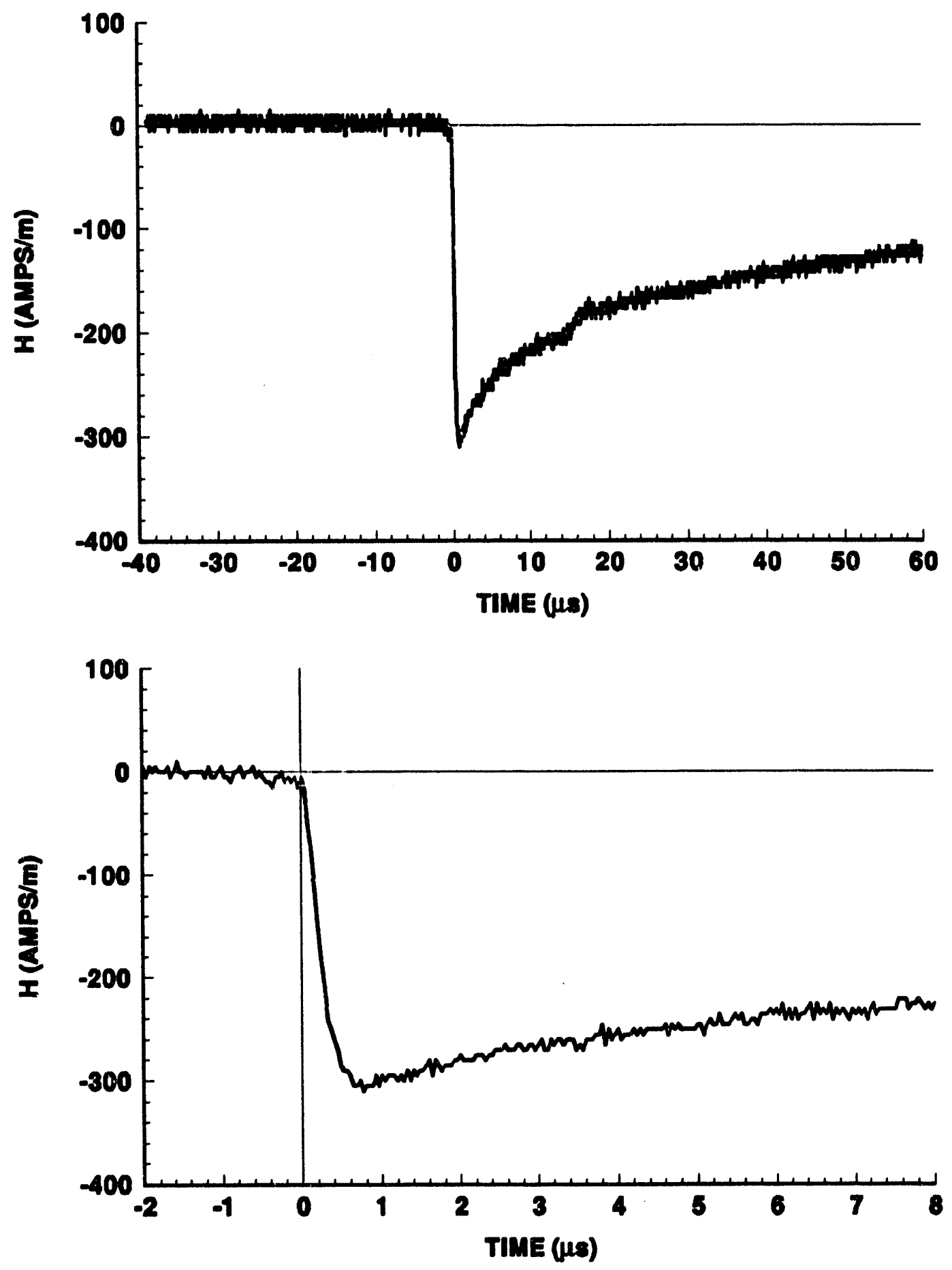


\section{STROKE 4}

\section{HS3}
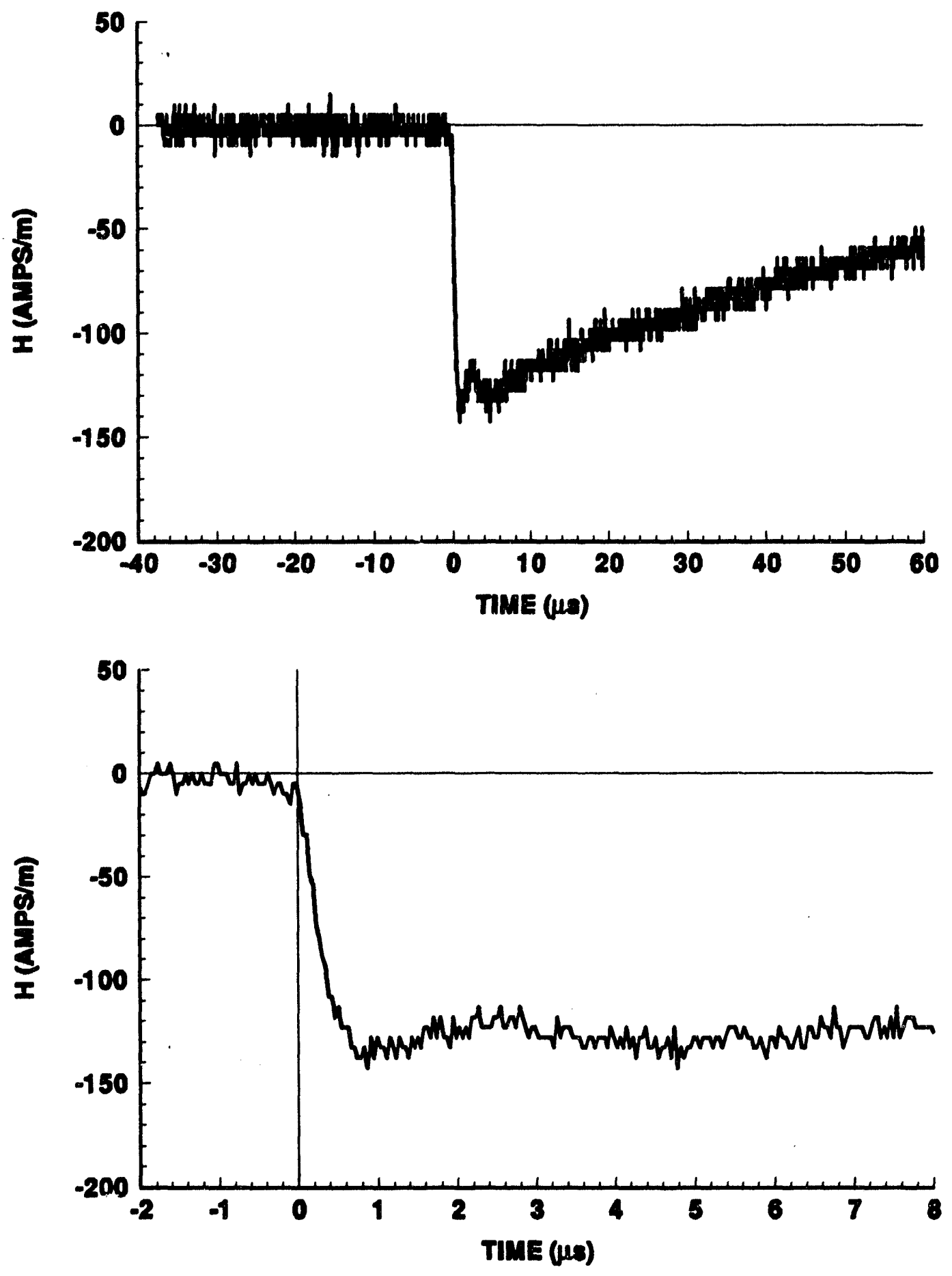


\section{STROKE 5}

HS3
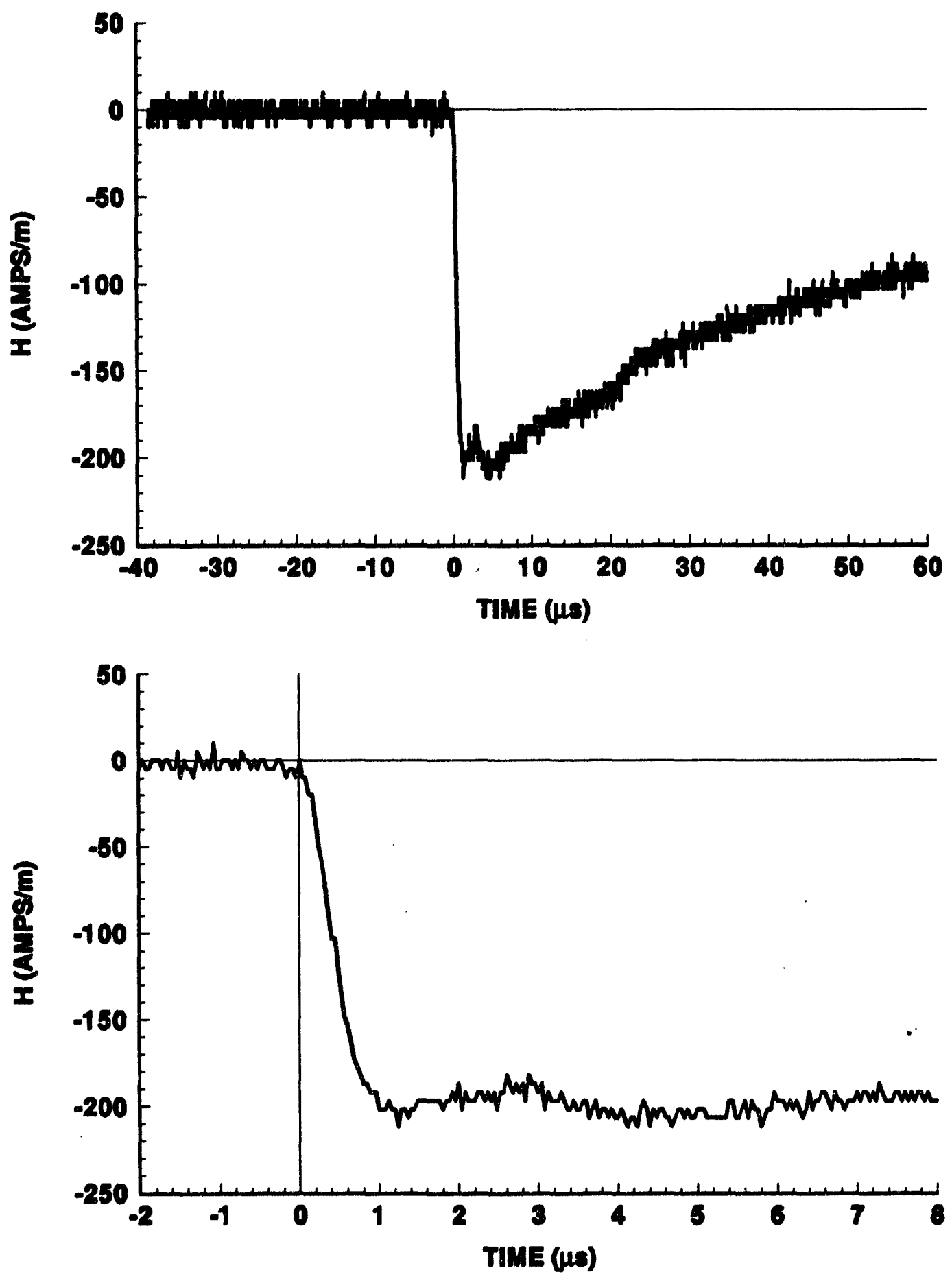


\section{STROKE 6 \\ HS3}
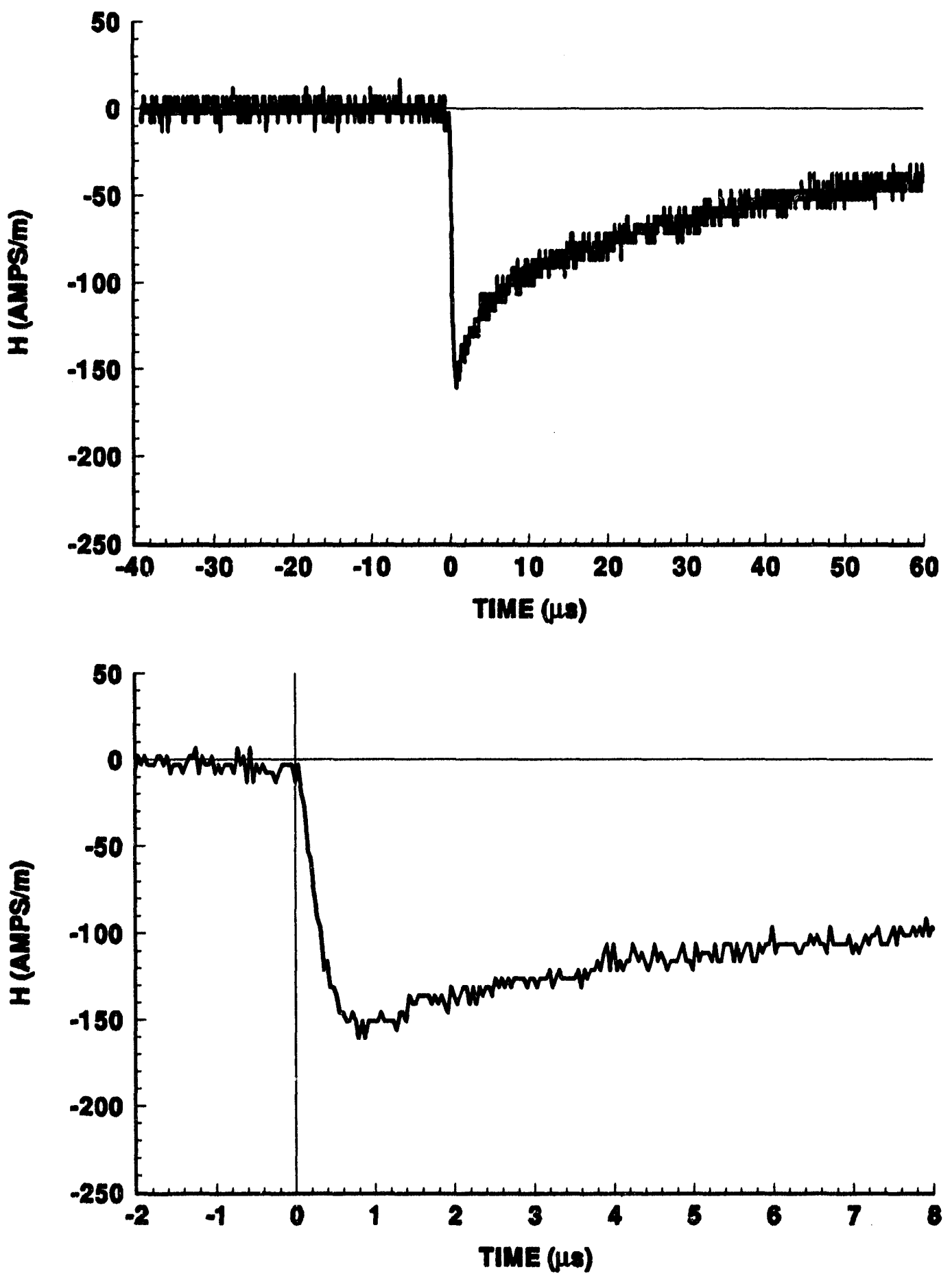


\section{STROKE 7 \\ HS3}
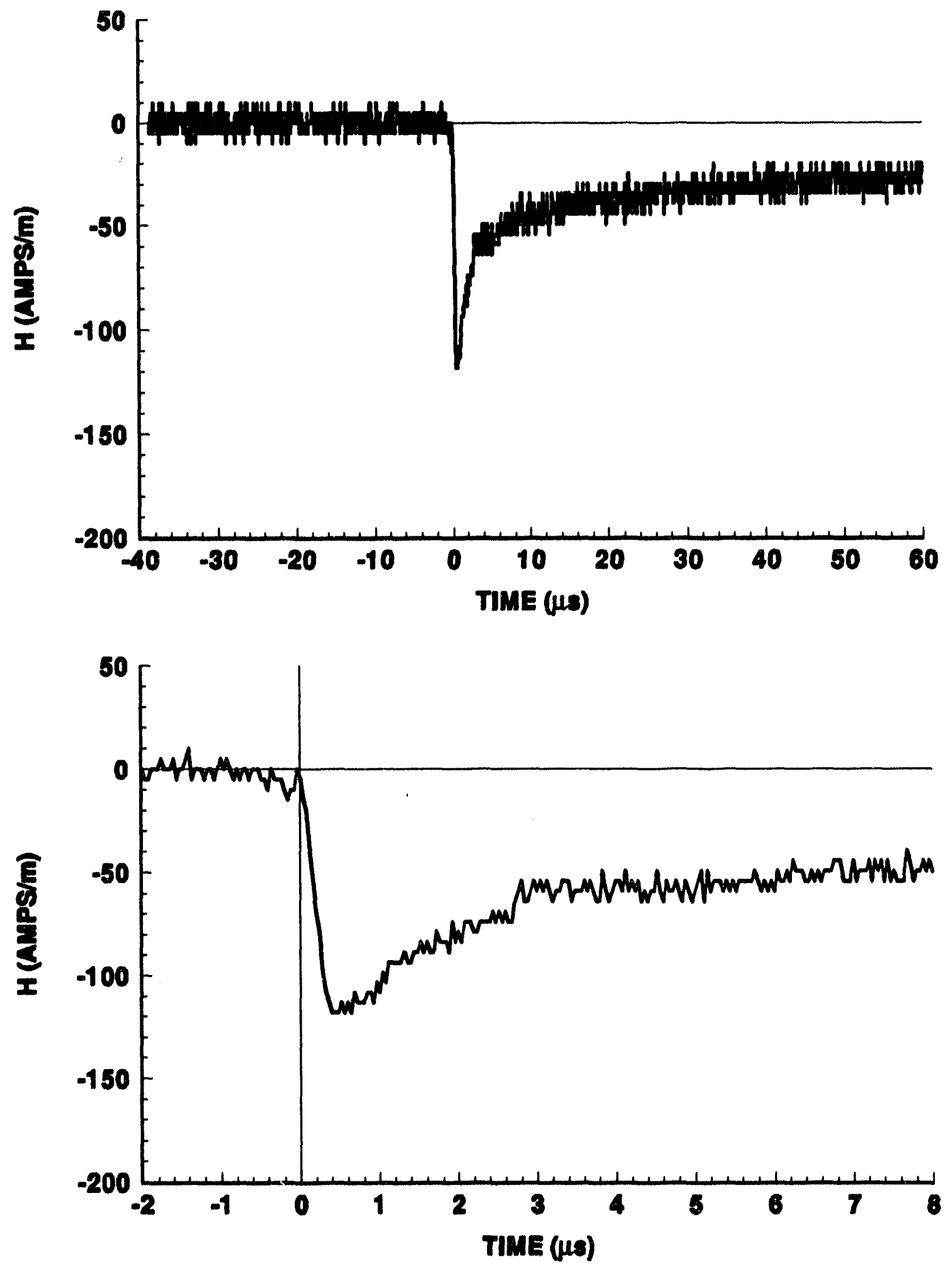


\section{STROKE 1}

ES1

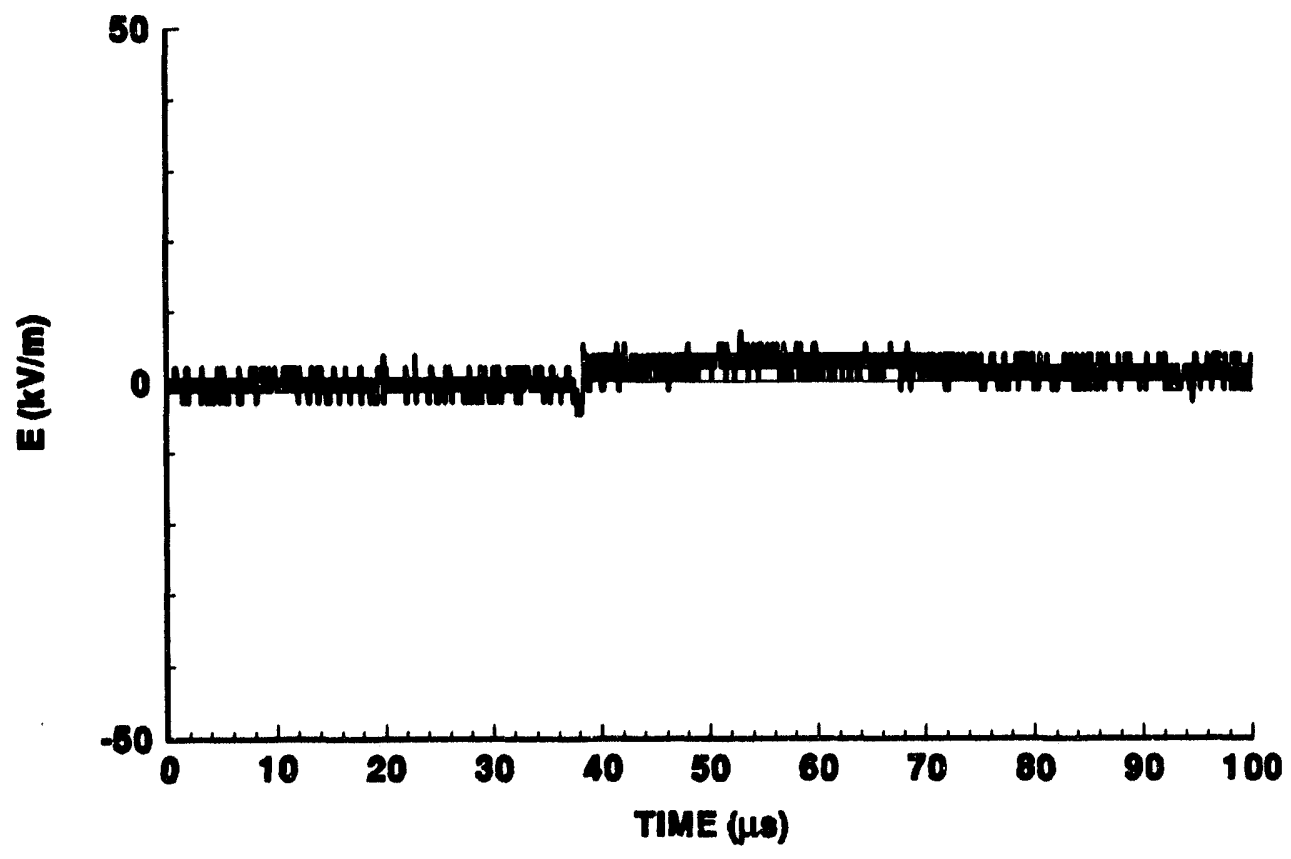

O302 STROKE 2

ES1

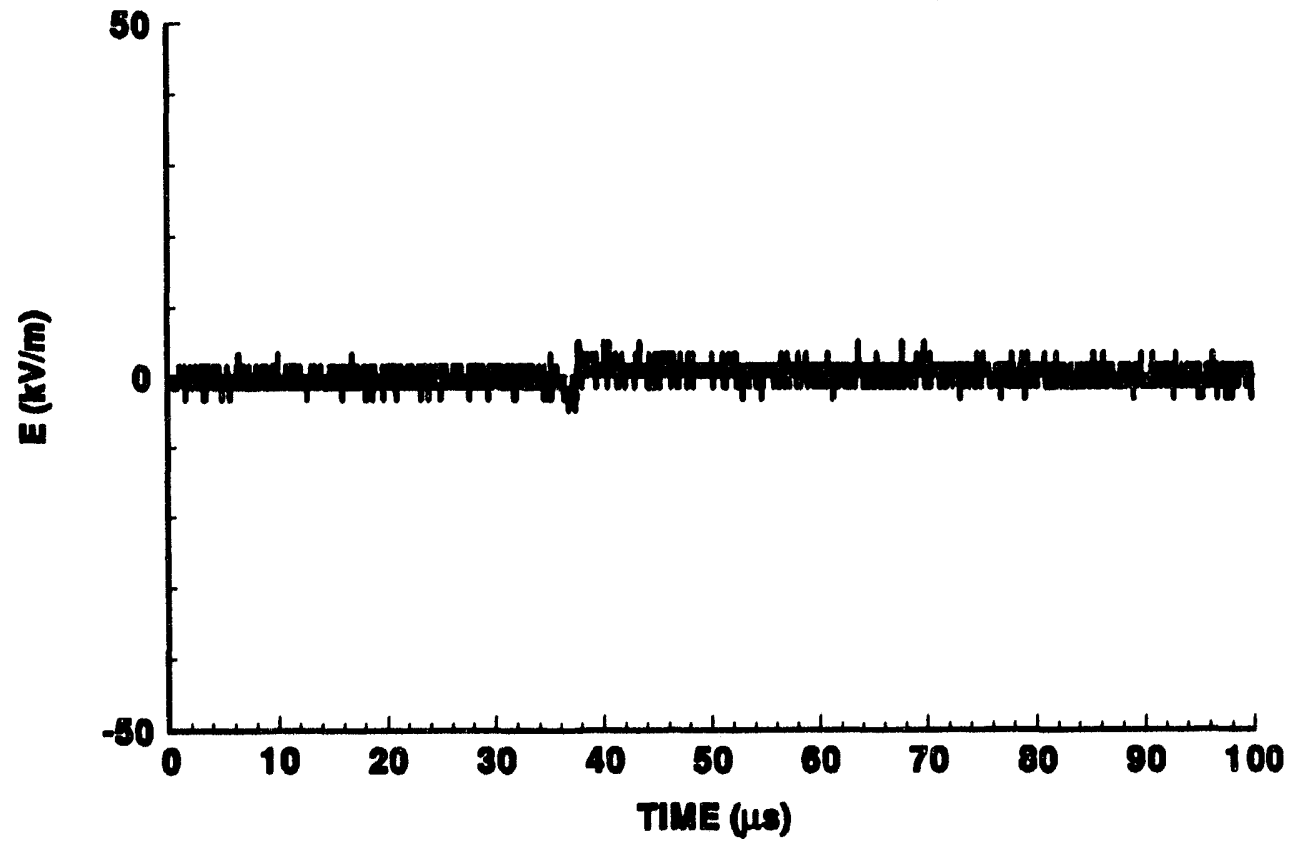




\section{STROKE 3}

ES1

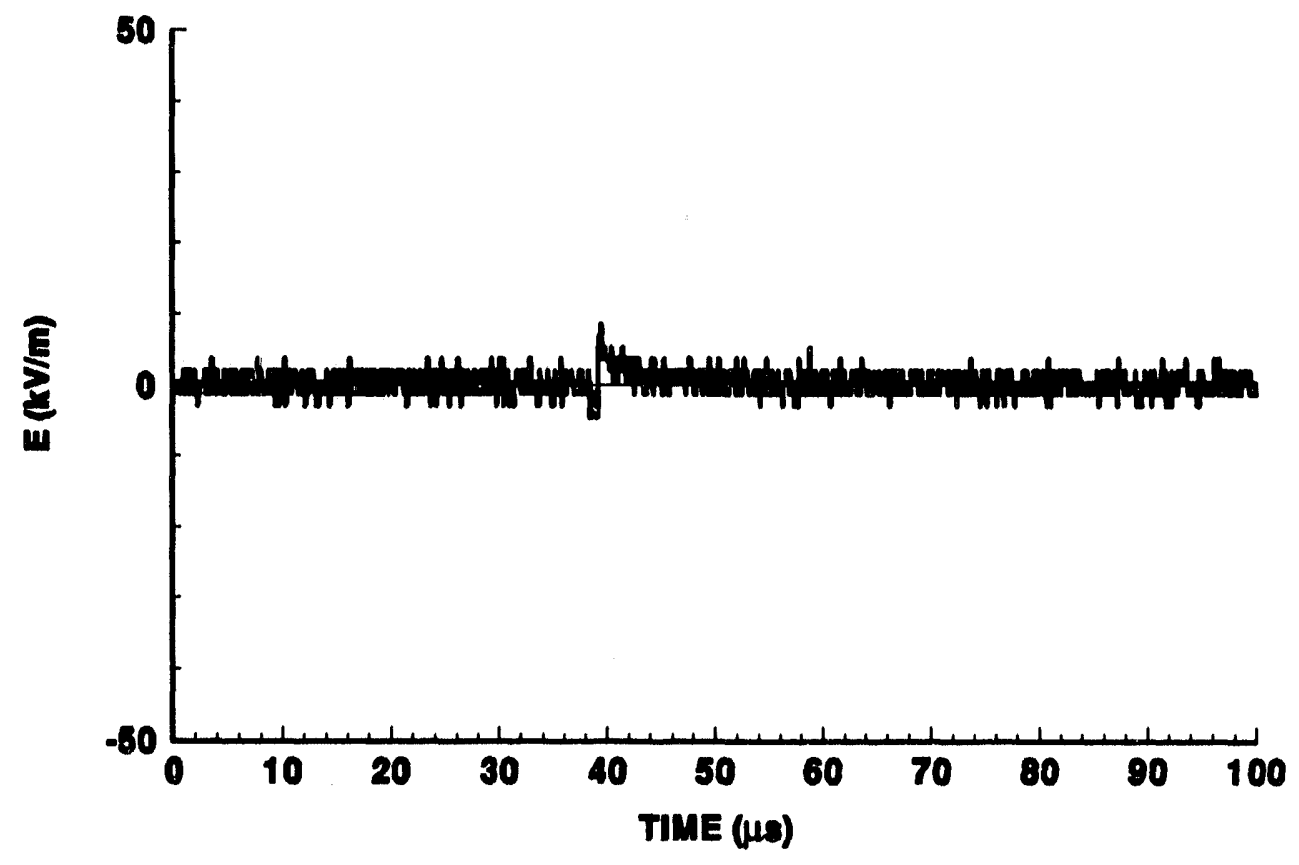

9302 STROKE 4

ES1

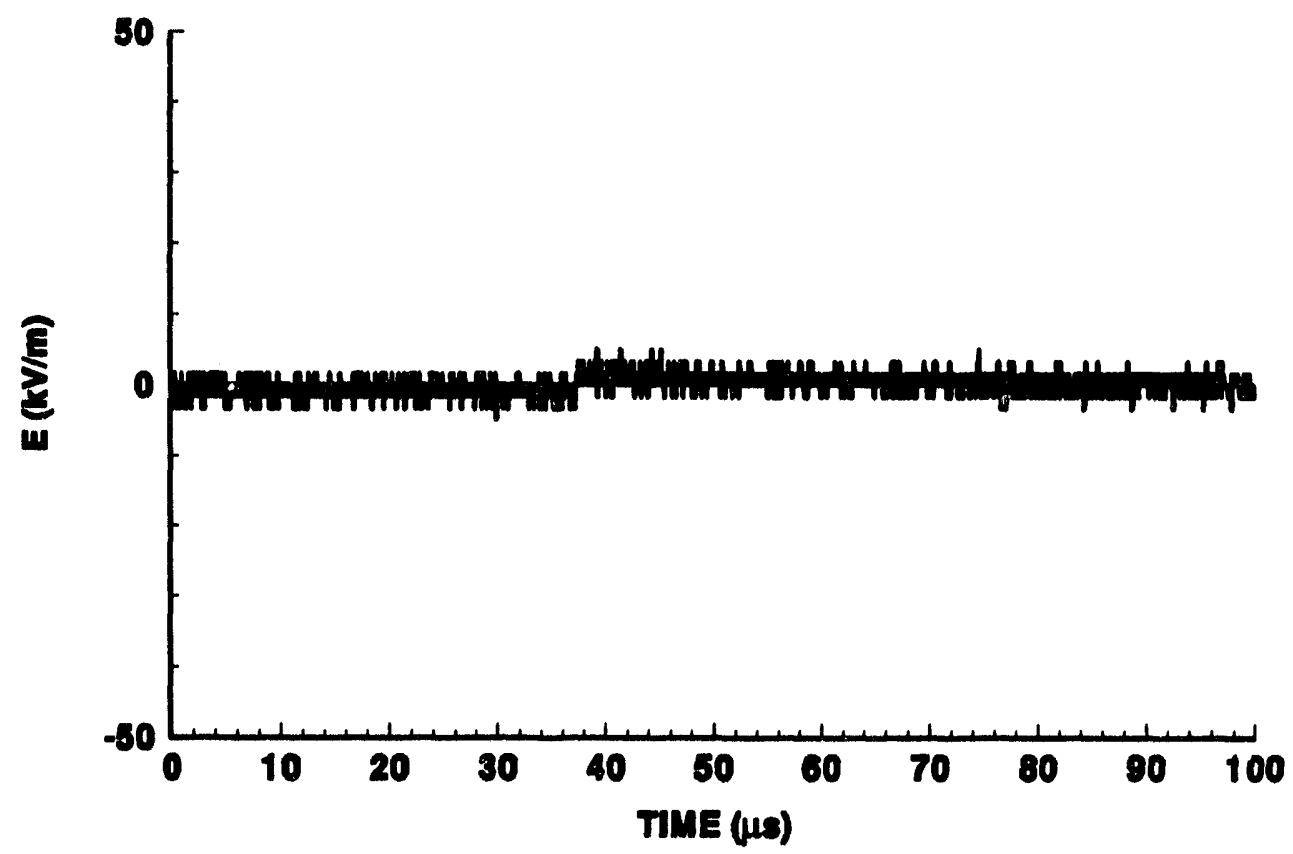




\section{STROKE 5}

ES1

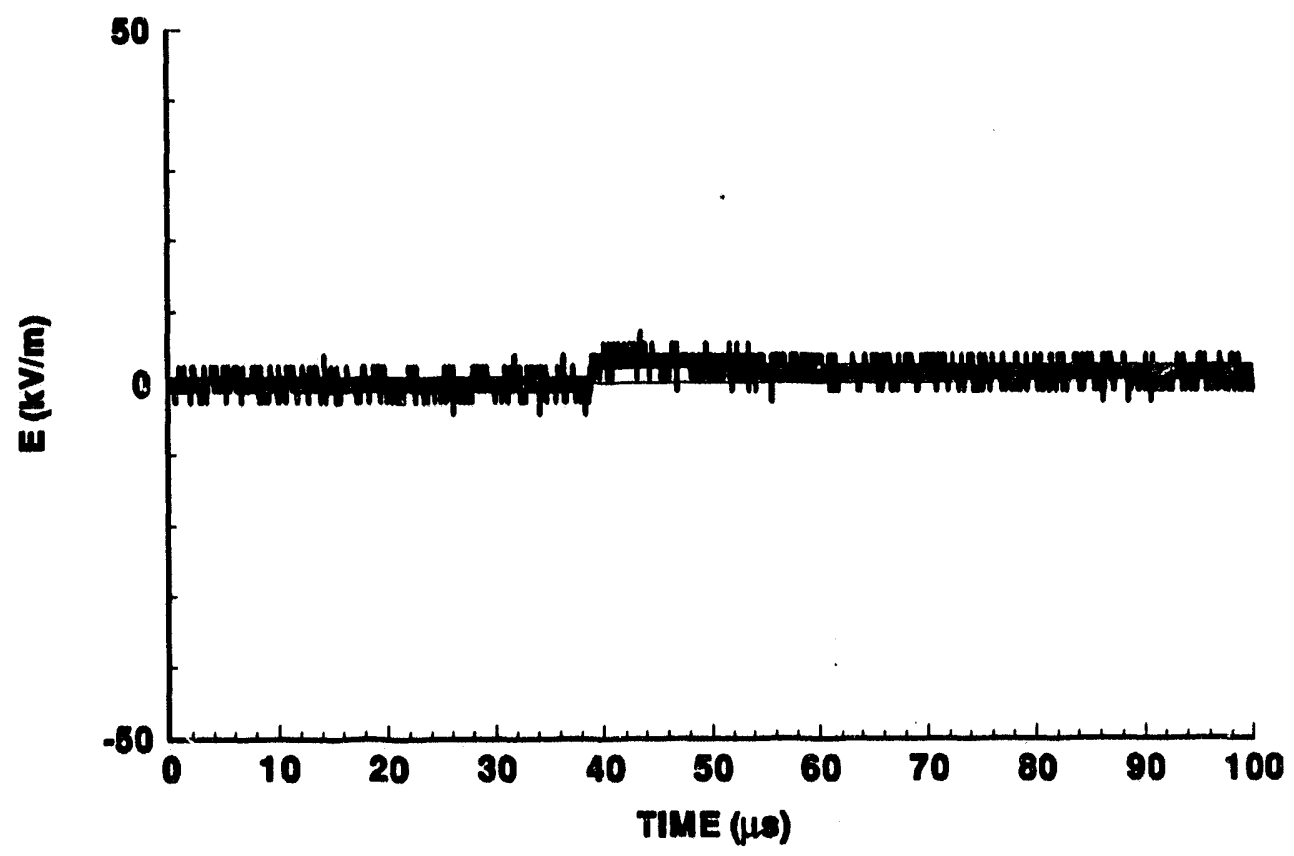

9302 STROKE 6

ES1

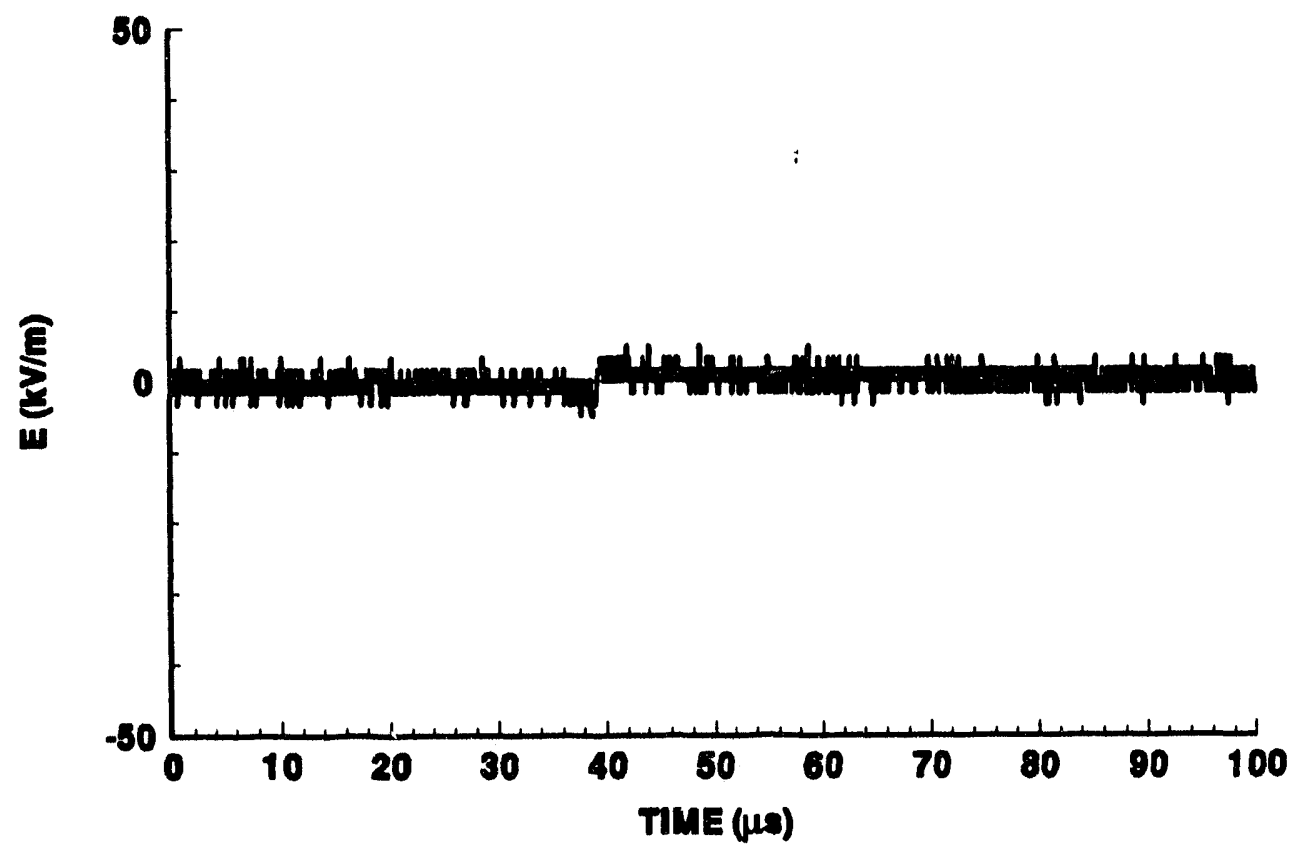




\section{STROKE 7}

ES1

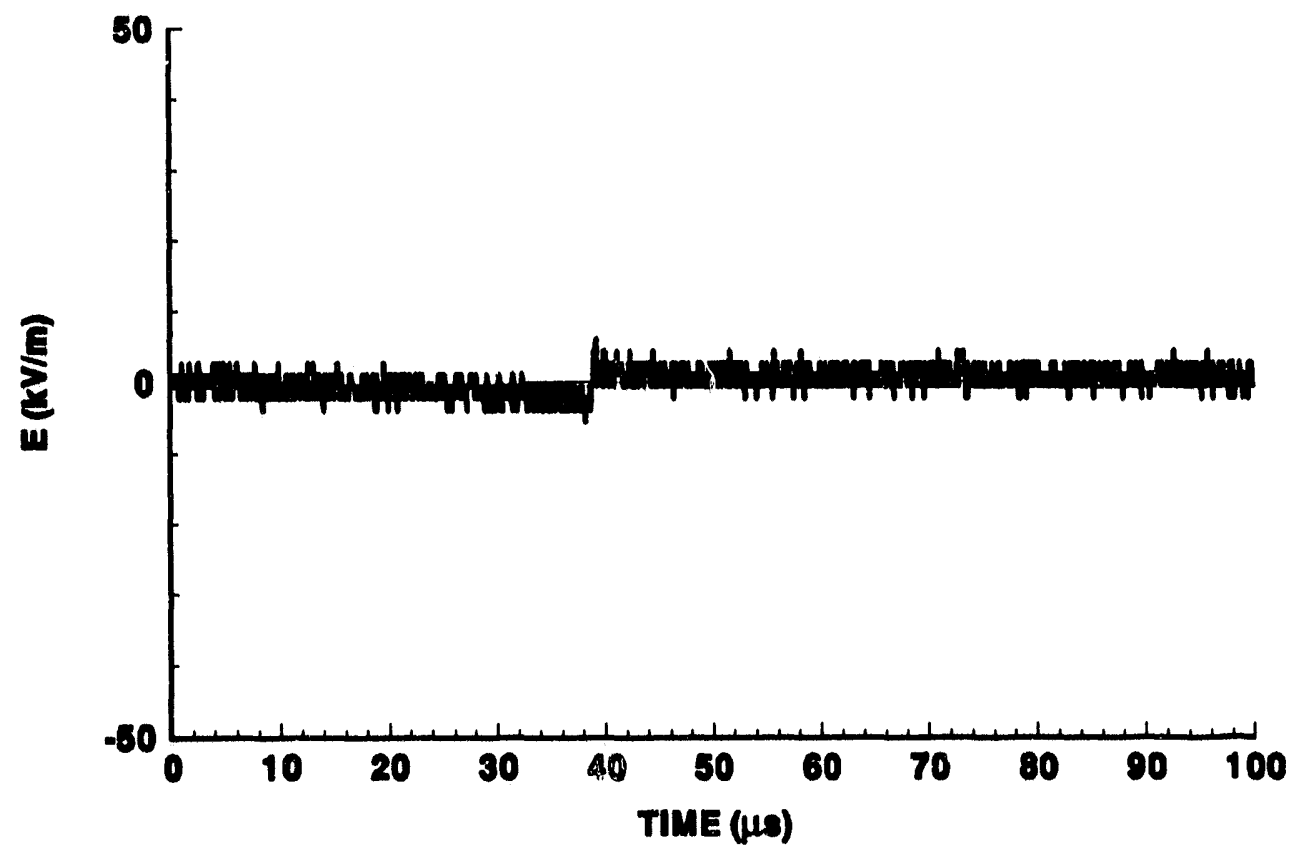




\section{STROKE 1}

ES2
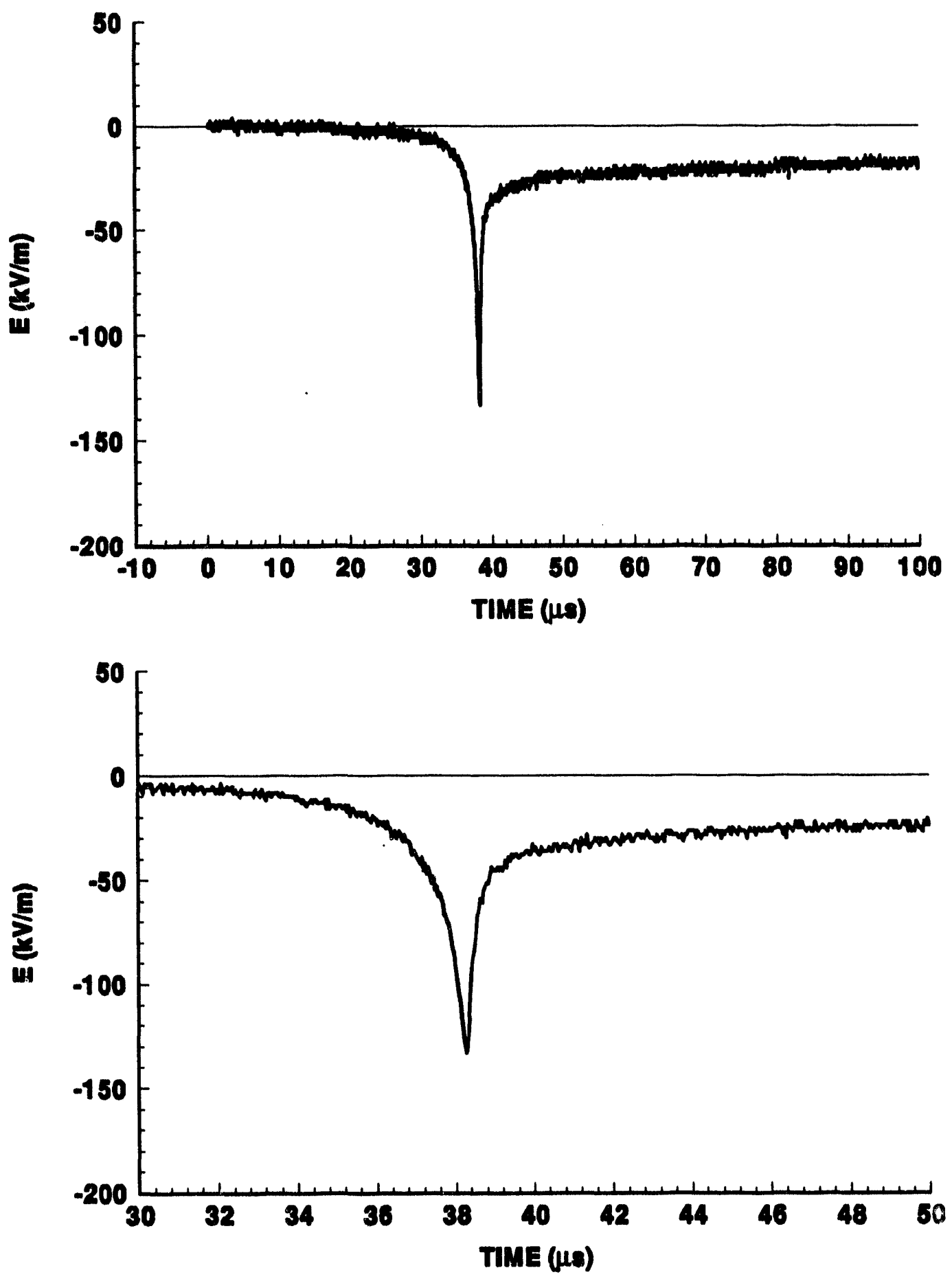


\section{STROKE 2}

\section{ES2}
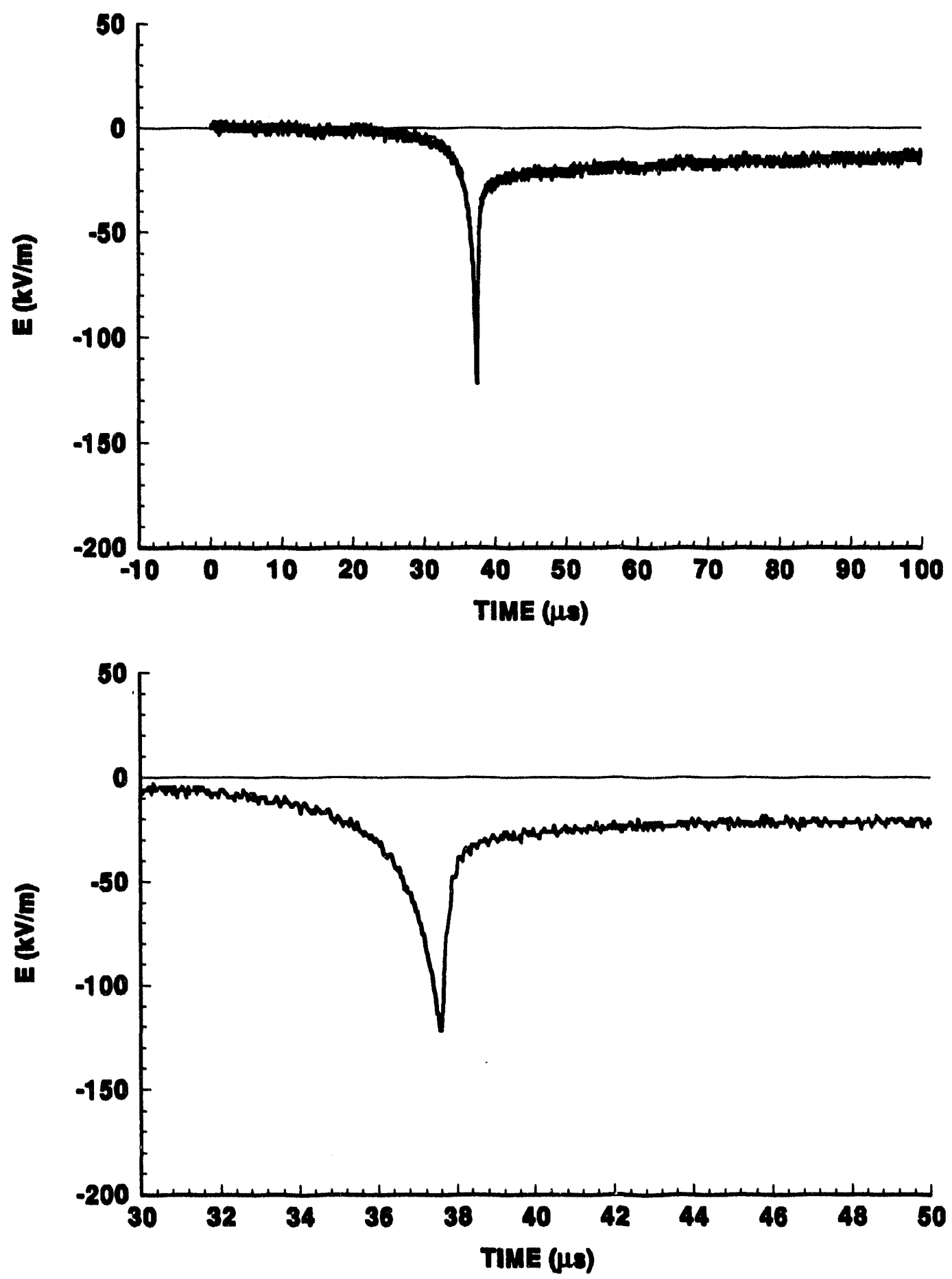


\section{STROKE 3}

ES2
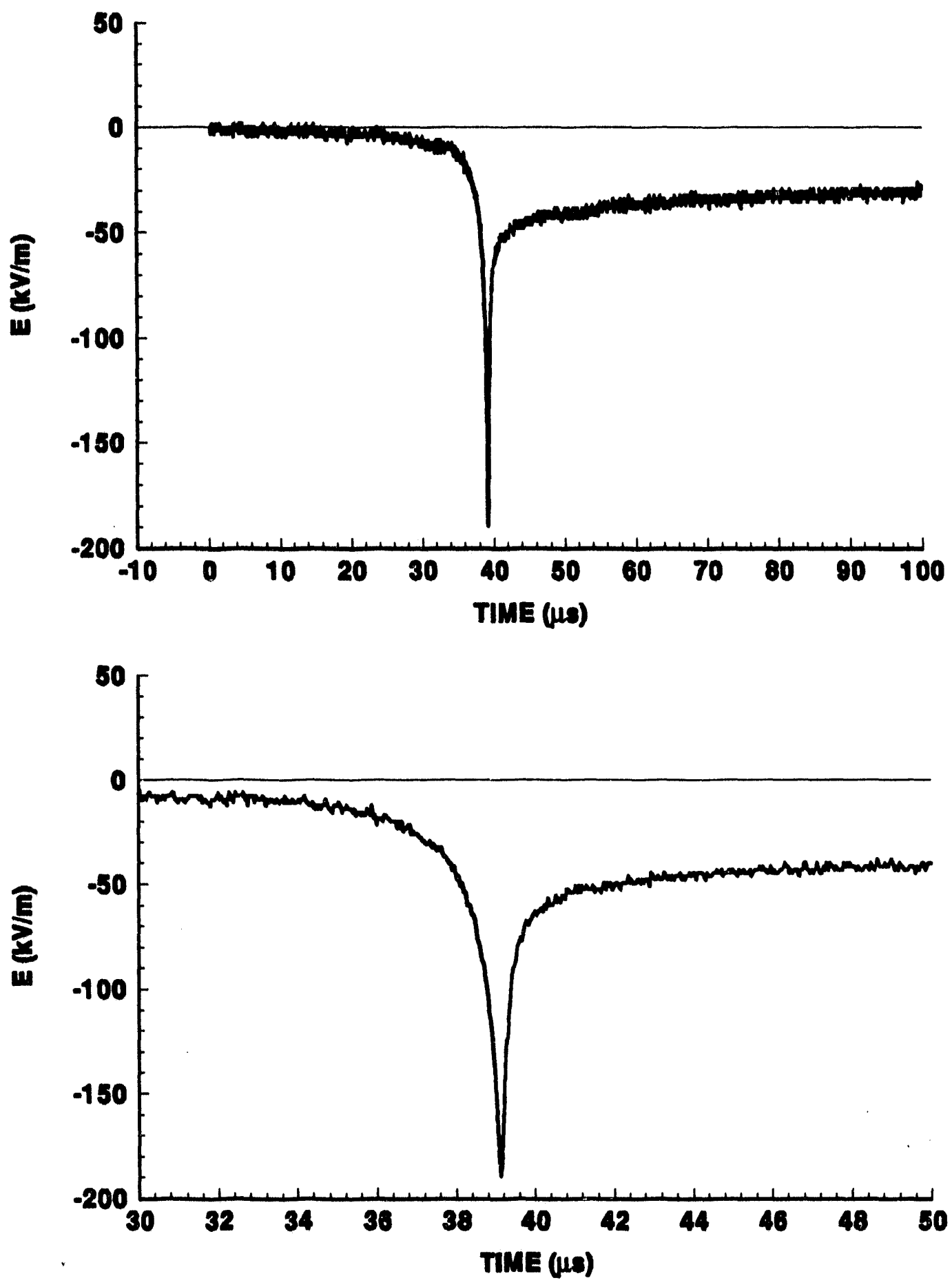


\section{STROKE 4}

E92
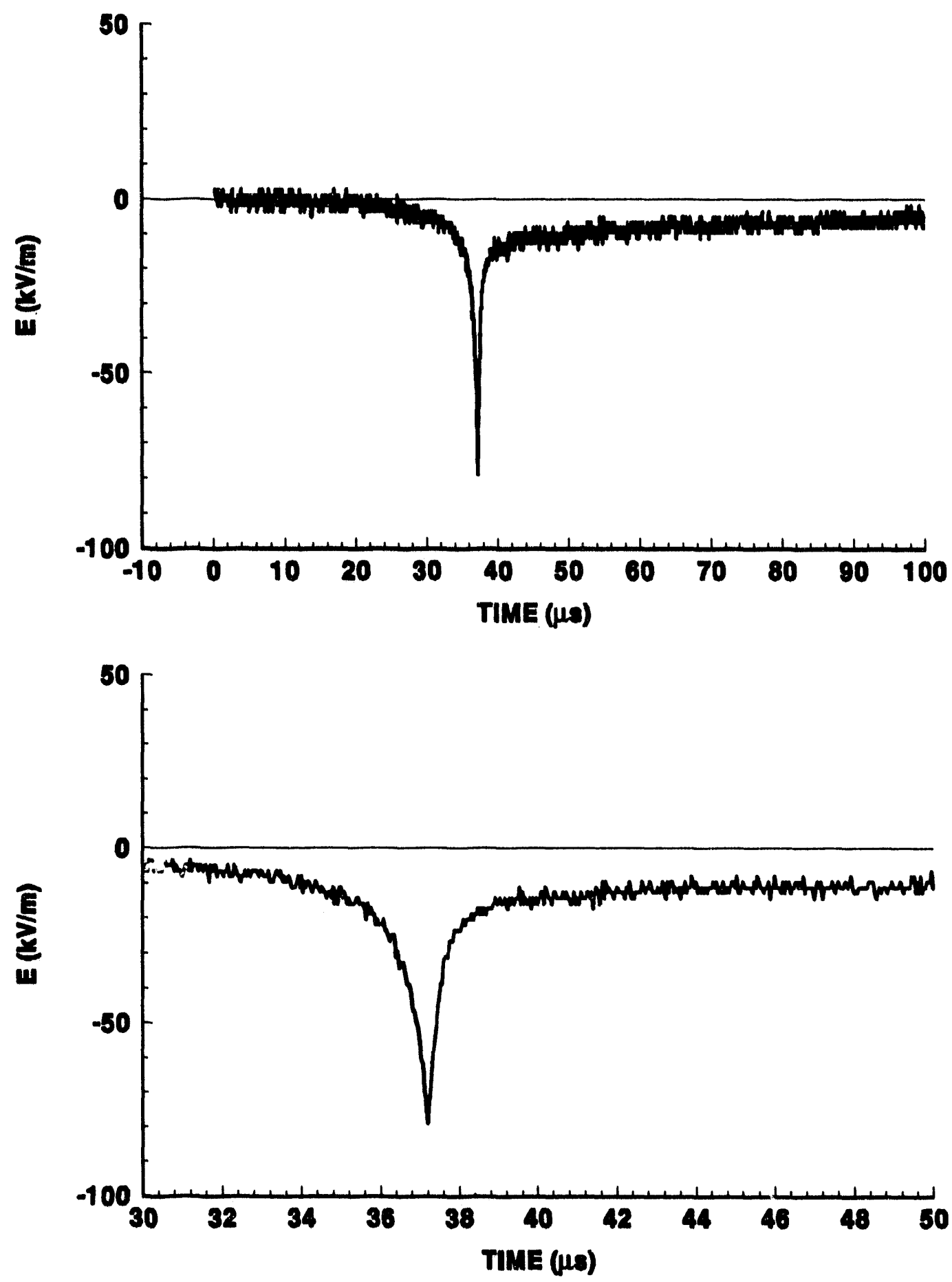


\section{STROKE 5}

Es2
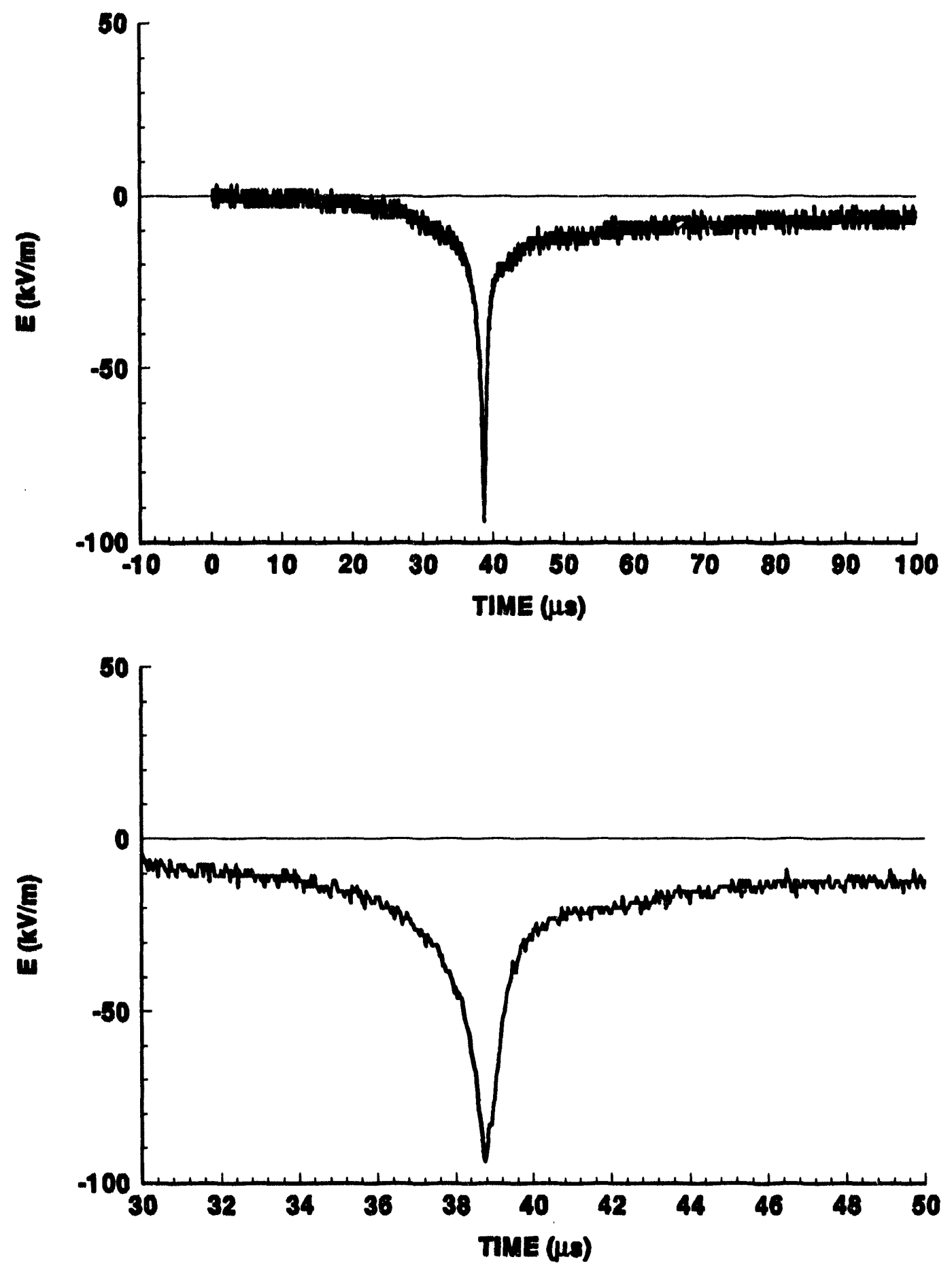


\section{STROKE 6}

ES2
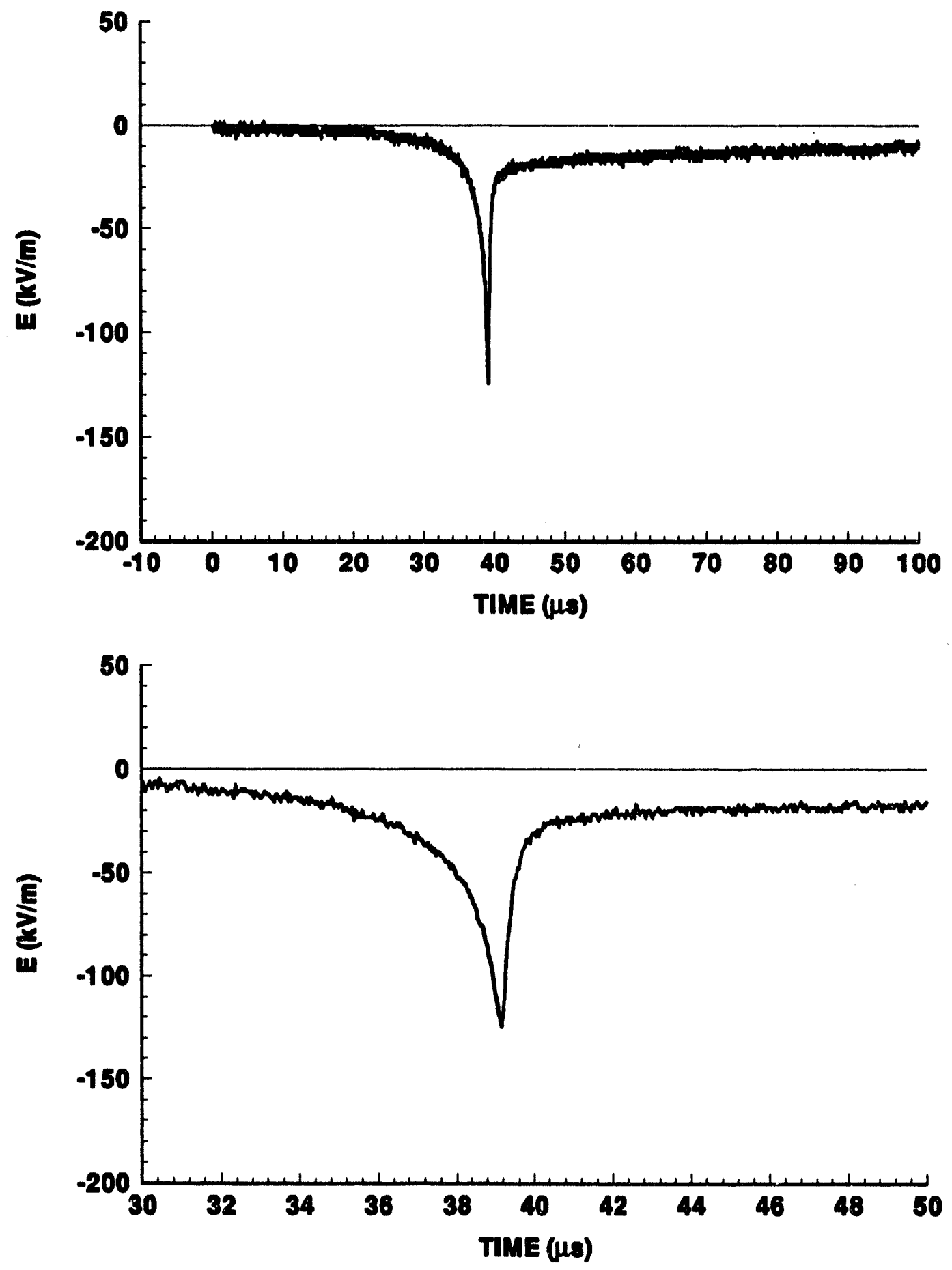


\section{STROKE 7}

ES2
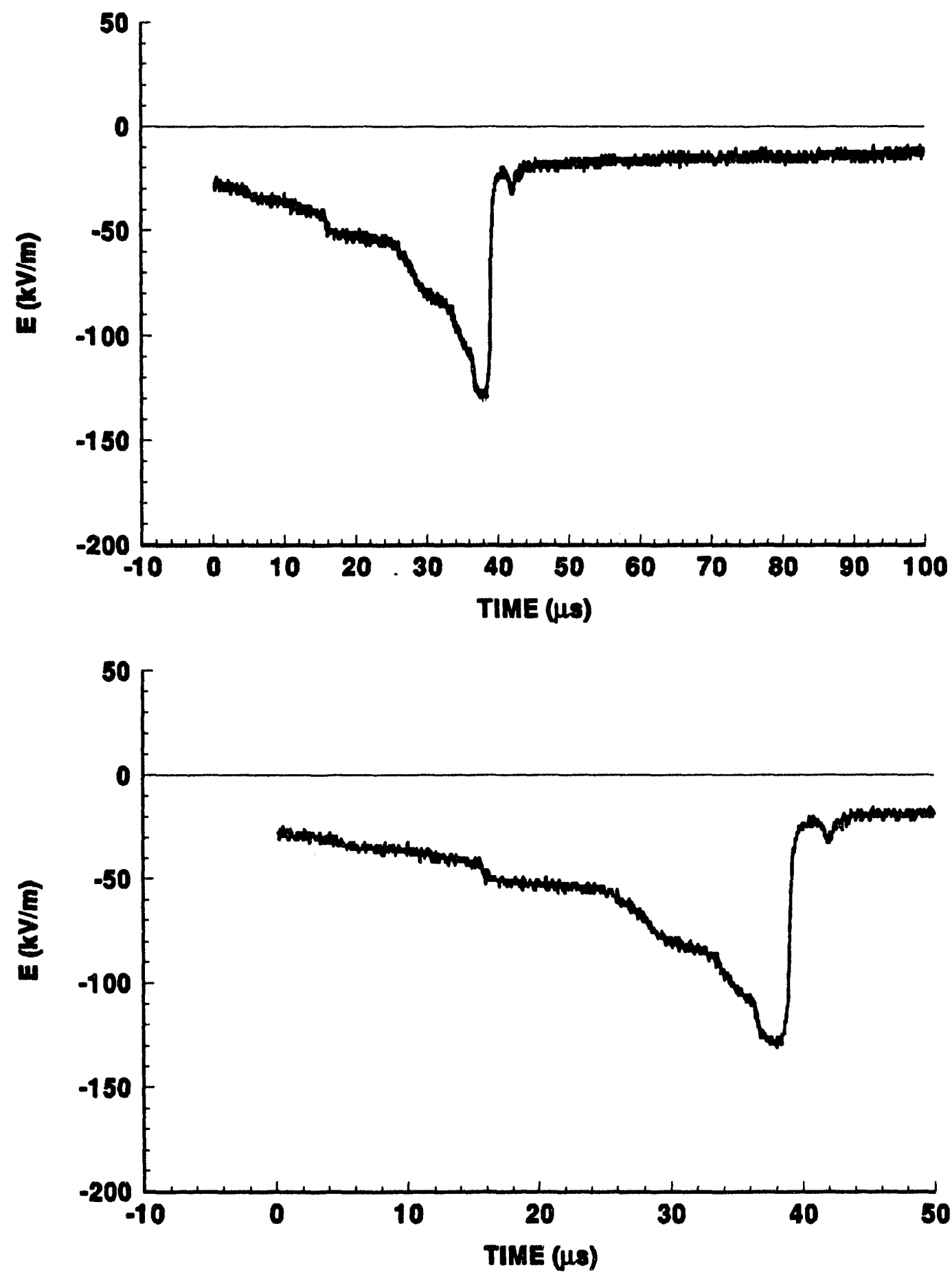


\section{STROKE 1 \\ ES3}
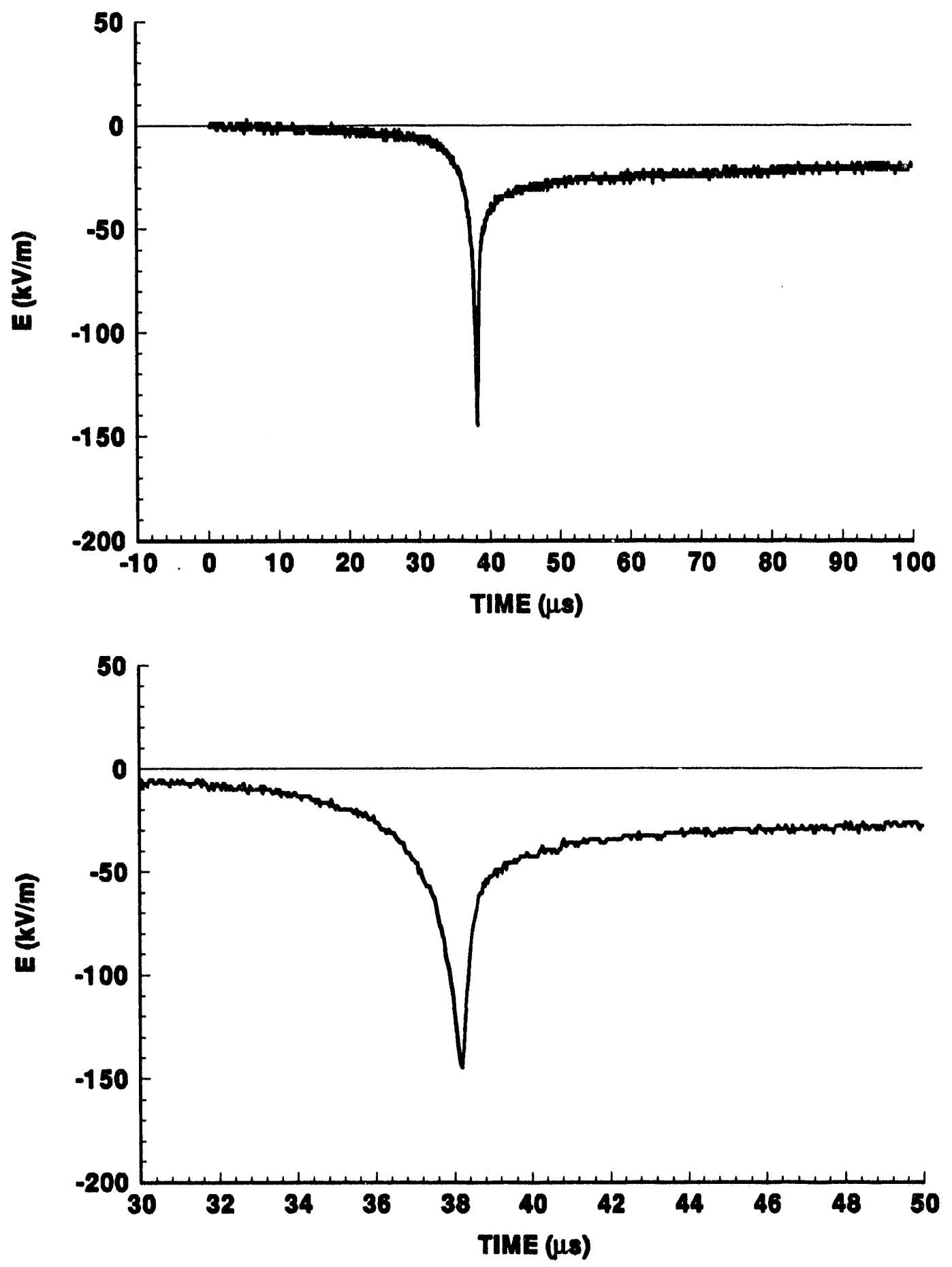


\section{STROKE 2}

ES3
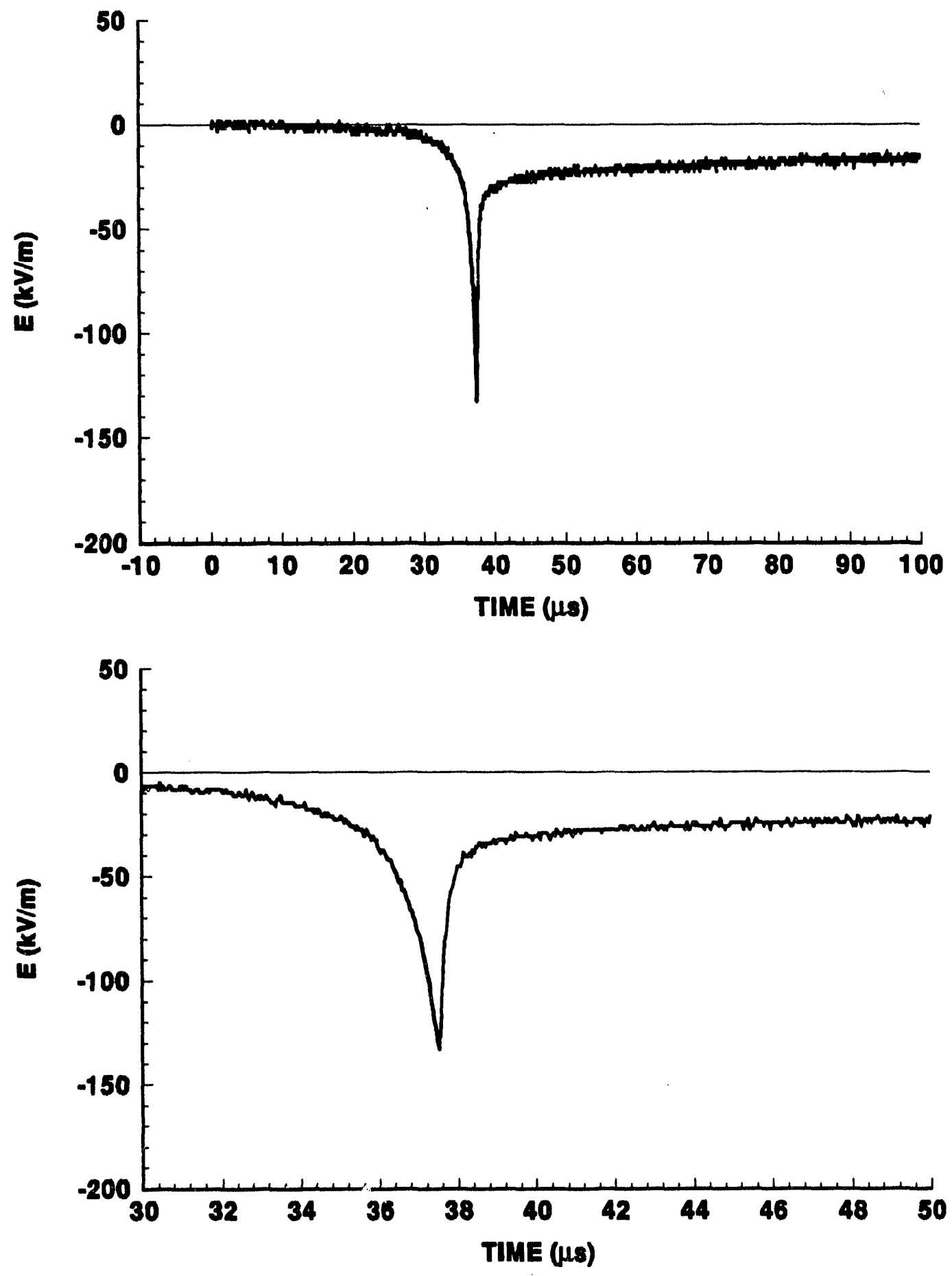


\section{STROKE 3}

ES3
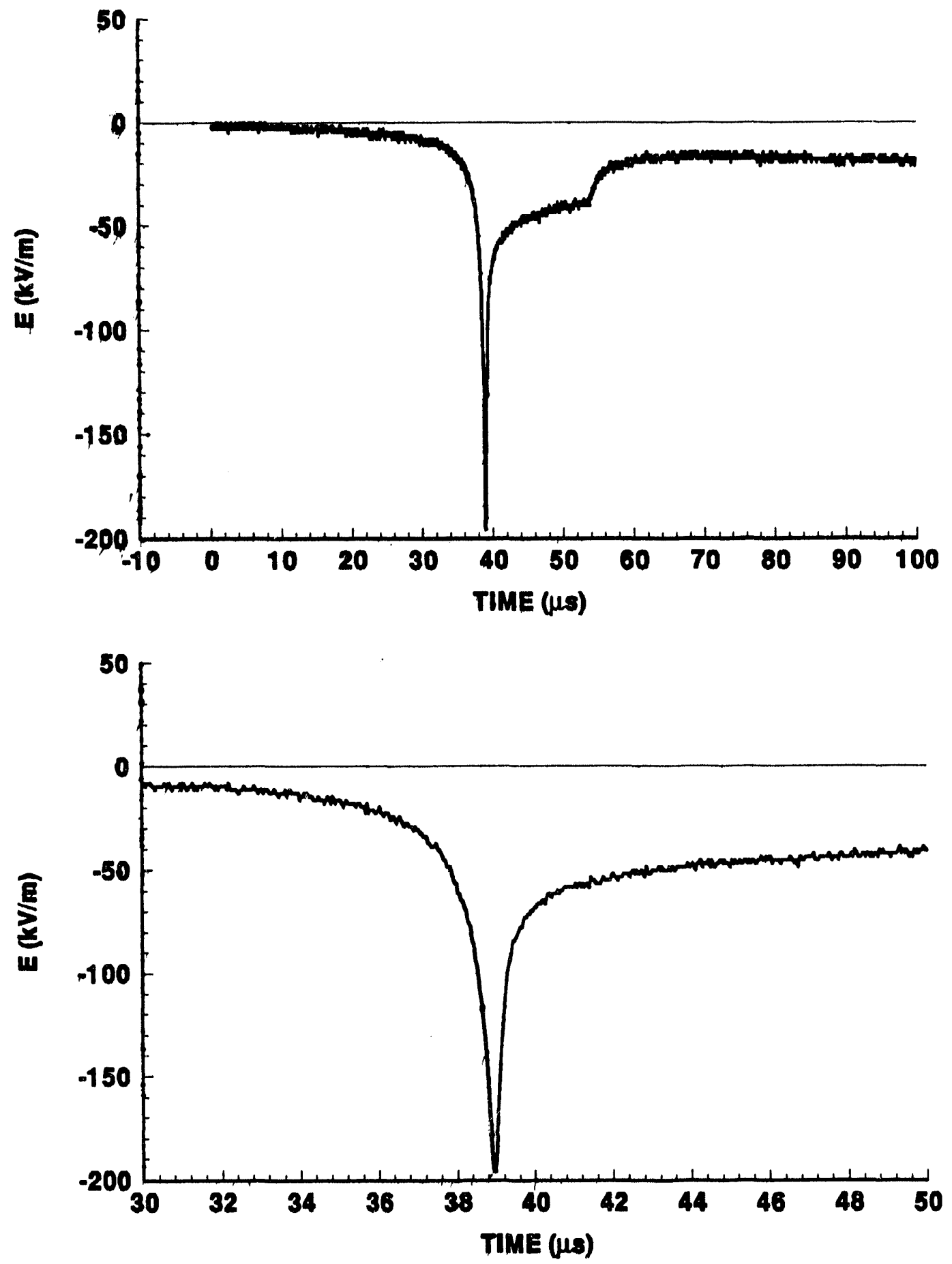


\section{STROKE 4}

ES3
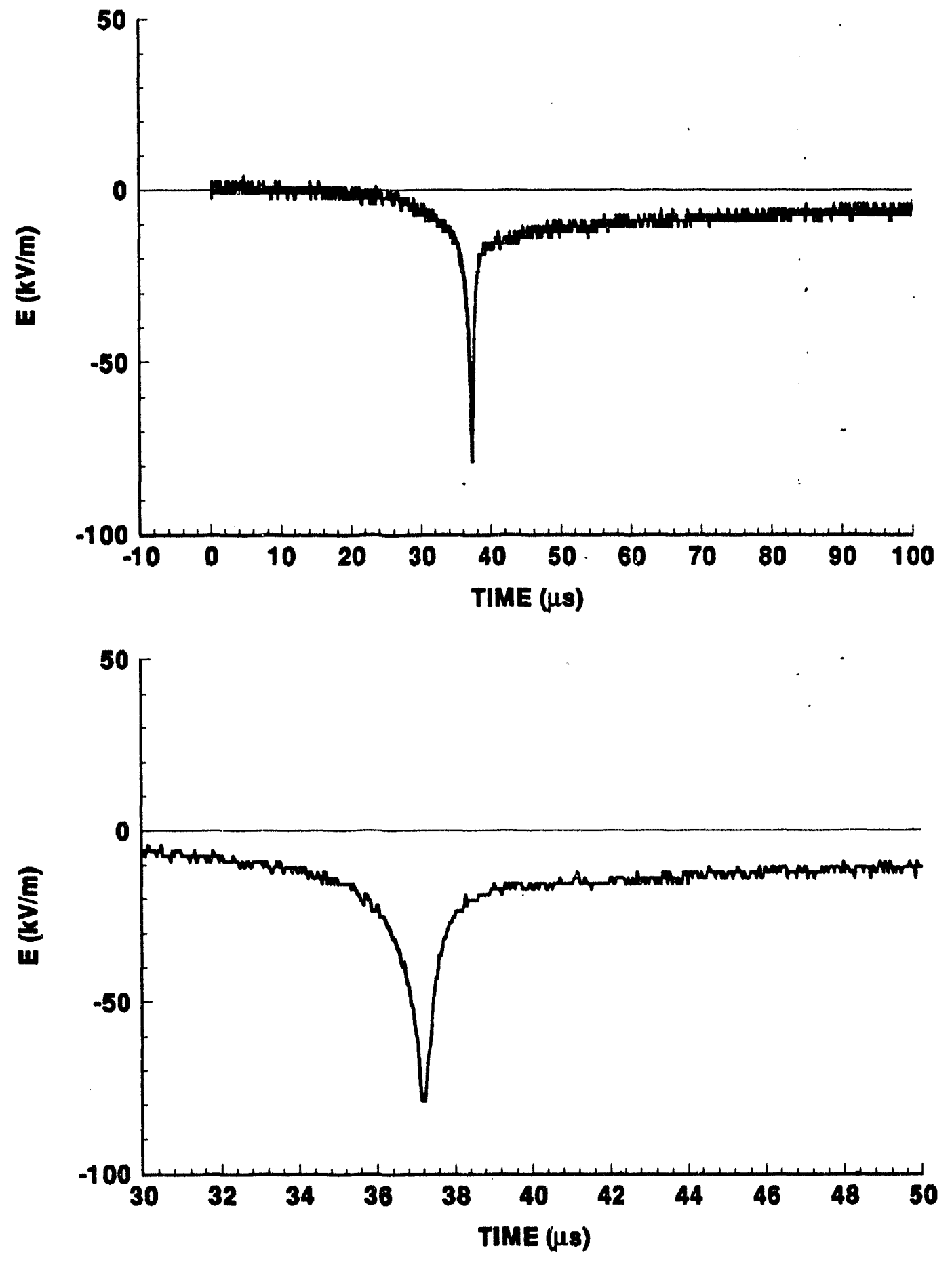


\section{STROKE 5}

ES3
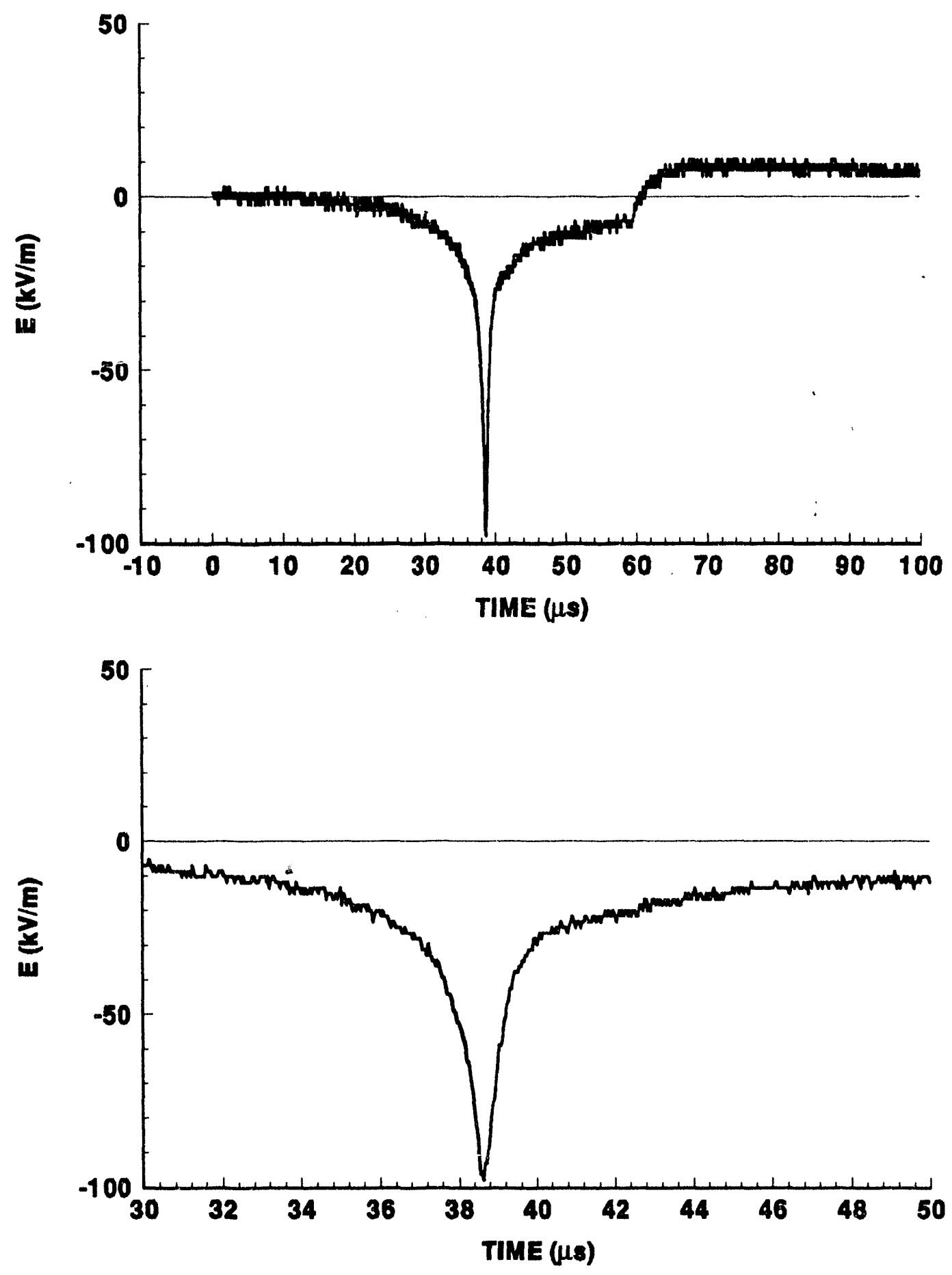


\section{STROKE 6 \\ ES3}
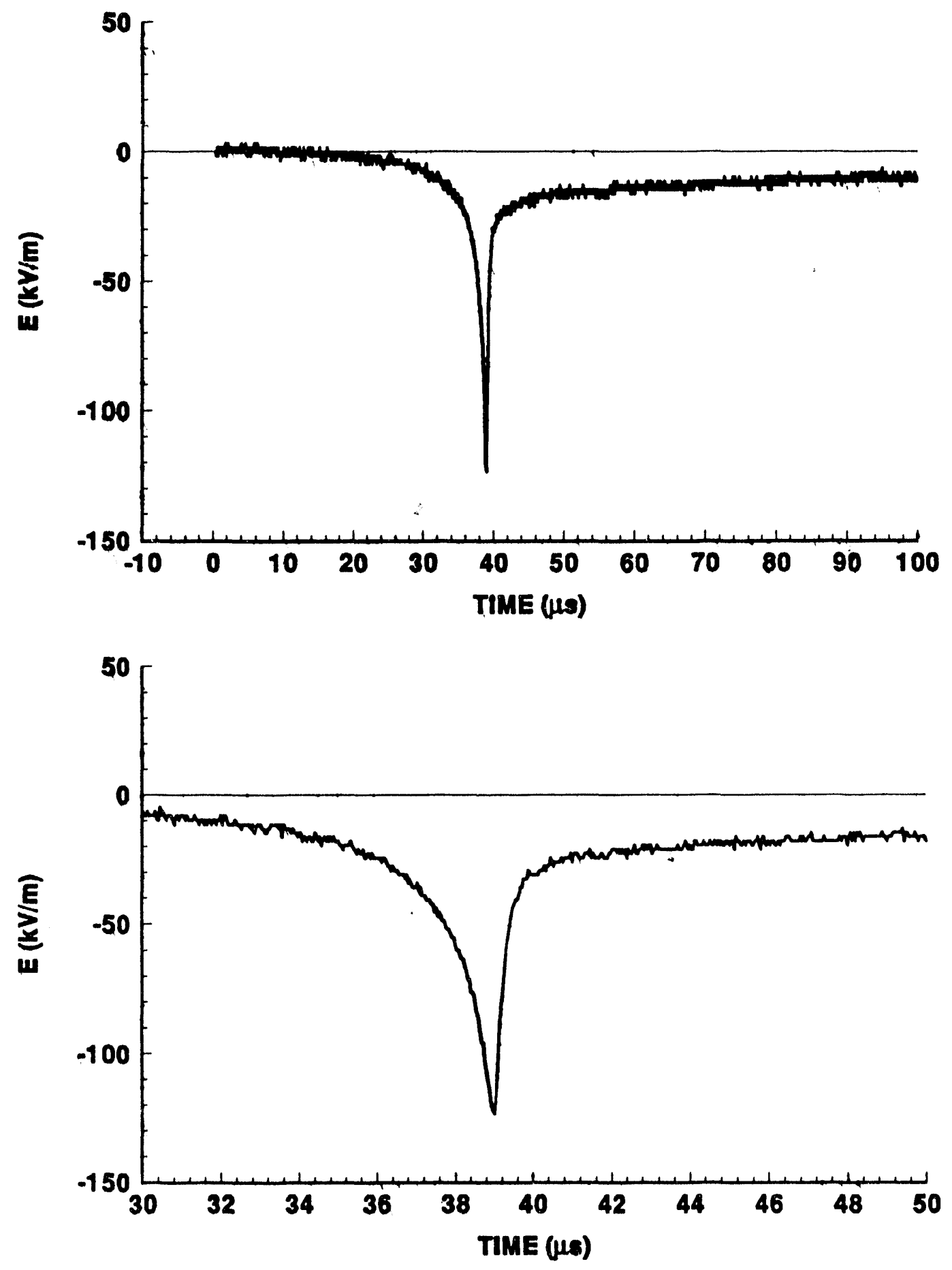


\section{STROKE 7 \\ ES3}
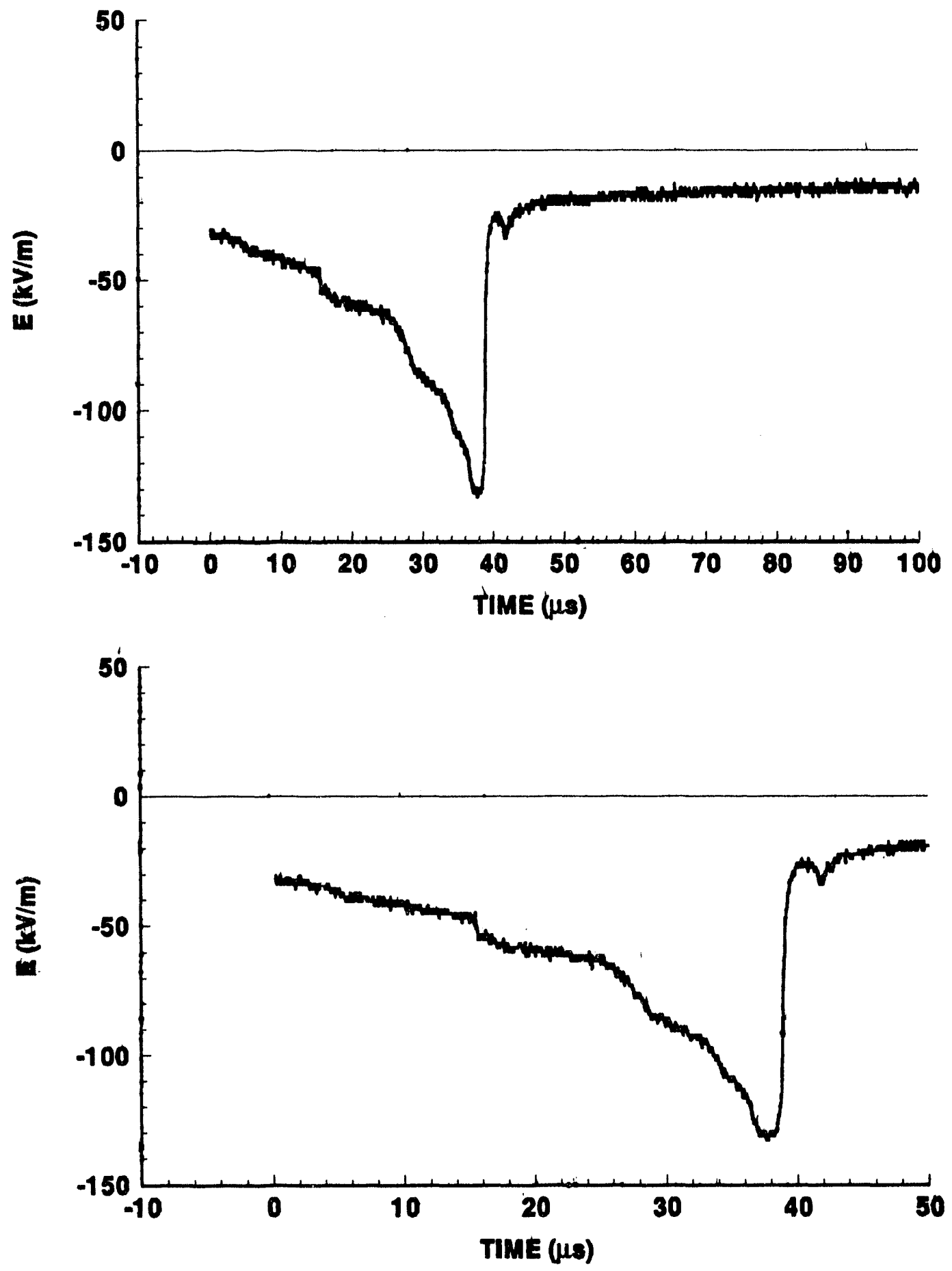


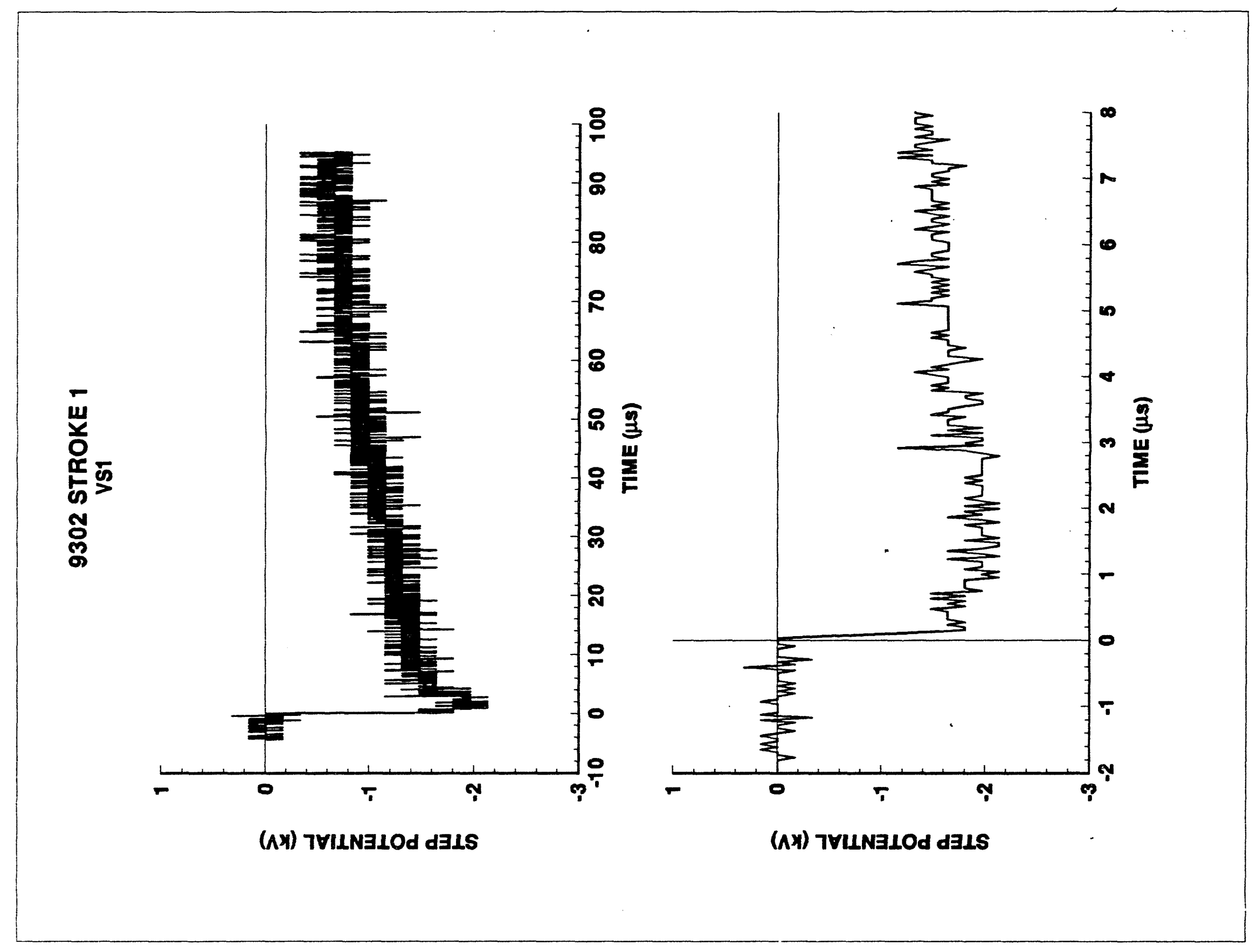


9302 STROKE 2

VS1
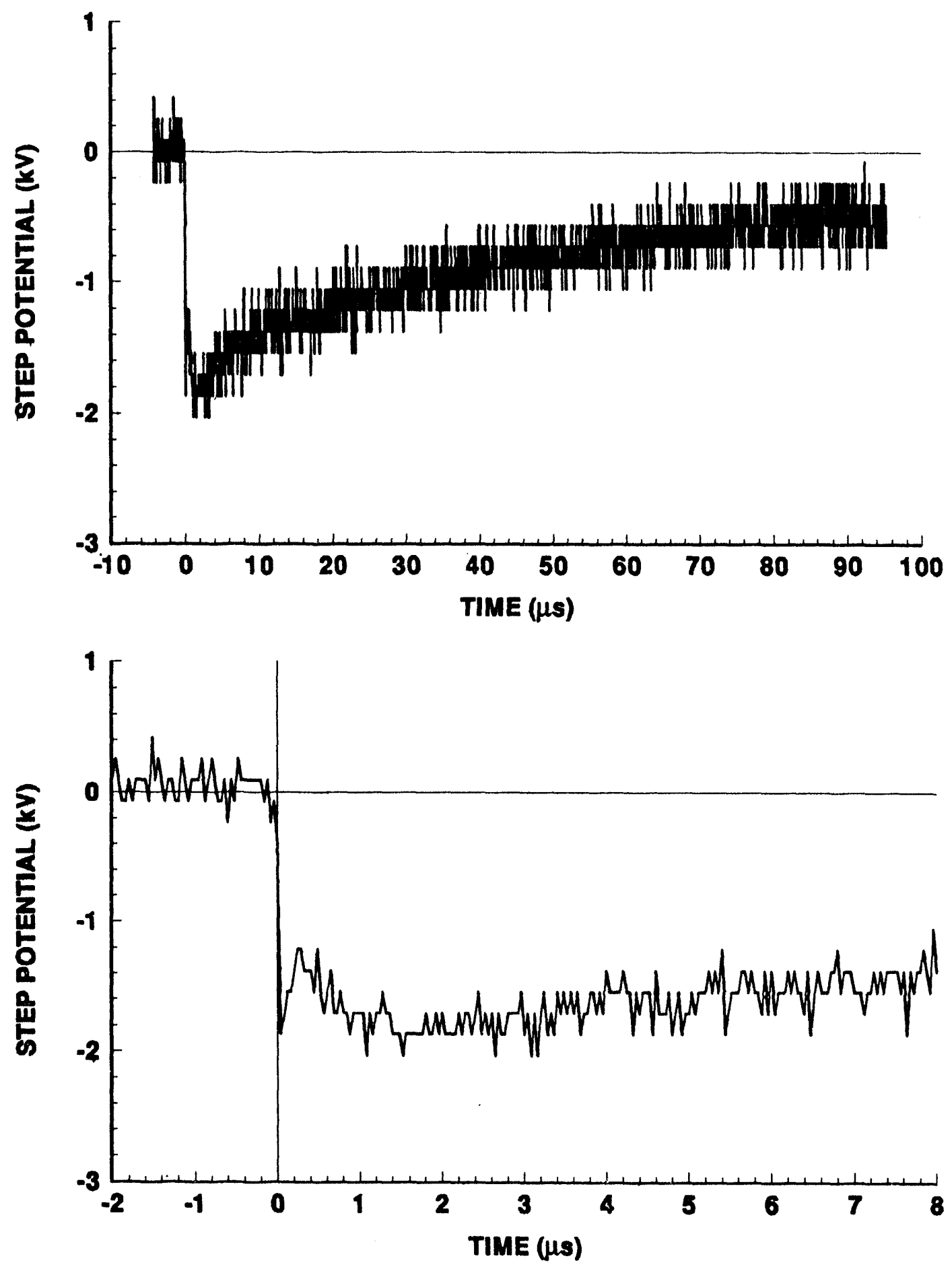

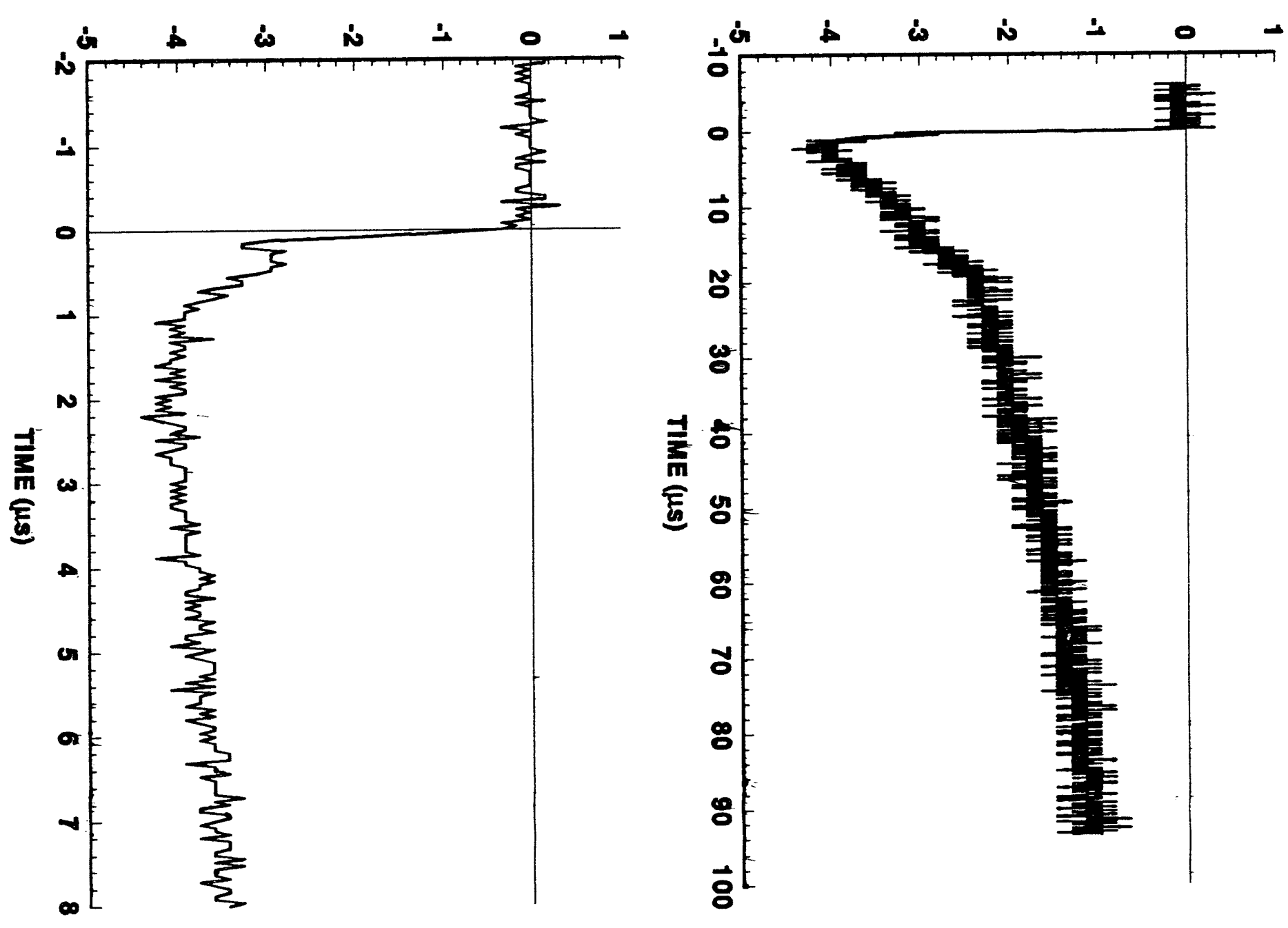


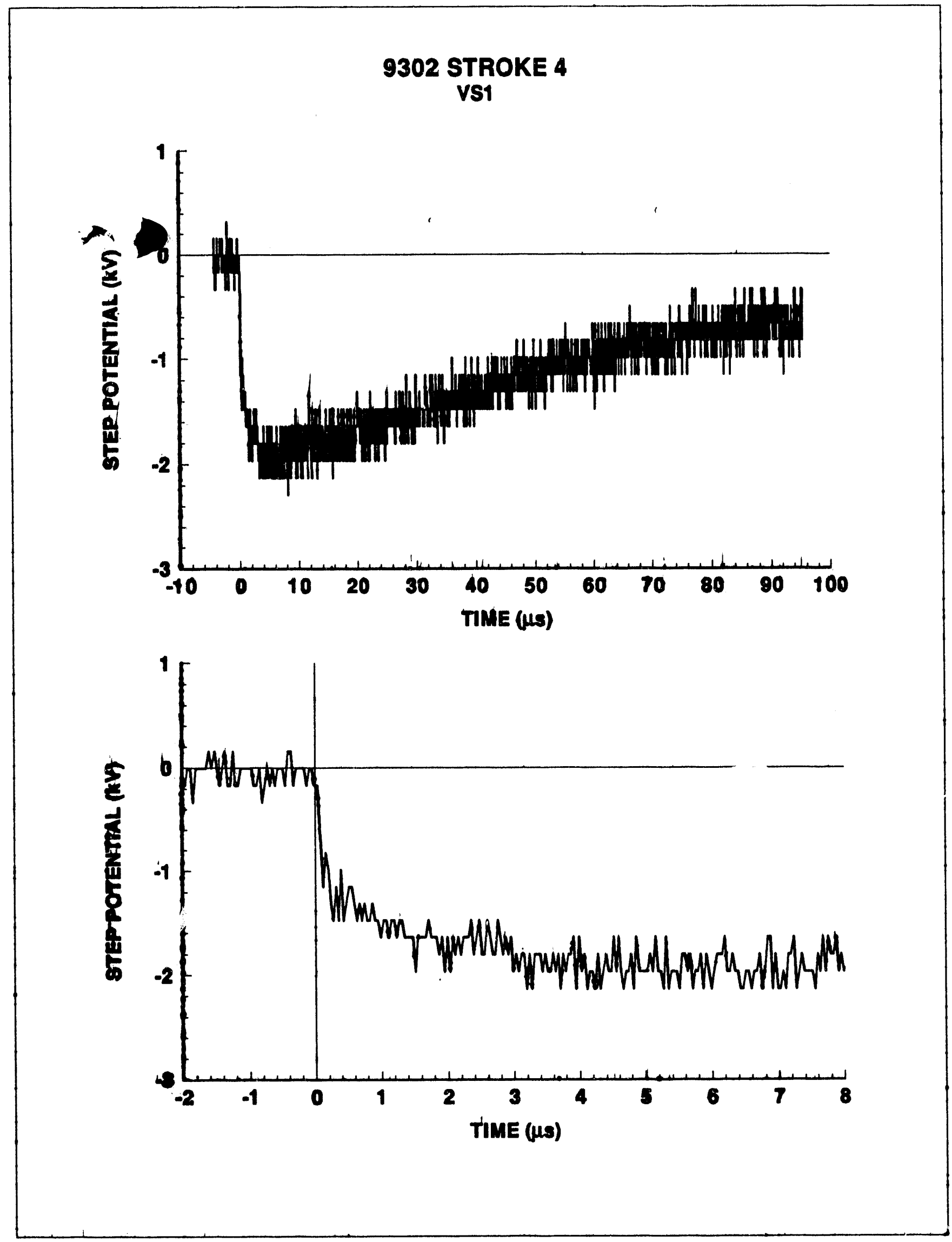




\section{STROKE 6 \\ vS1}
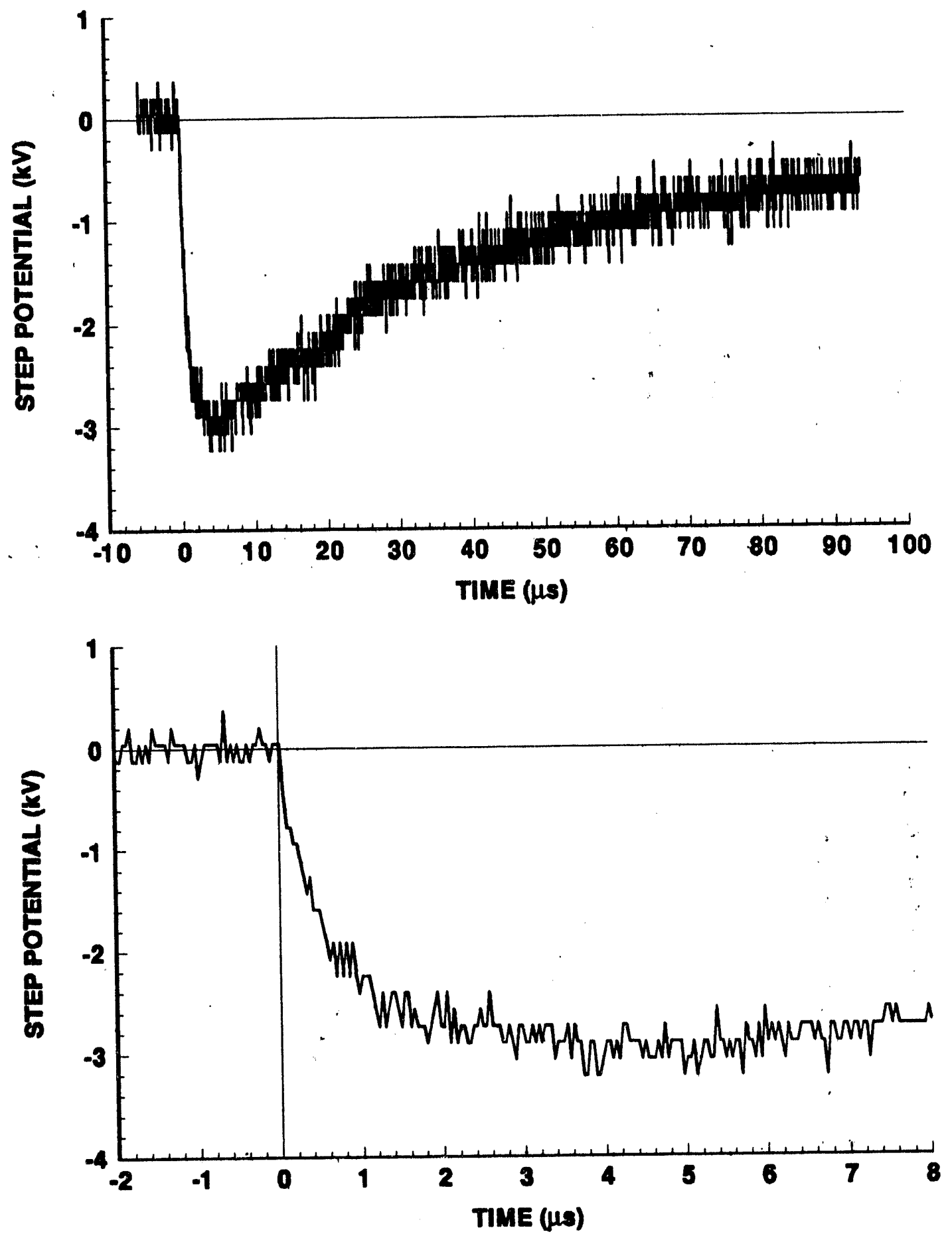


\section{STROKE 6 \\ VS1}
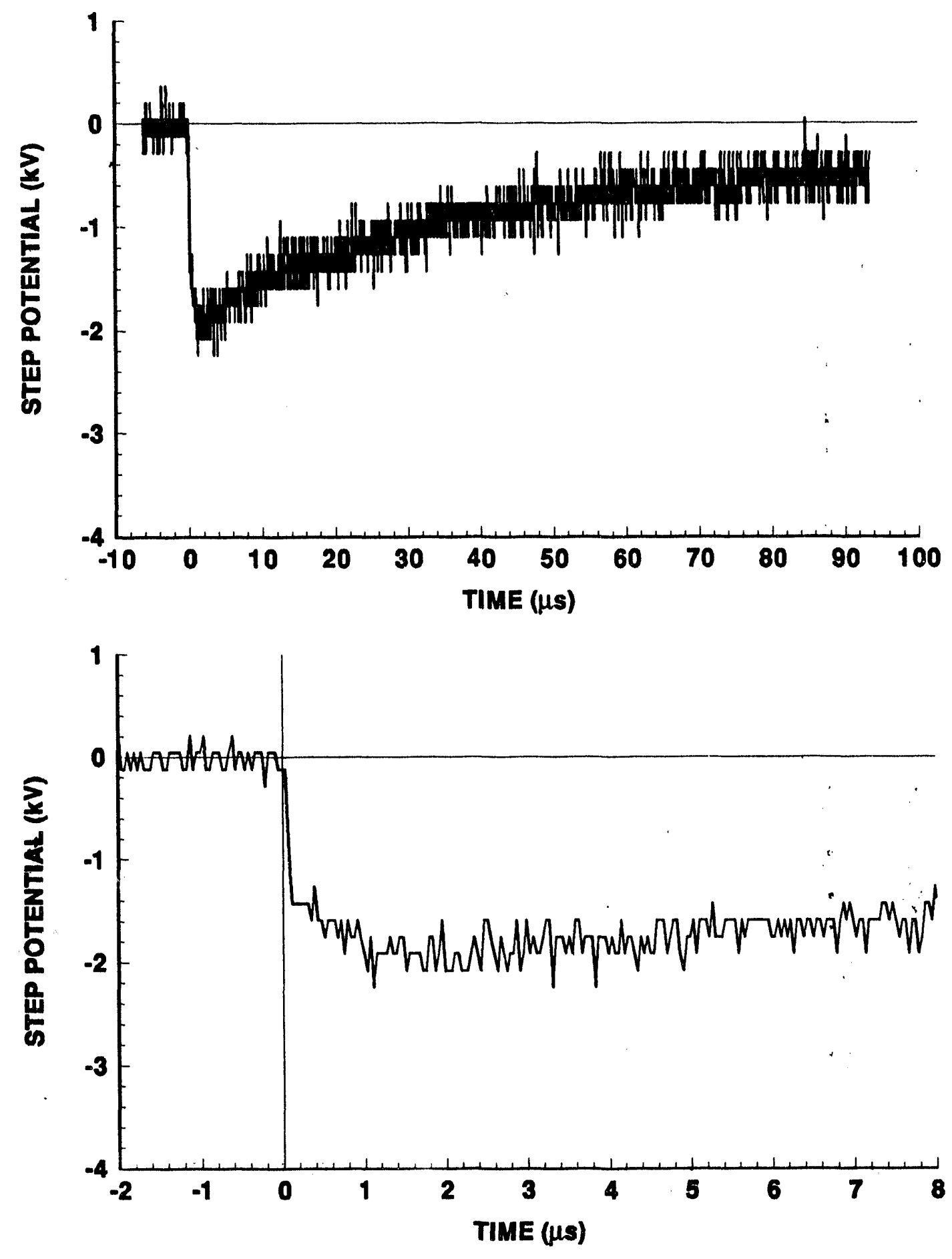


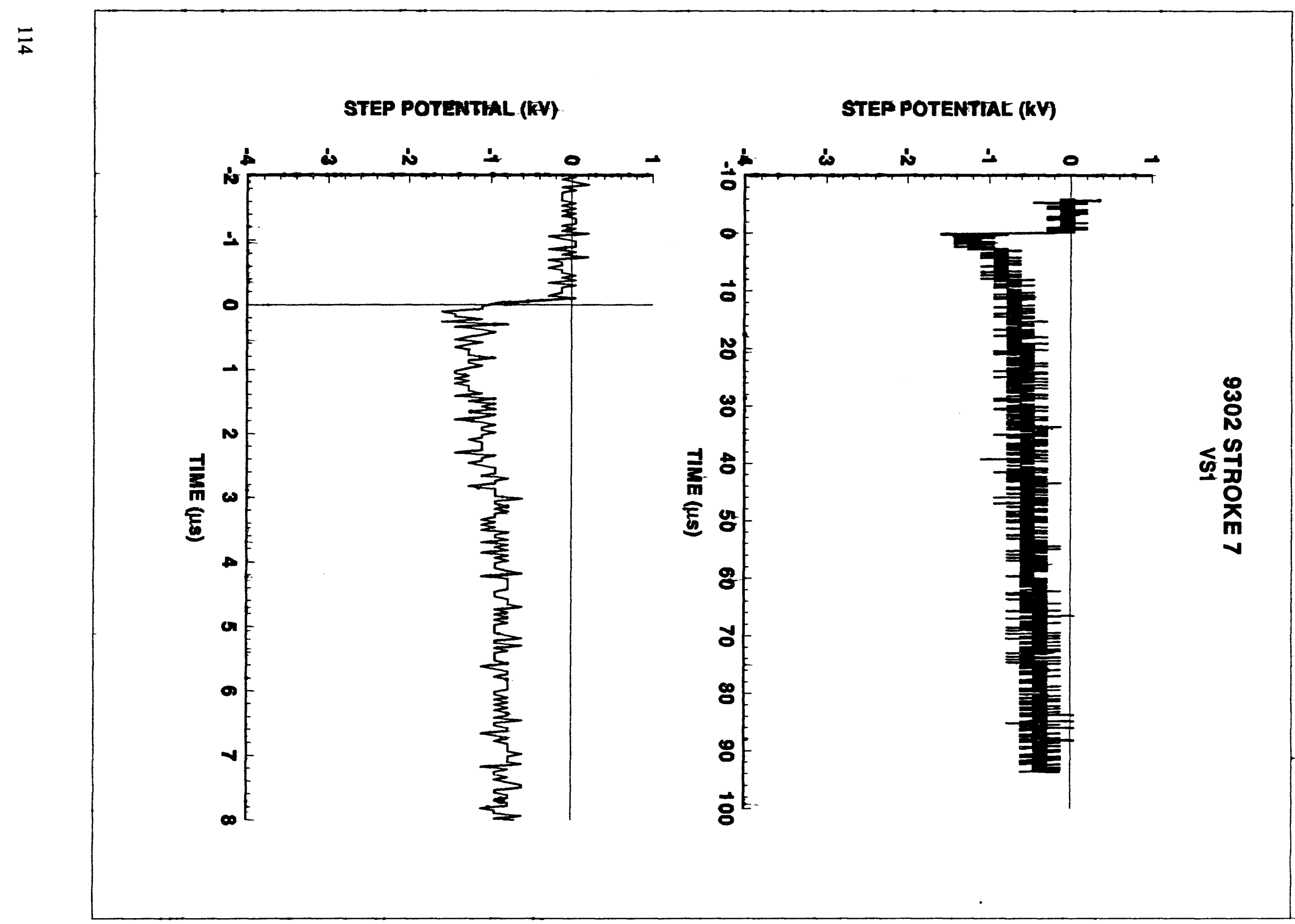




\section{STROKE 1 \\ vs2}
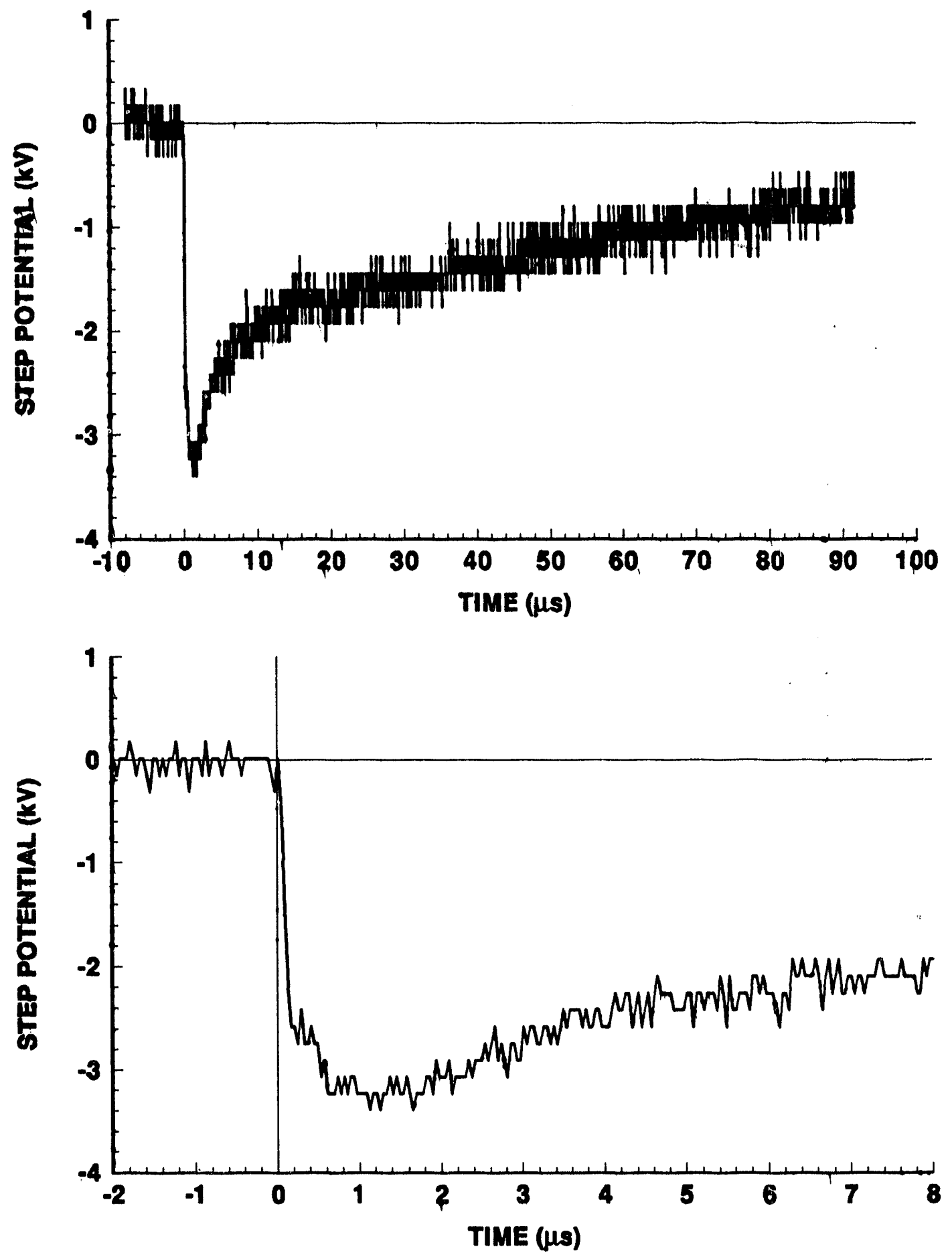


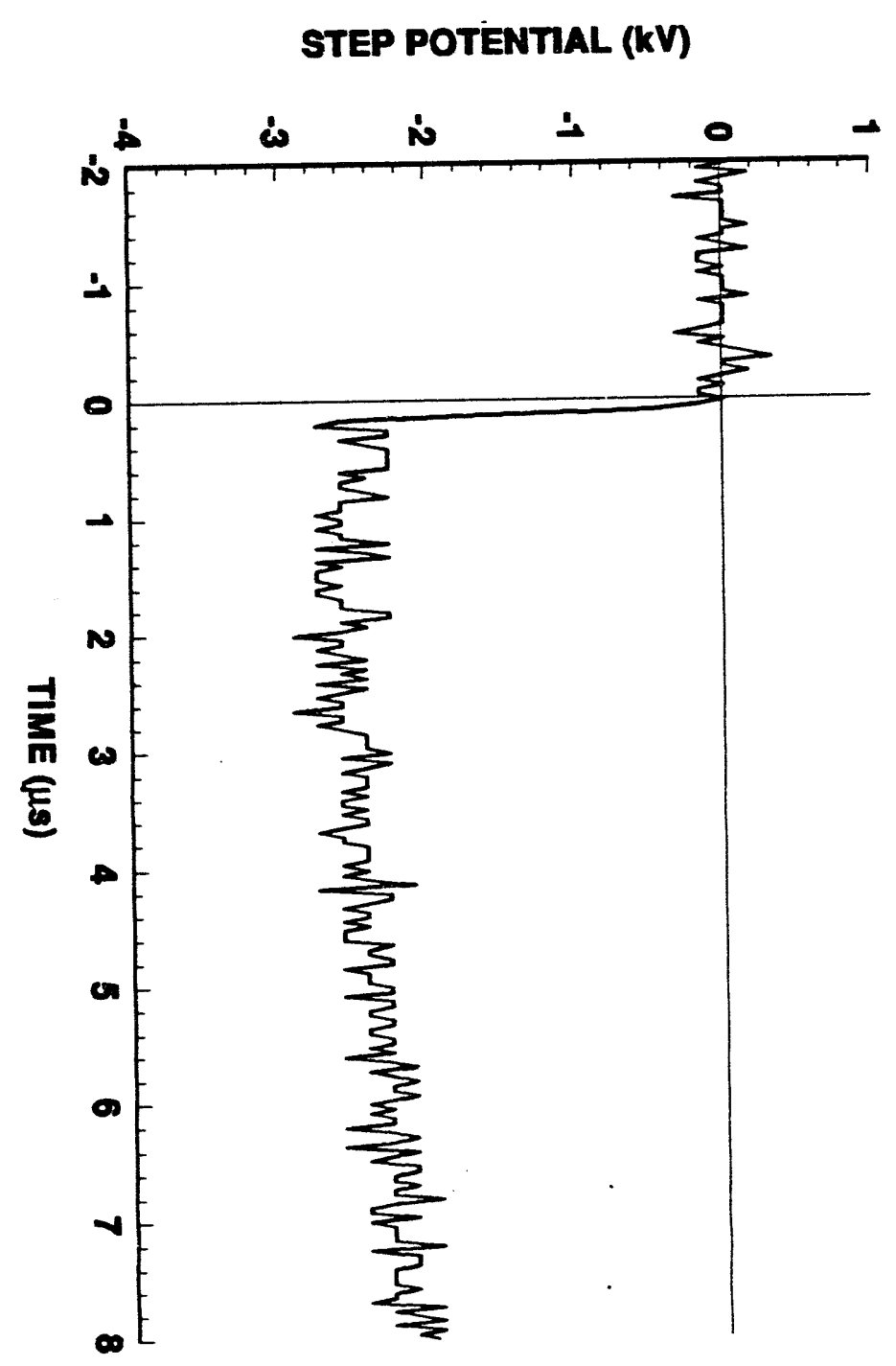

STEP POTENTIAL (kV)

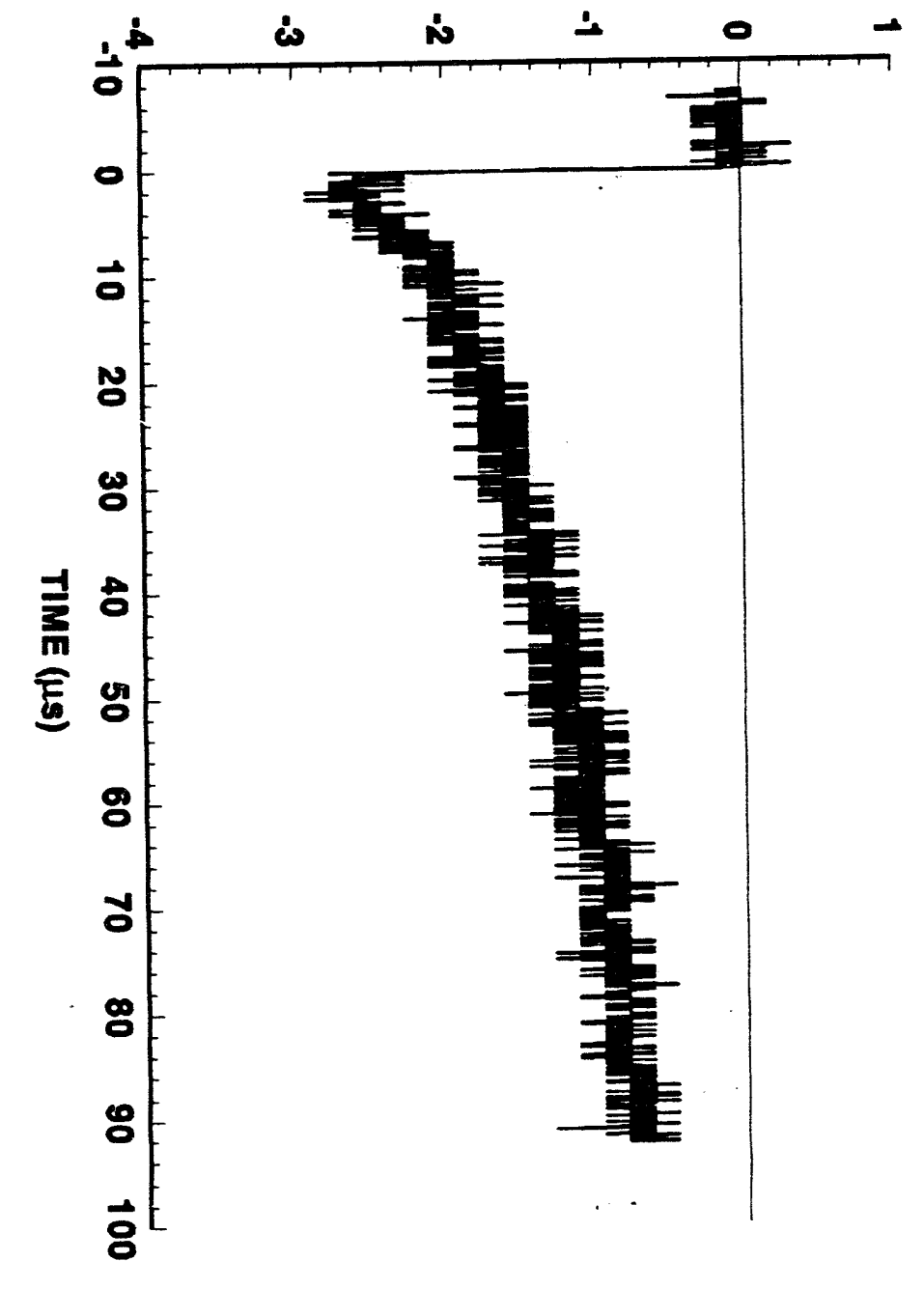




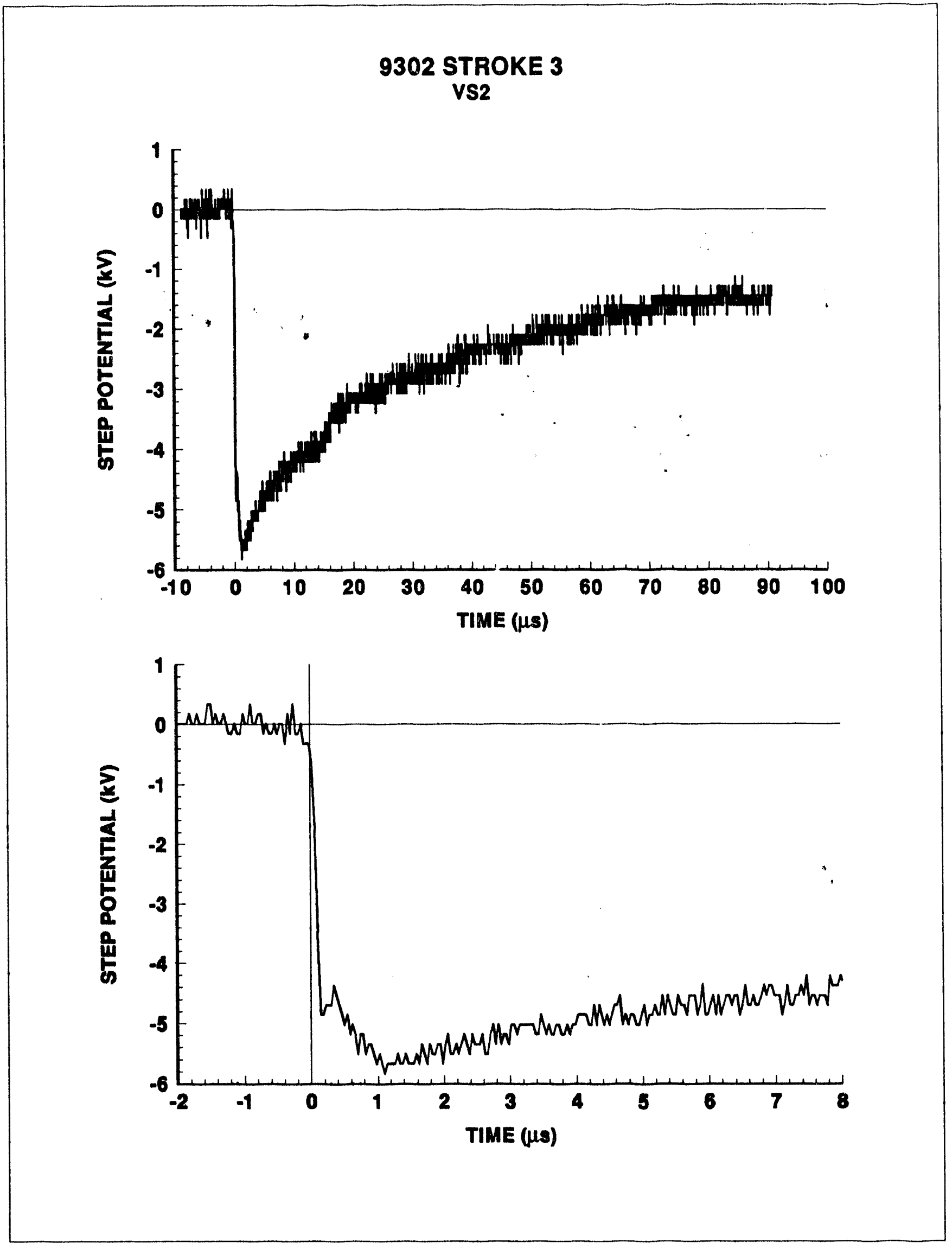




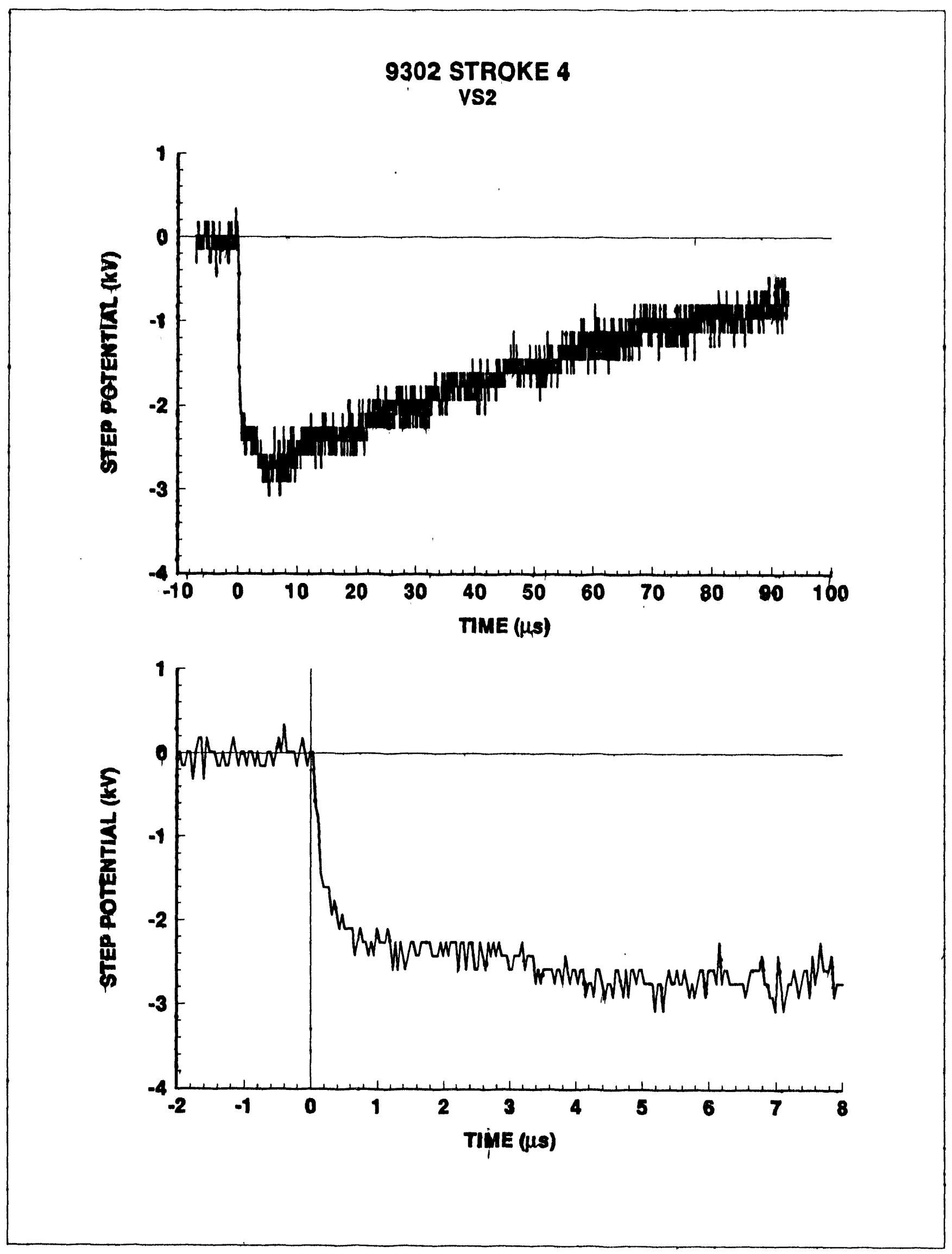




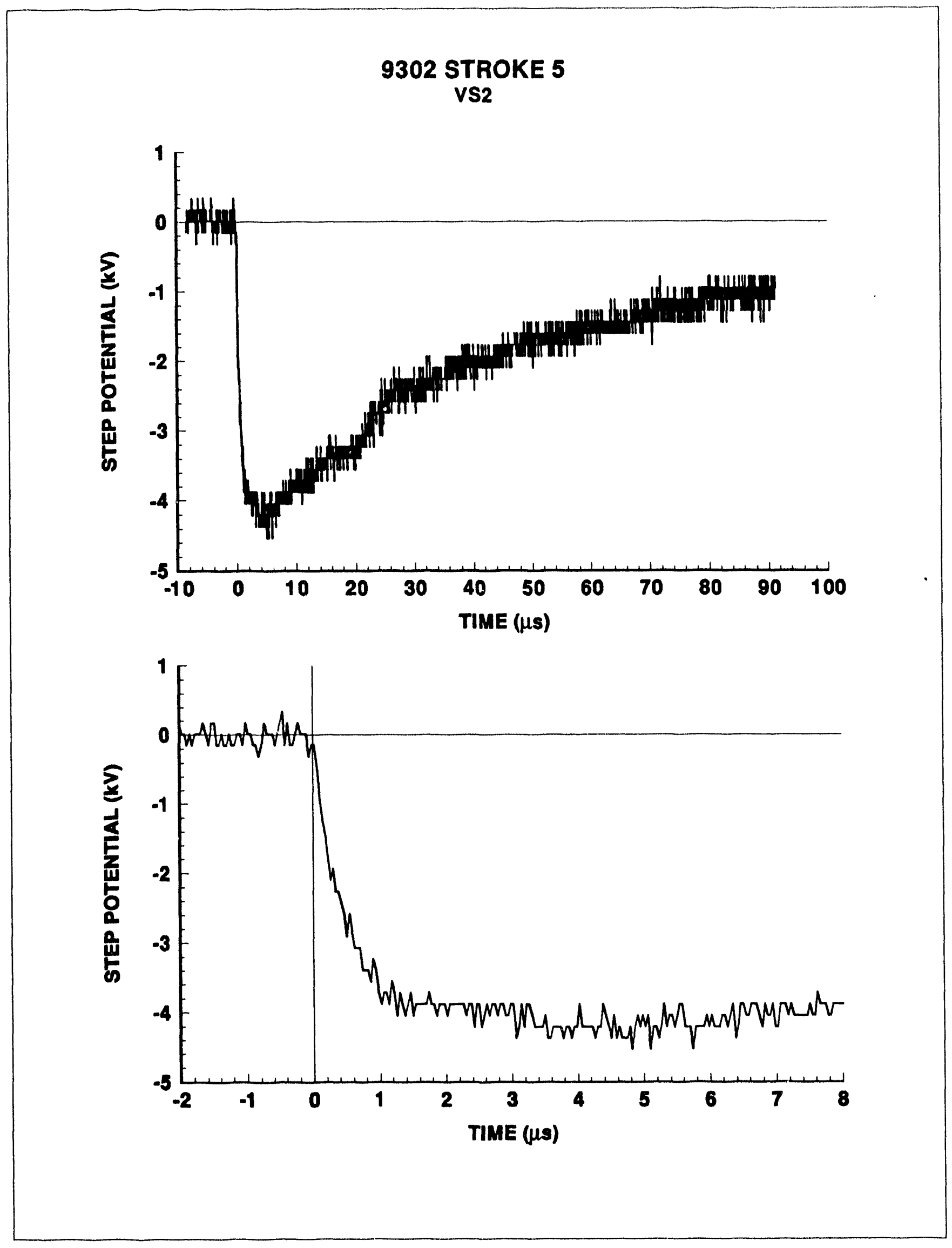


STEP POTENTIAL (kV)

STEP POTENTIAL (kV)
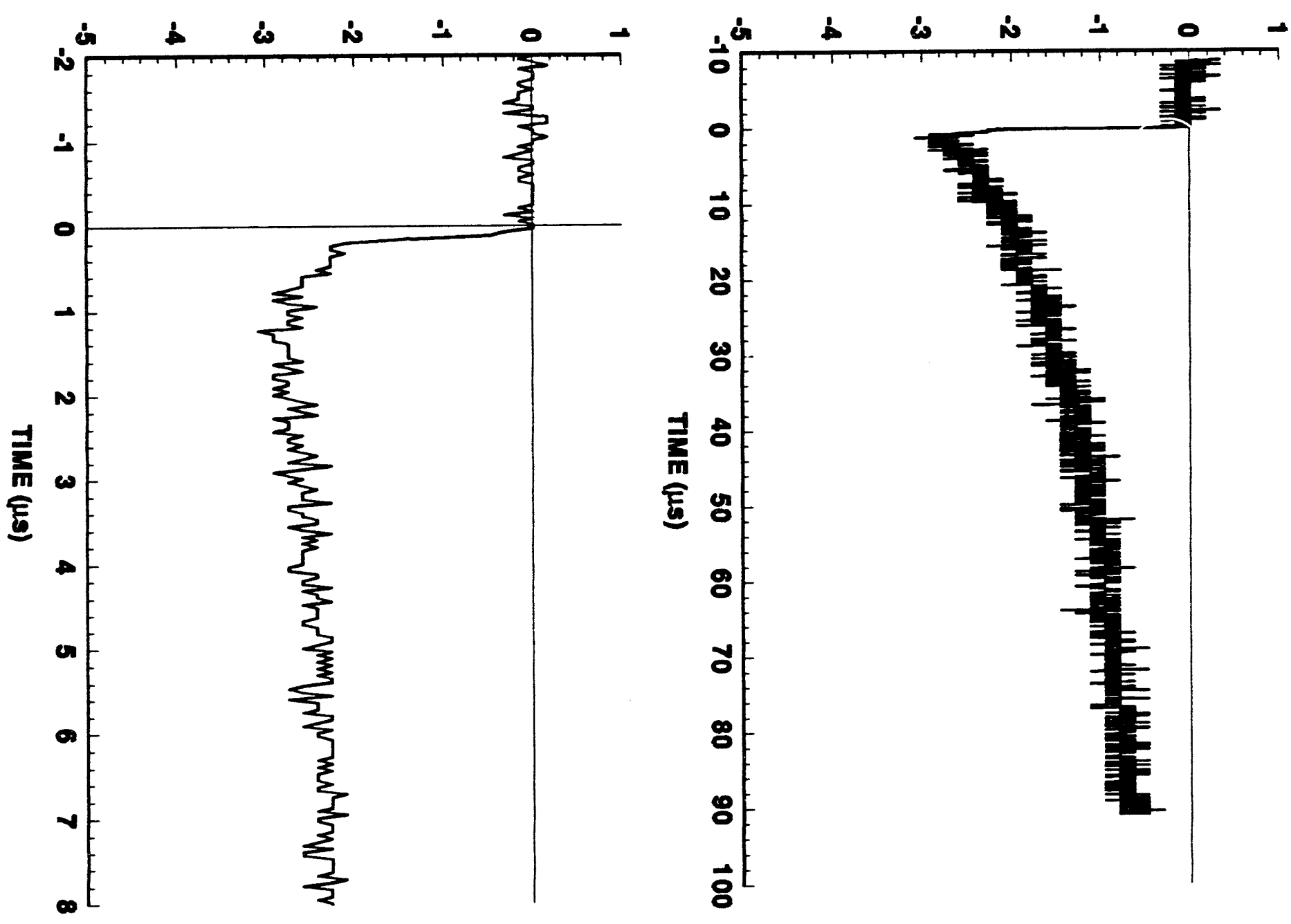

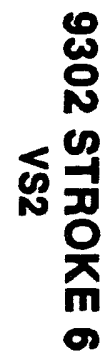


STEP POTENTIAL (kV)

STEP POTENTIAL (KV)
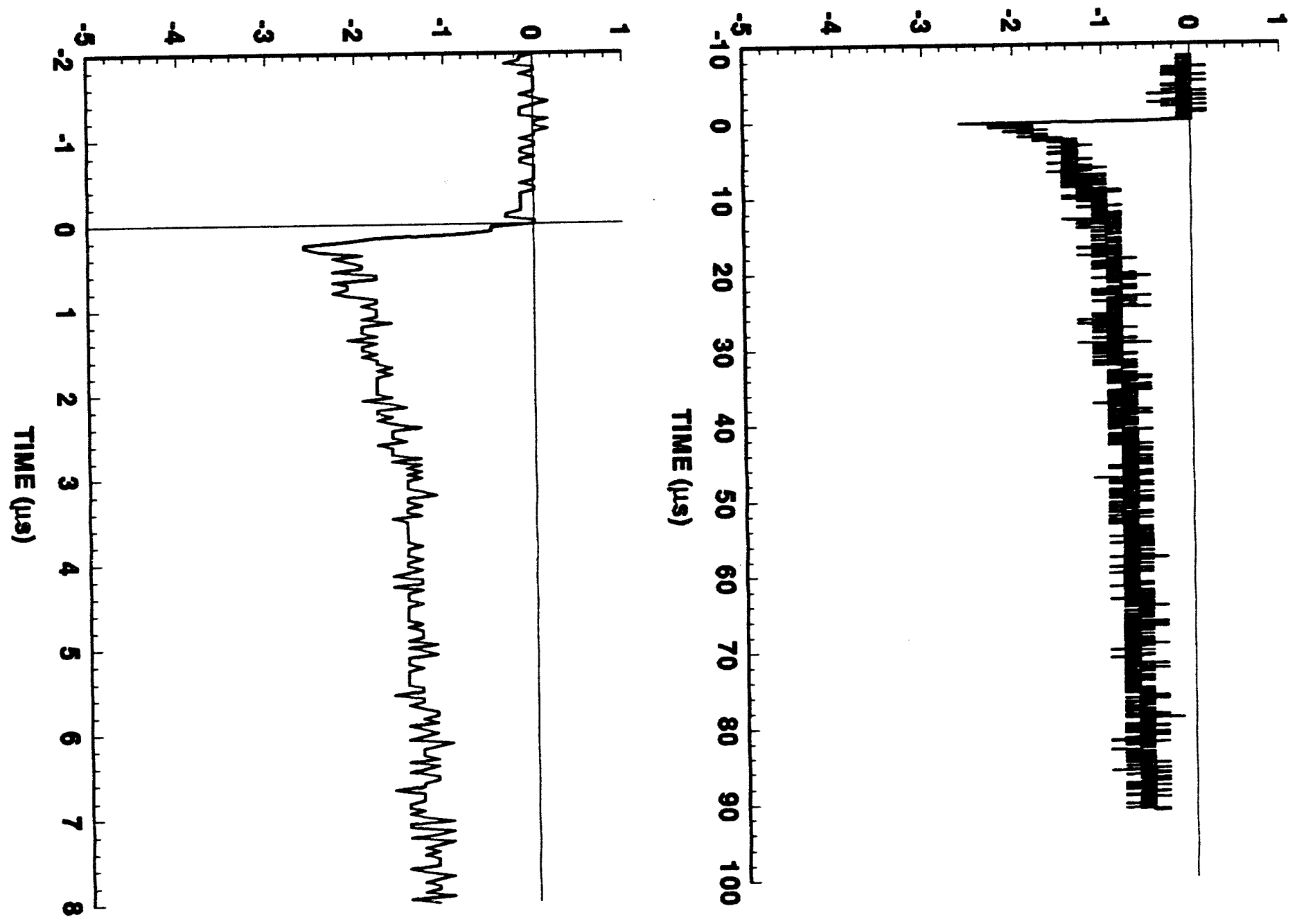

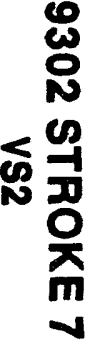




\section{STROKE 1}

vS3
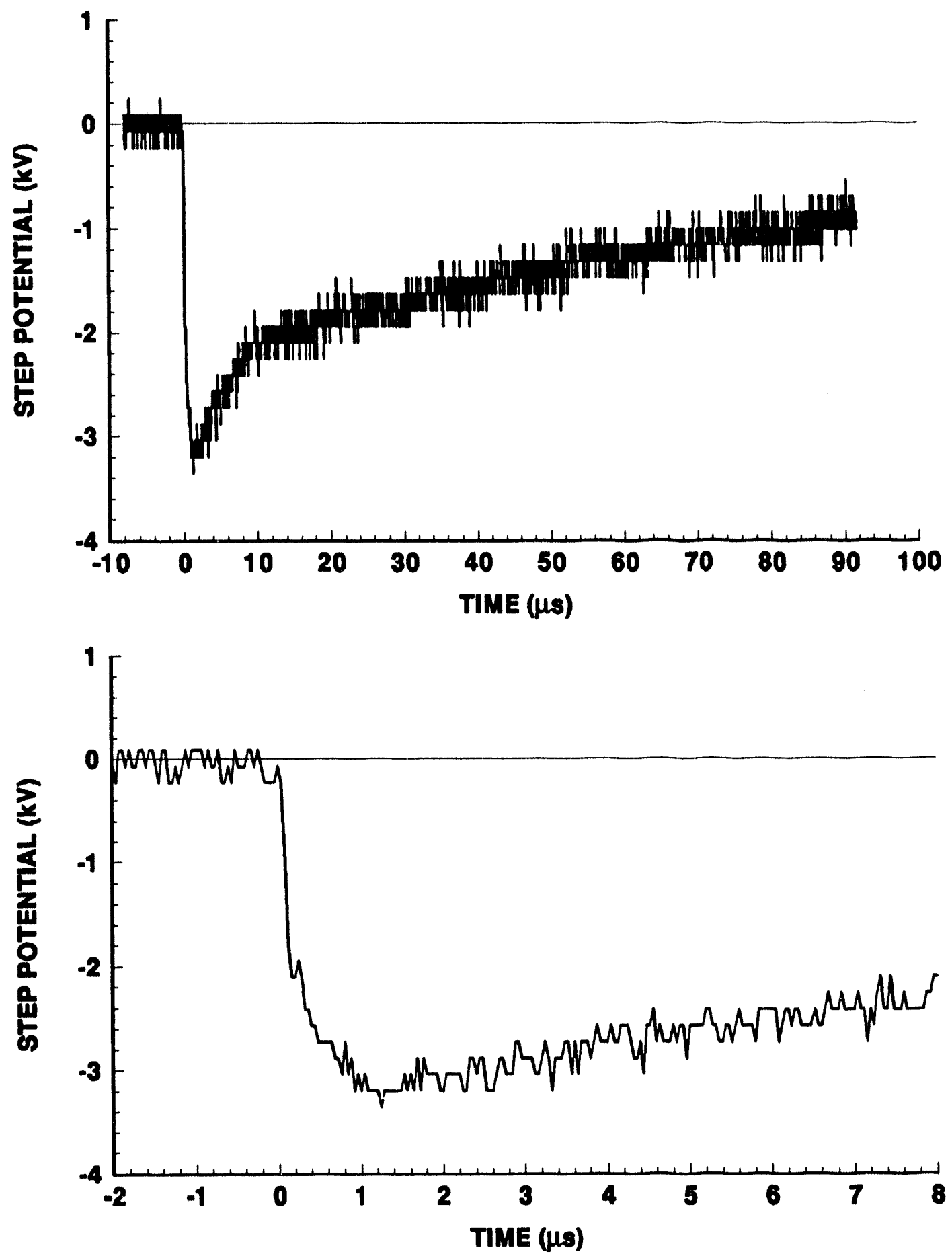
9302 STROKE 2

vS3
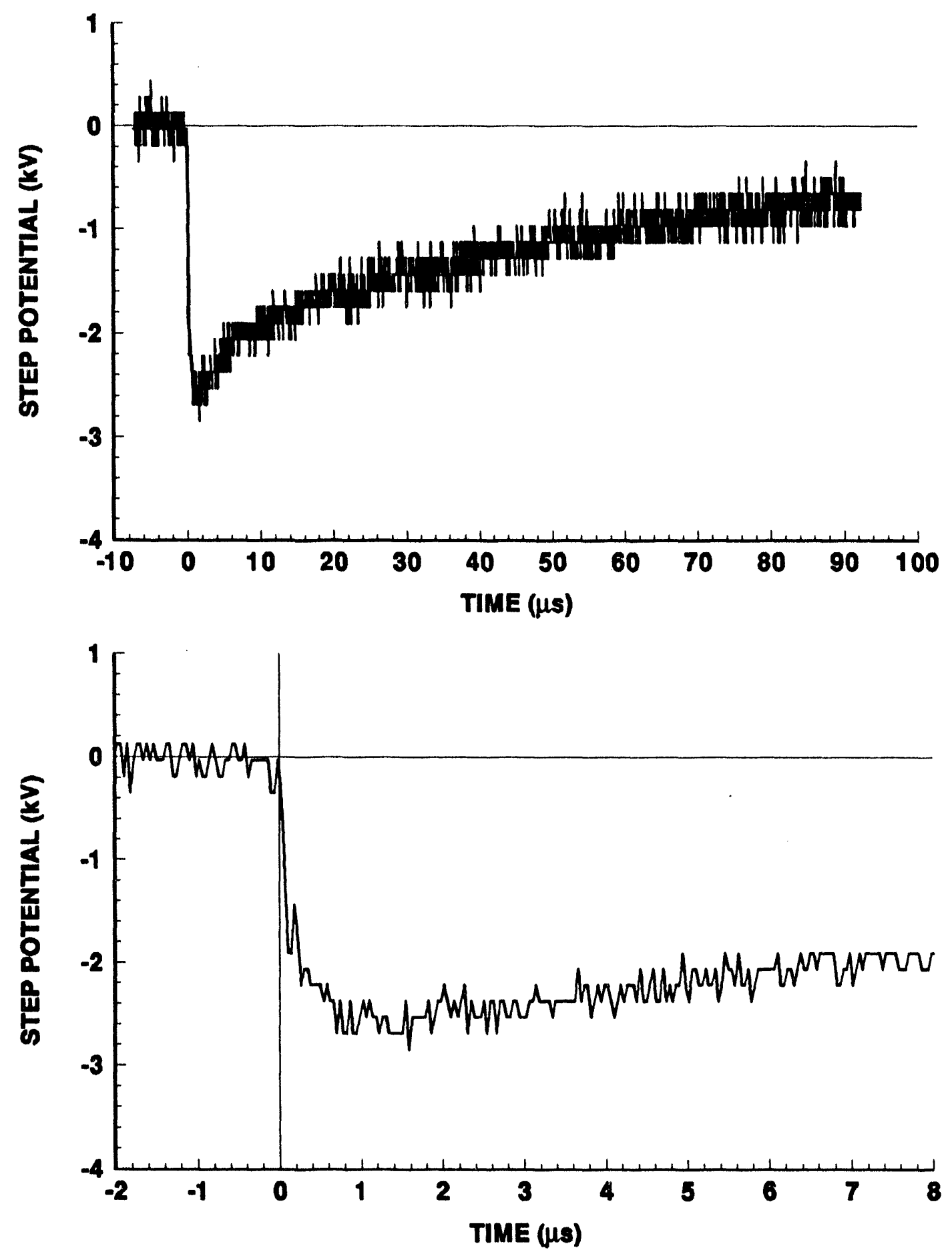

123 


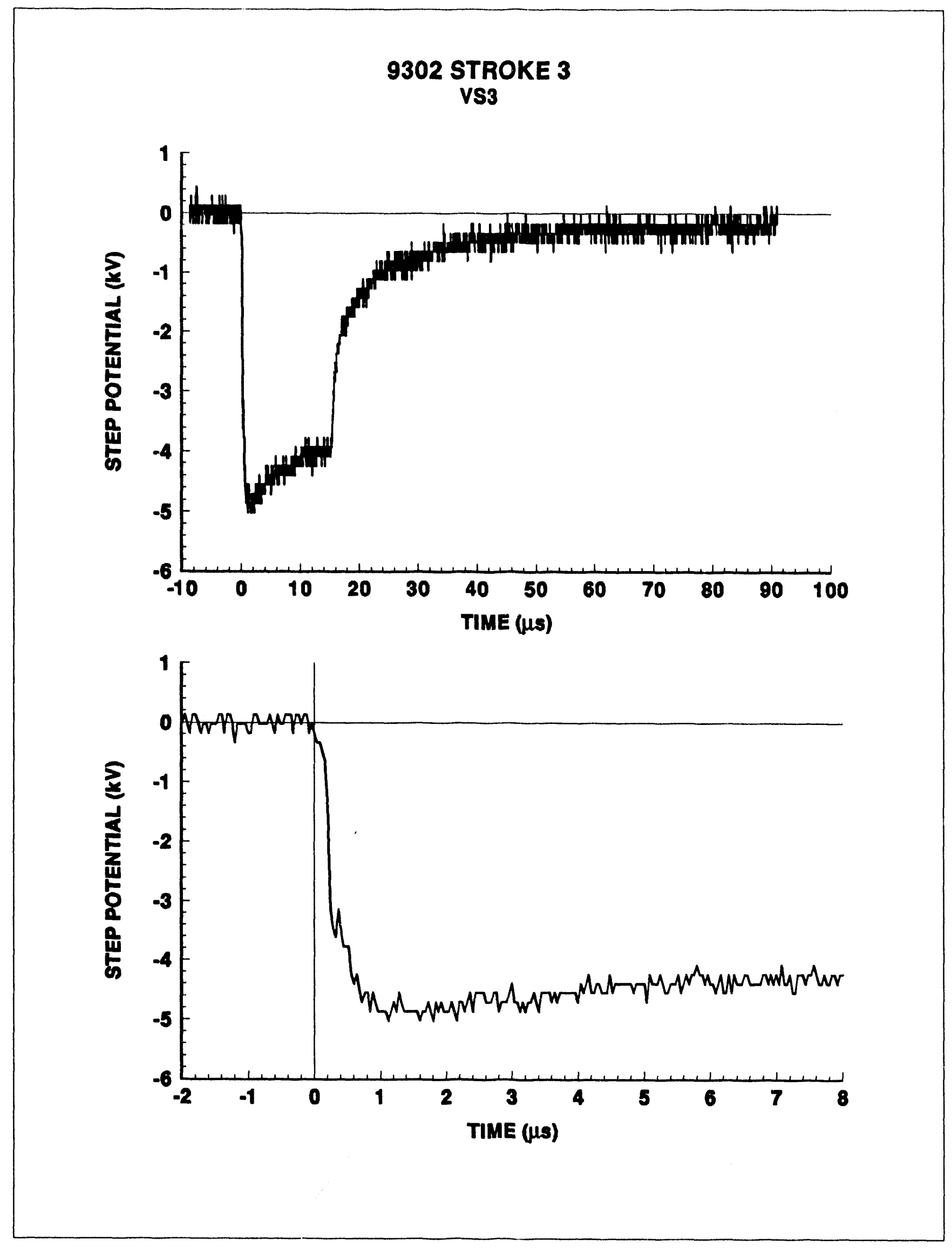




\section{STROKE 4}

VS3
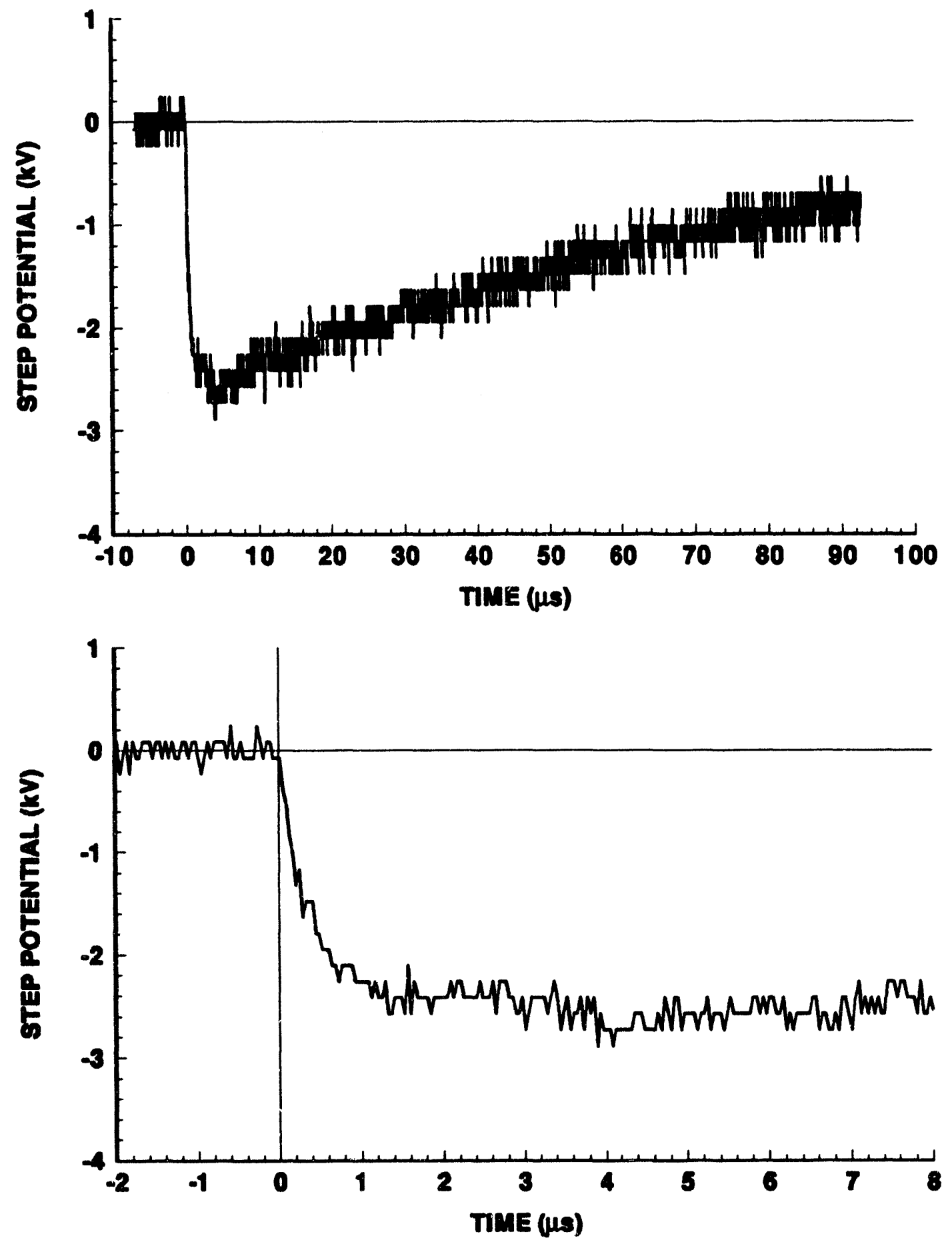


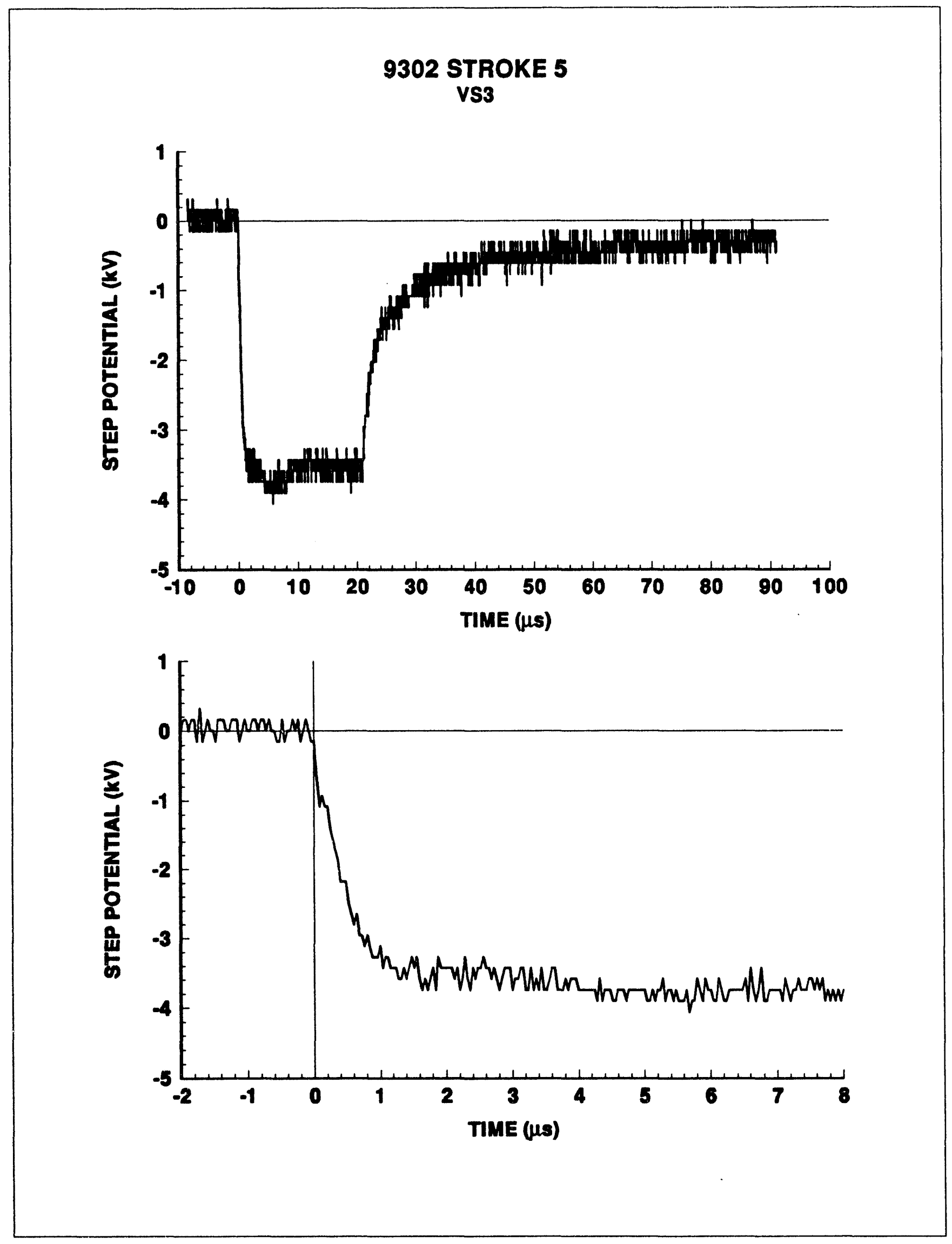




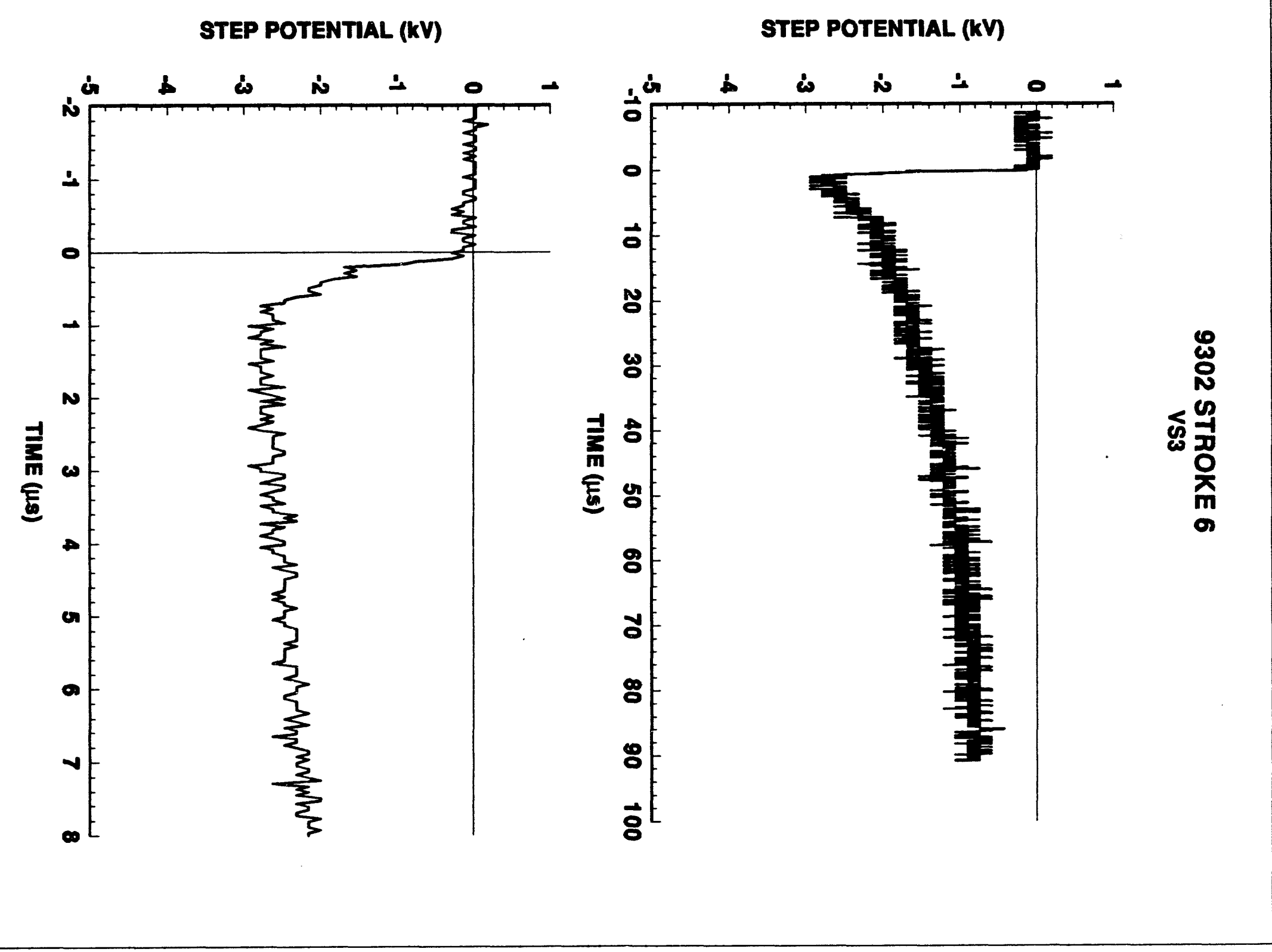




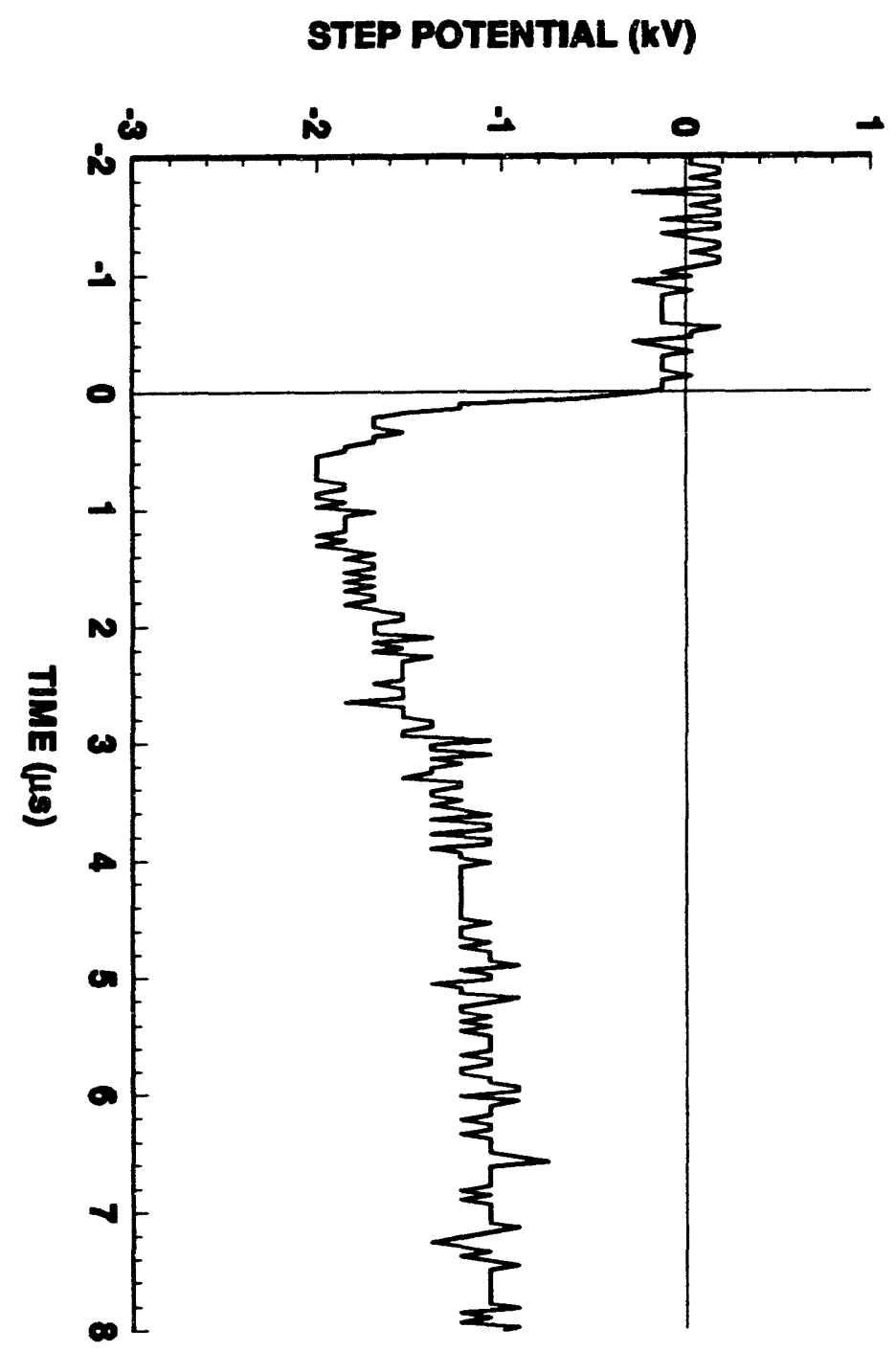

STEP POTENTIAL (kV)

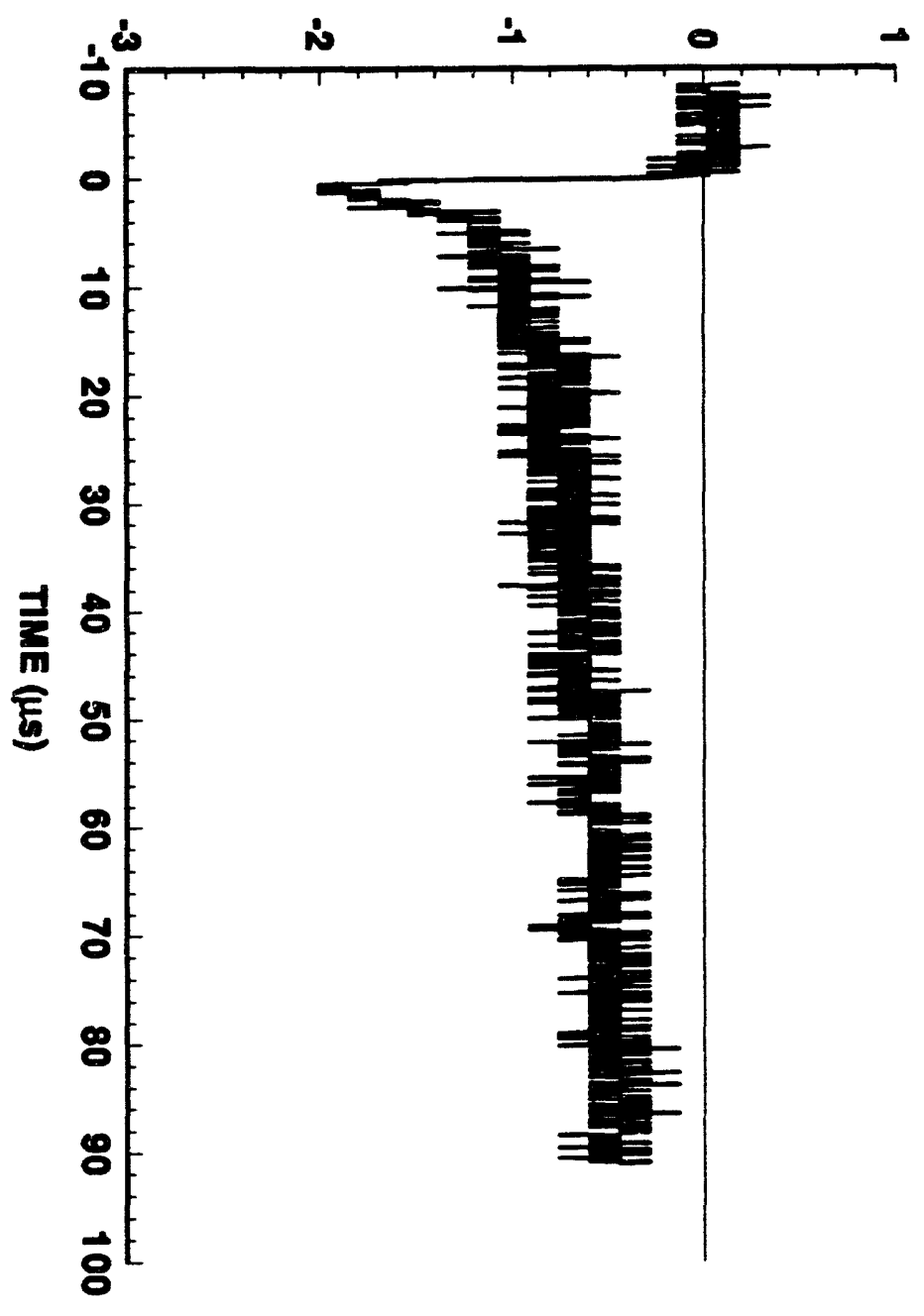

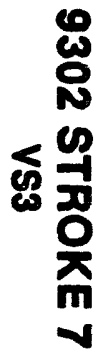




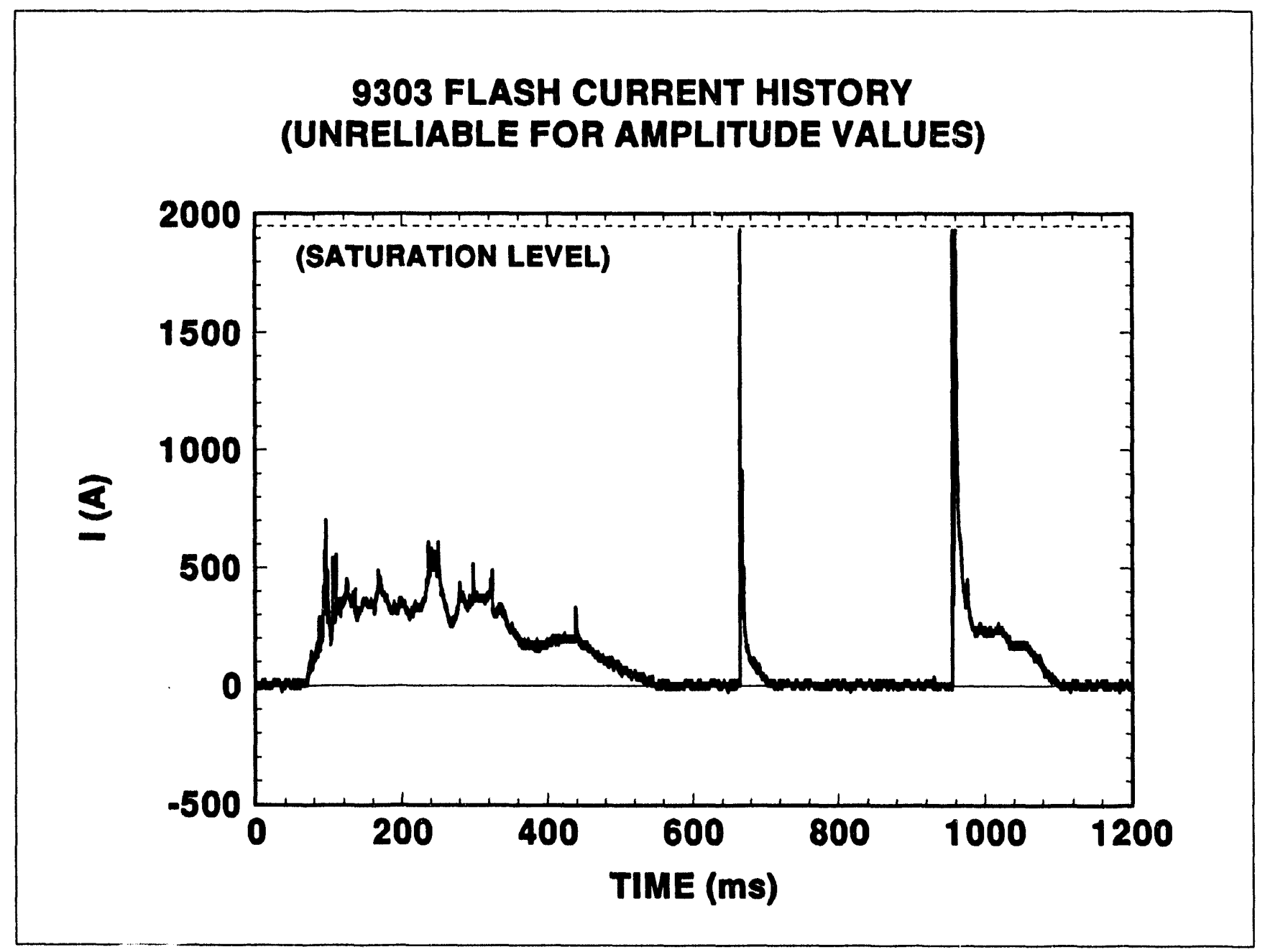




\section{STROKE 1}

HS1
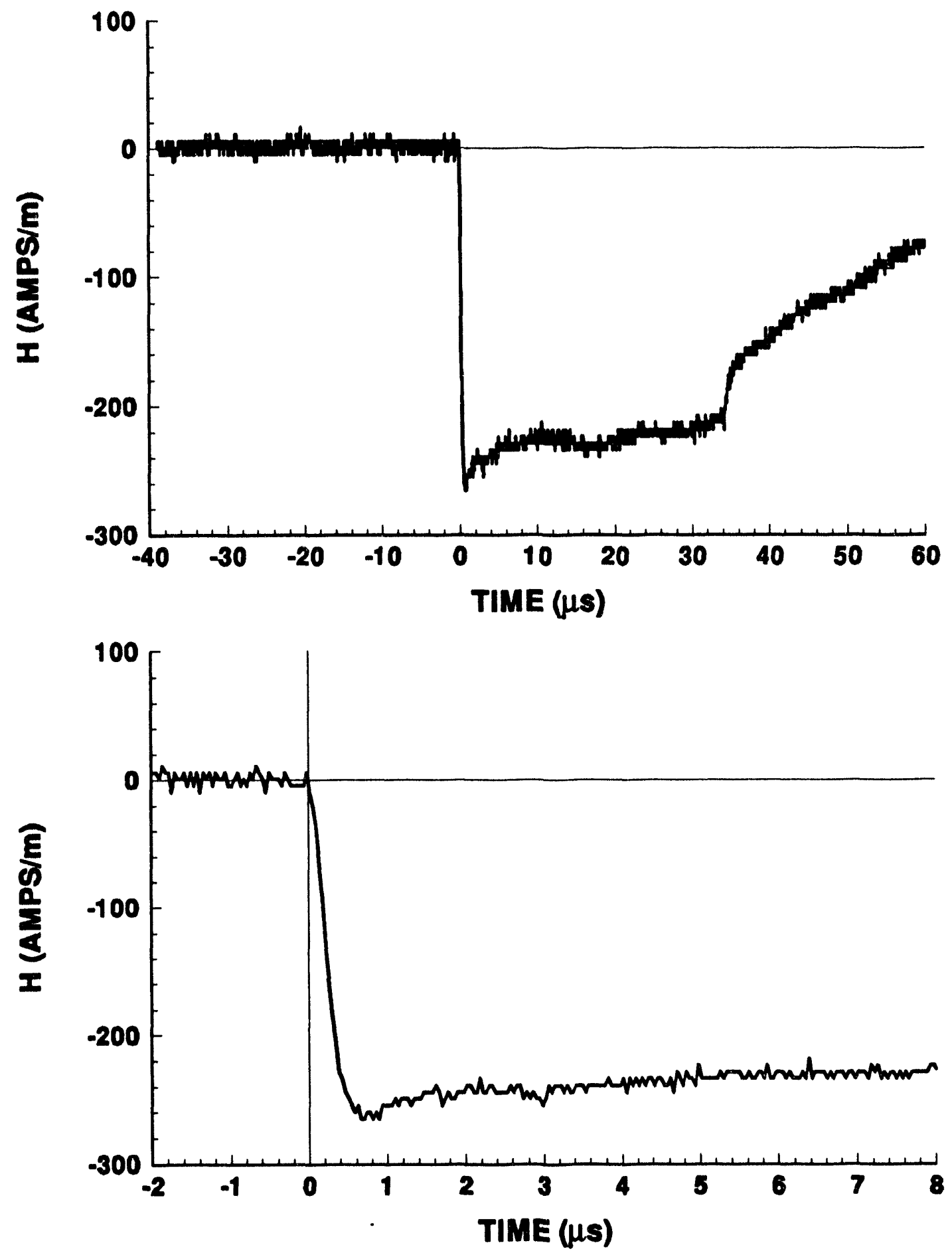


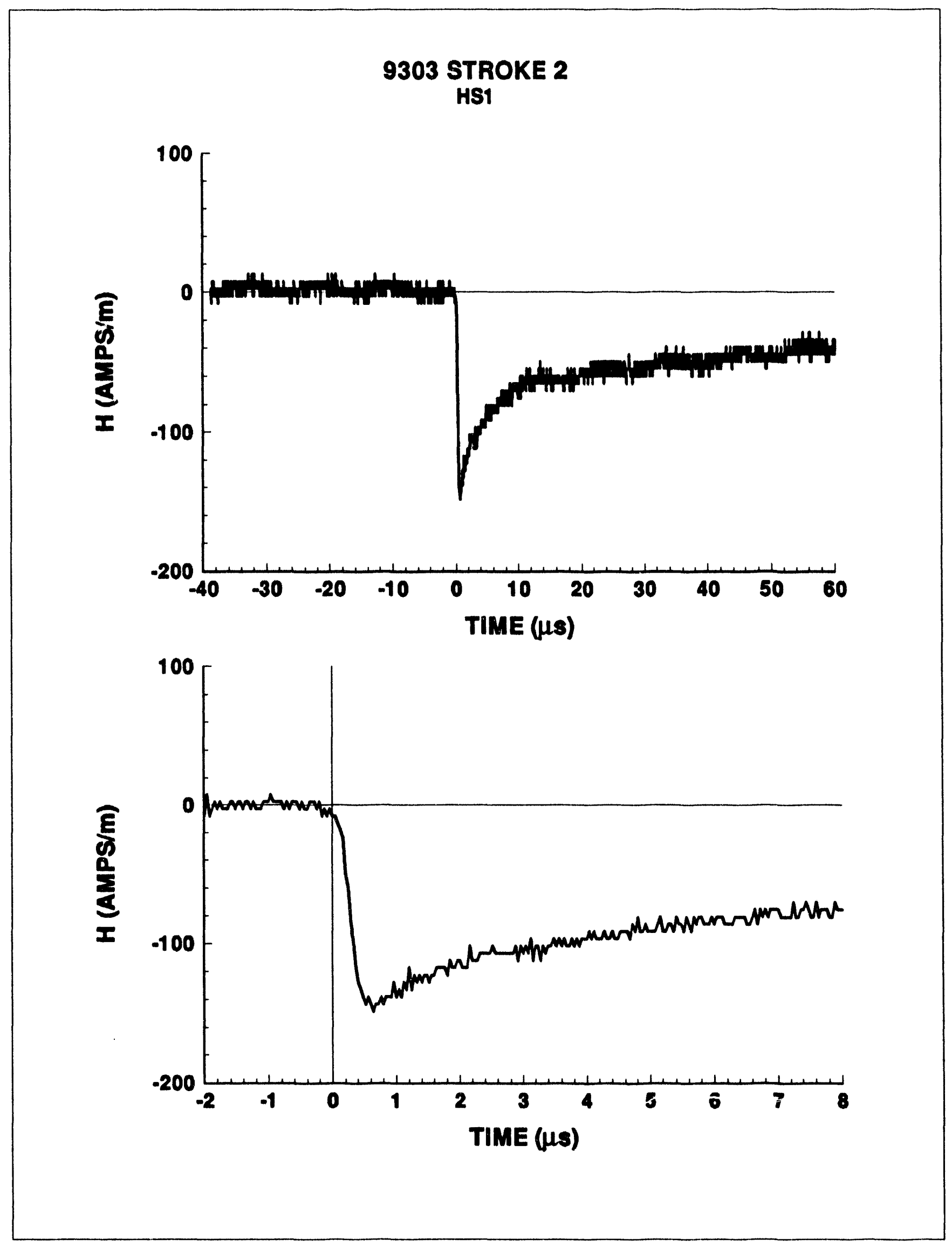




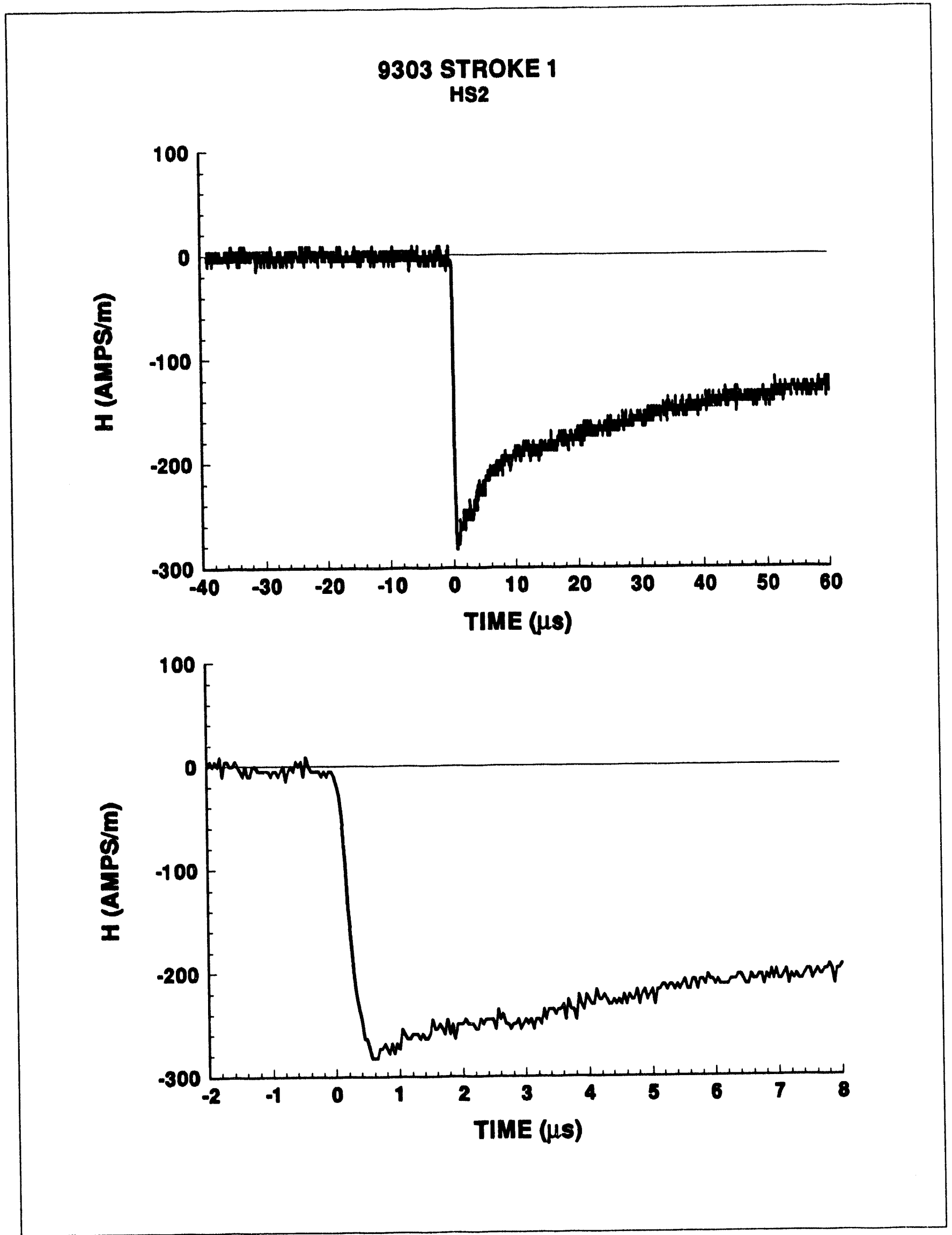




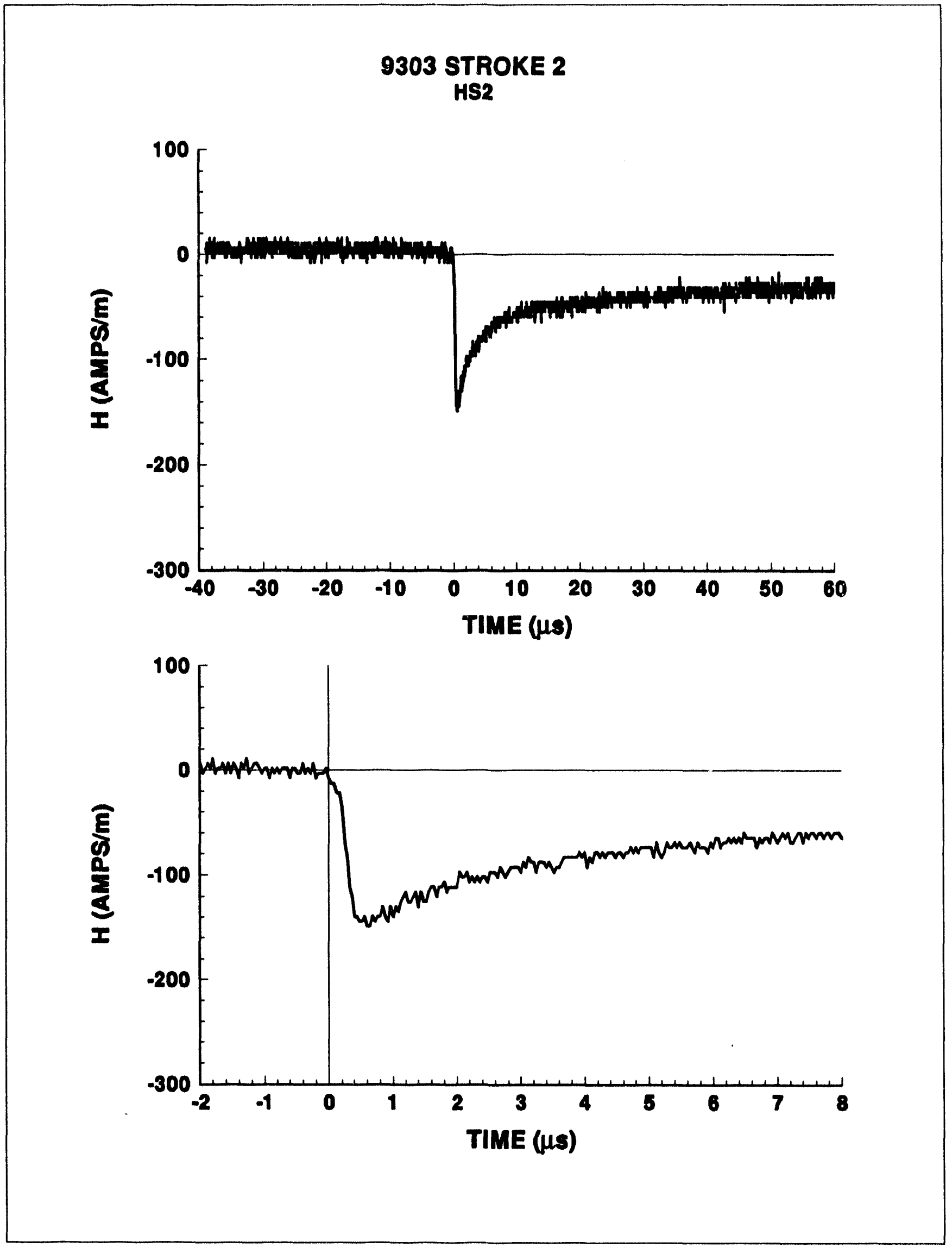




\section{STROKE 1}

\section{HS3}
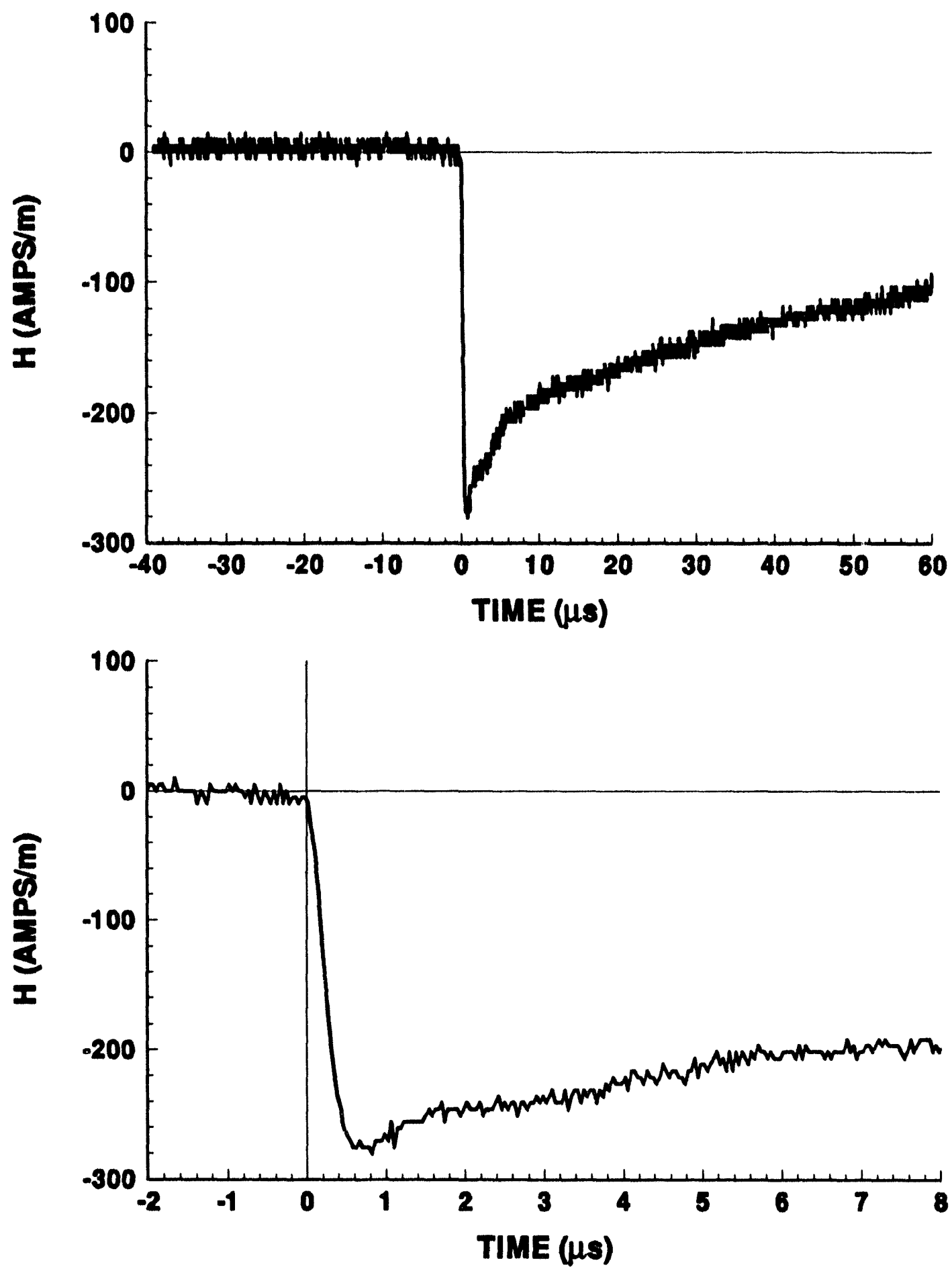


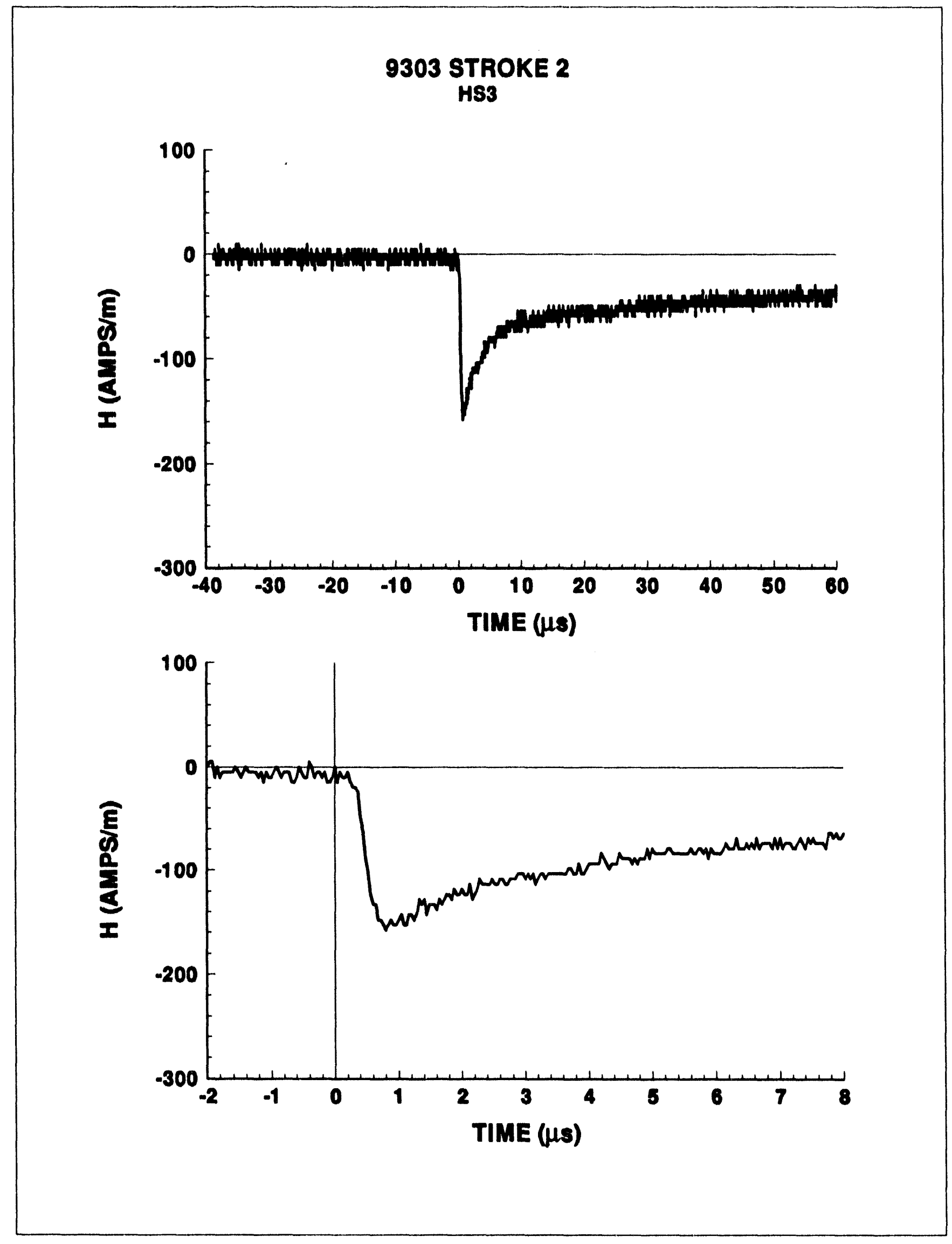




\section{STROKE 1}

ES1

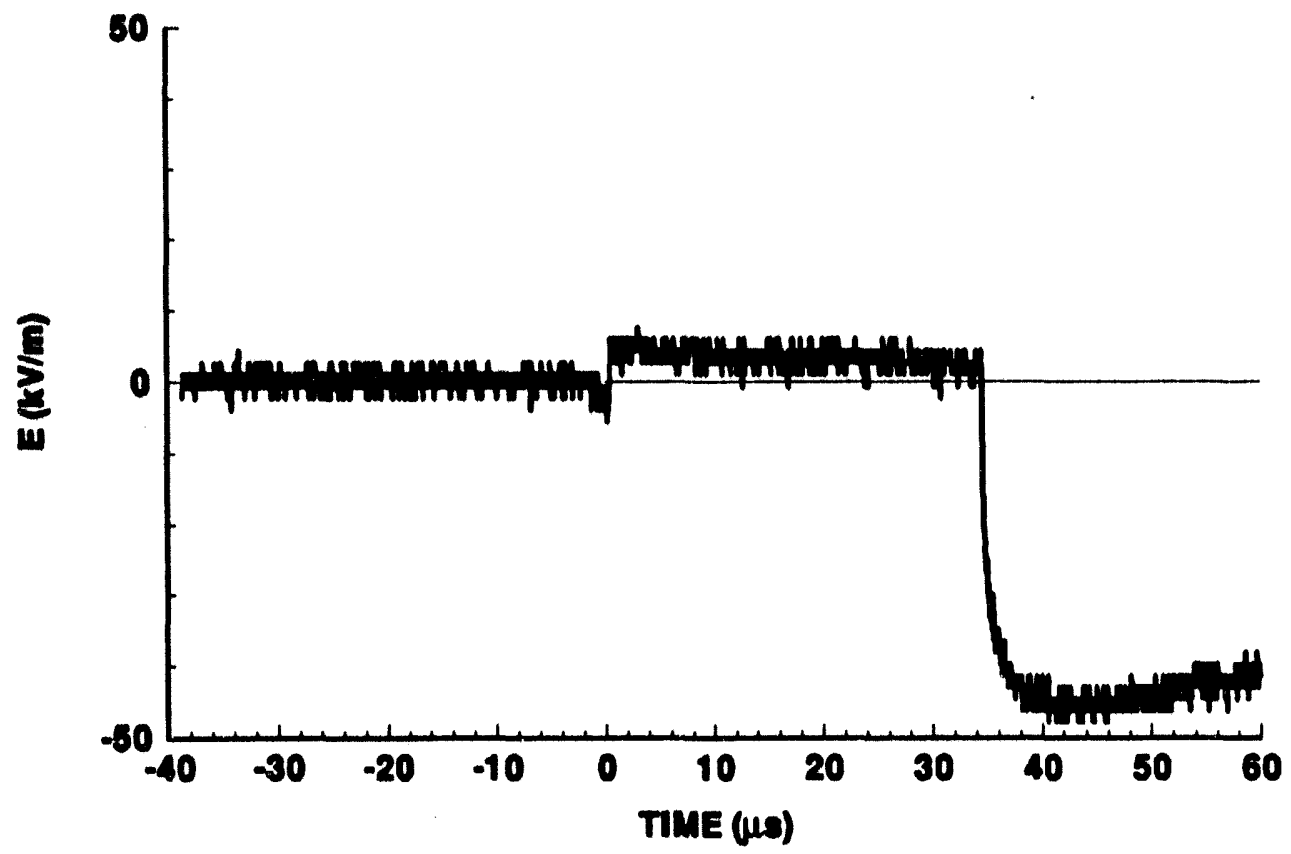

9303 STROKE 2

ES1

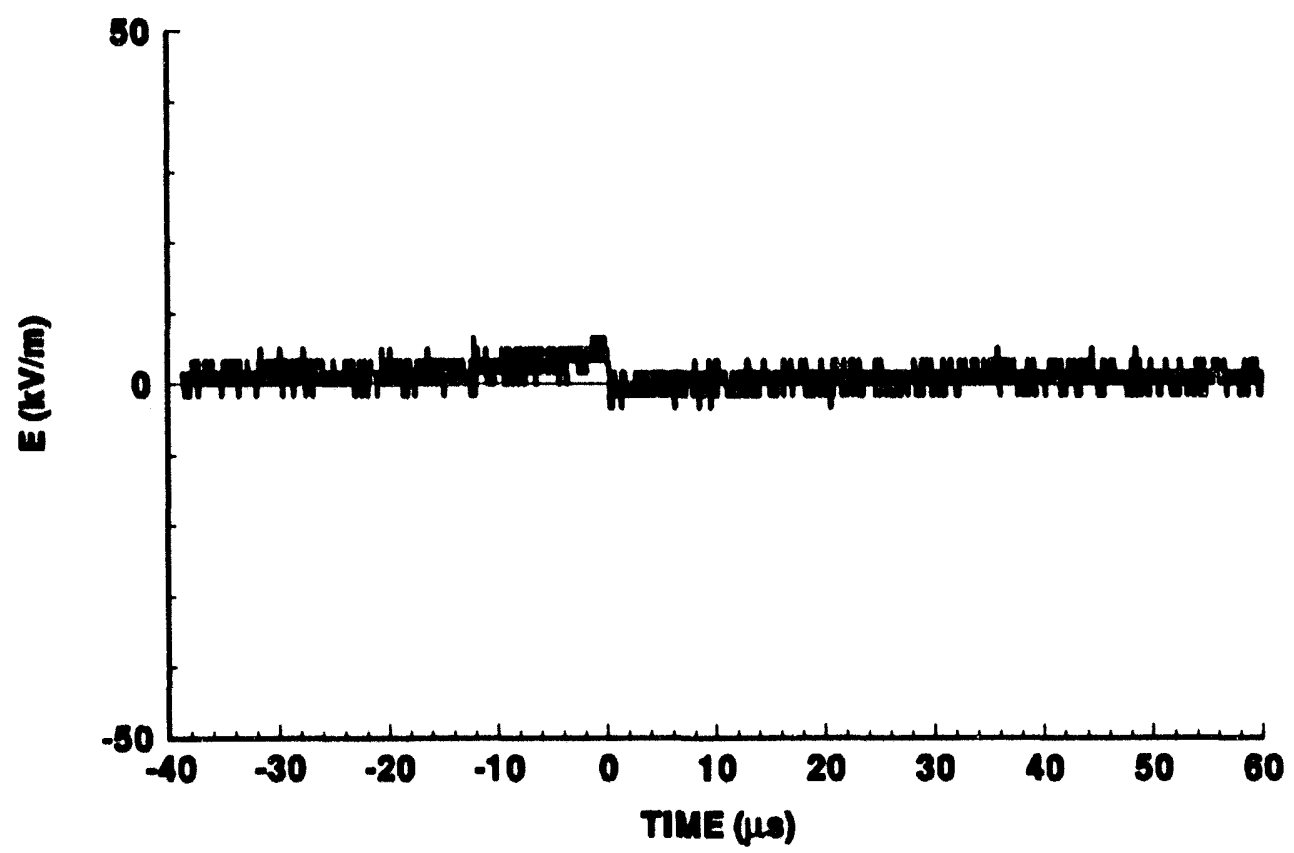




\section{STROKE 1}

ES2
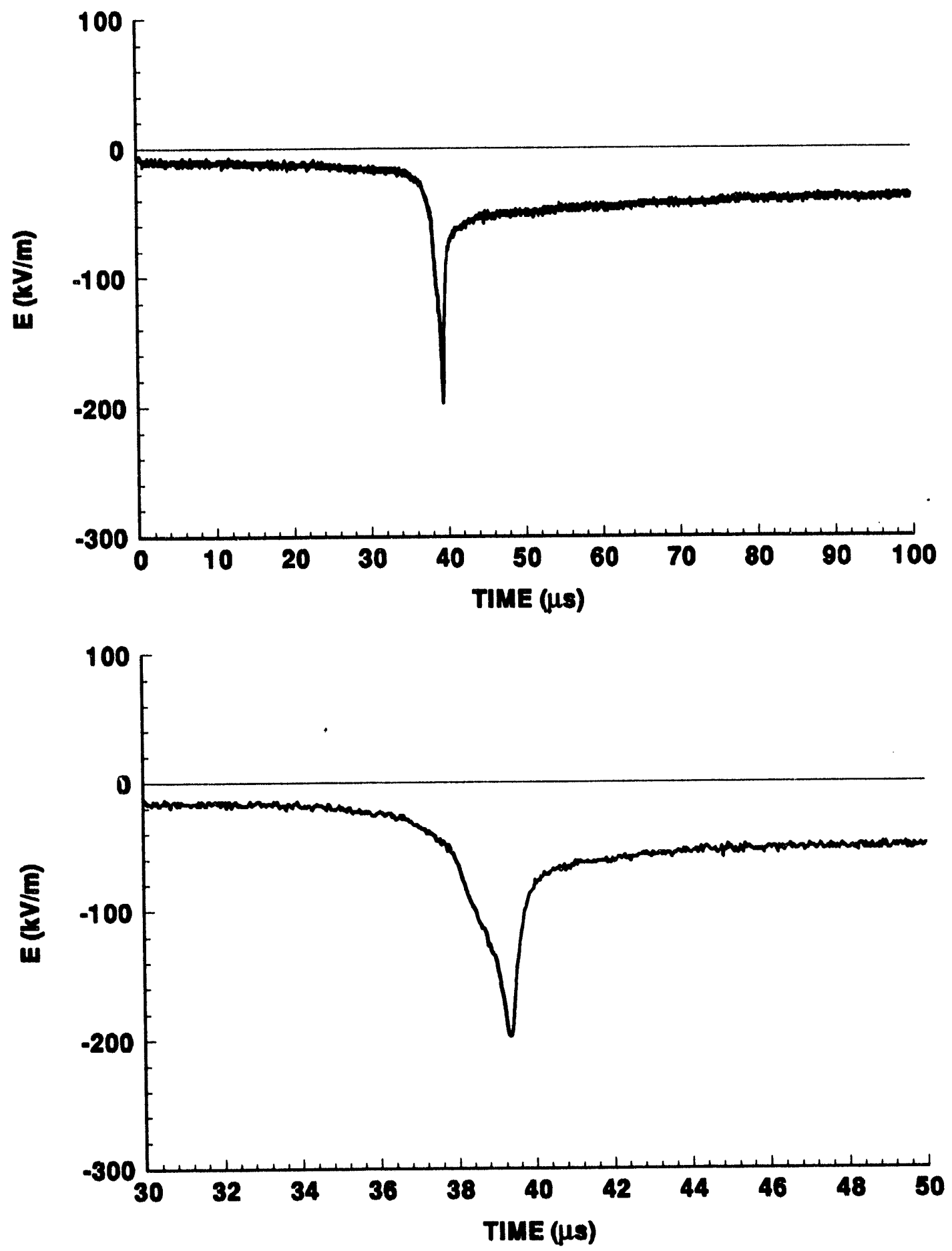


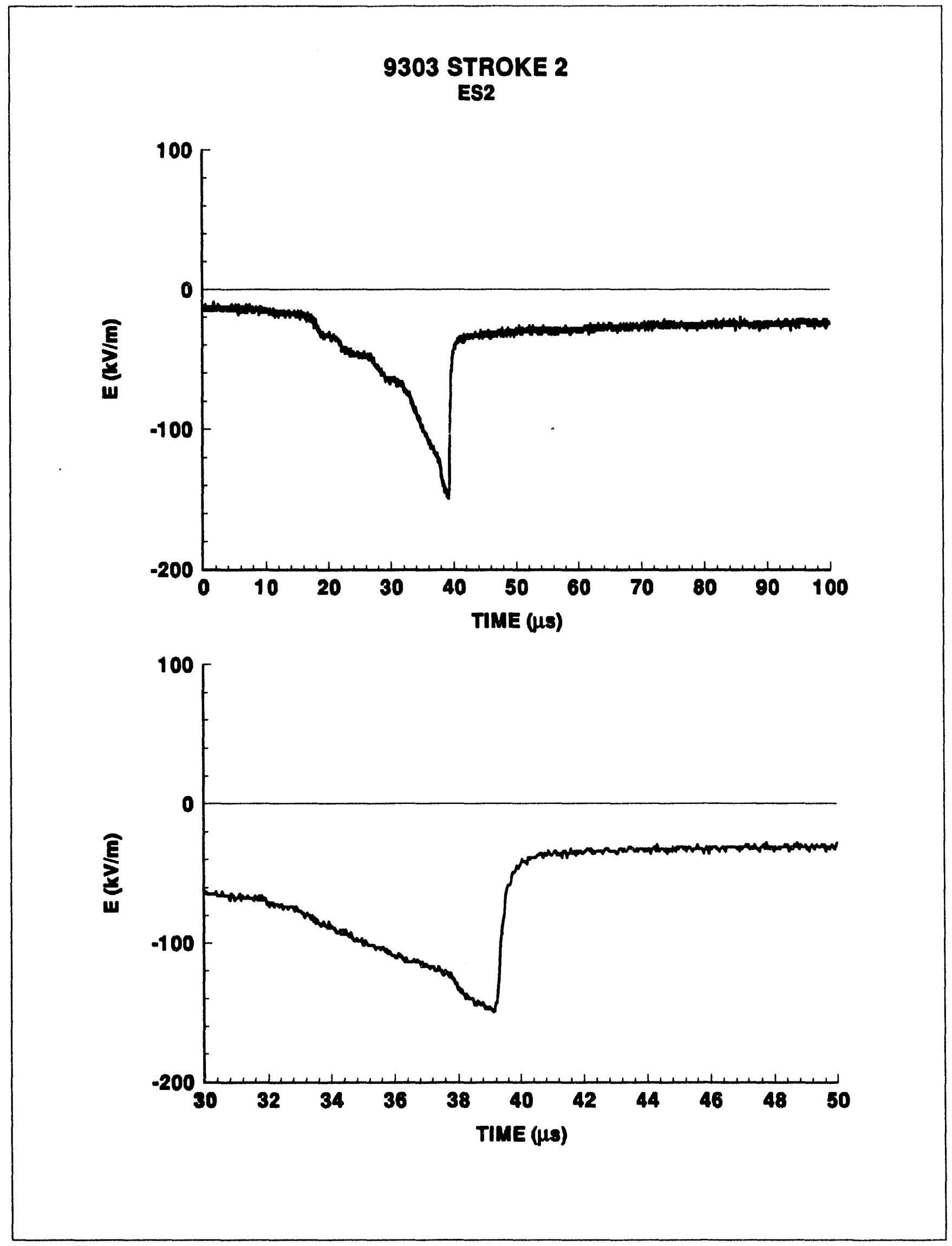




\section{STROKE 1}

ES3
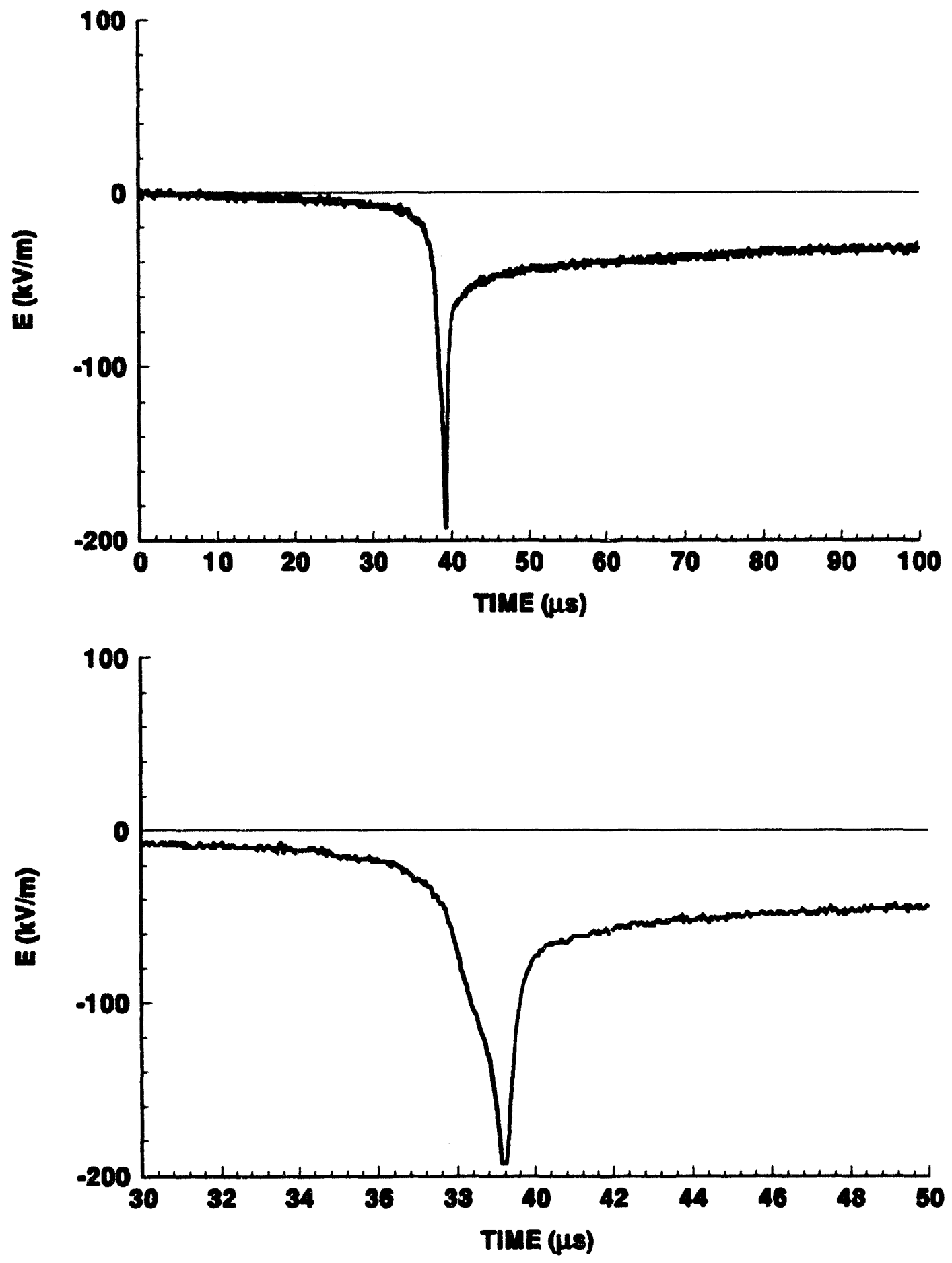


\section{STROKE 2}

ES3
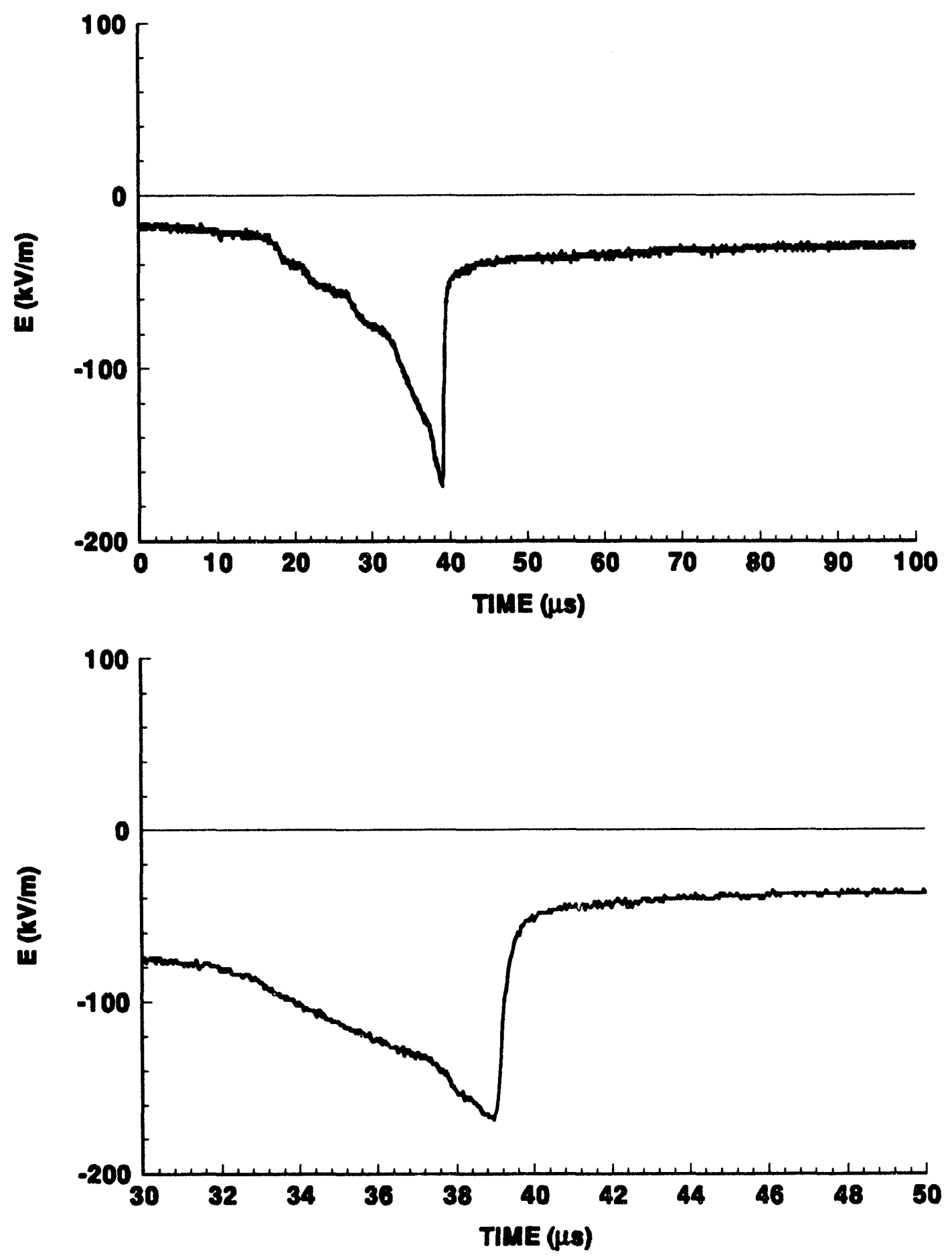


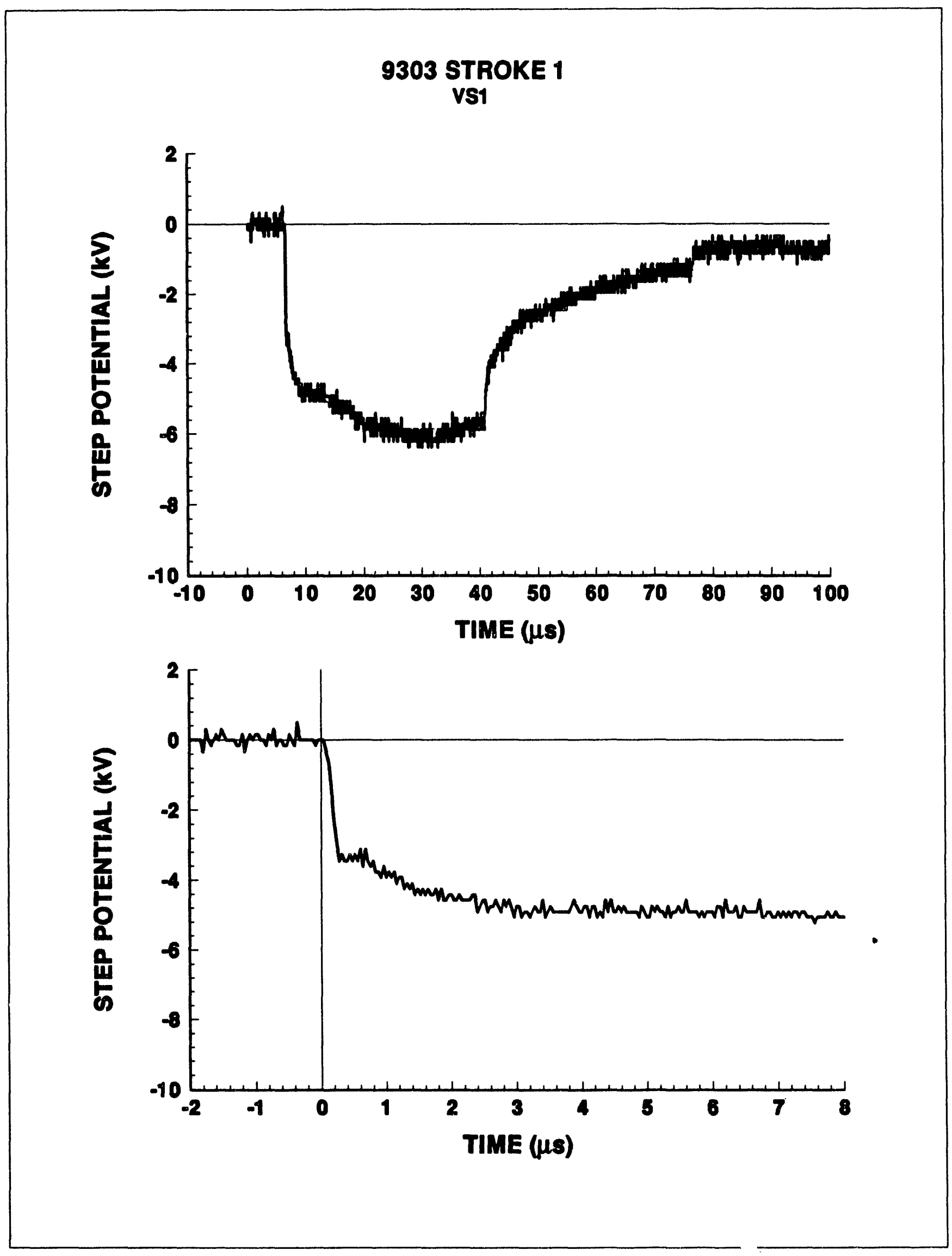



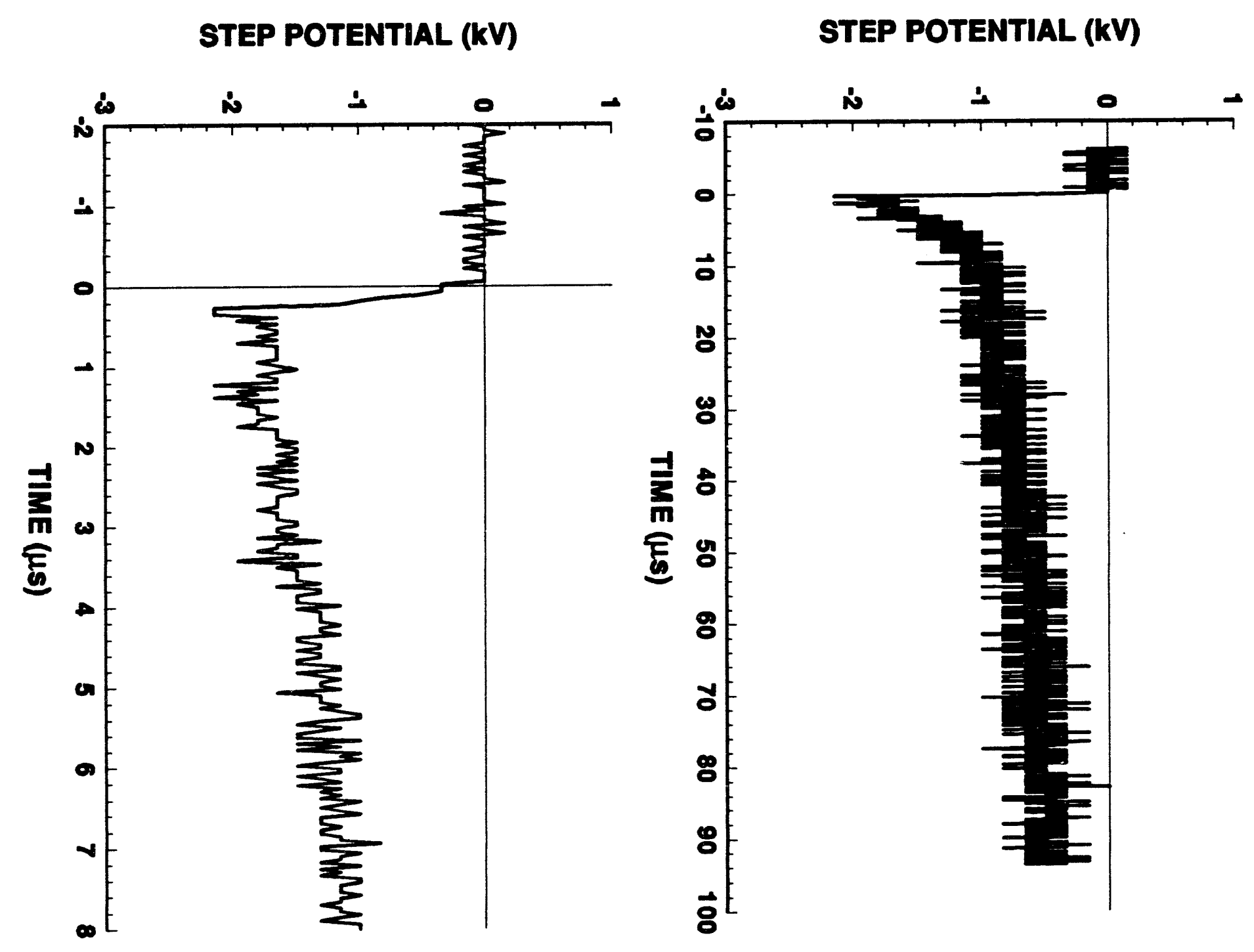


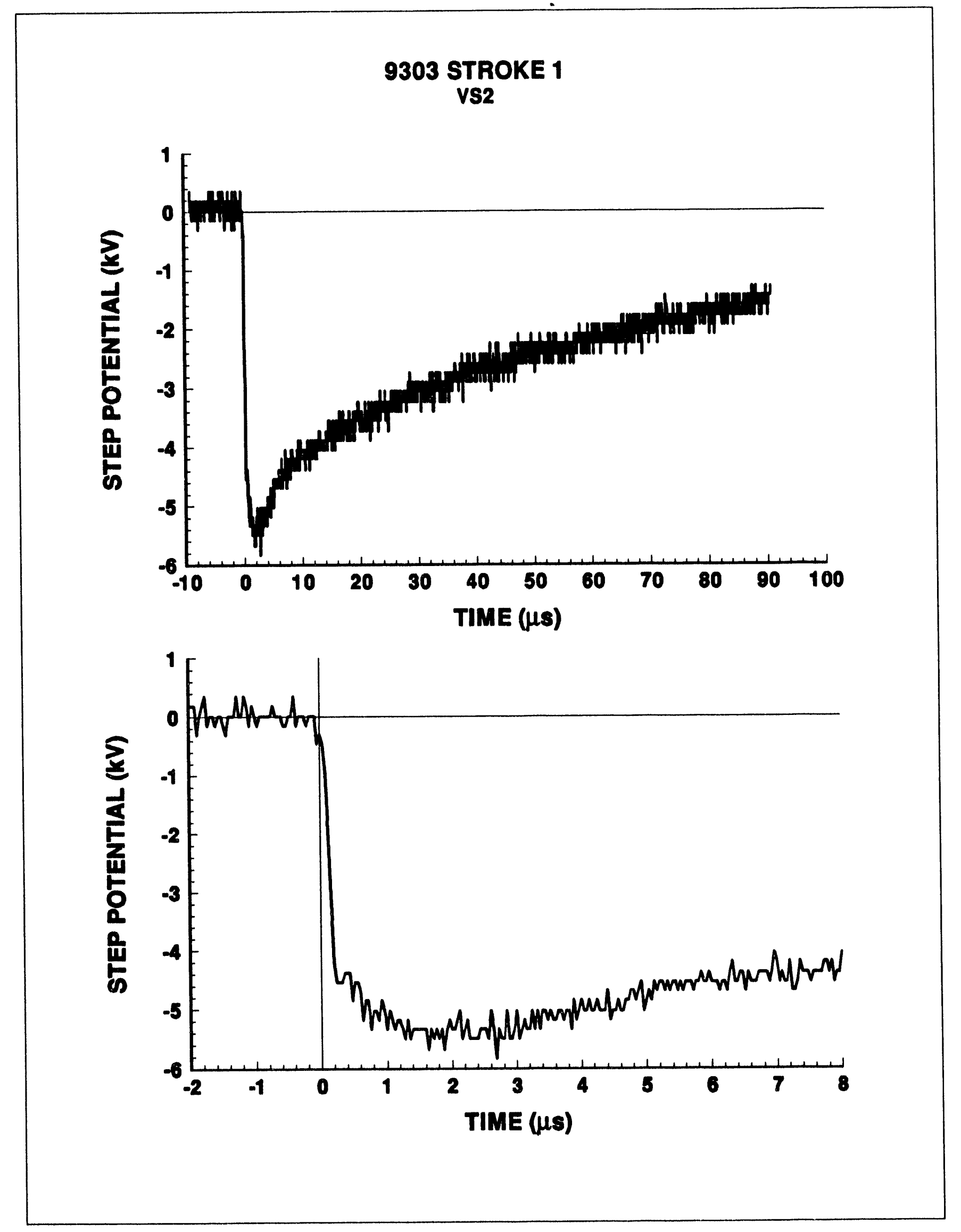

143 

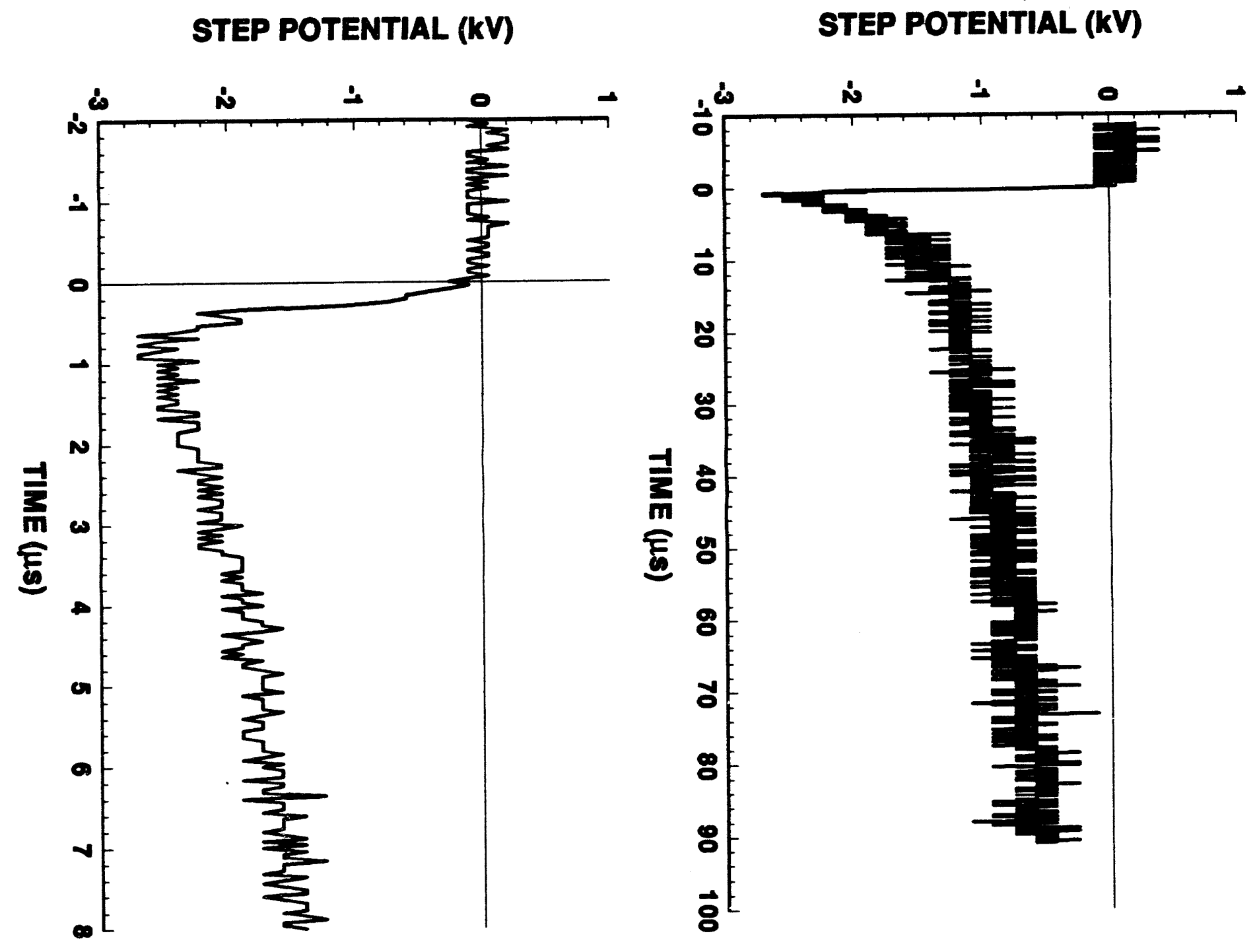


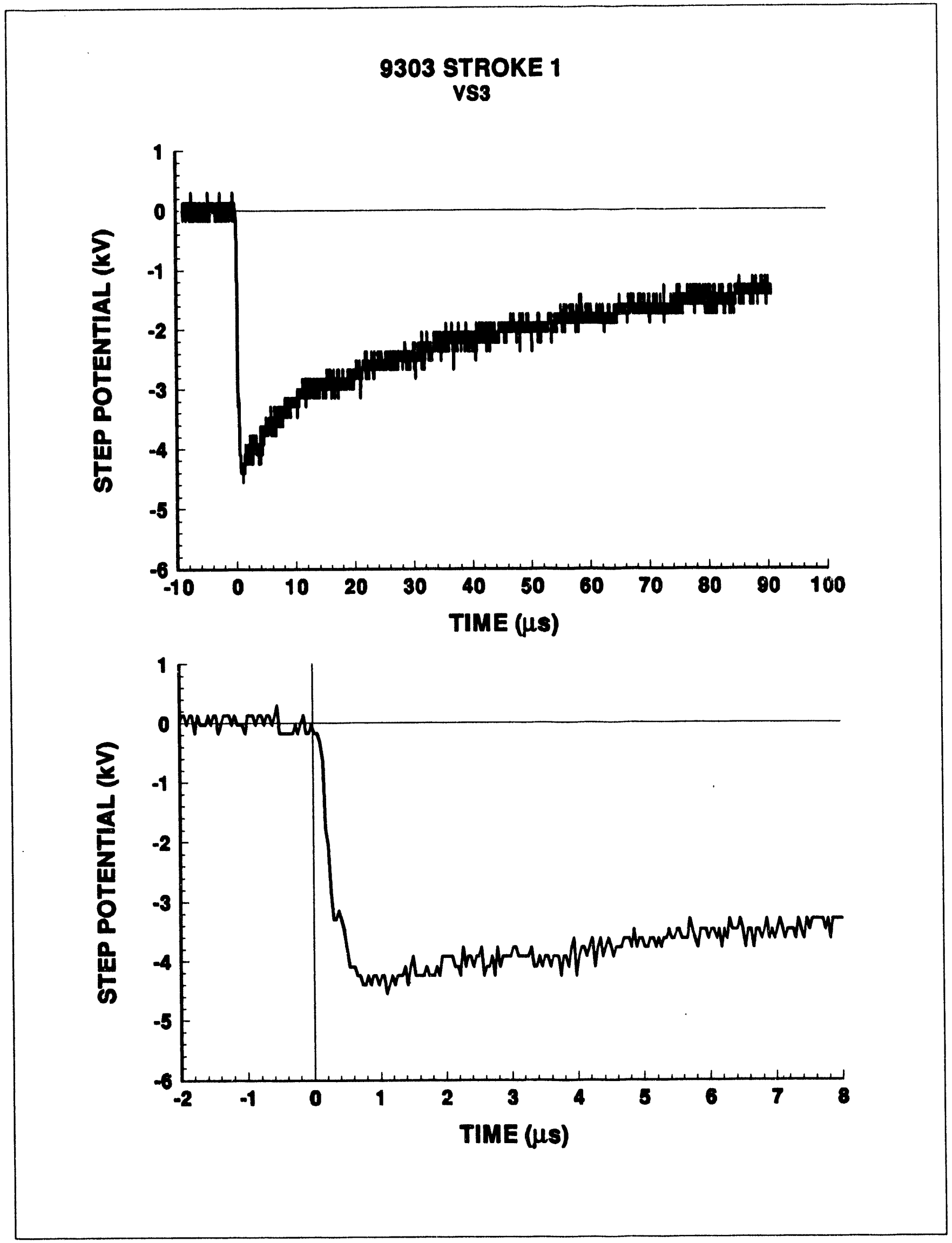




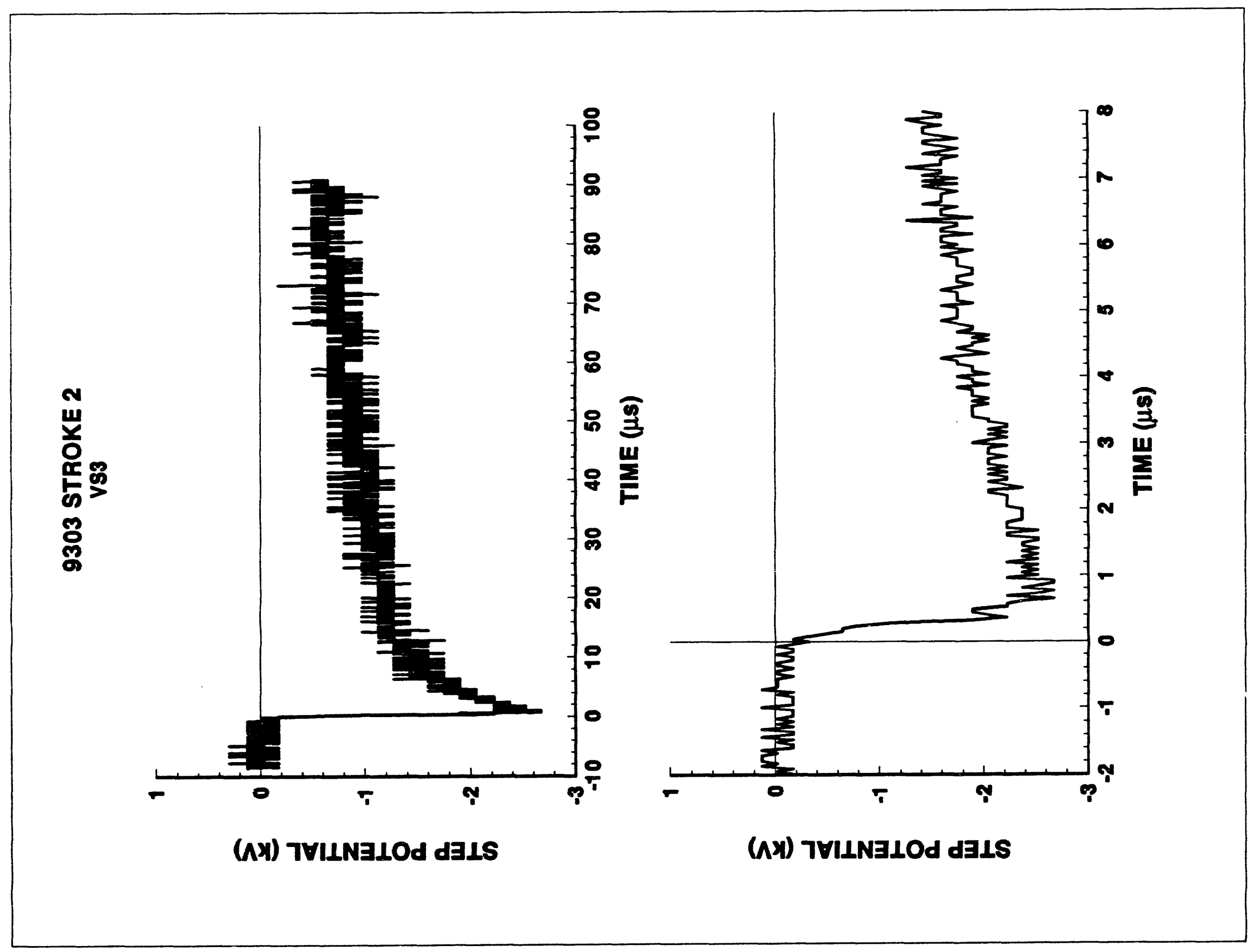




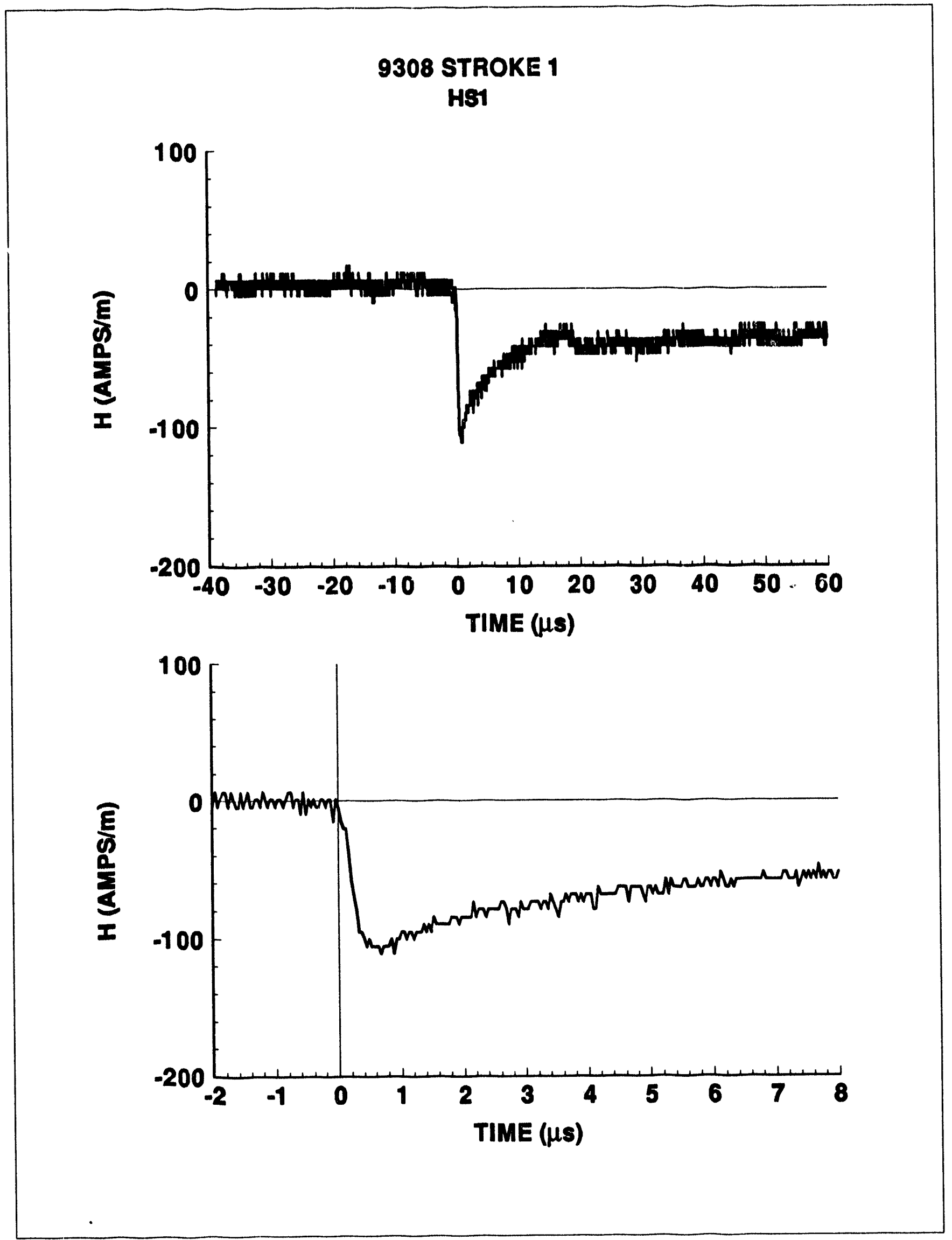




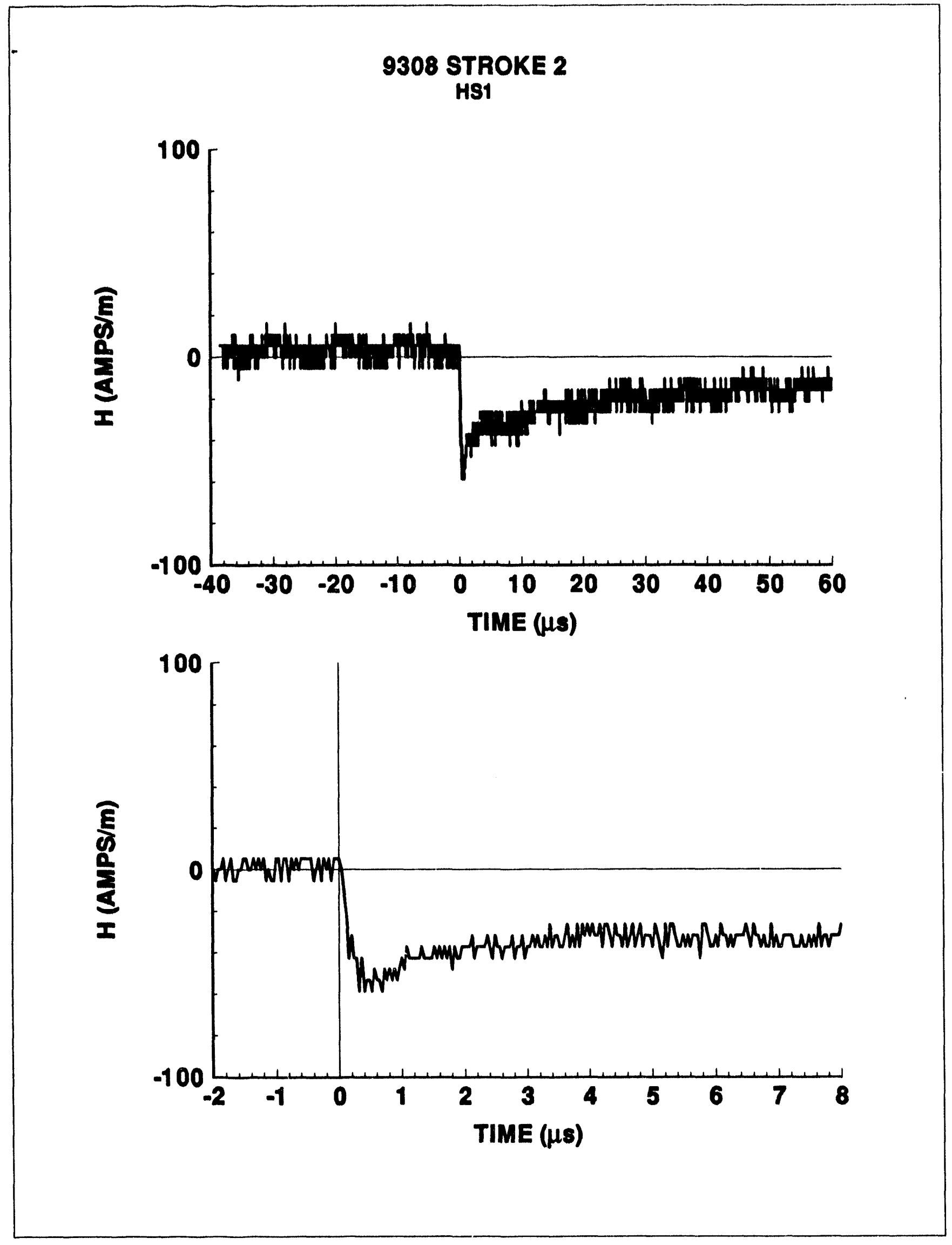




\section{STROKE 3 \\ HS1}
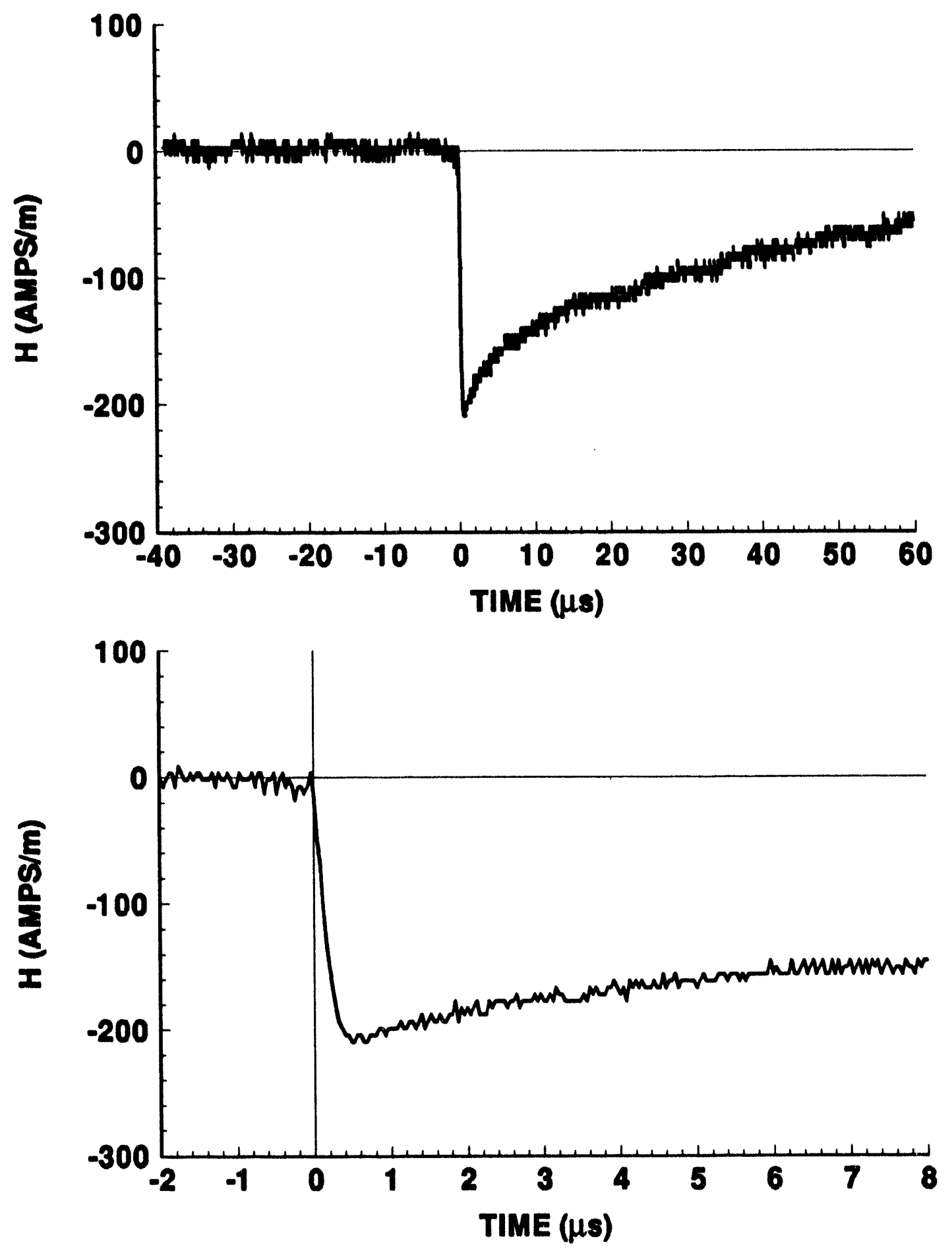


\section{STROKE 4}

HS1
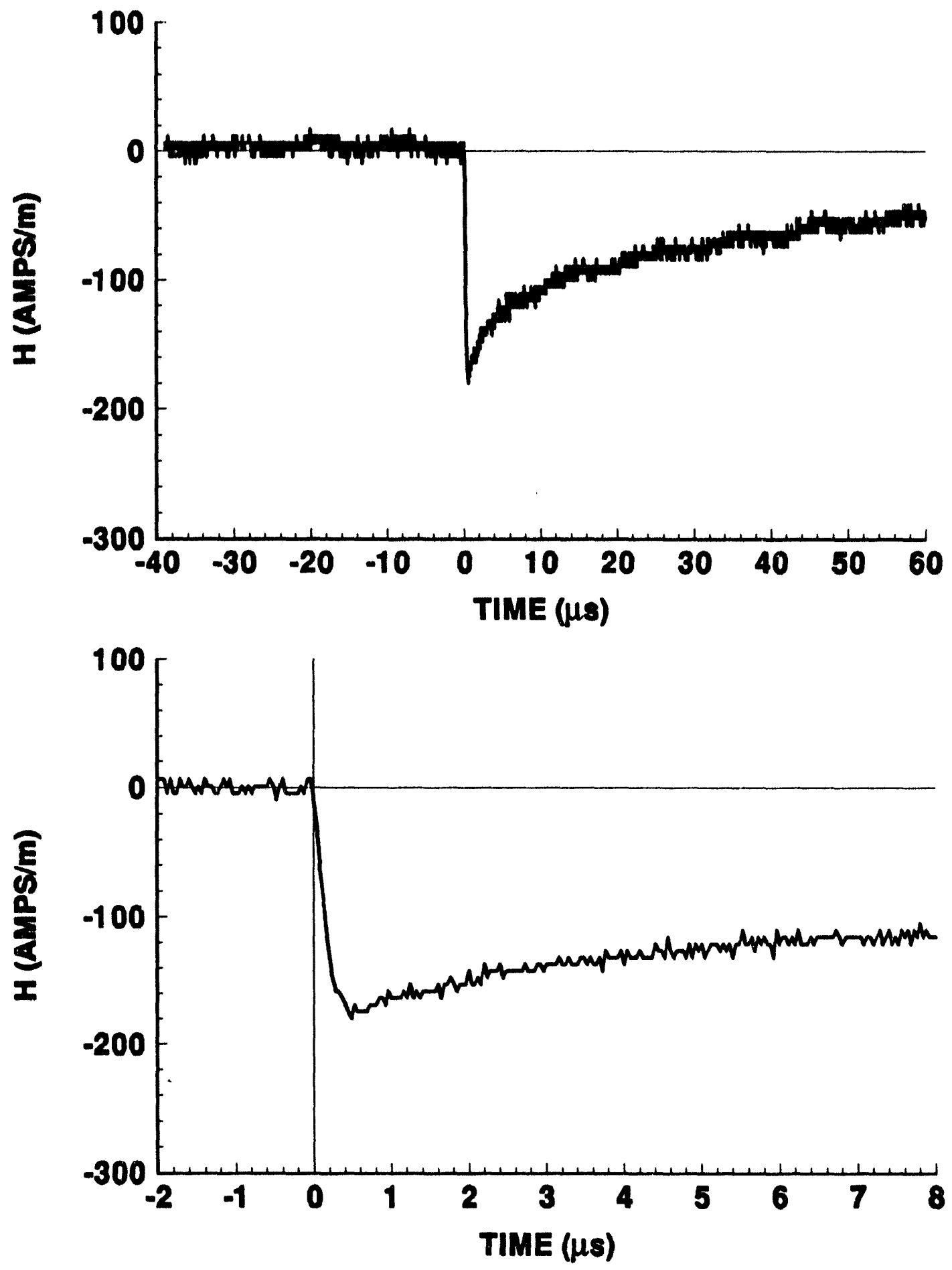


\section{STROKE 5}

HS1
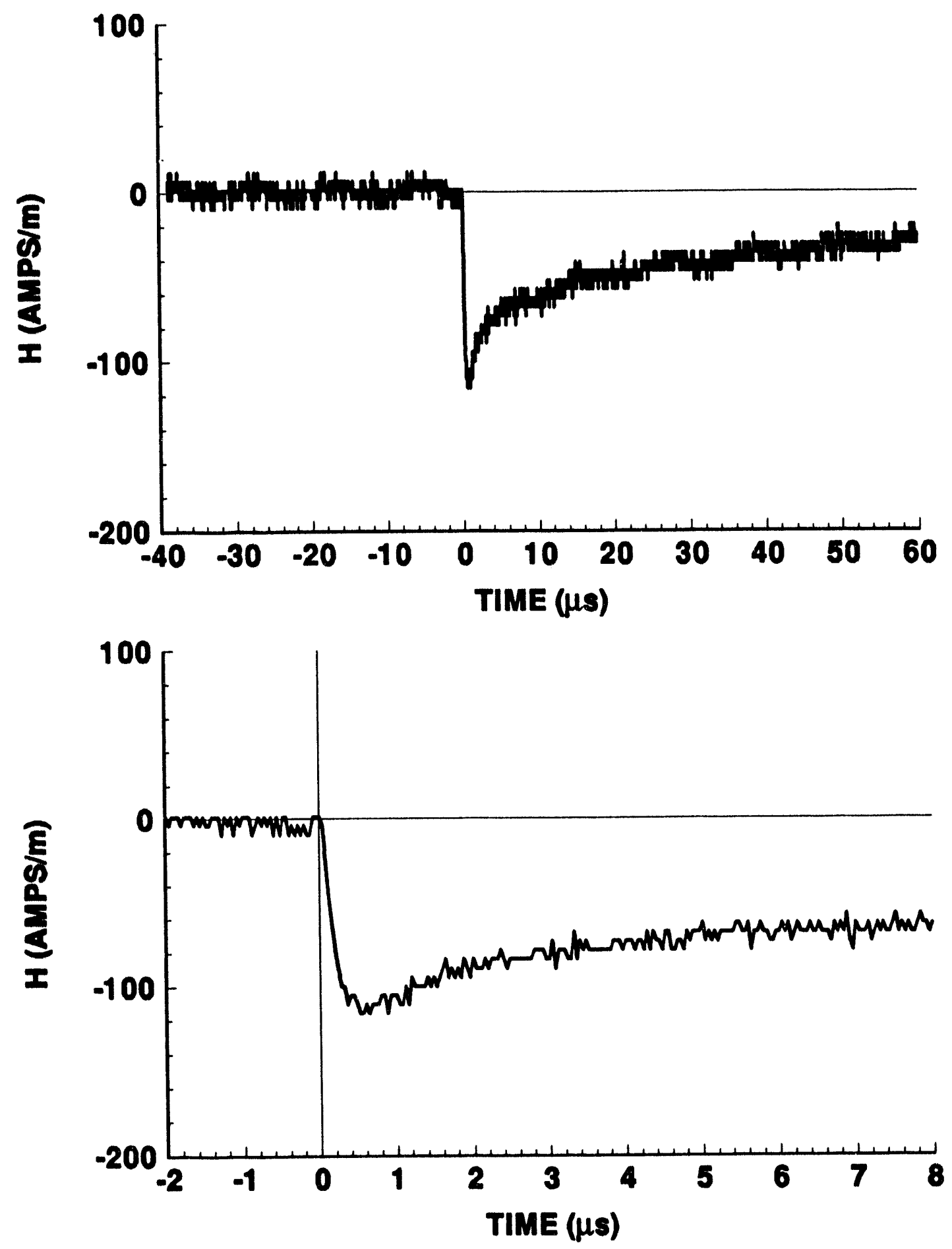


\section{STROKE 6 \\ HS1}
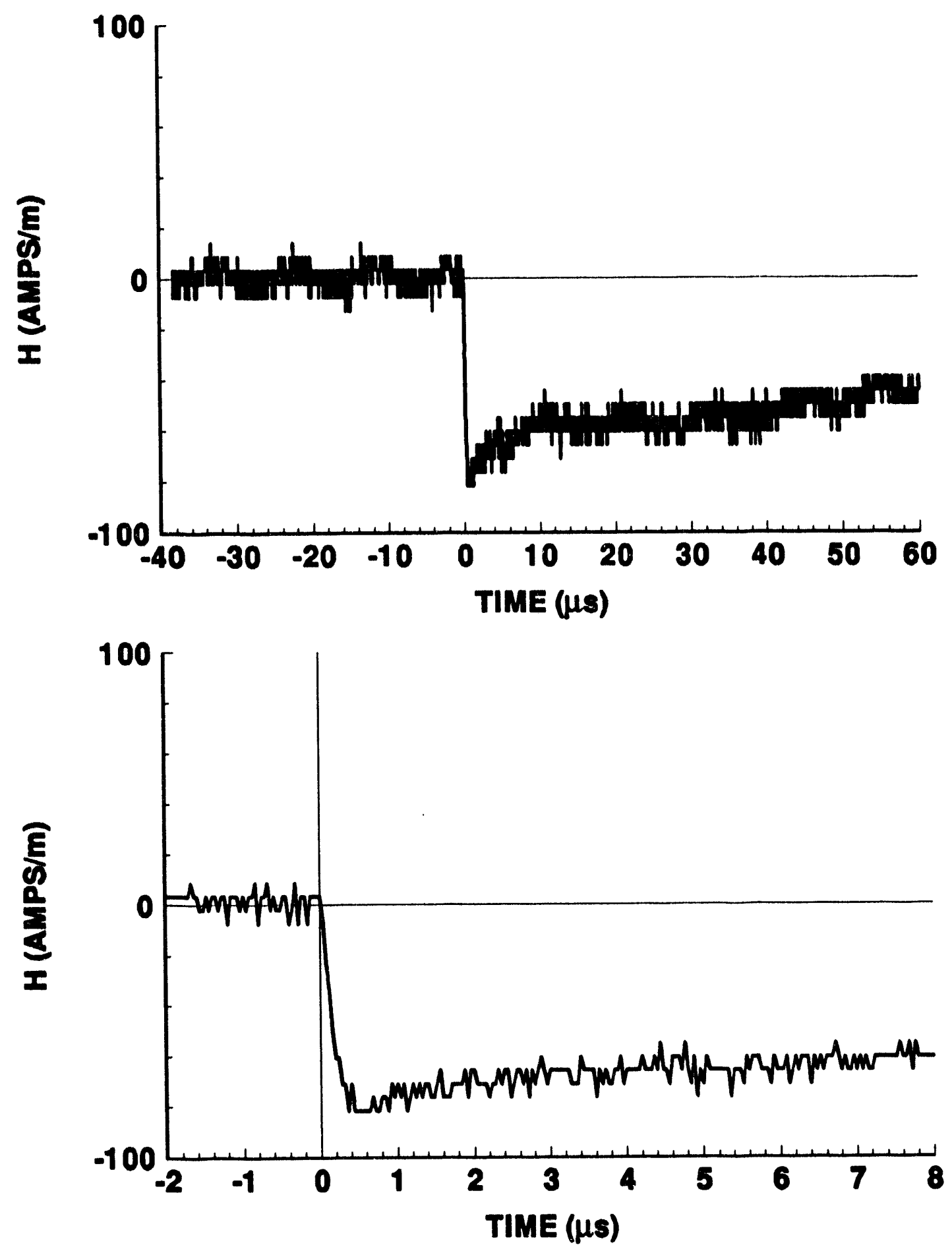


\section{STROKE 7}

HS1
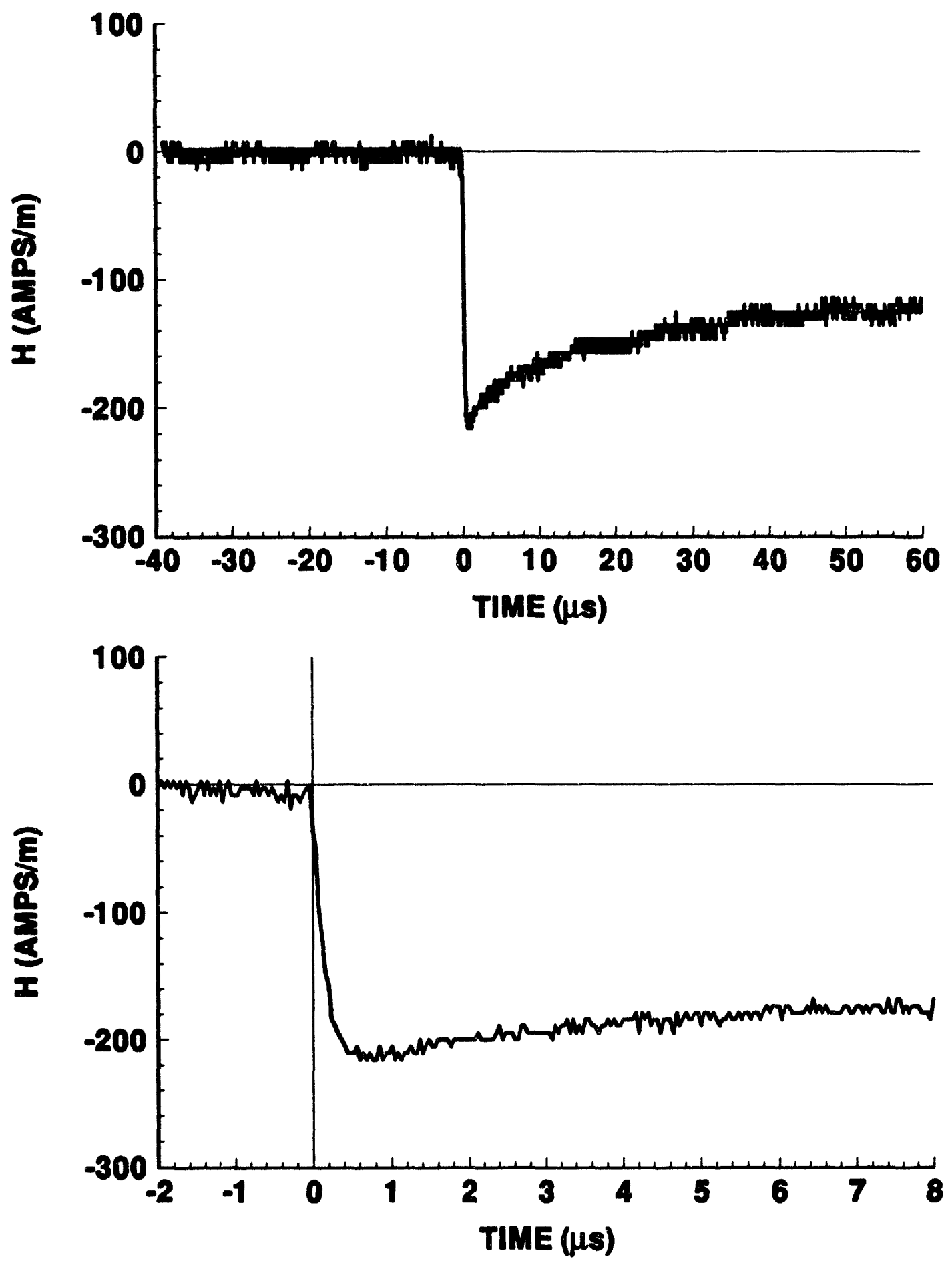


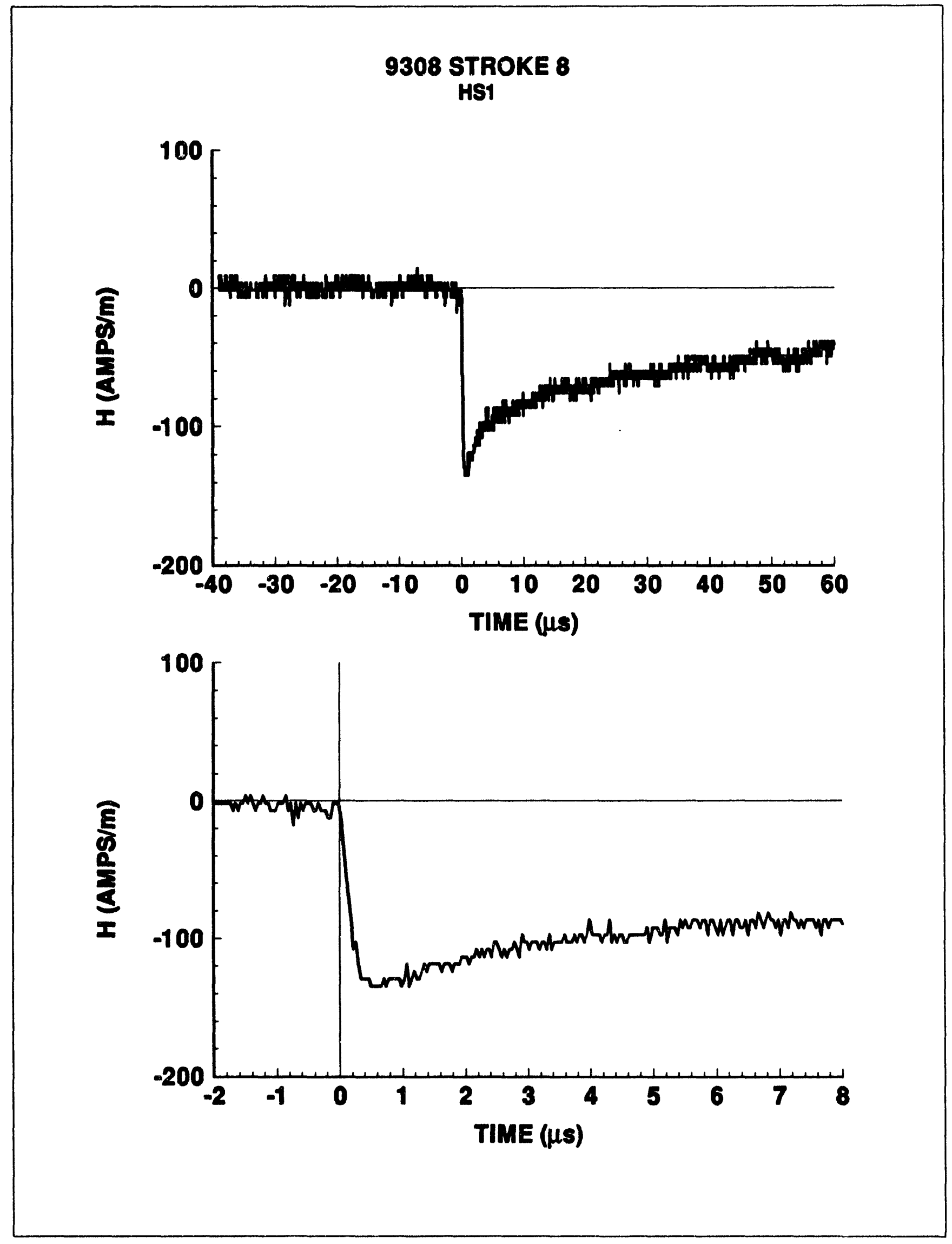




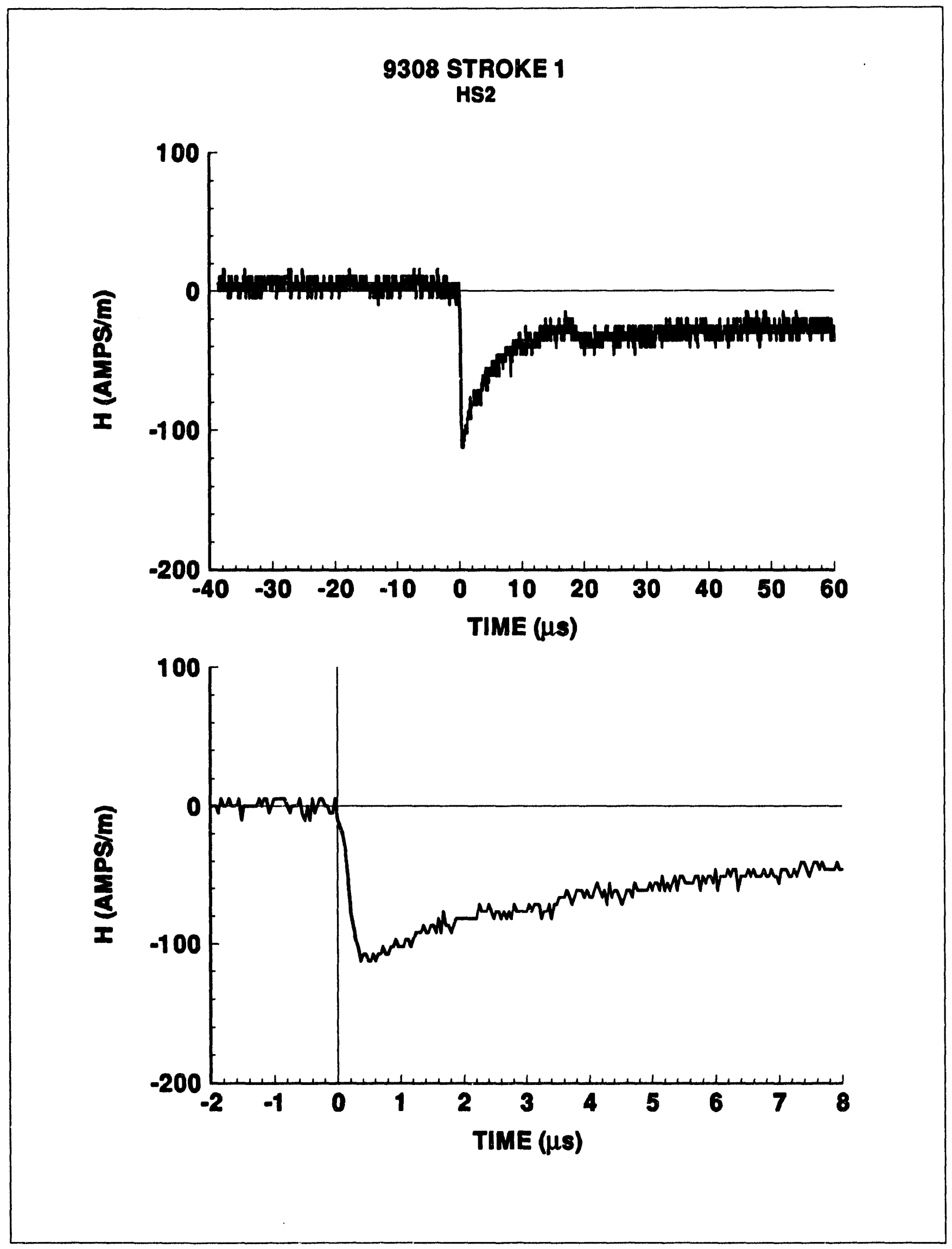




\section{STROKE 2}

HS2
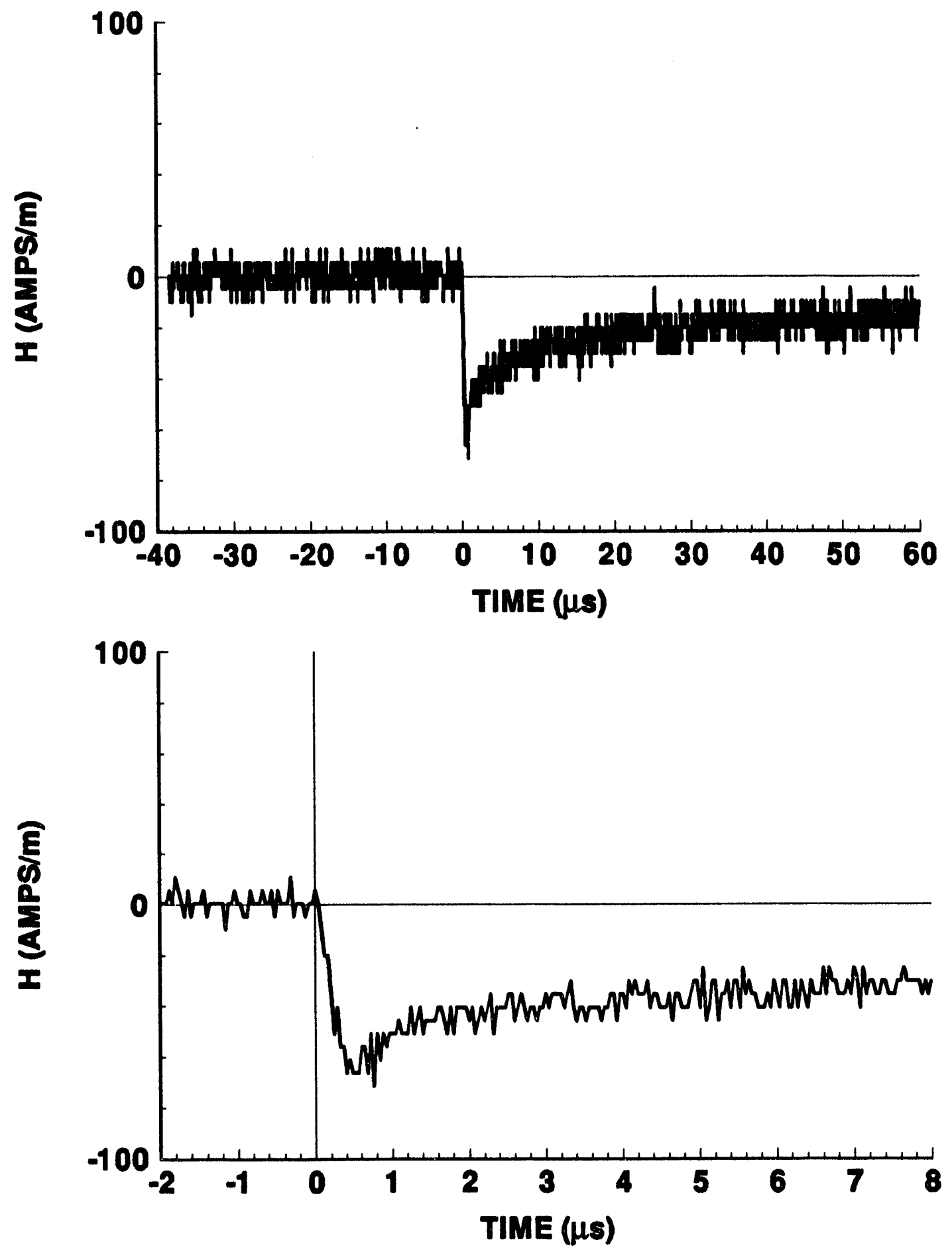


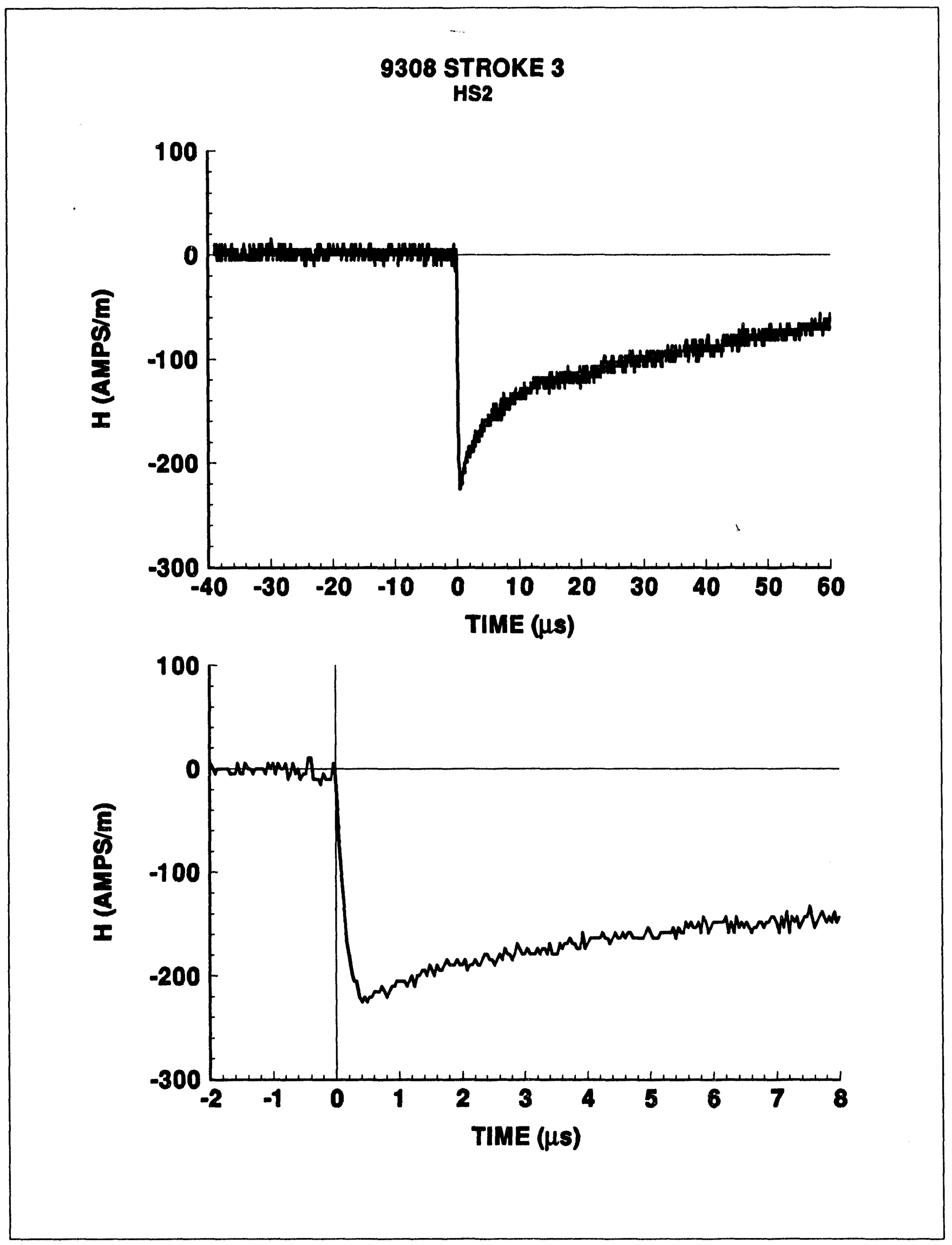




\section{STROKE 4 \\ HS2}
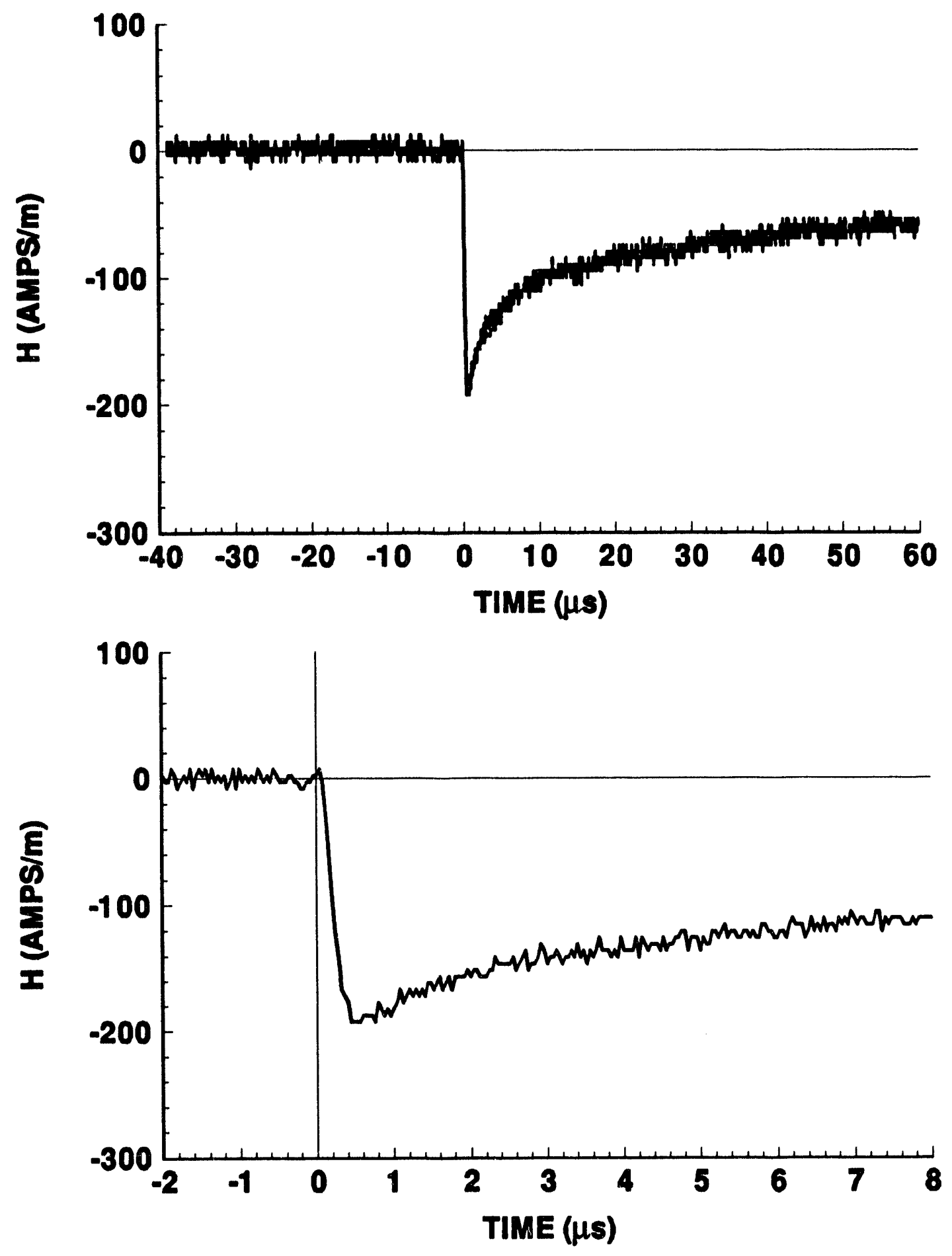


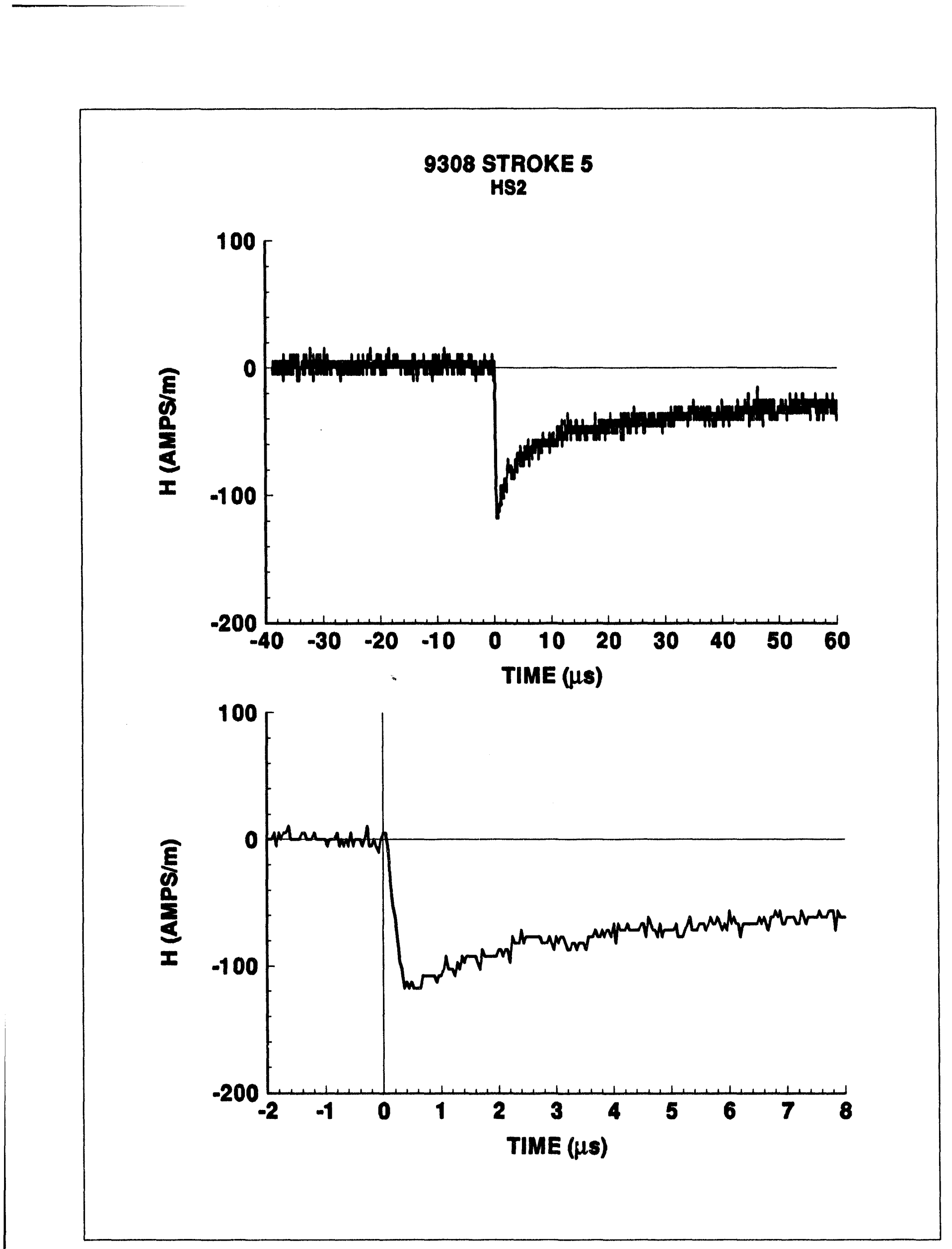

159 


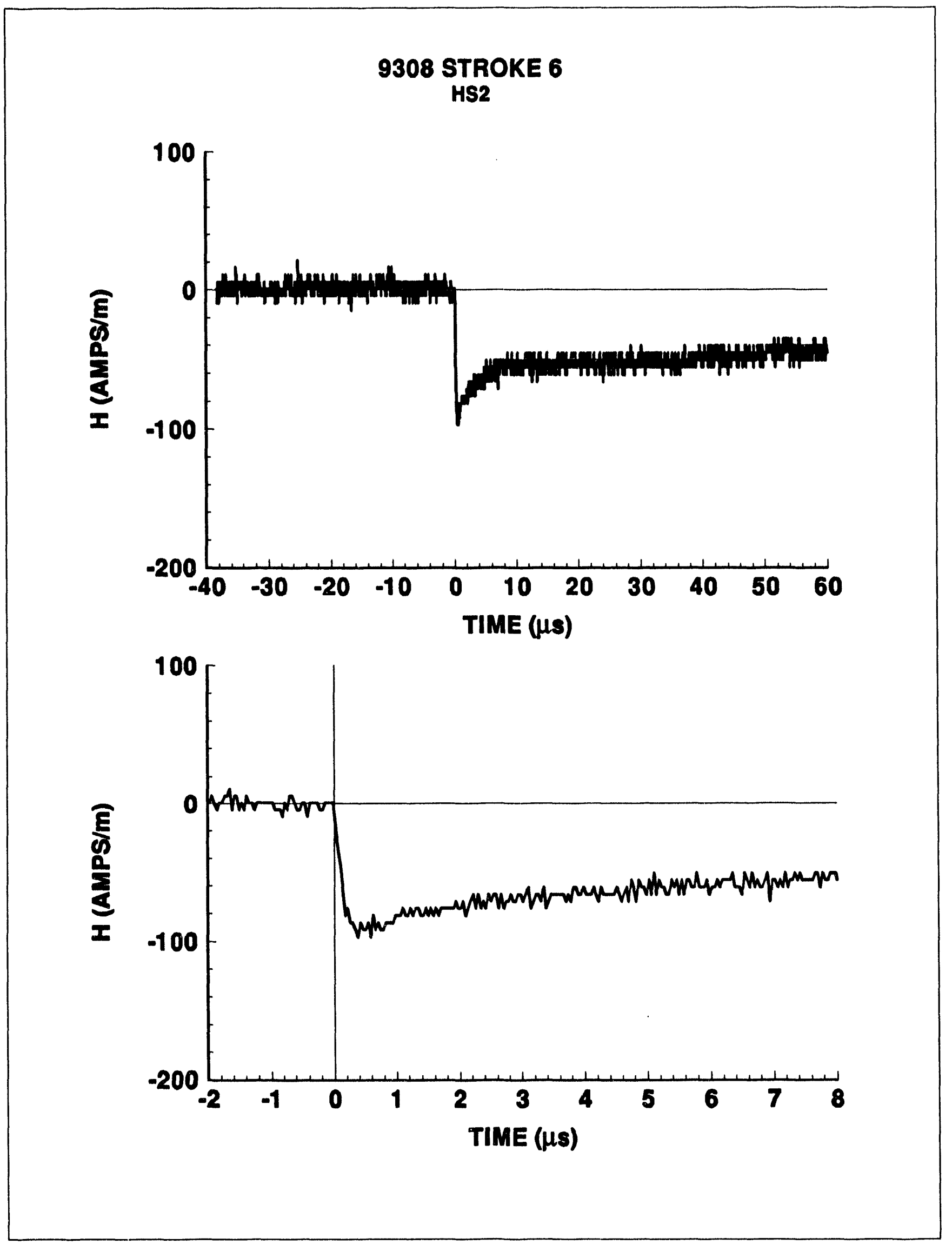




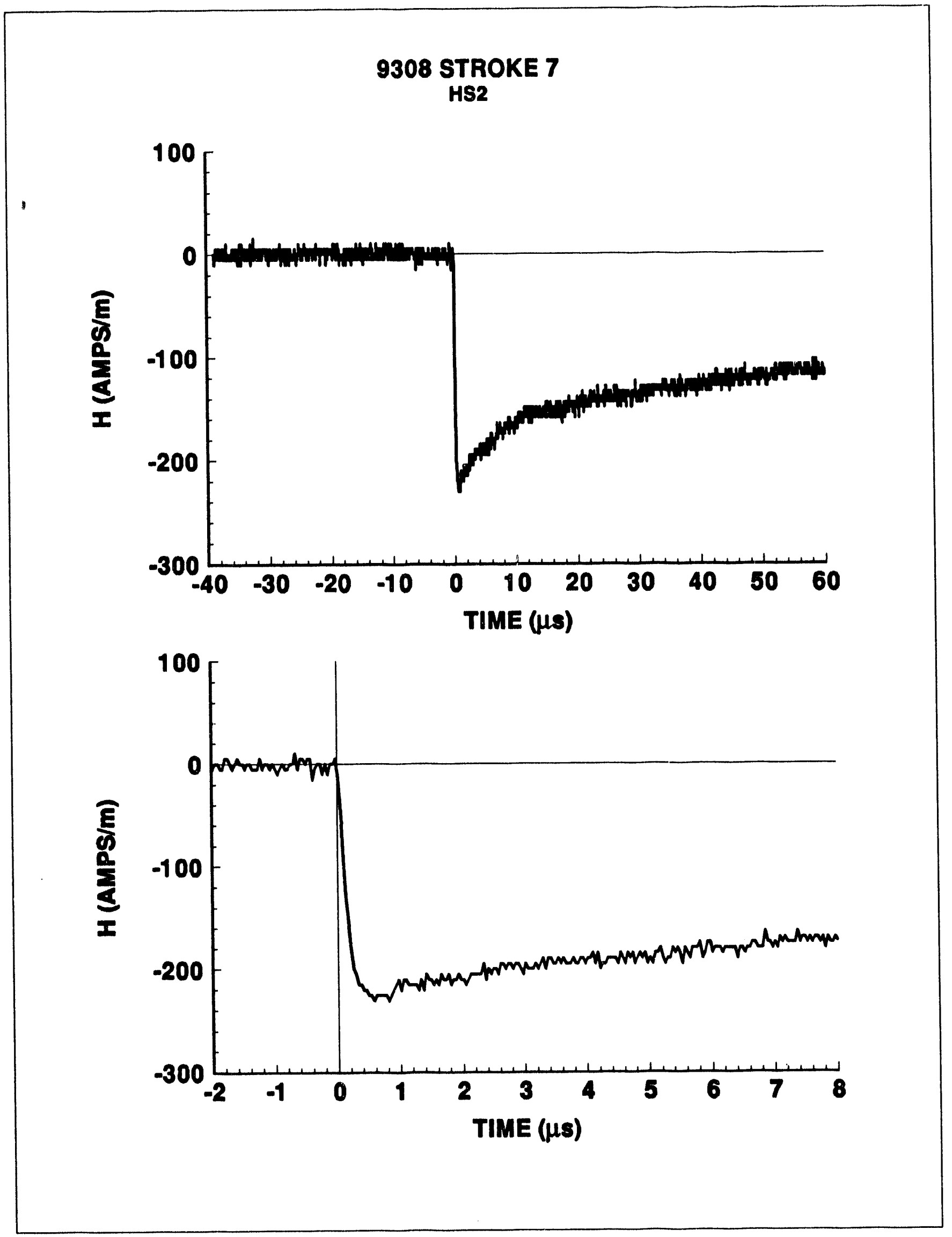


H (AMPS/m)

H (AMPS/m)

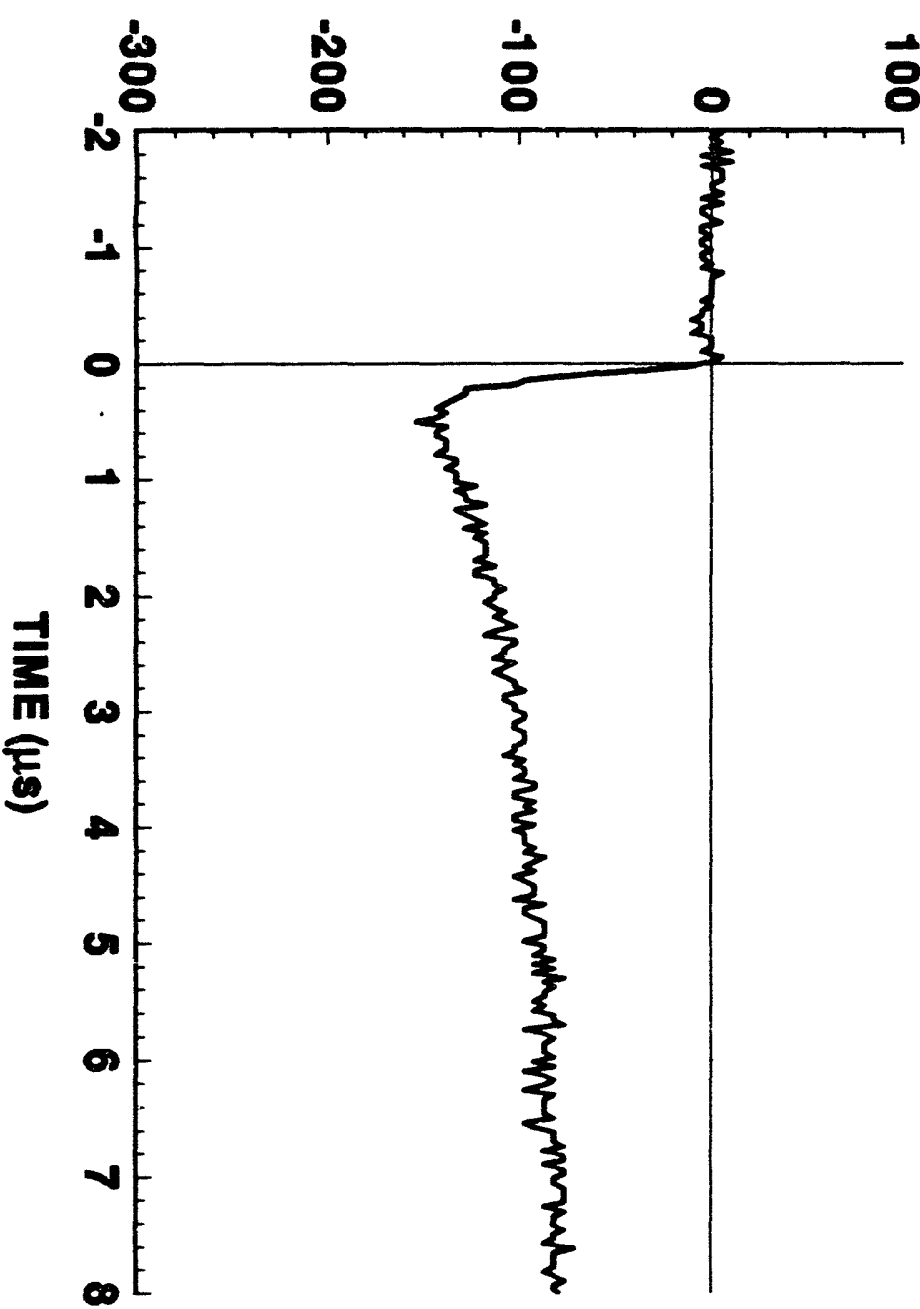

용

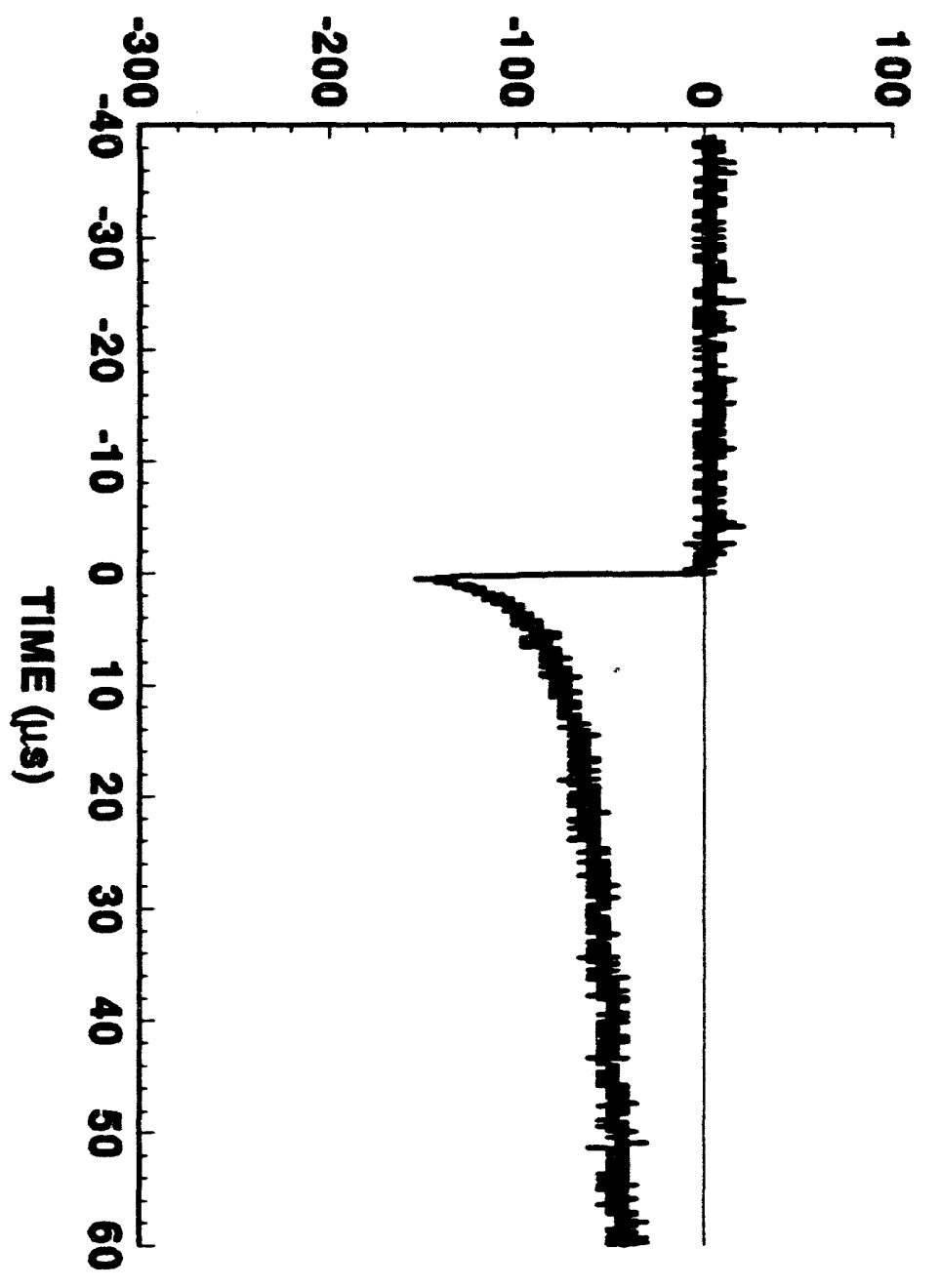

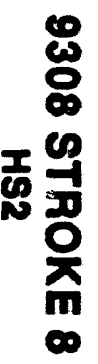




\section{STROKE 1}

ES1
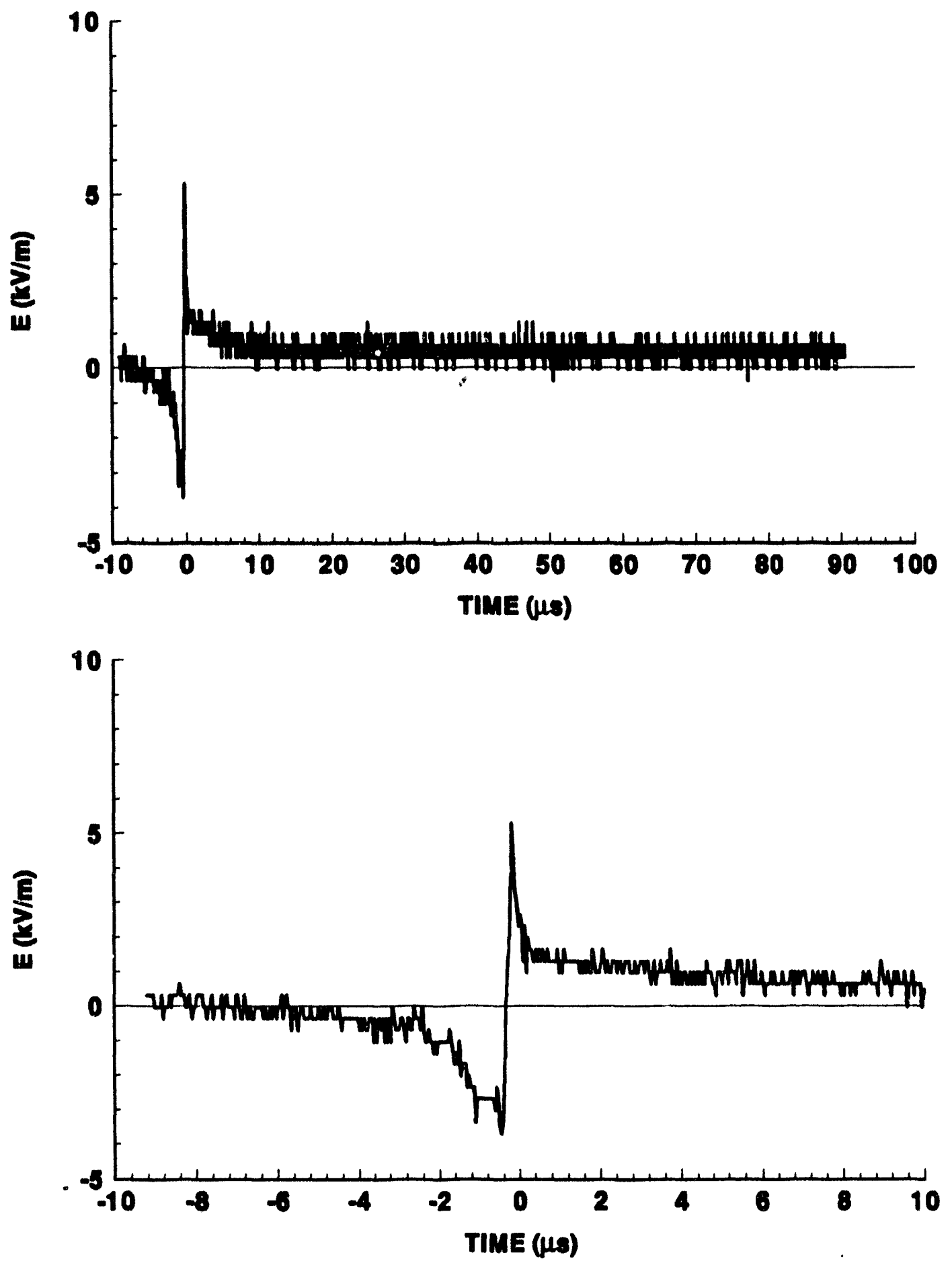


\section{STROKE 2 \\ E81}
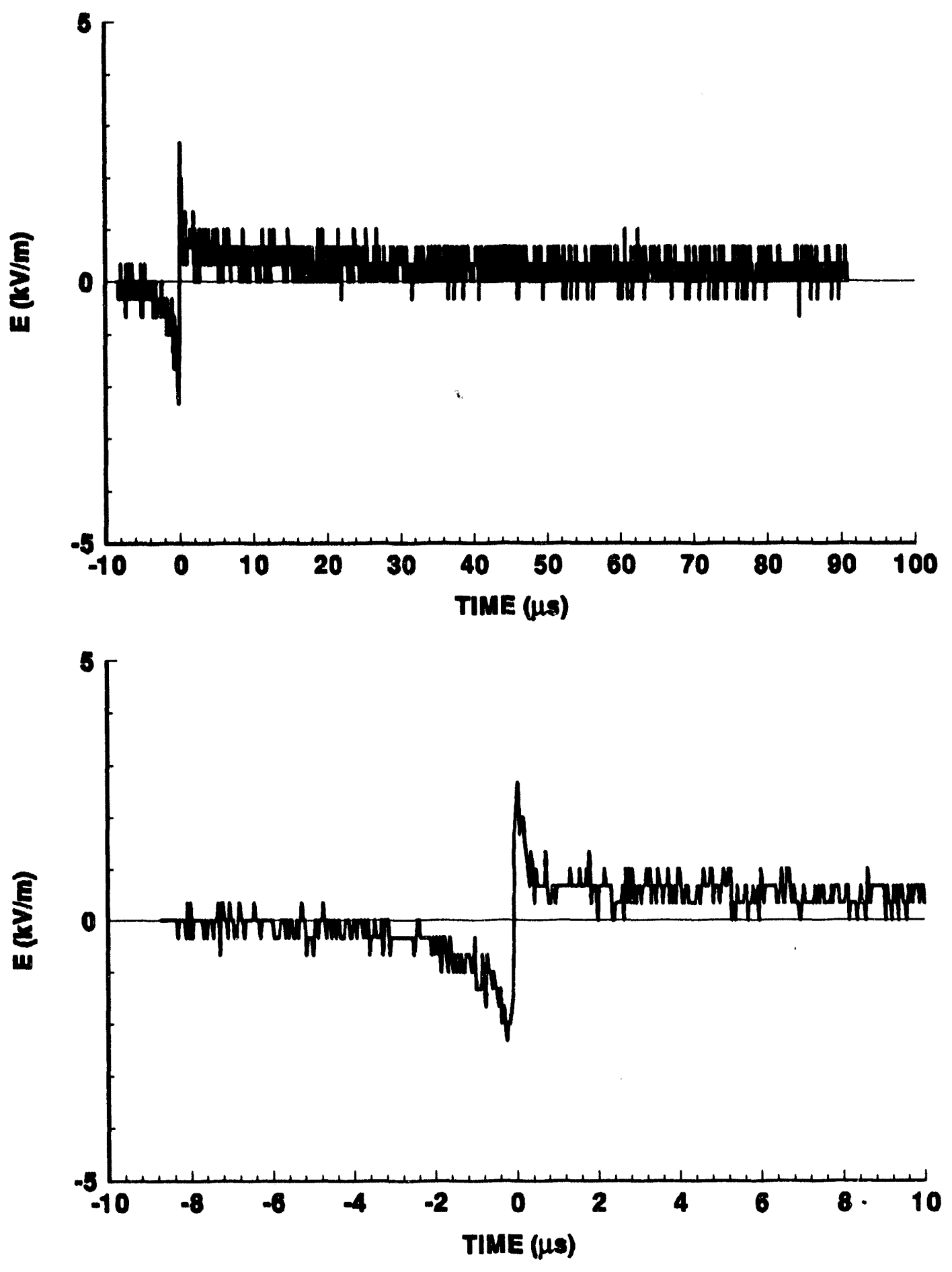


\section{STROKE 3}

\section{ES1}
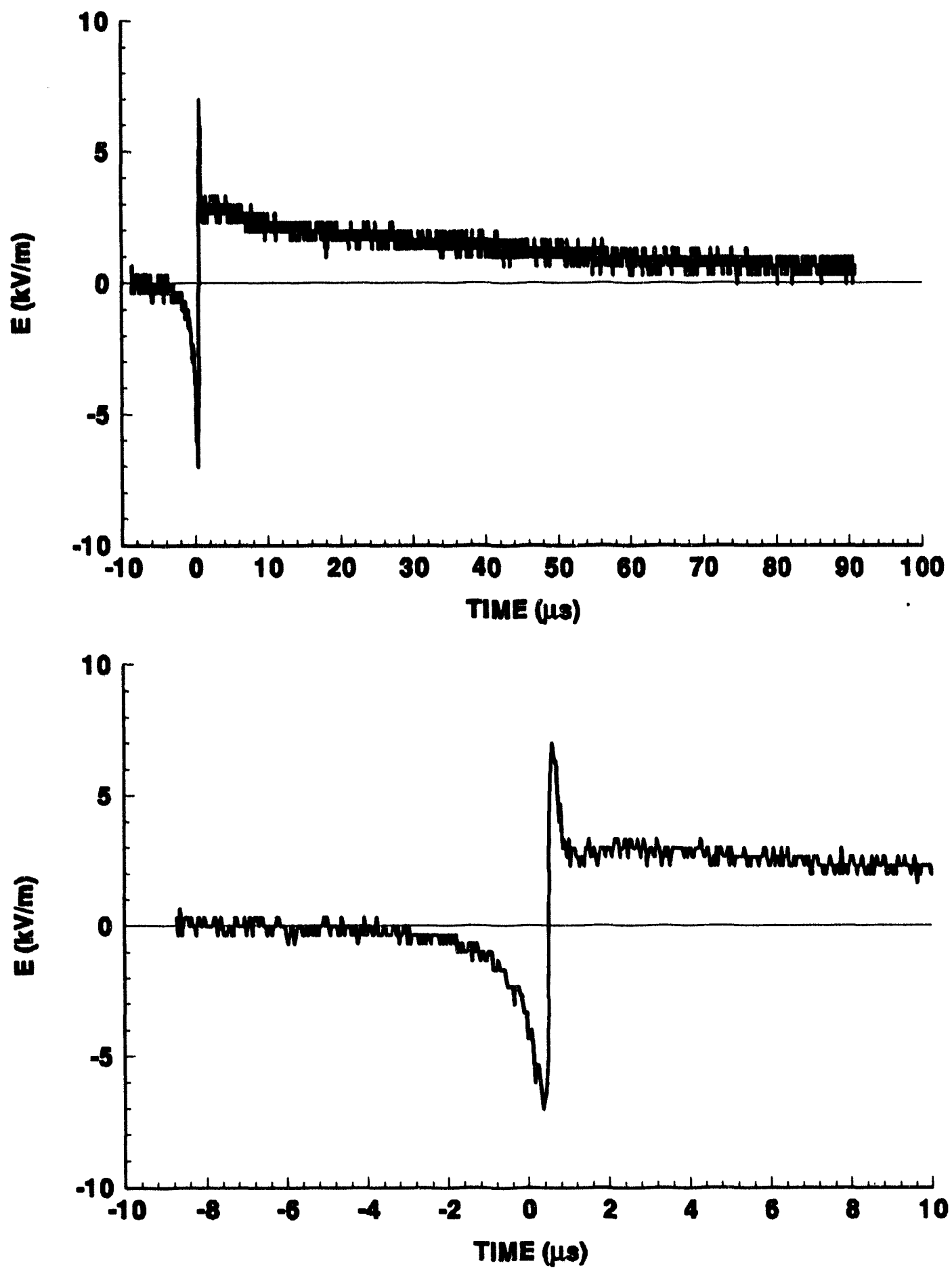


\section{STROKE 4}

ES1
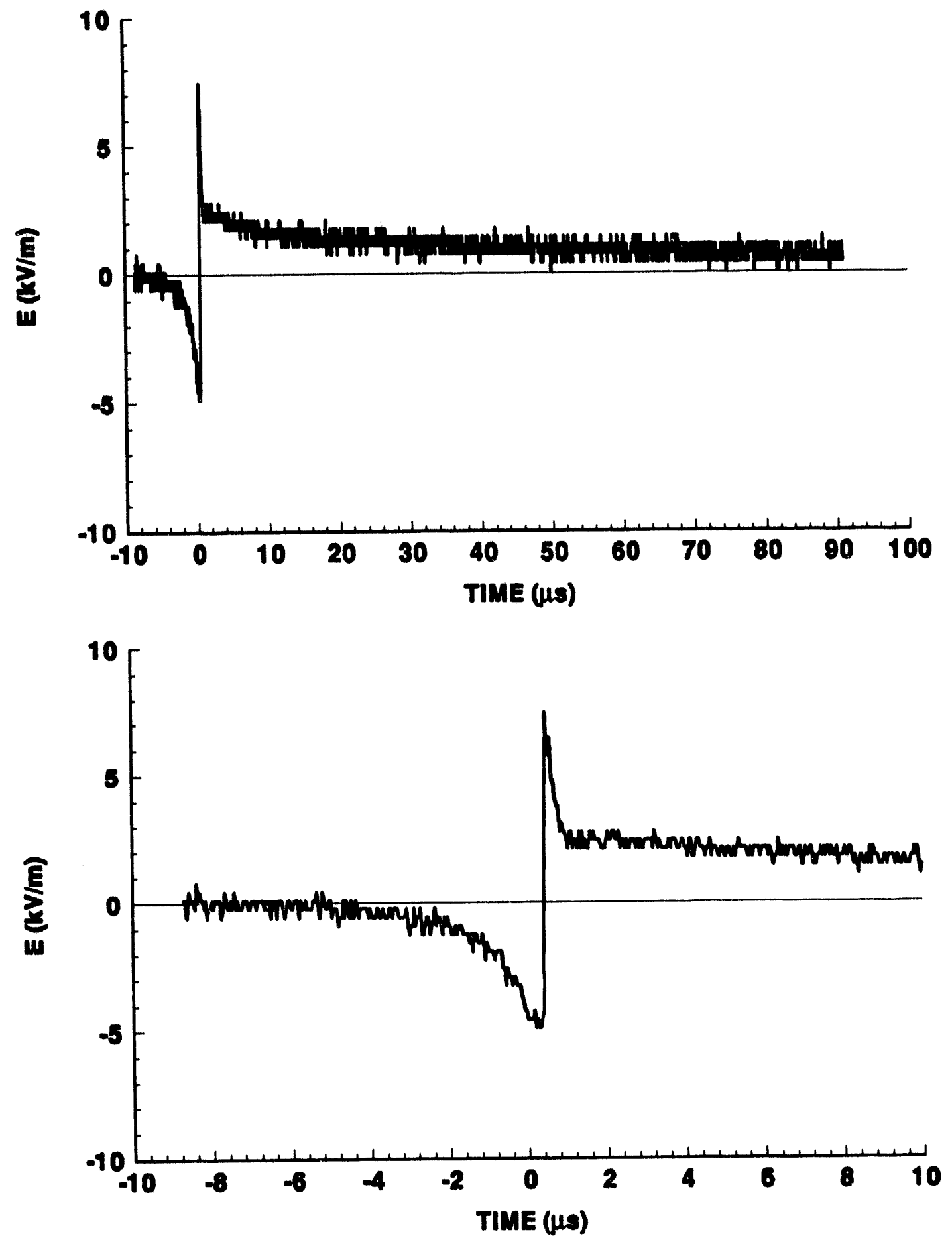


\section{STOKE 5}

ES1
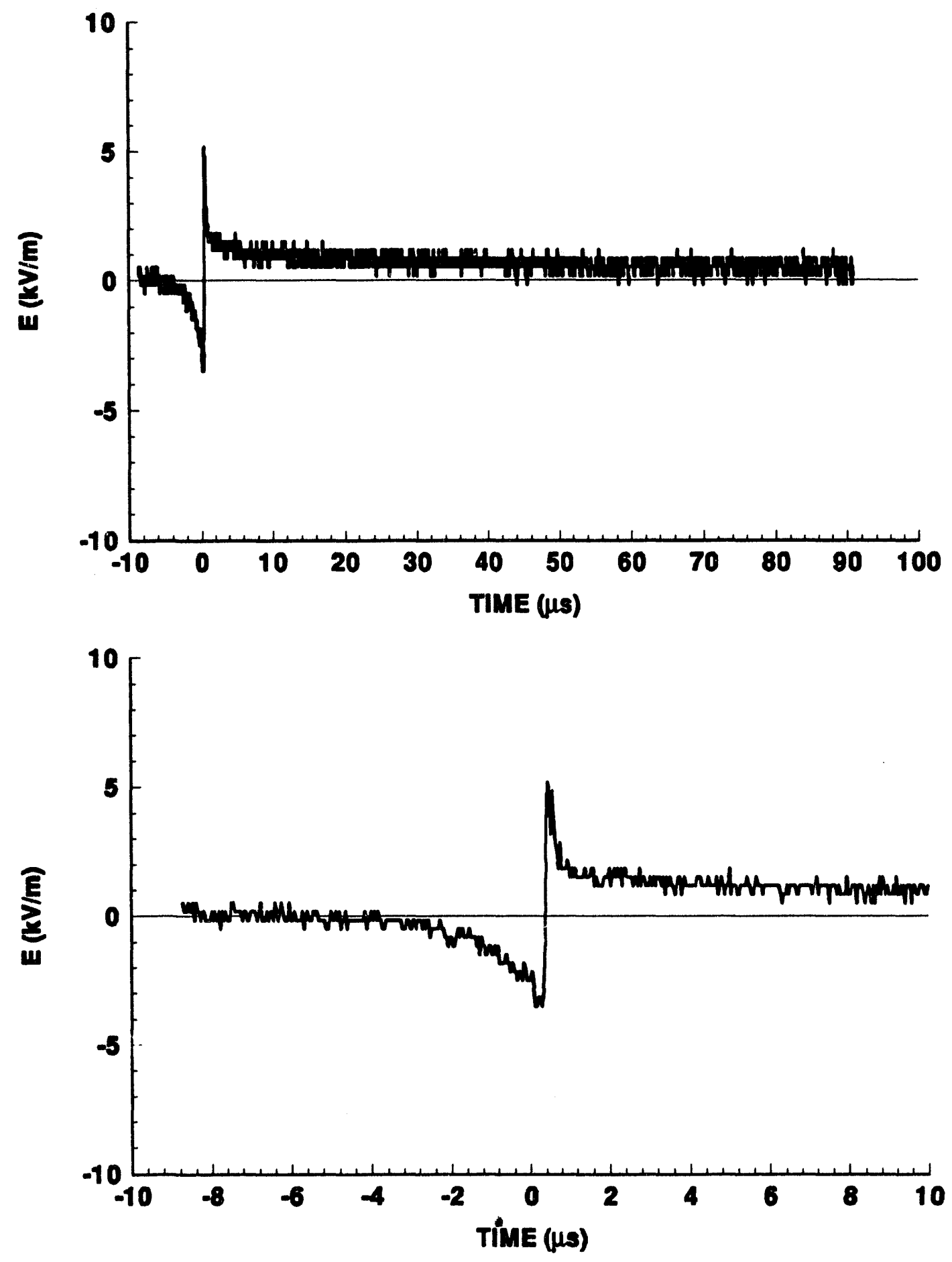


\section{STOKE 6 \\ ES1}
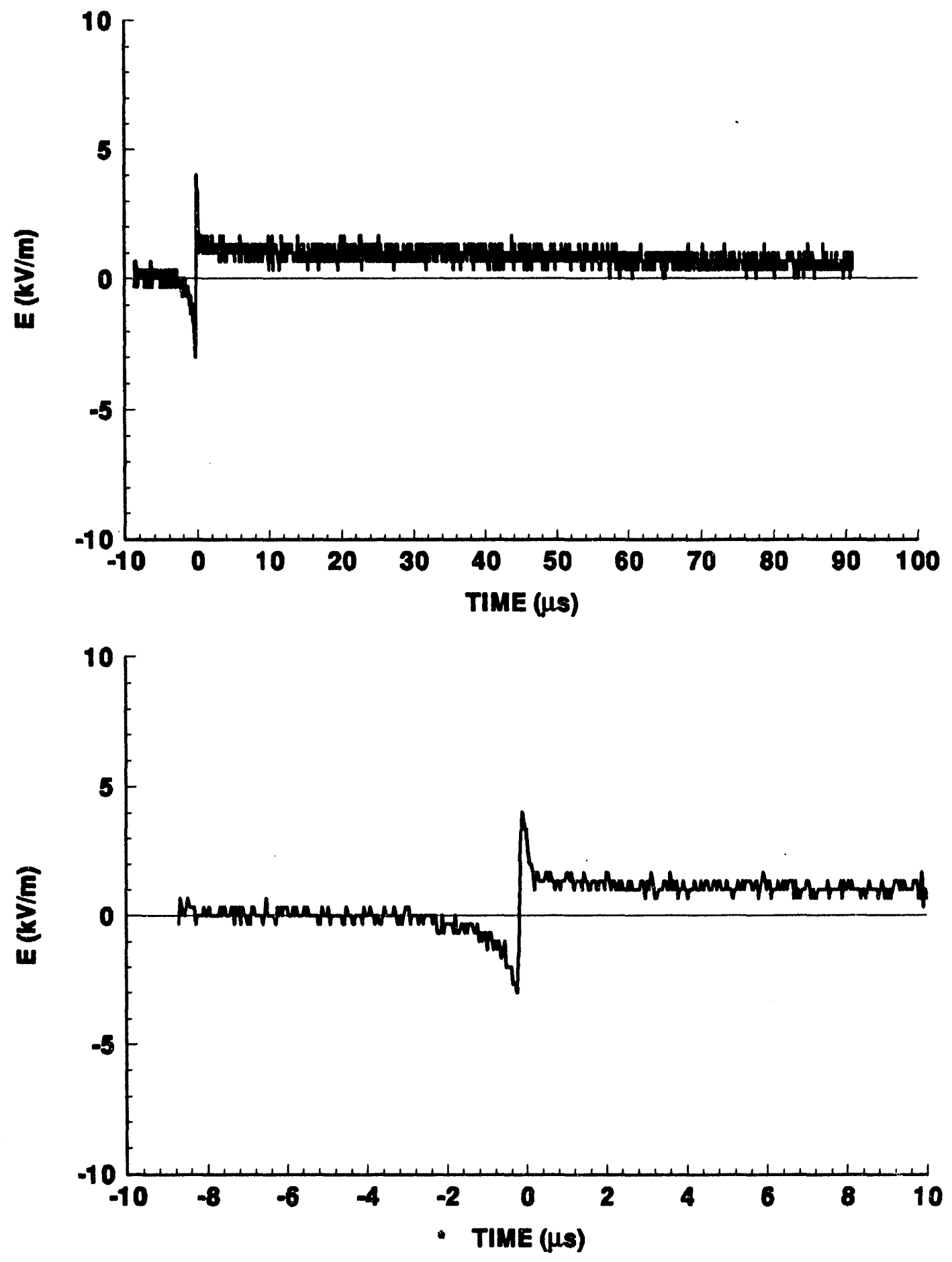


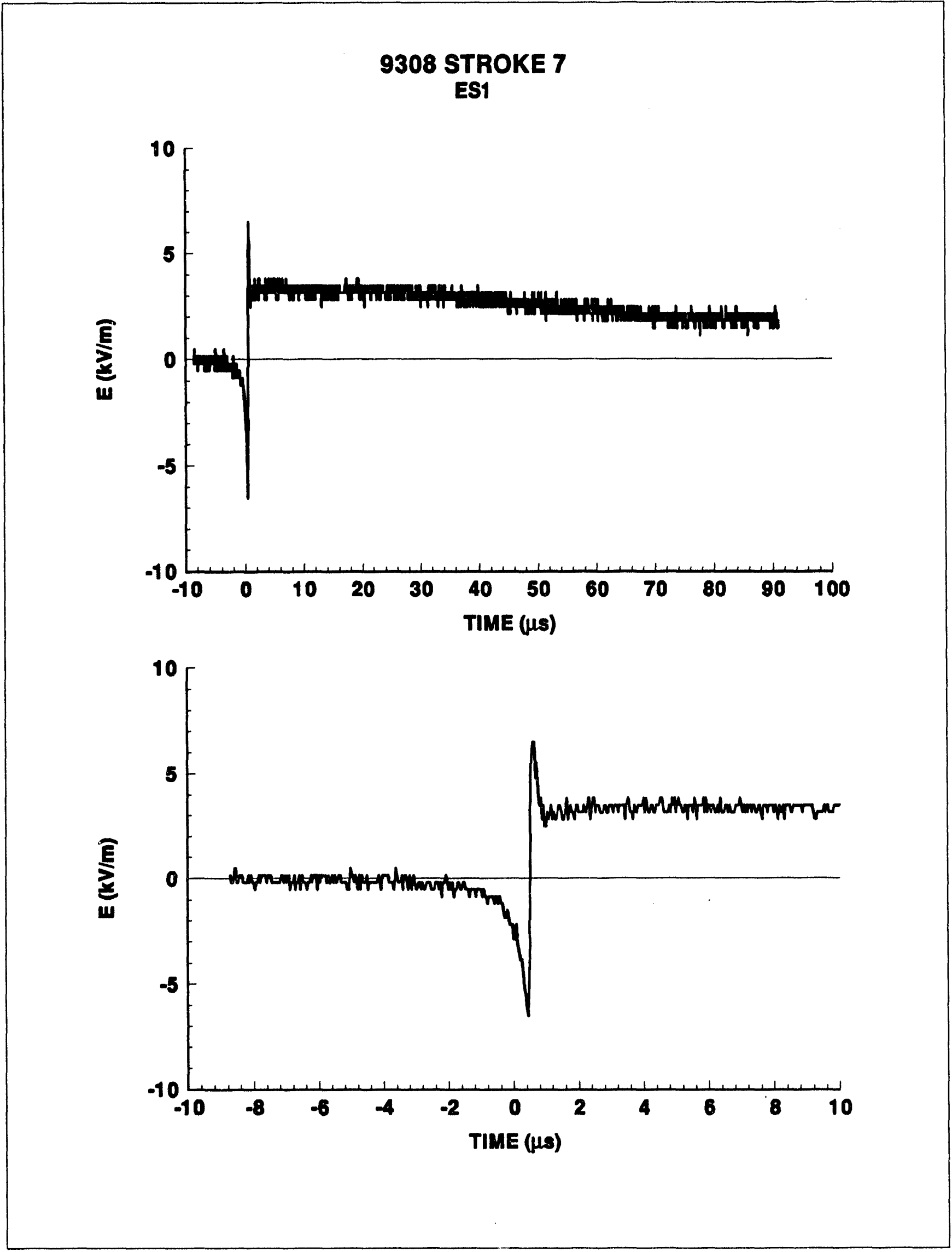




\section{STROKE 8}

ES1
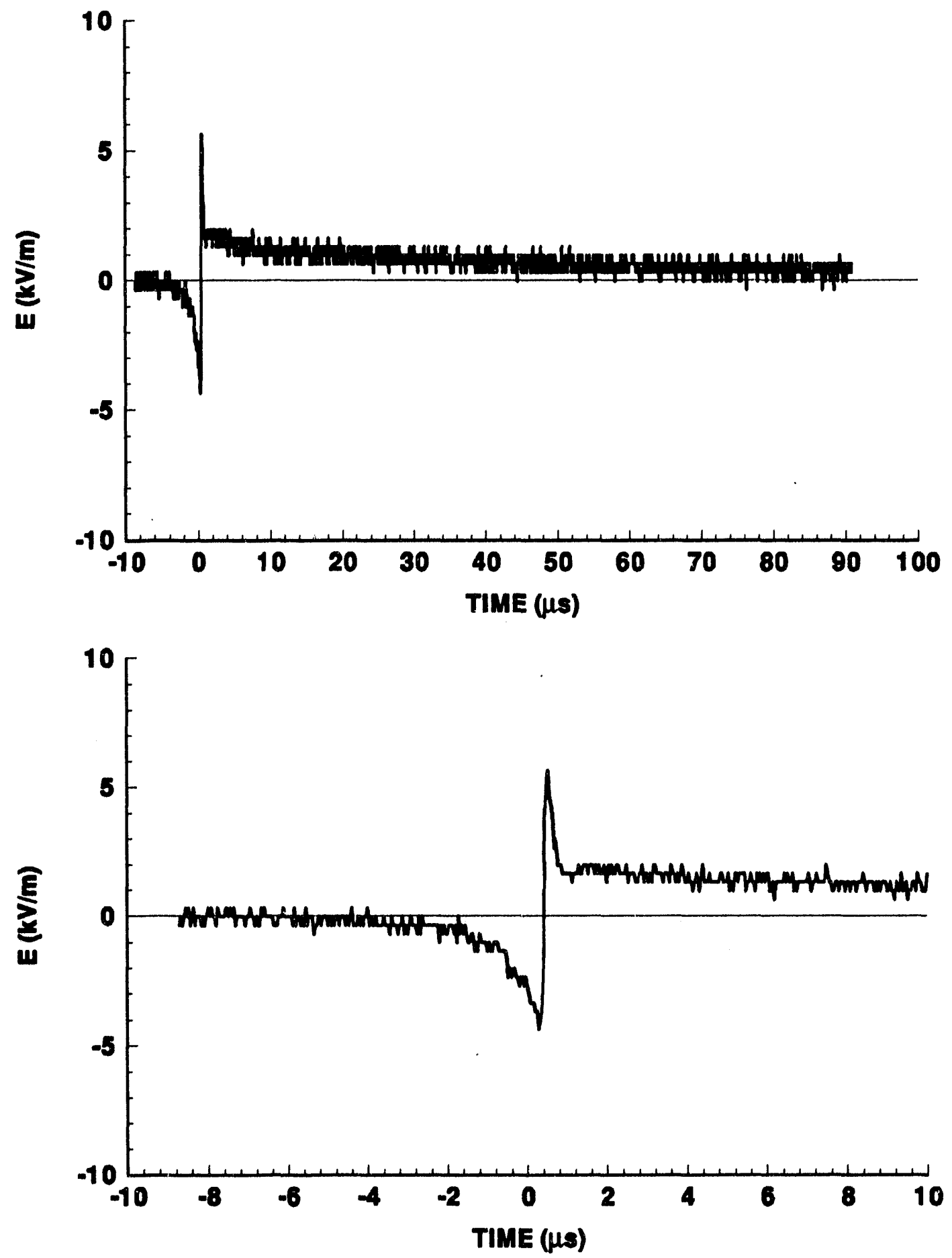


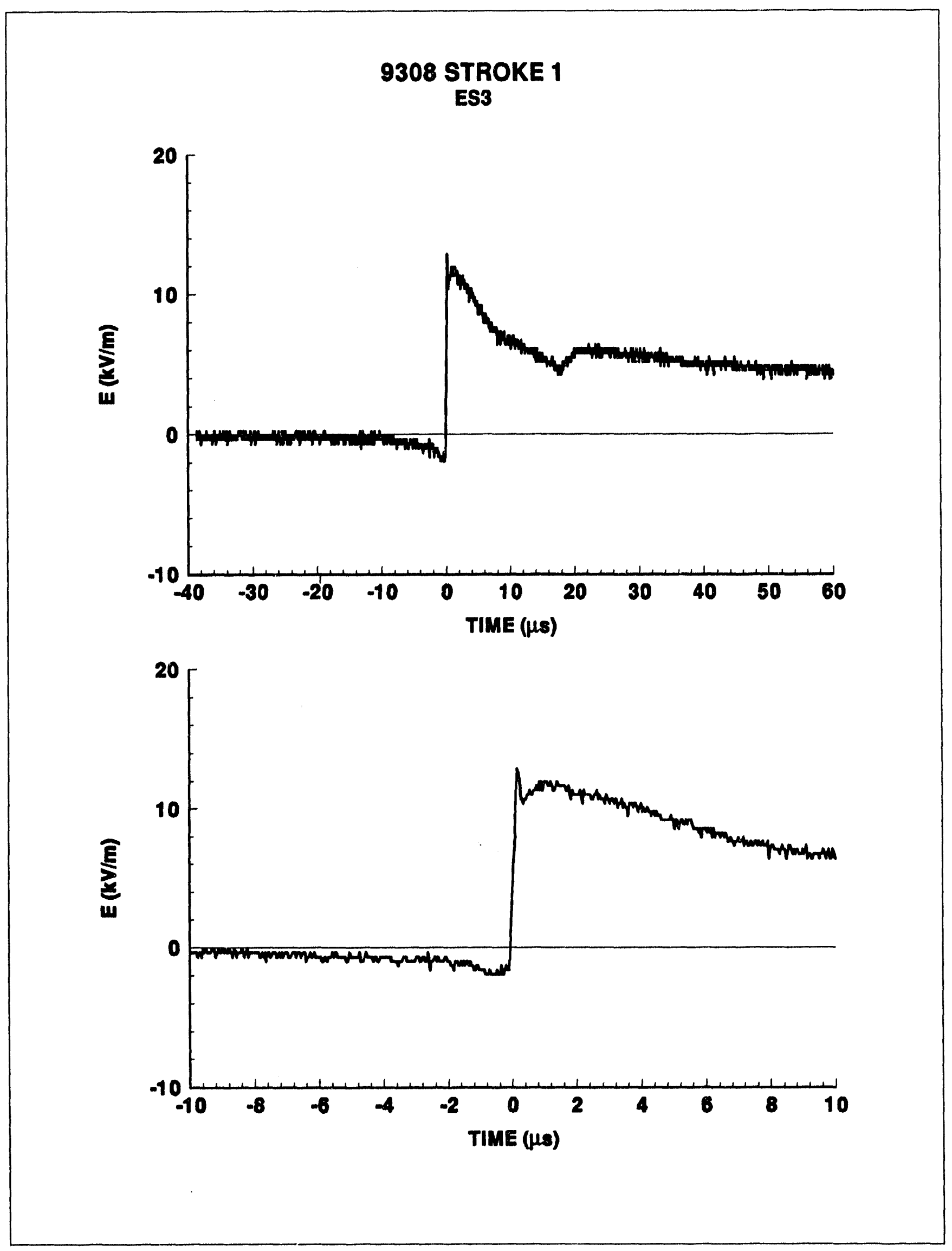




\section{STROKE 2}

ES3
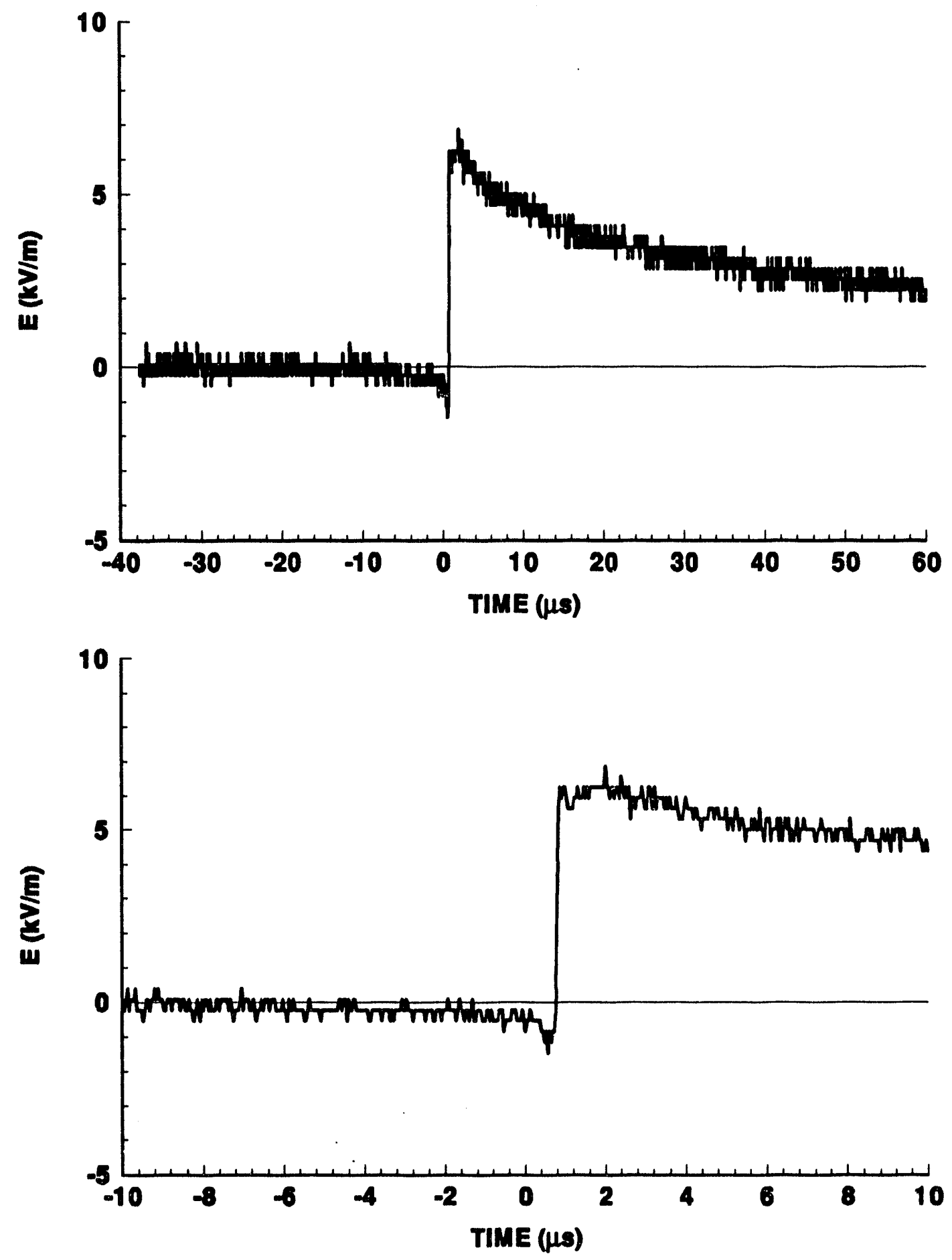
9308 STROKE 3

ES3
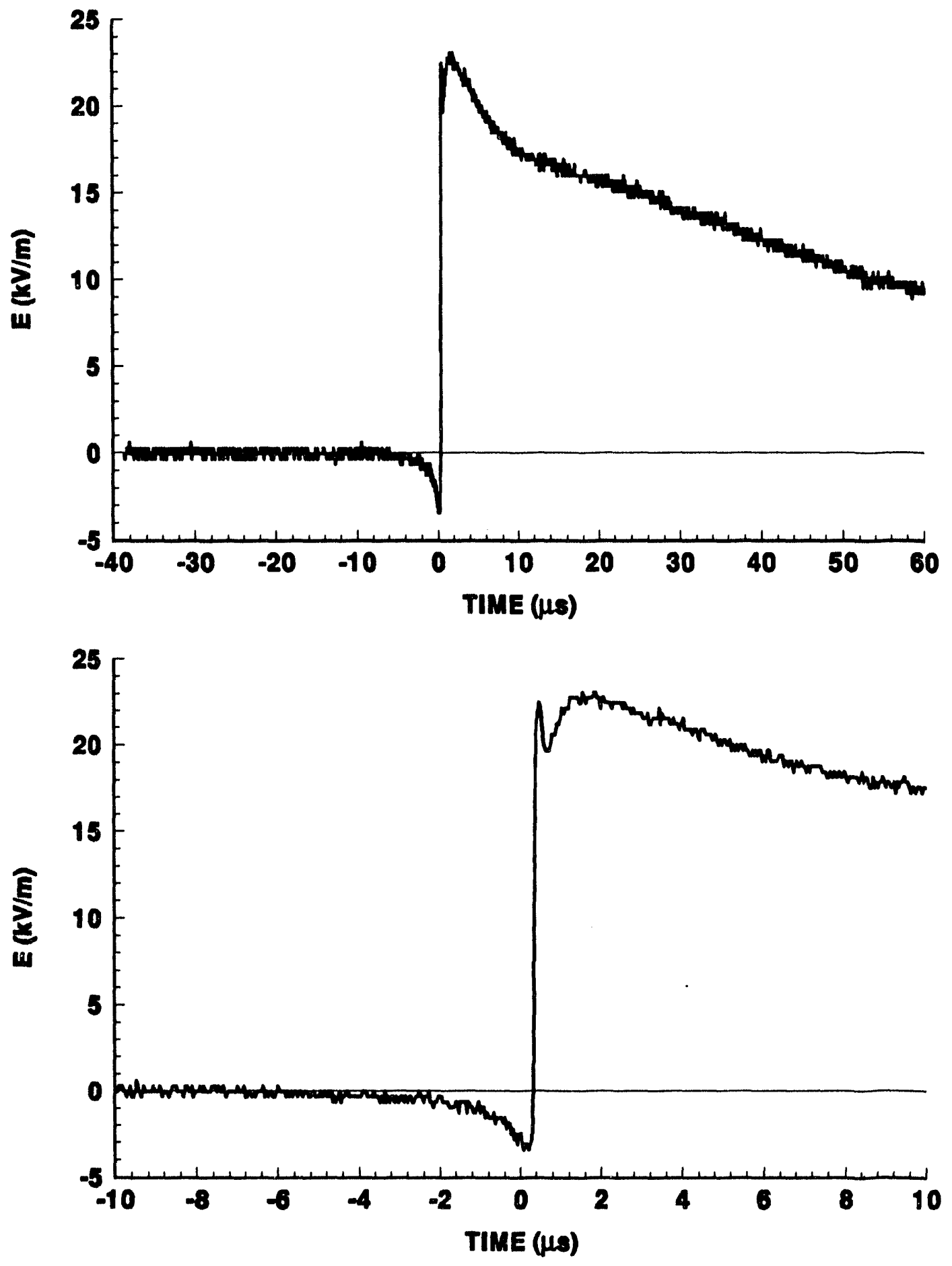


\section{STROKE 4}

ES3
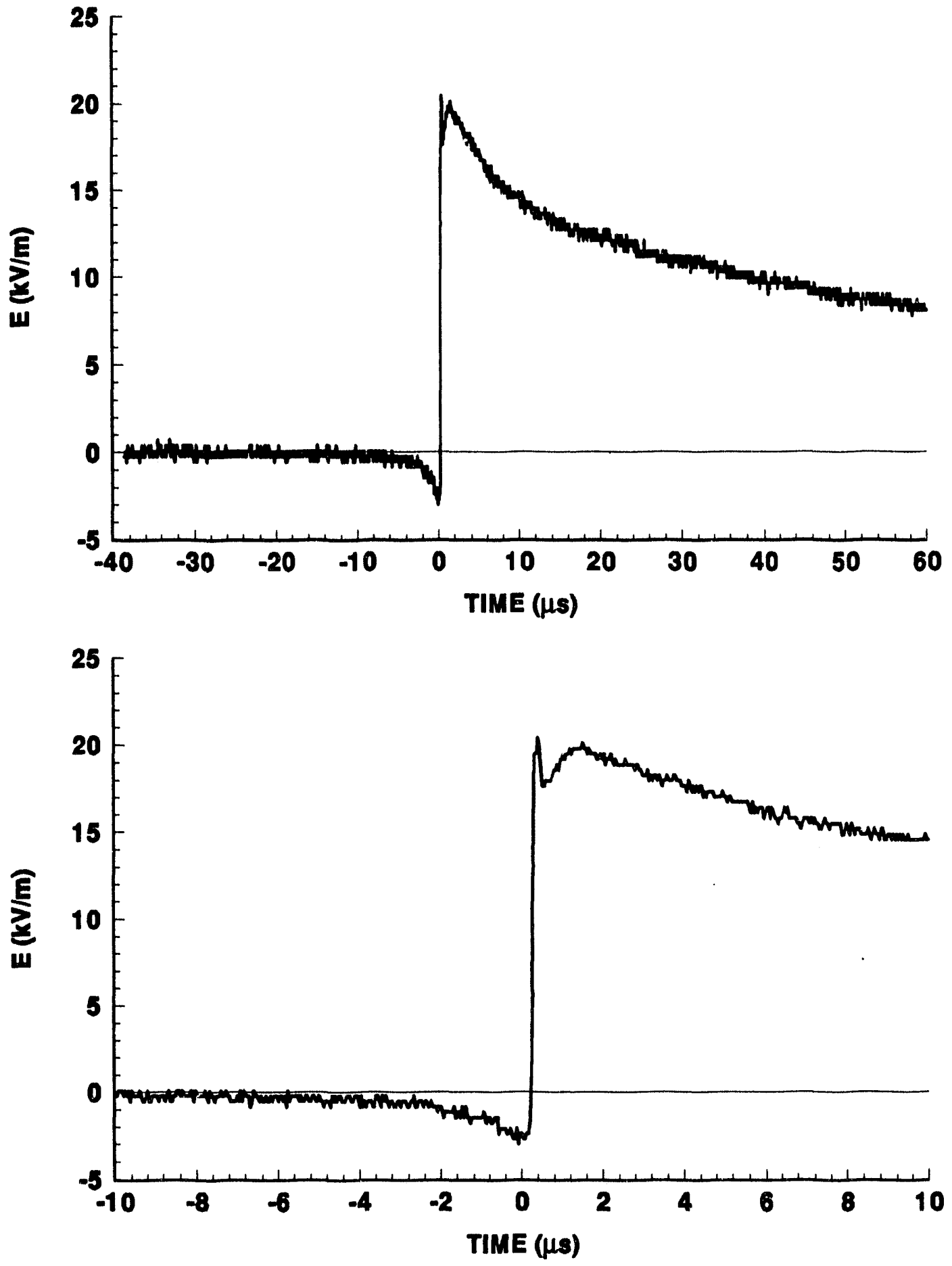


\section{STROKE 5}

ES3
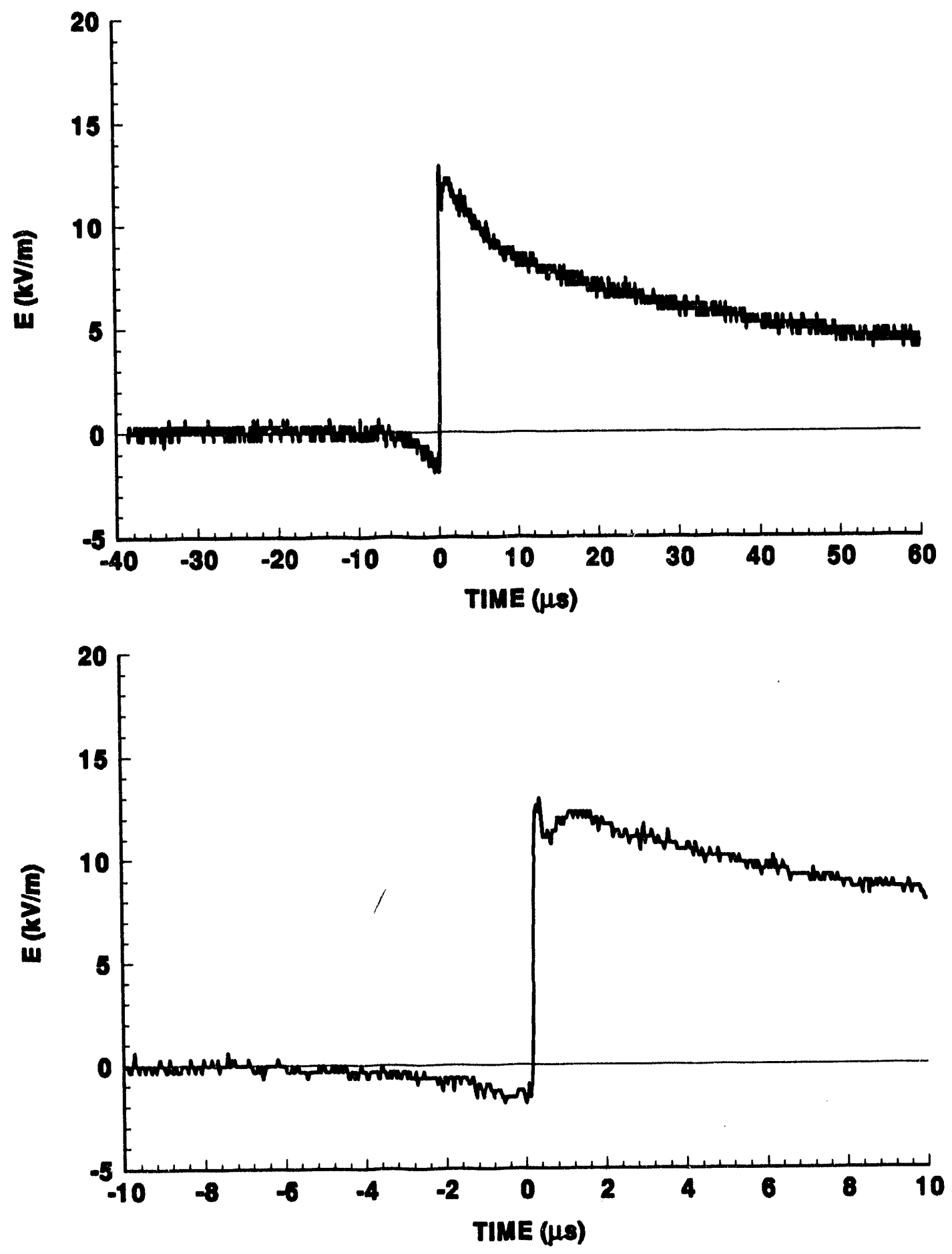


\section{STROKE 6}

ES3
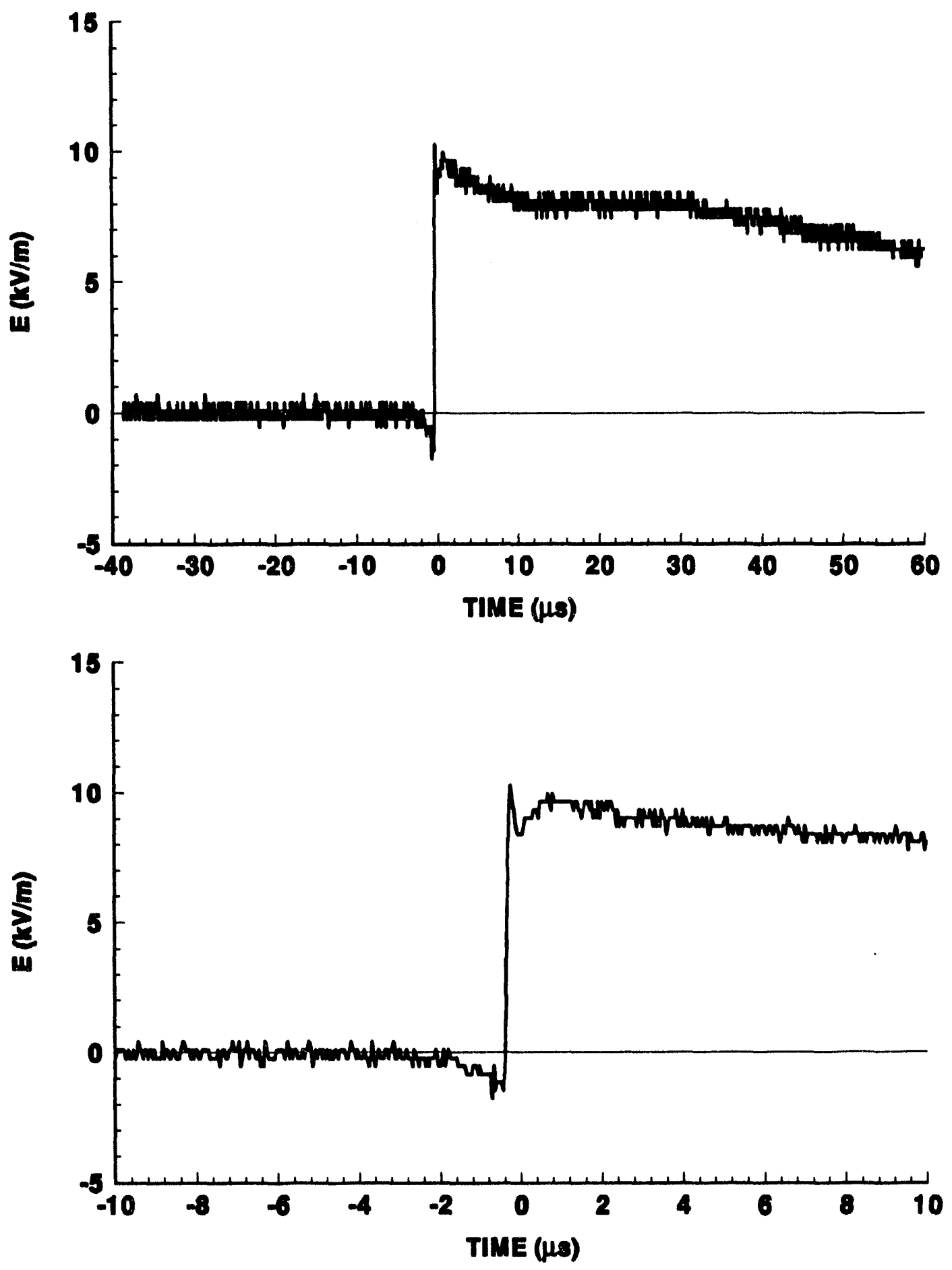


\section{STROKE 7}

ES3
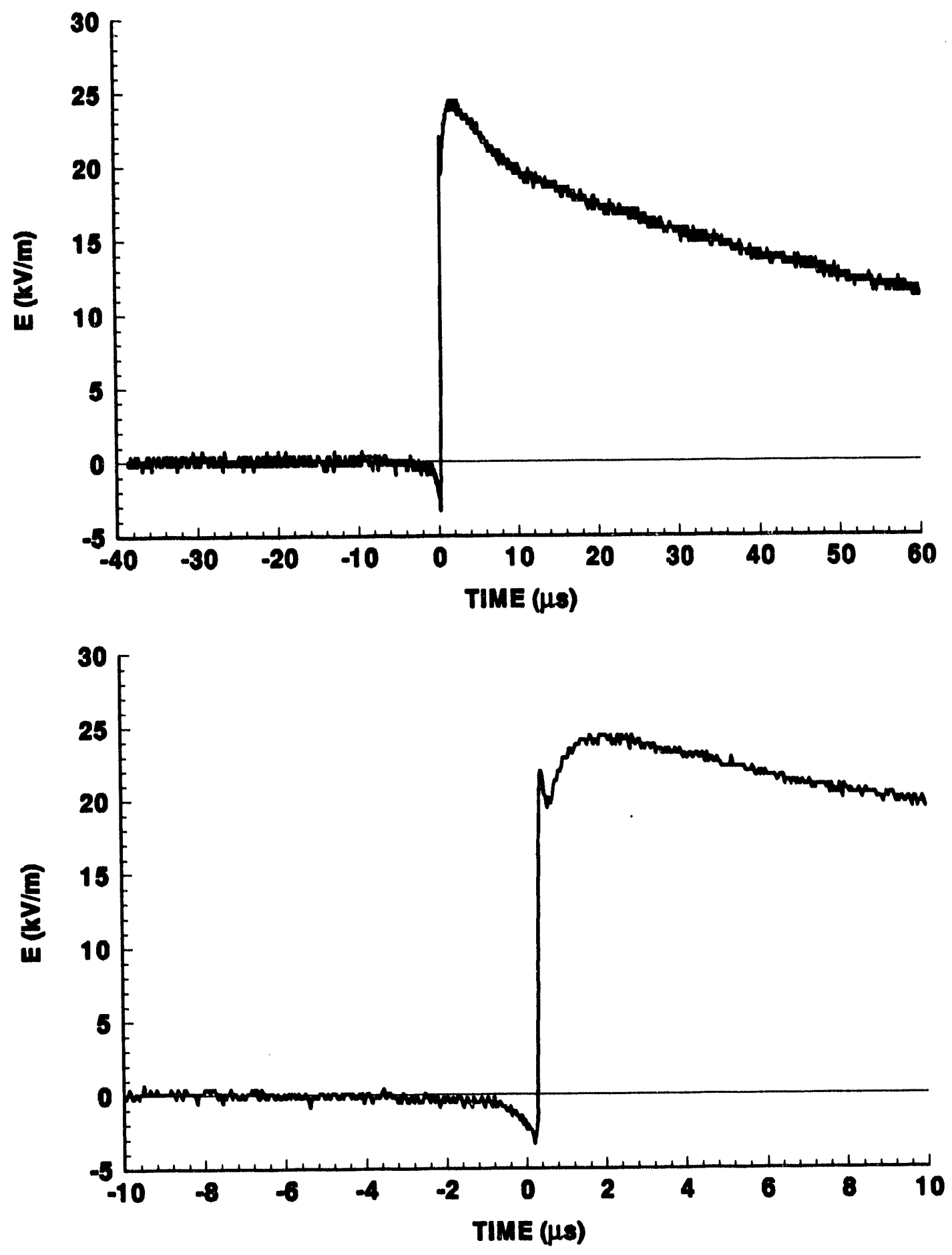


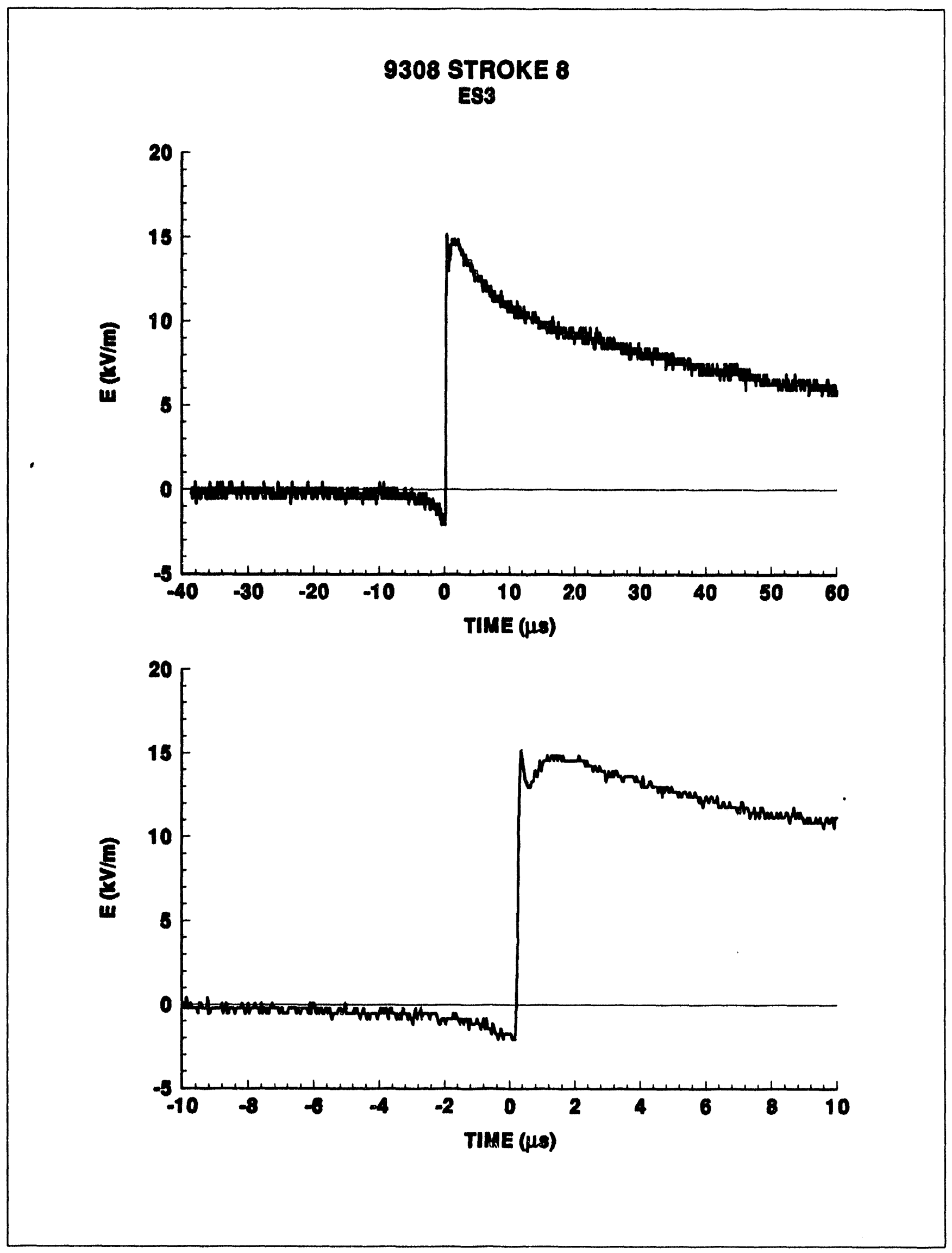




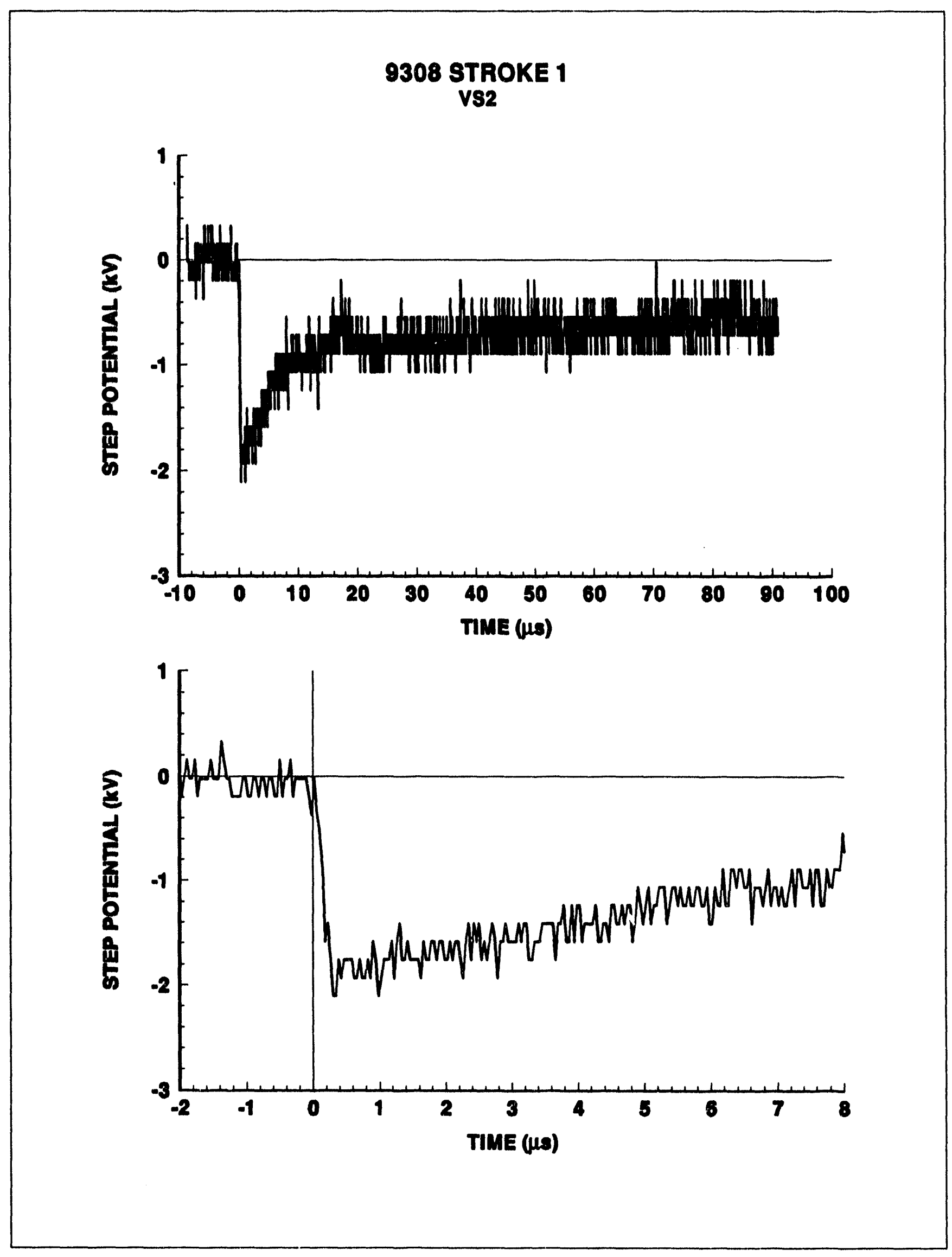




\section{STROKE 2}

v92
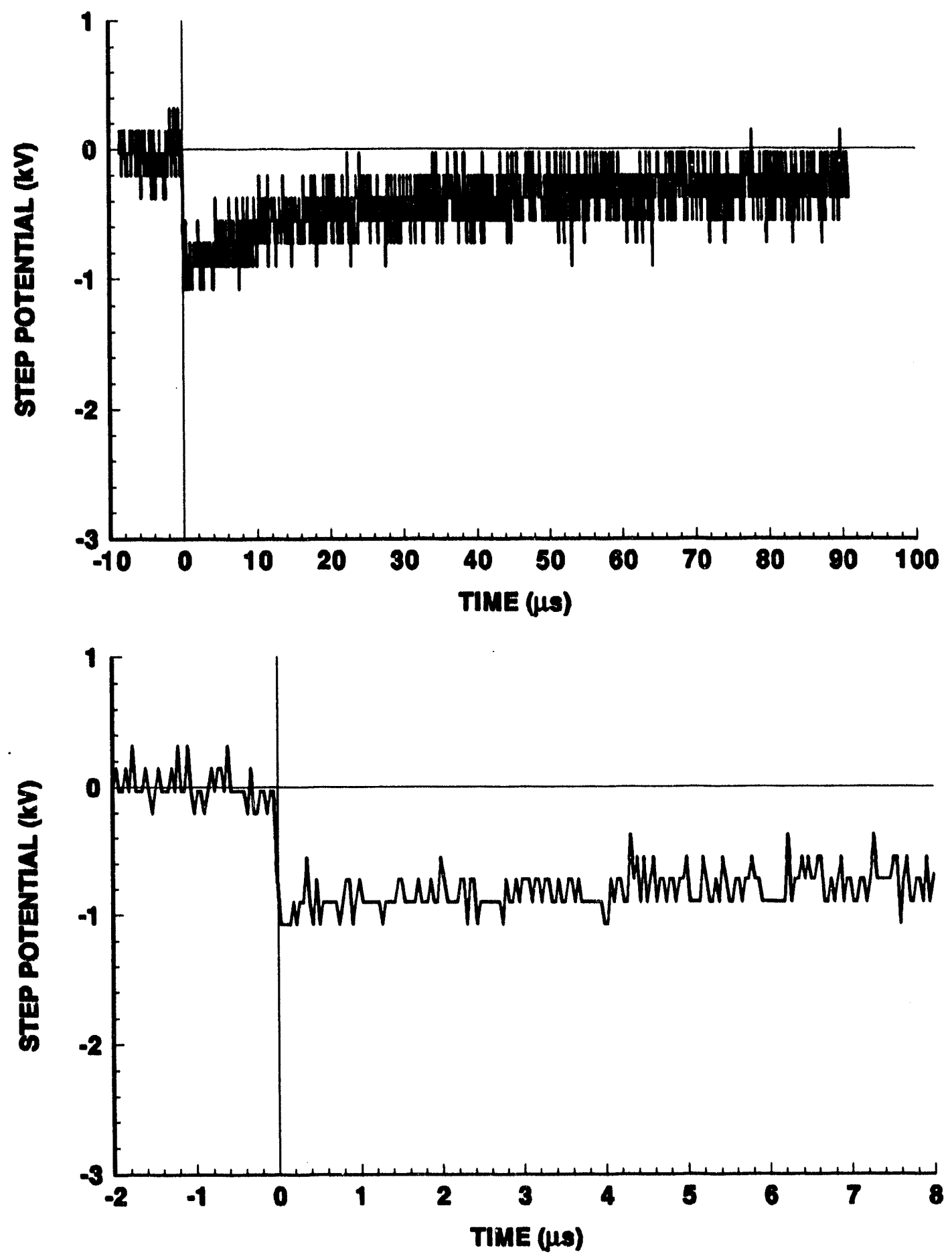


\section{STROKE 8 \\ vs2}
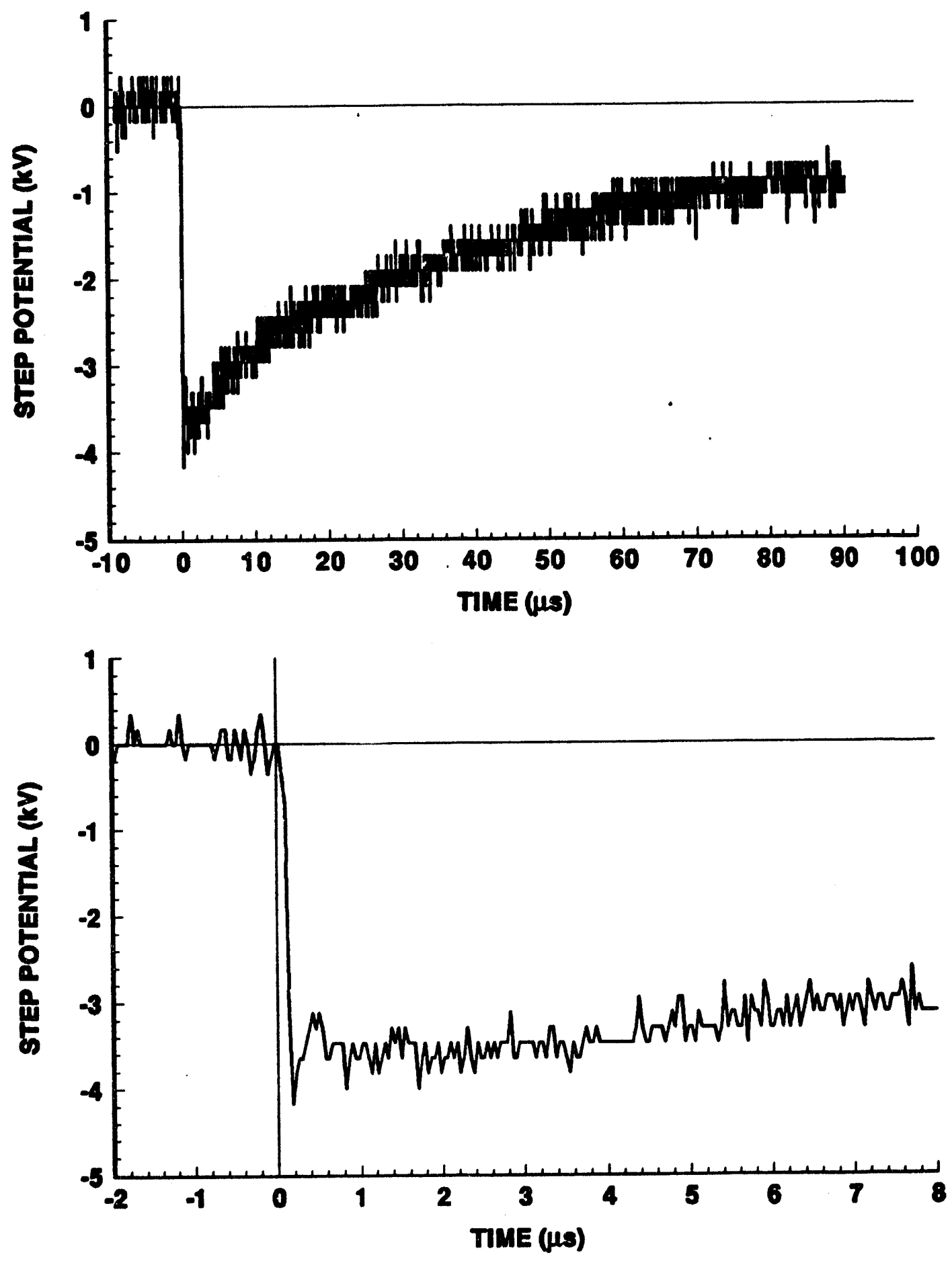
9308 STROKE 4

vse
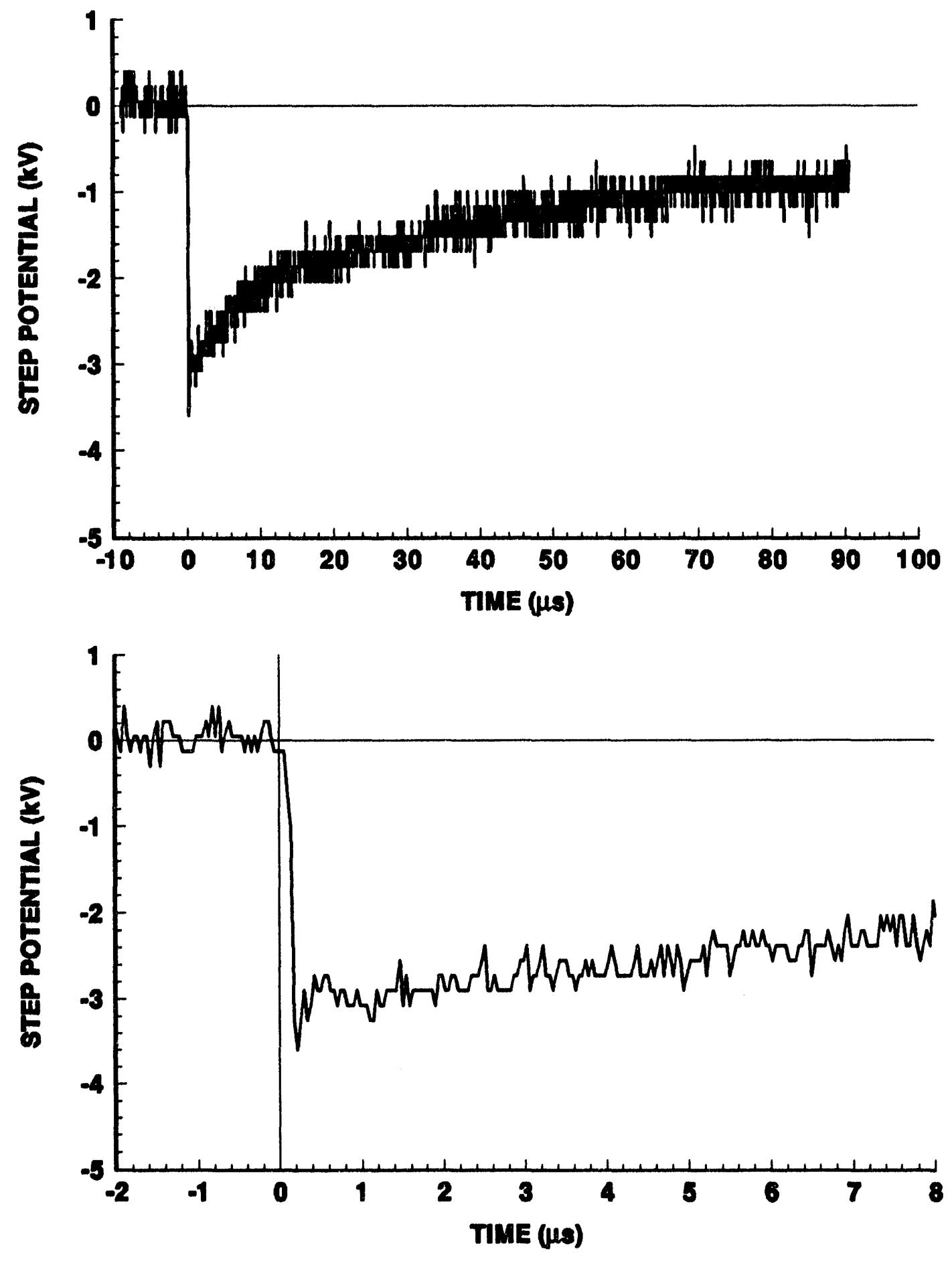


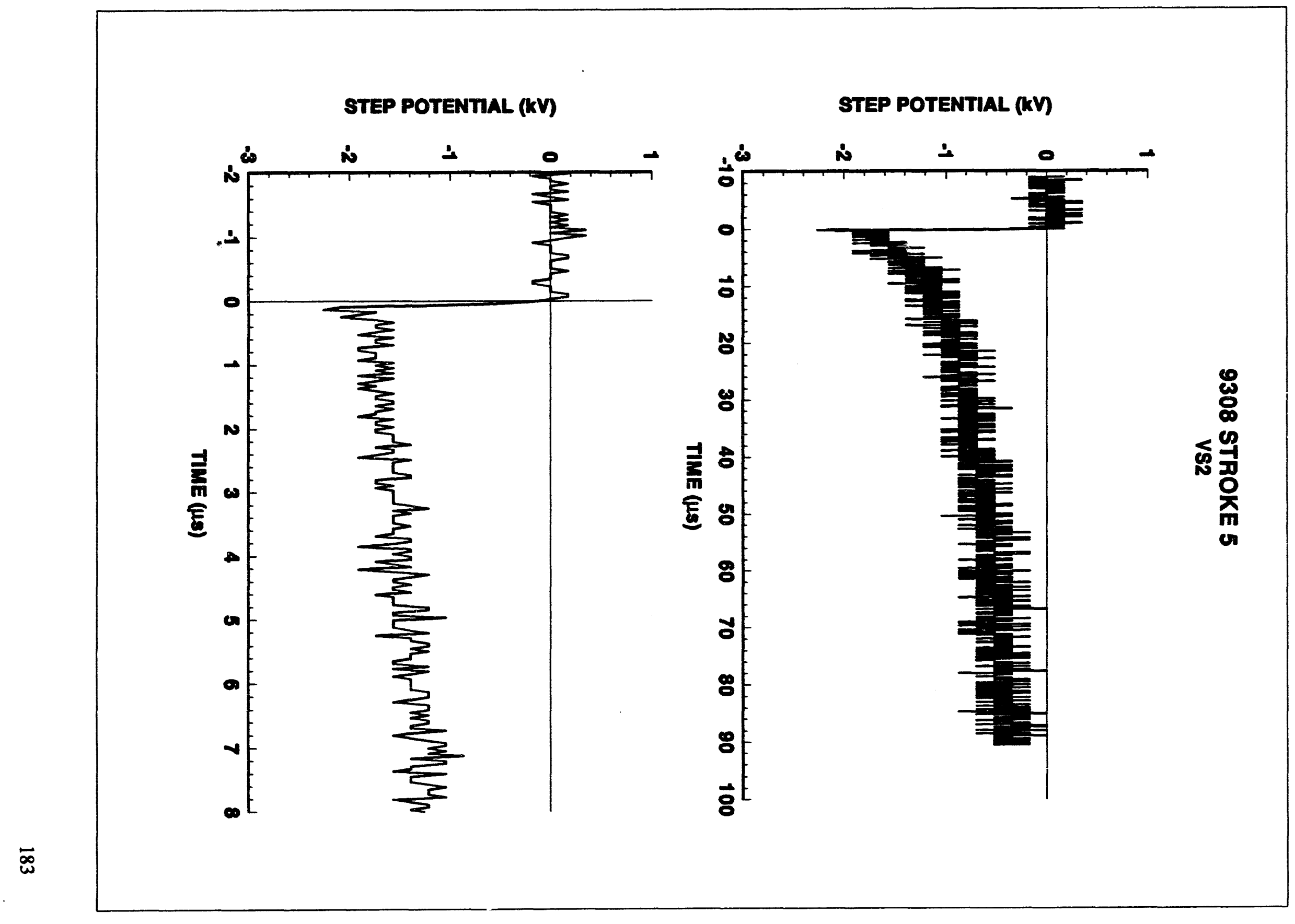


STEP POTENTIAL (KV)

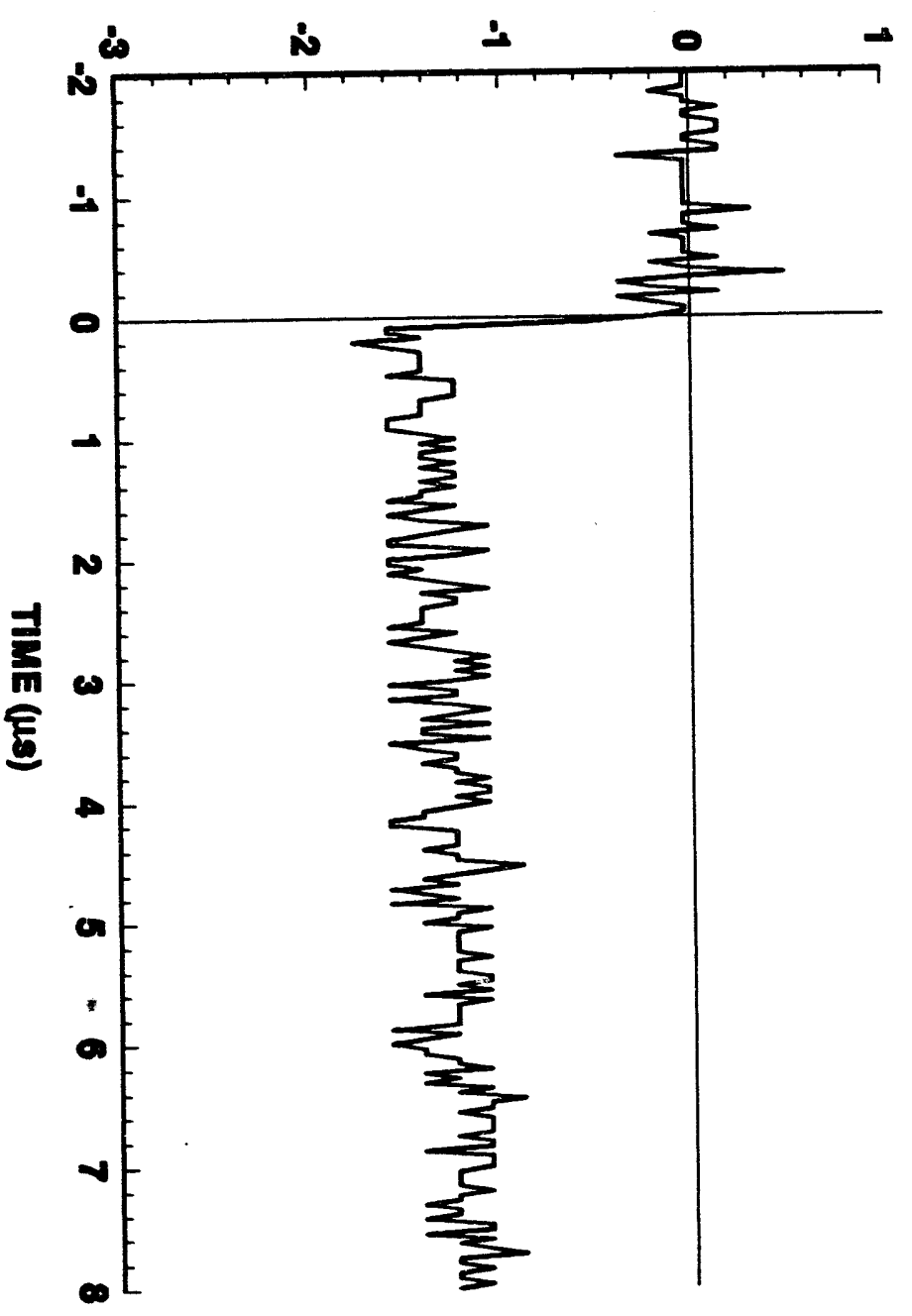

STEP POTENTIAL (KV)

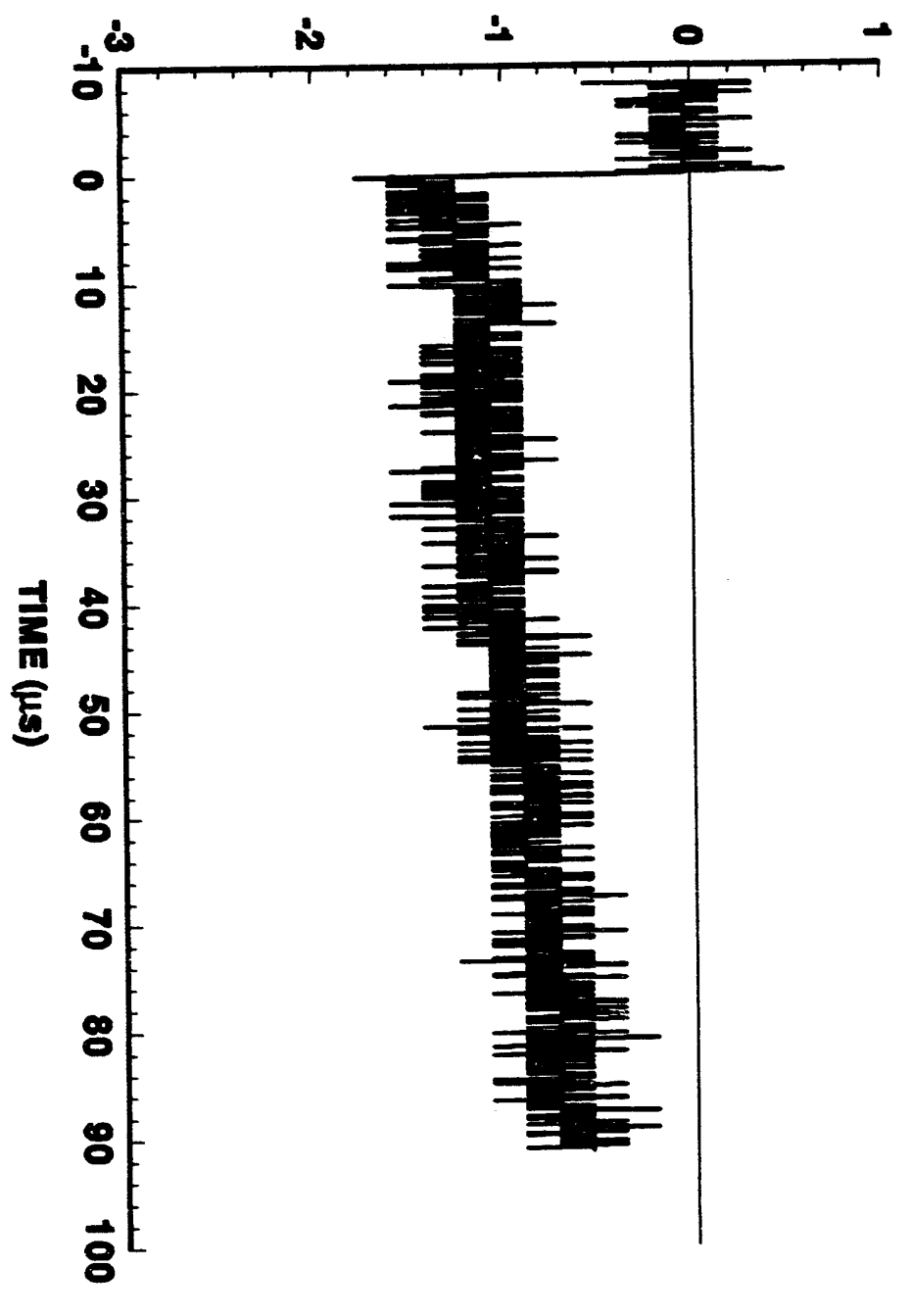




\section{STROKE 7 \\ VS2}
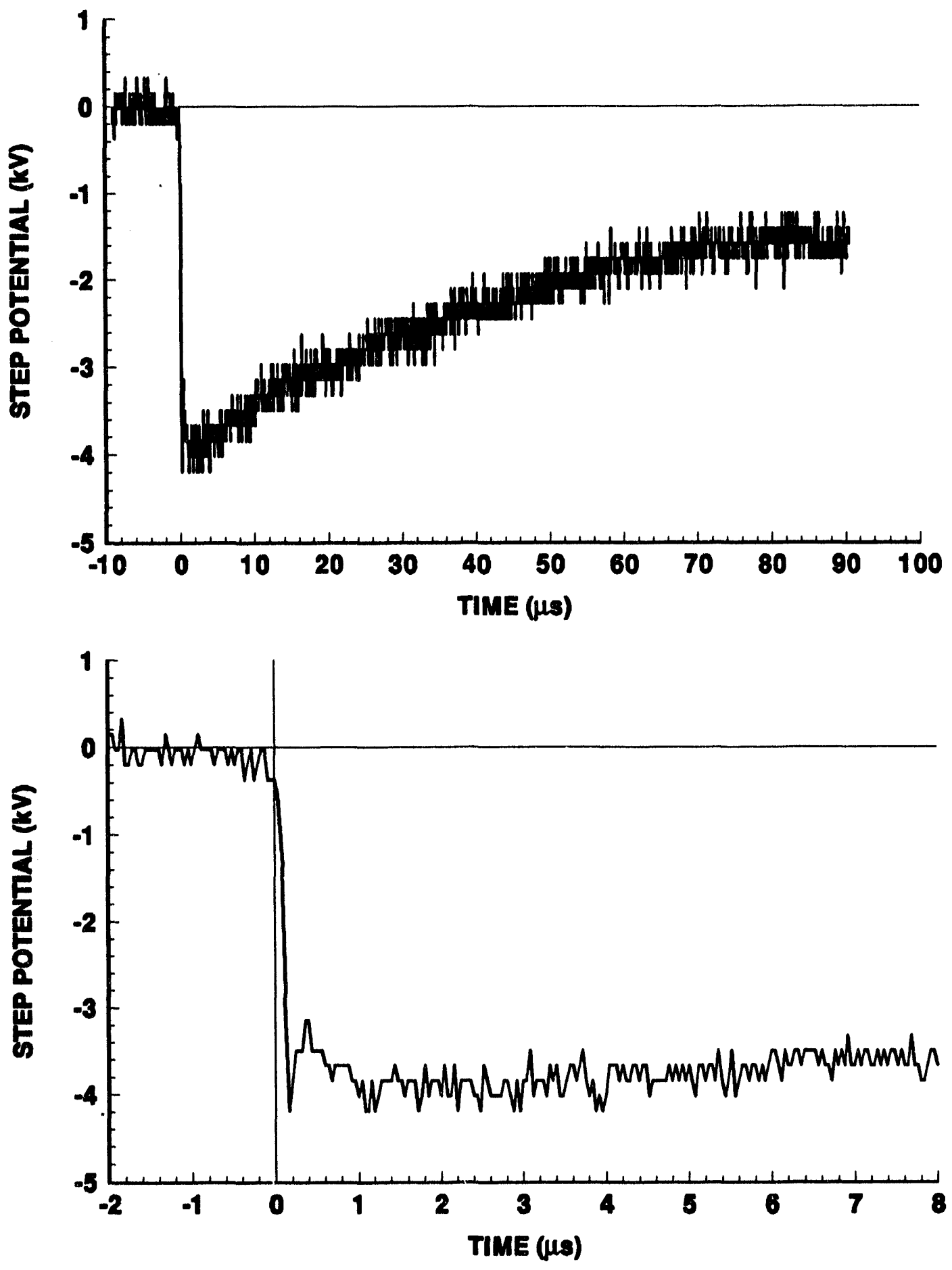


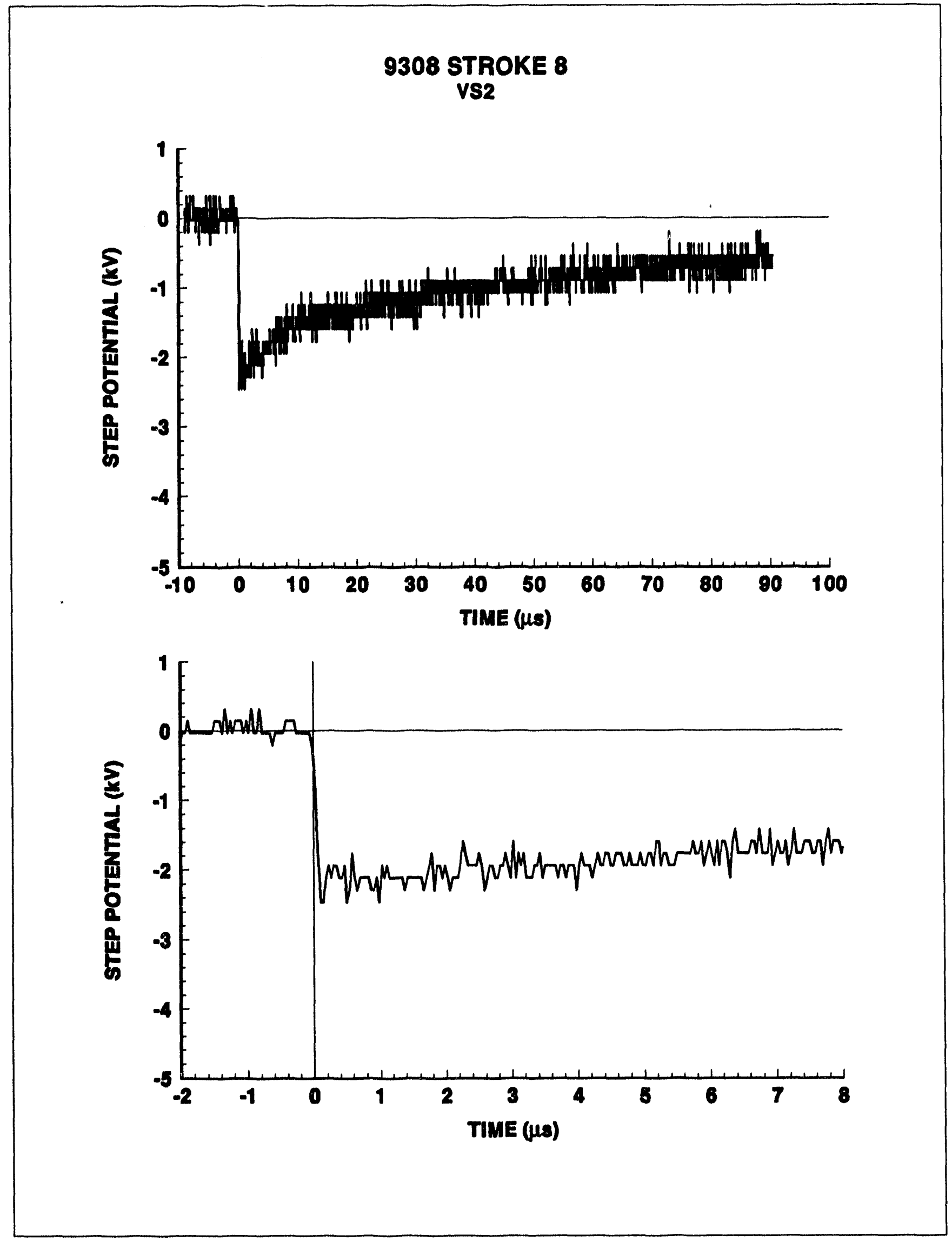




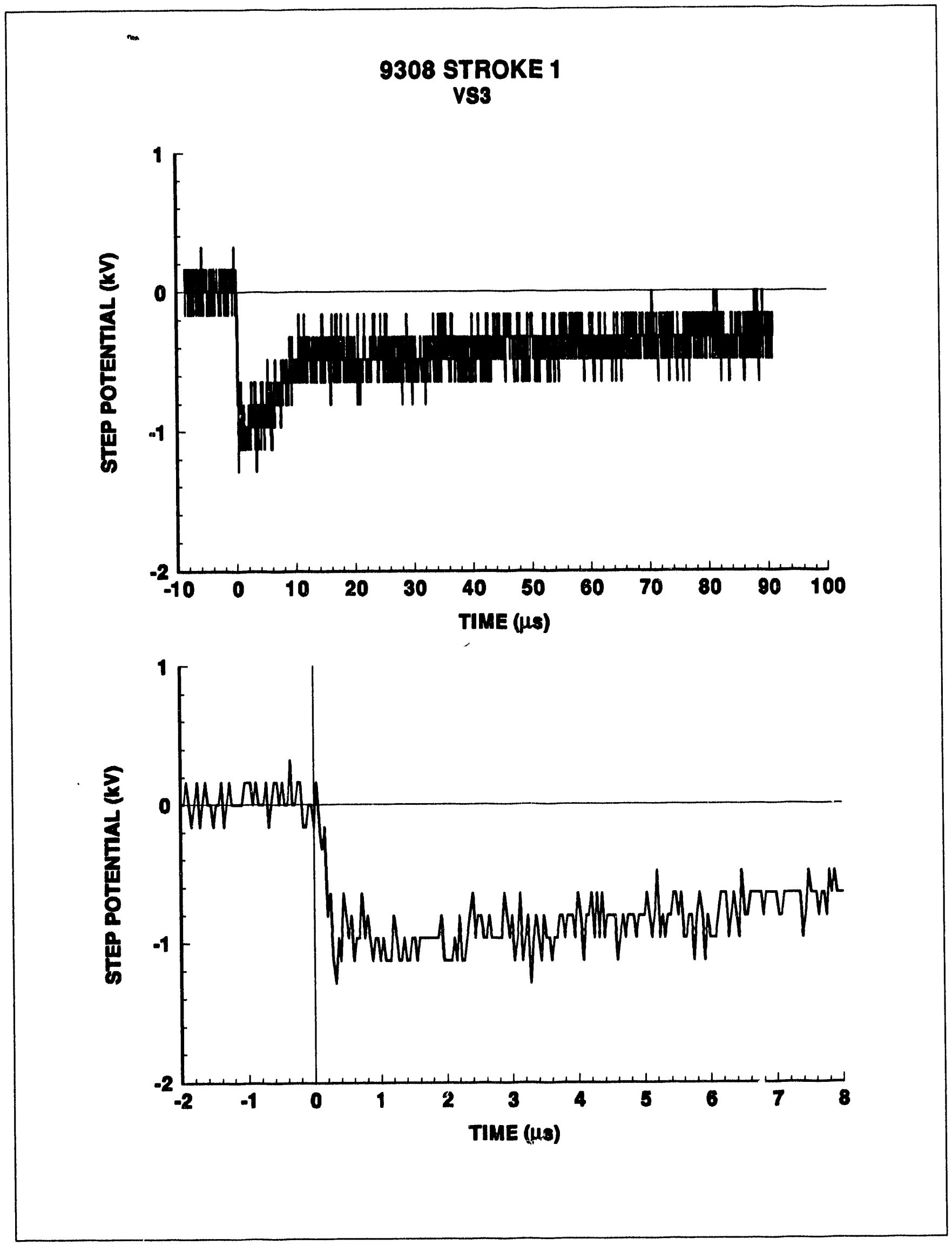


9308 STROKE 2

vS3
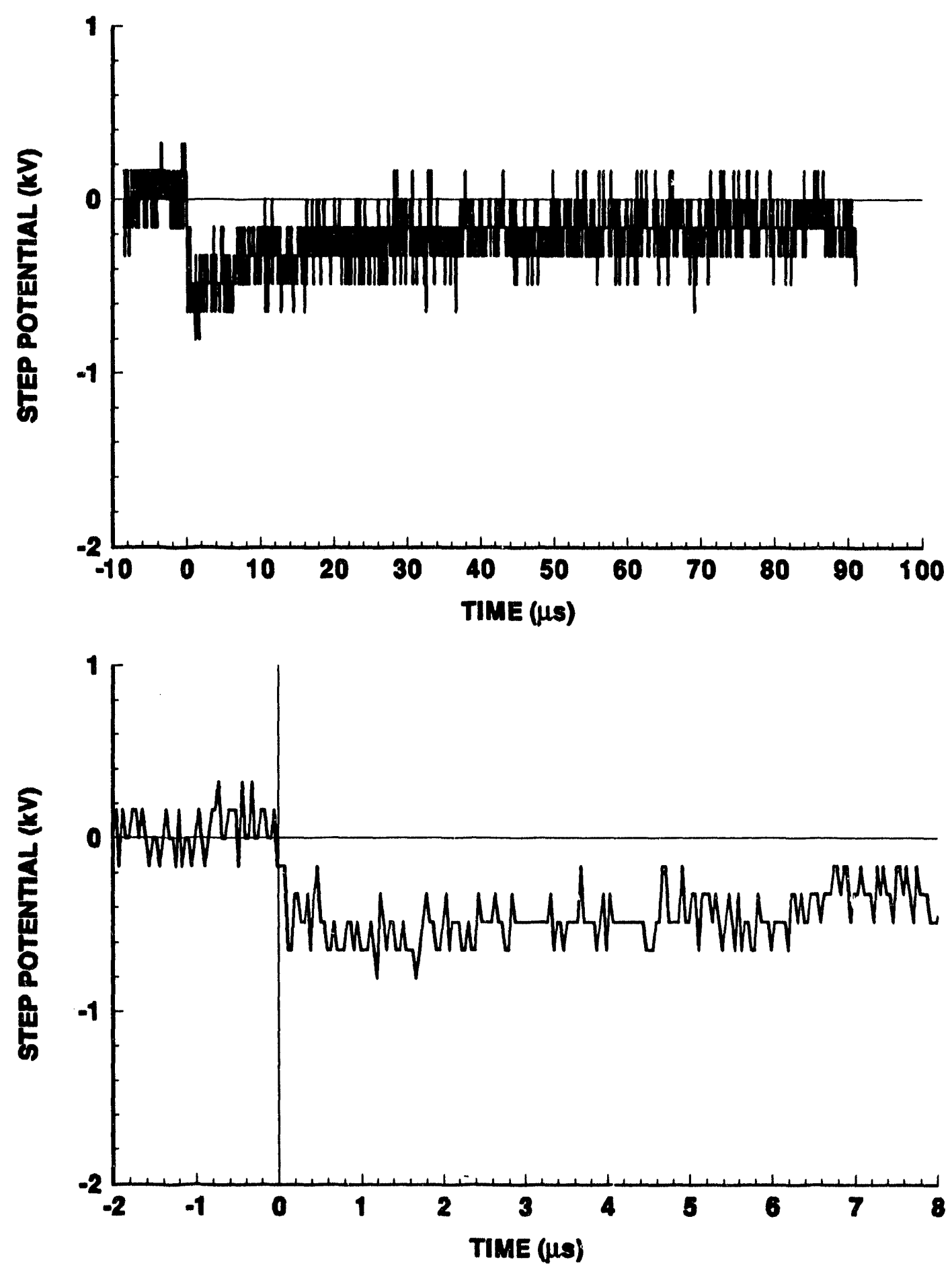


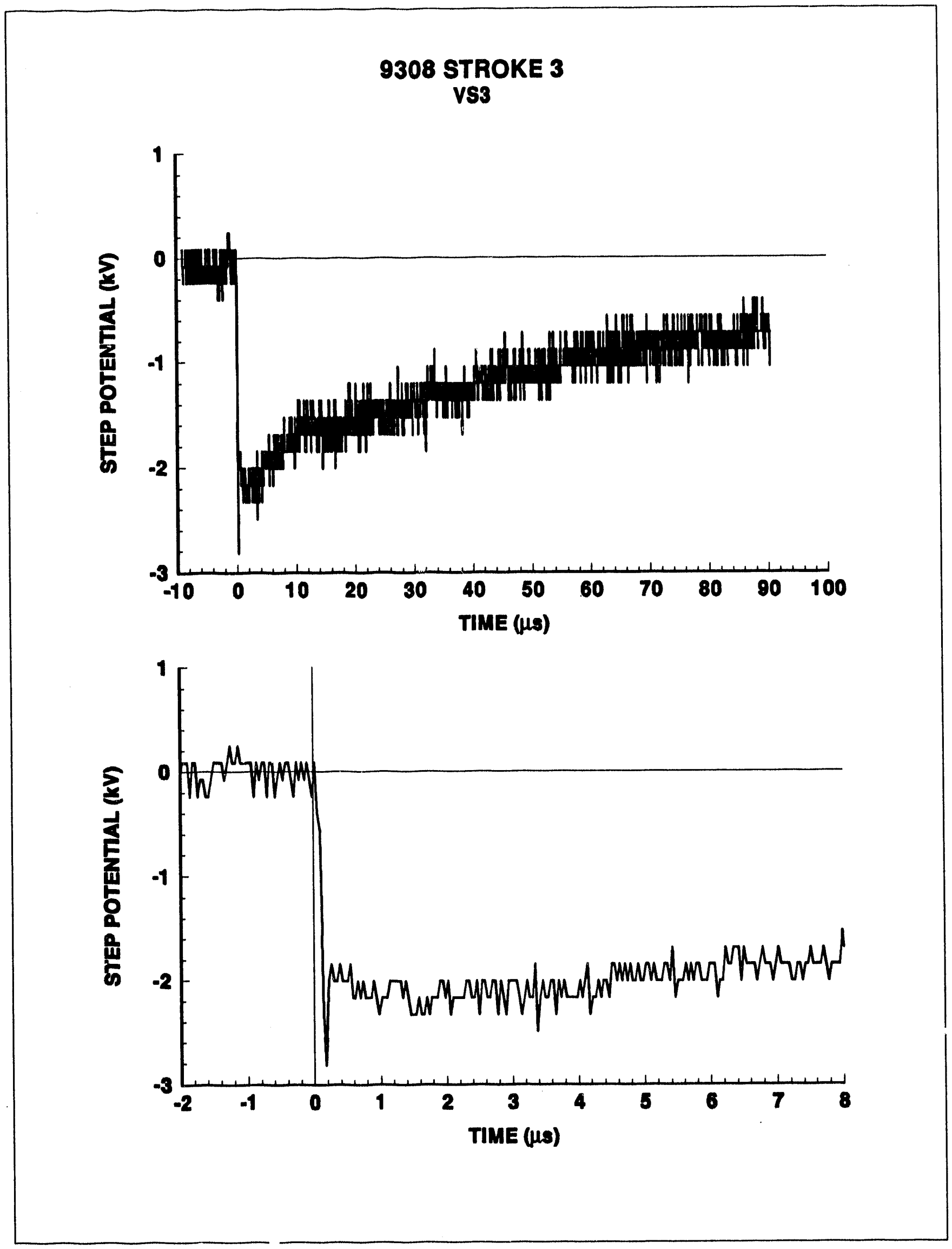


9308 STROKE 4

vS3
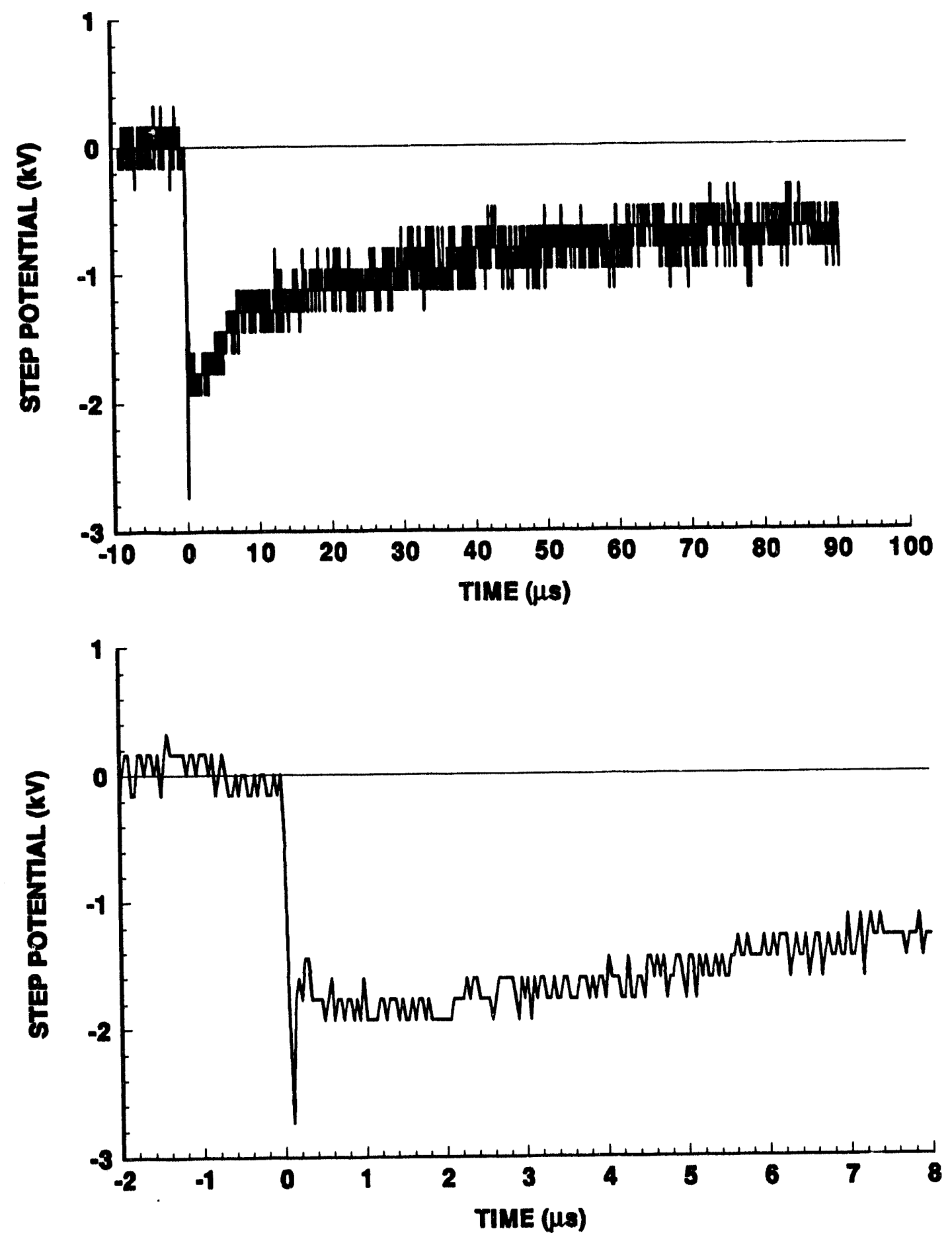


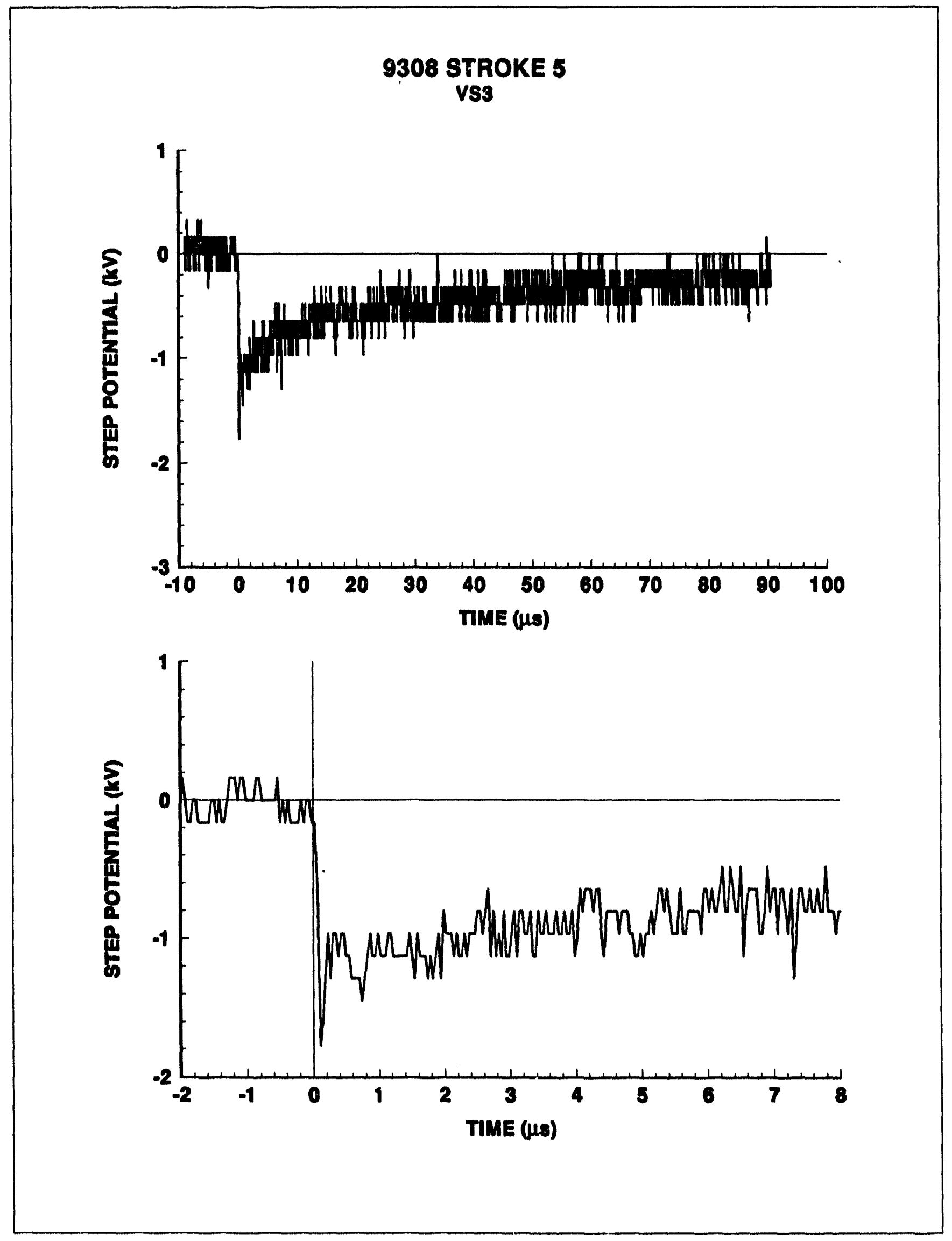




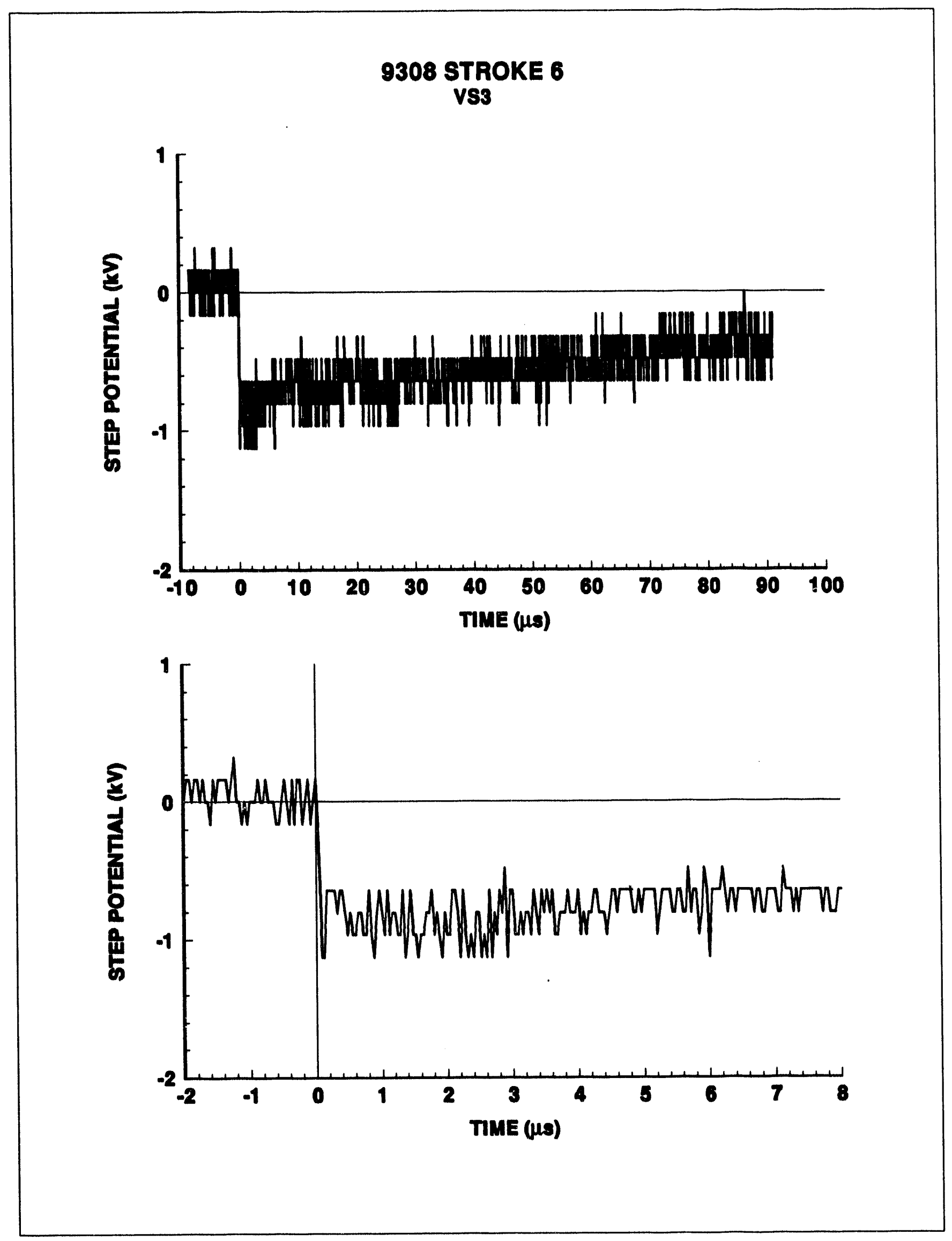




\section{STROKE 7 \\ vS3}
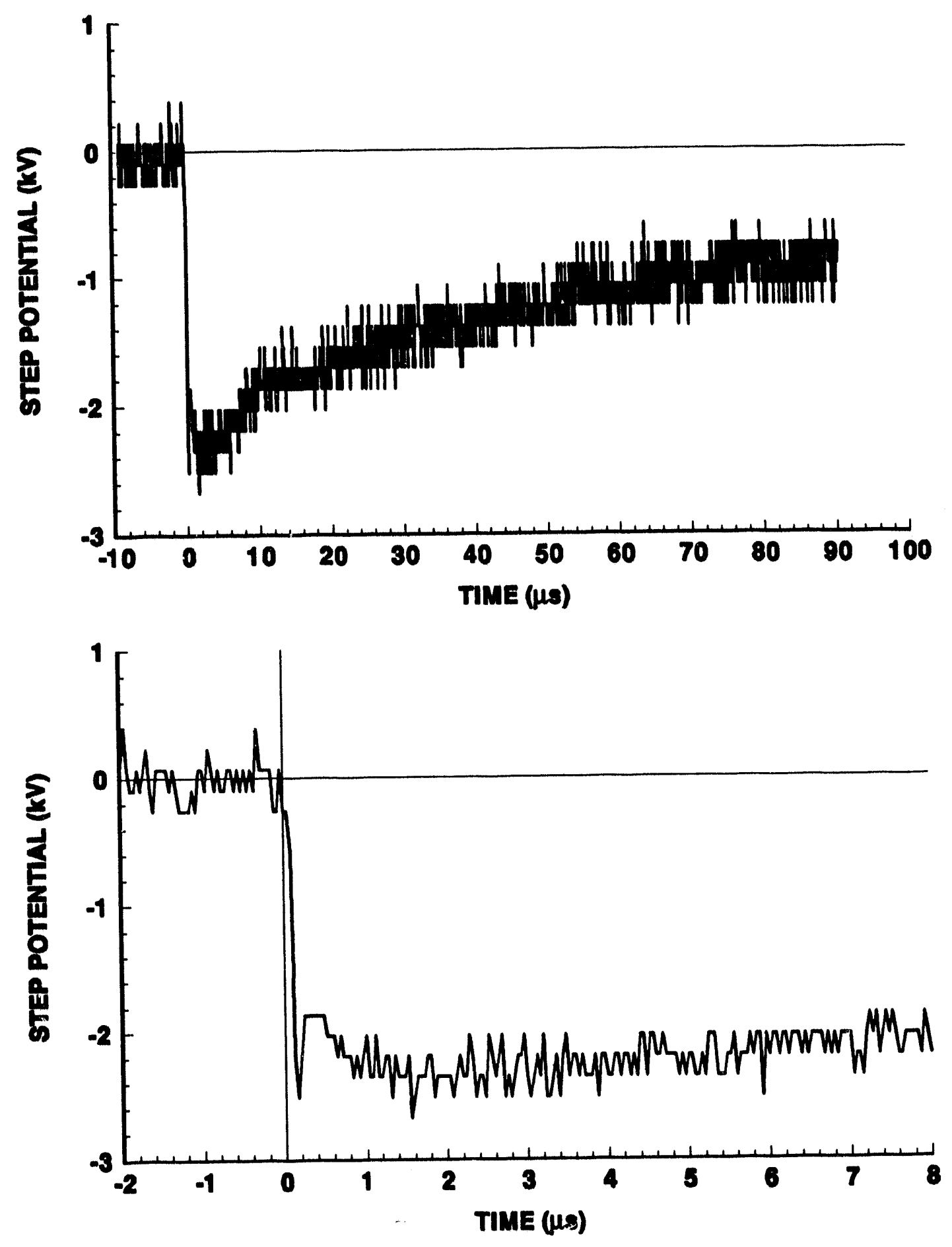


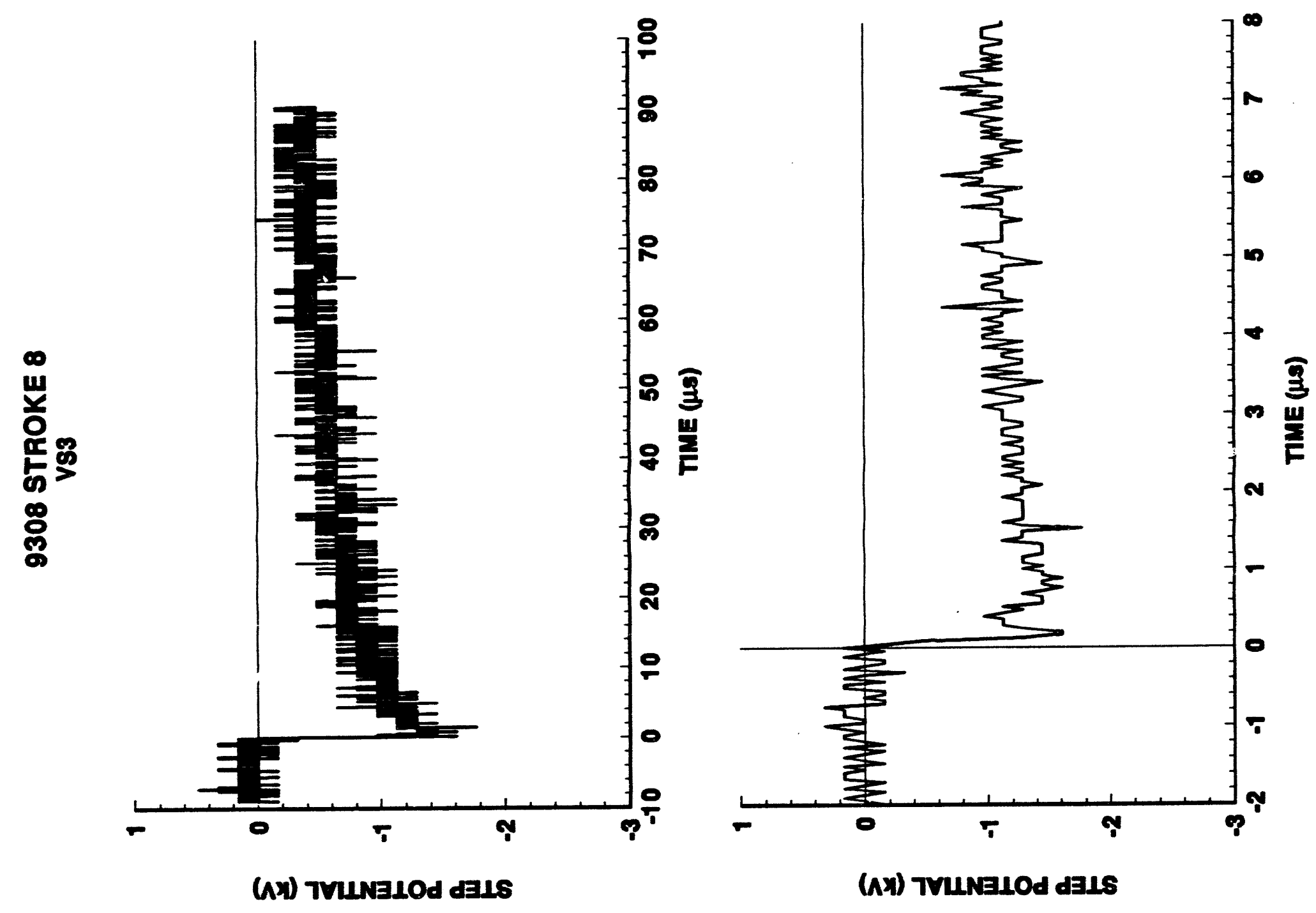




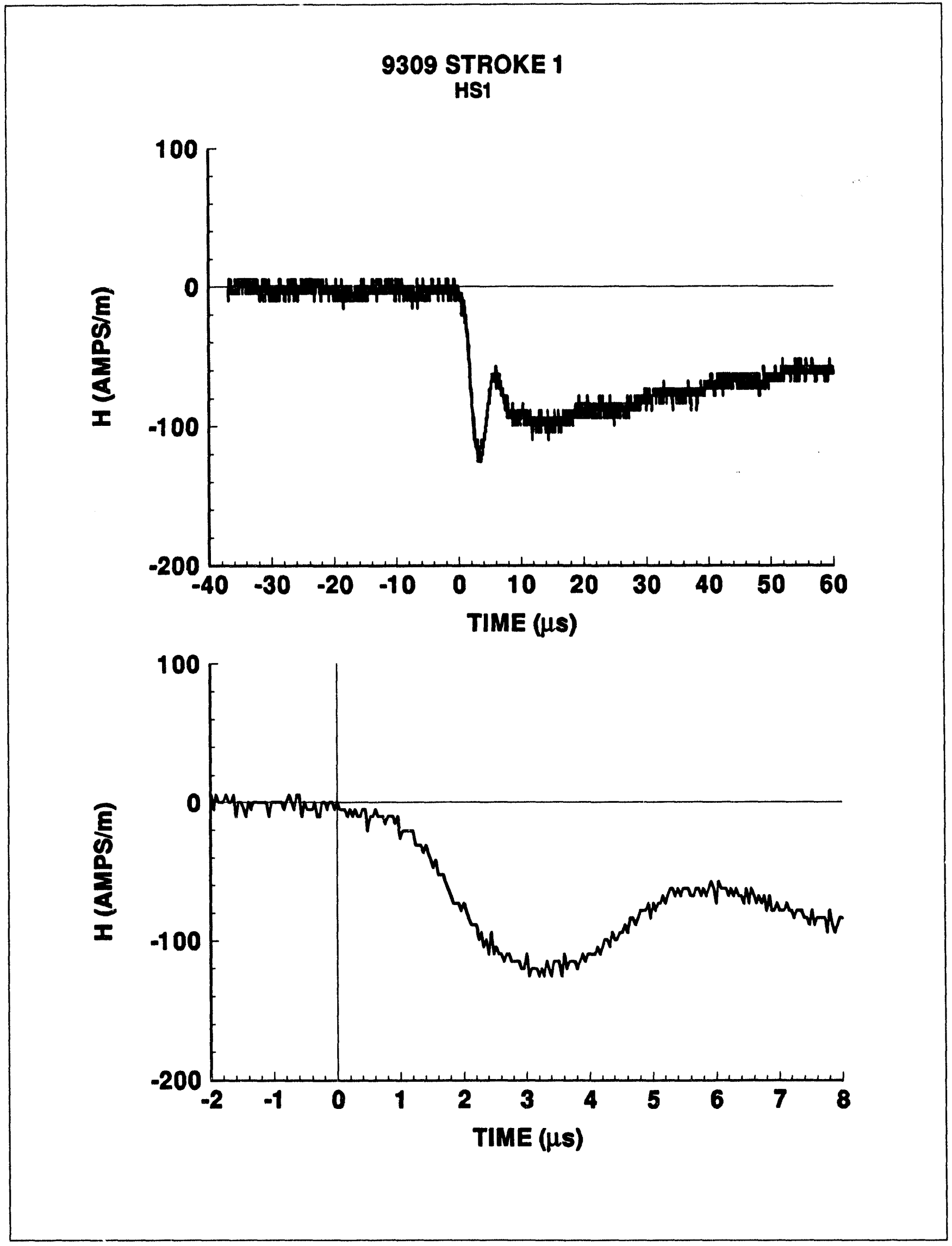




\section{STROKE 2 \\ HS1}
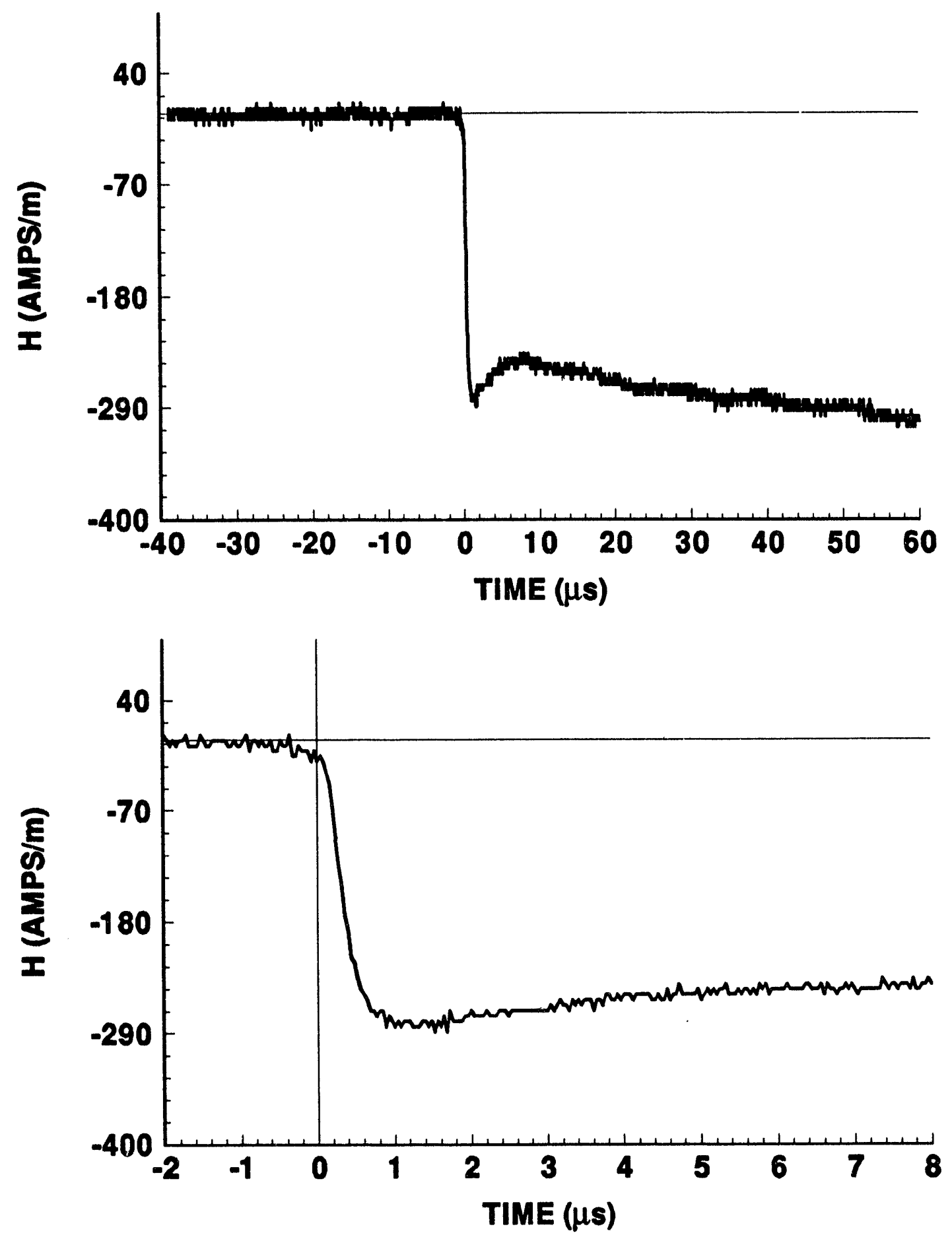


\section{STROKE 3}

HS1
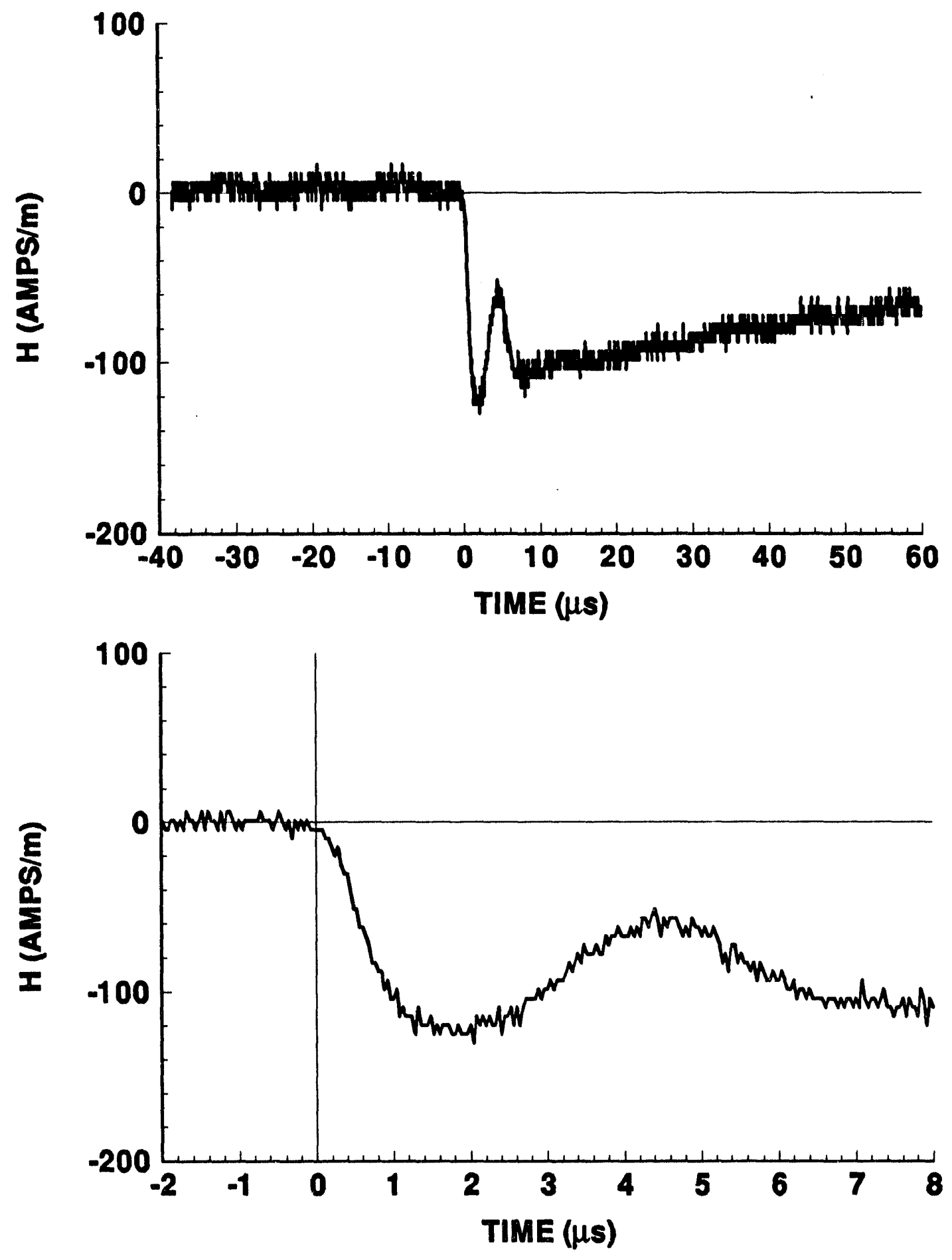


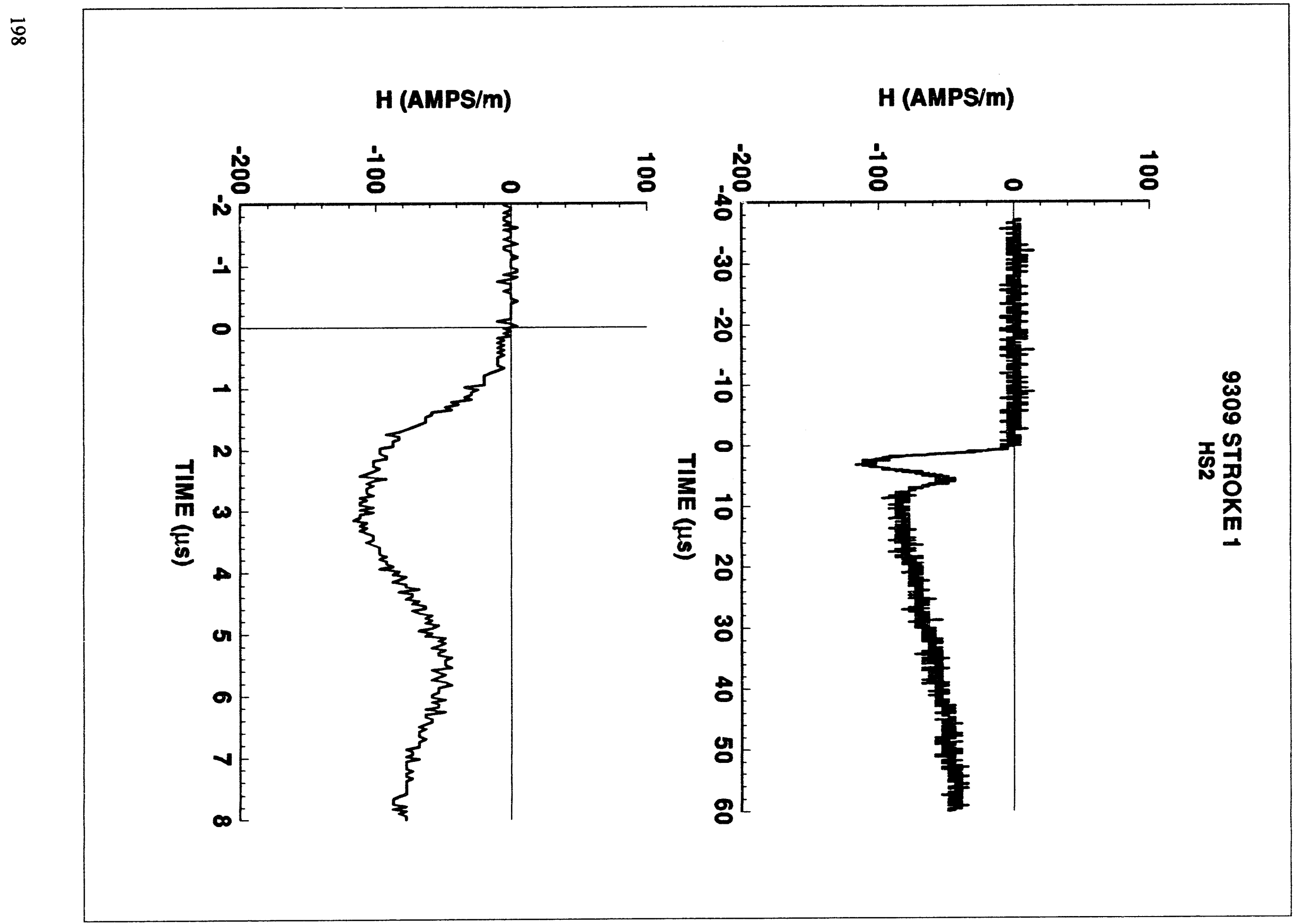




\section{STROKE 2}

HS2
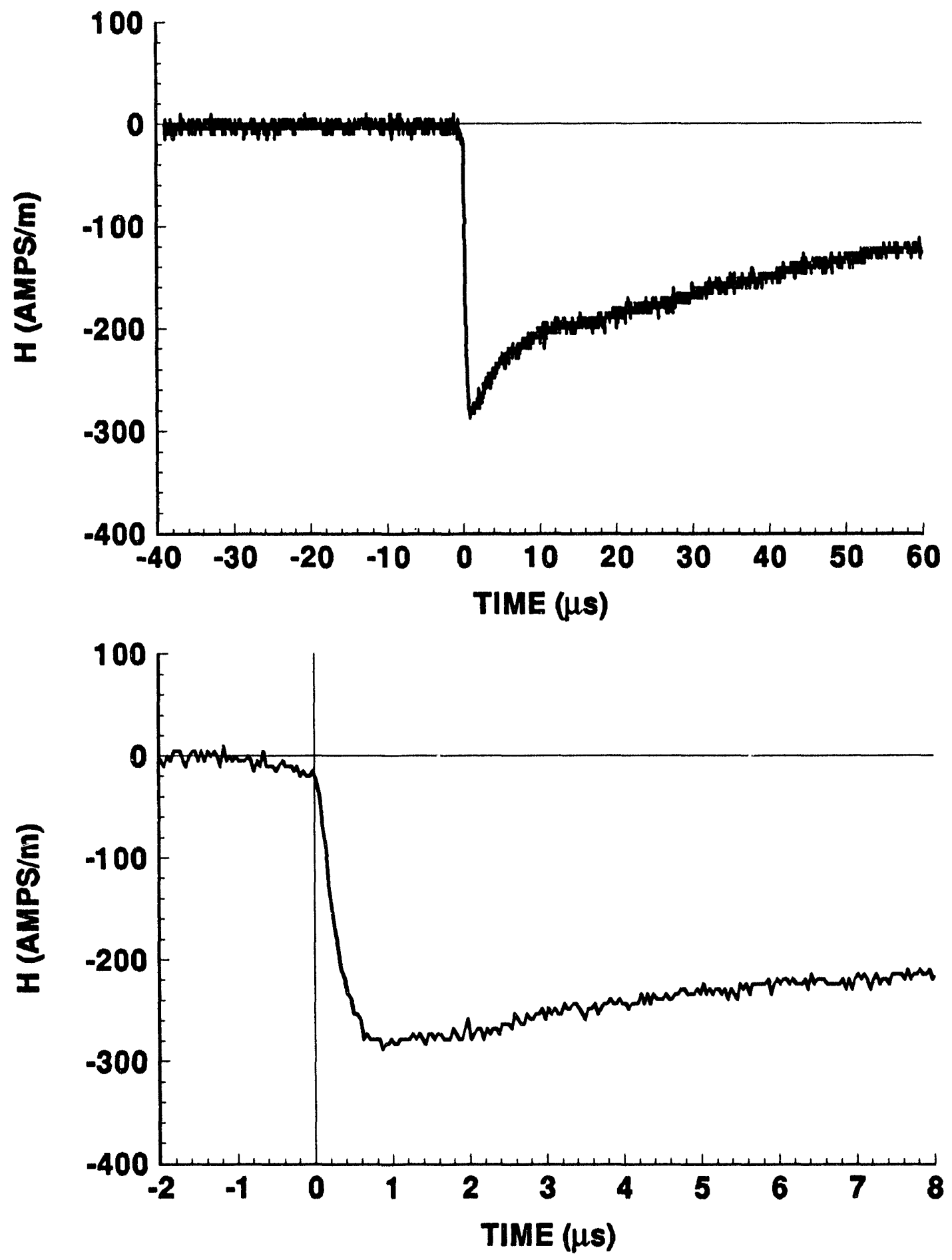


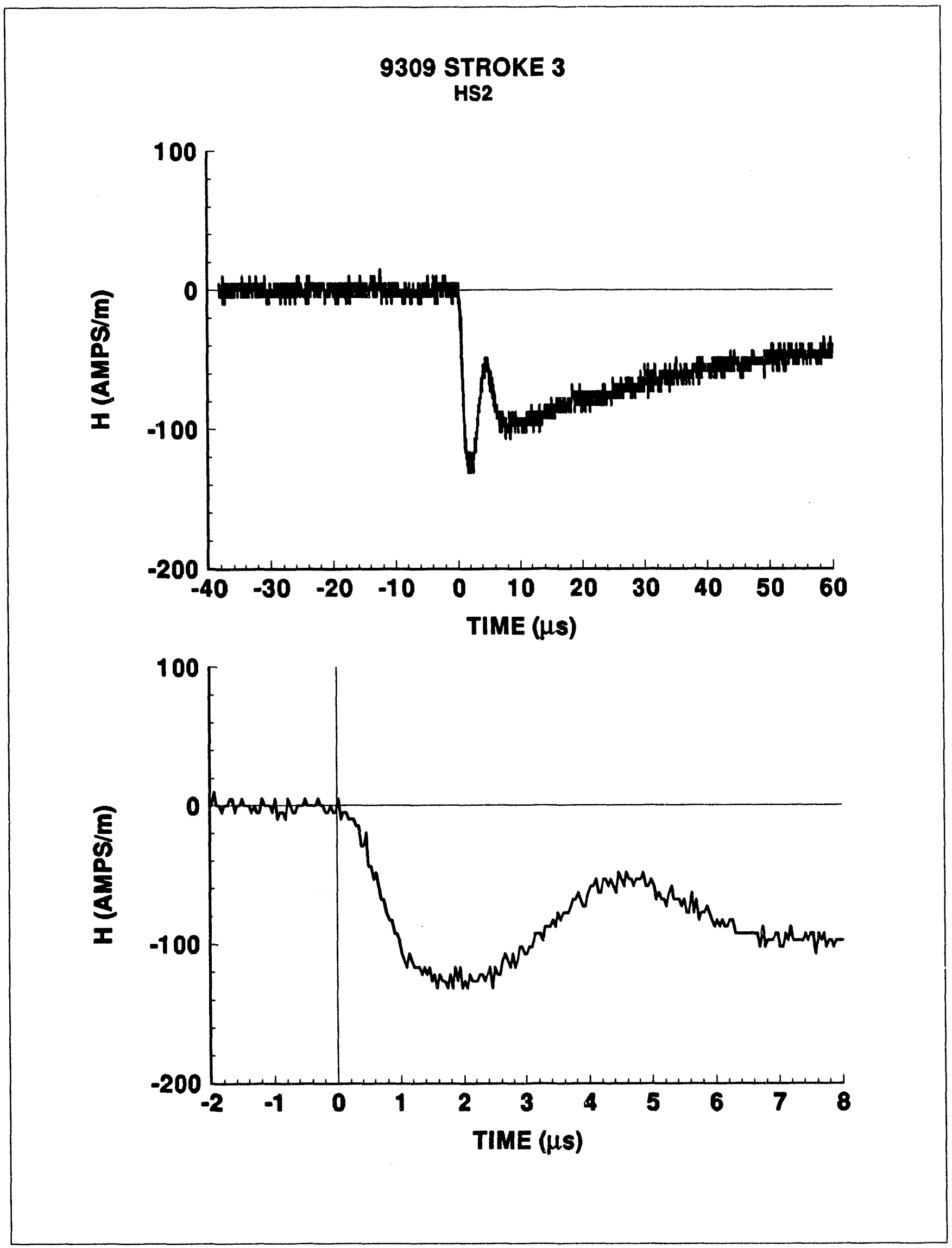




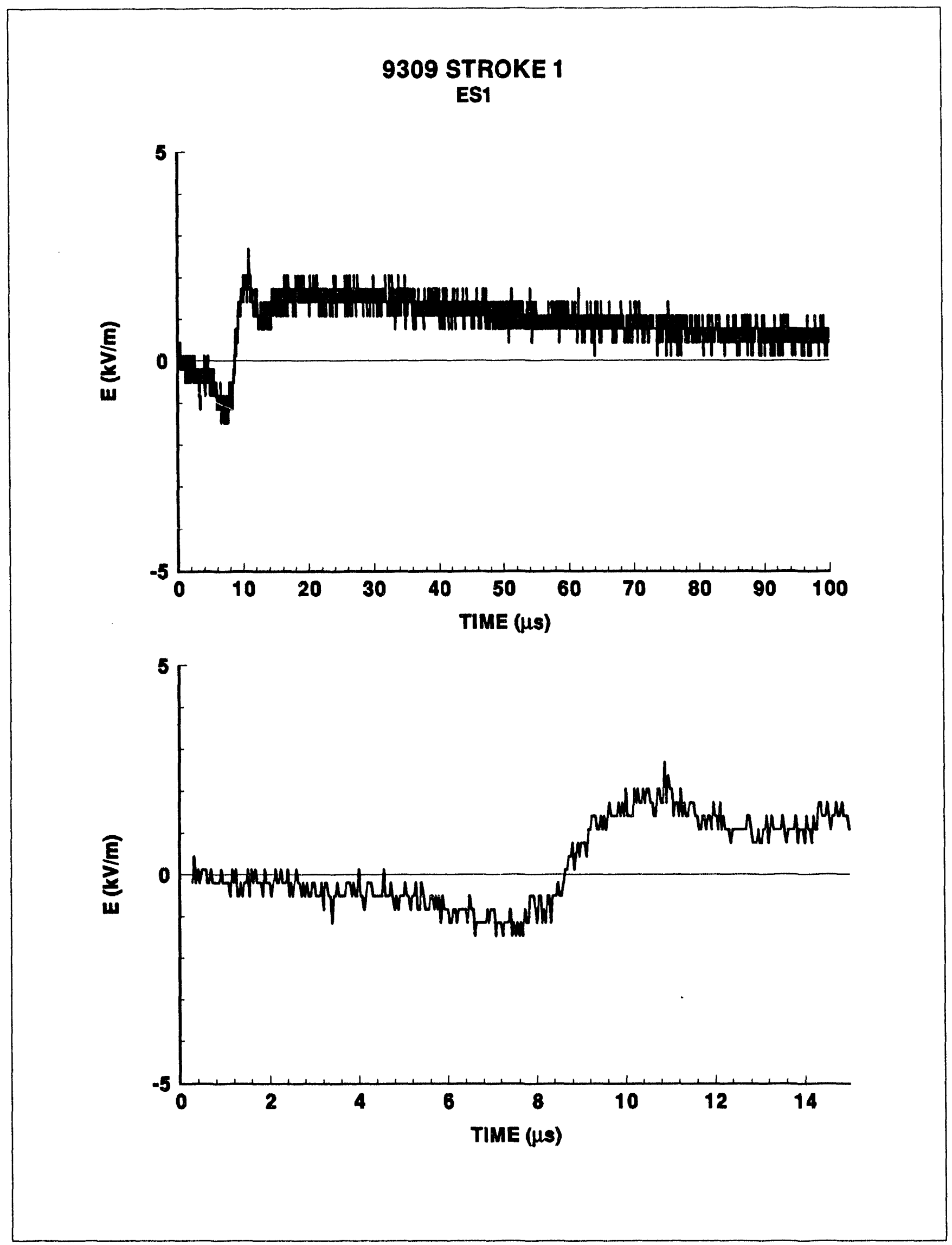




\section{STROKE 2 \\ ES1}
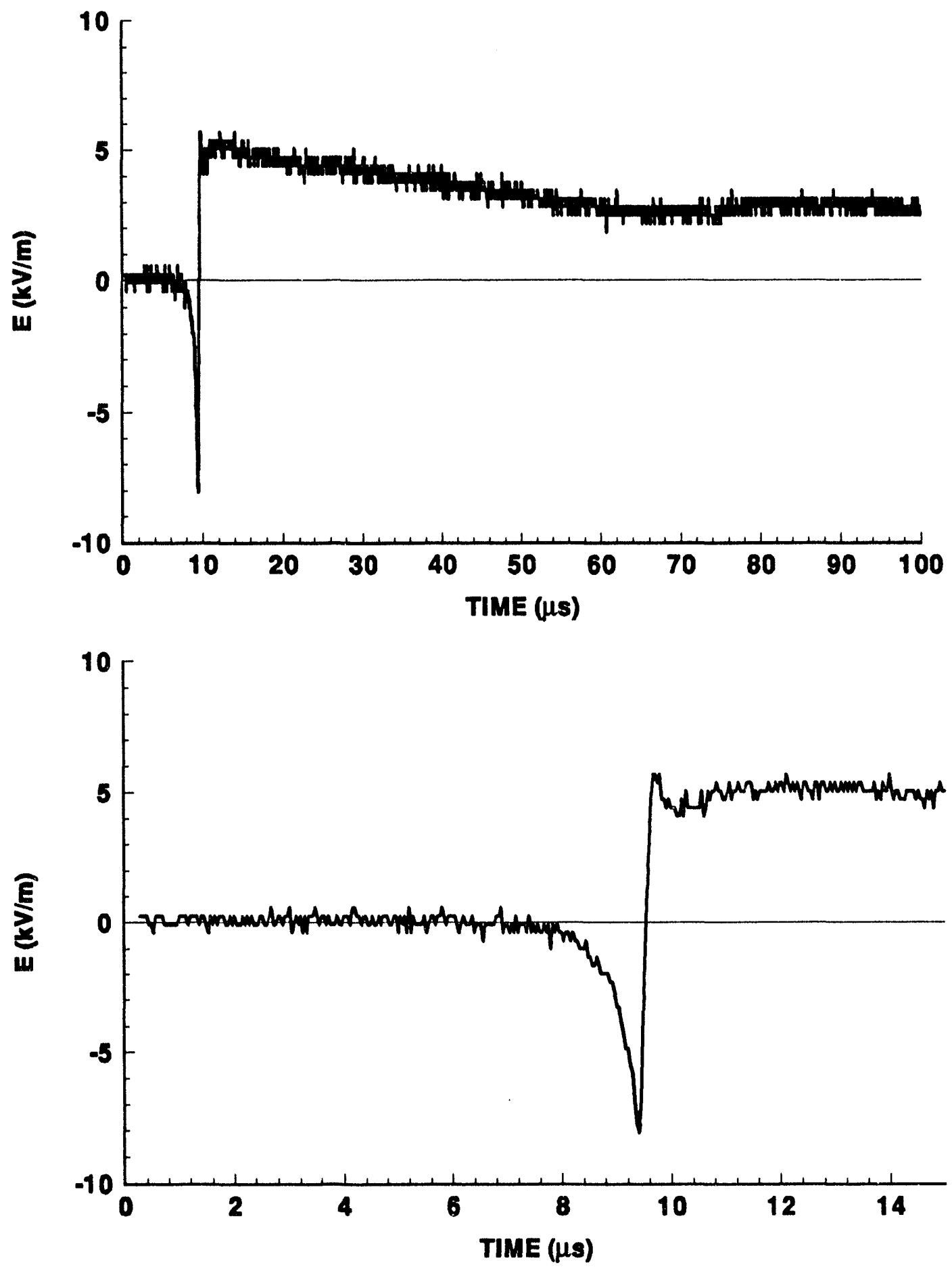


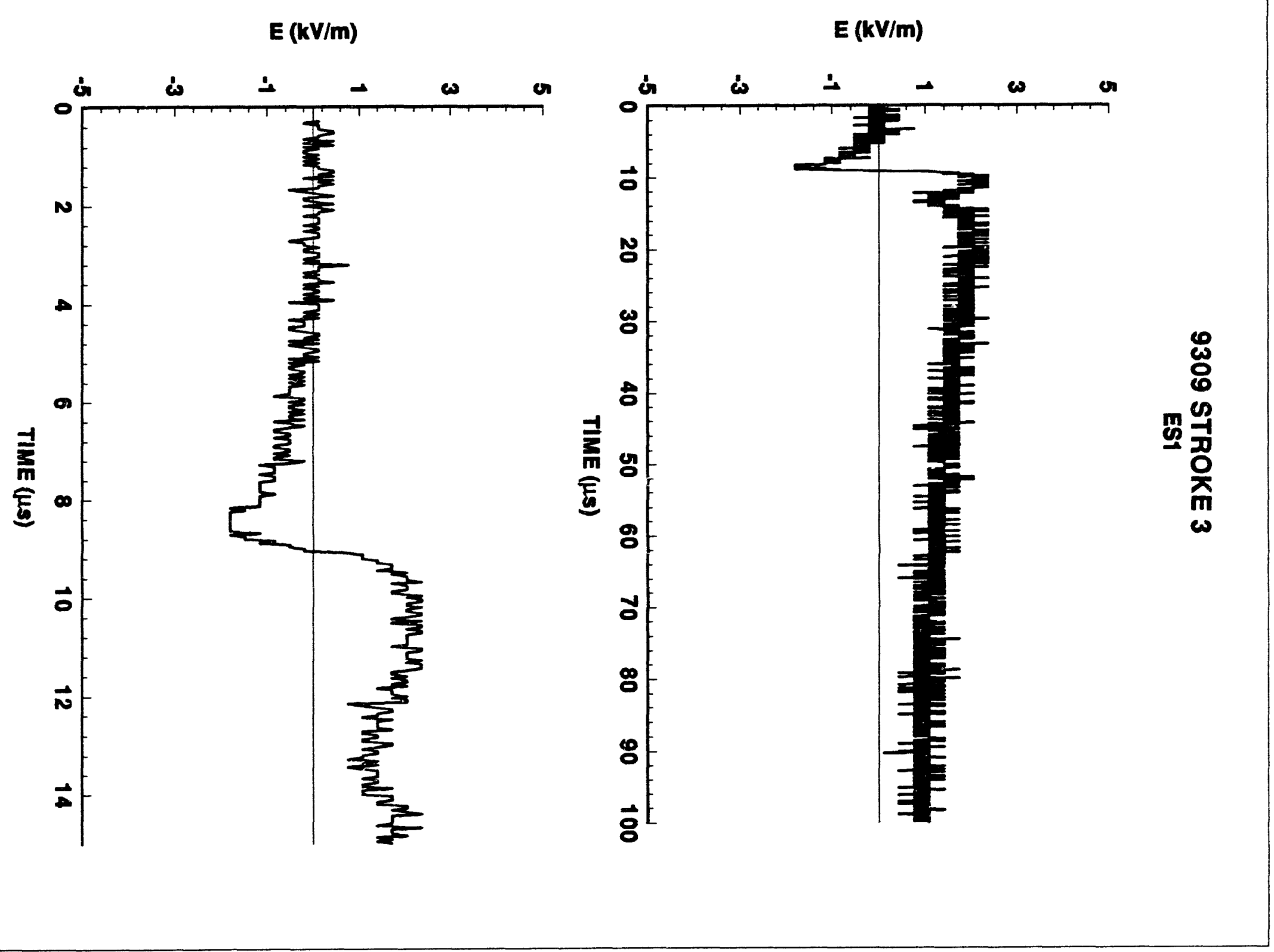




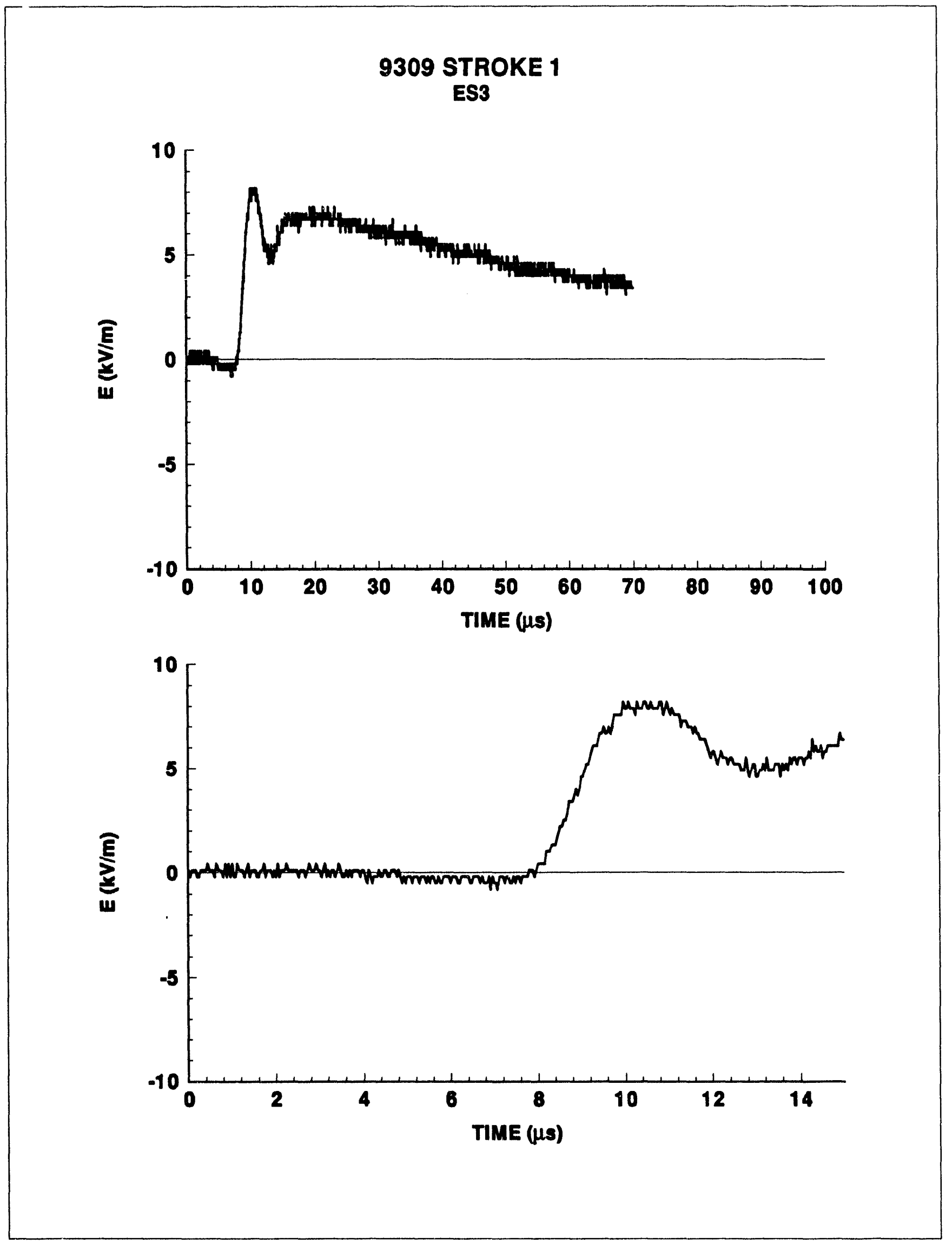




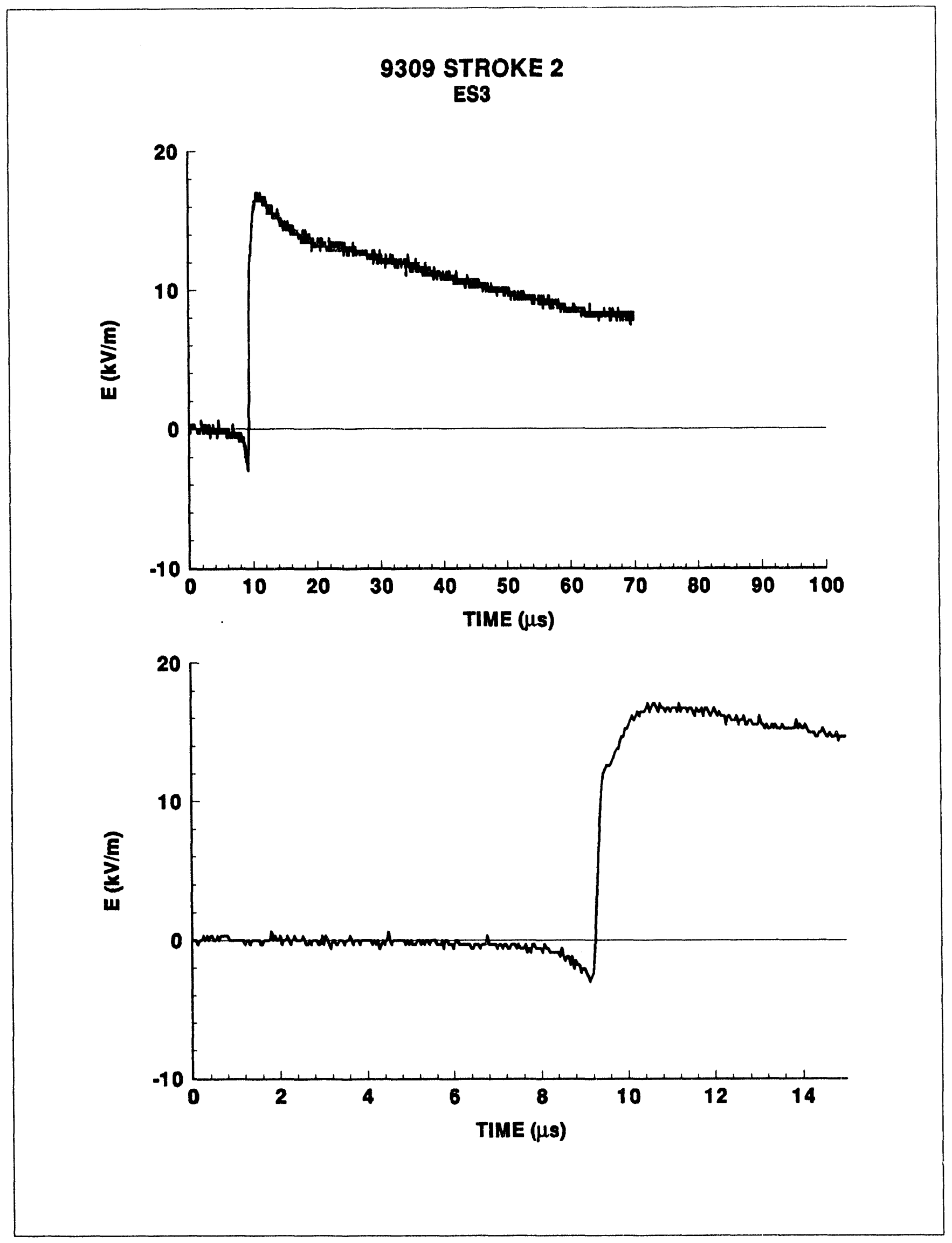




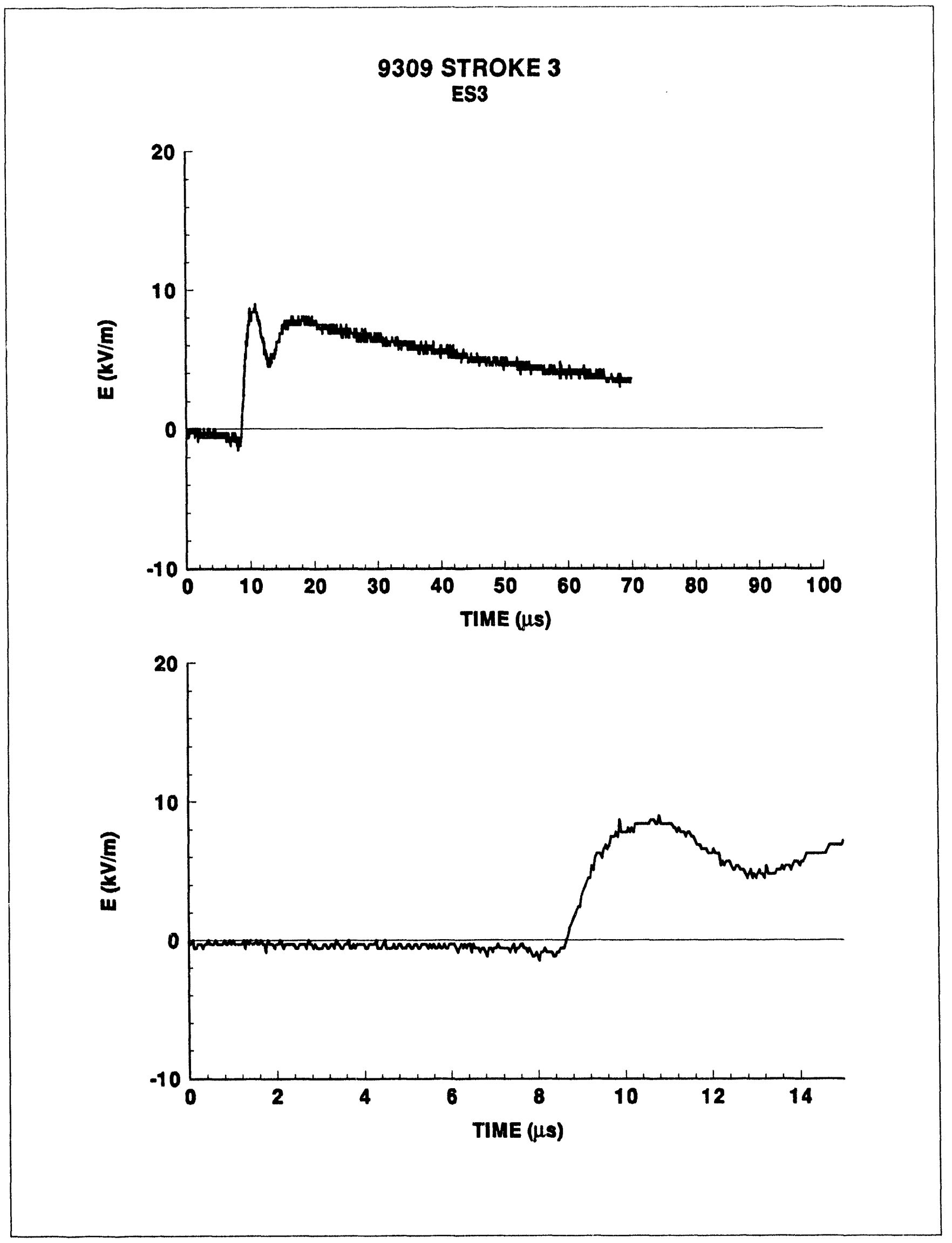




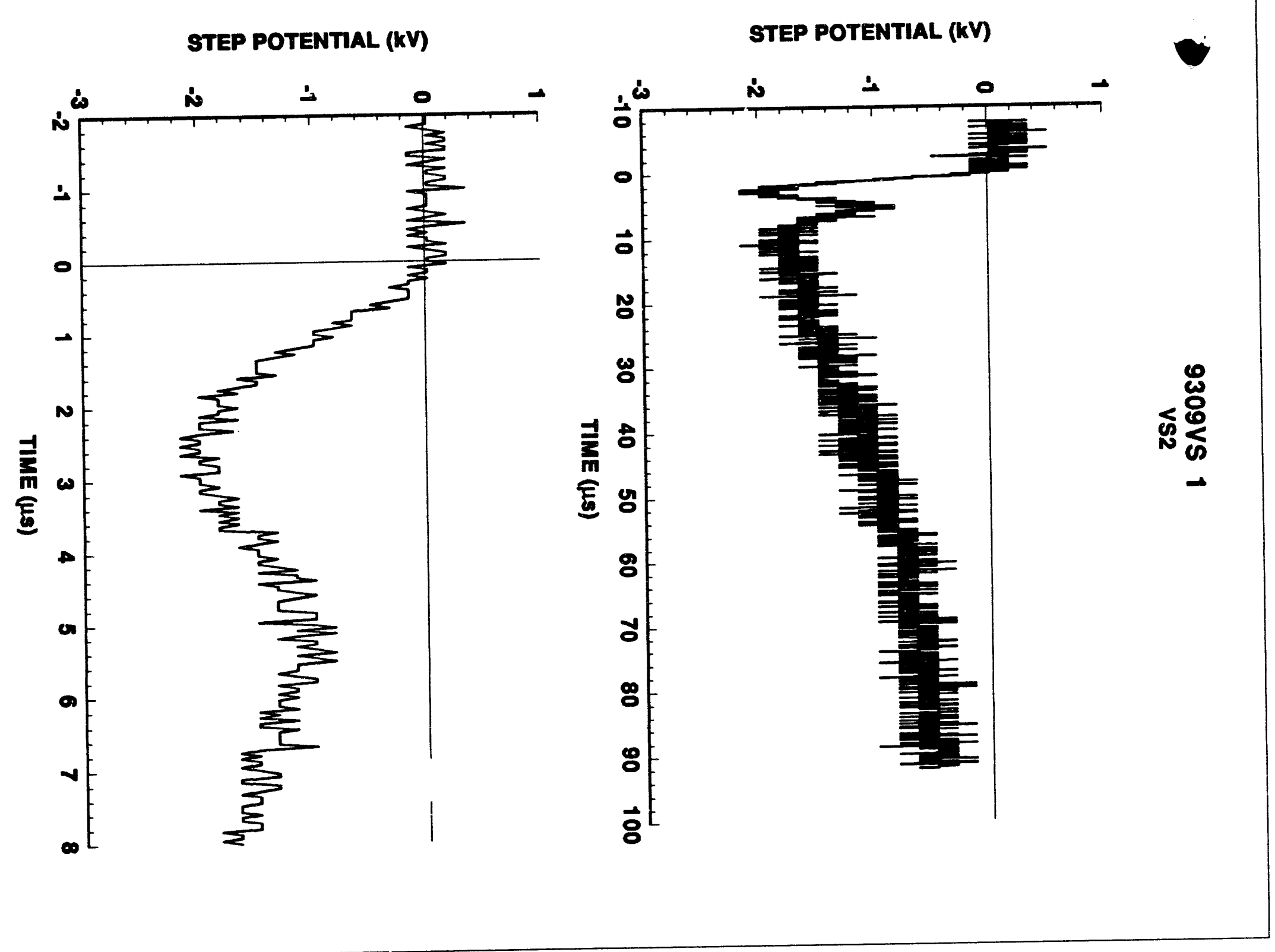




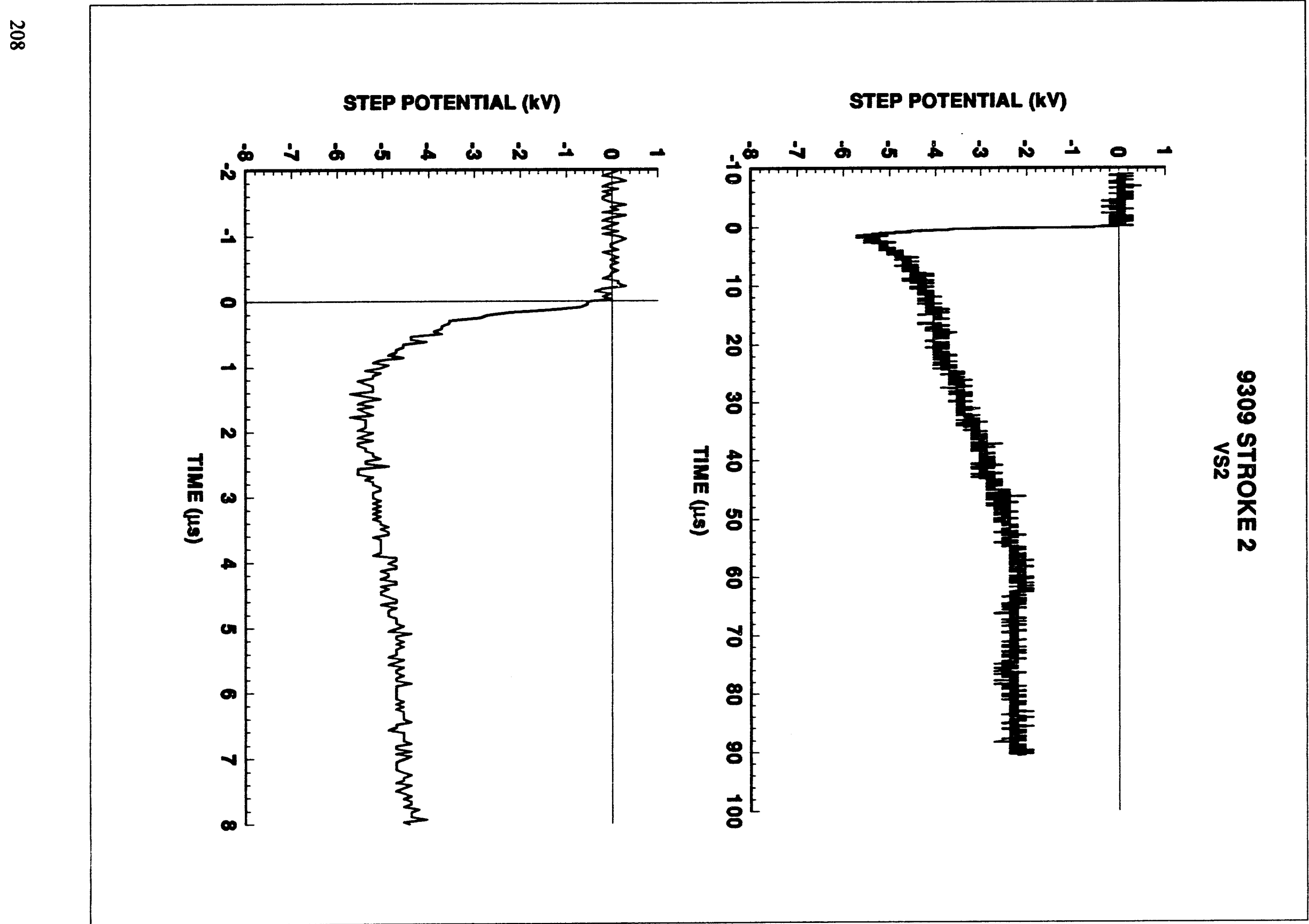


STEP POTENTIAL (kV)

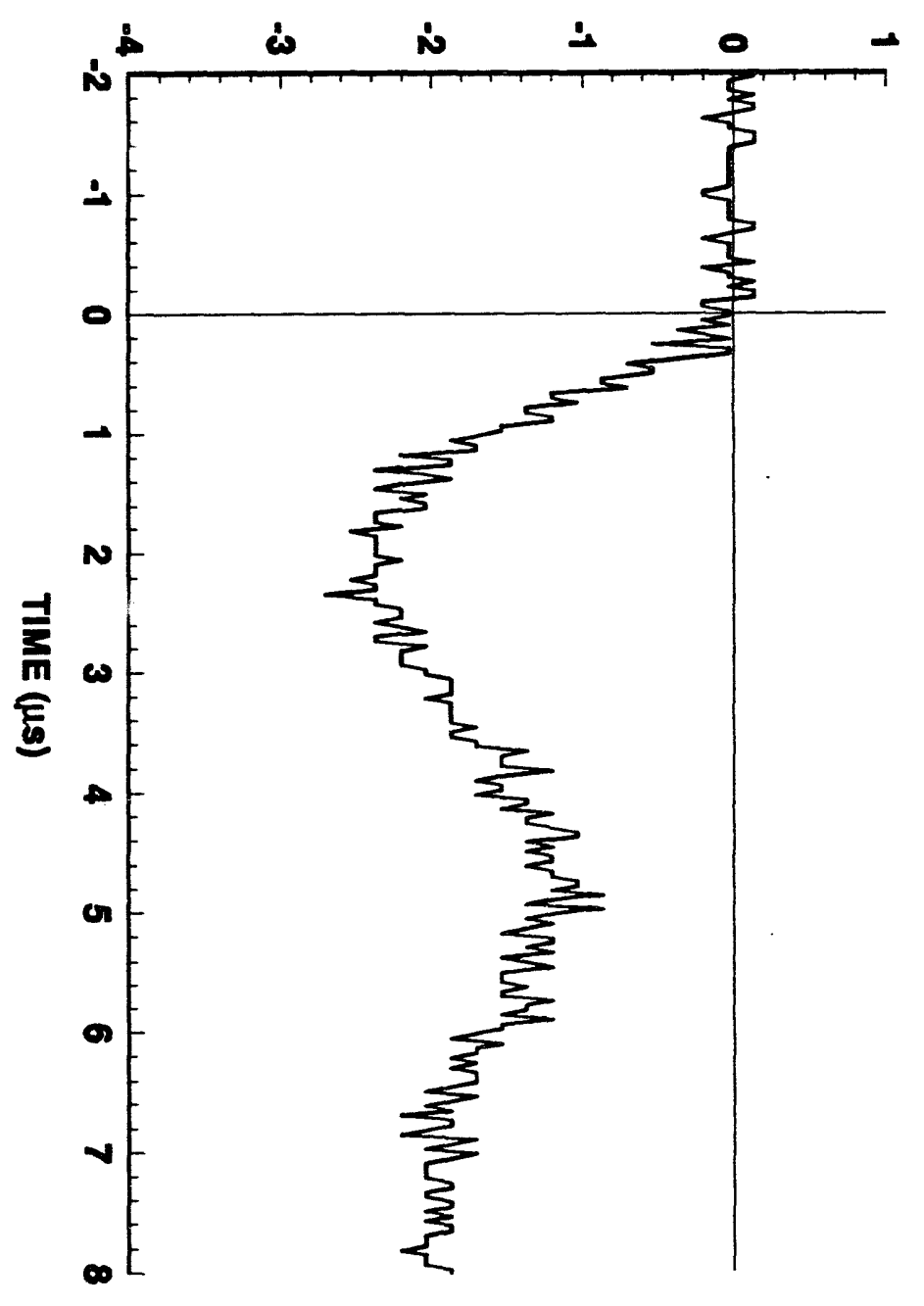

STEP POTENTIAL (kV)

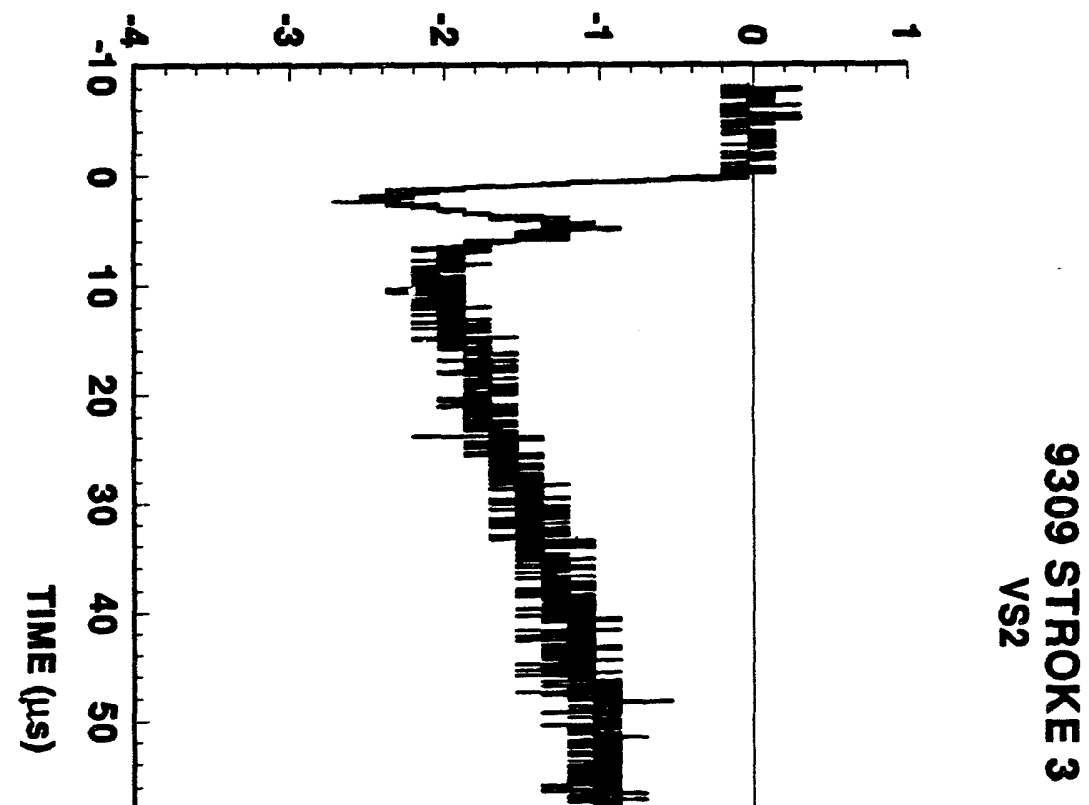


9309 STROKE 1

VS3
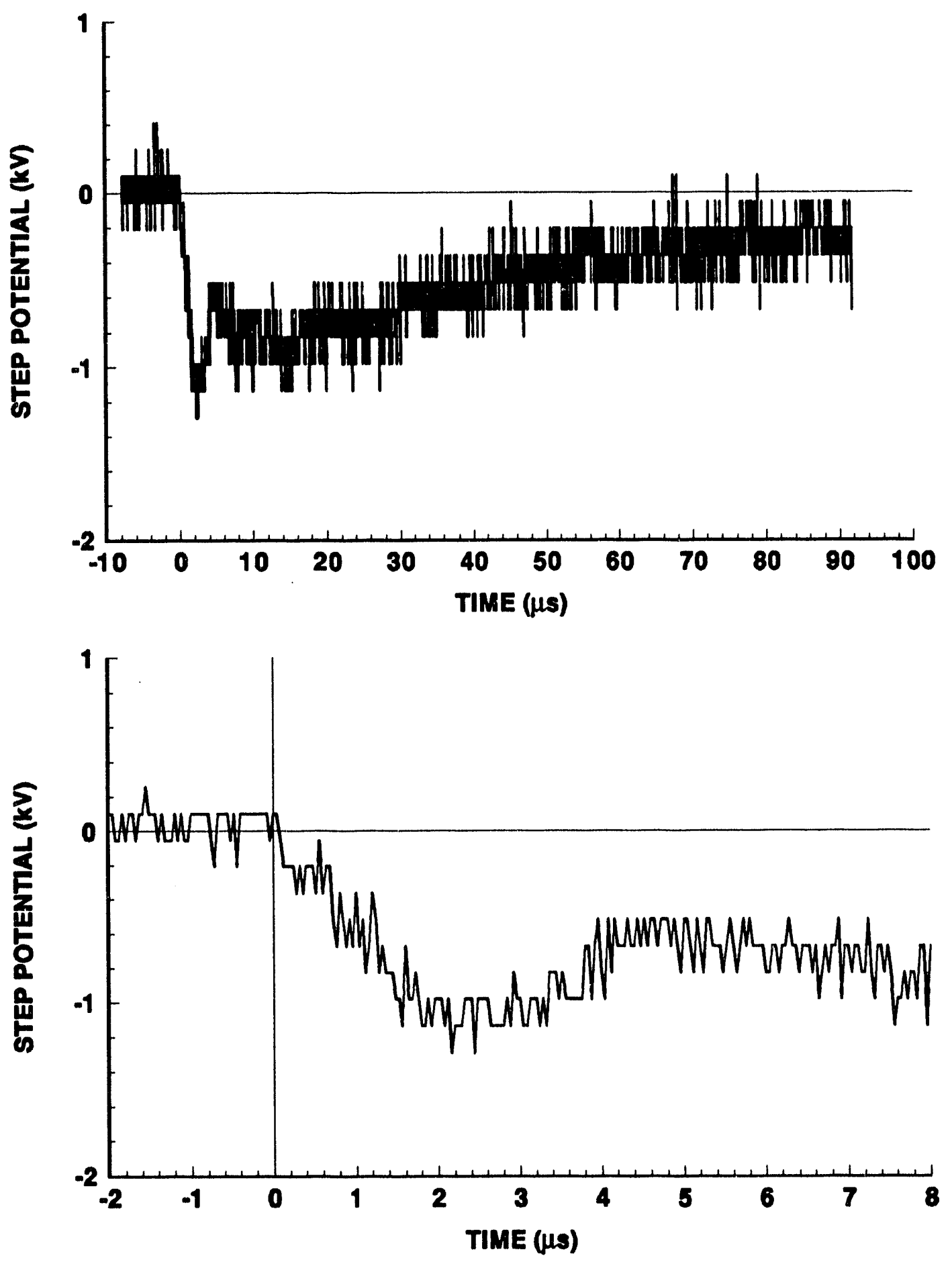


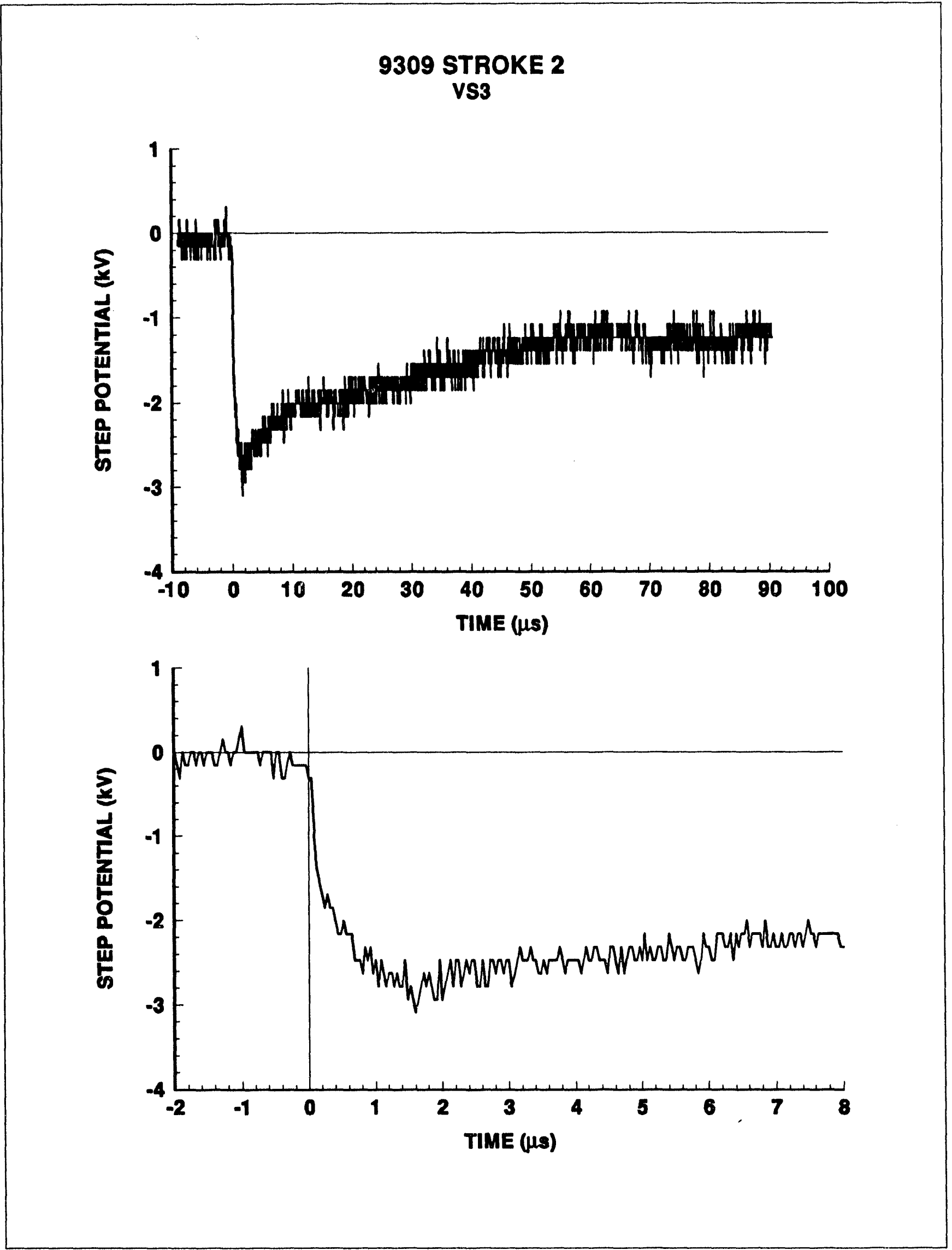


STEP POTENTIAL (kV)

STEP POTENTIAL (kV)
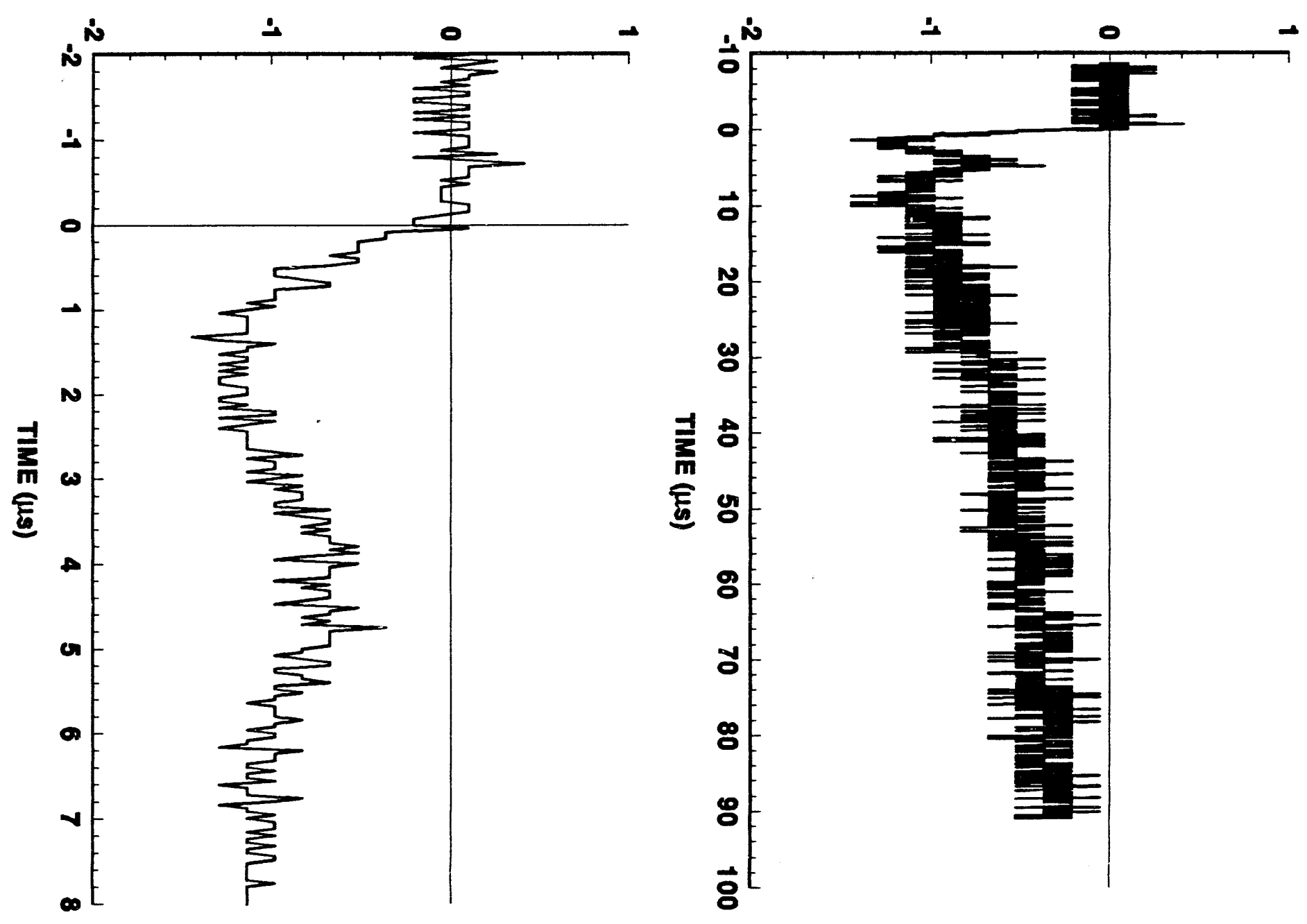


\section{3-12 STROKE 1}

INCIDENT CURRENT
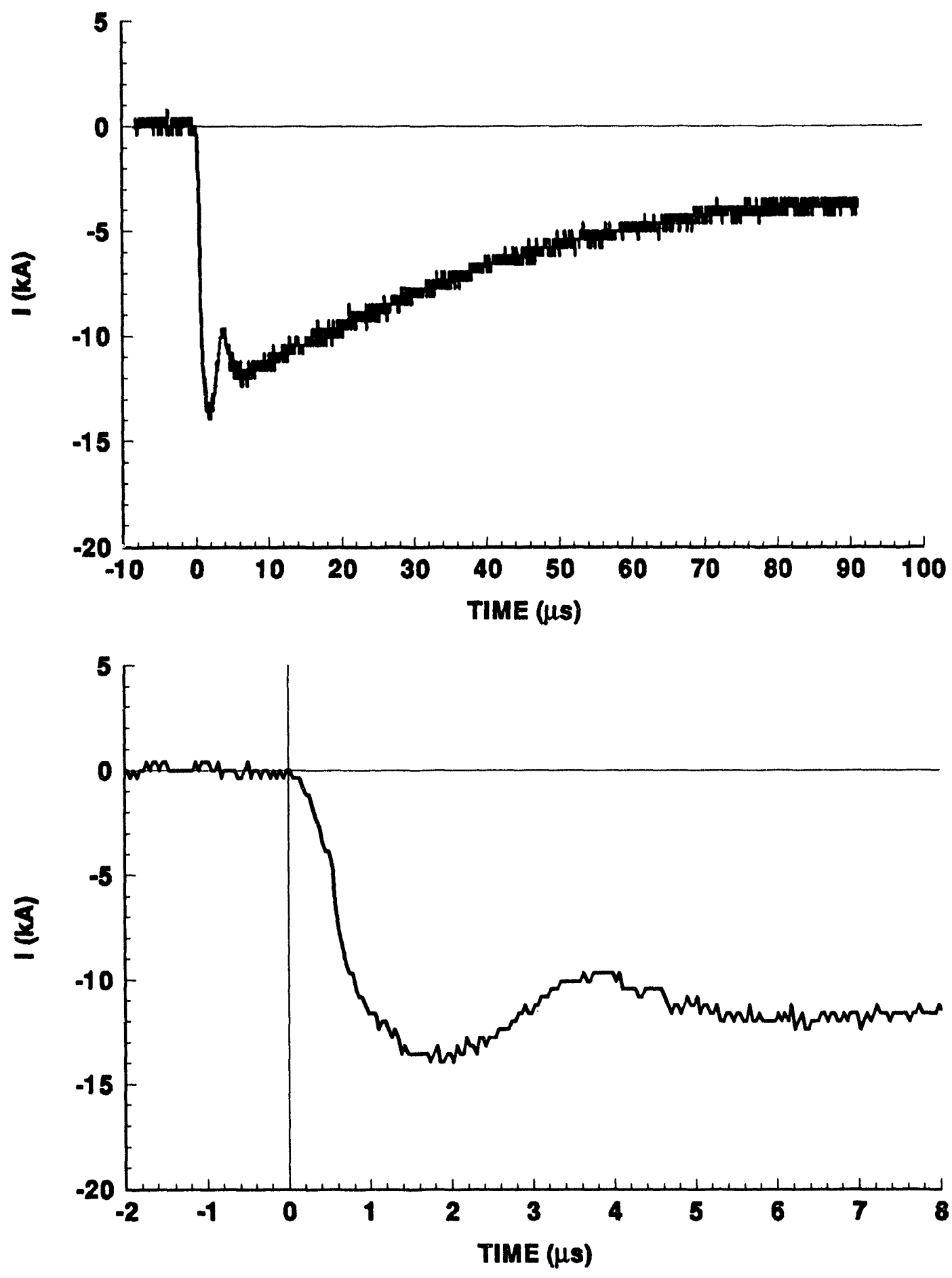


\section{3-12 STROKE 2}

INCIDENT CURRENT
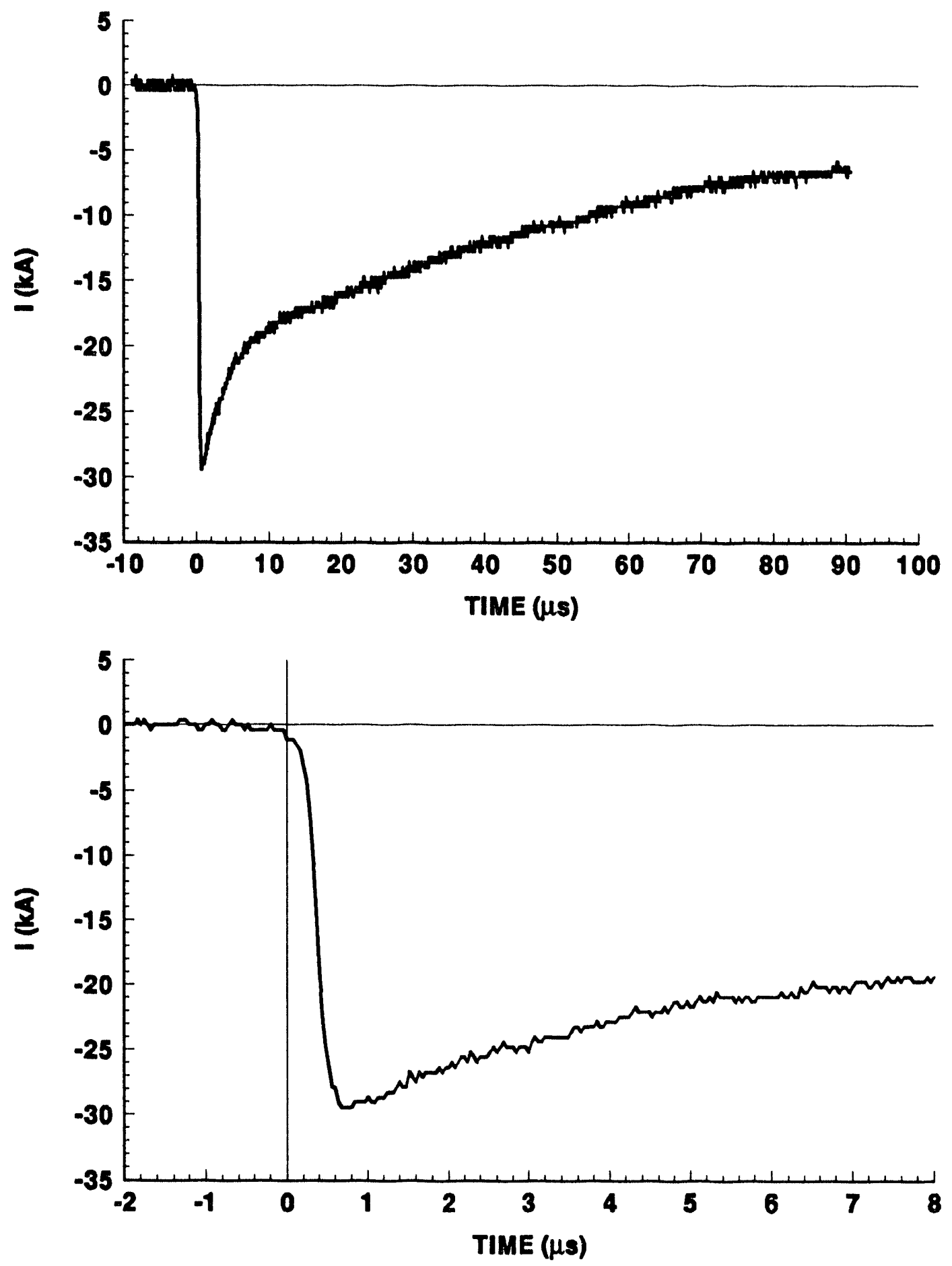


\section{3-12 STROKE 1}

HS1
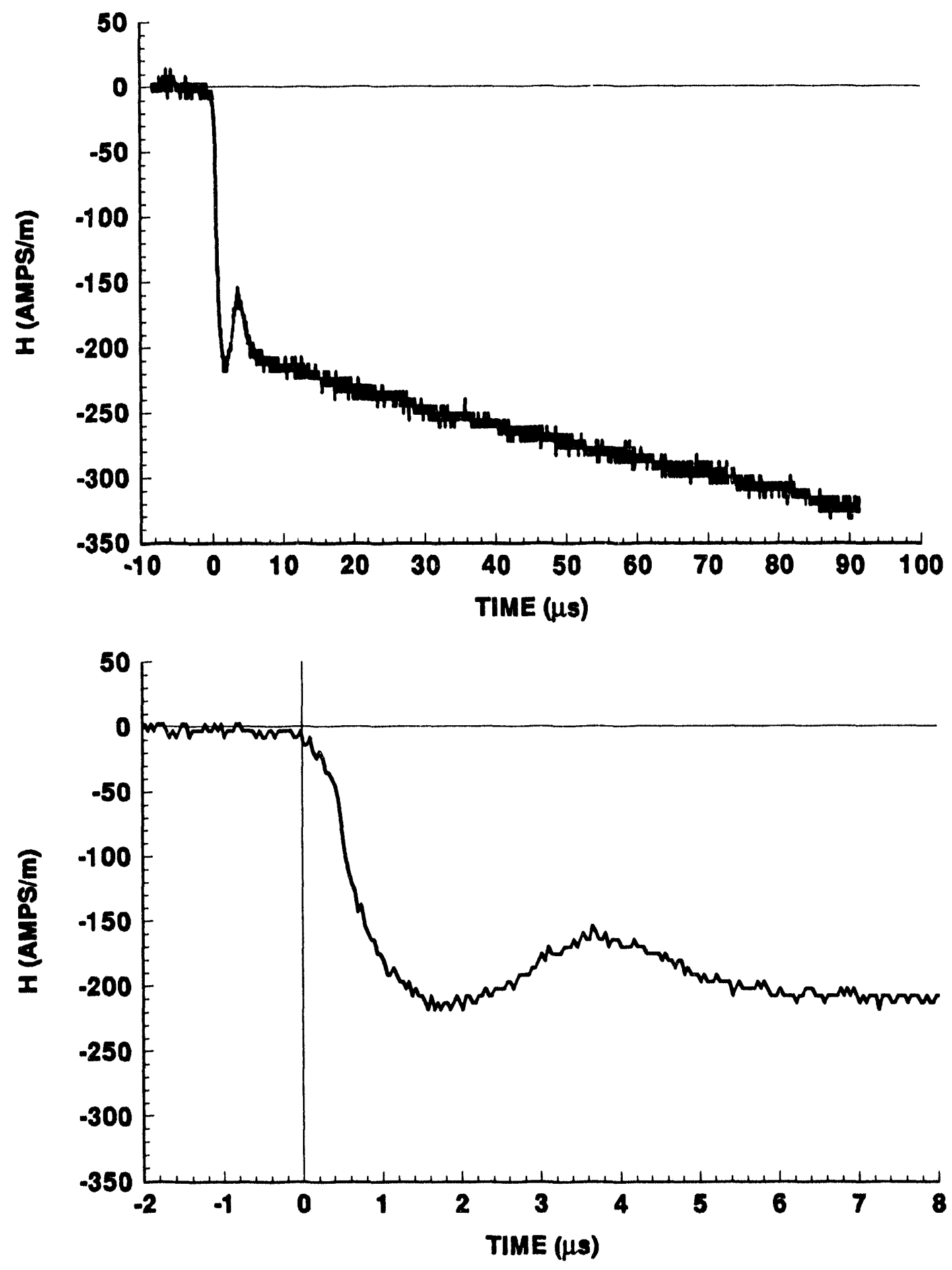


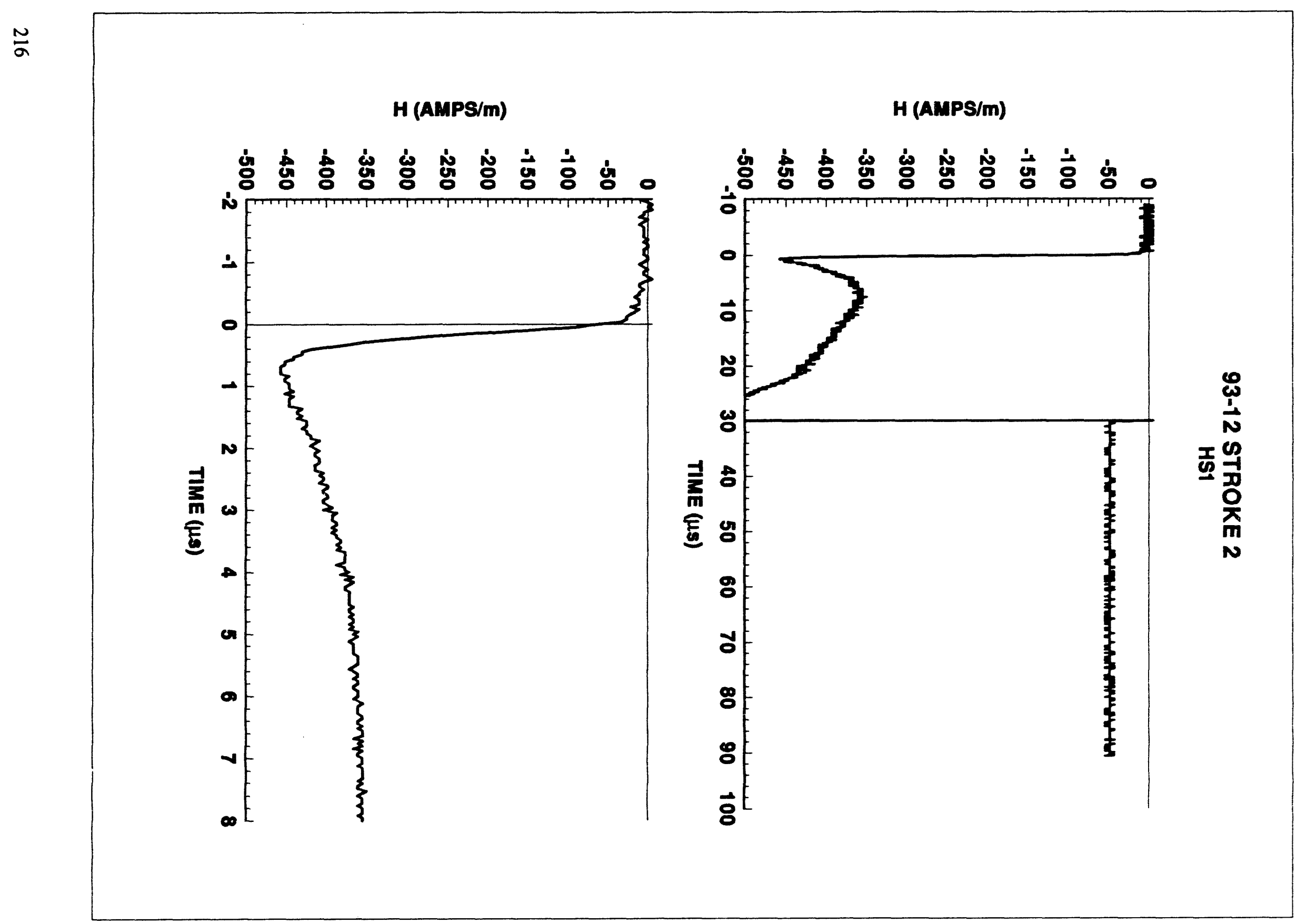




\section{3-12 STROKE 1}

HS4
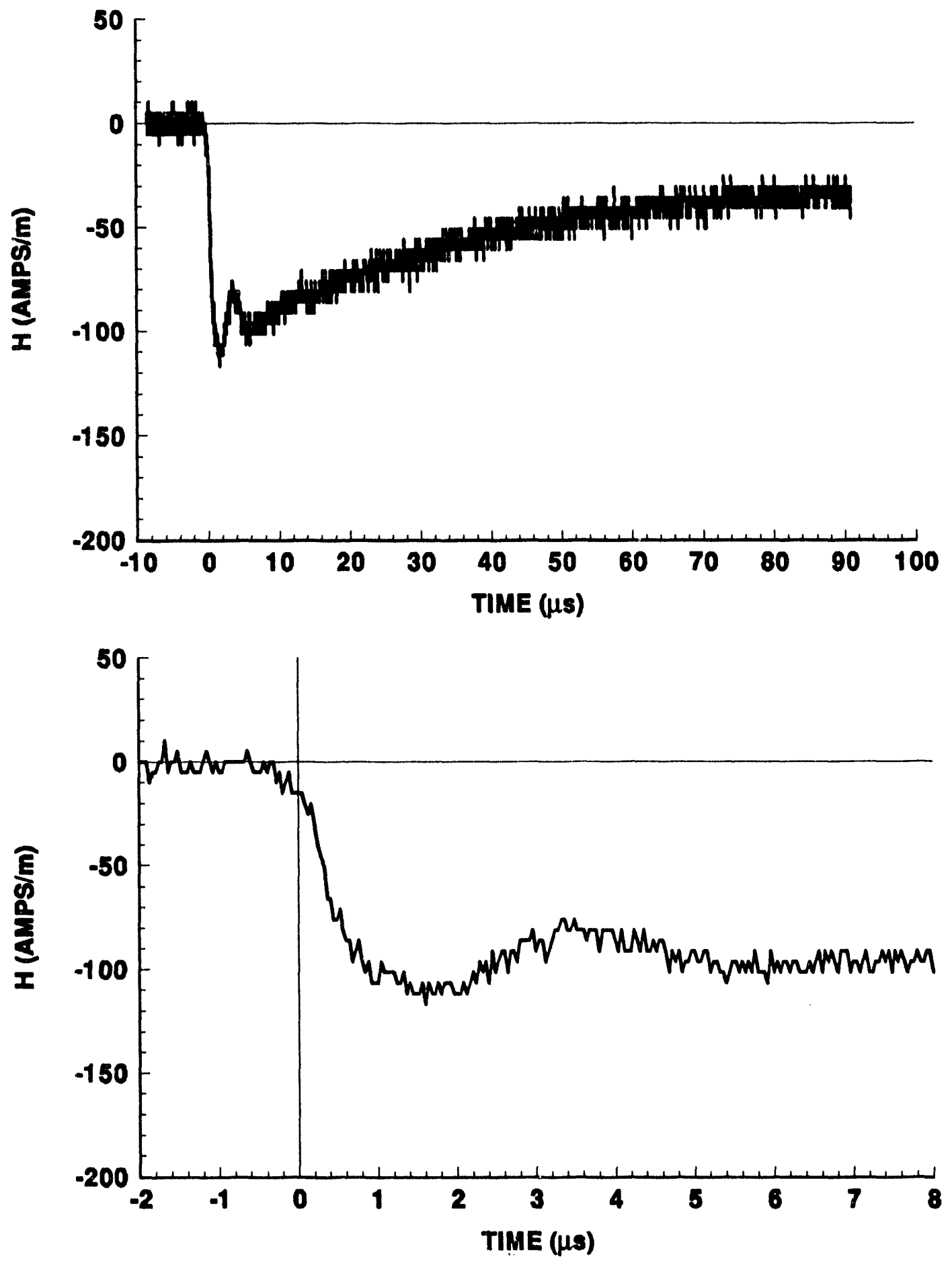


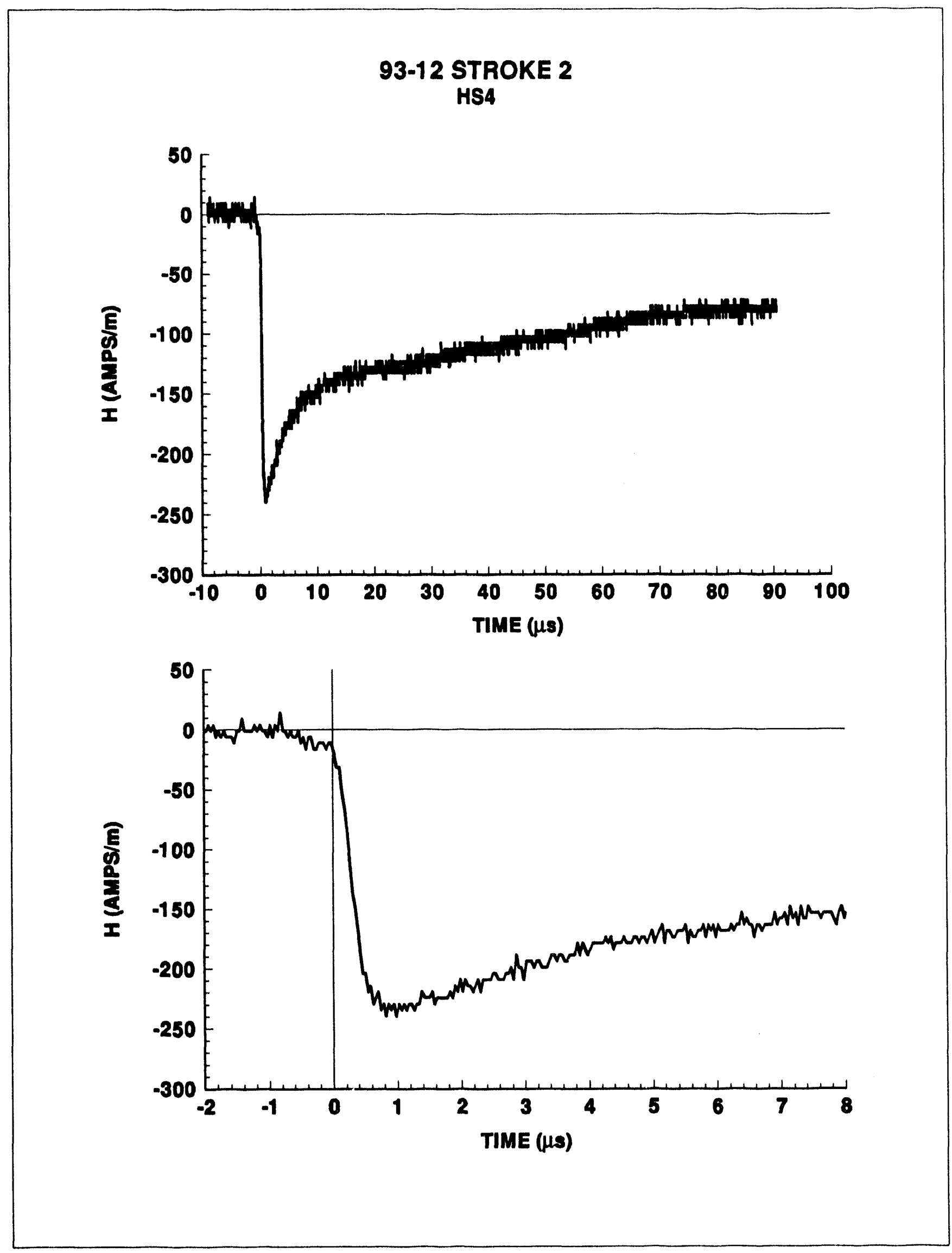




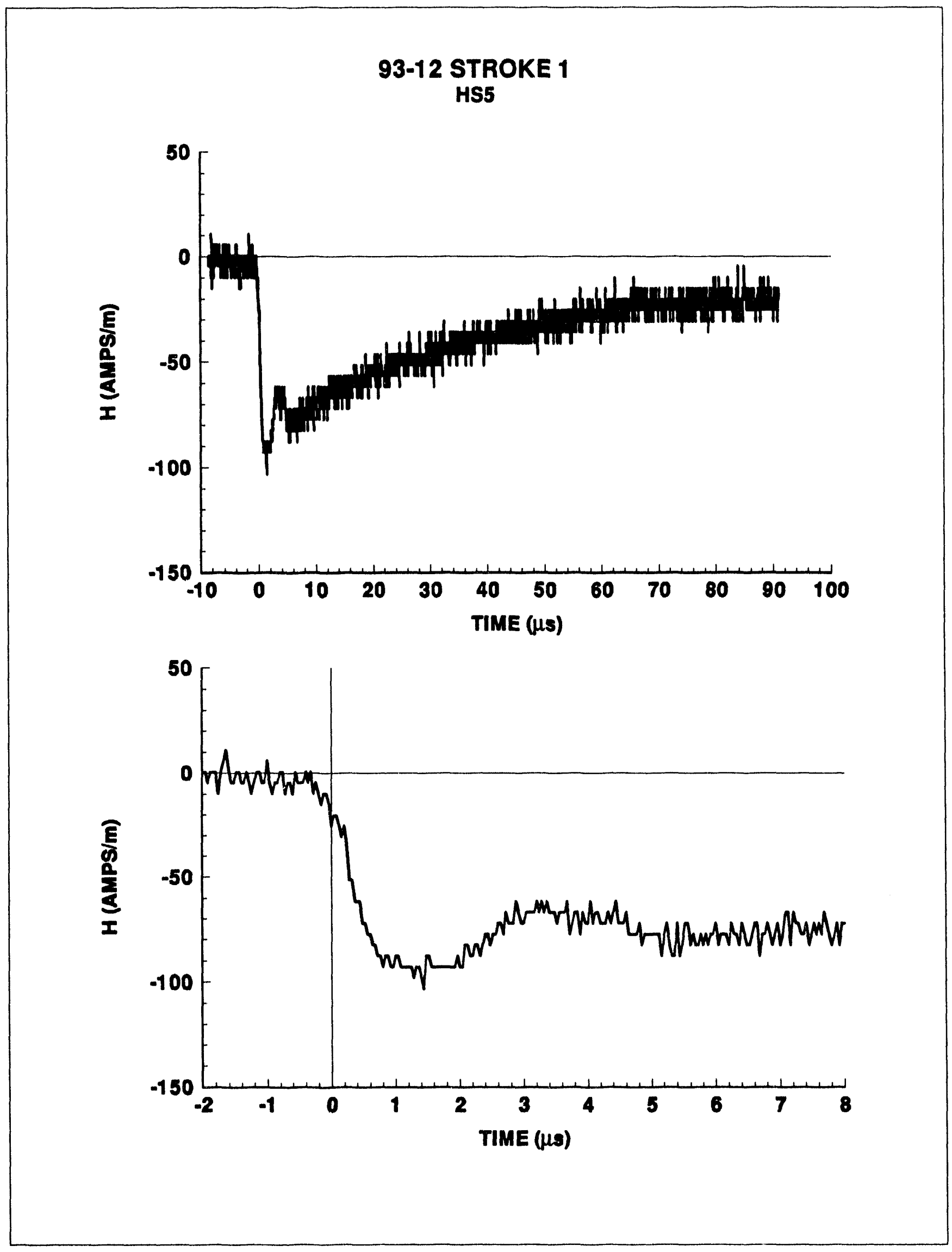




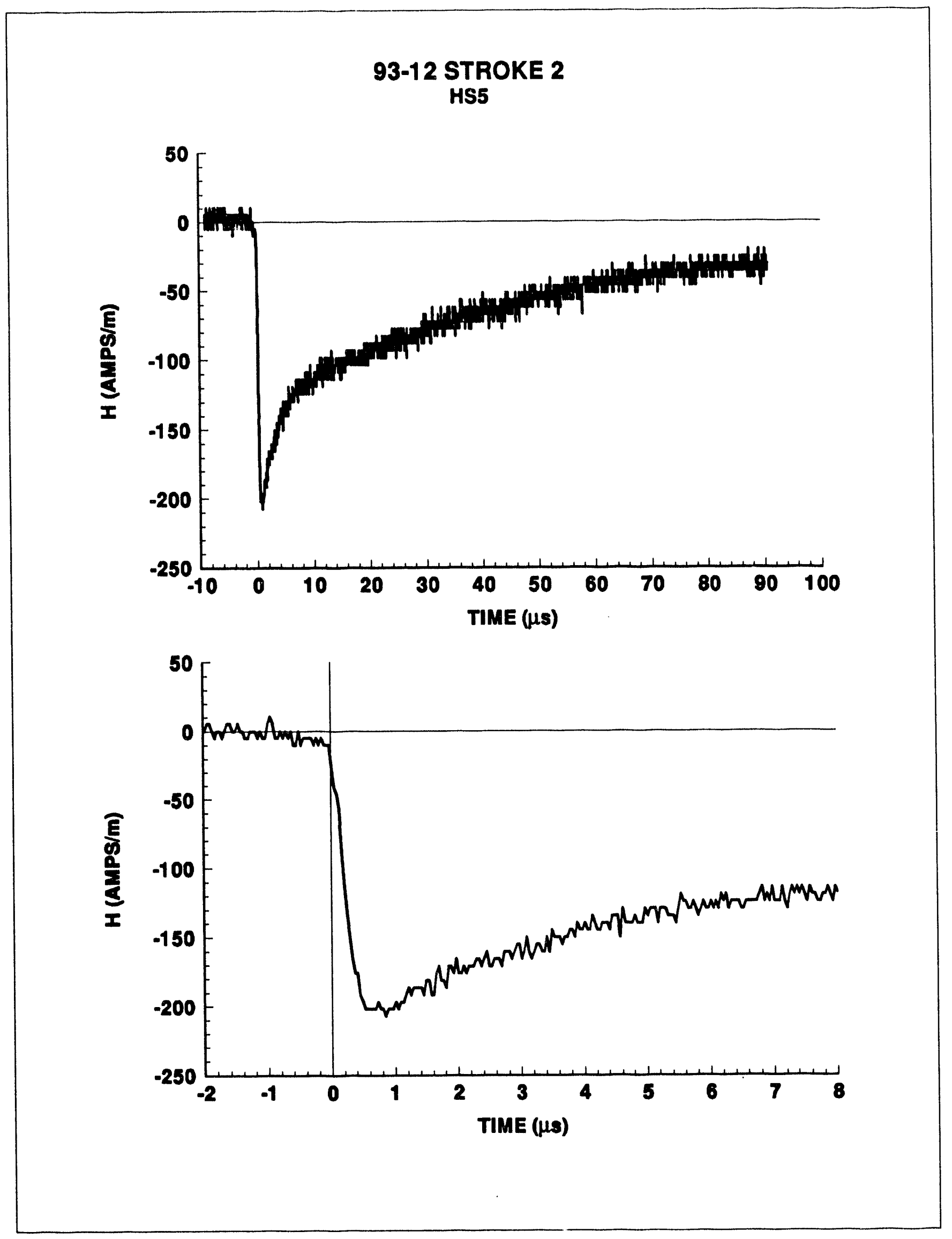




\section{STROKE 1}

ES1
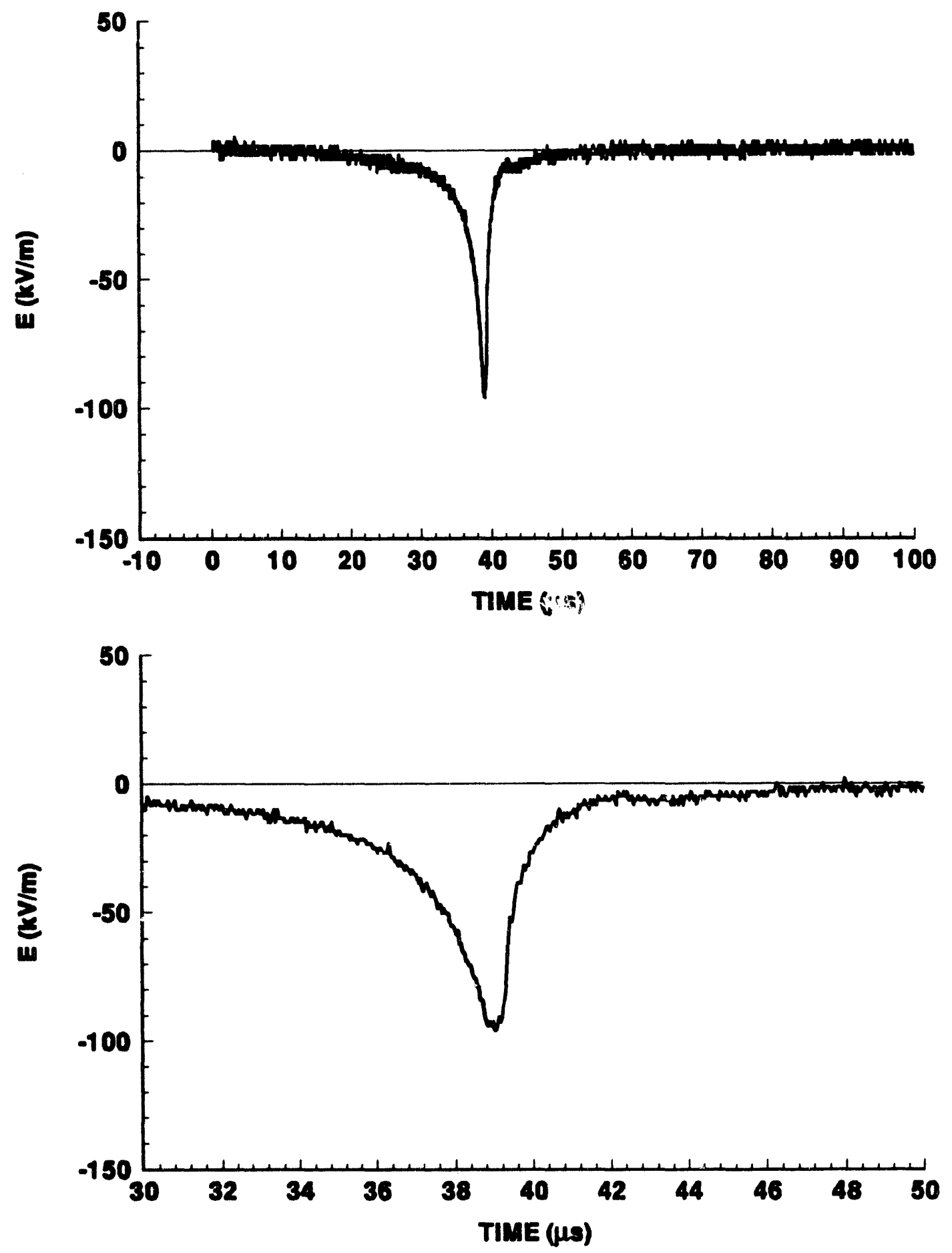


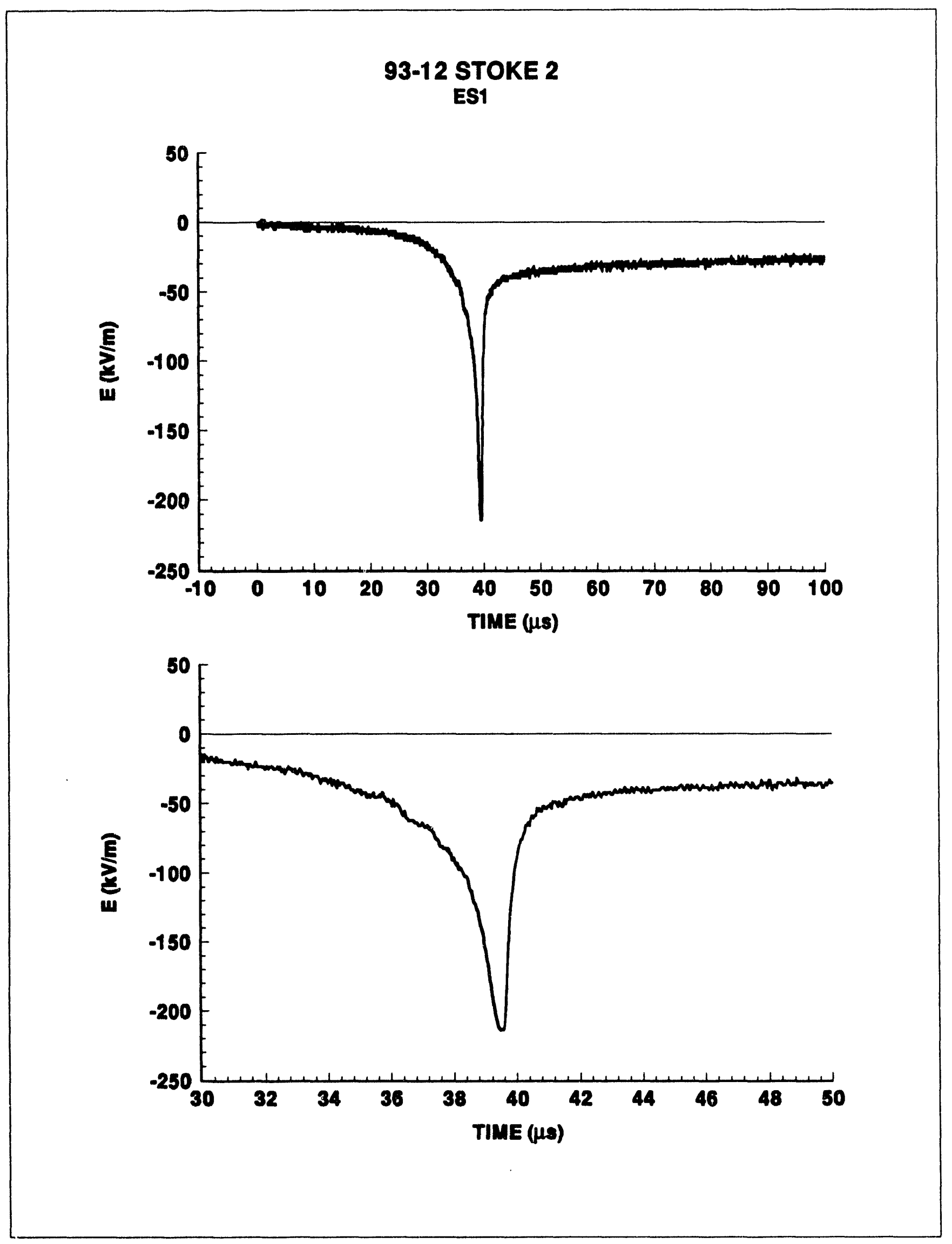




\section{3-12 STROKE 1}

ES4
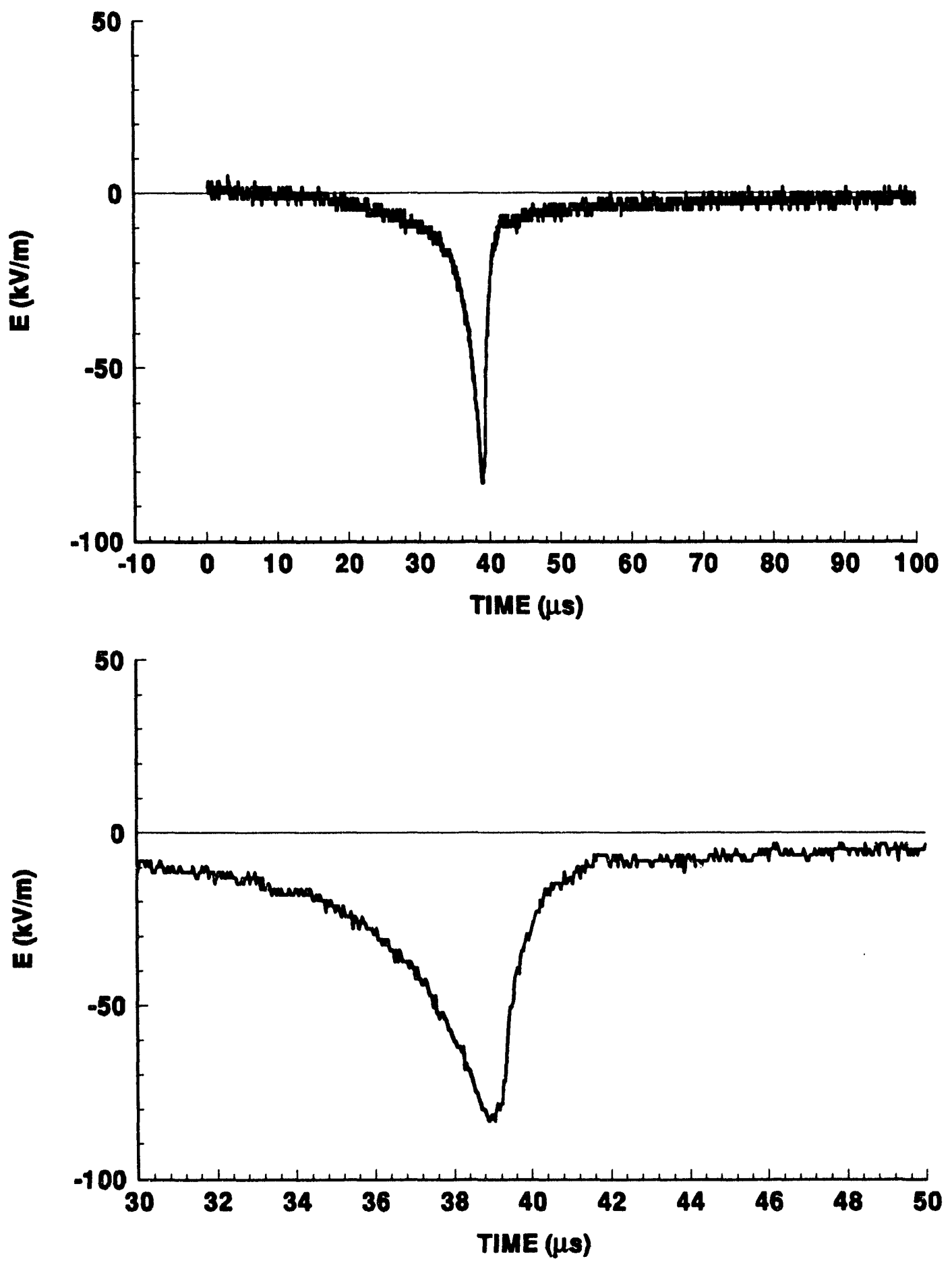


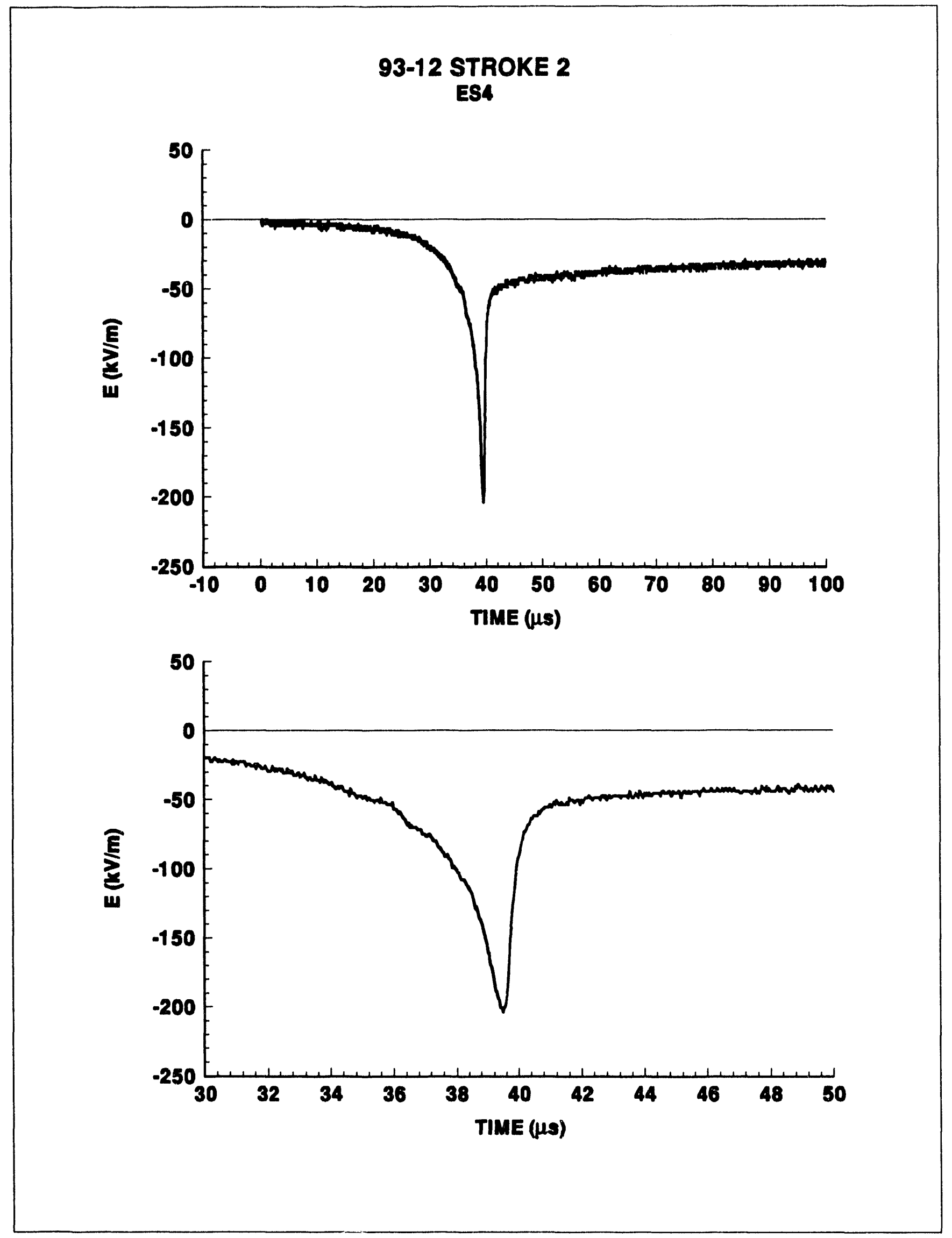




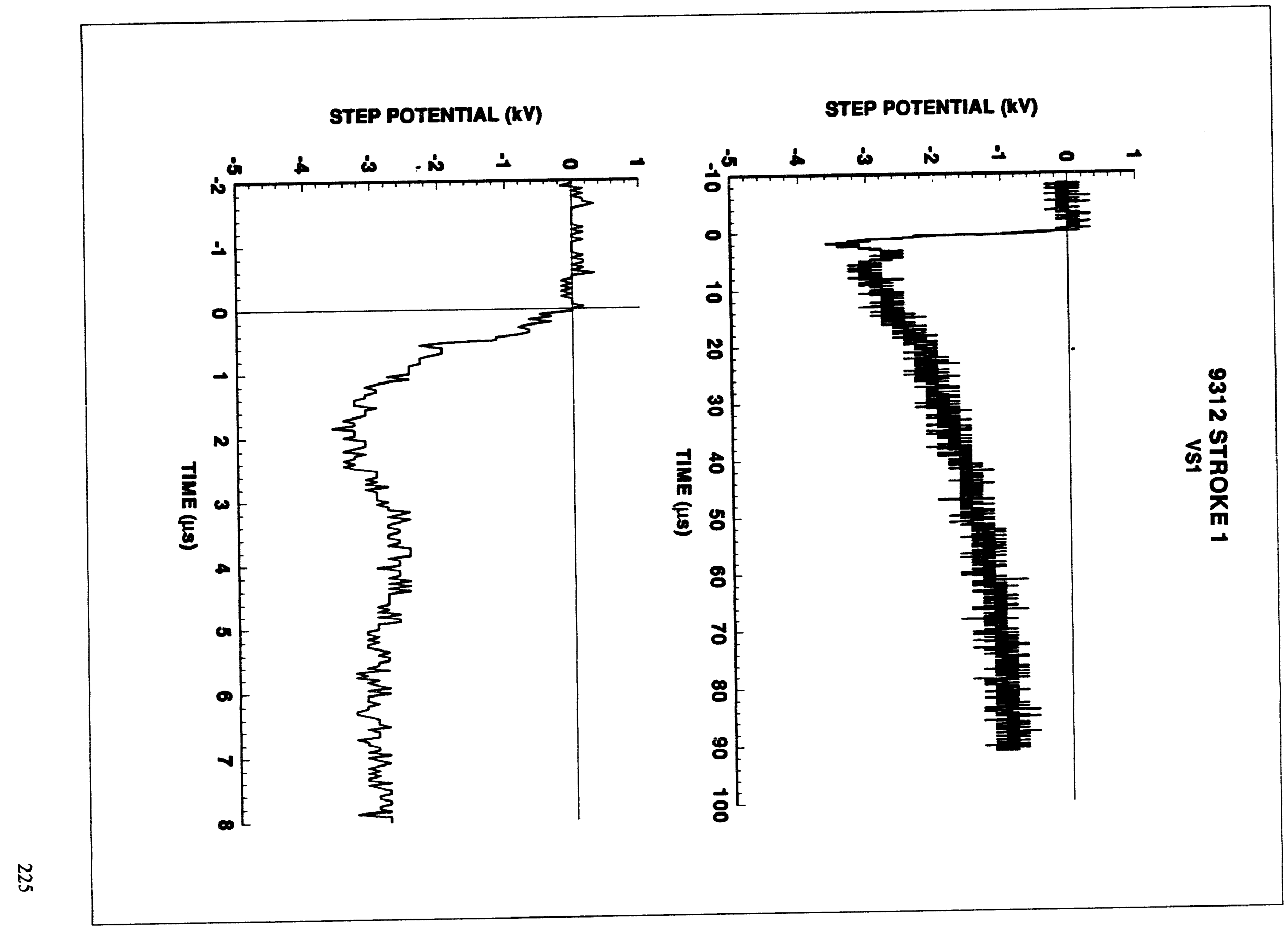



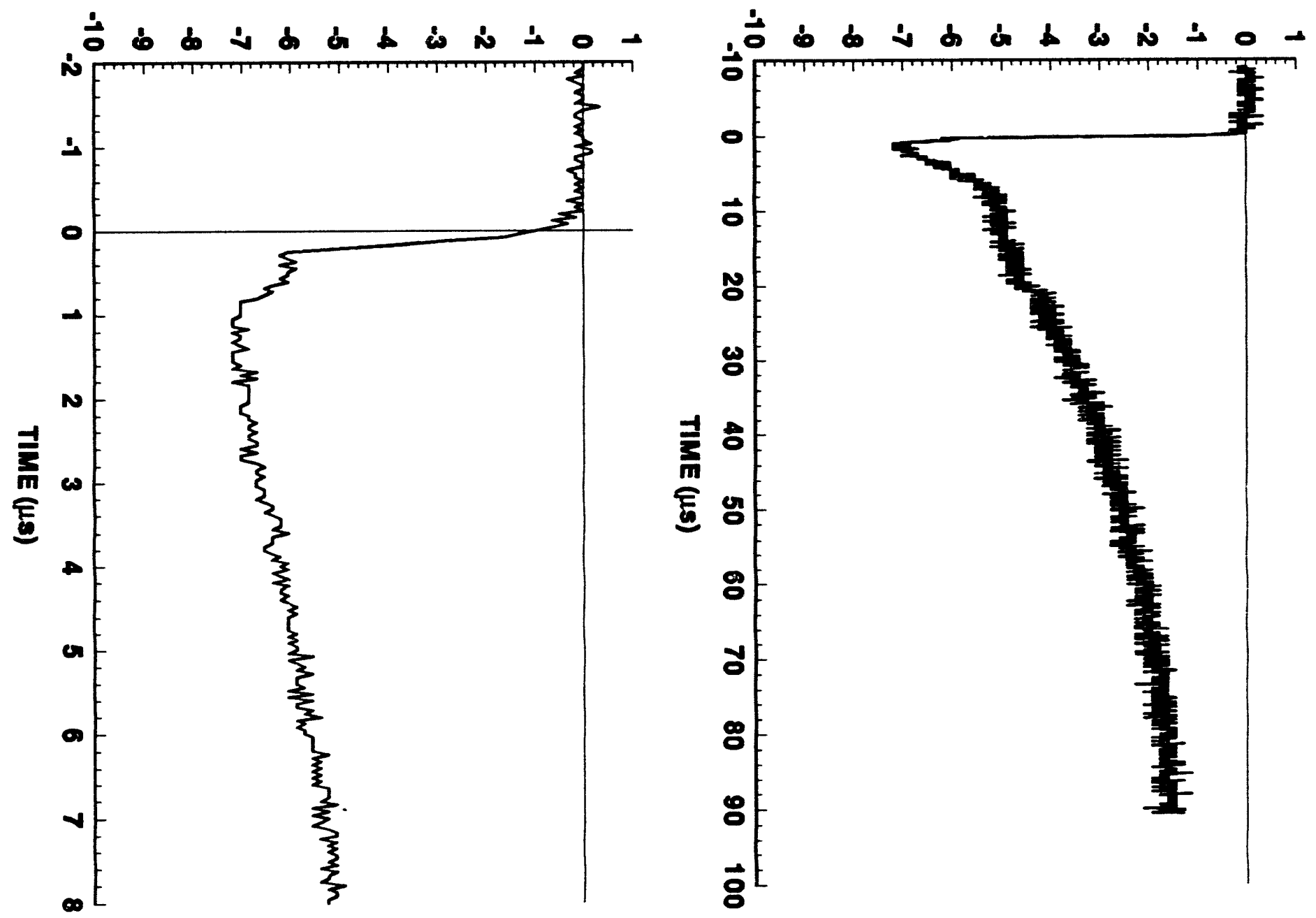

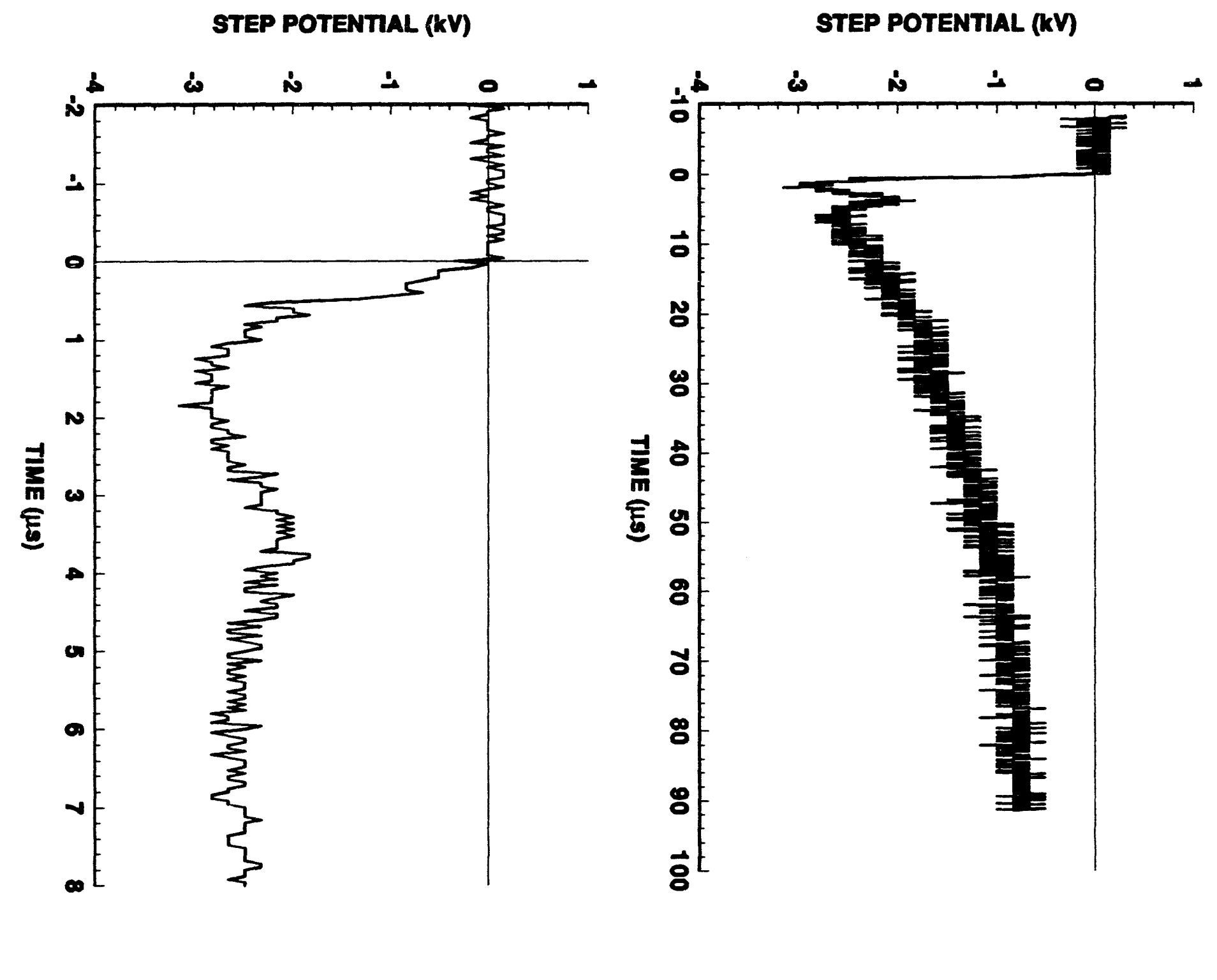

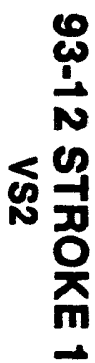


STEP POTENTIAL (KV)

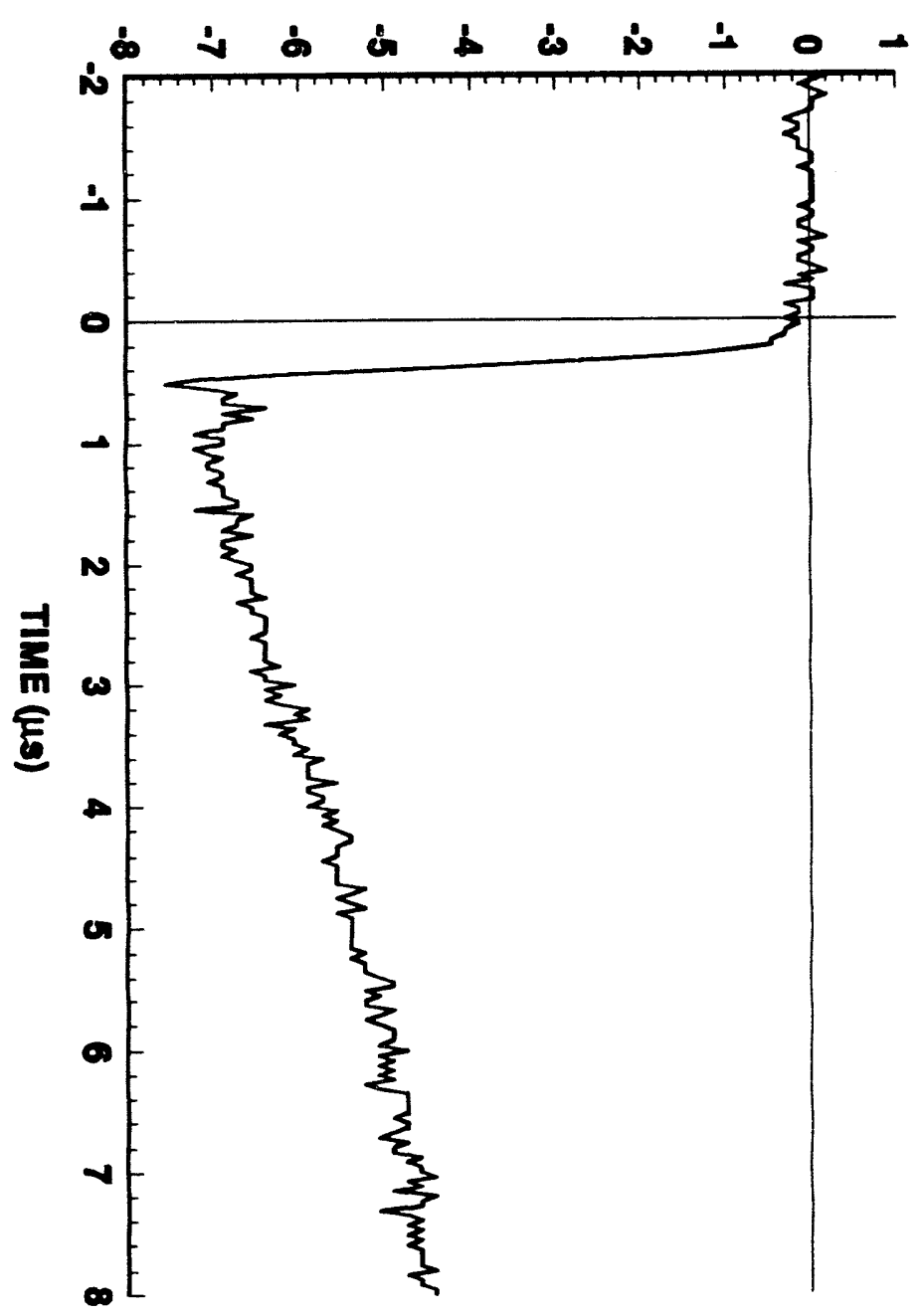

STEP POTENTIAL (KV)

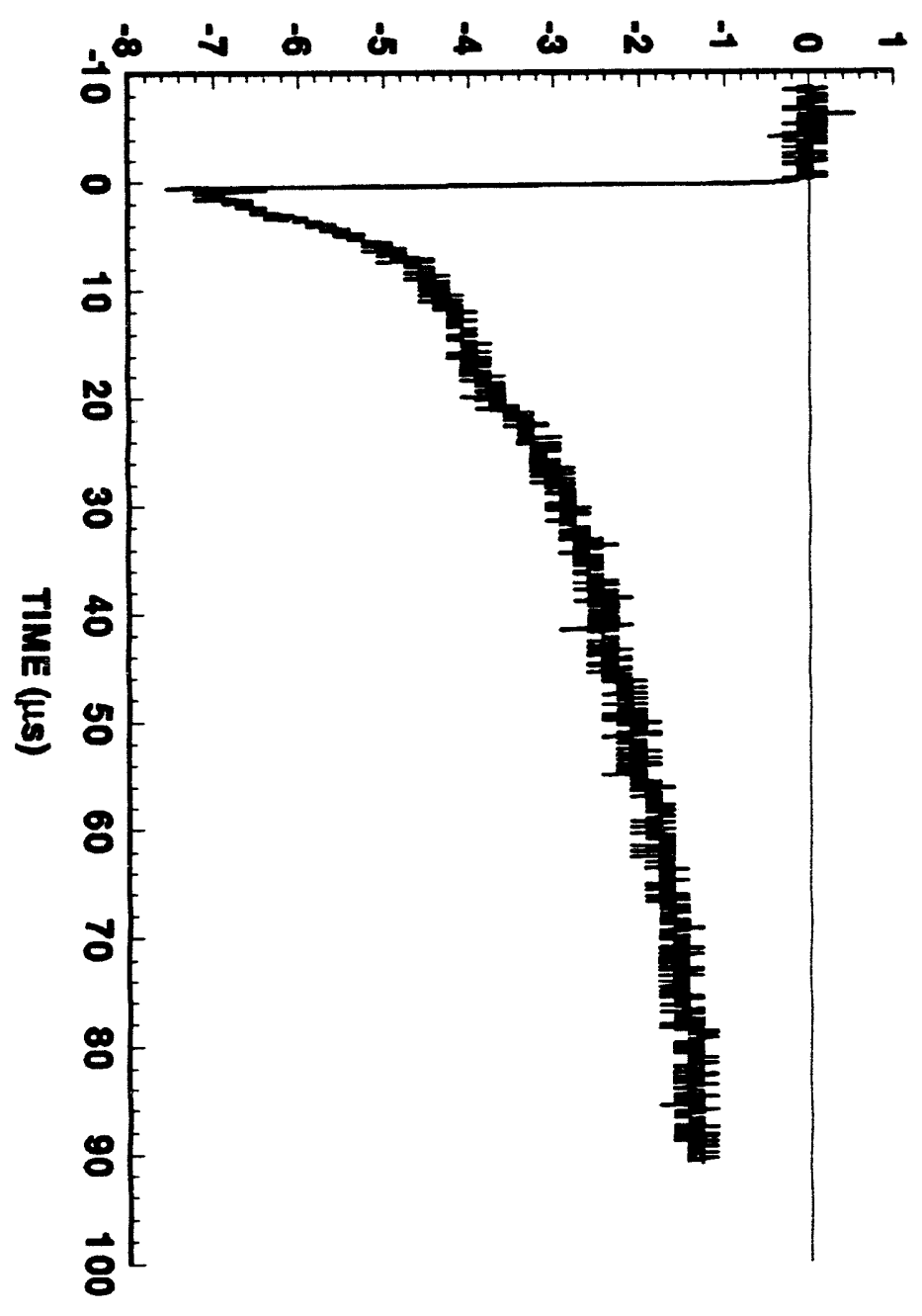




\section{STROKE 1 \\ VS4}
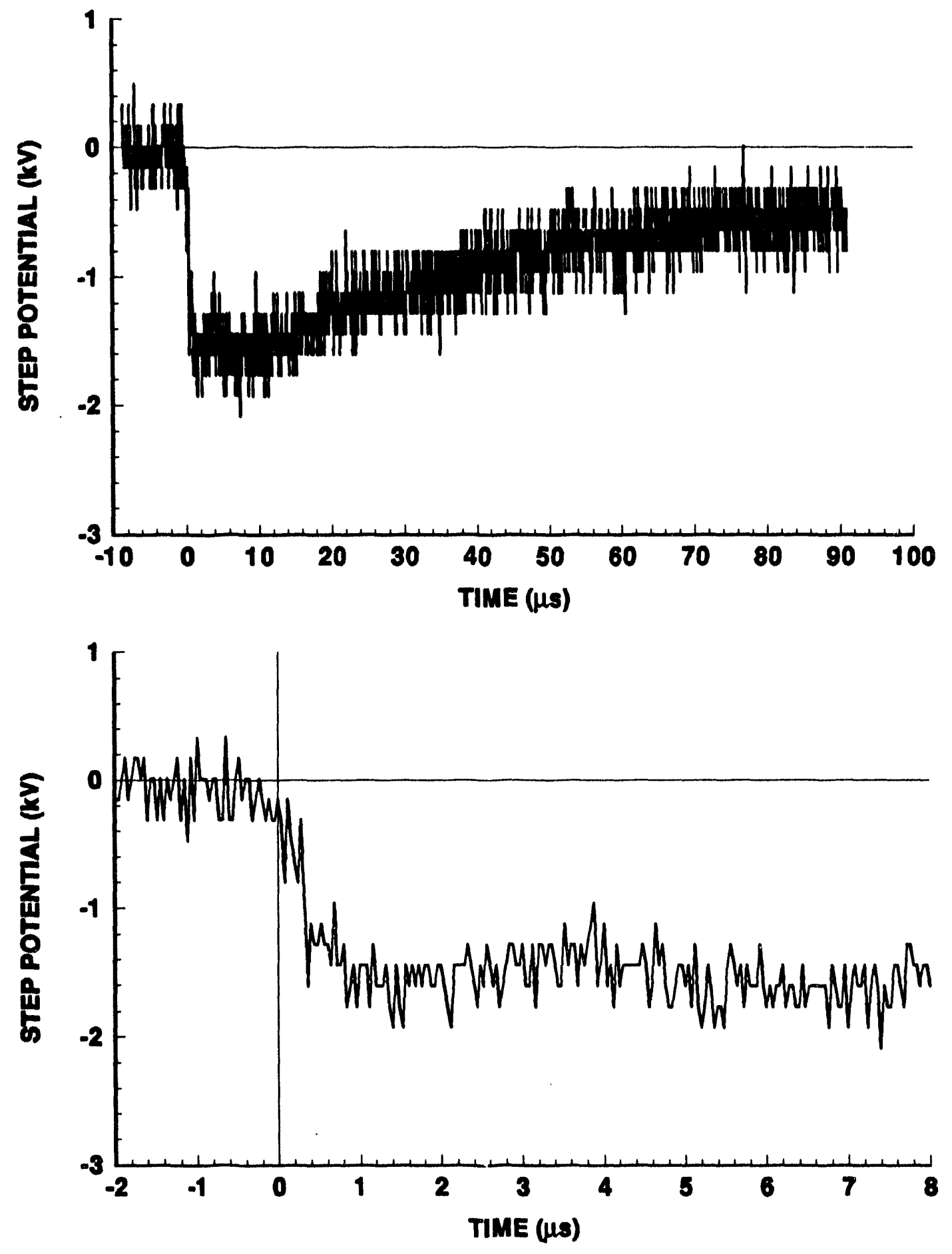
93-12 STROKE 2
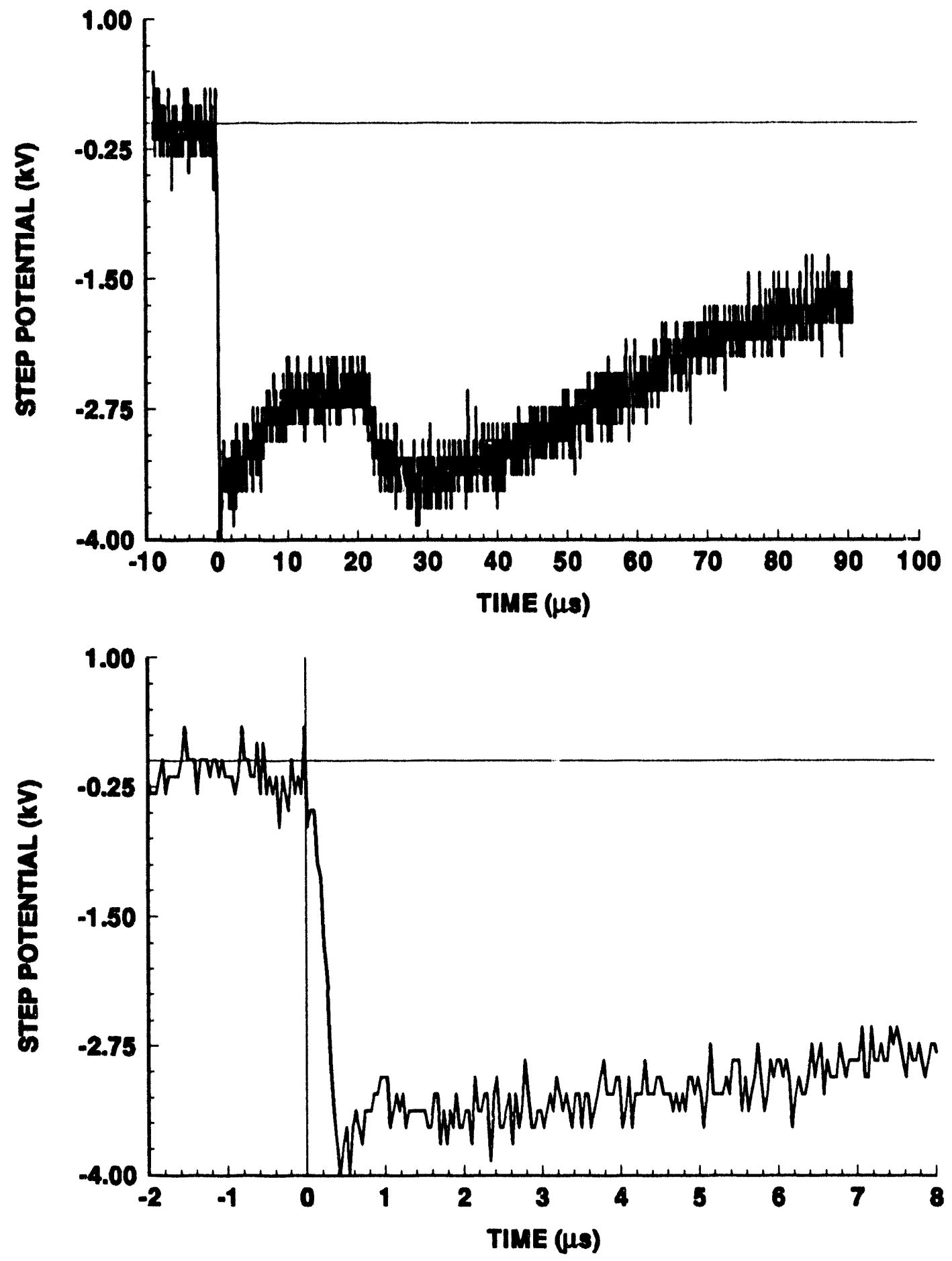


\section{3-12 STROKE 1 \\ VS5}
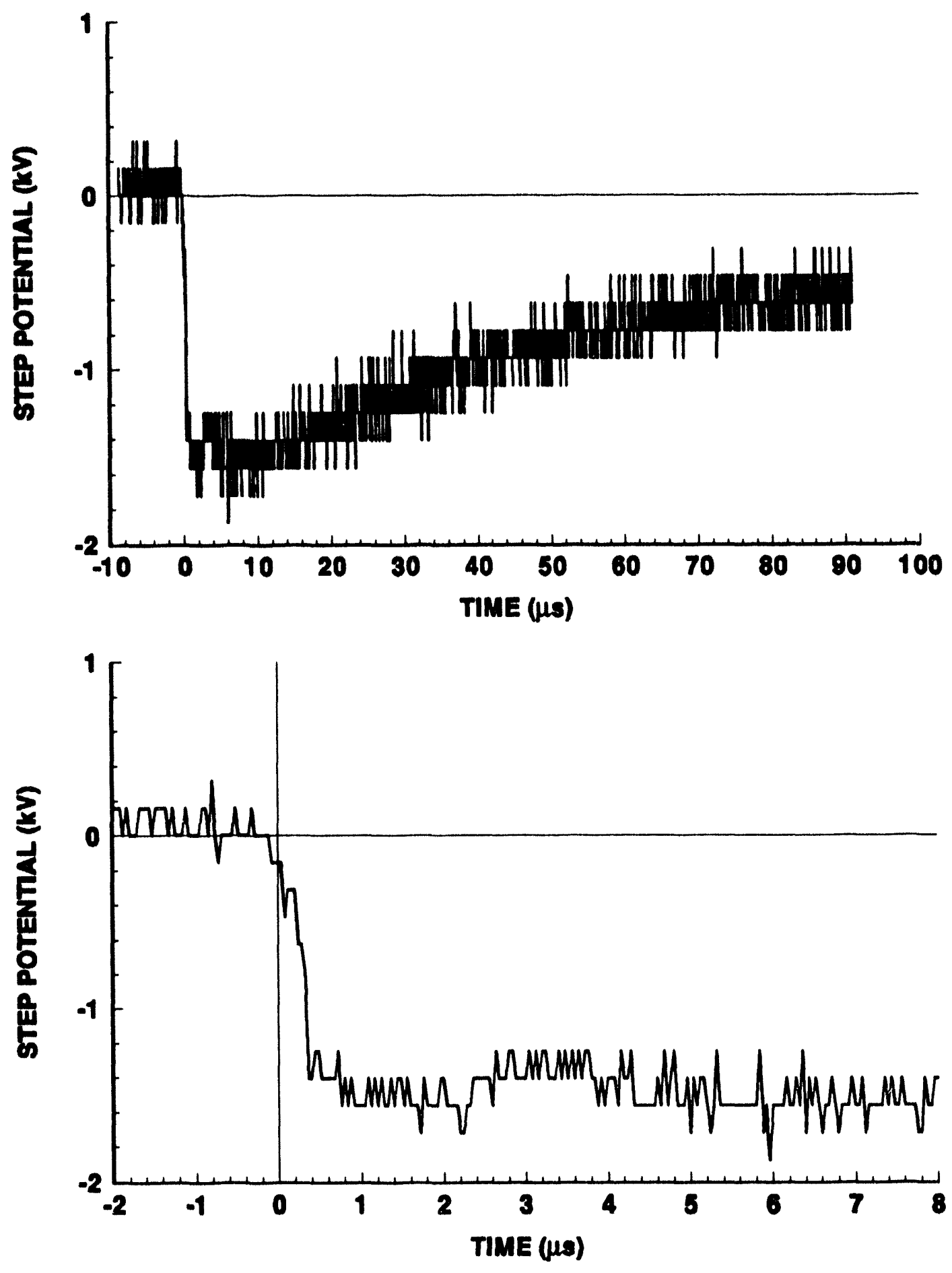


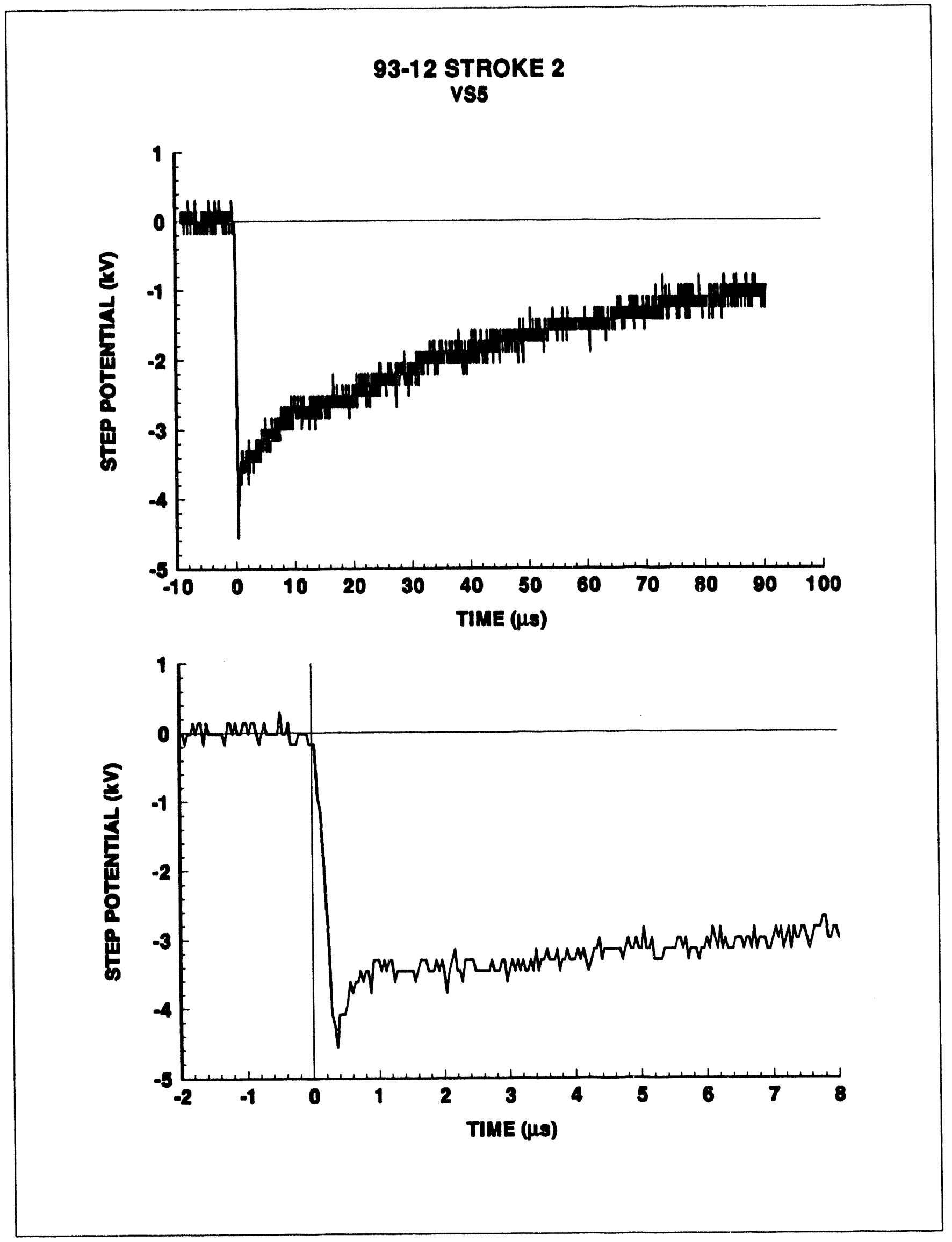


93-14 STROKE 1

INCIDENT CURRENT
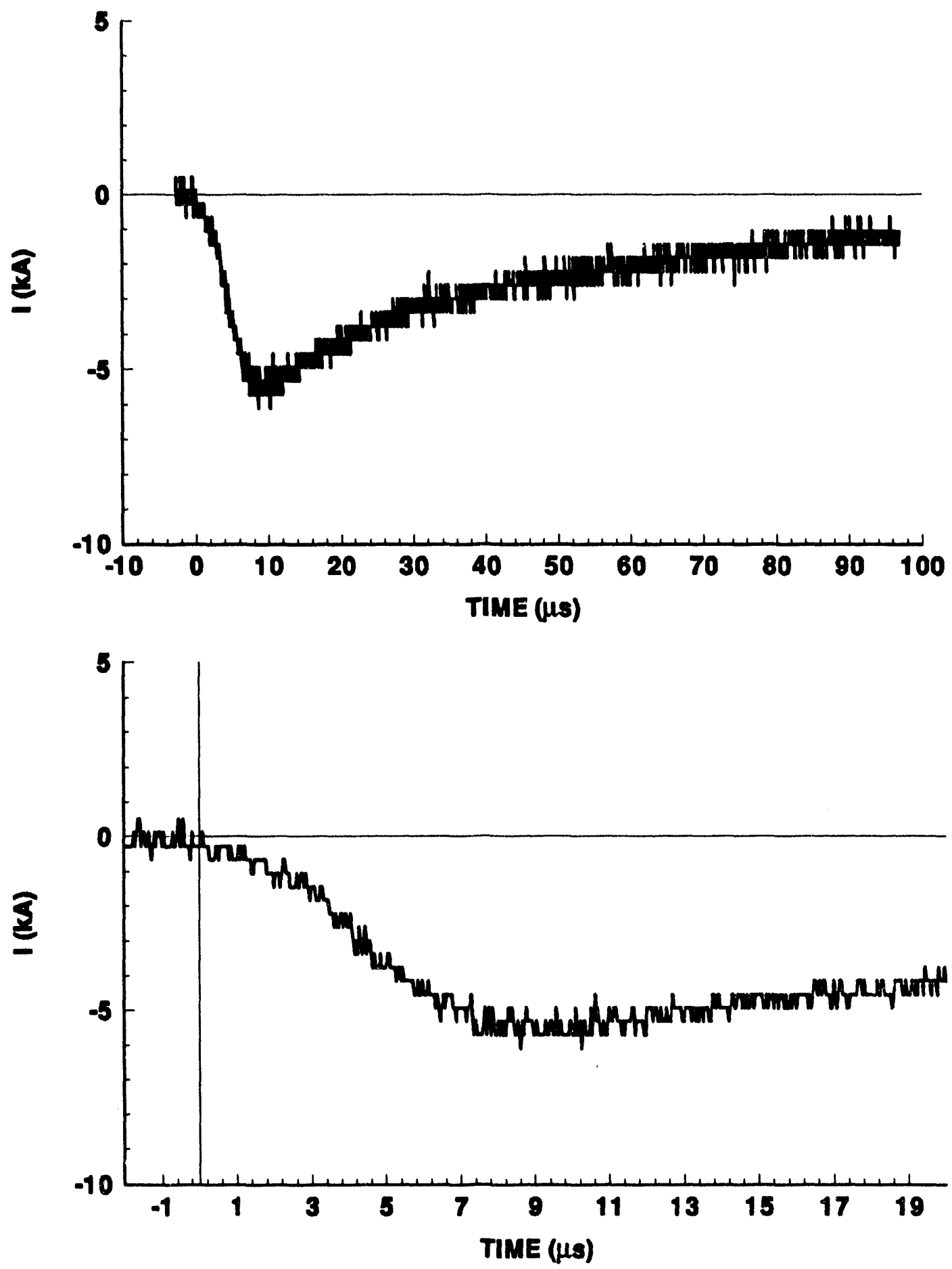


\section{3-14 STROKE 1}

HS1
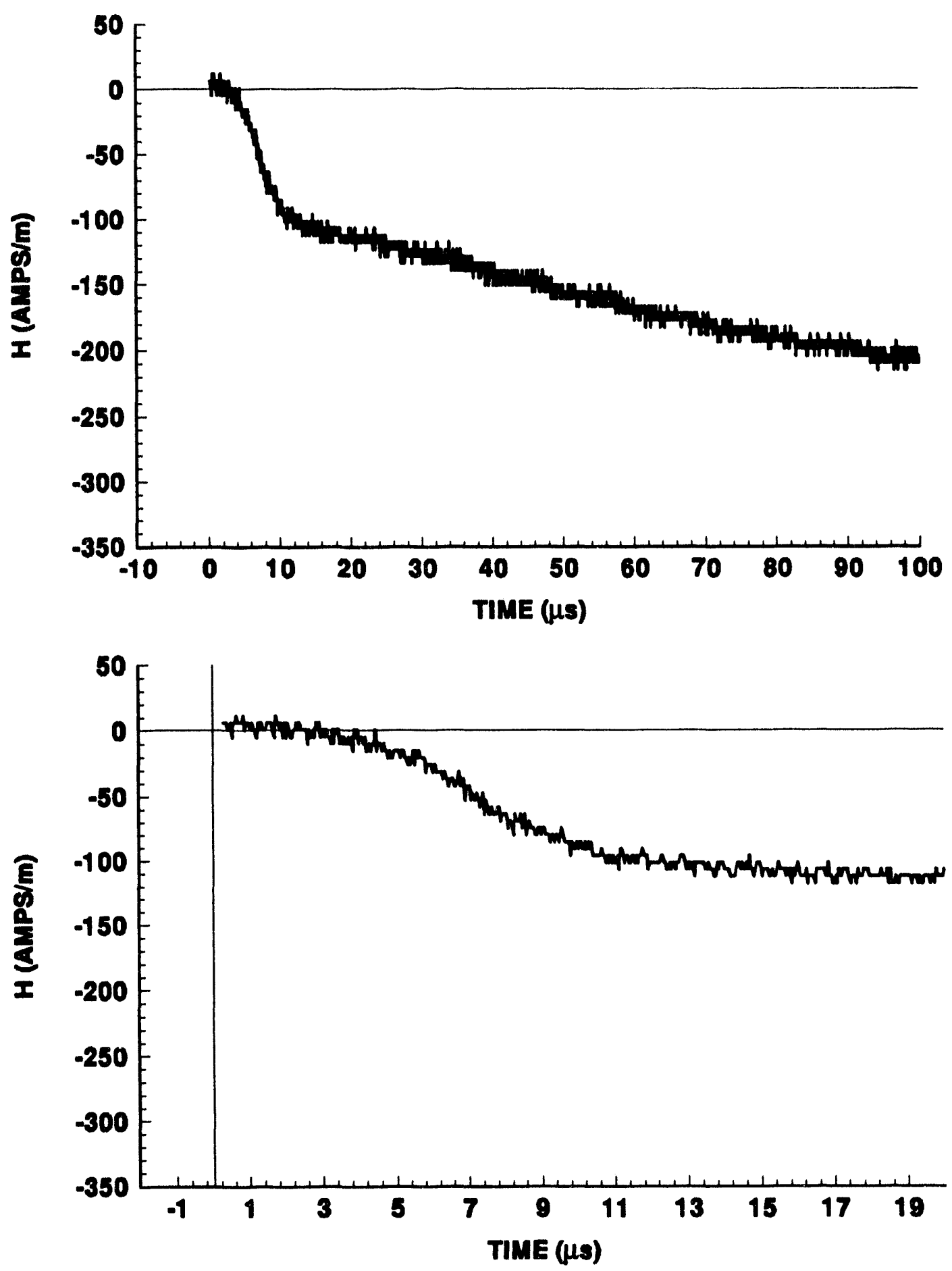


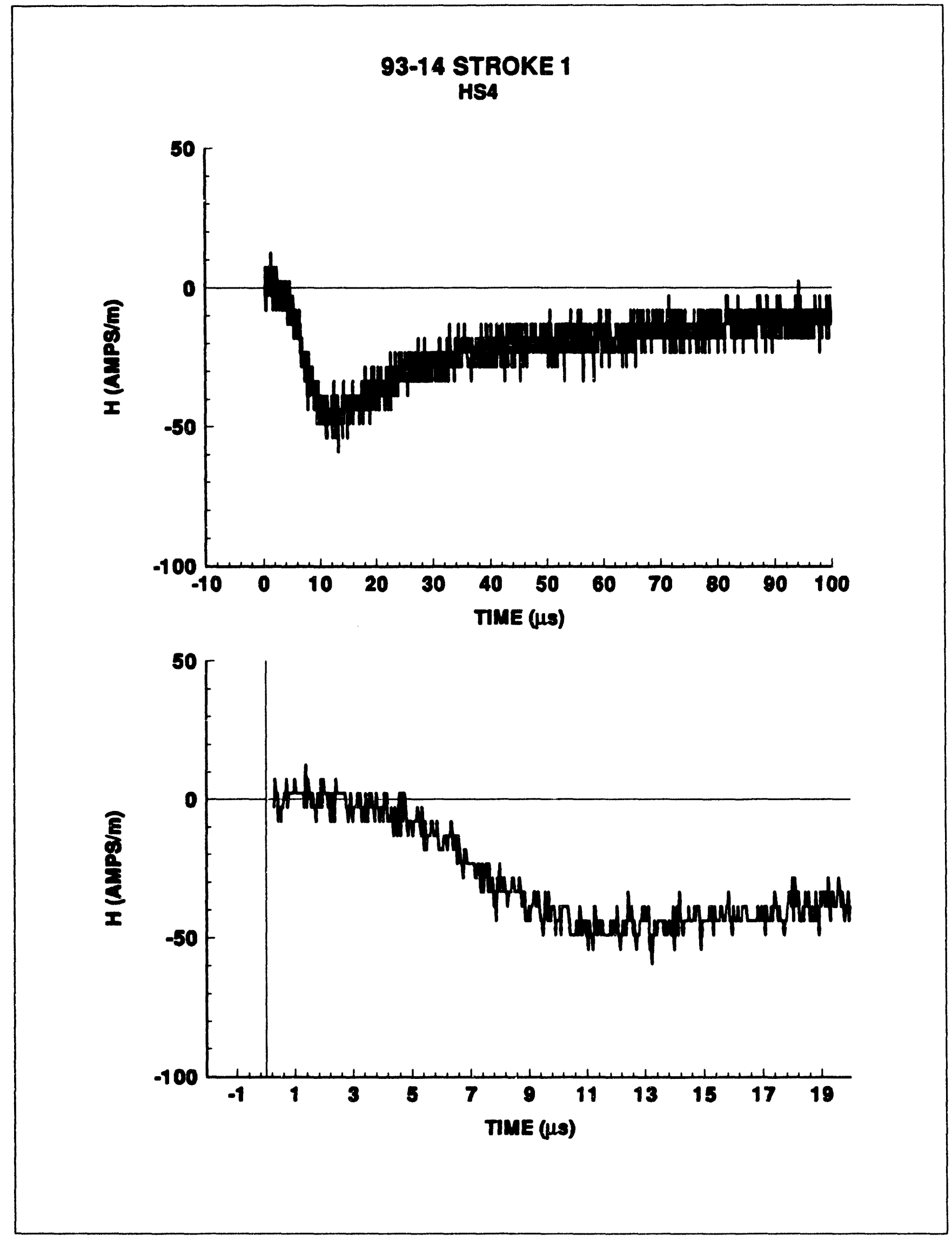




\section{3-14 STROKE 1 \\ HS5}
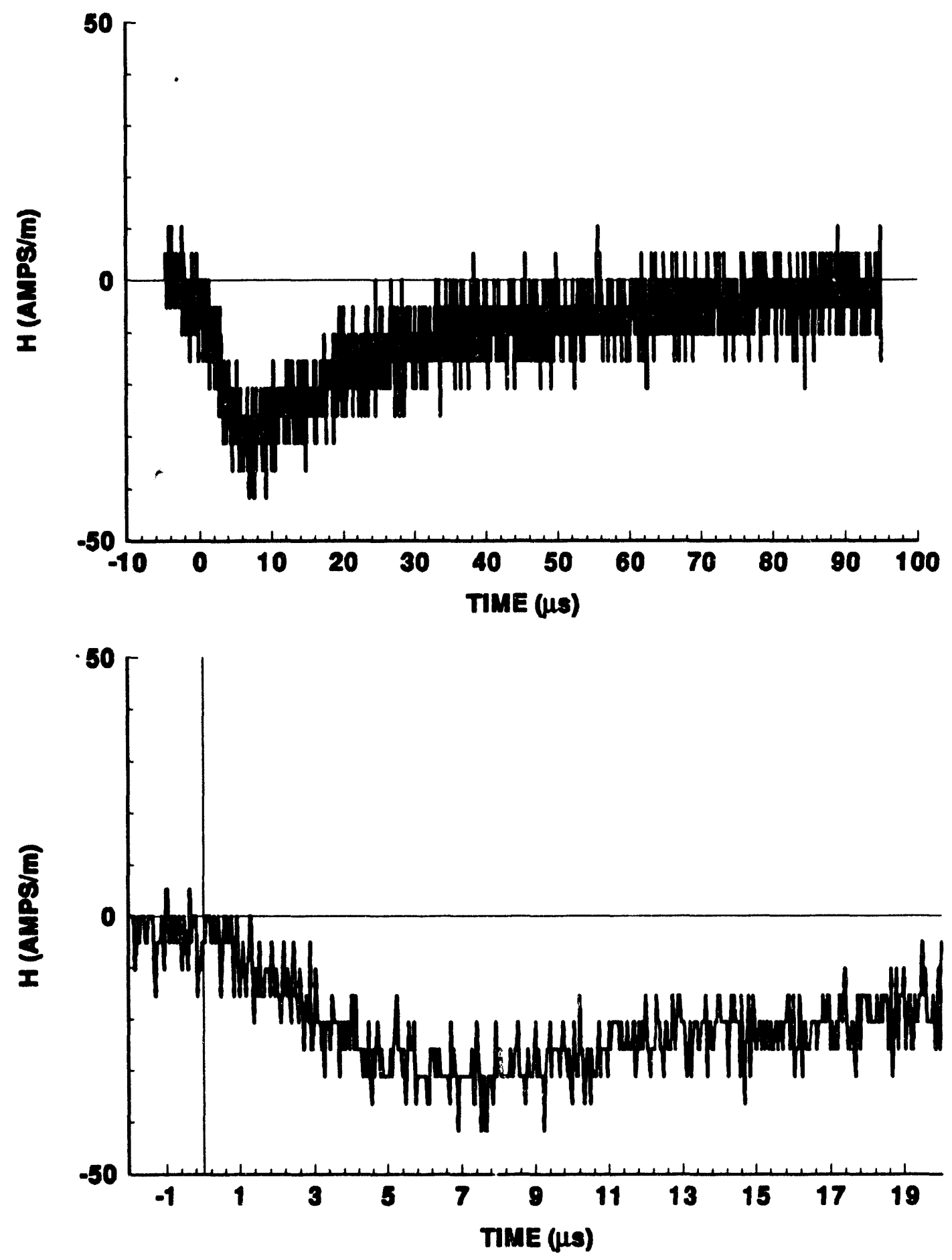


\section{3-14 STROKE 1}

\section{ES1}

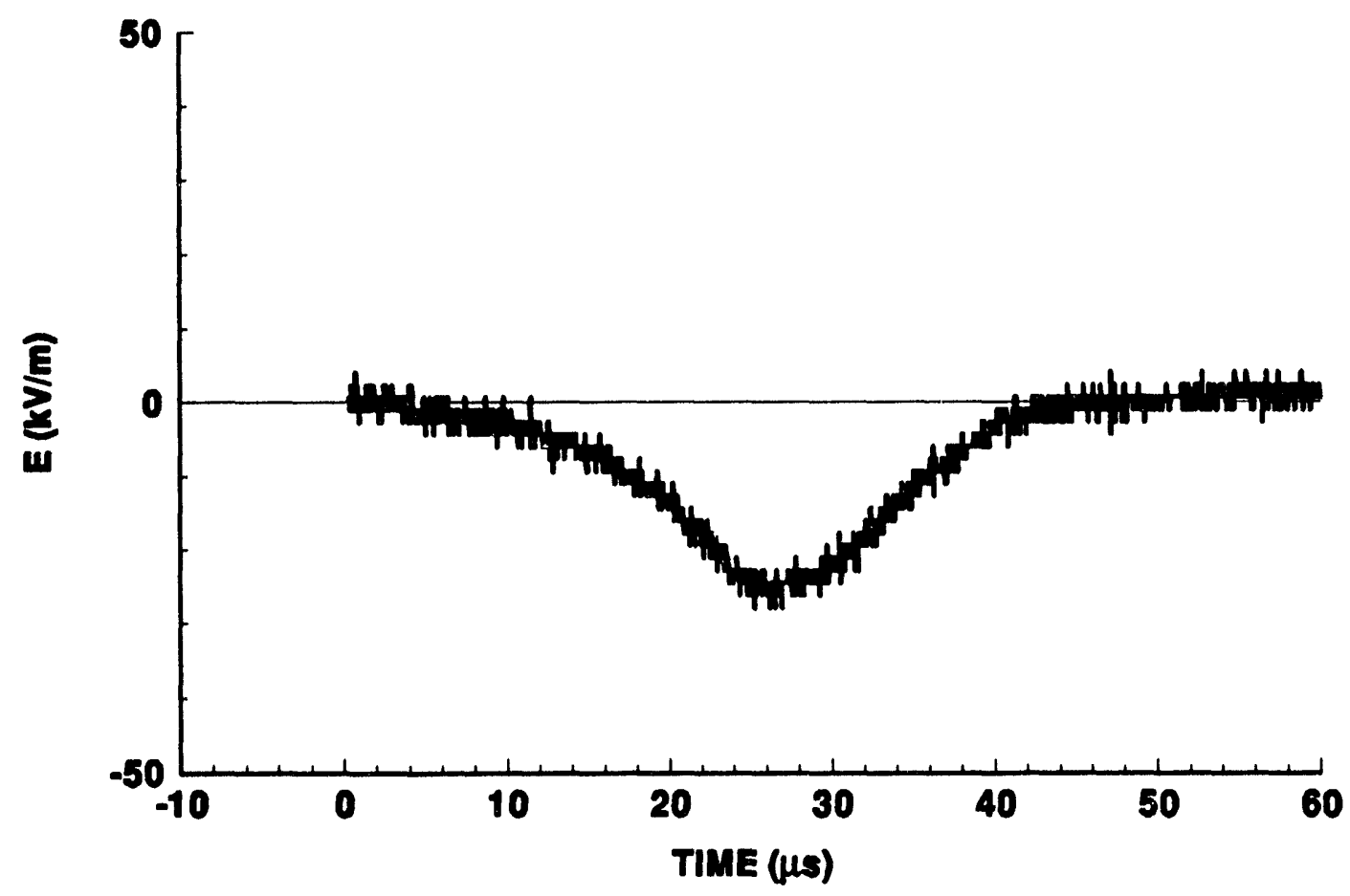




\section{3-14 STROKE 1}

ES4

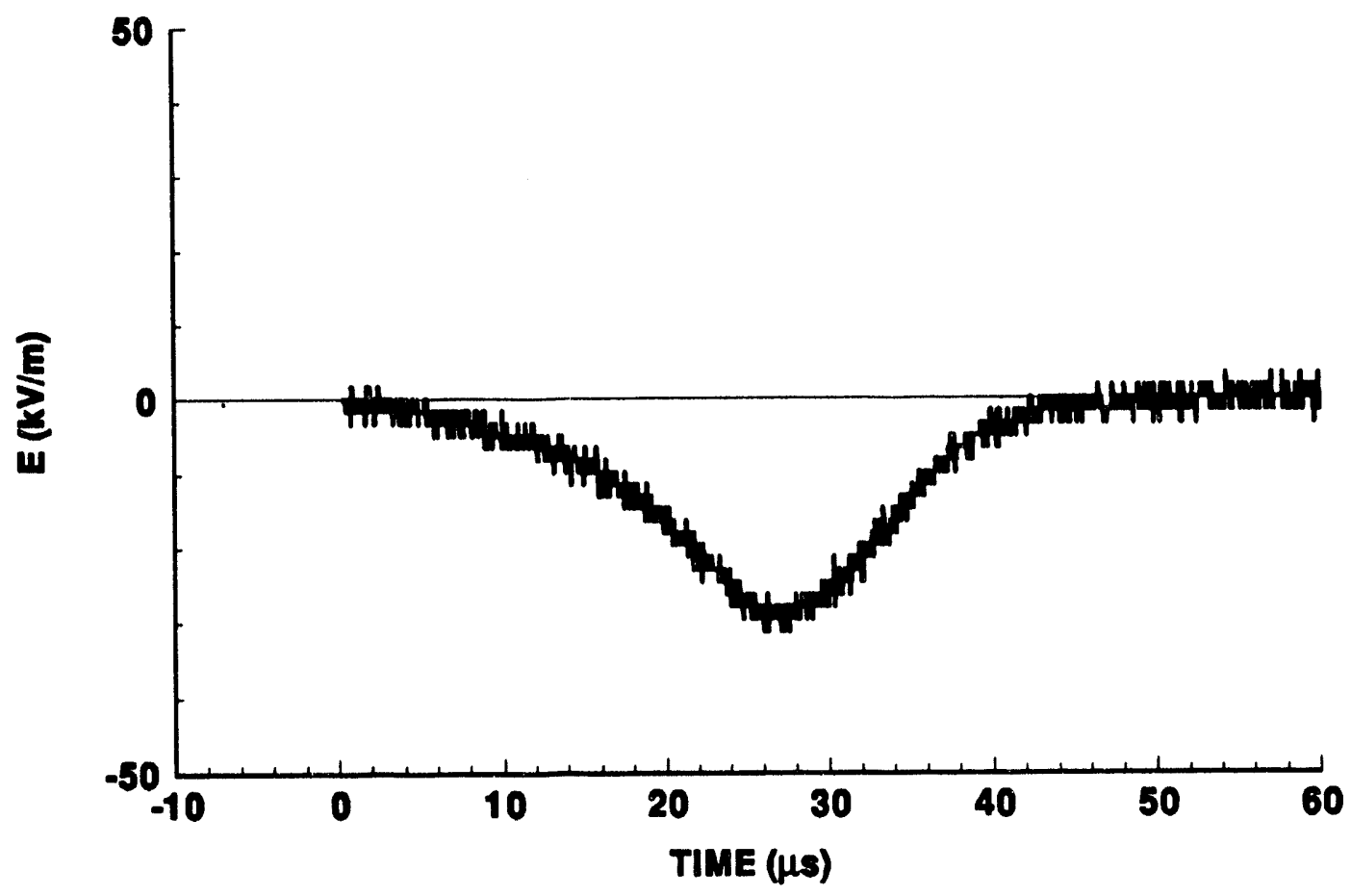



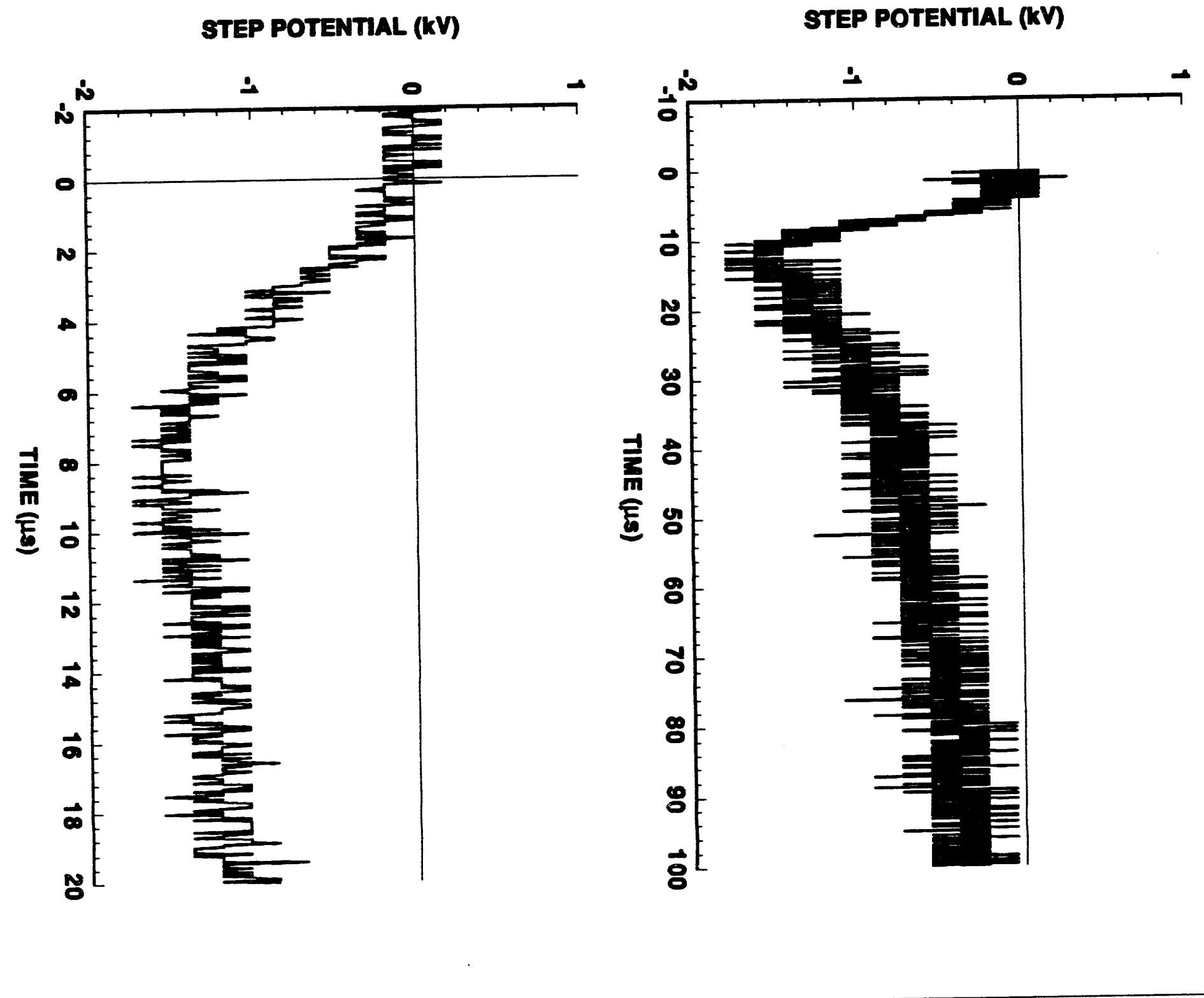


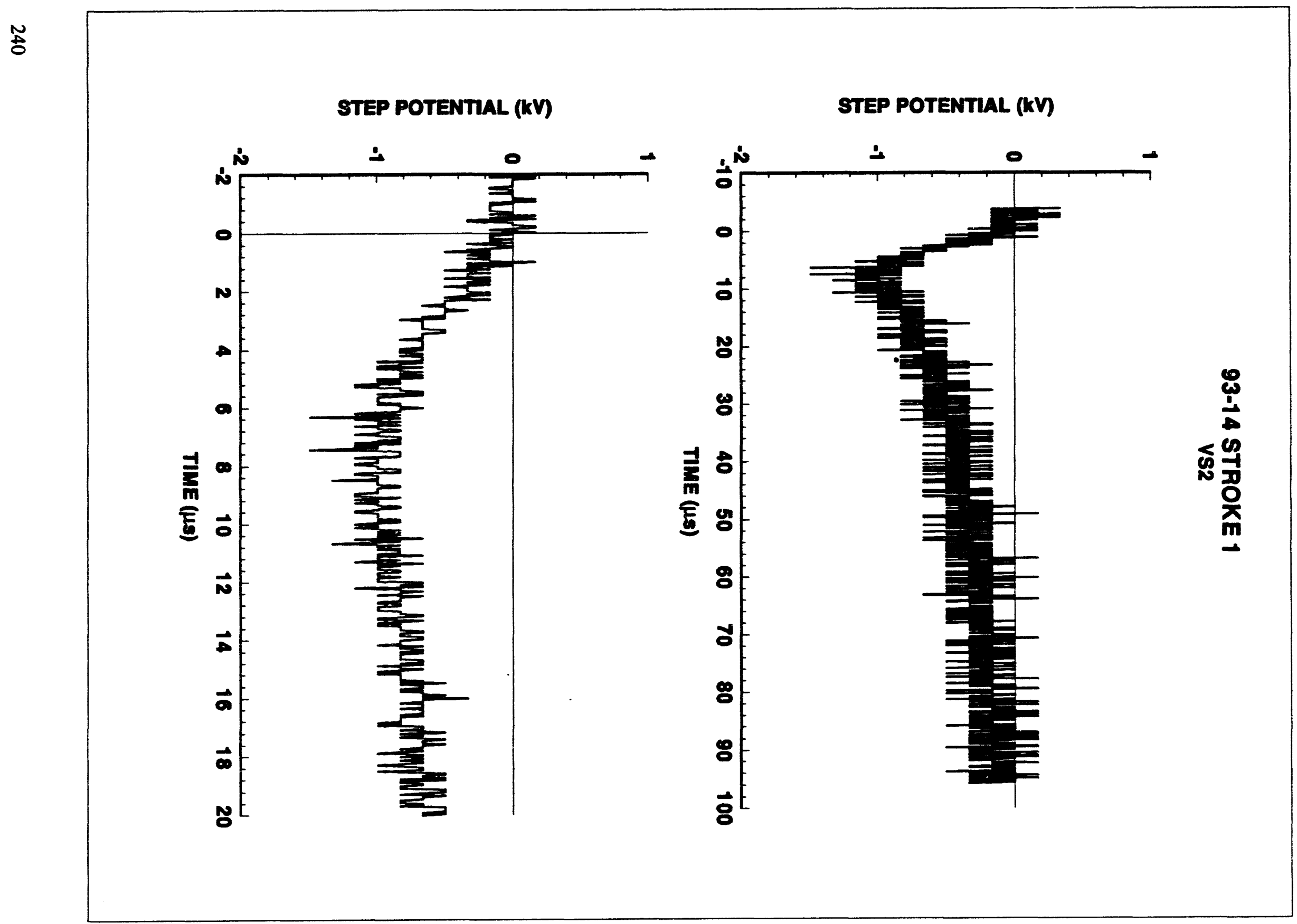




\section{3-14 STROKE 1 \\ vS4}
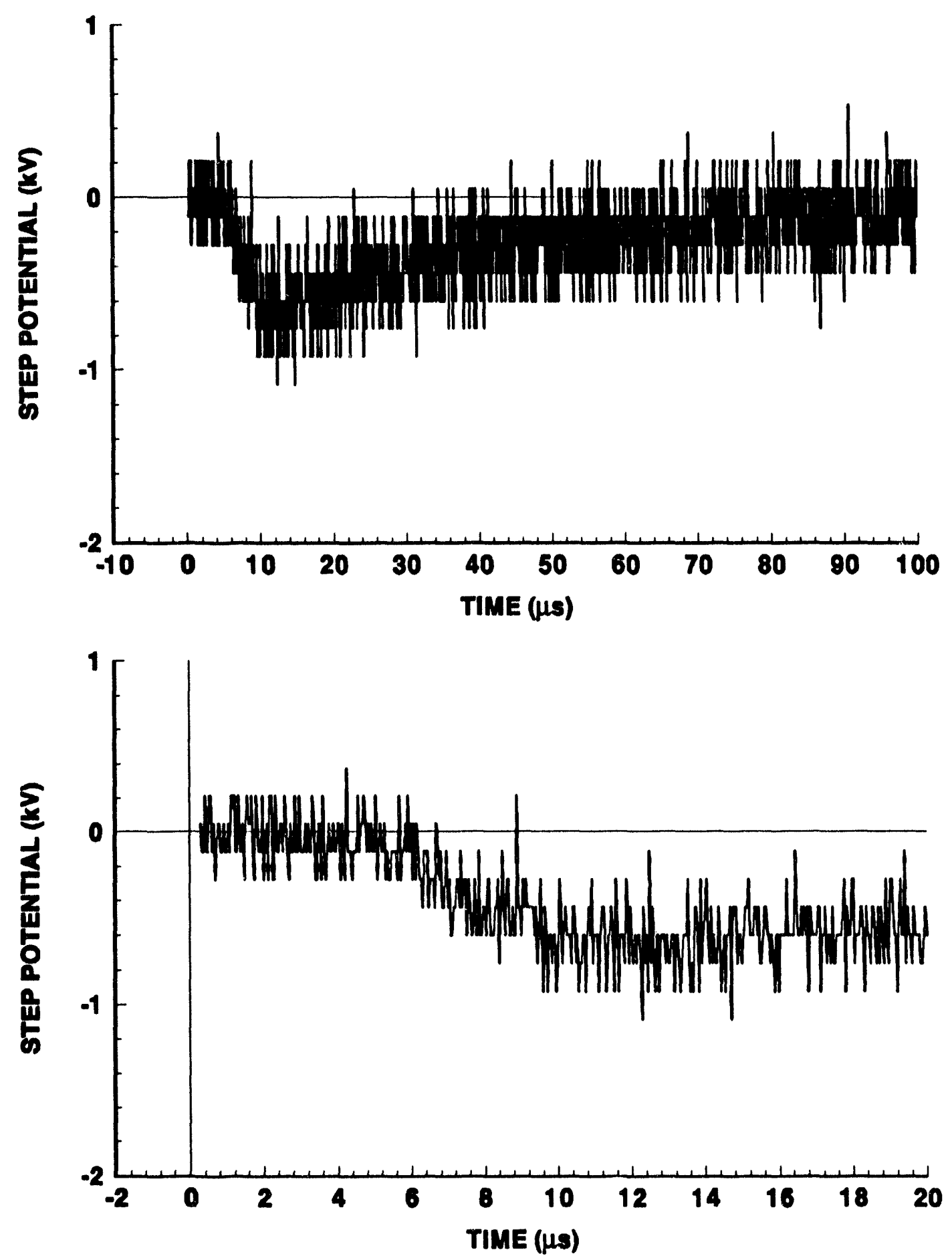


\section{STROKE 1}

v85
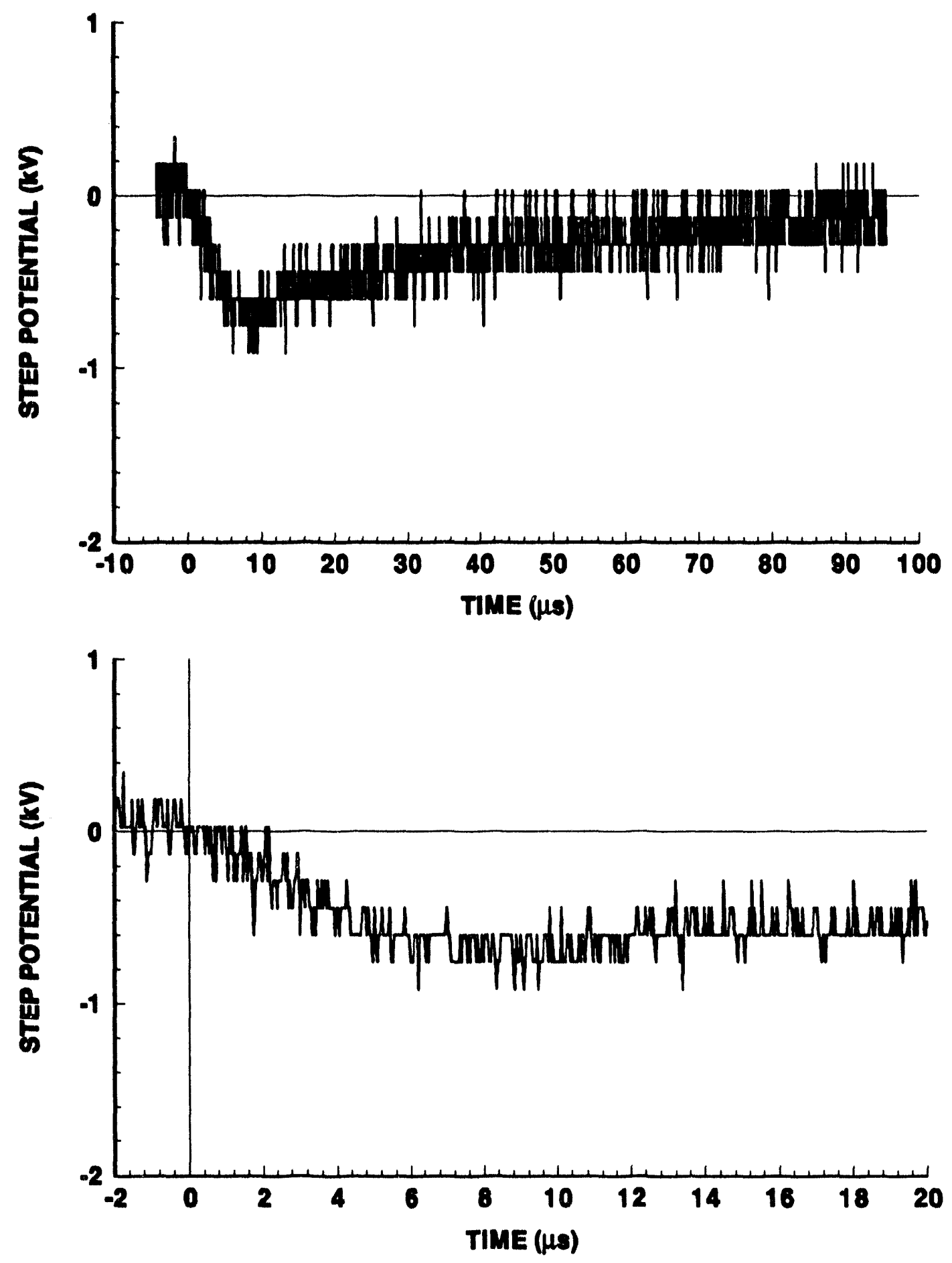
93-15 STROKE 1

INCIDENT CURRENT
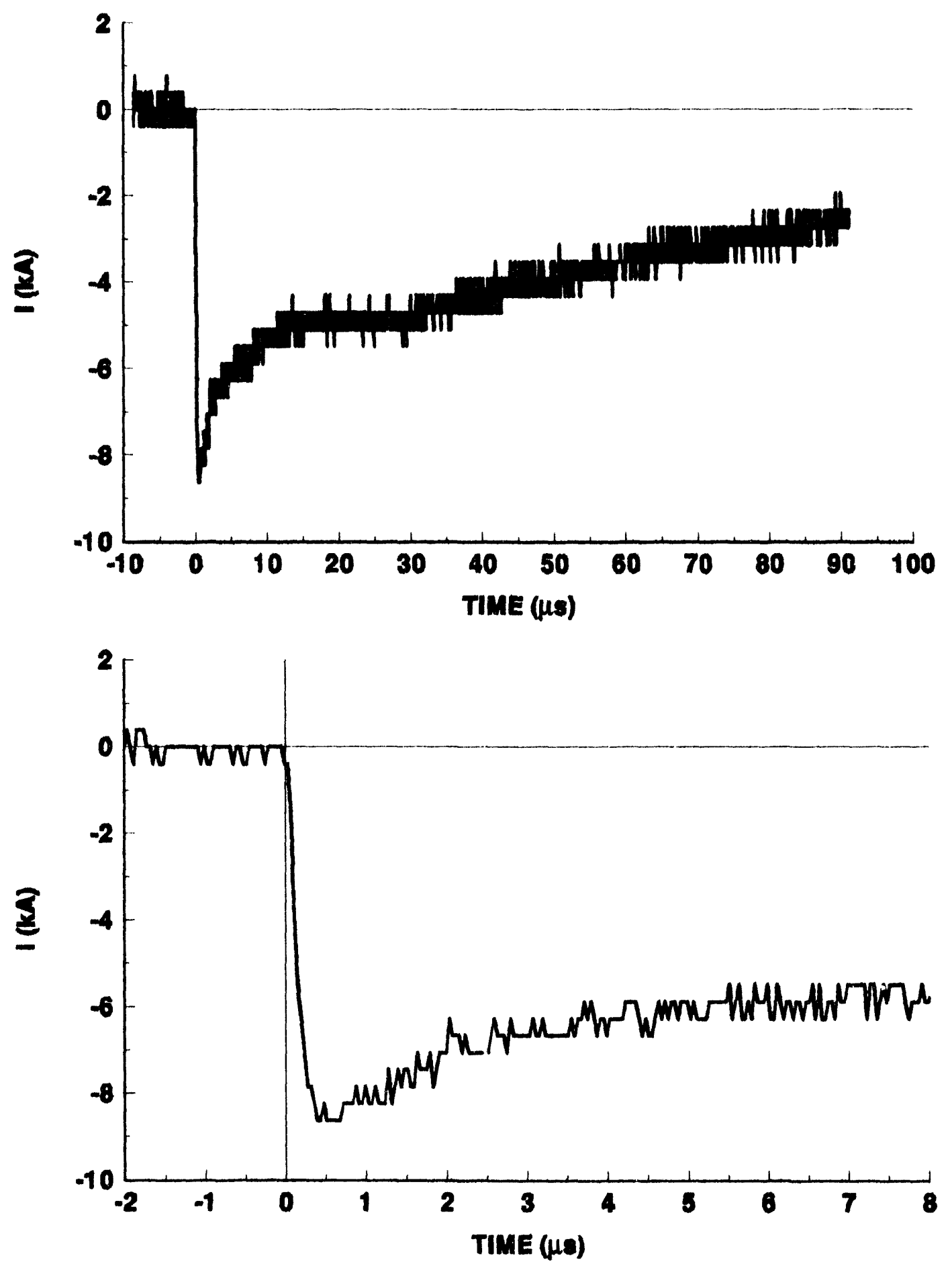
93-15 STROKE 2

INCIDENT CURRENT
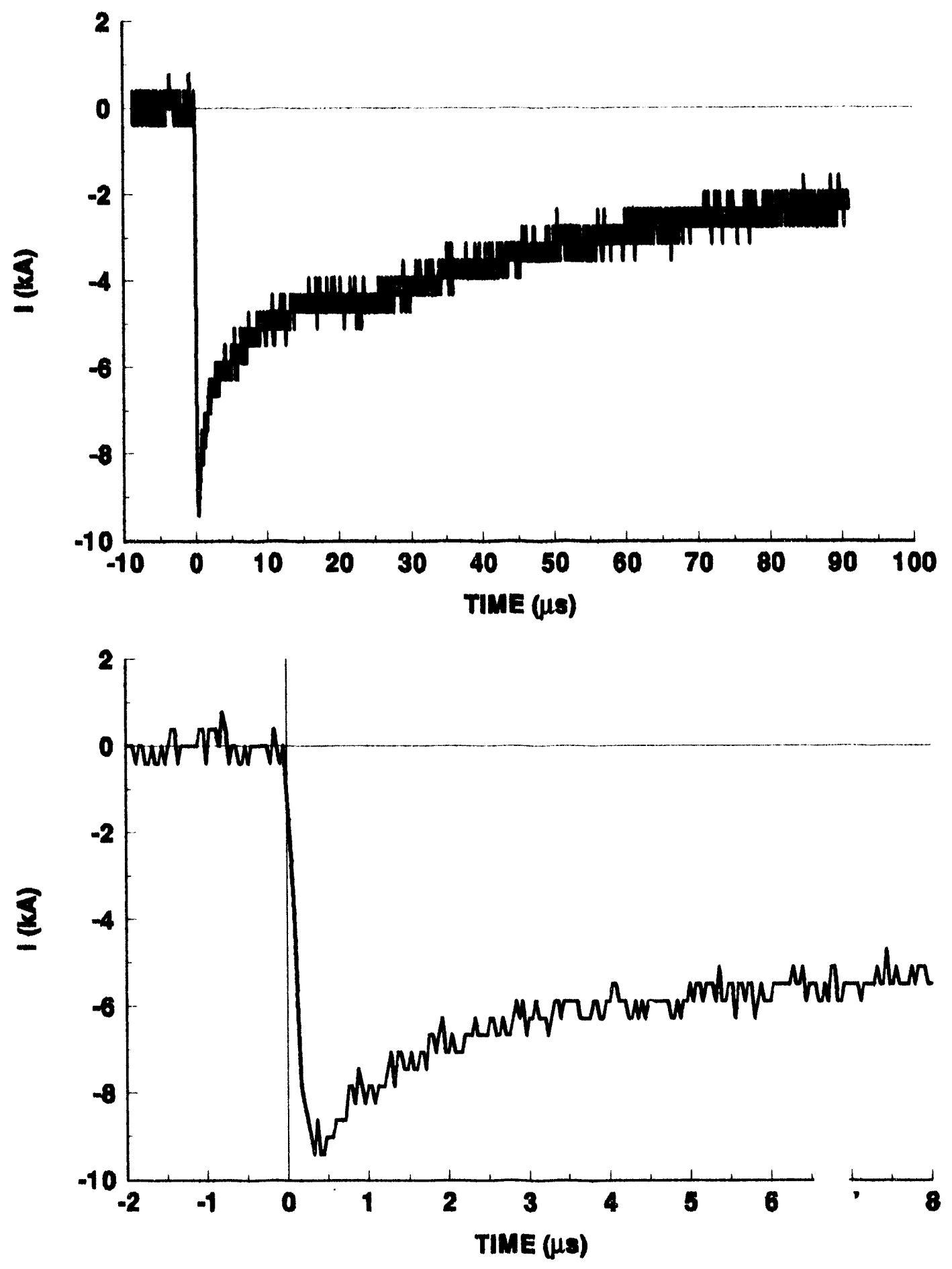
93-15 STROKE 3

INCIDENT CURRENT
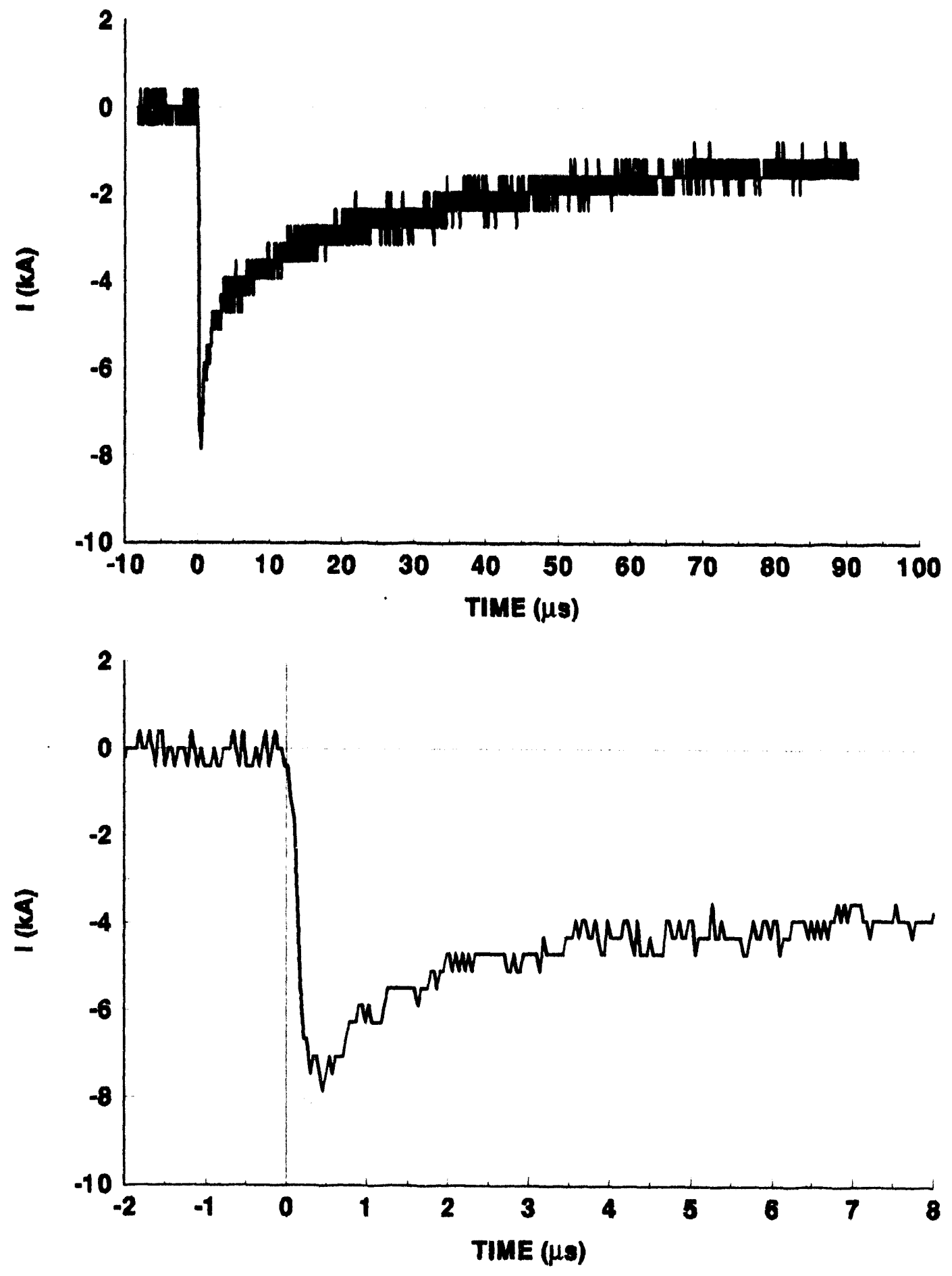


\section{STROKE 4}

INCIDENT CURRENT
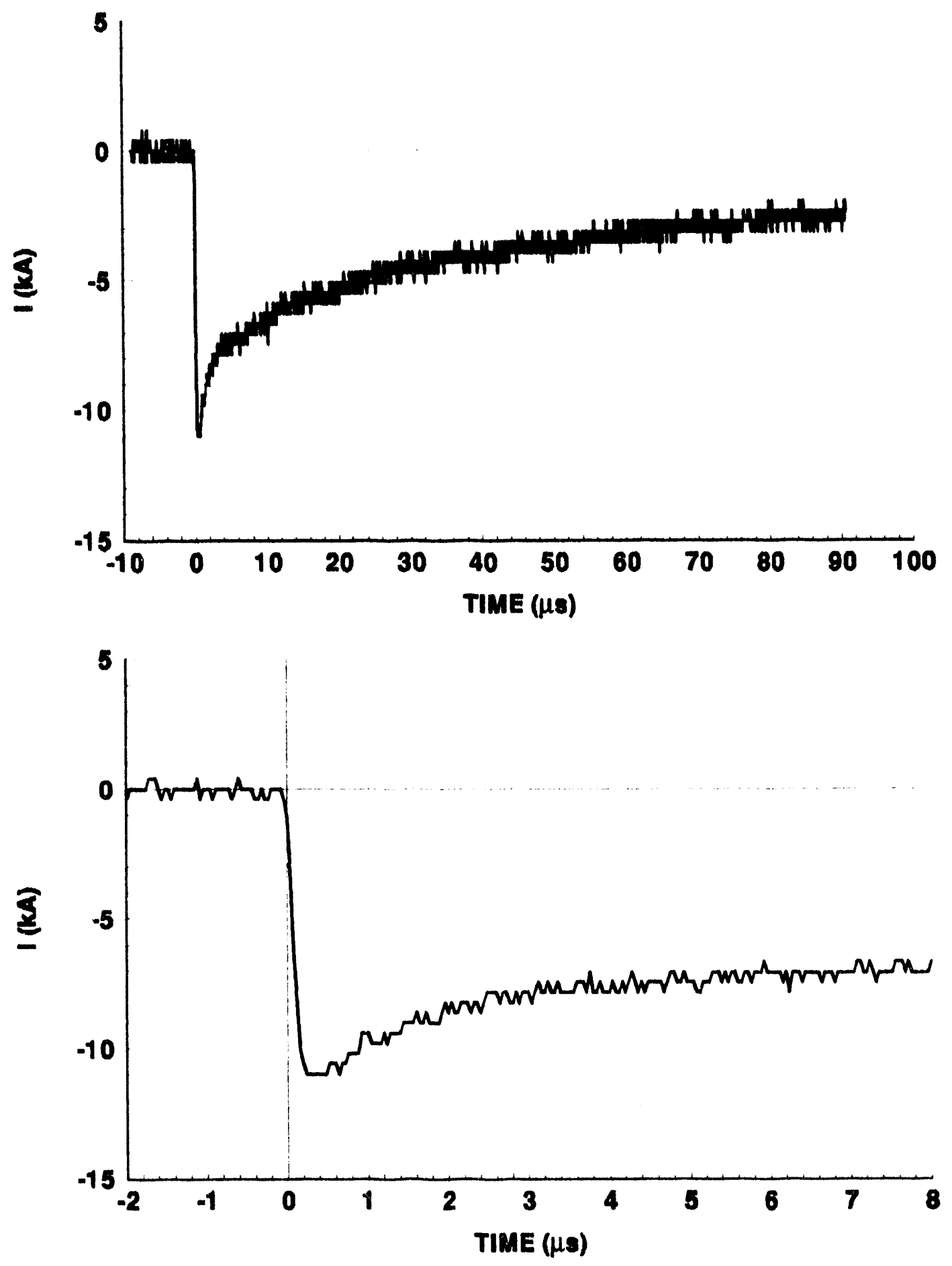


\section{STROKE 5}

INCIDENT CURRENT
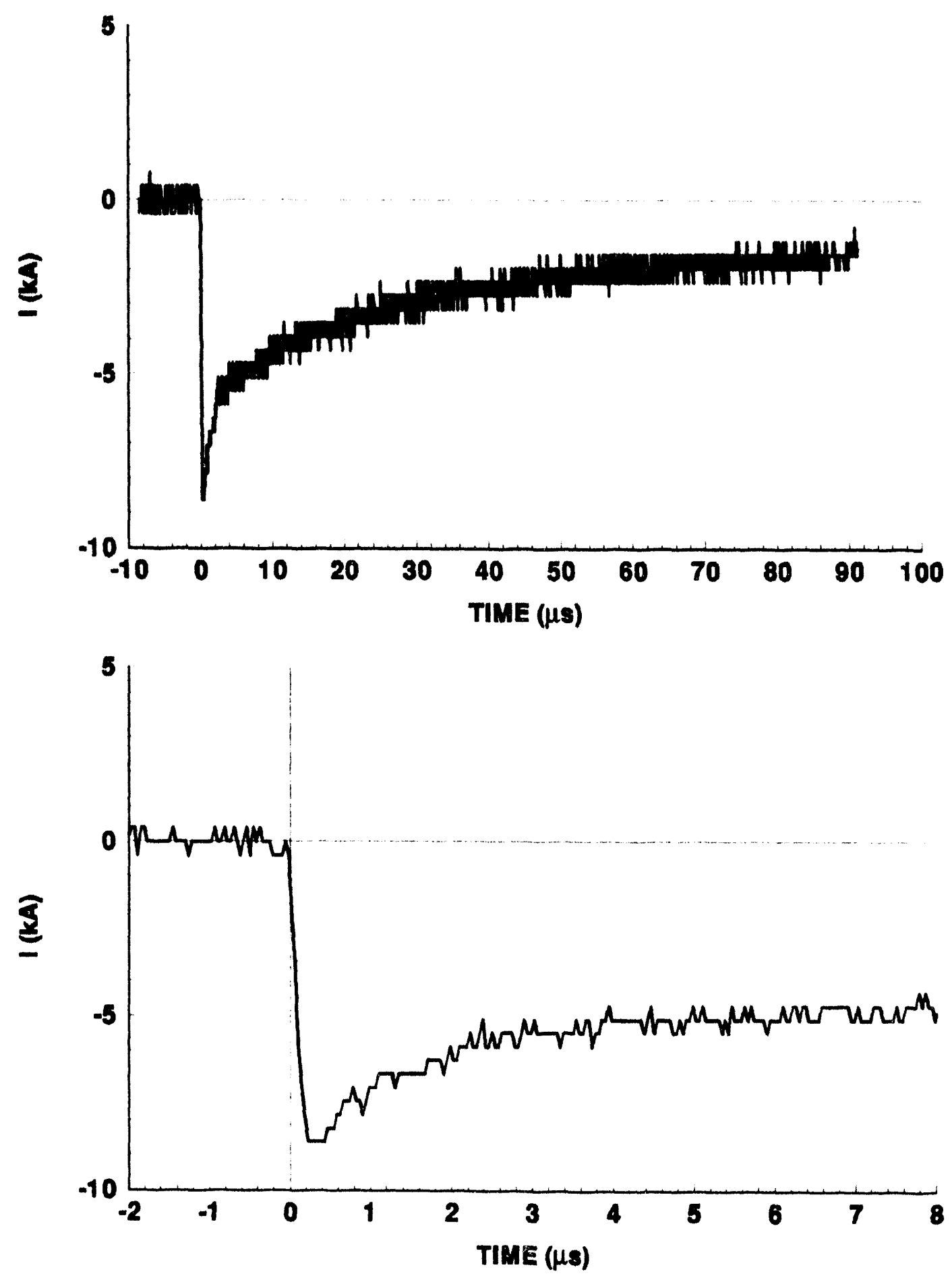


\section{3-15 STROKE 6}

INCIDENT CURRENT
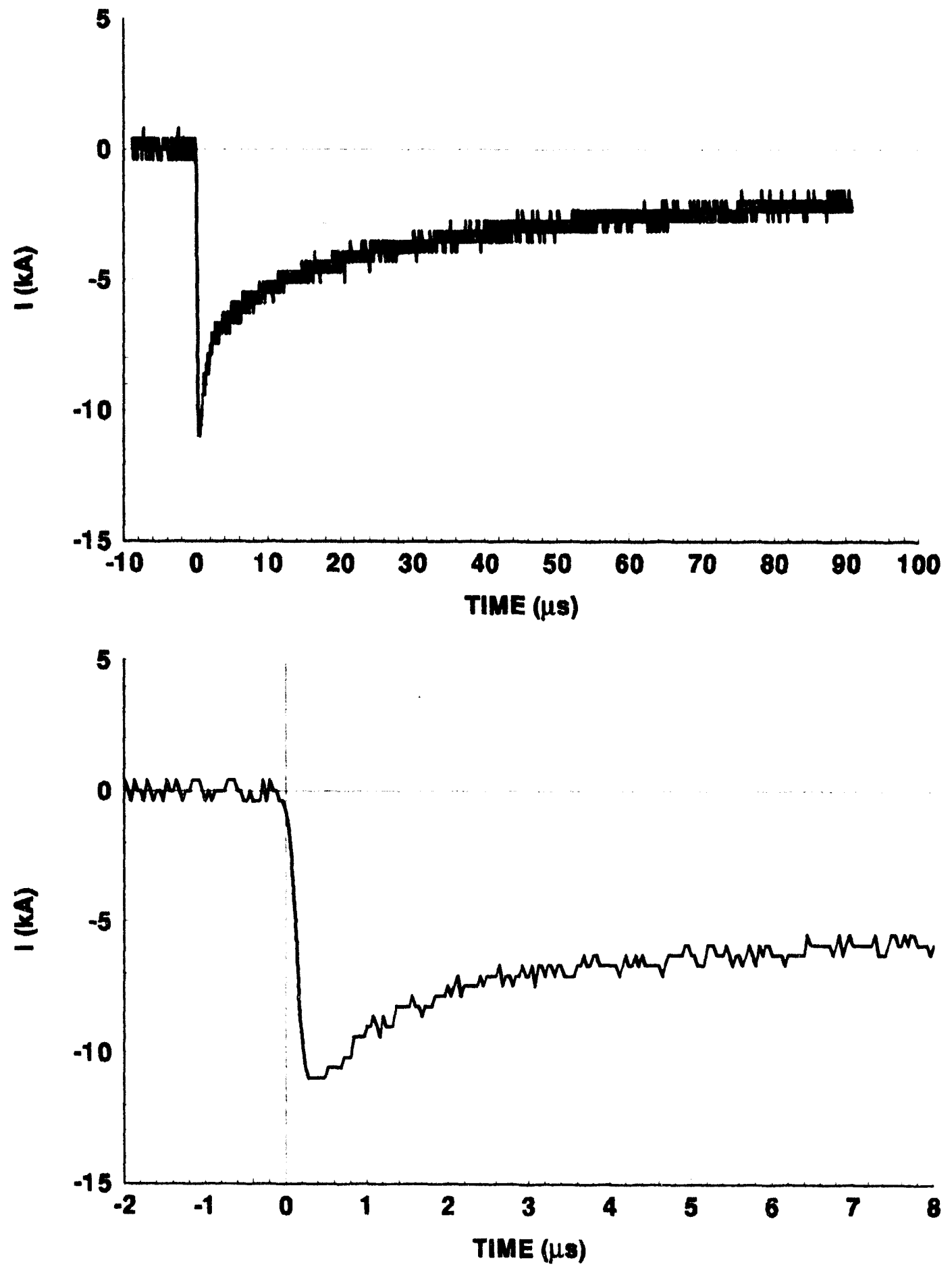
93-15 STROKE 7

INCIDENT CURRENT
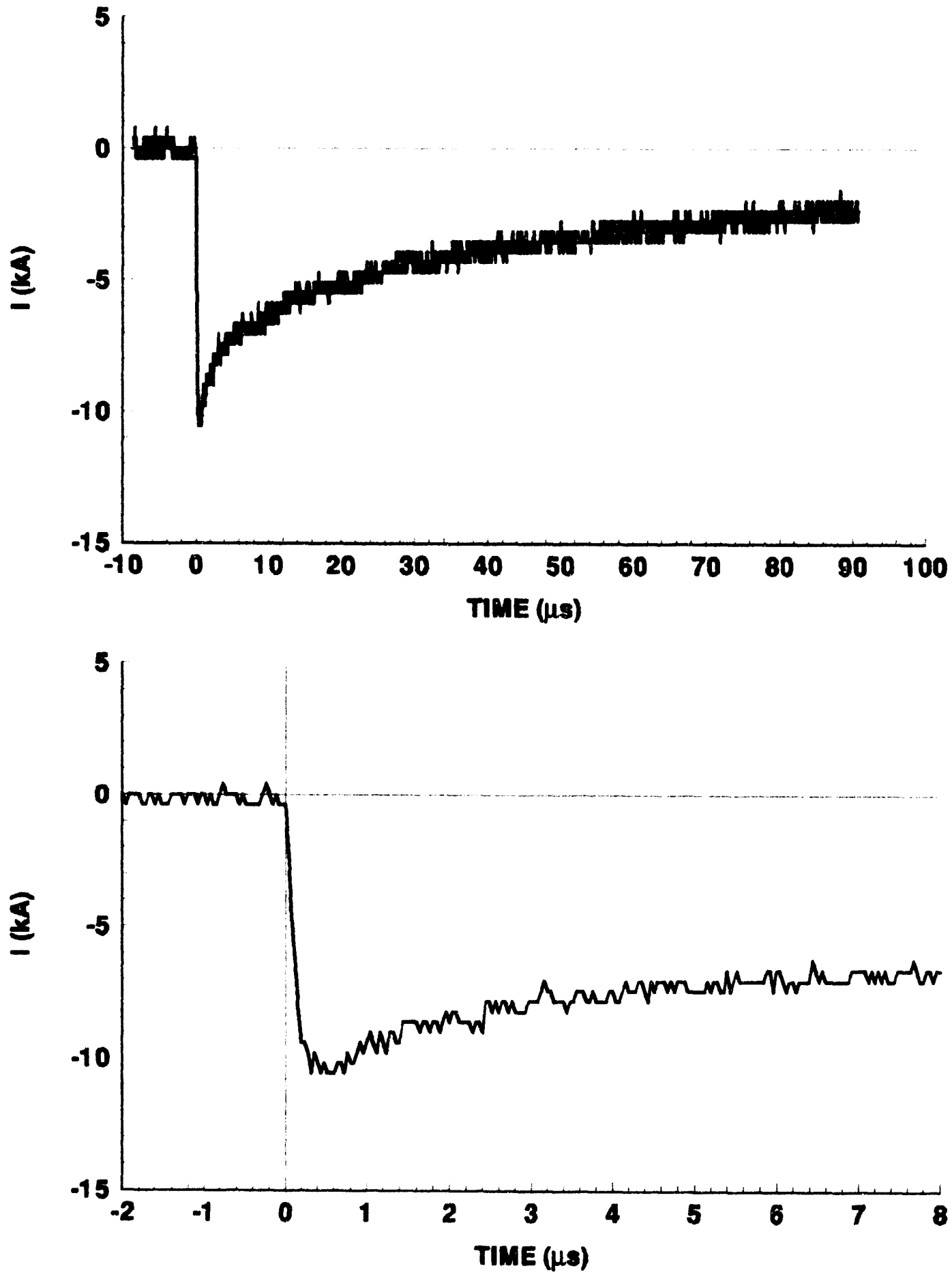


\section{3-15 STROKE 8}

INCIDENT CURRENT
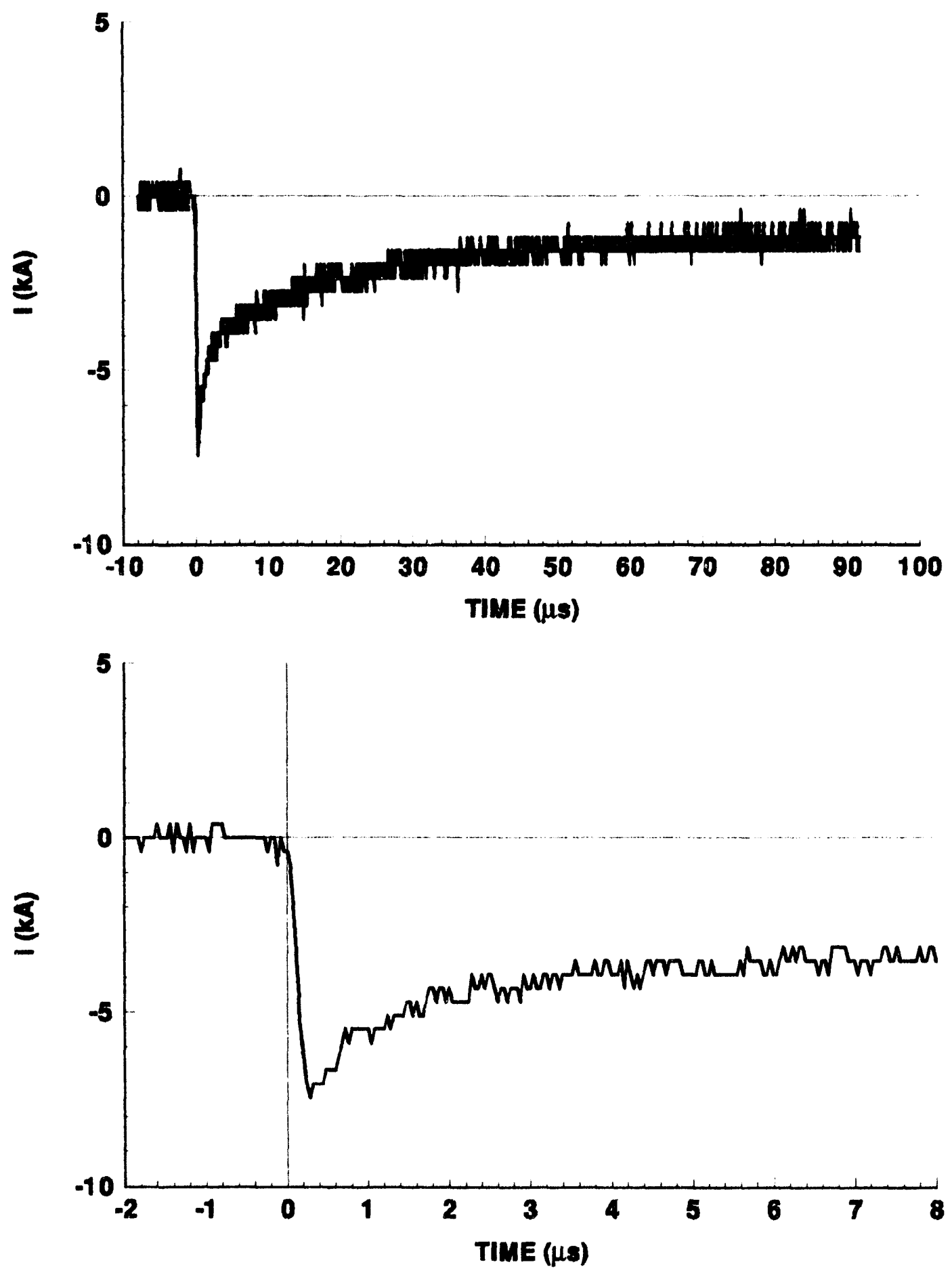


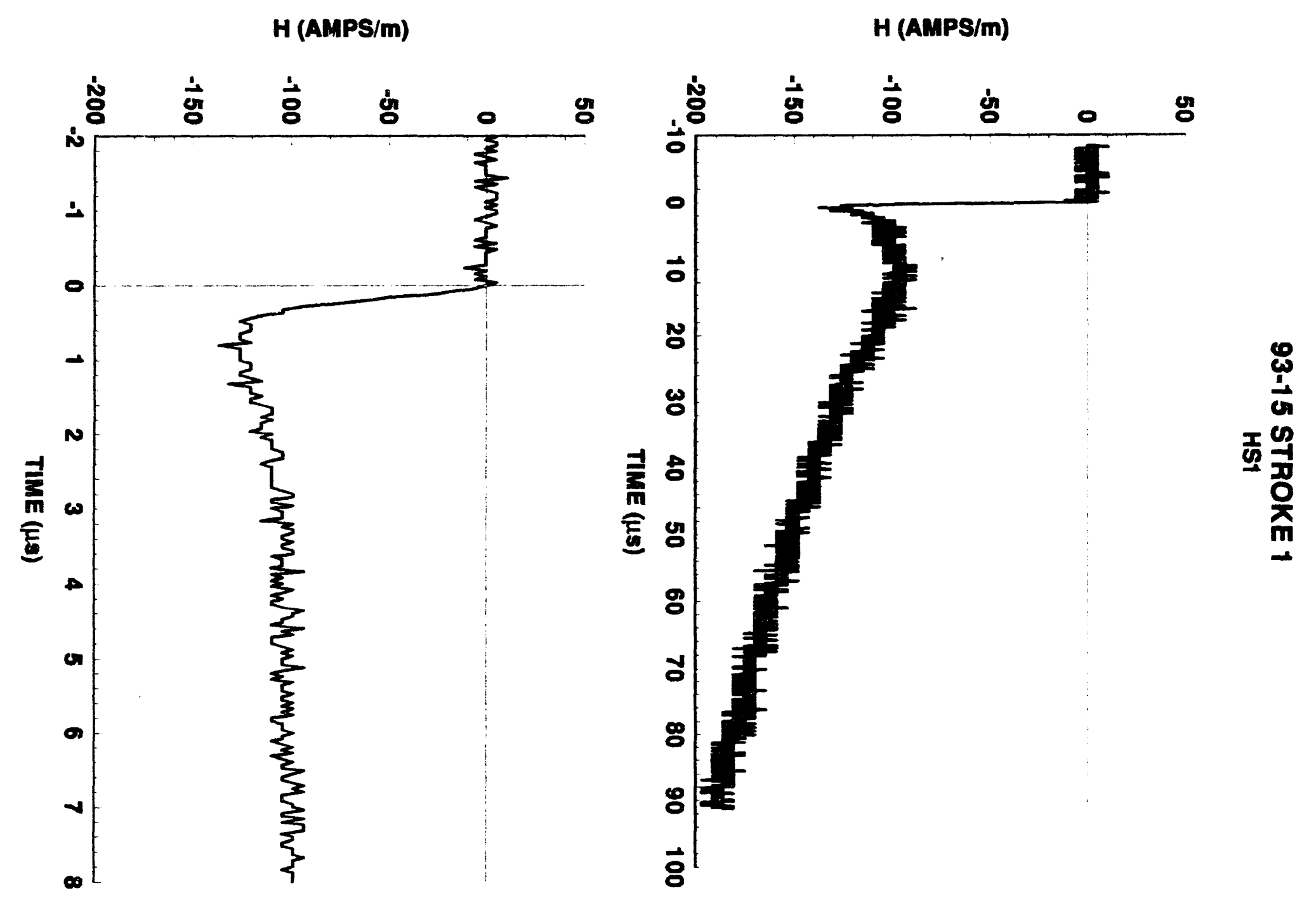



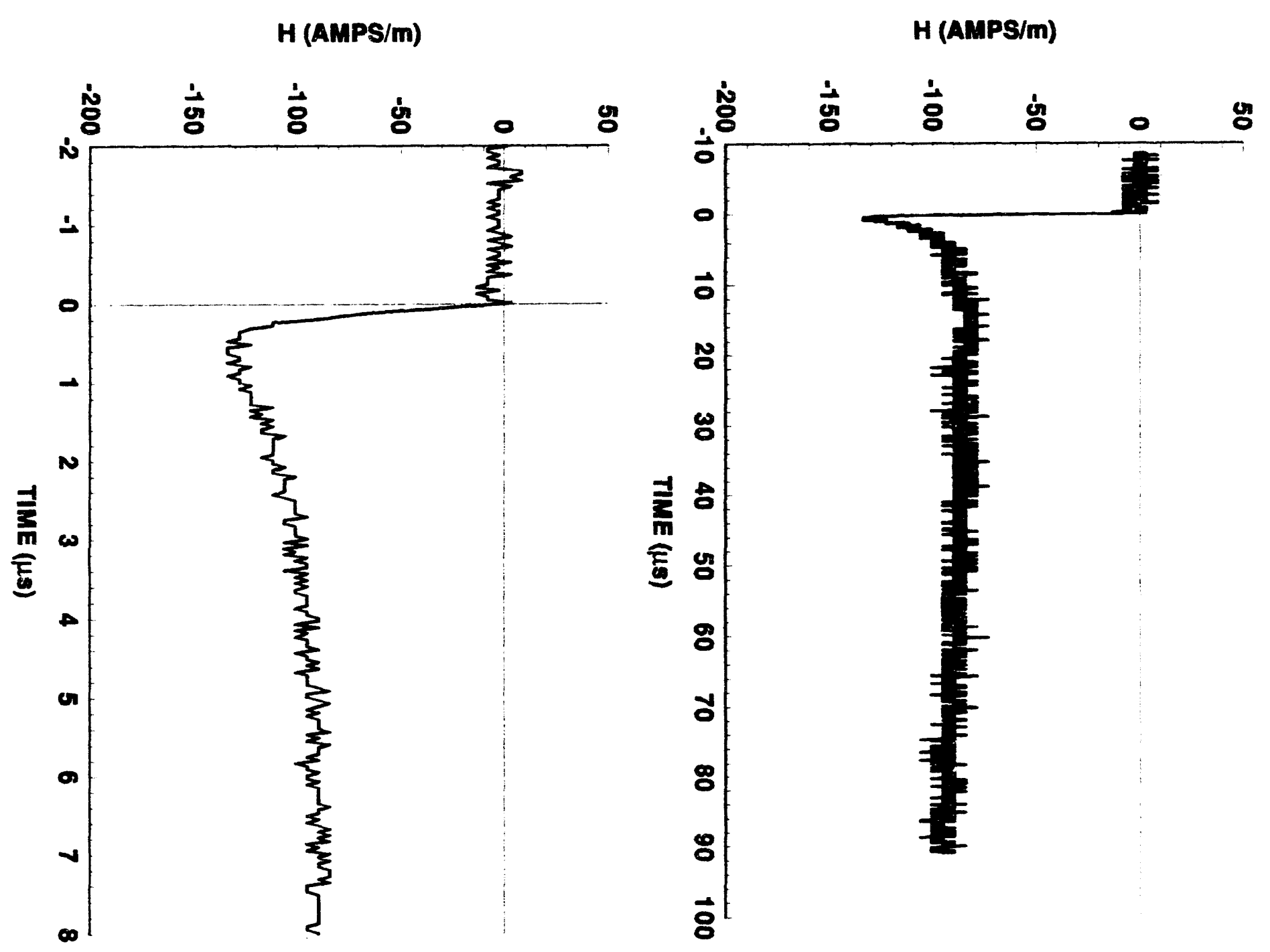


\section{3-15 STROKE 3}

HS1
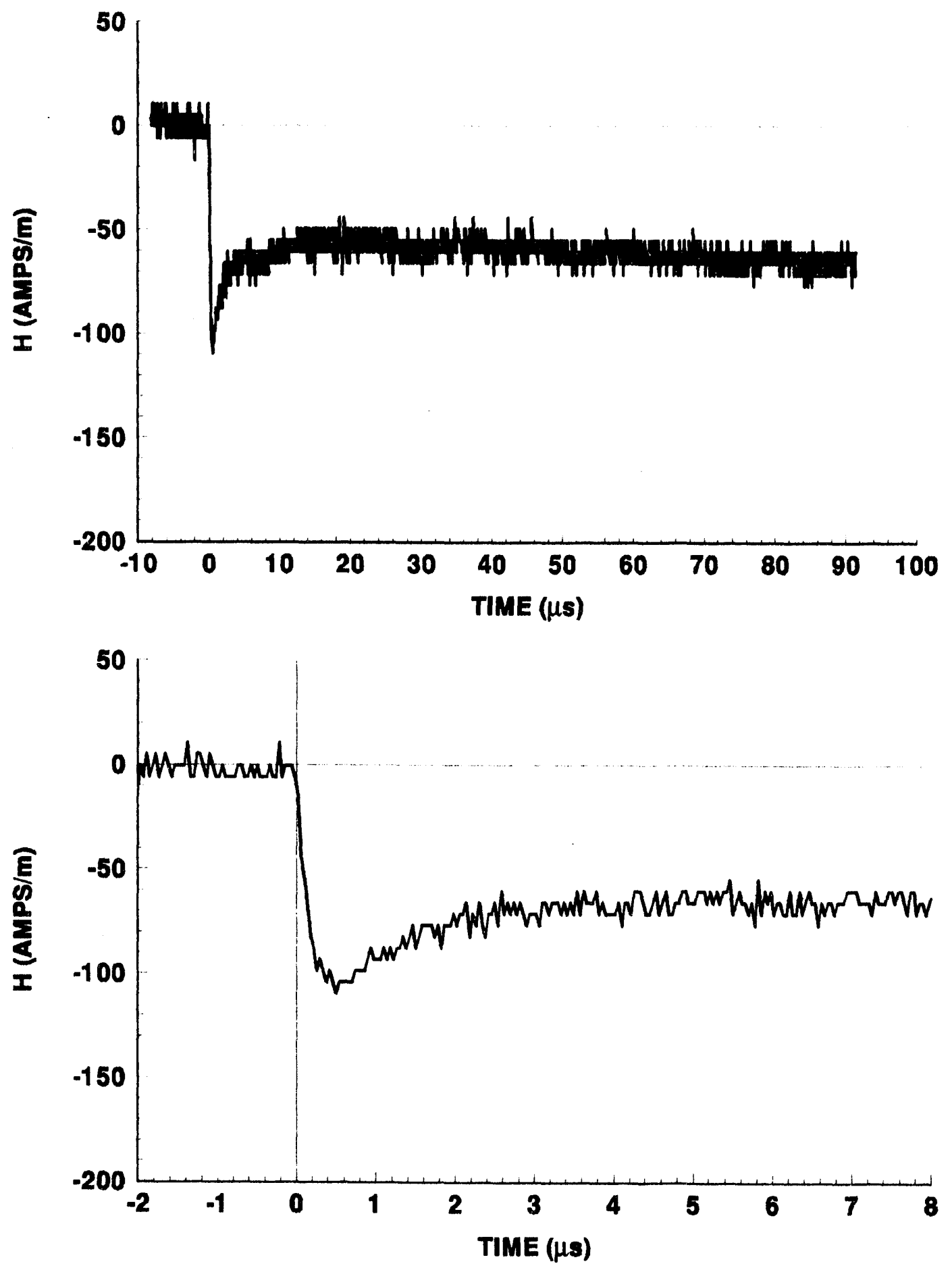


\section{3-15 STROKE 4}

HS1
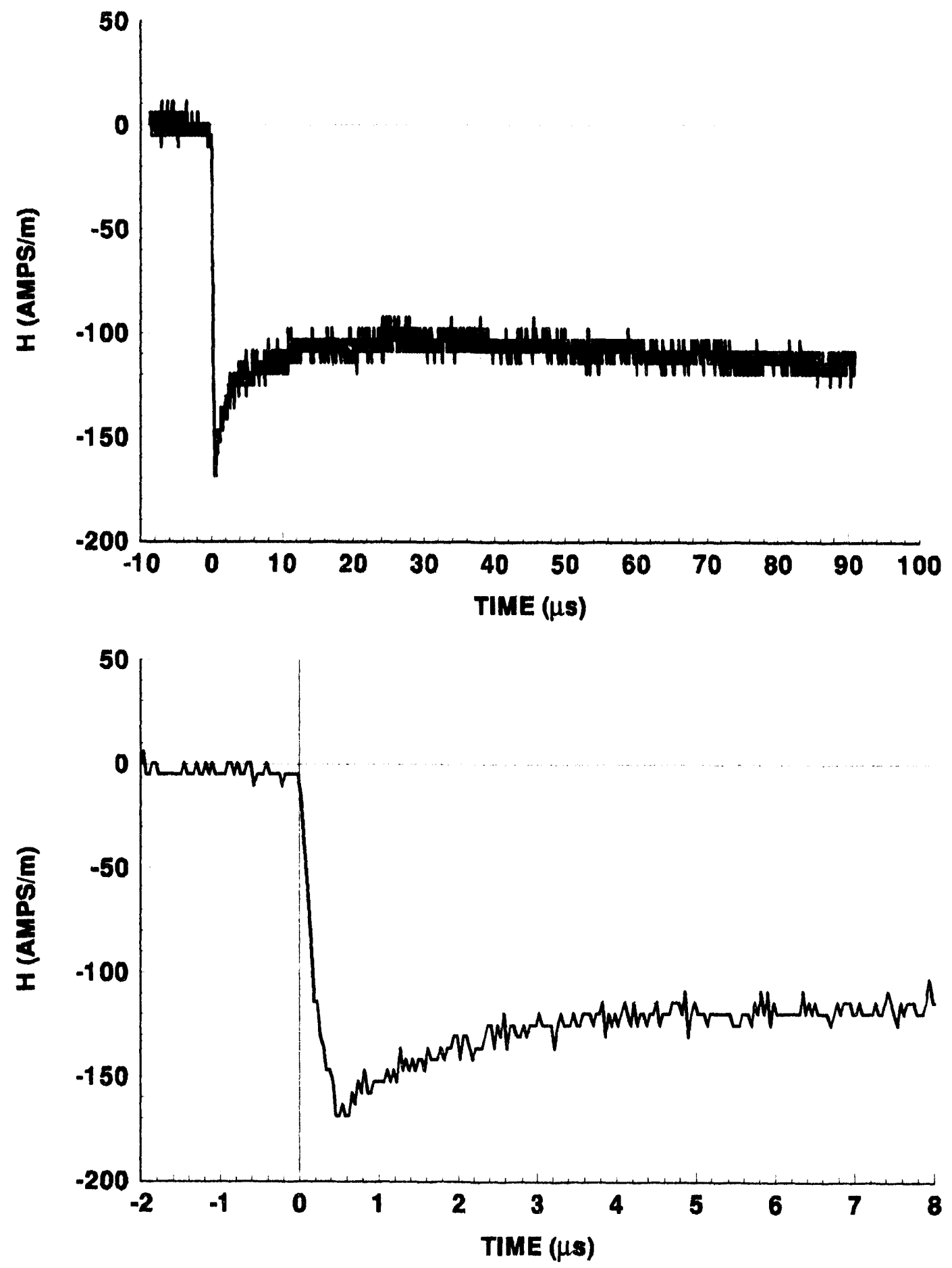
93-15 STROKE 5

HS1
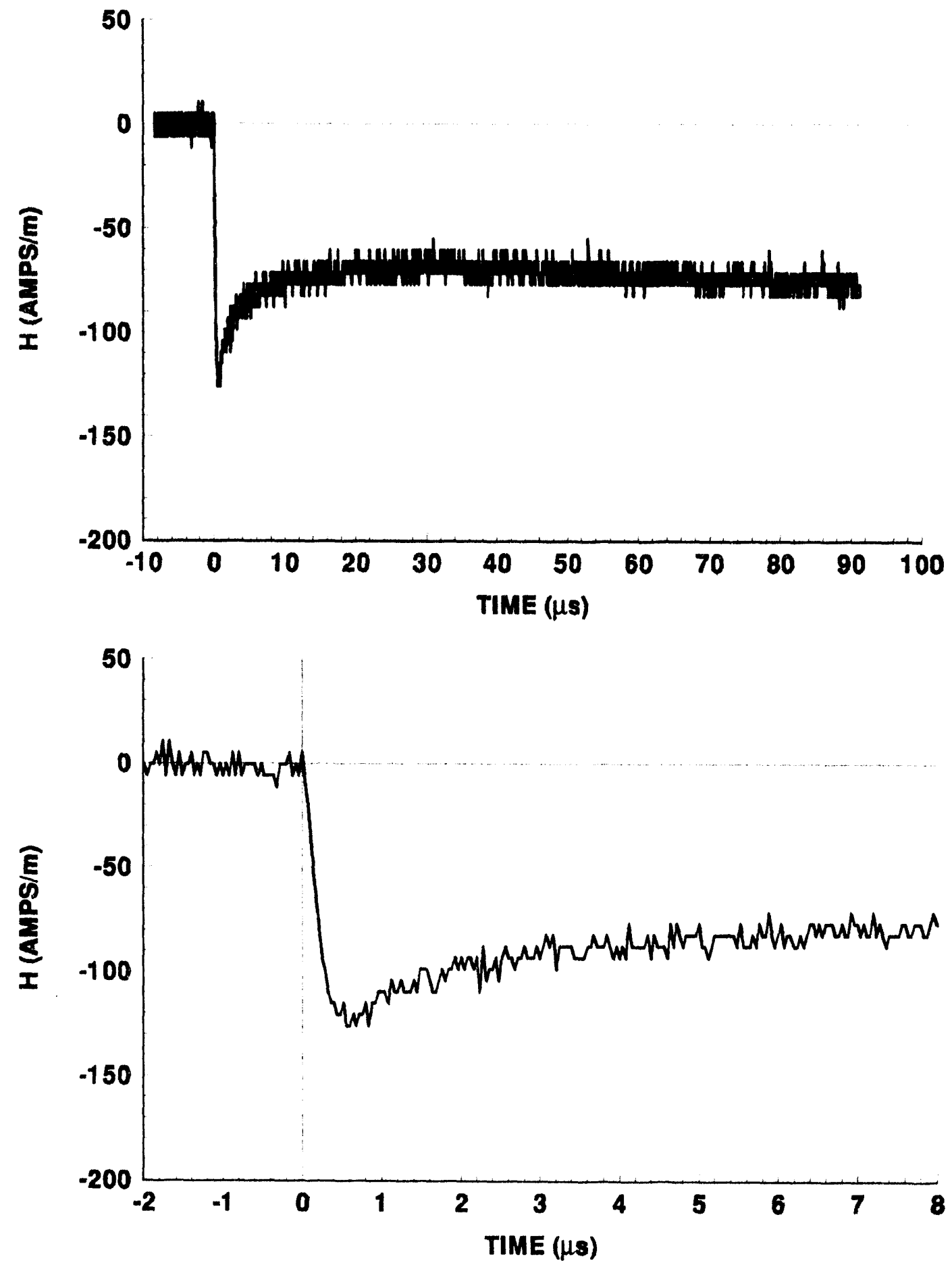


\section{3-15 STROKE 6}

HS1
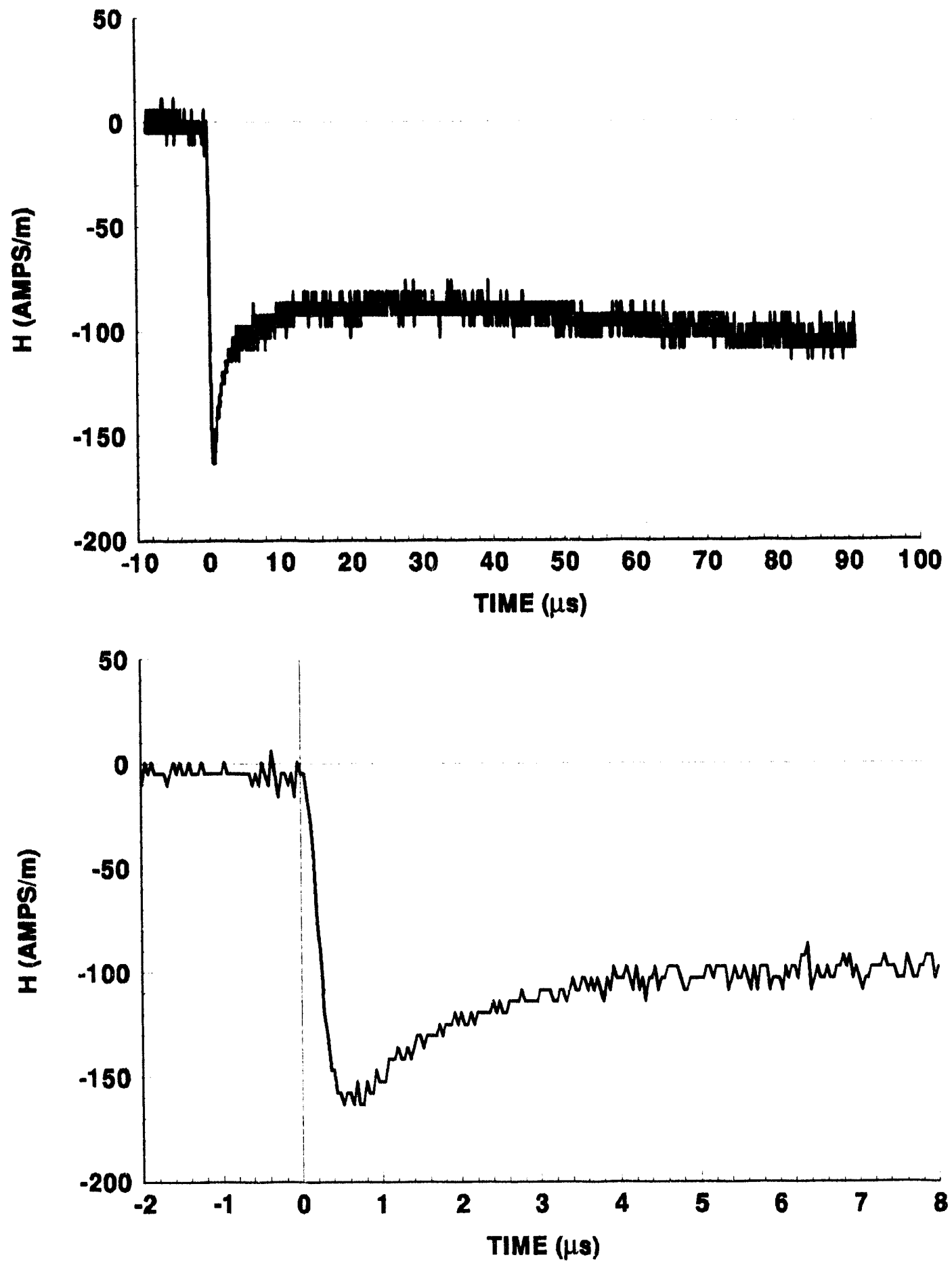
93-15 STROKE 7

HS1
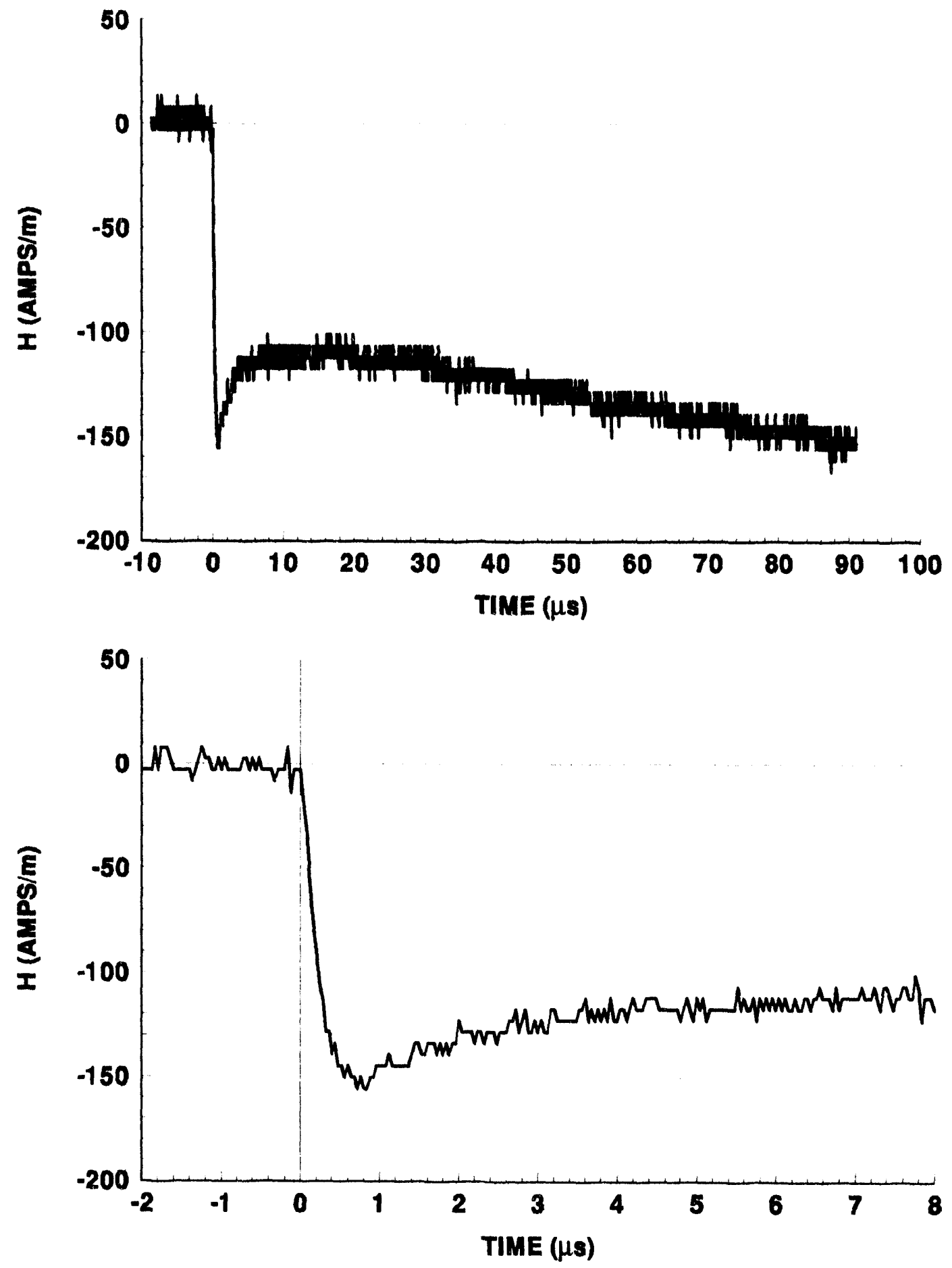

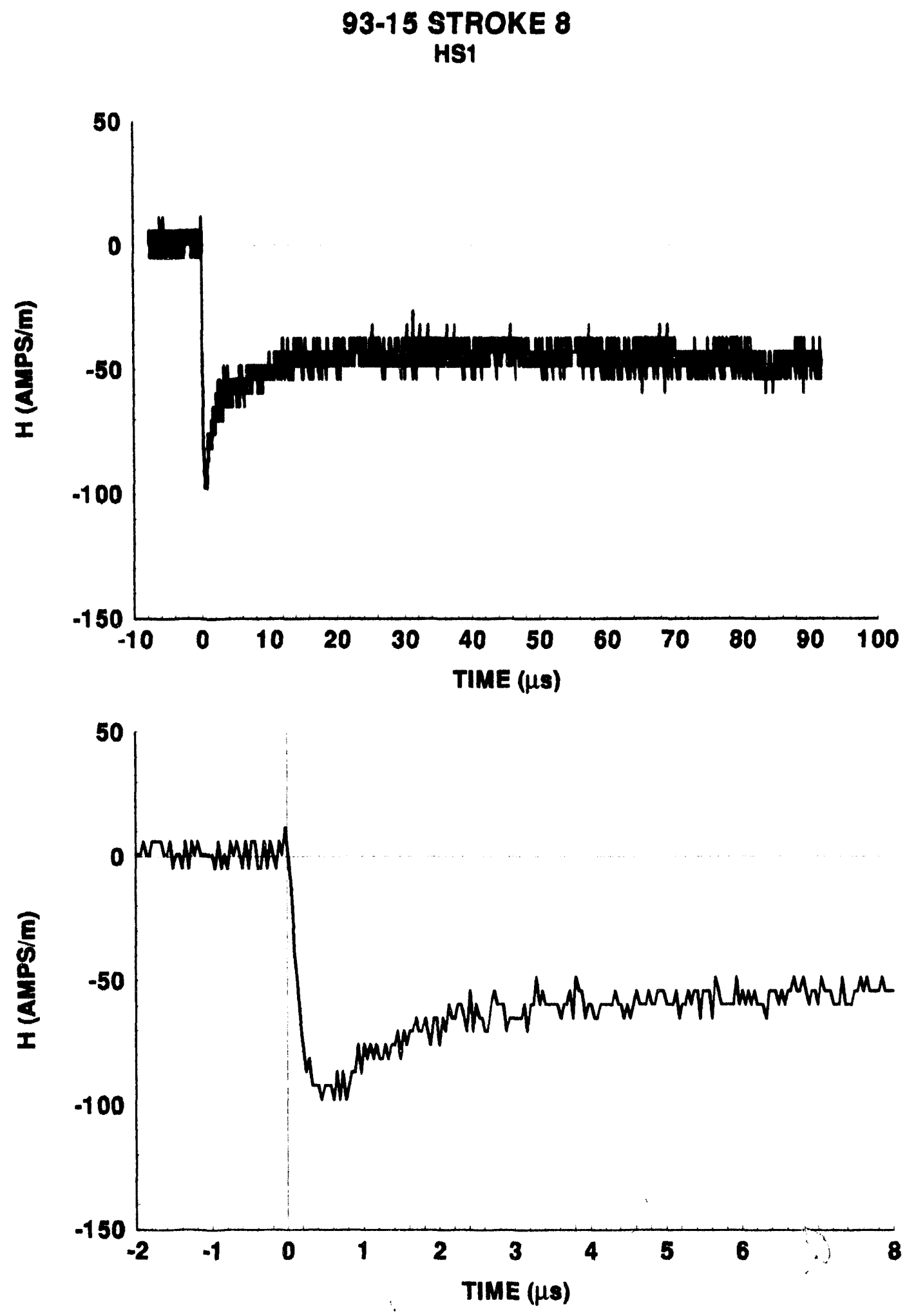

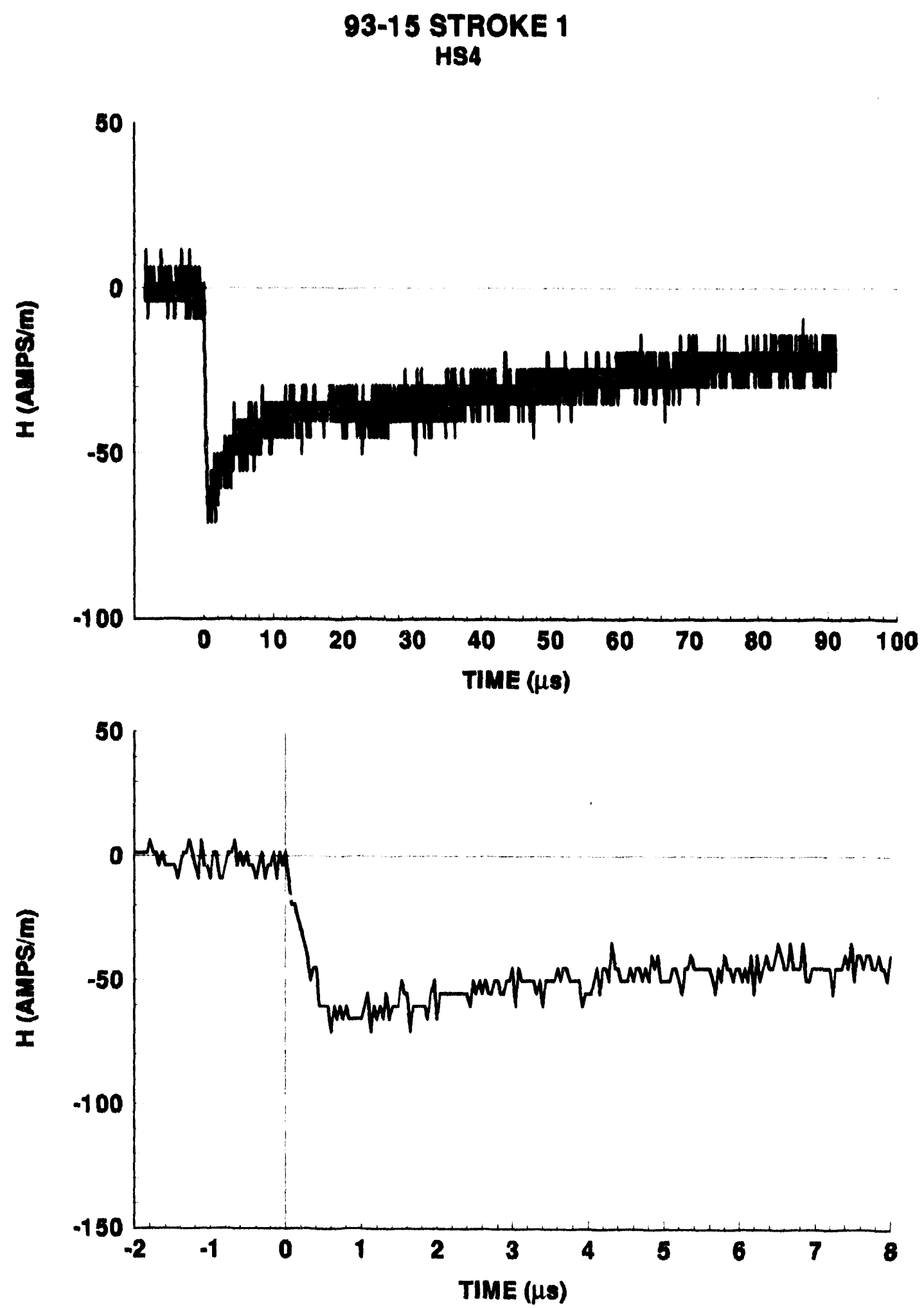
93-15 STROKE 2

HS4
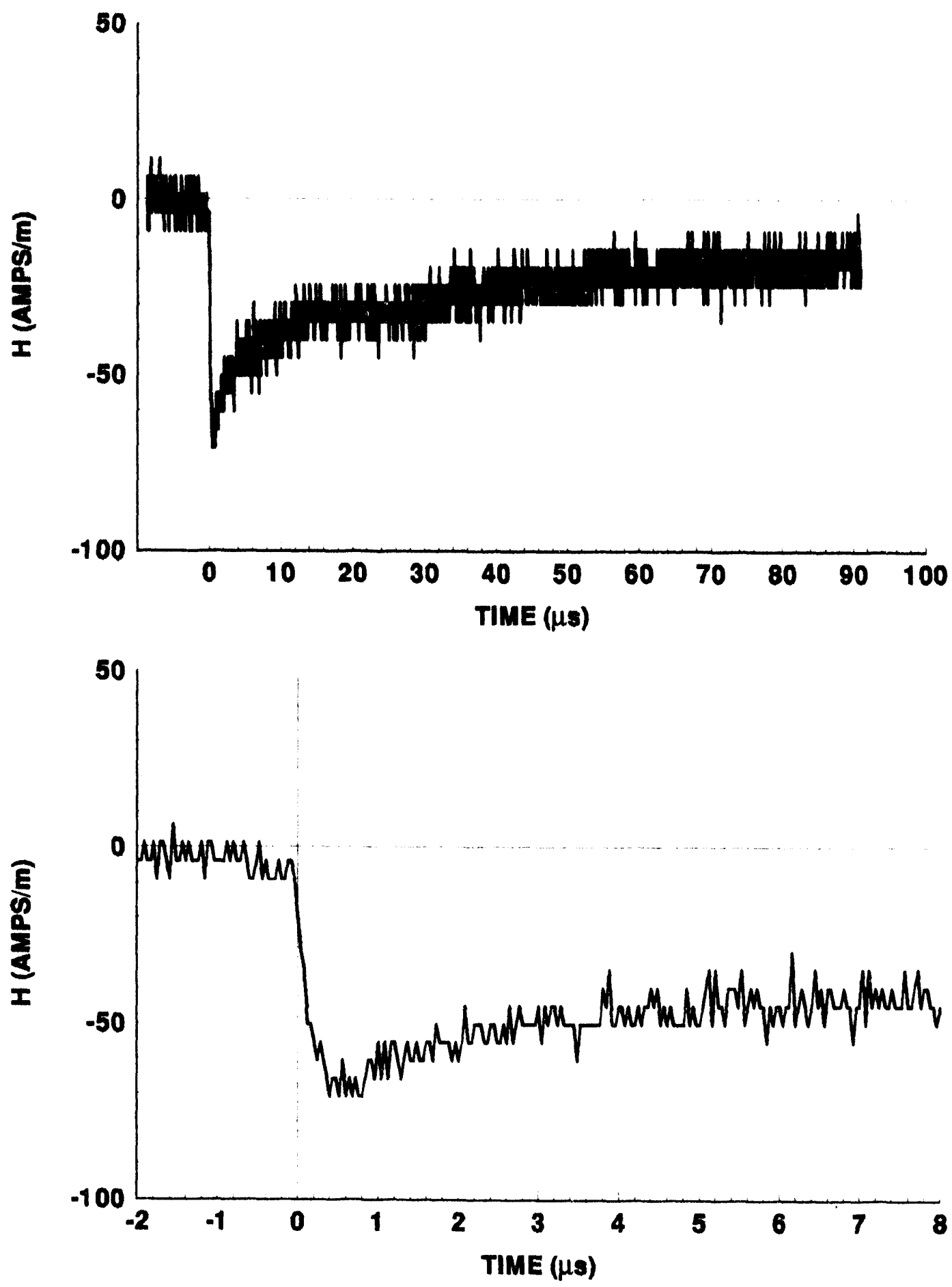

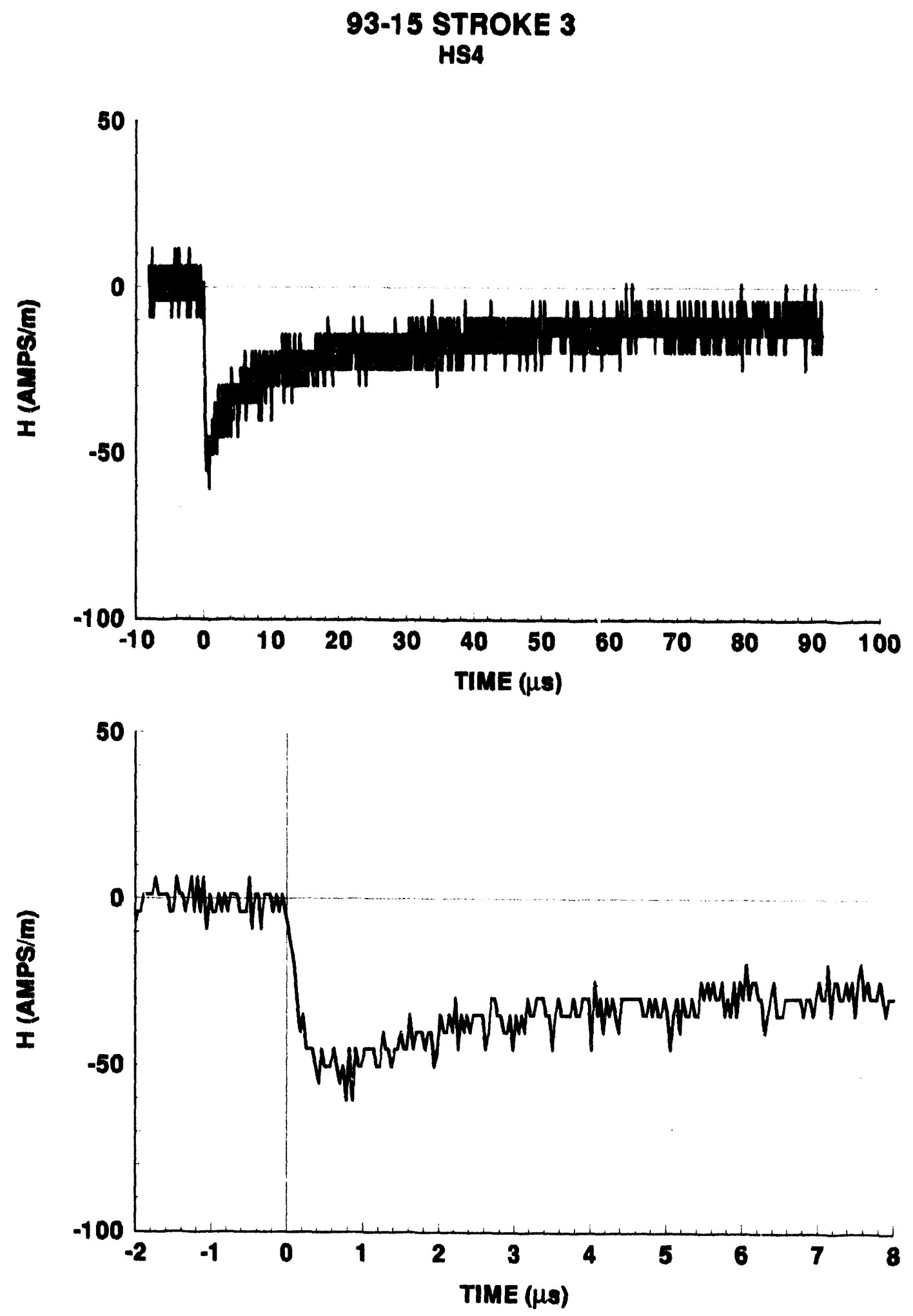


\section{3-15 STROKE 4}

HS4
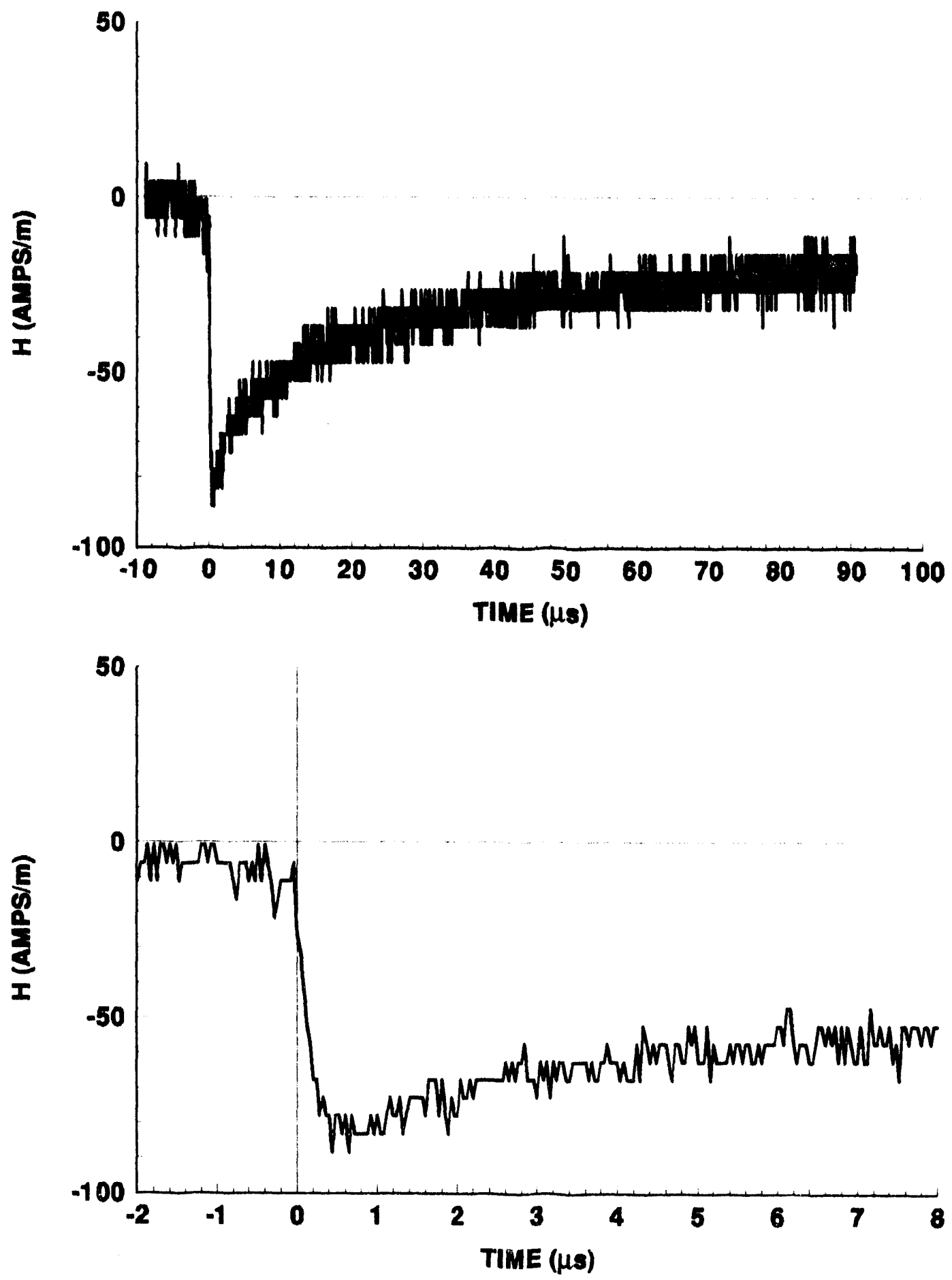


\section{3-15 STROKE 5}

HS4
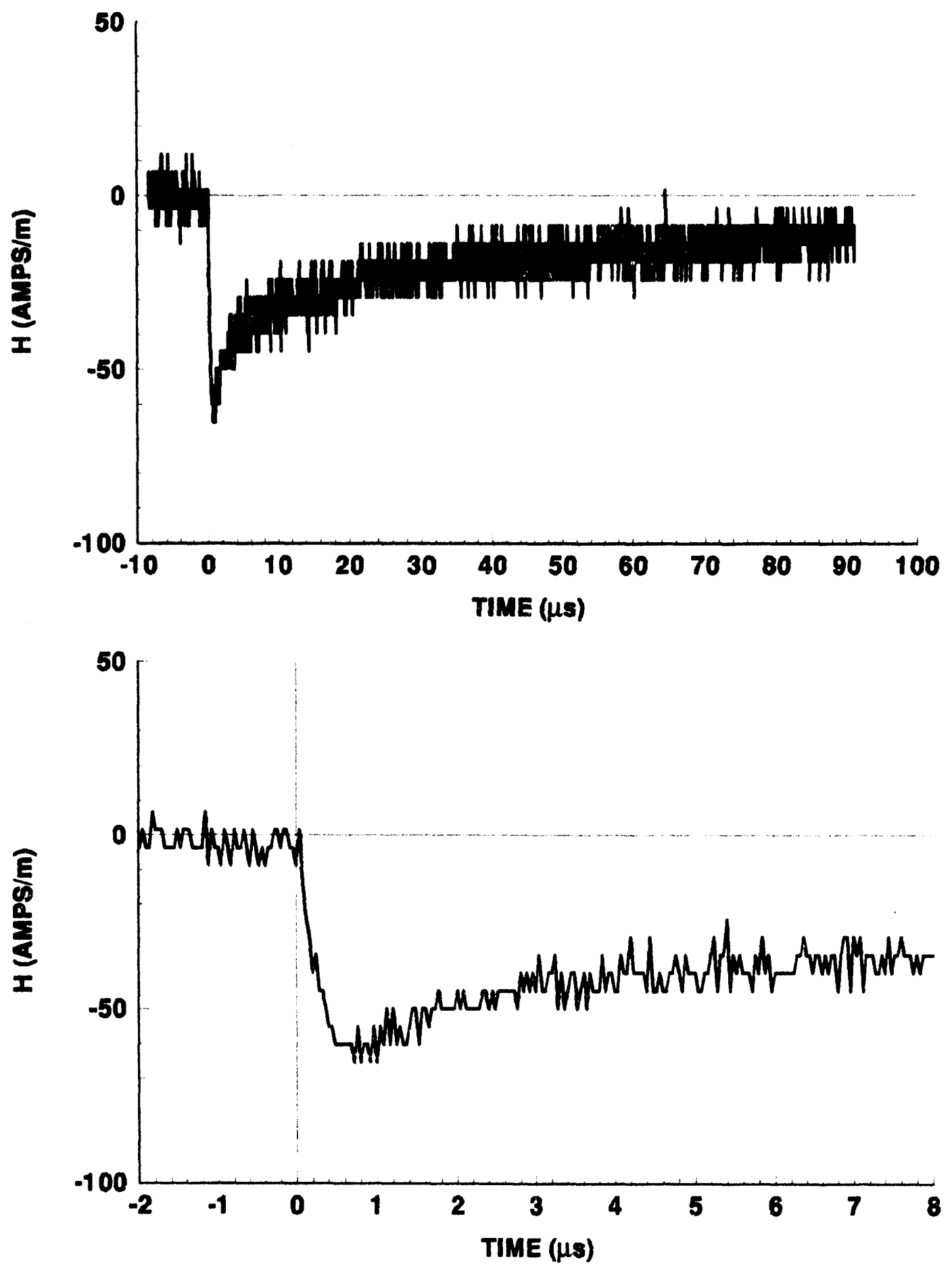

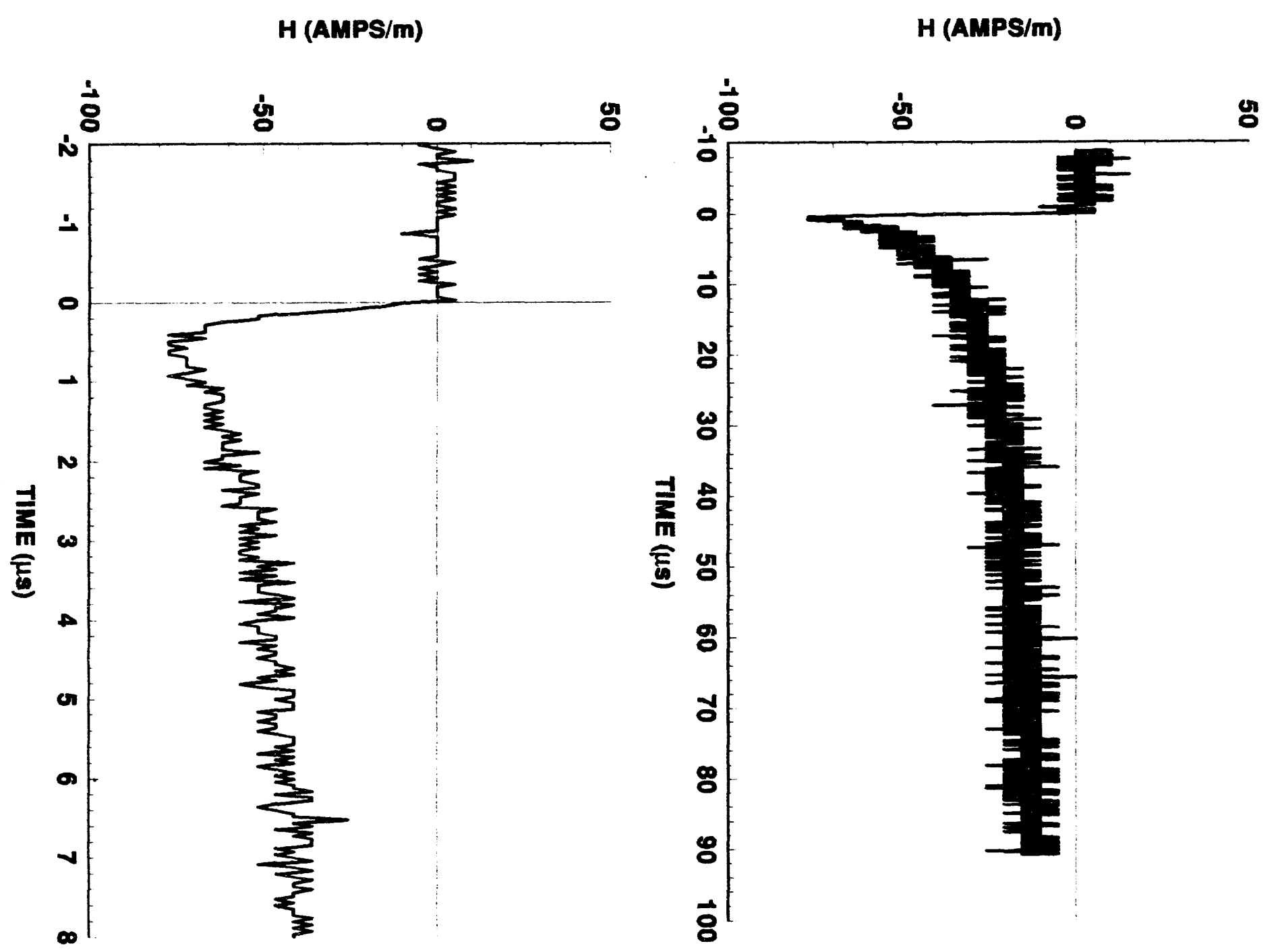


\section{3-15 STROKE 7}

HS4
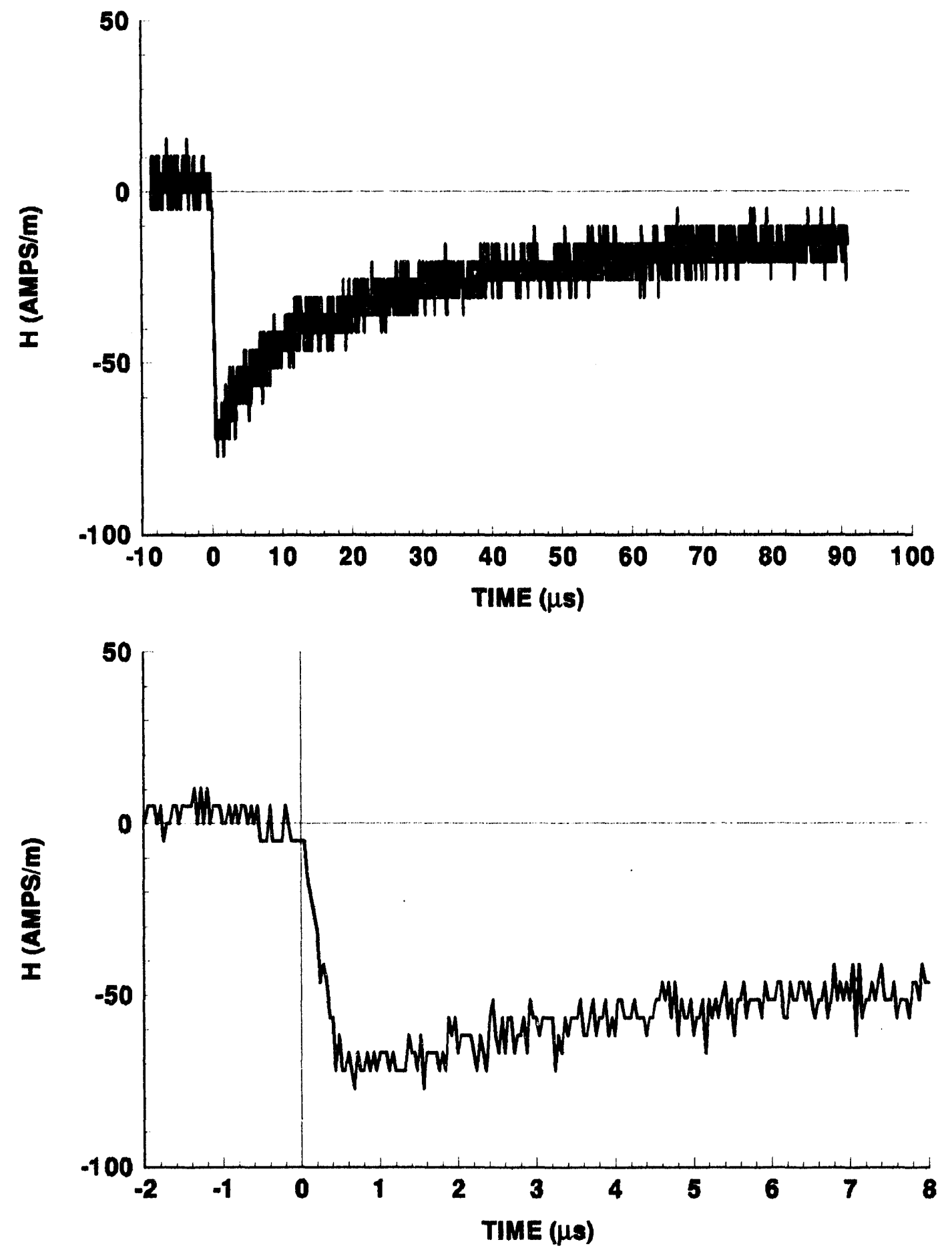

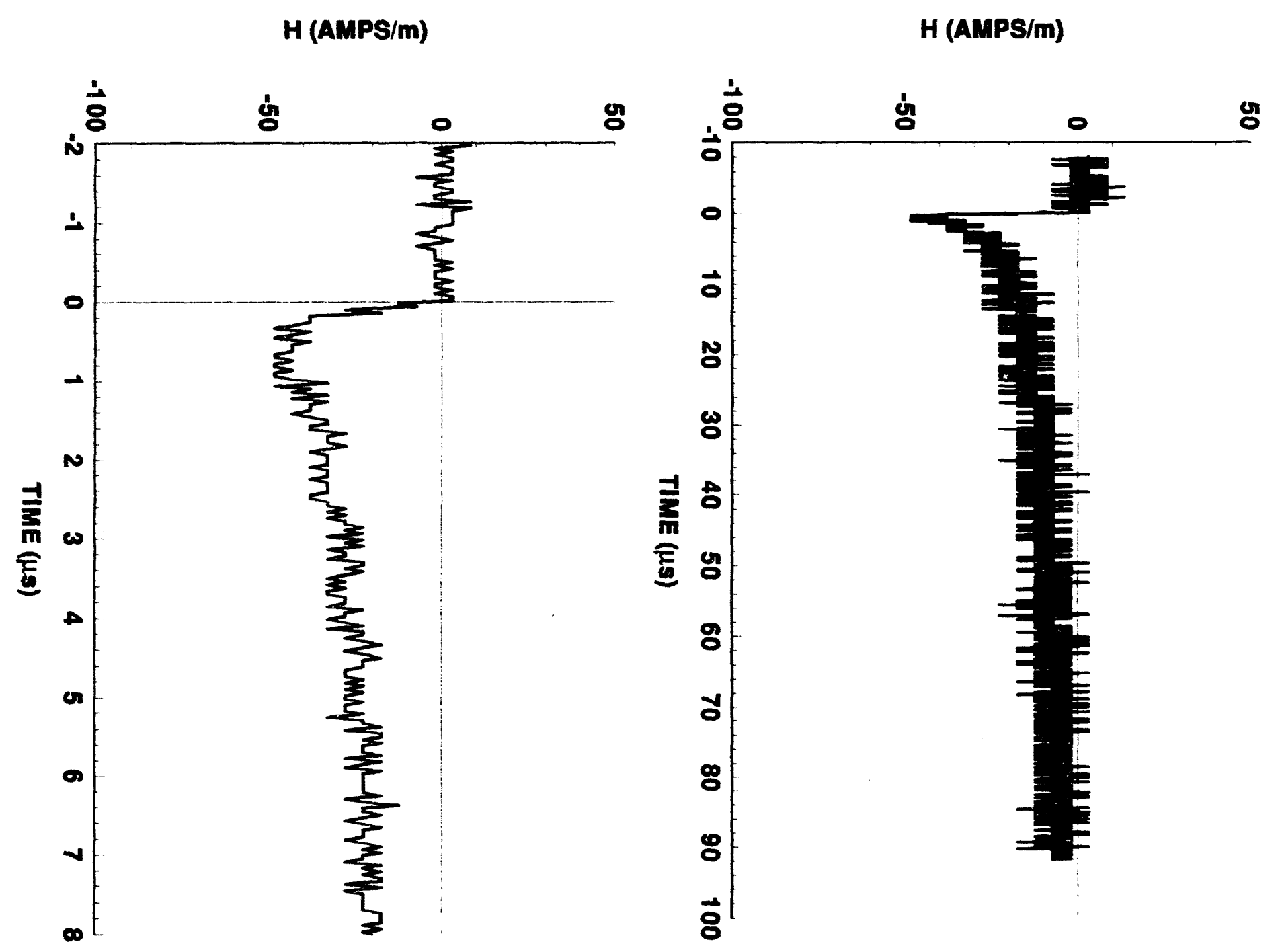

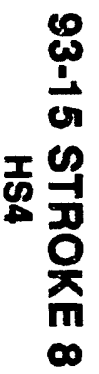




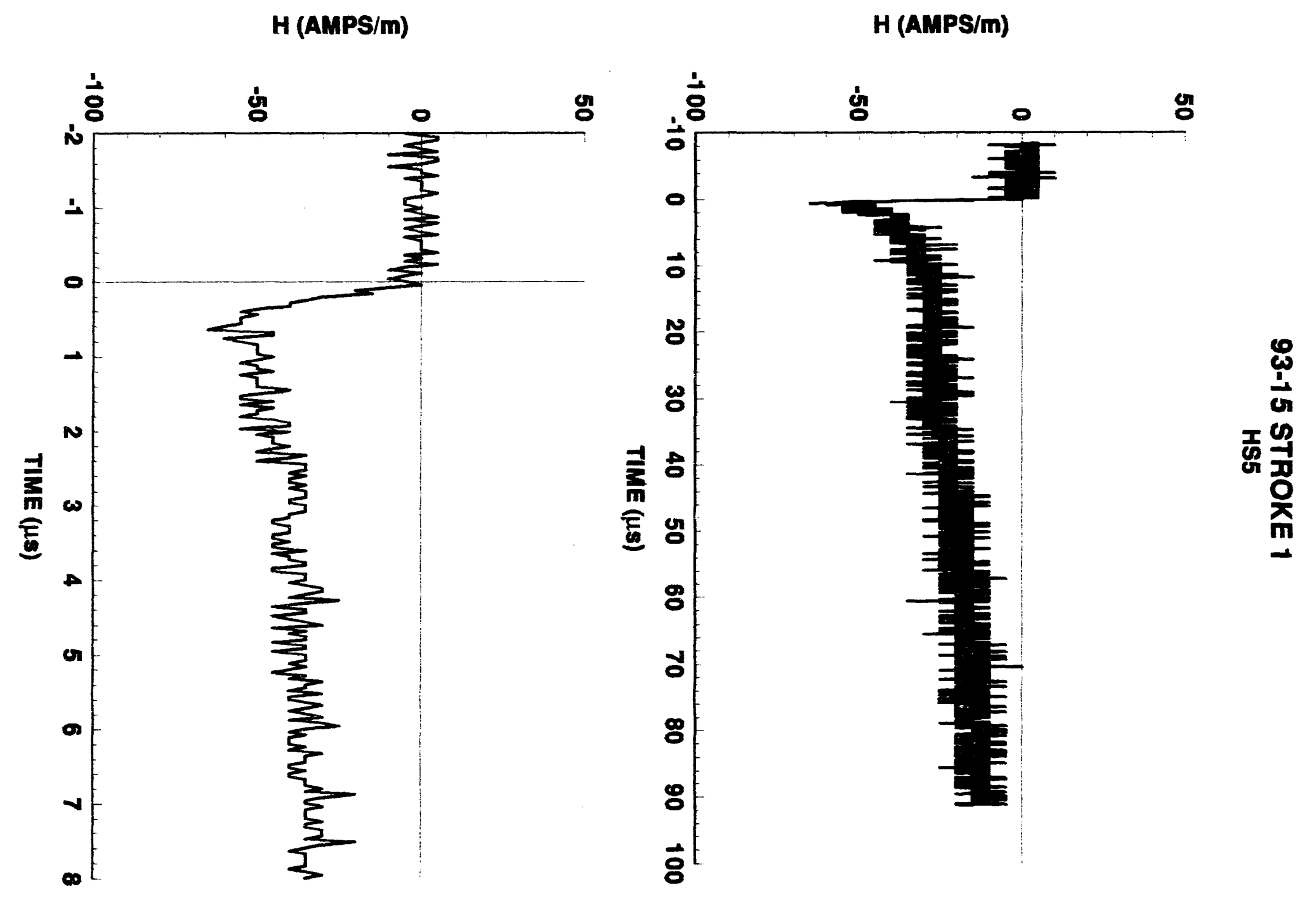




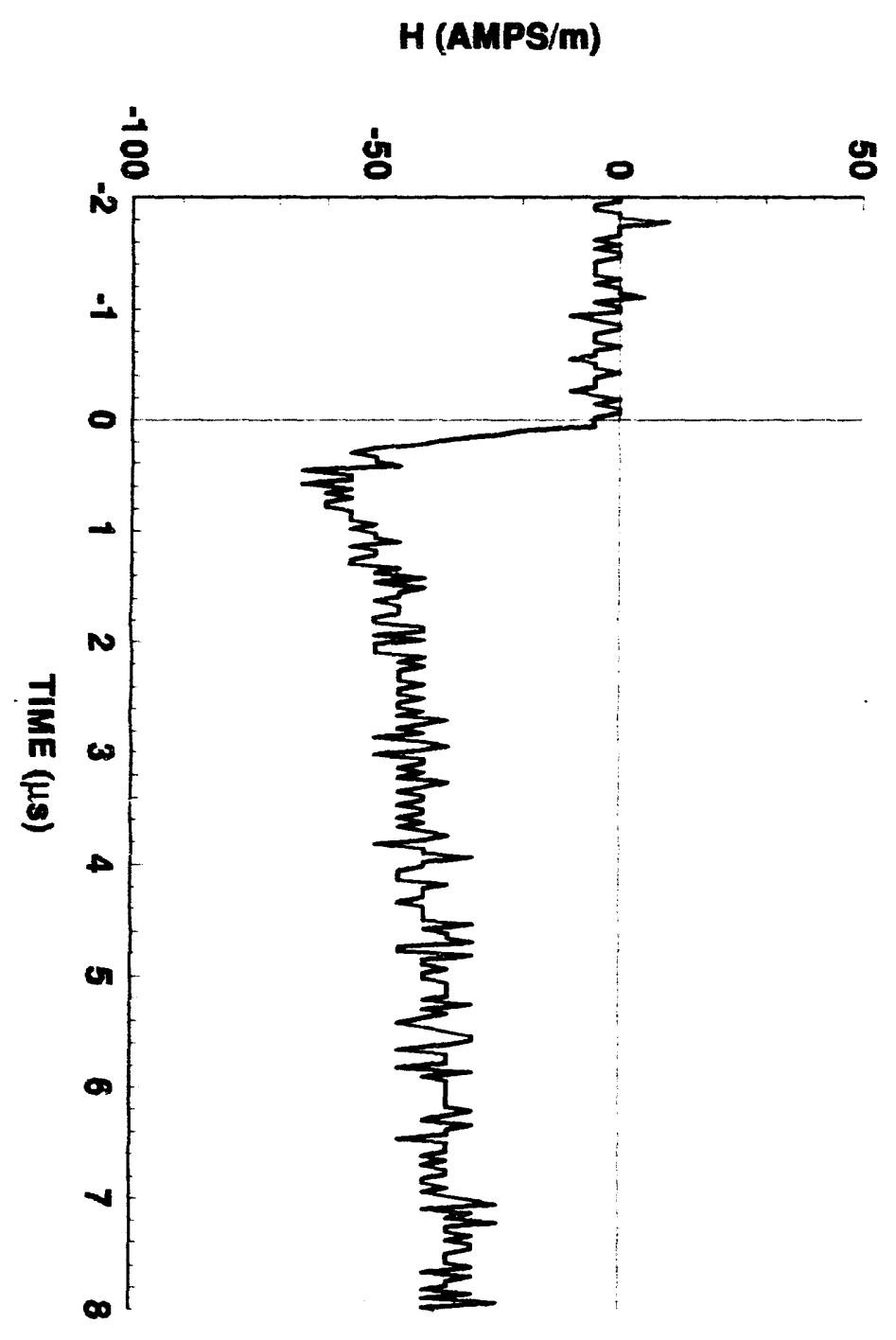

H (AMPS/m)

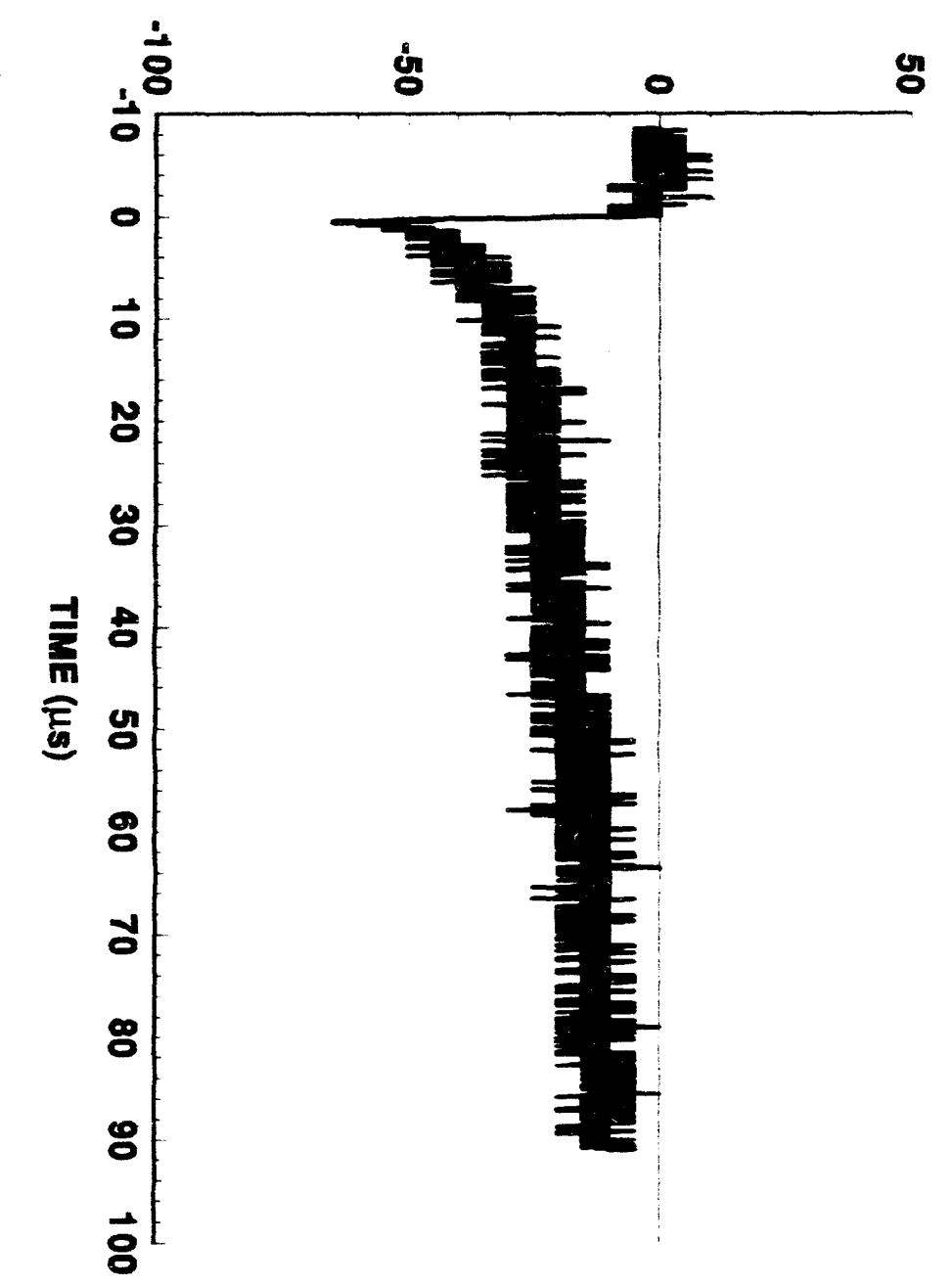

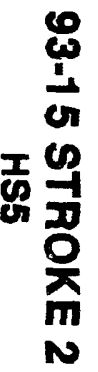




\section{3-15 STROKE 3 \\ HS5}
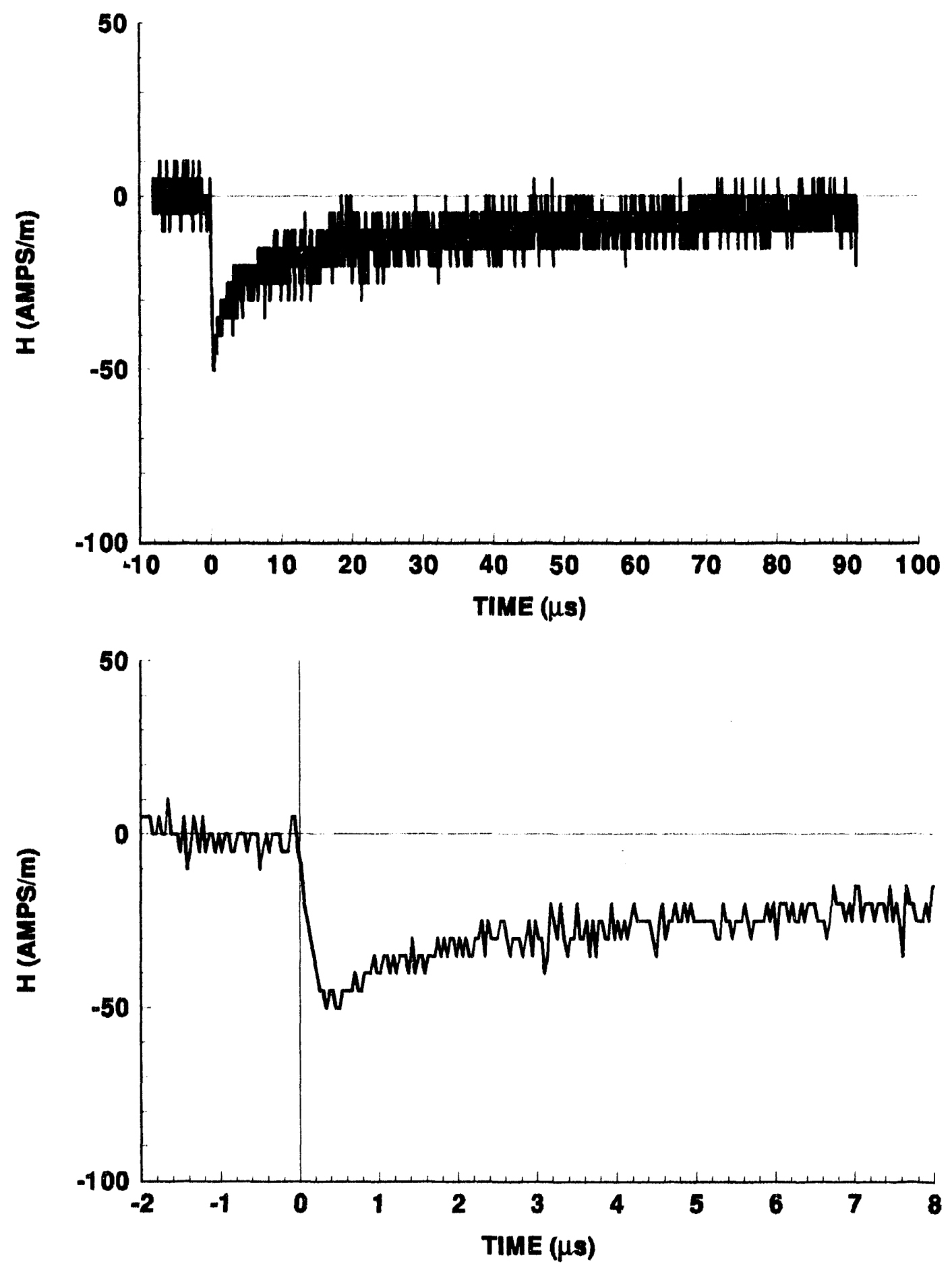

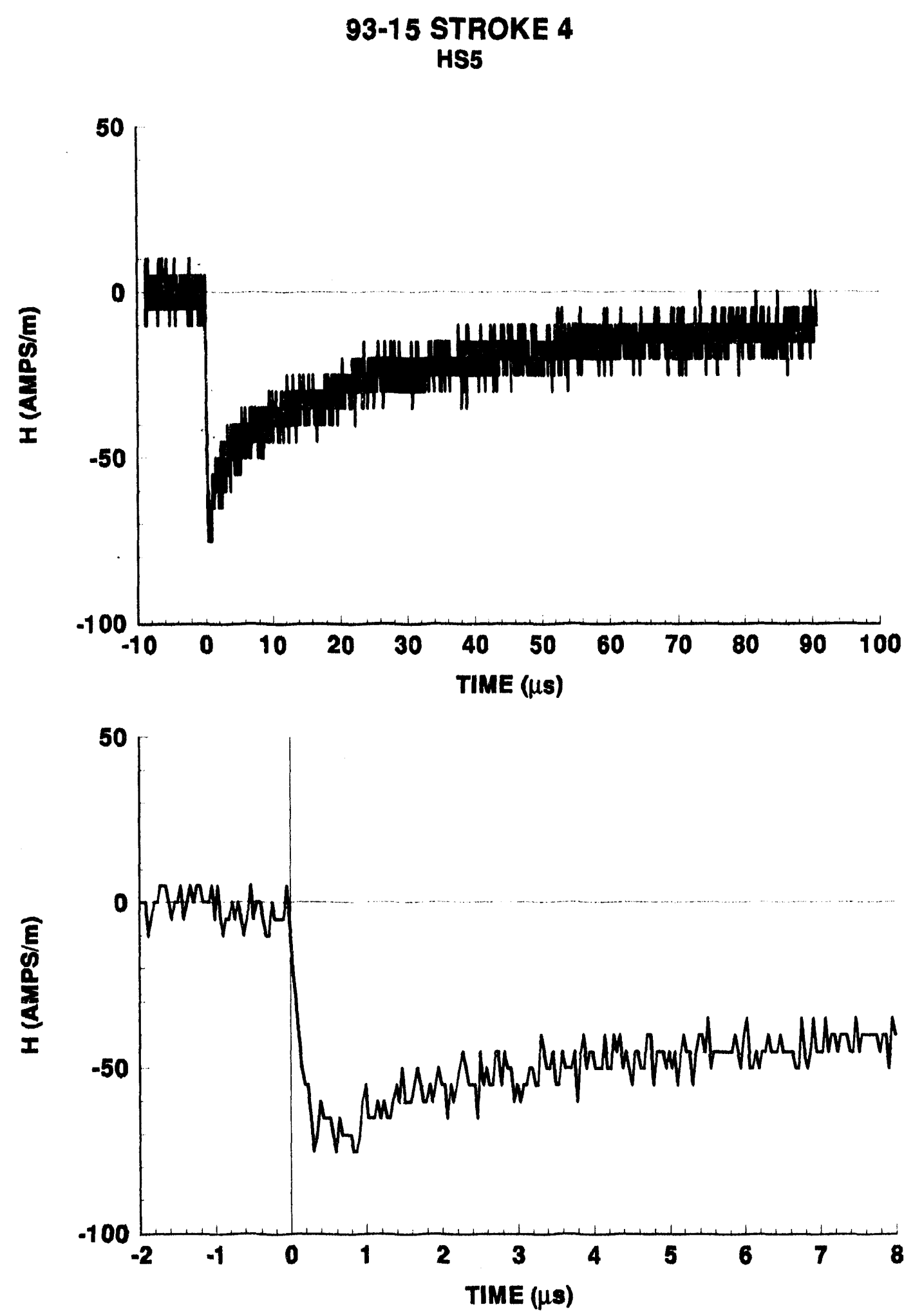


\section{3-15 STROKE 5}

HS5
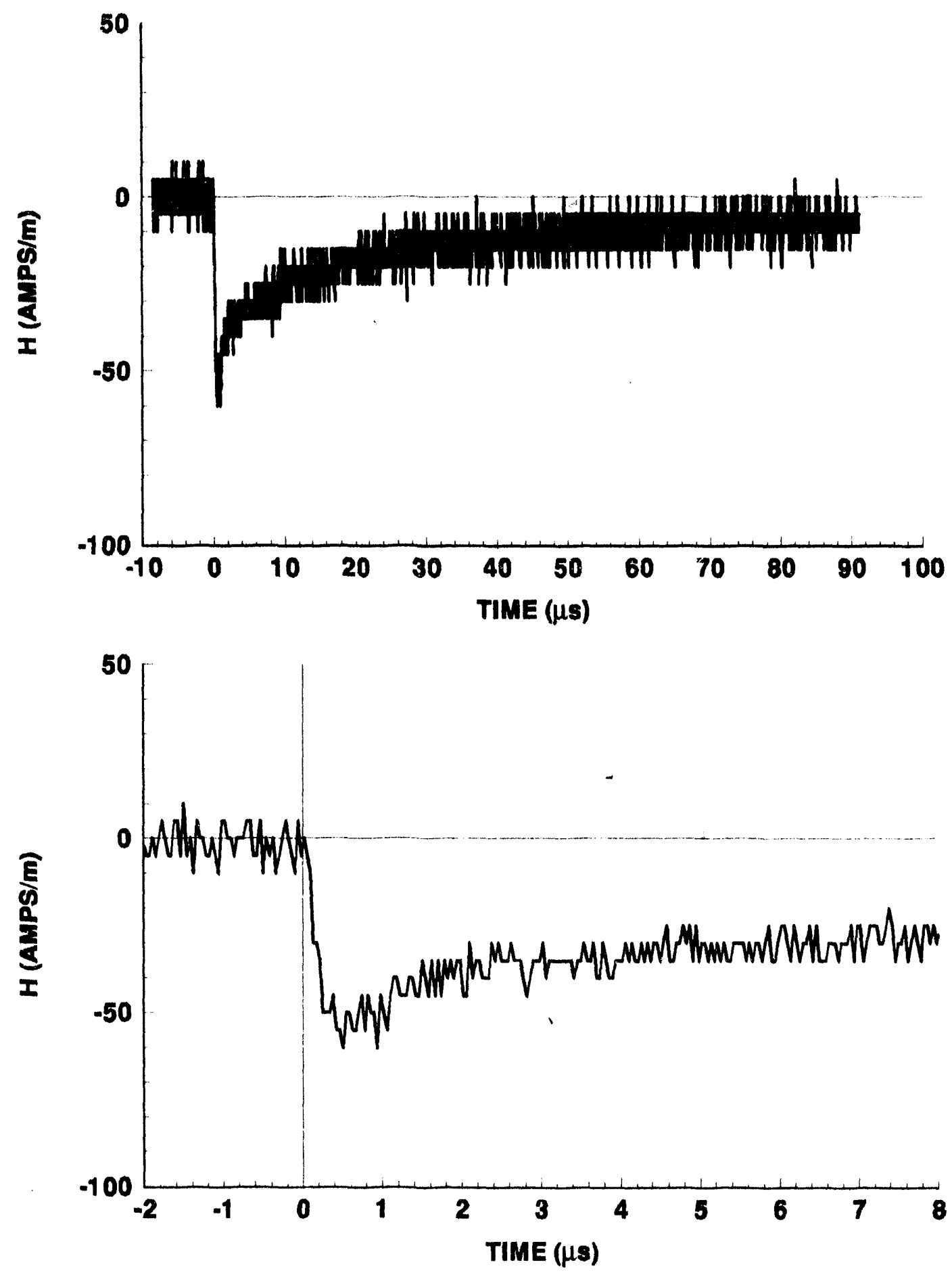


\section{3-15 STROKE 6 \\ HS5}
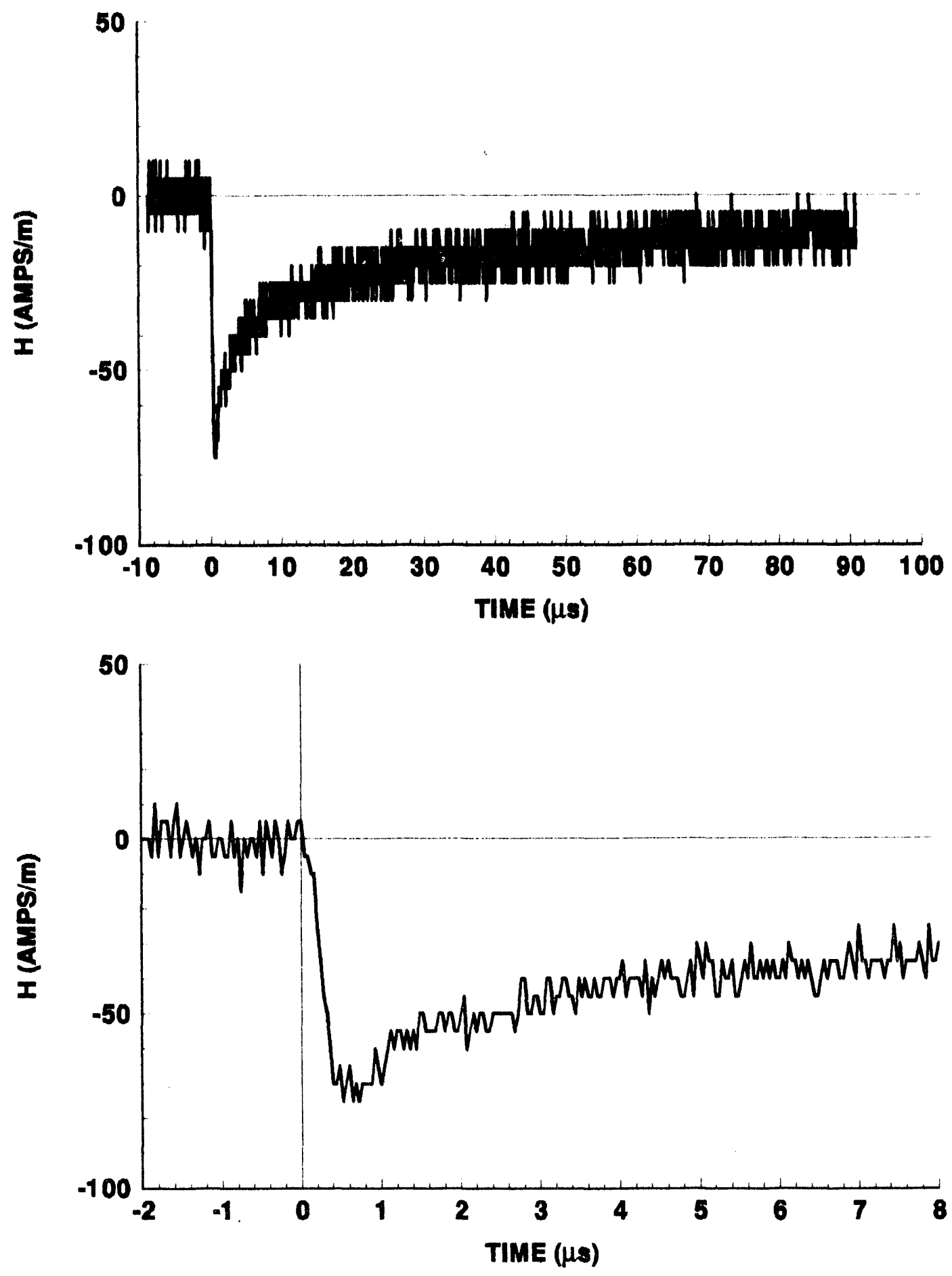


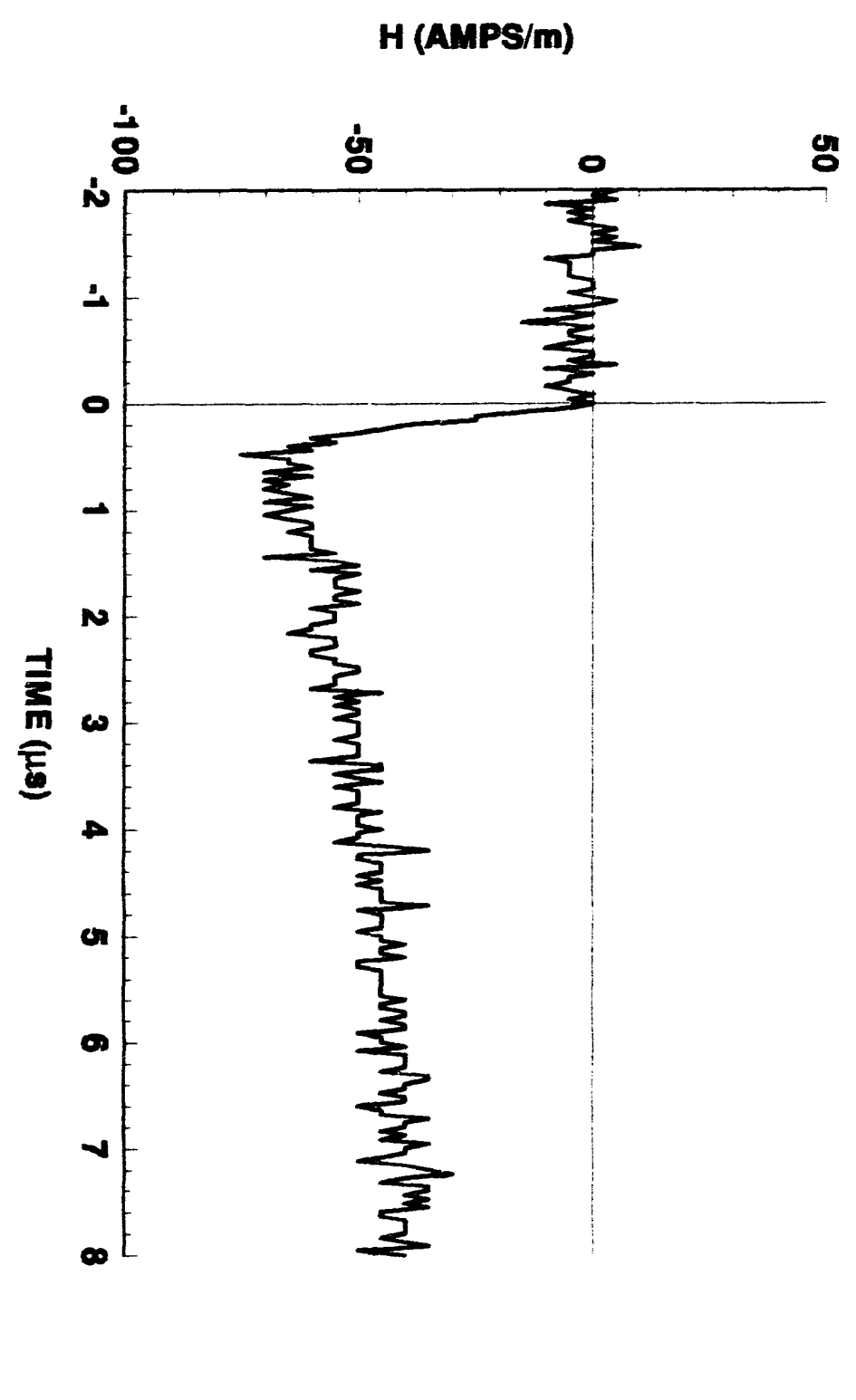

H (AMPS/m)

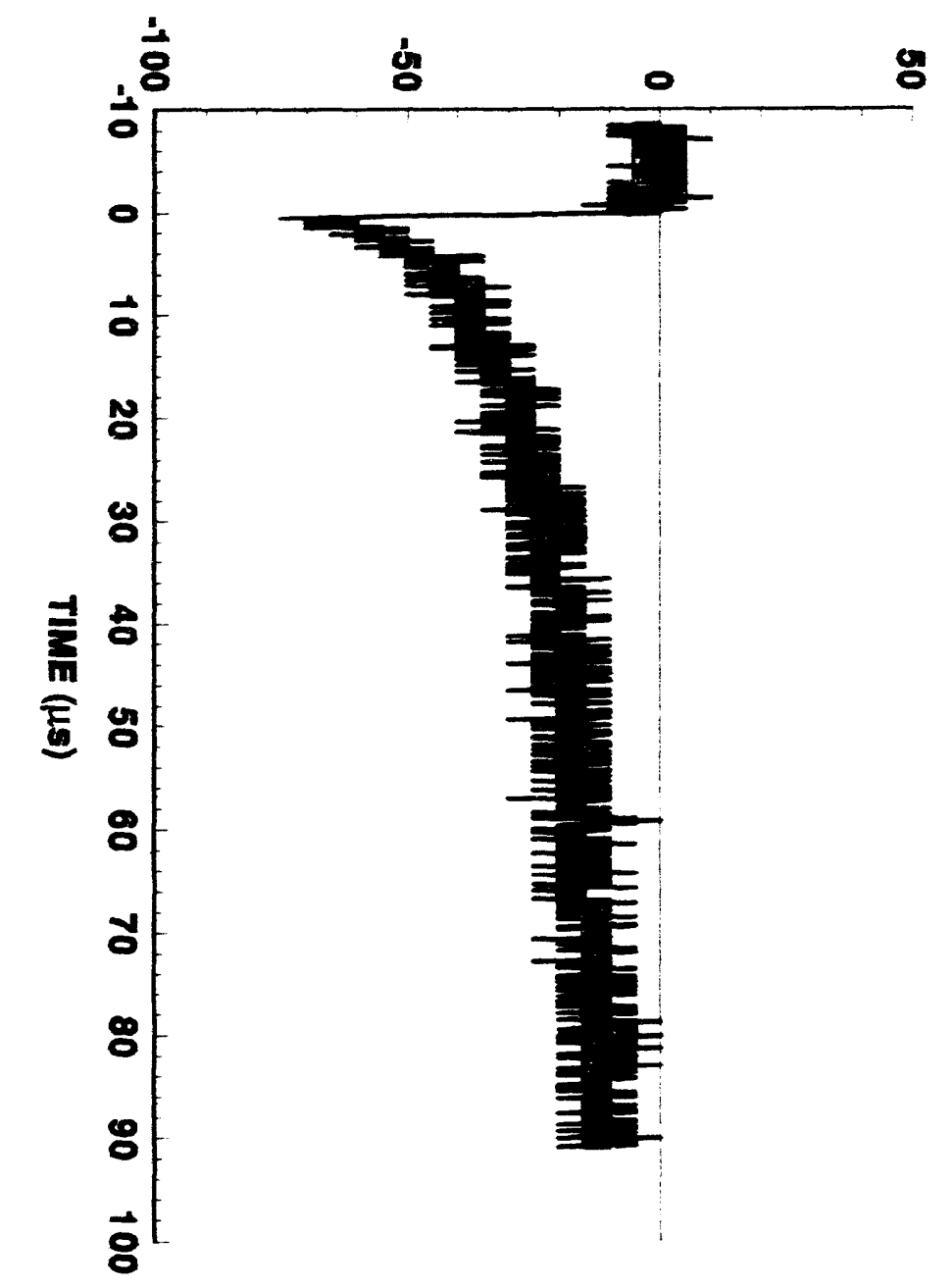

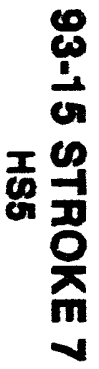




\section{3-15 STROKE 8}

HSS
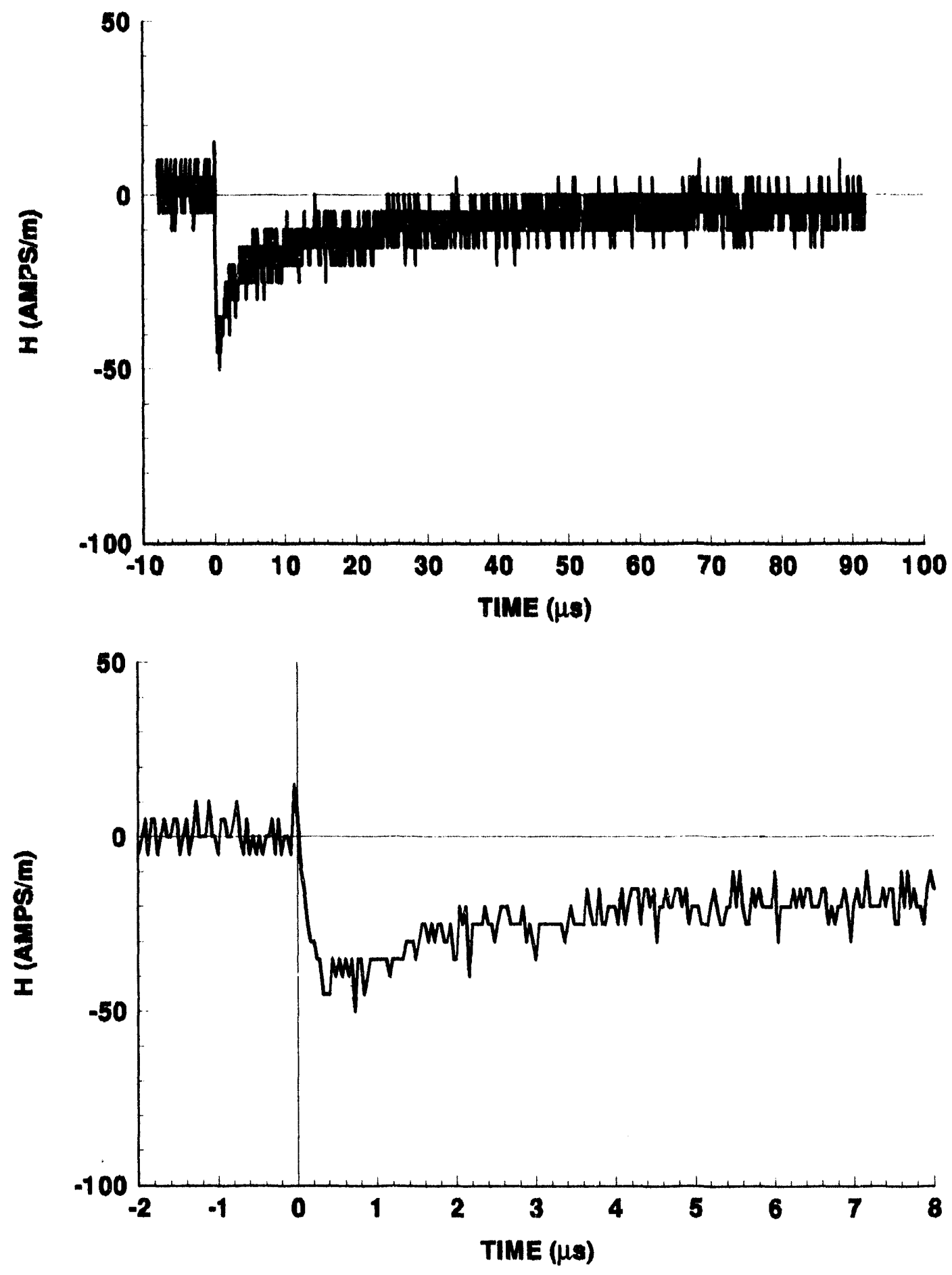


\section{3-15 STROKE 1}

ES1
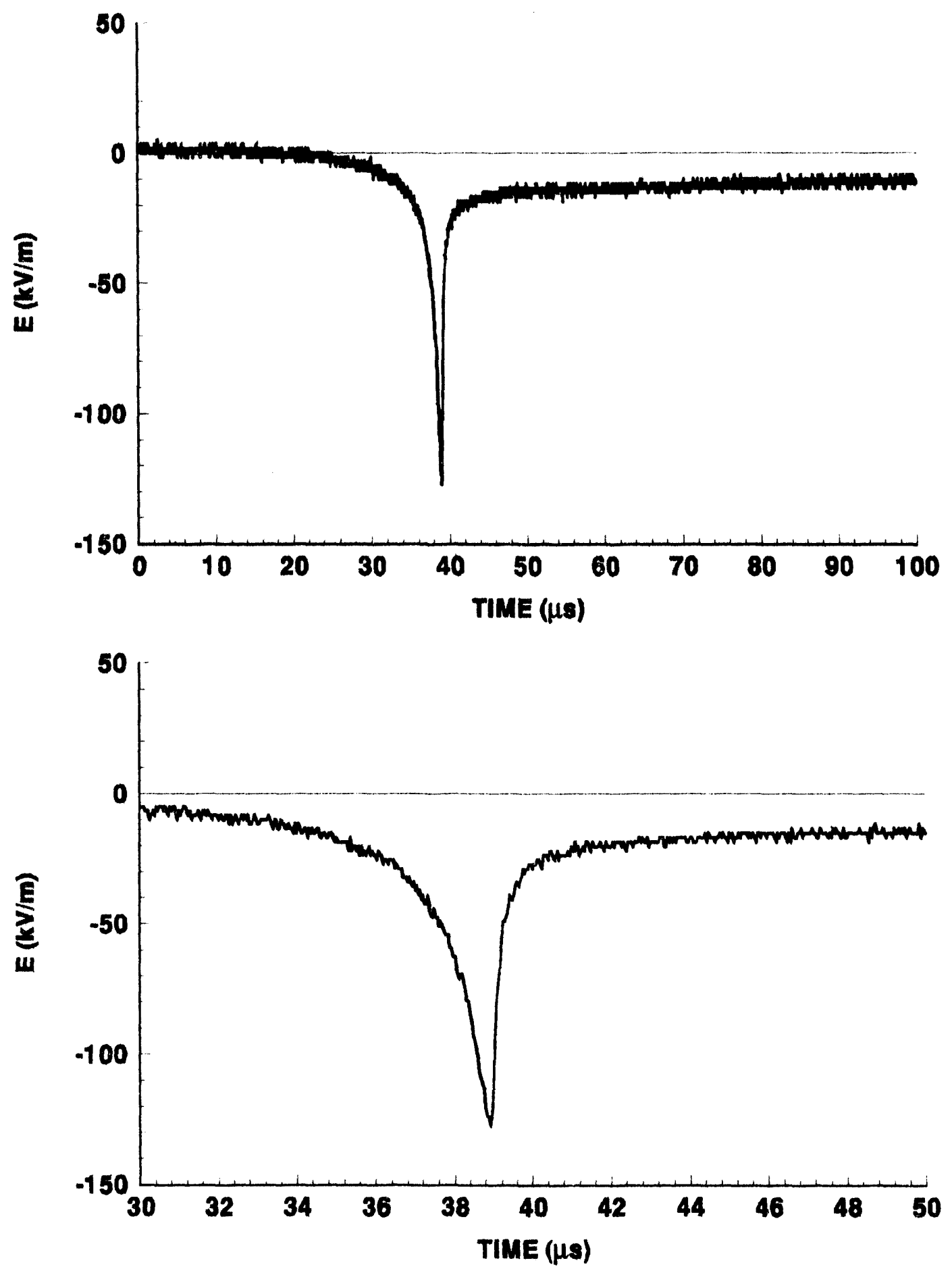


\section{3-15 STROKE 2}

ES1
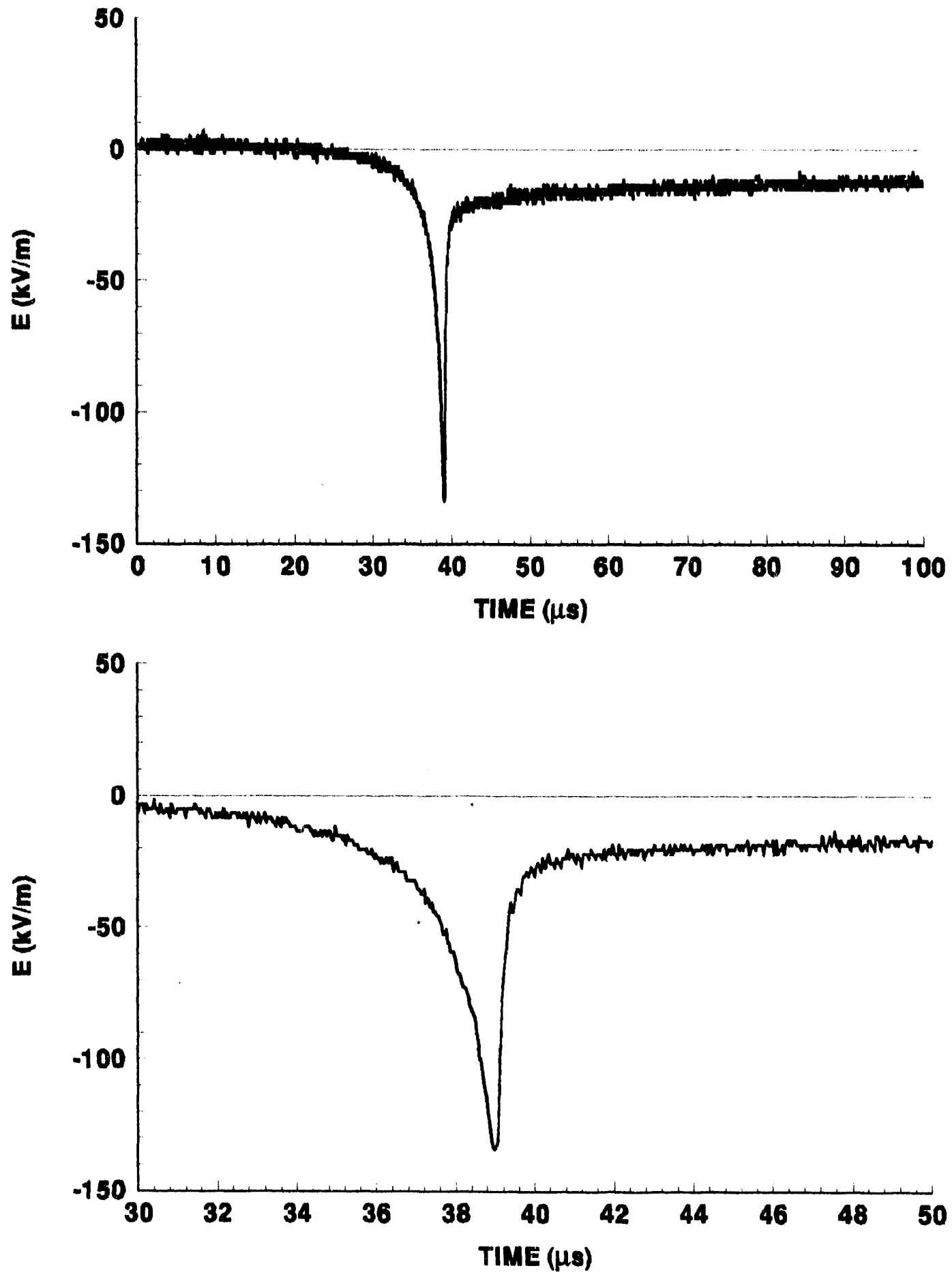


\section{3-15 STROKE 3}

ES1
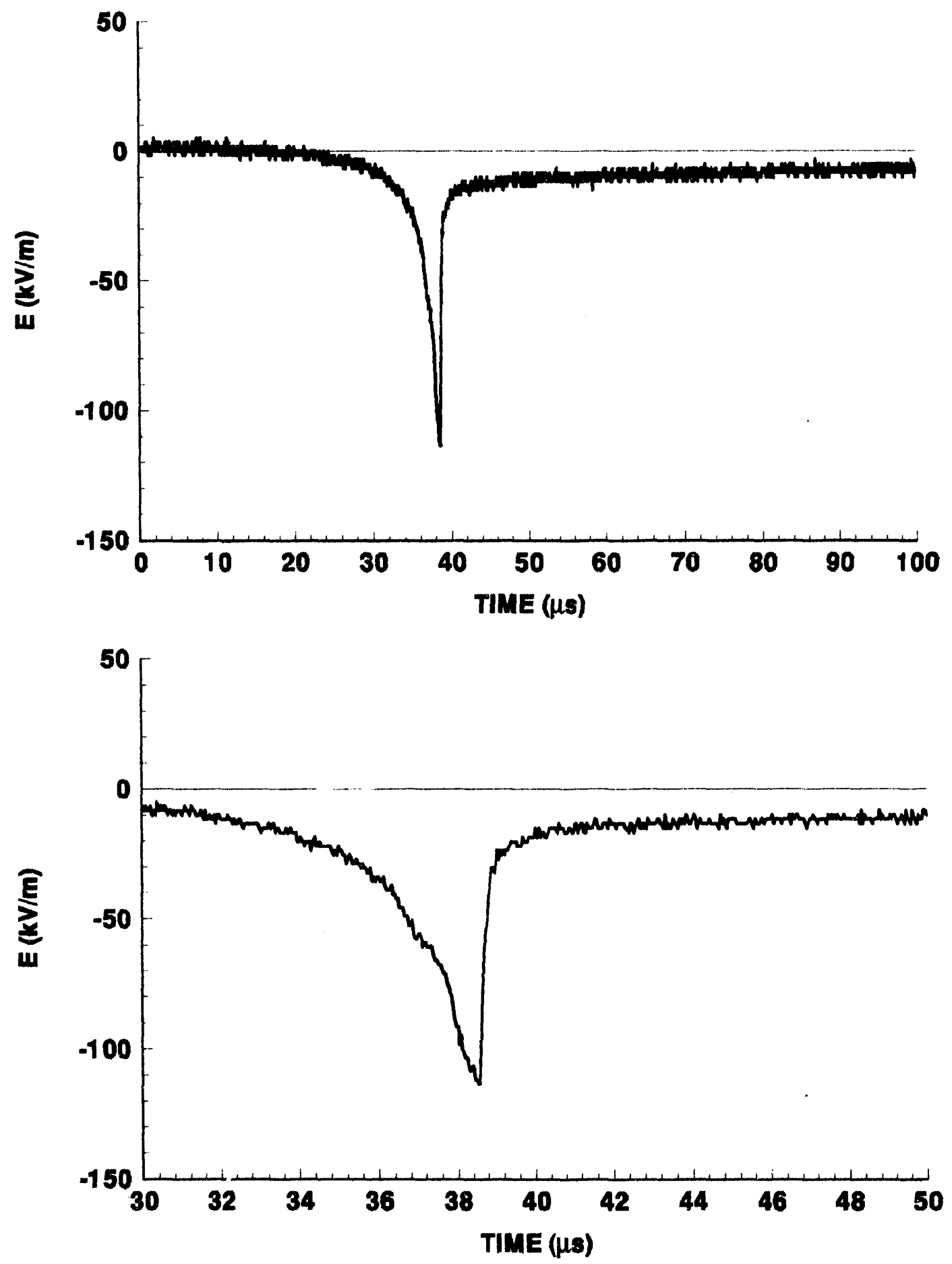


\section{3-15 STROKE 4 \\ ES1}
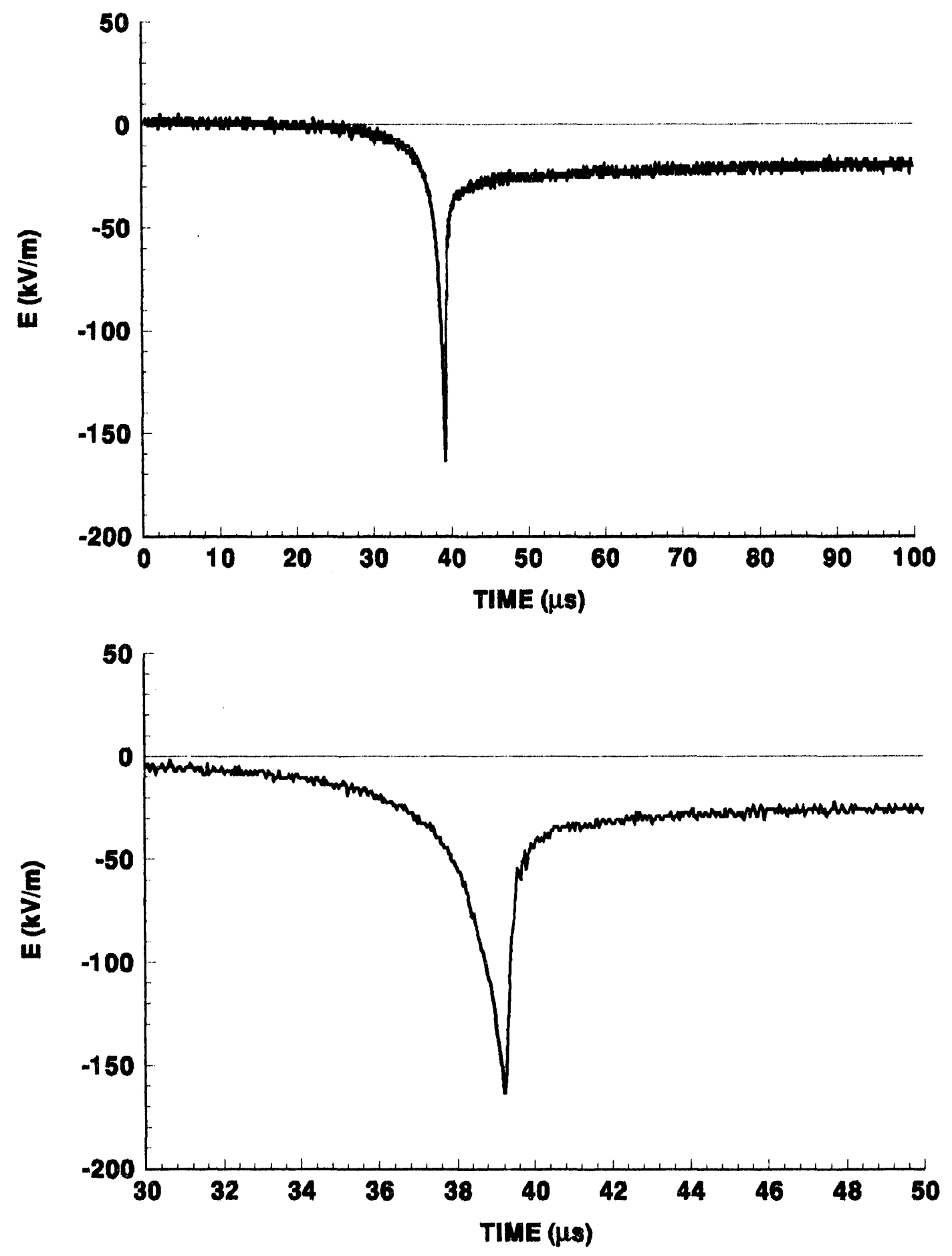


\section{3-15 STROKE 5}

ES1
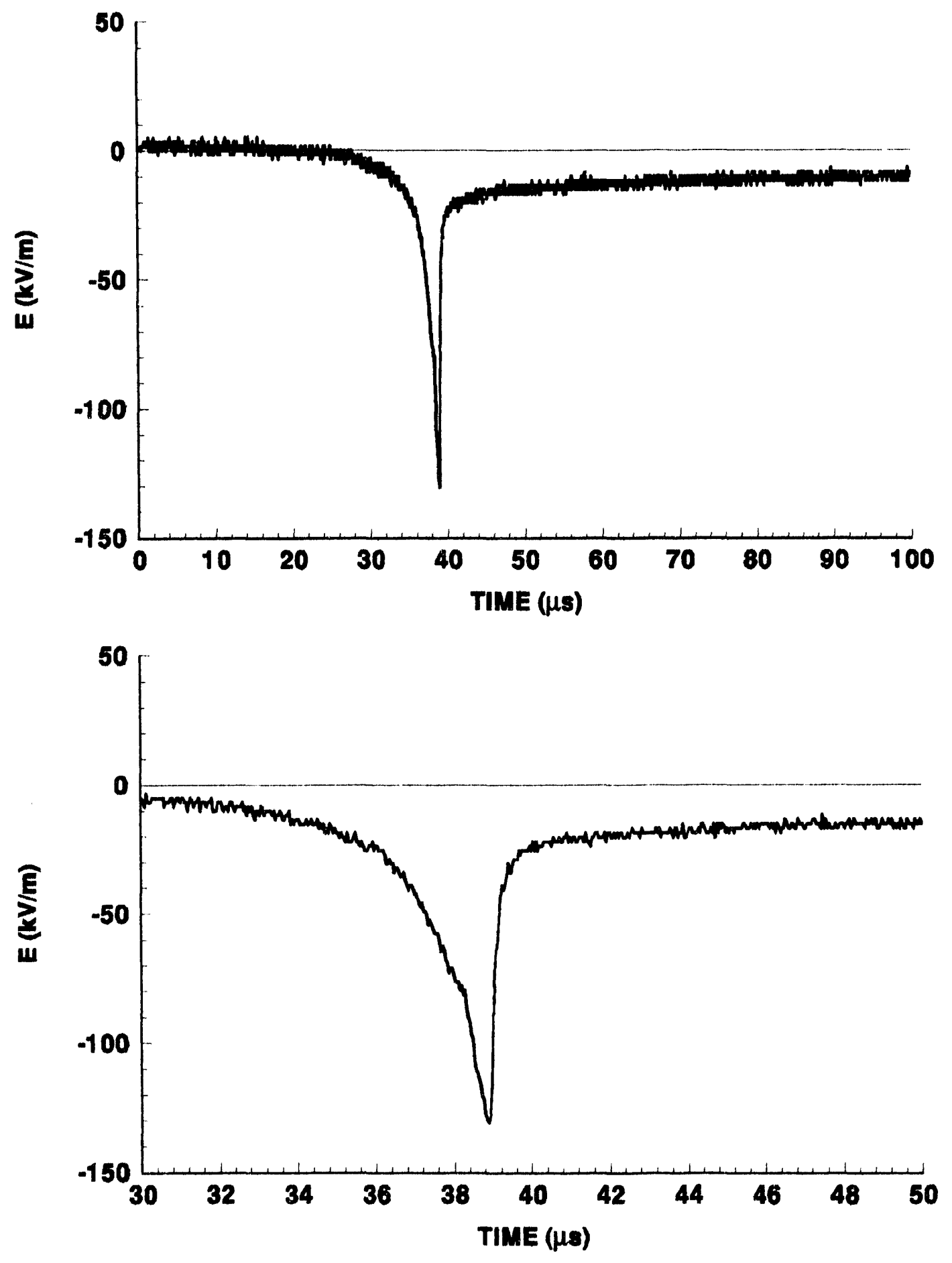


\section{3-15 STROKE 6 \\ ES1}
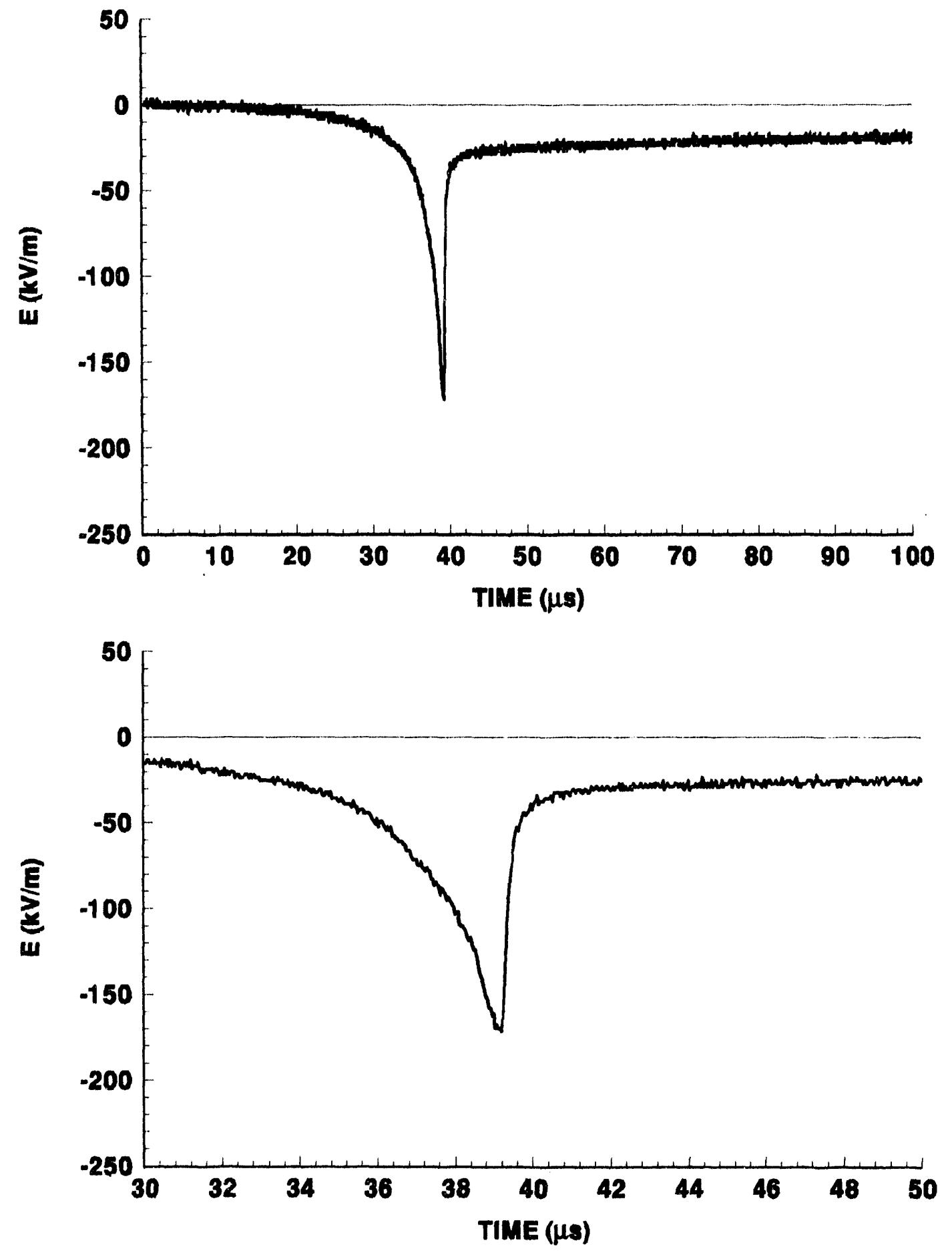


\section{3-15 STROKE 7}

ES1
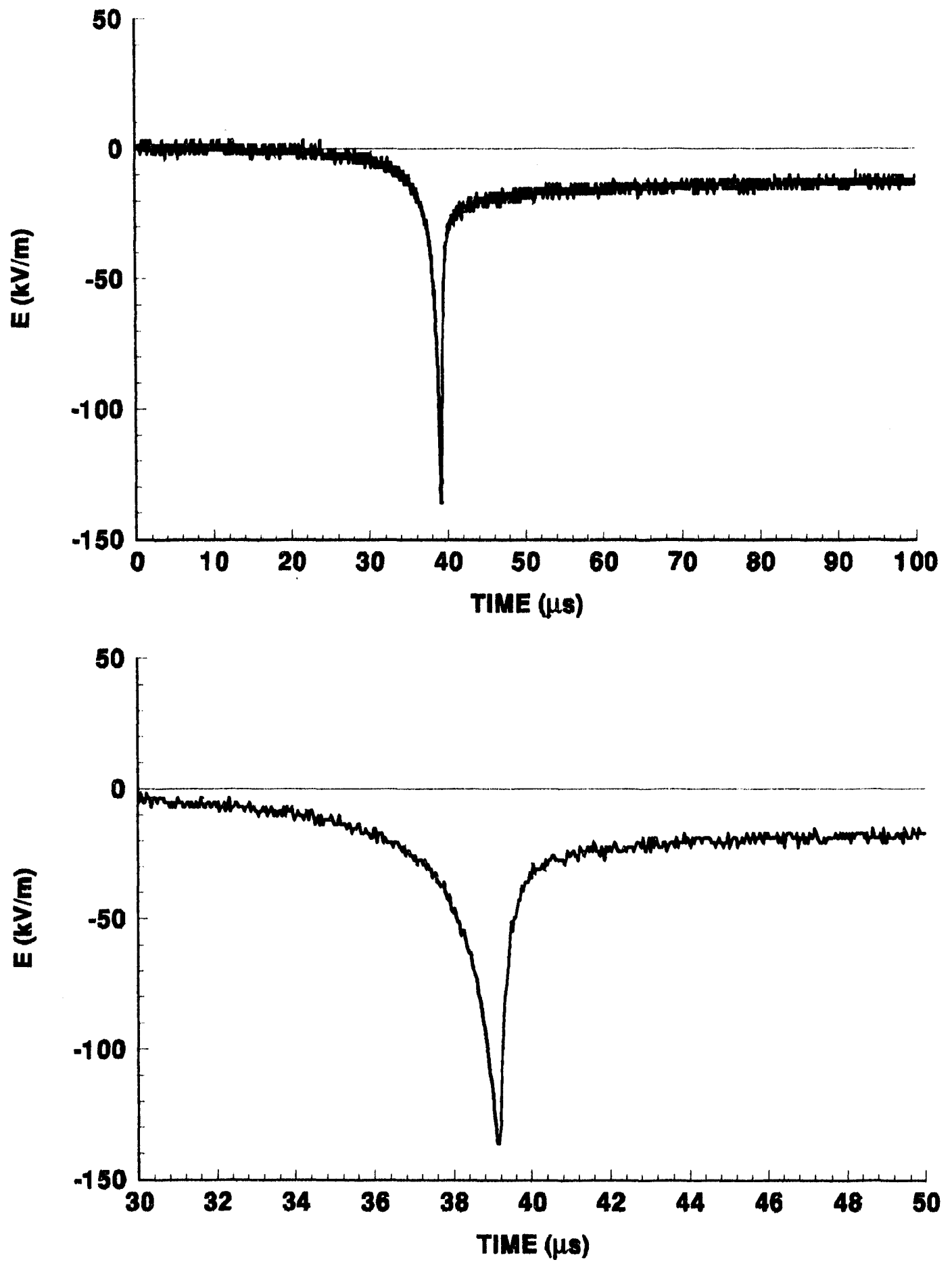


\section{3-15 STROKE 8 \\ ES1}
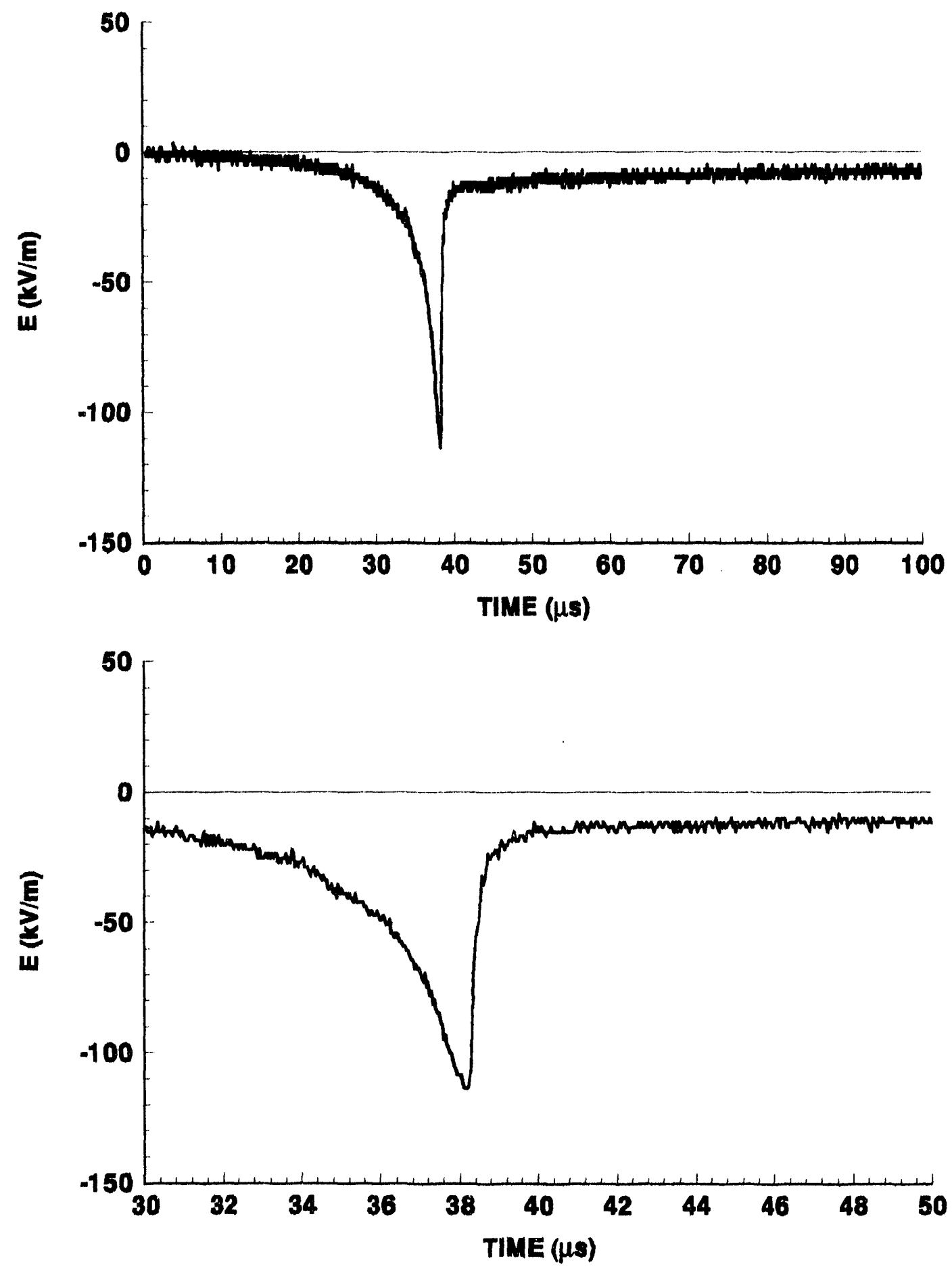


\section{3-15 STROKE 1}

ES4
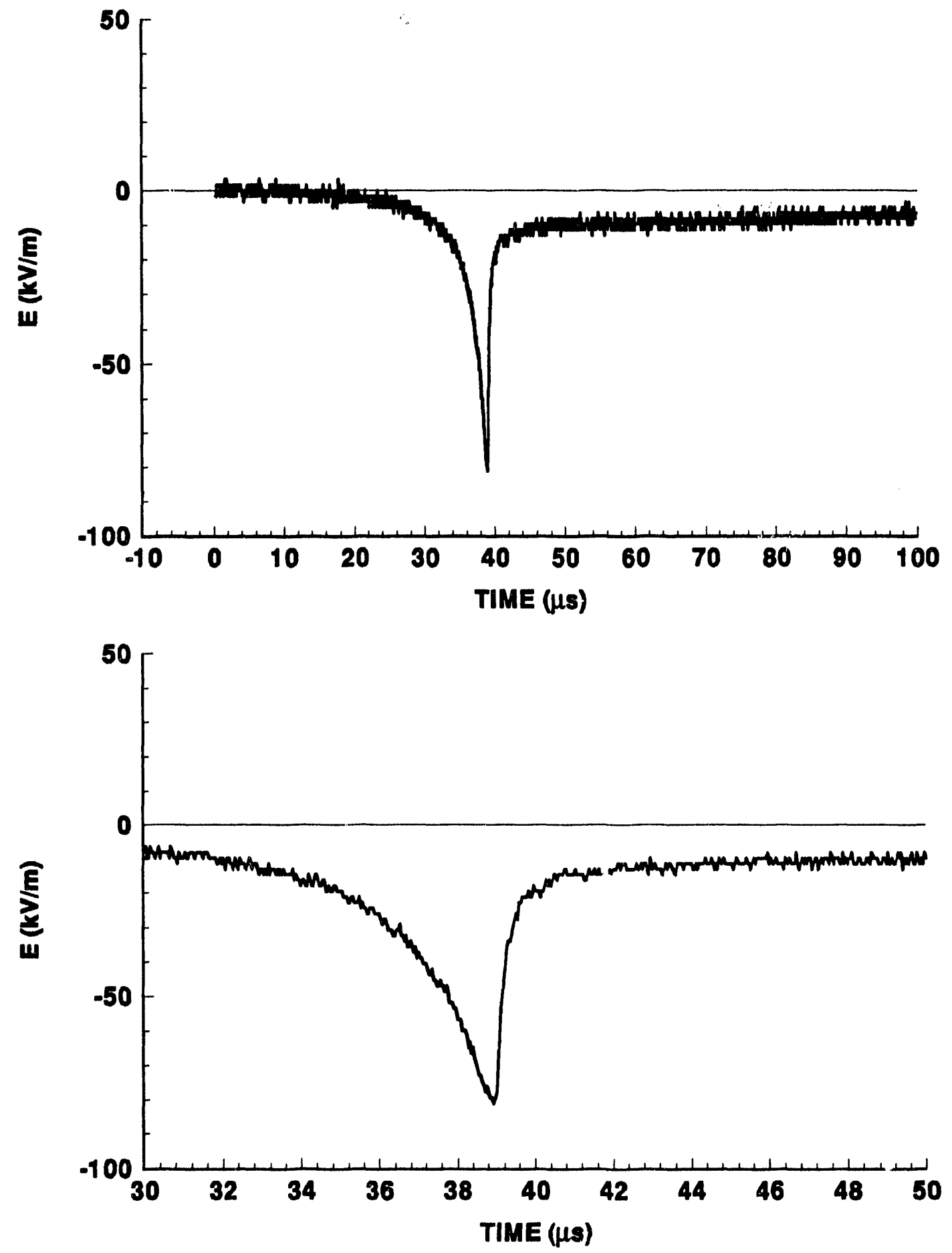


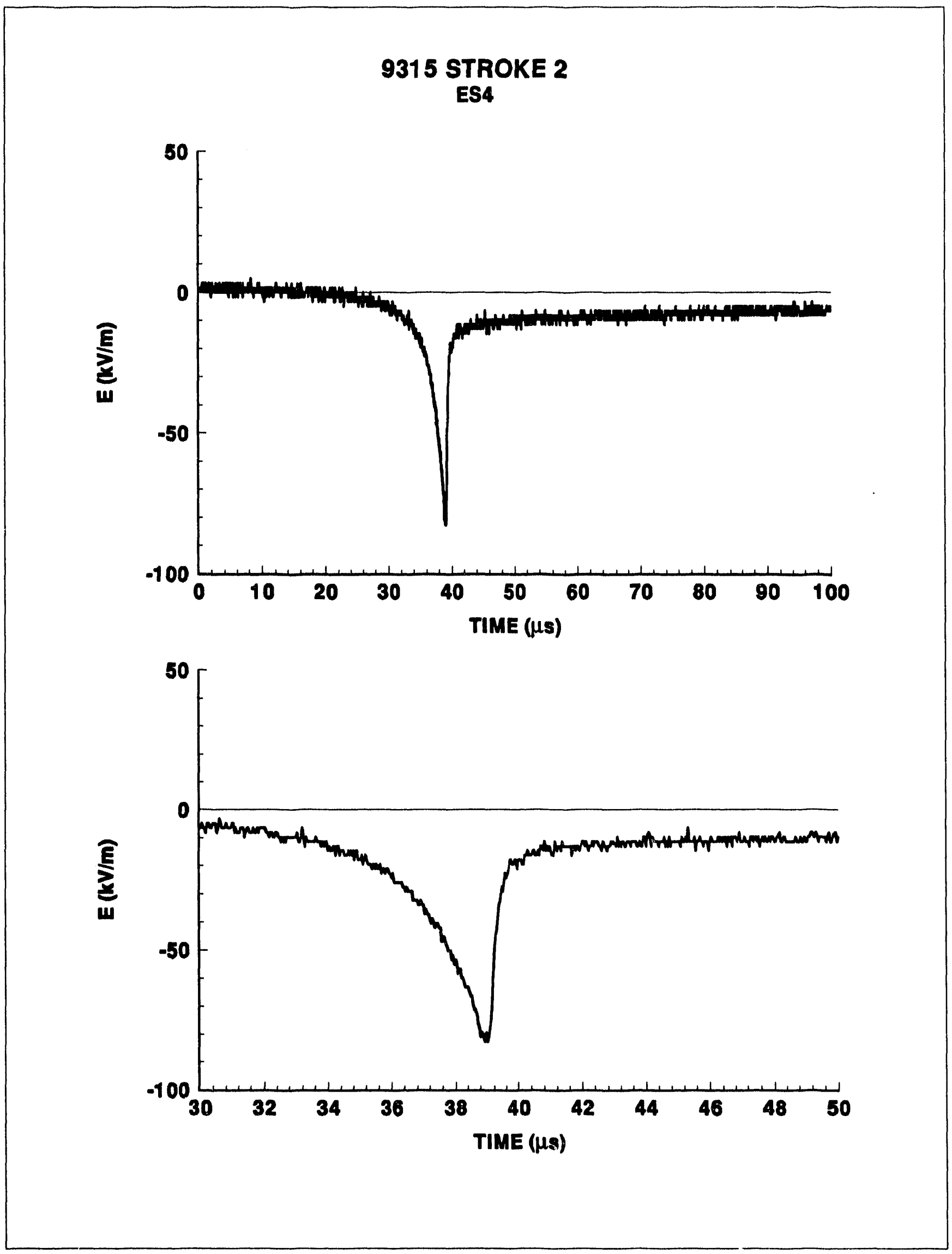




\section{STROKE 3 \\ ES4}
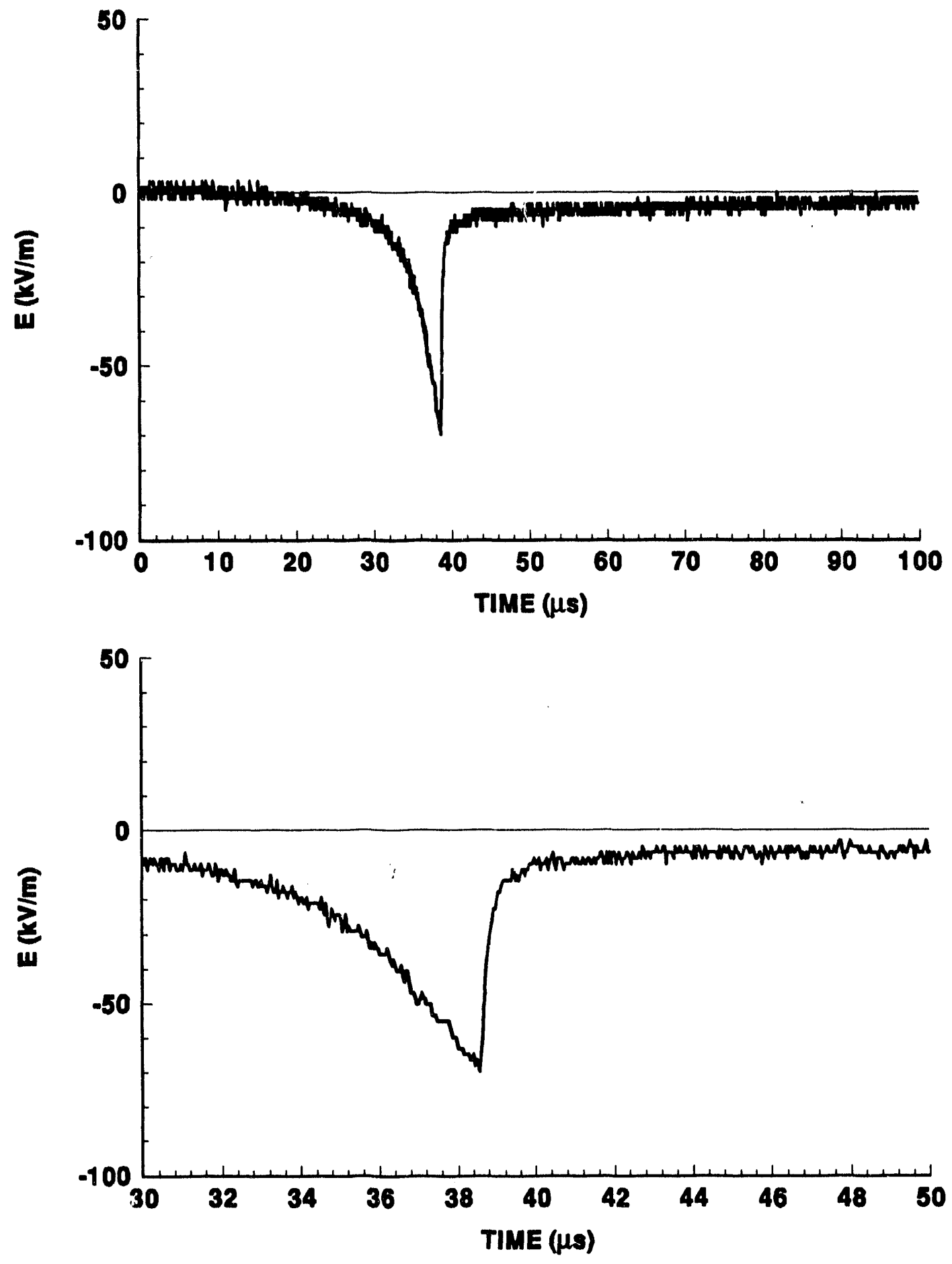


\section{3-15 STROKE 4 \\ ES4}
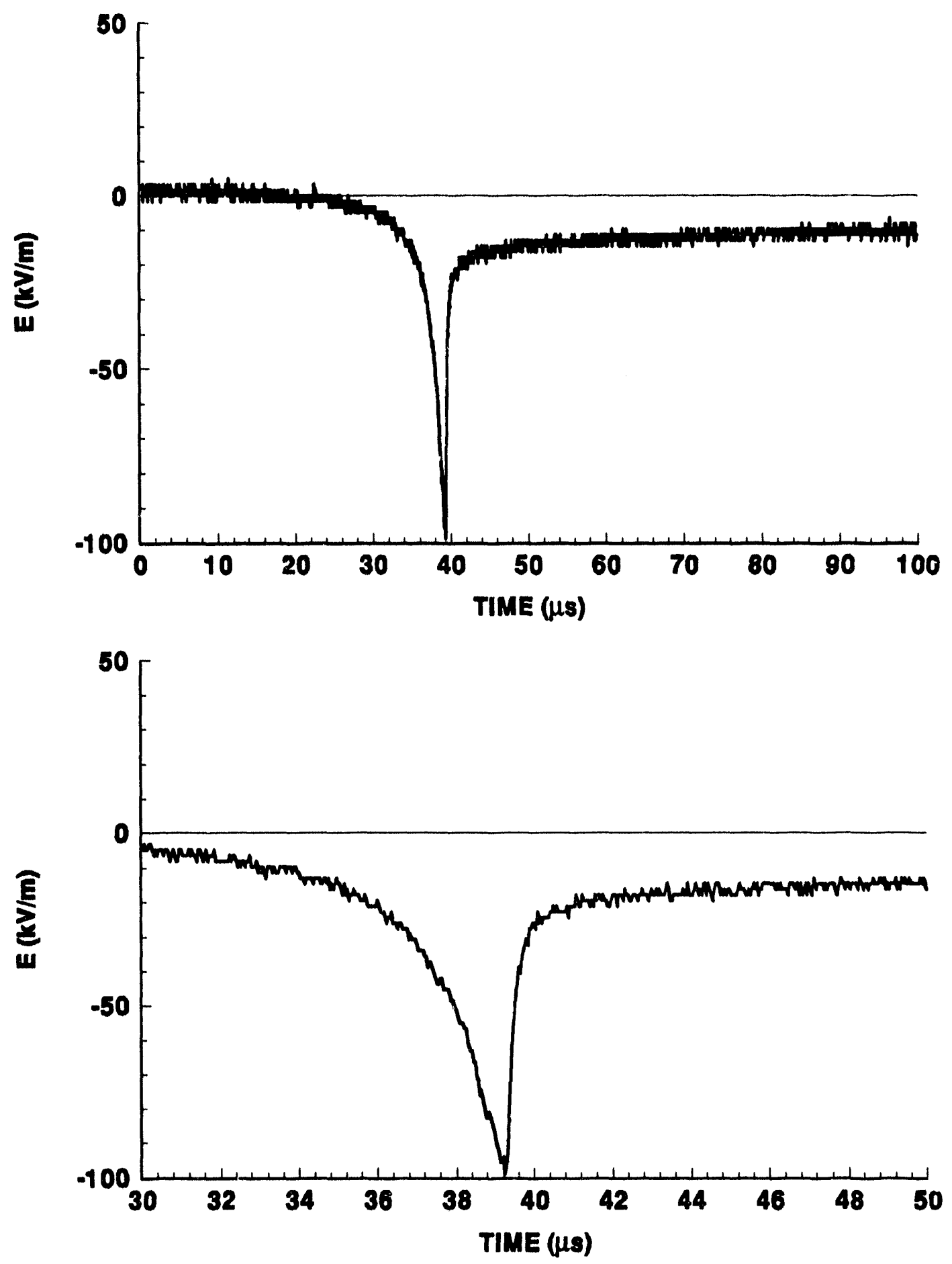


\section{3-15 STROKE 5 \\ ES4}
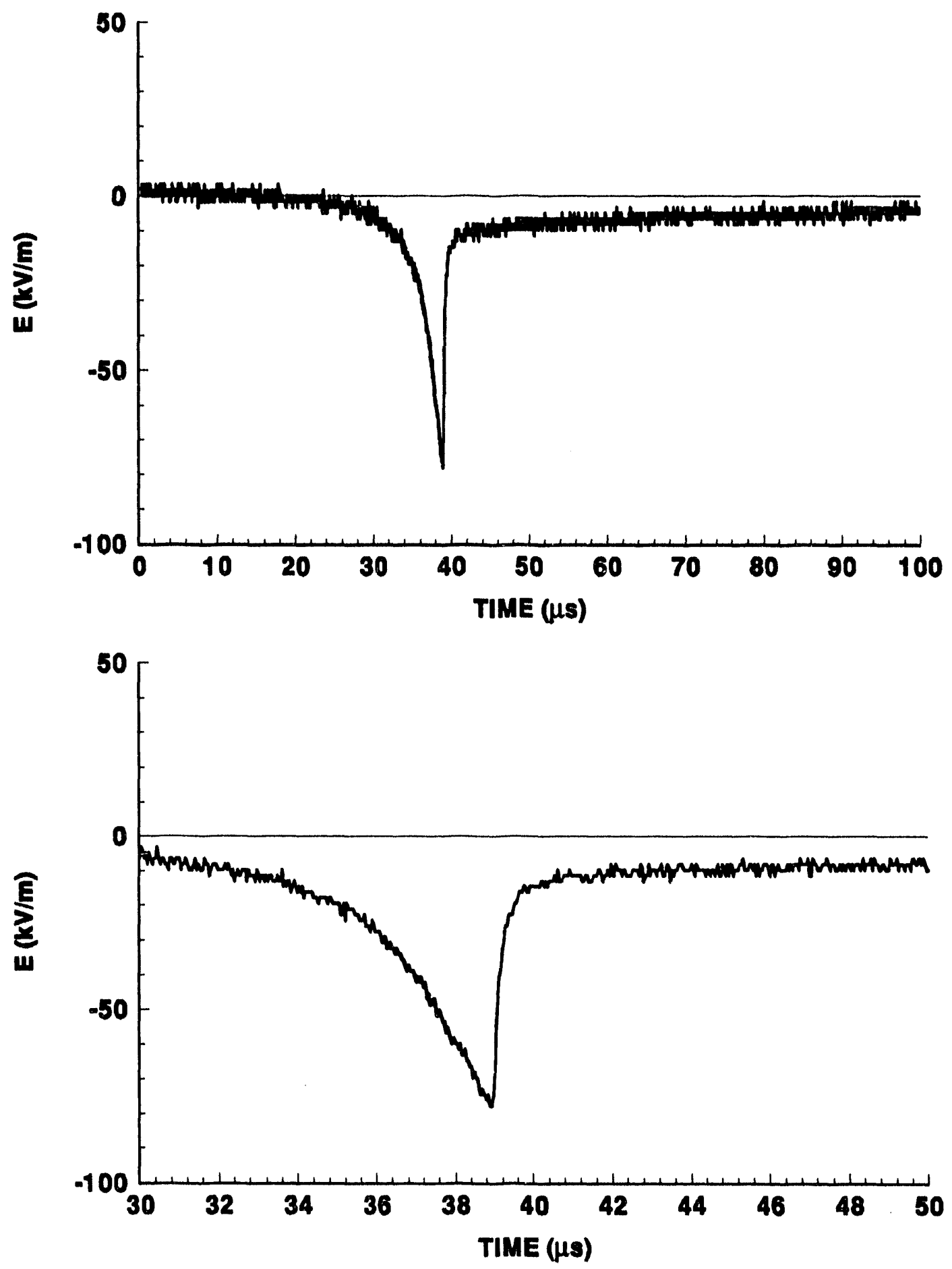


\section{3-15 STROKE 6}

ES4
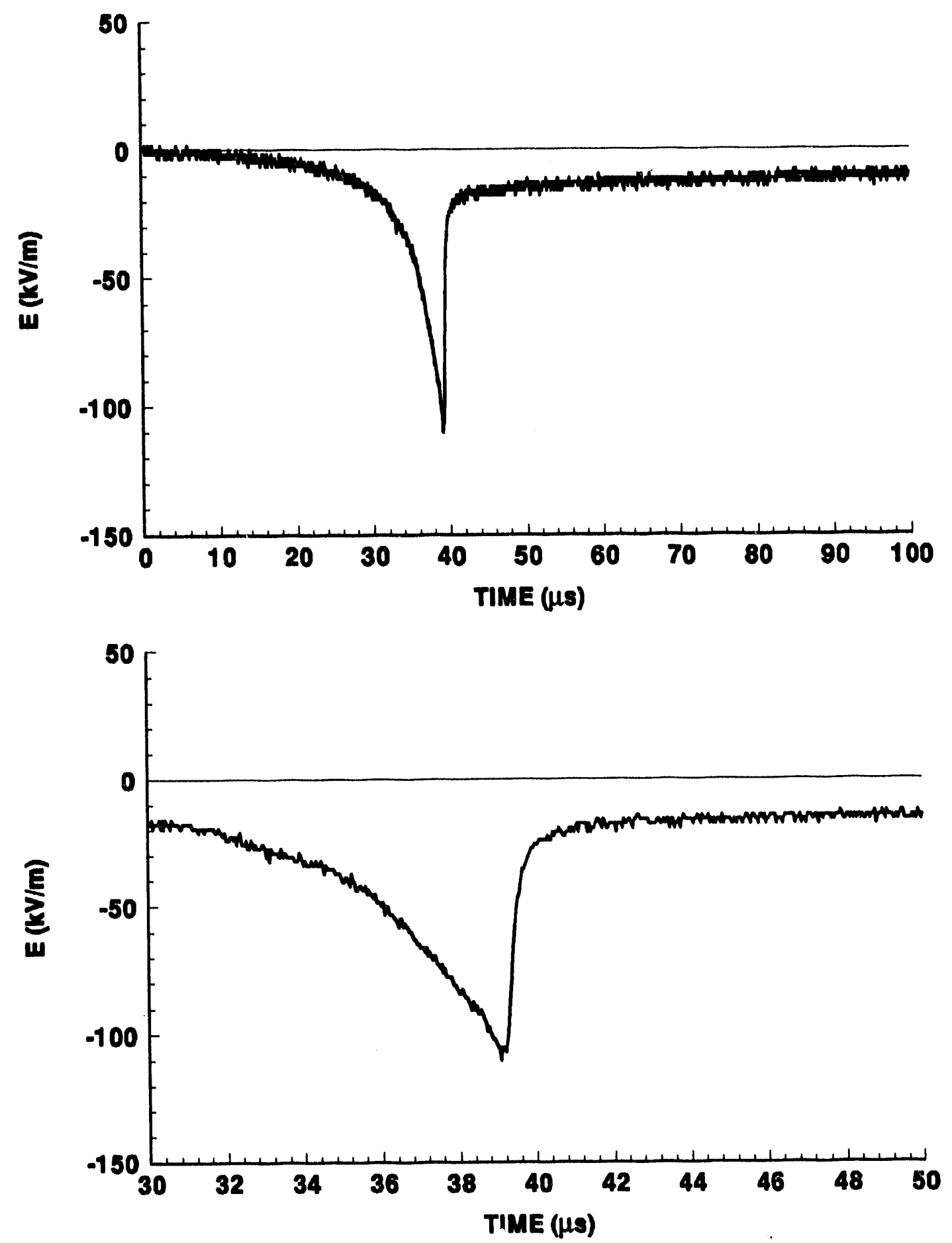


\section{3-15 STROKE 7}

ES4
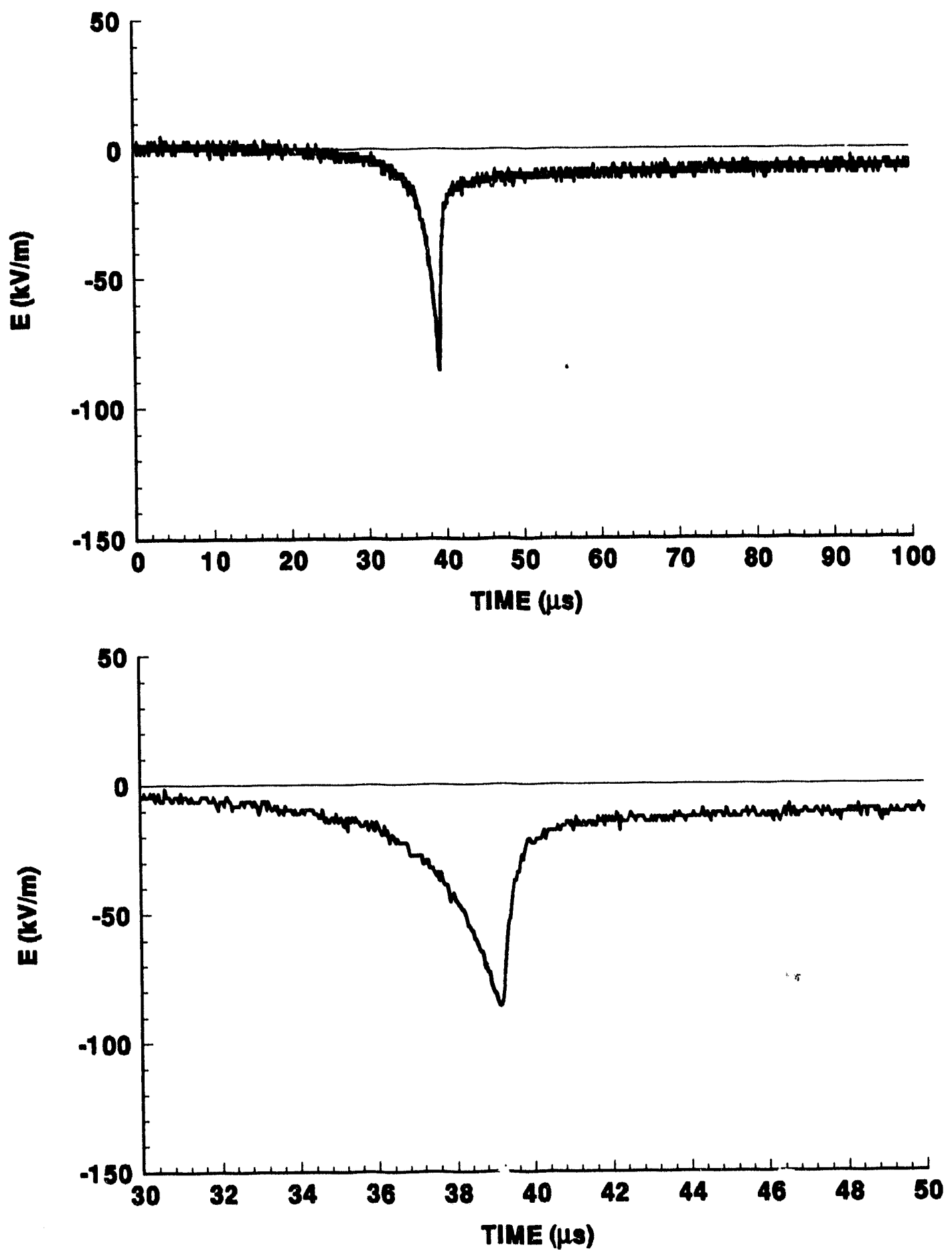


\section{3-15 STROKE 8}

ES4
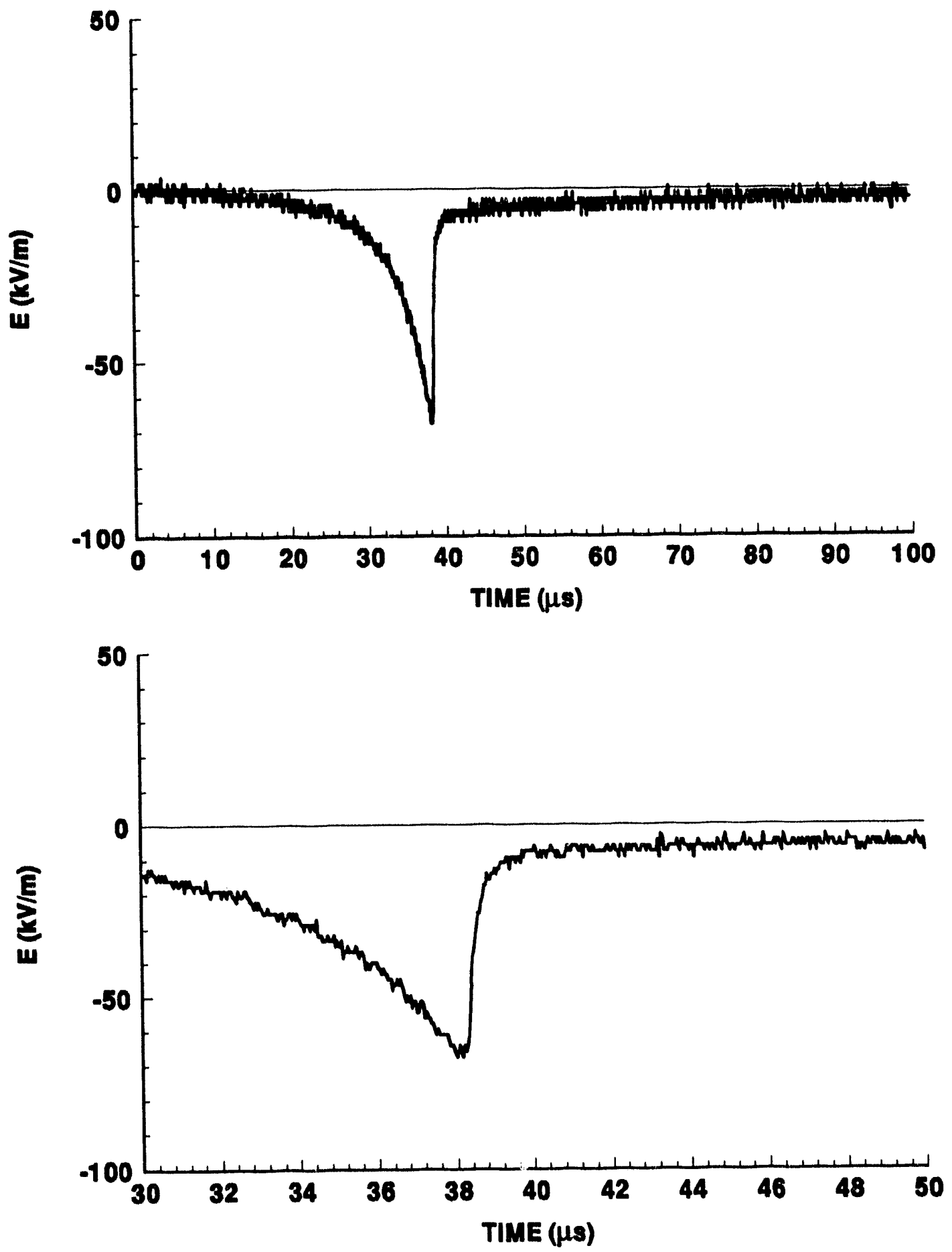
STEP POTENTIAL (kV)

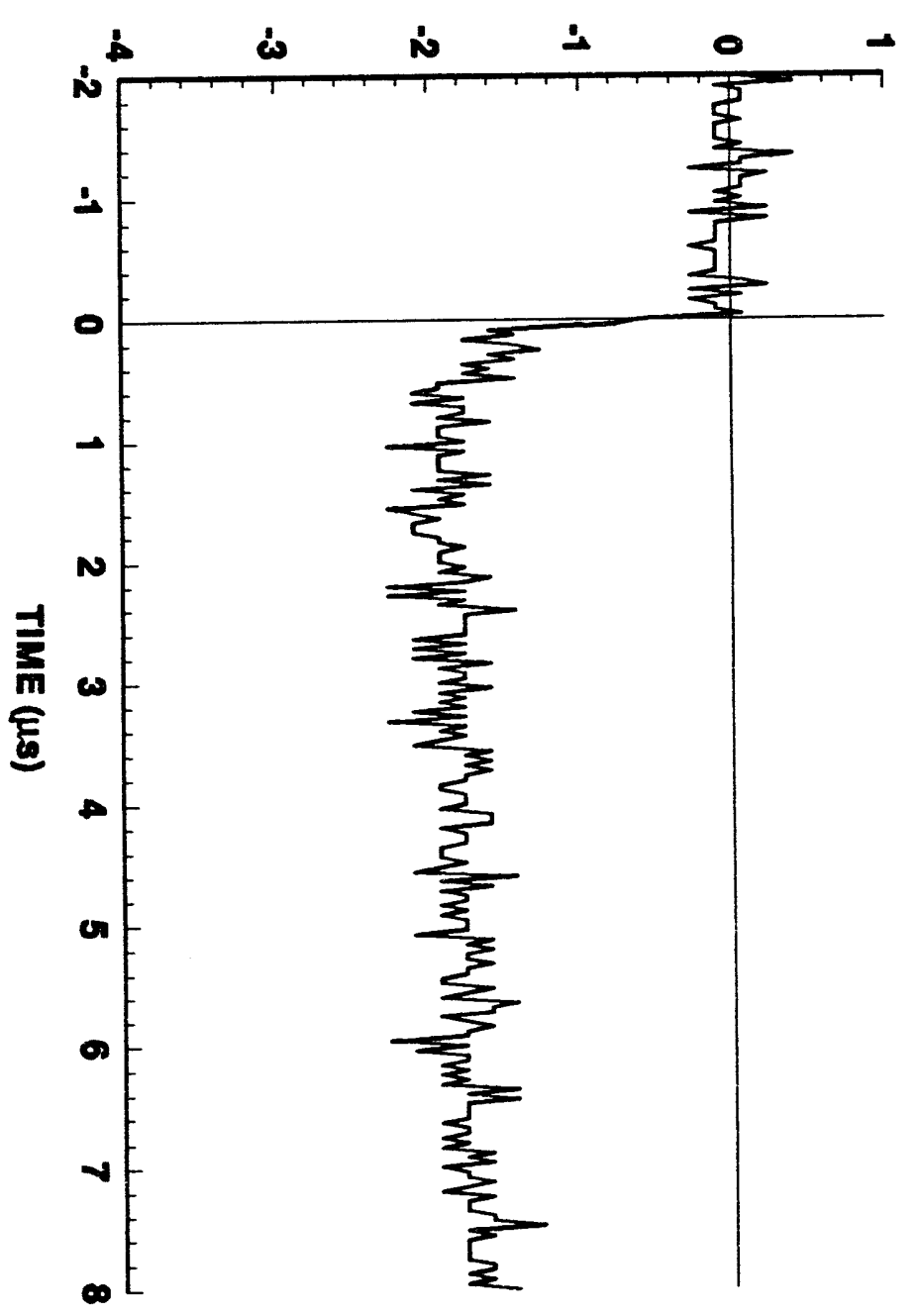

STEP POTENTIAL (KV)

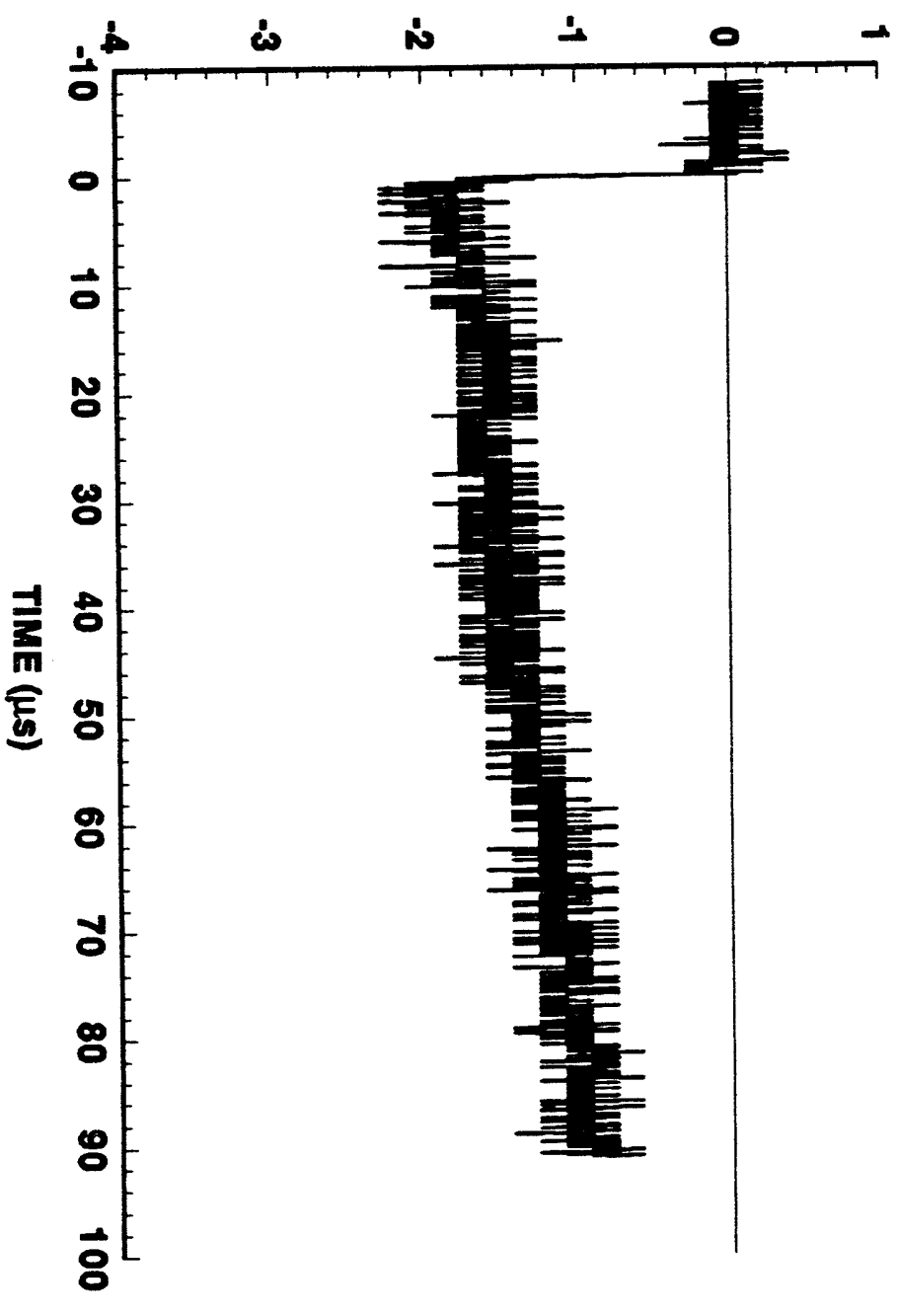

$\stackrel{\infty}{\dot{a}}$ ธำ 웅 


\section{3-15 STROKE 2}

vS1
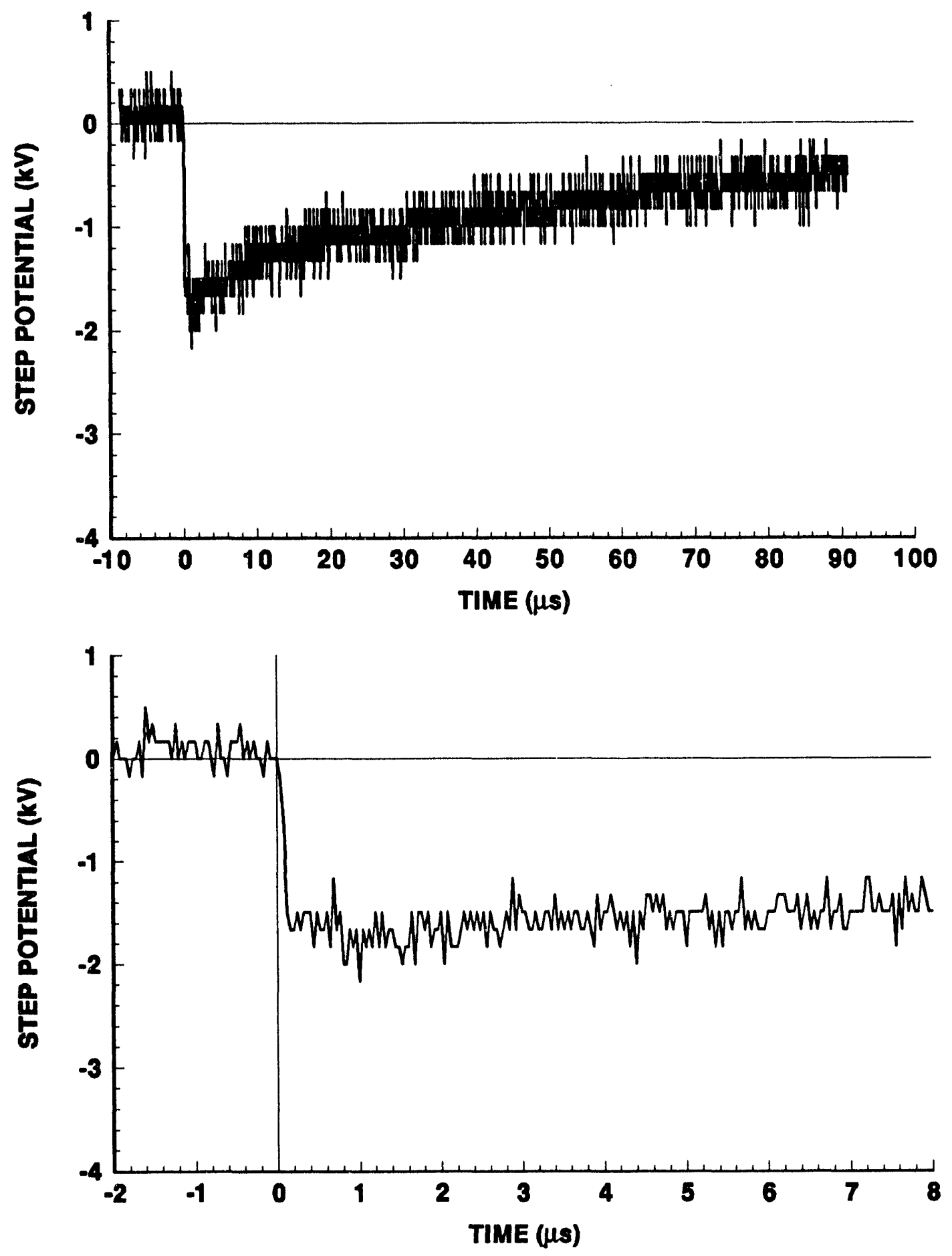


\section{3-15 STROKE 3}

vS1
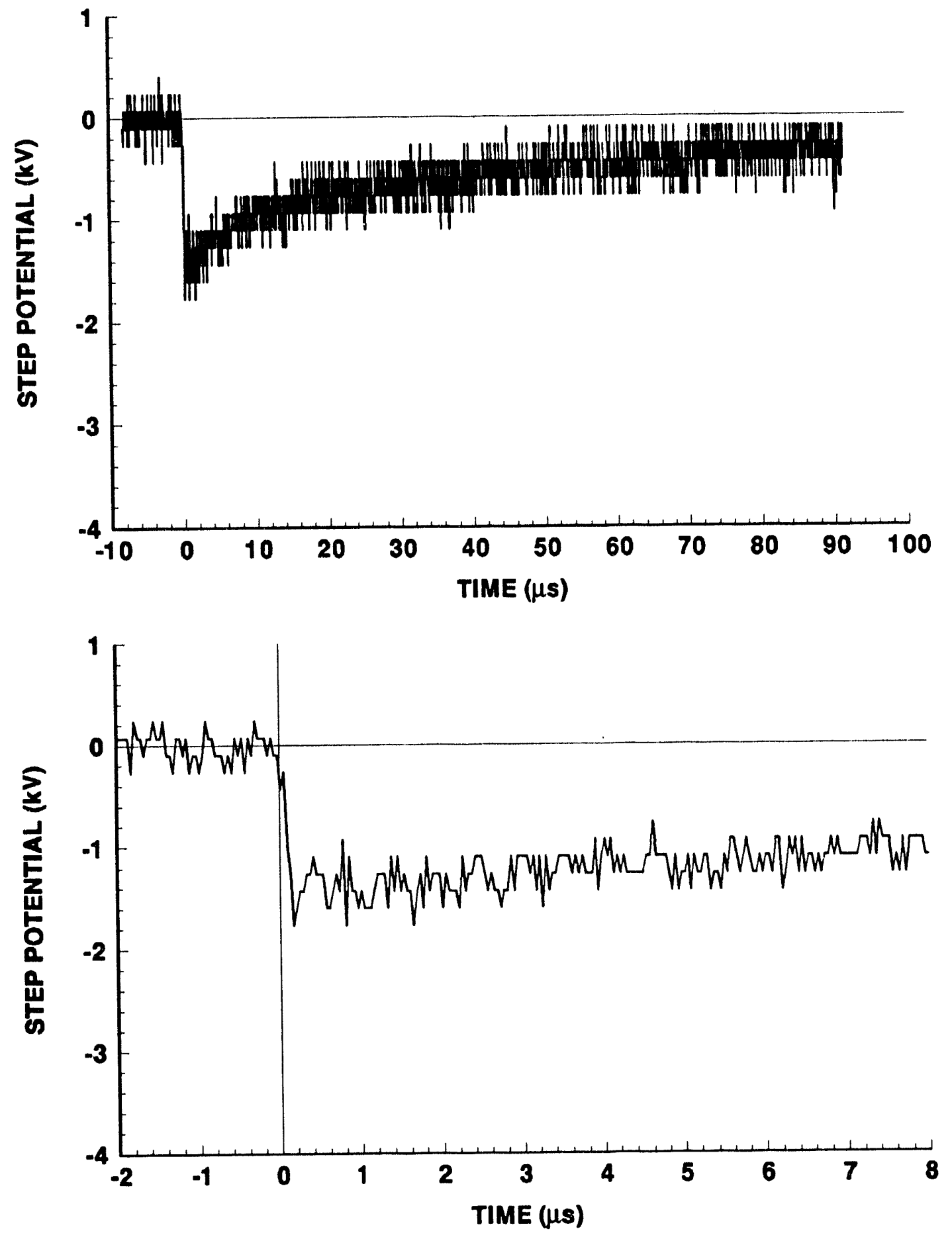


\section{3-15 STROKE 4 \\ VS1}
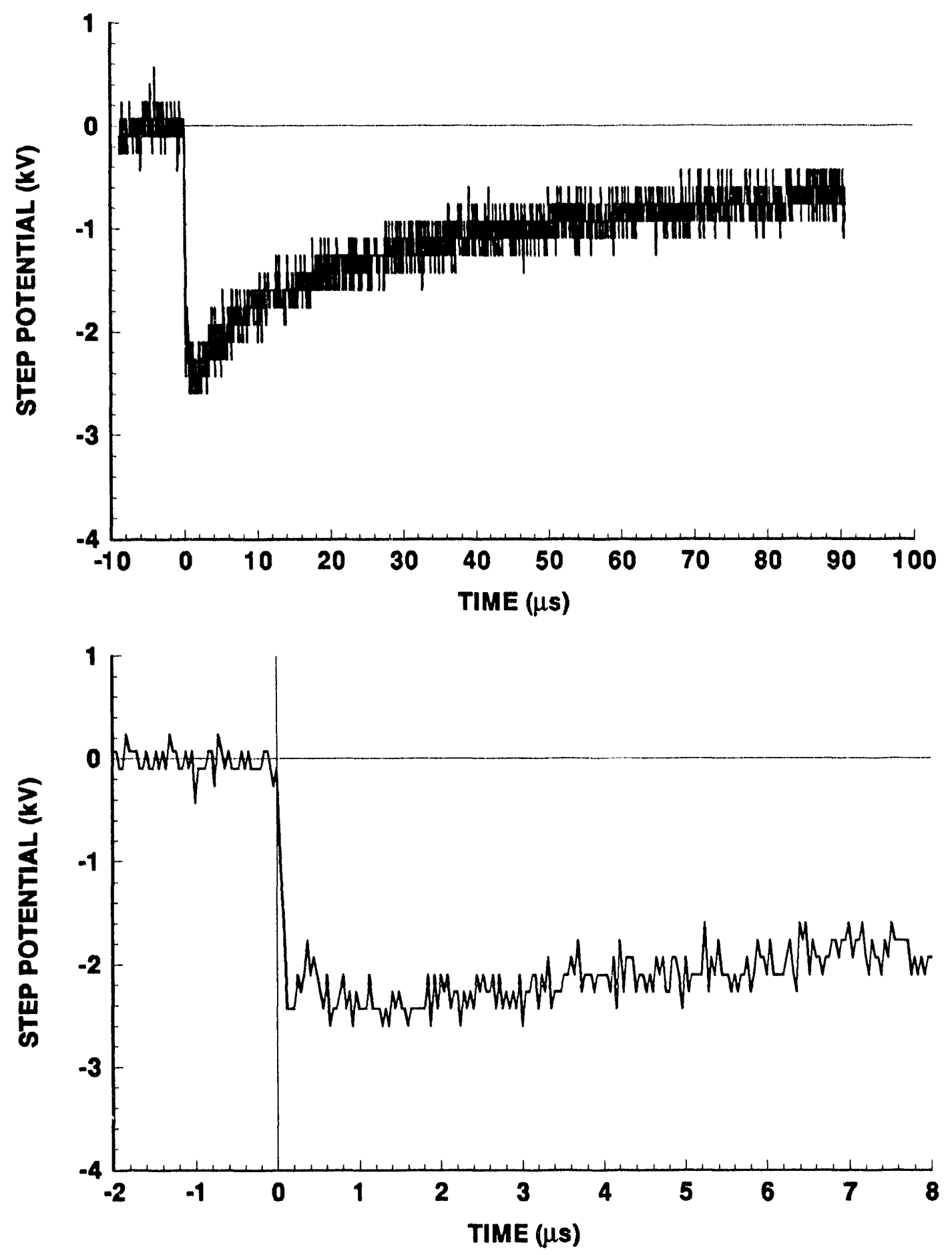


\section{3-15 STROKE 5 \\ VS1}
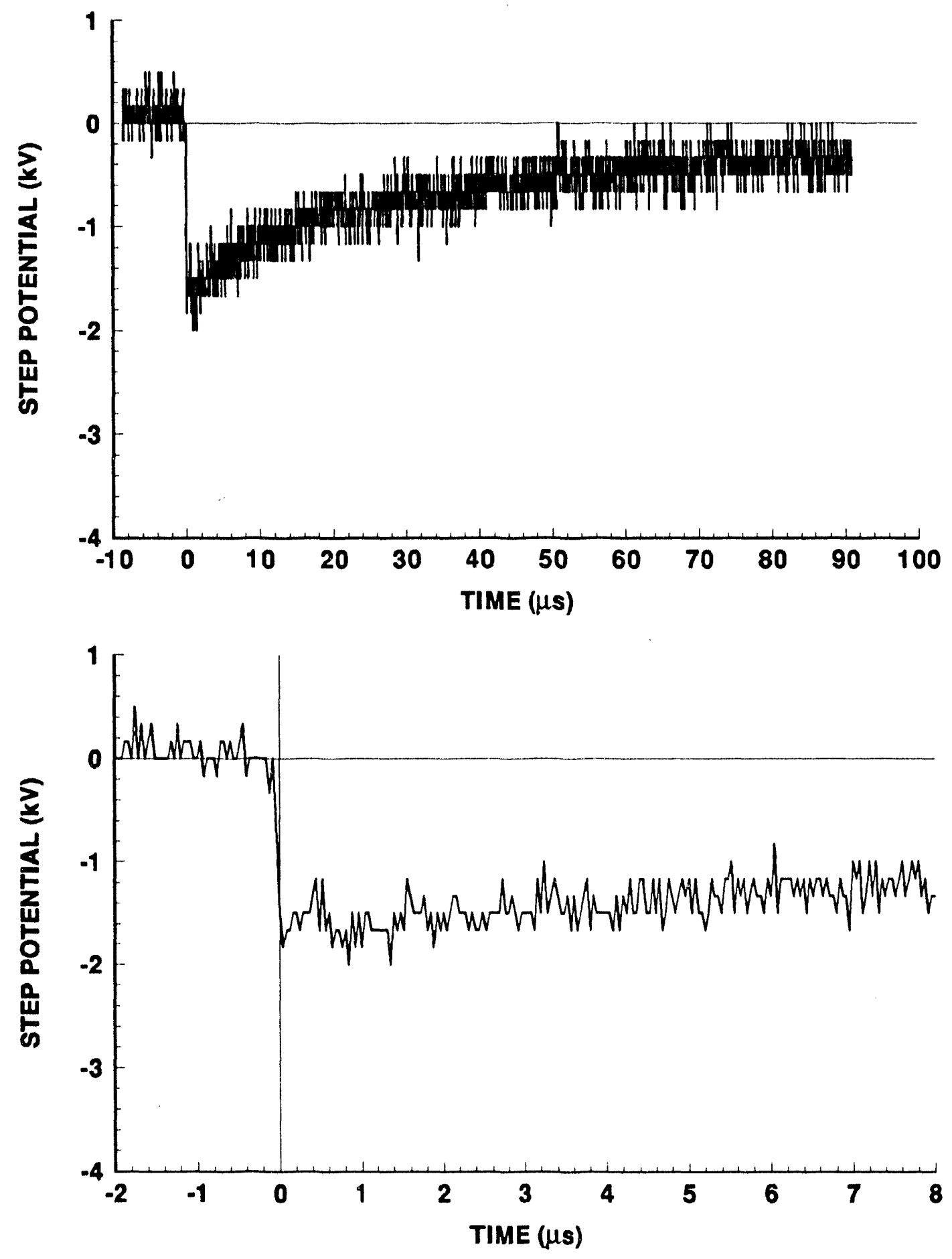


\section{3-15 STROKE 6}

VS1
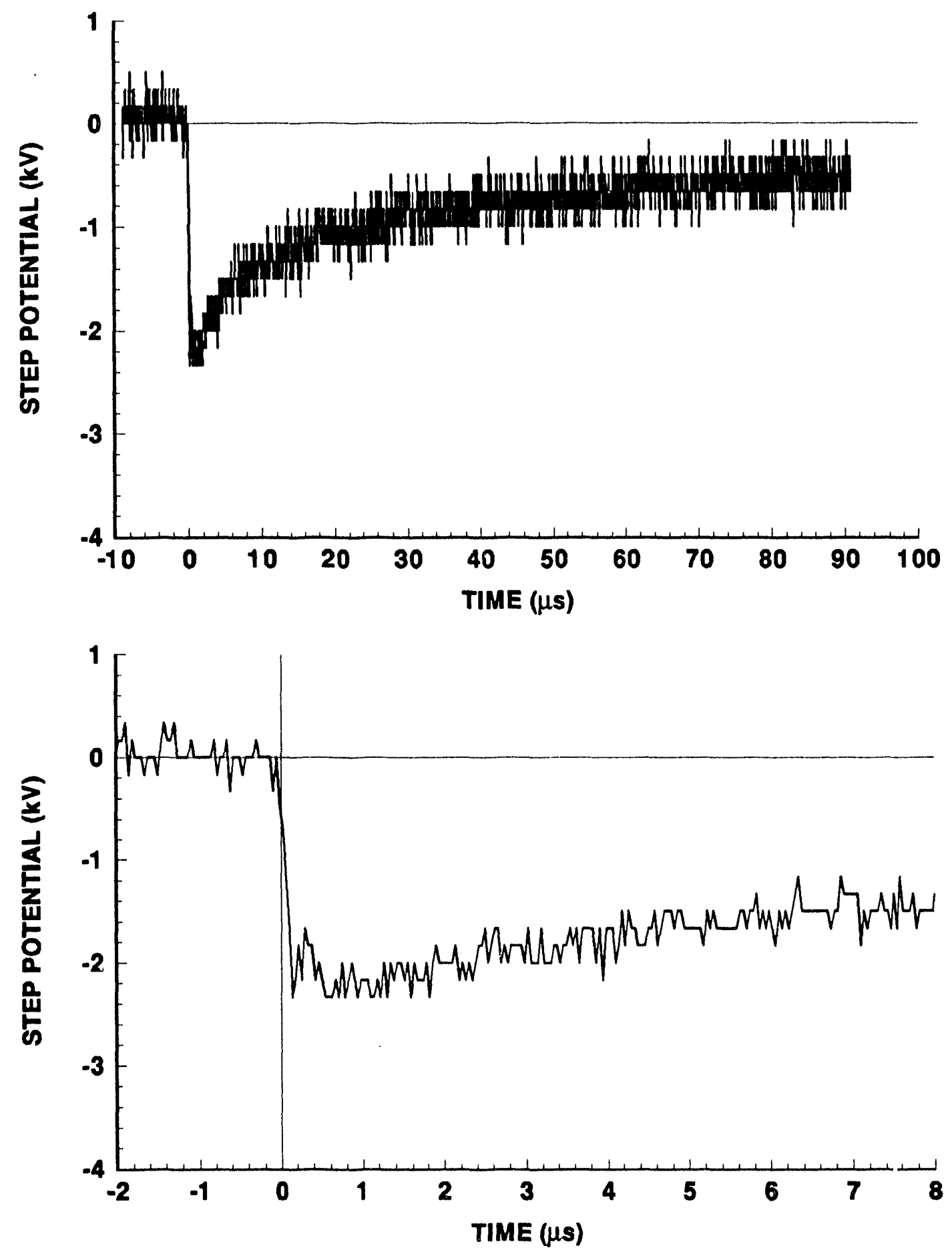


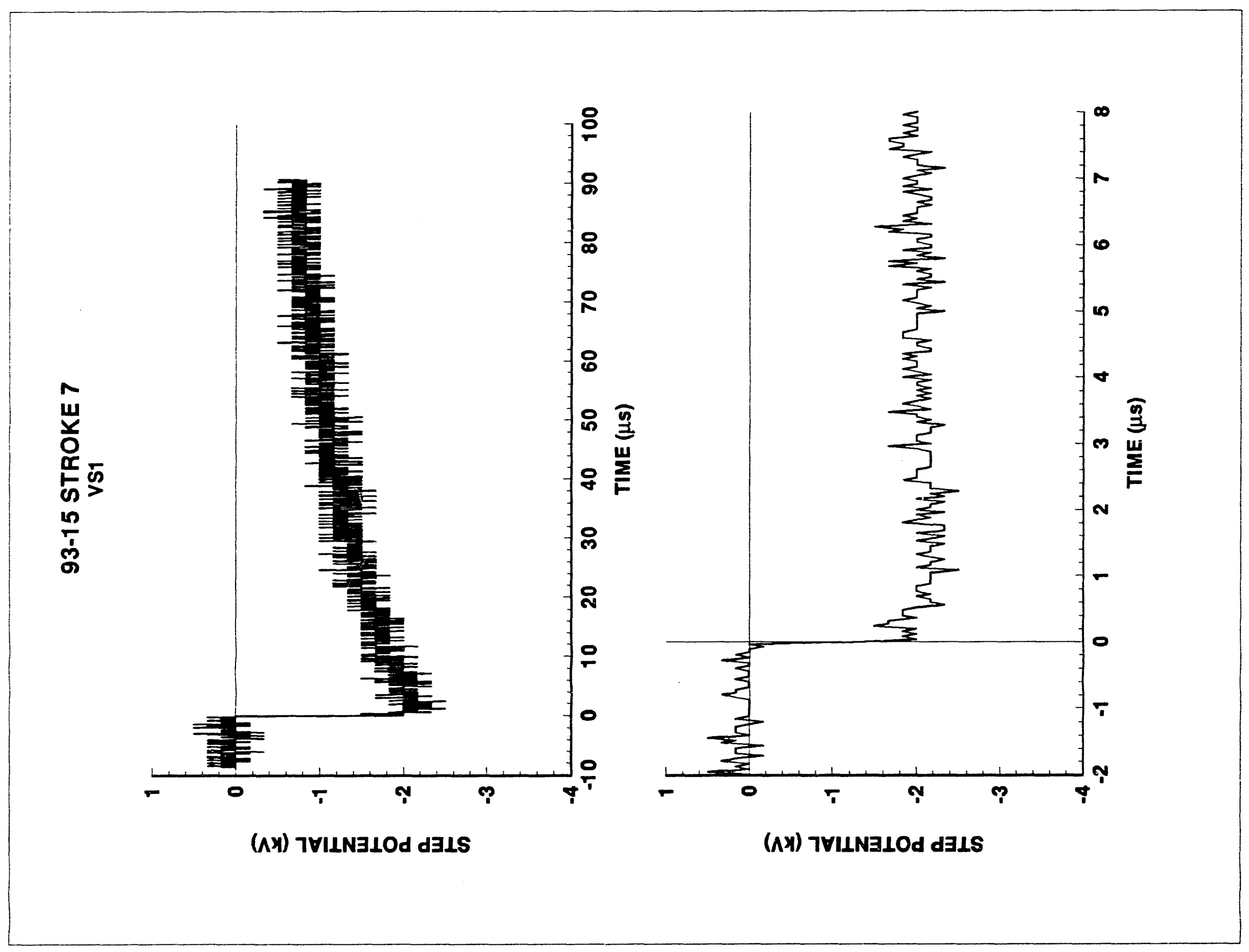




\section{3-15 STROKE 8 \\ VS1}
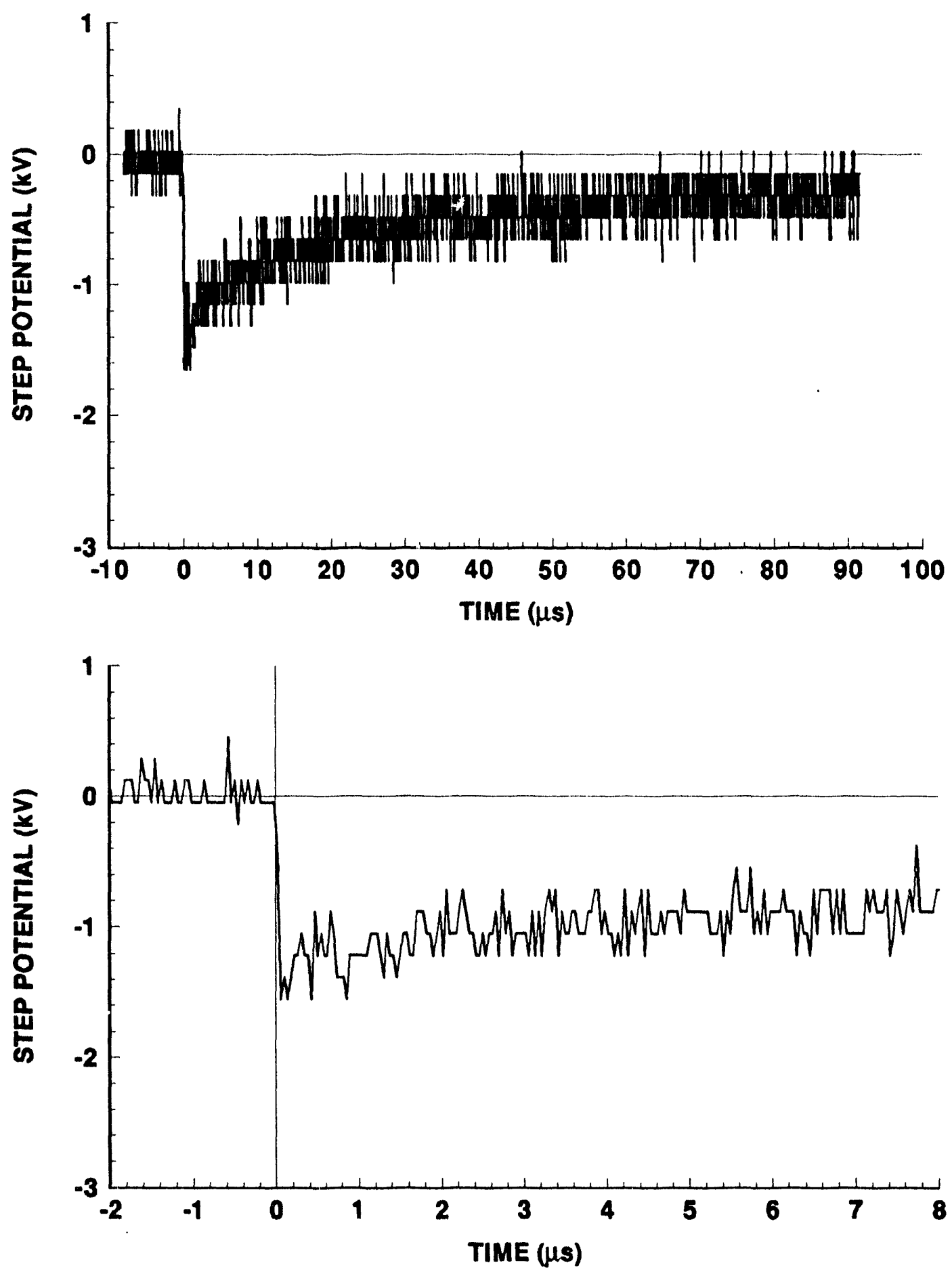


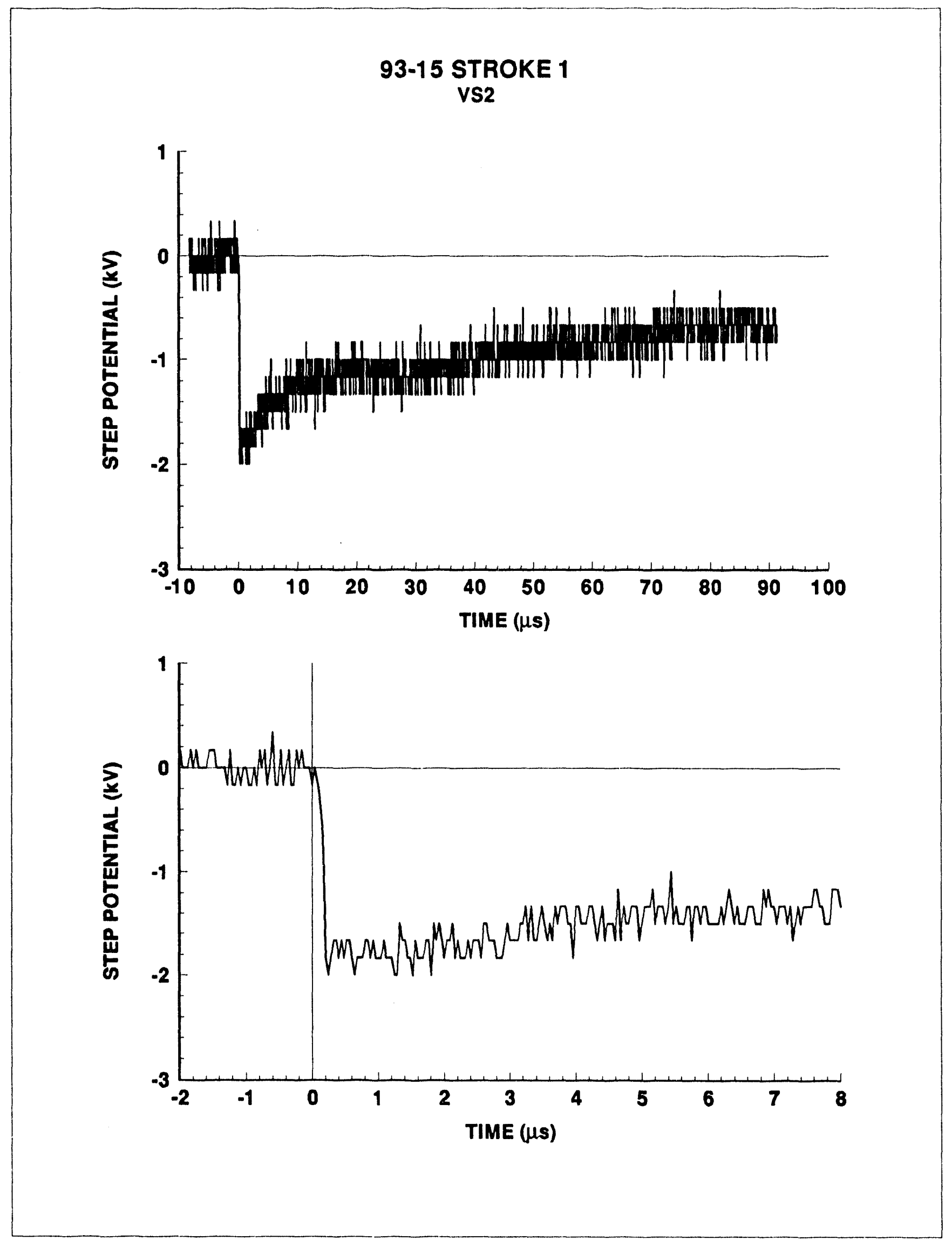



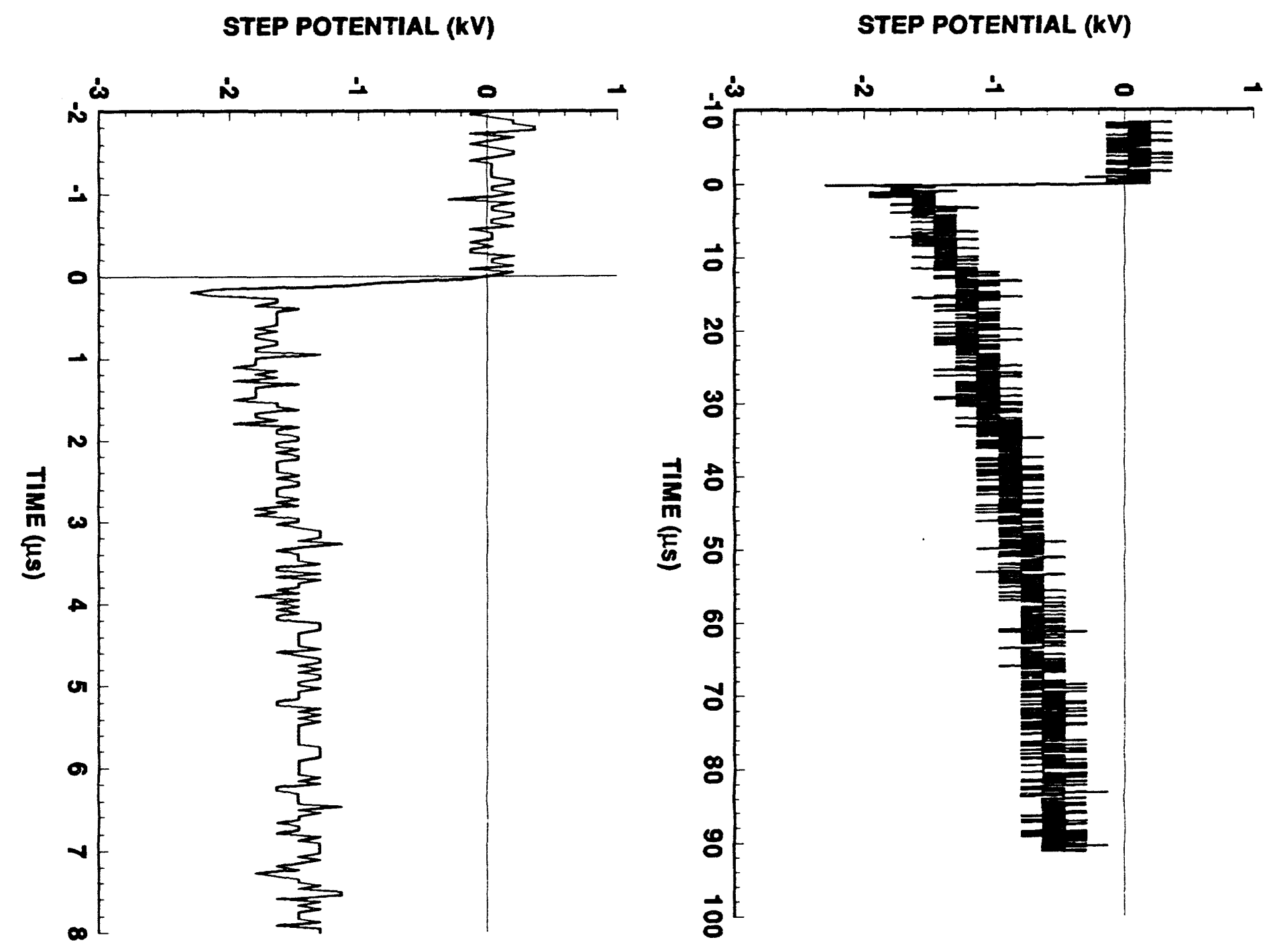


\section{3-15 STROKE 3 \\ vS2}
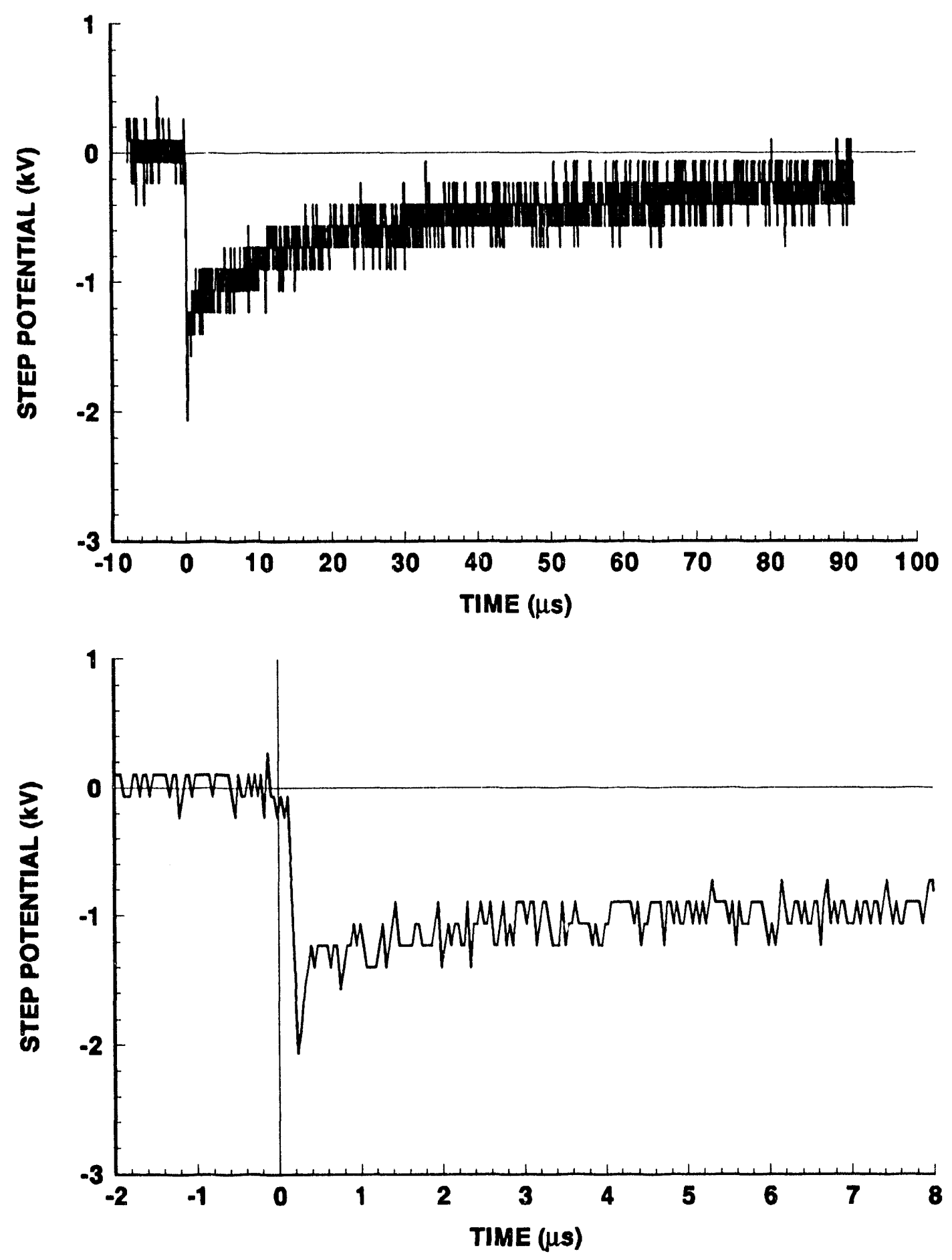


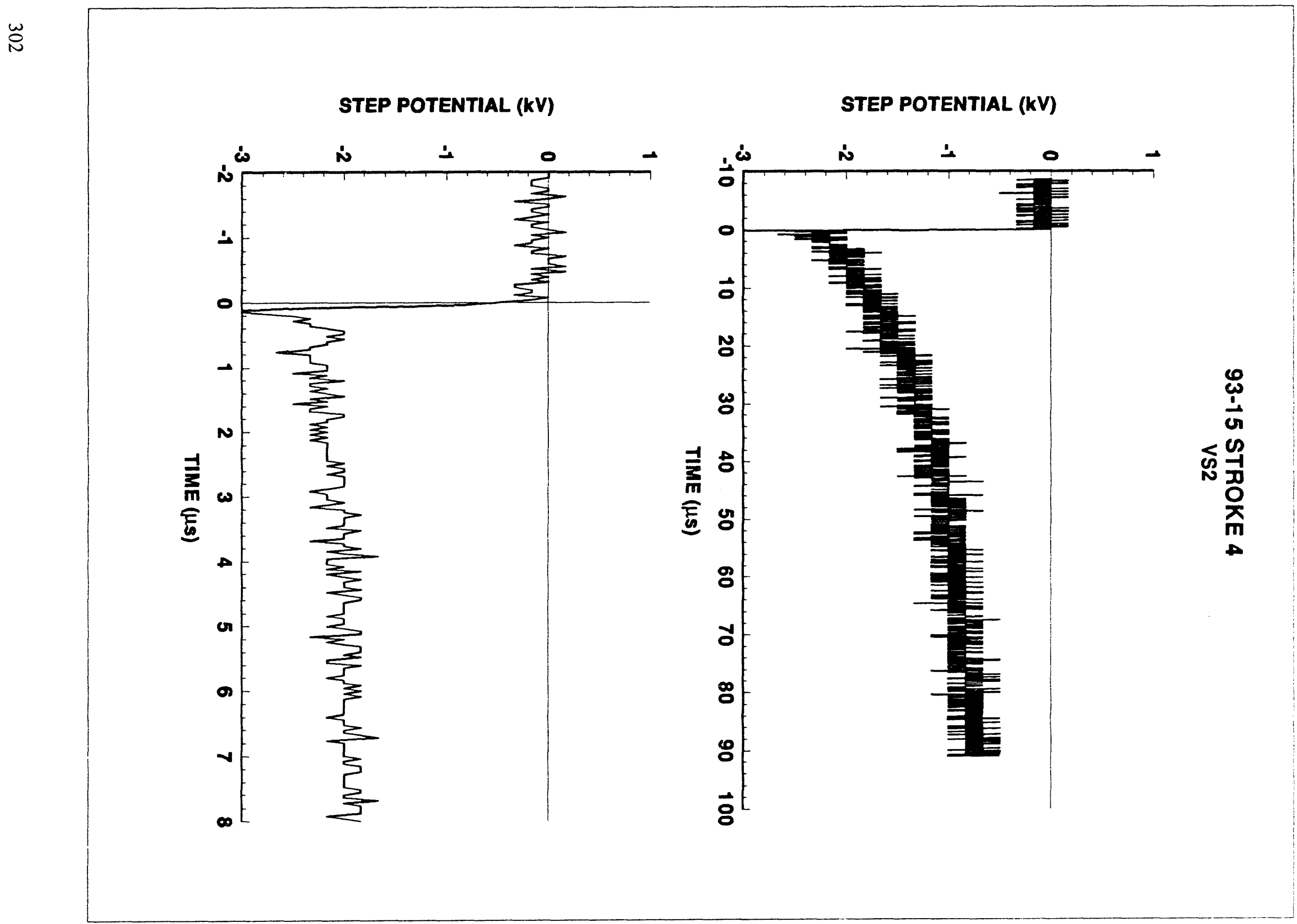




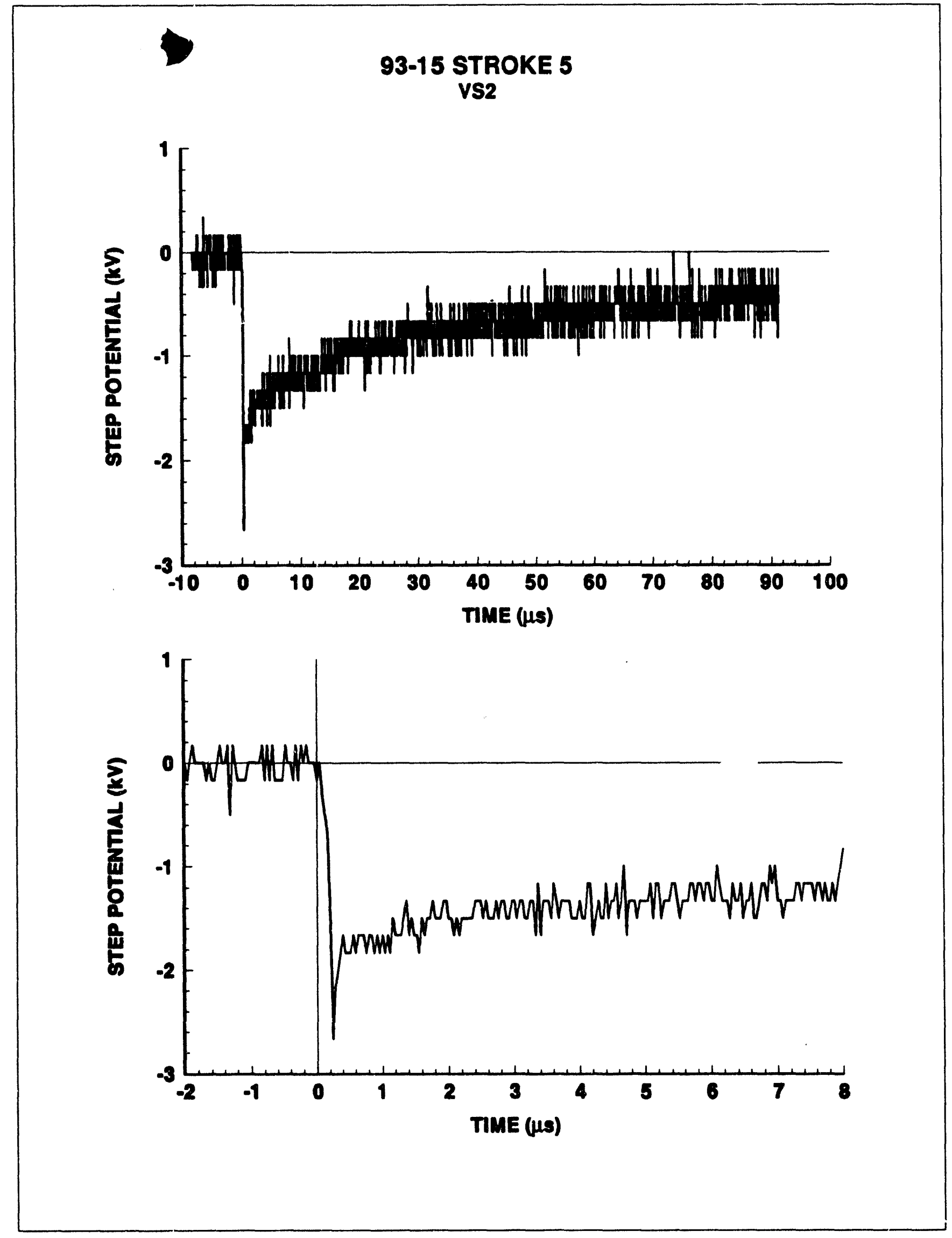


93-15 STROKE 6

vS2
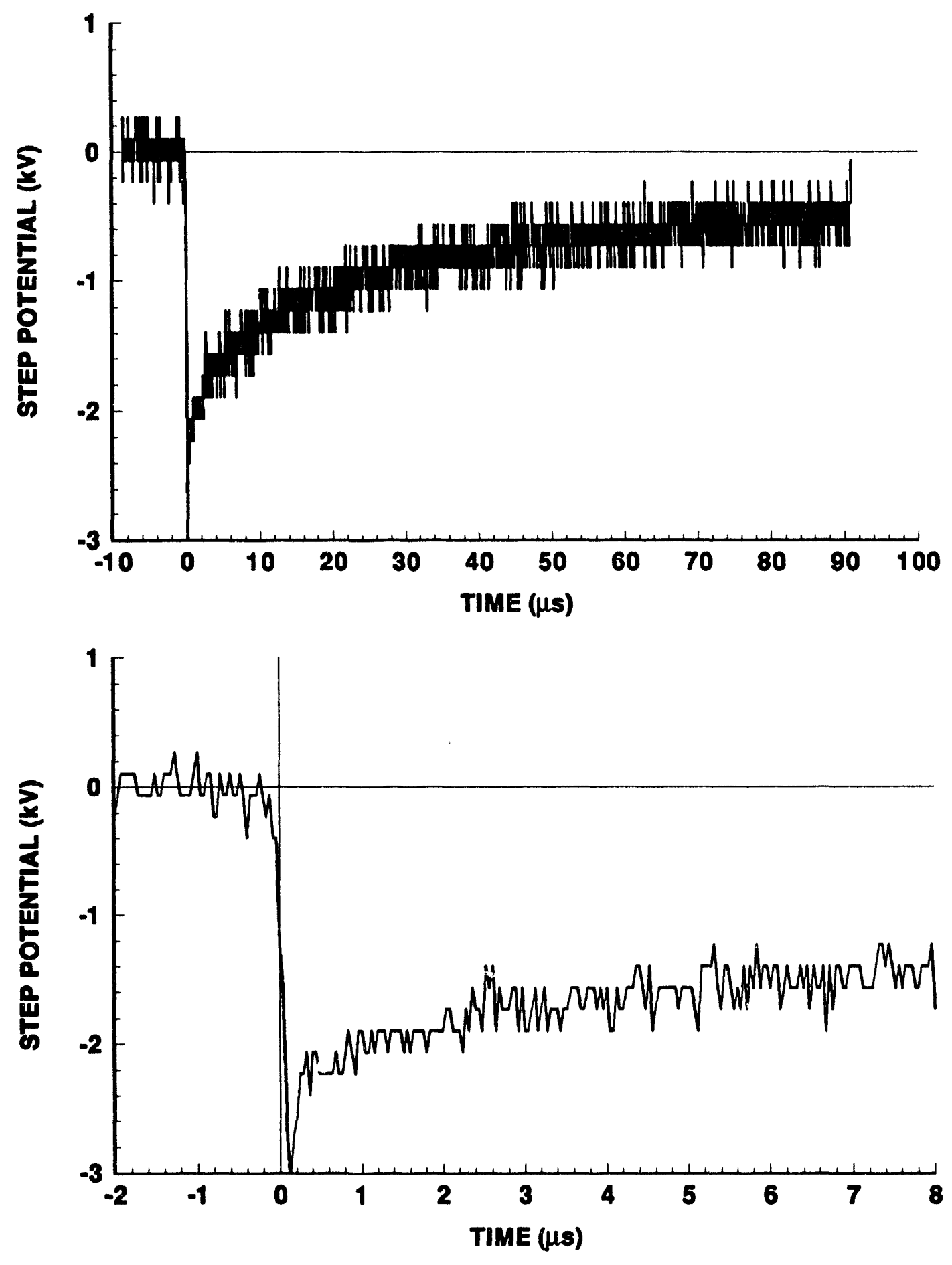


\section{3-15 STROKE 7}

VS2
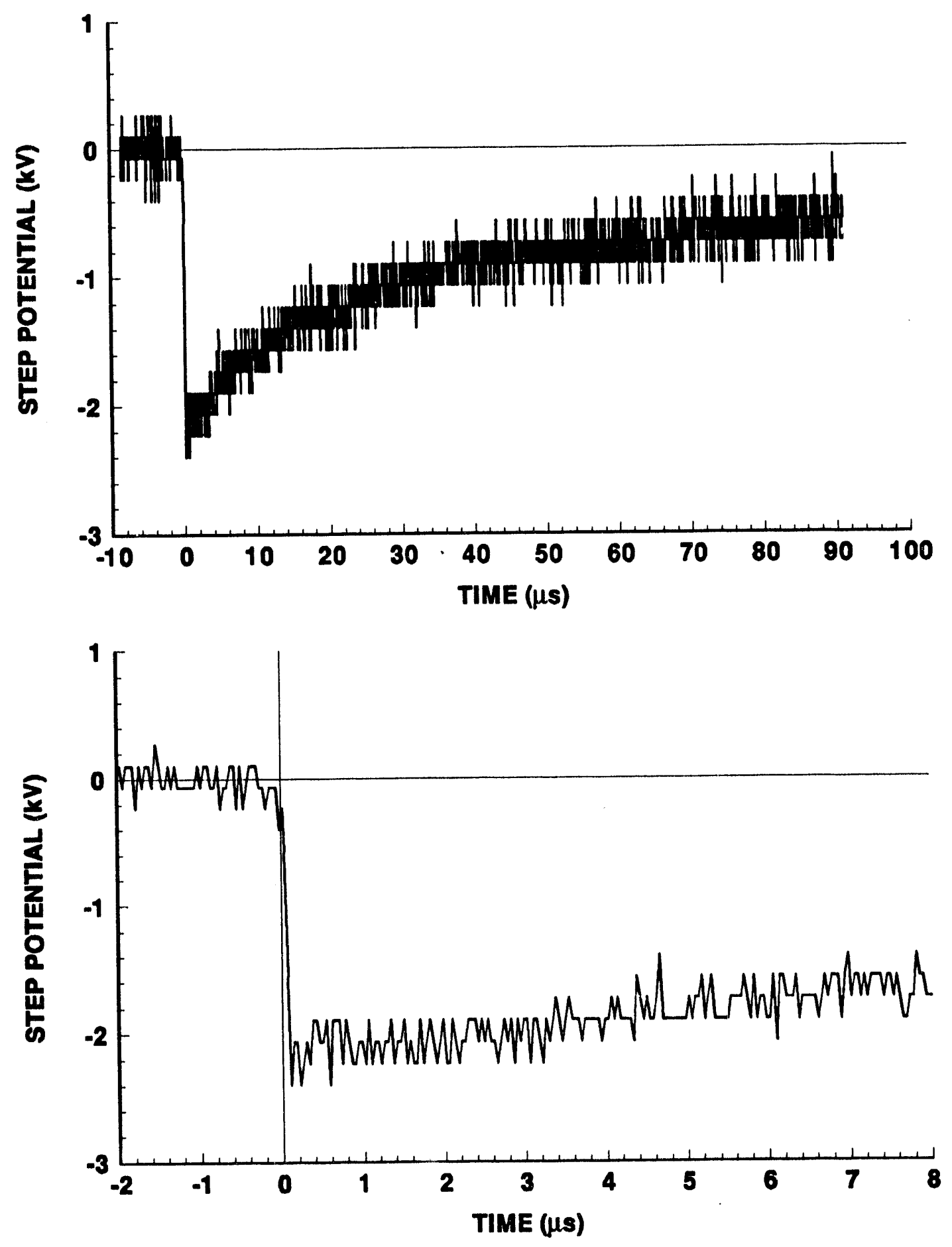


\section{3-15 STROKE 8 \\ VS2}
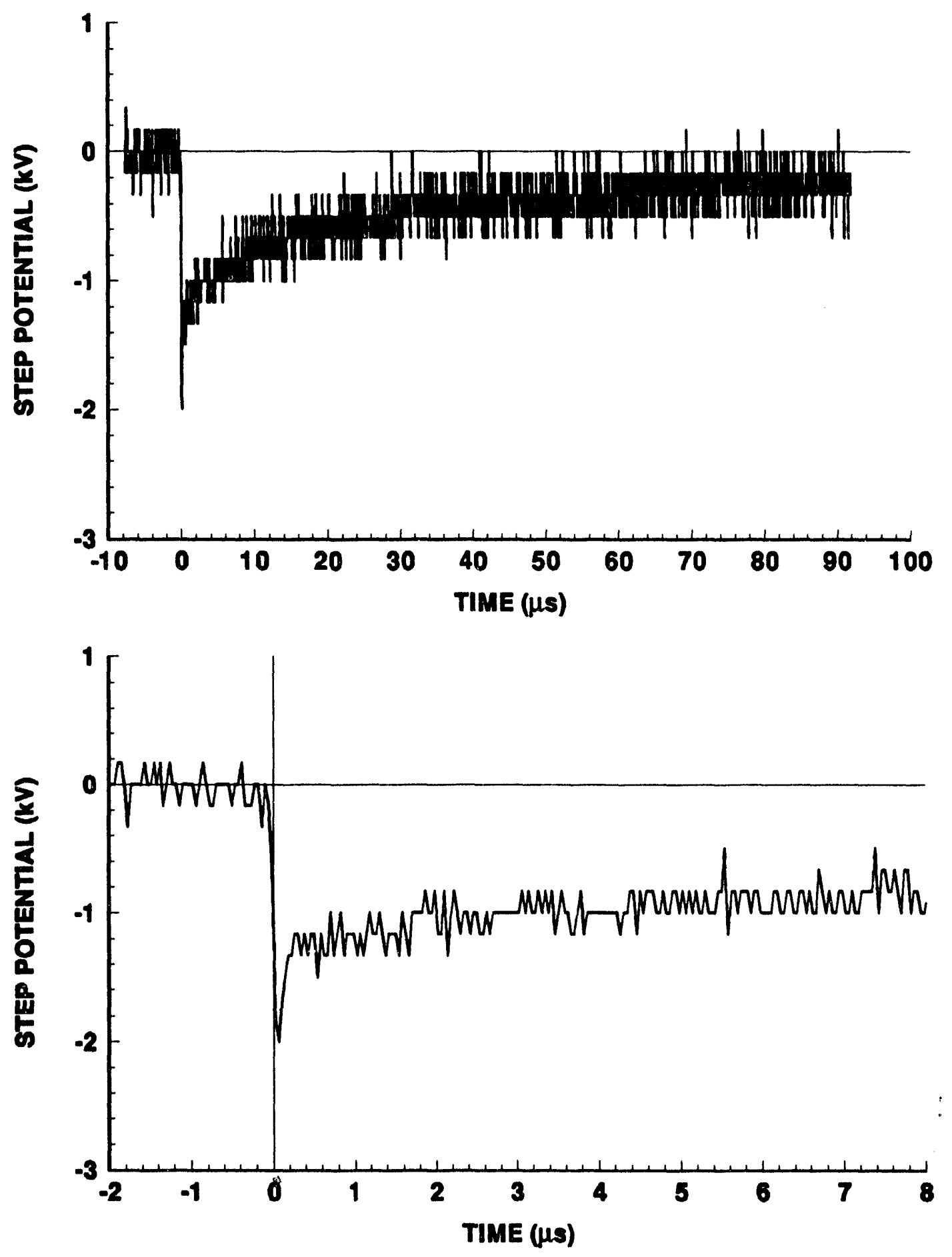


\section{3-15 STROKE 1 \\ vS4}
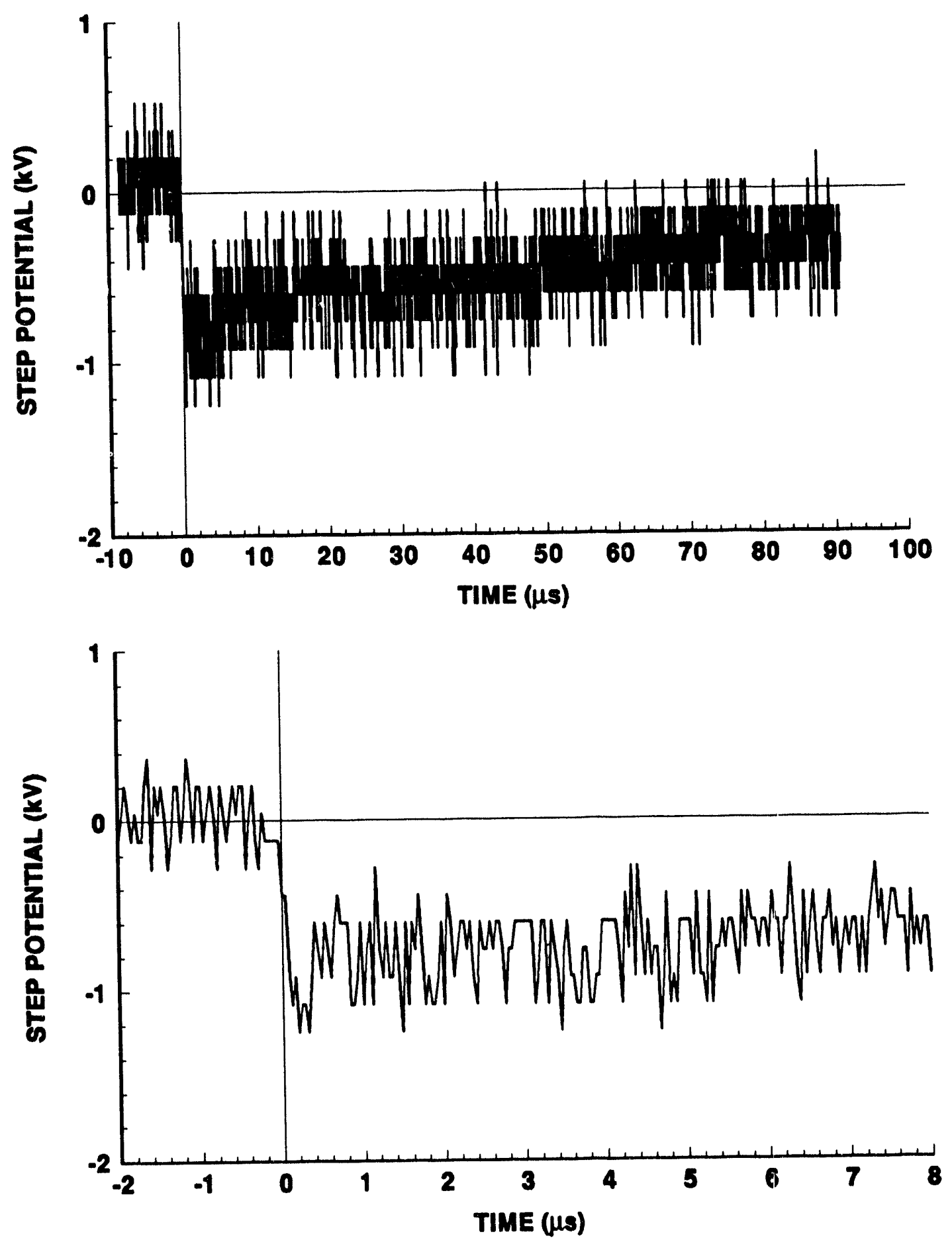
STEP POTENTIAL (KV)

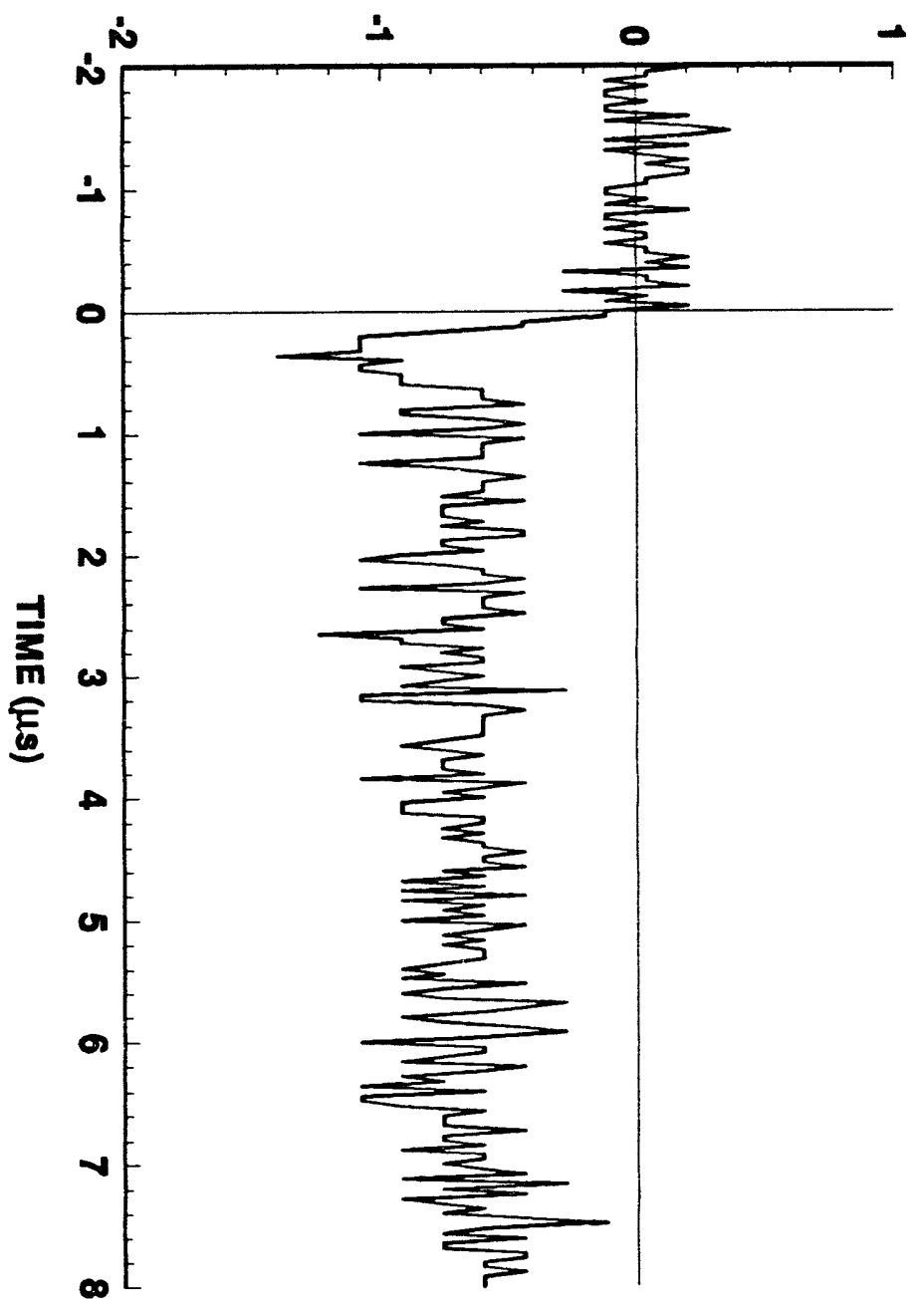

STEP POTENTIAL (kV)

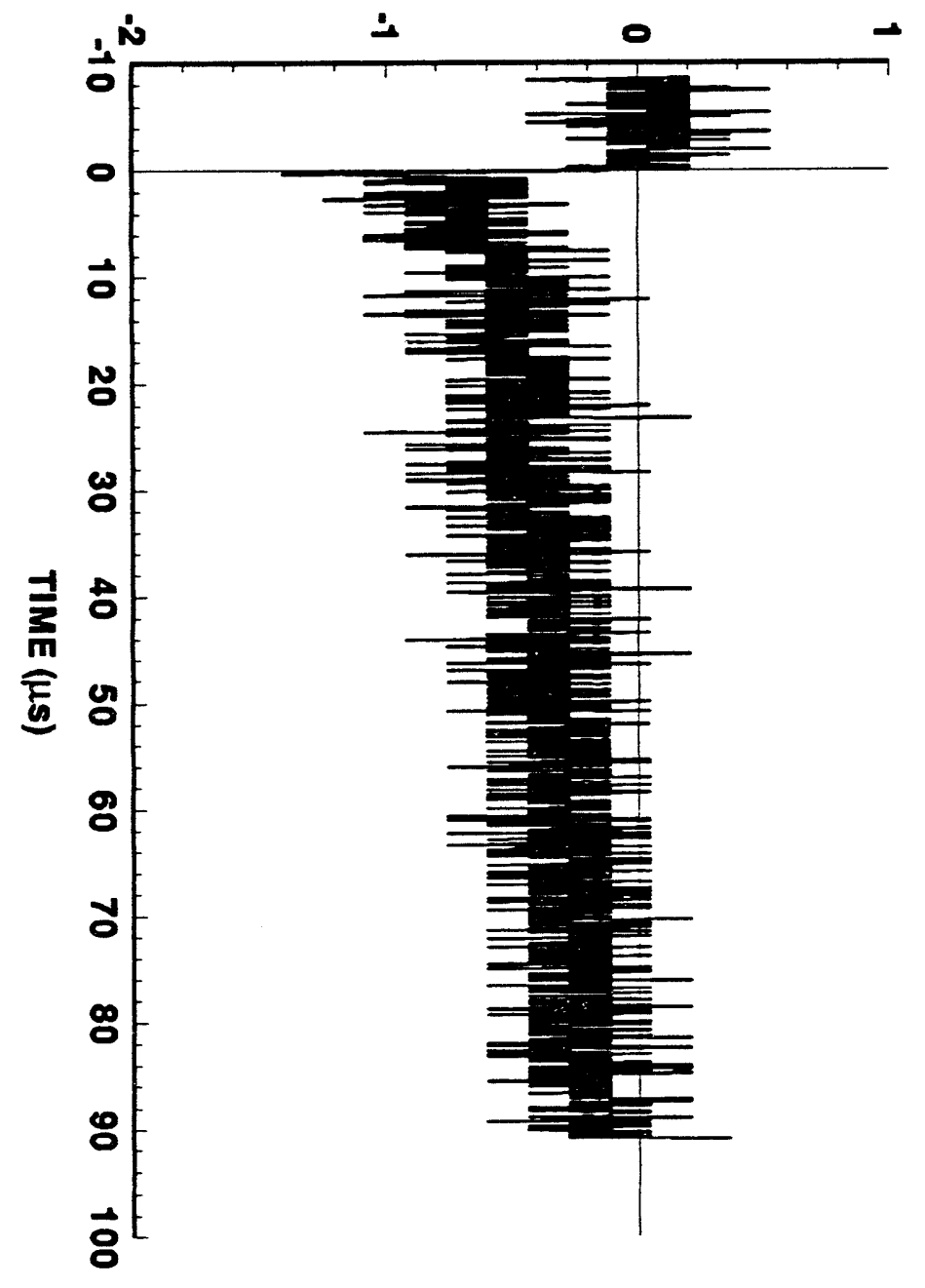

岕 क윽 ग्ग 중

N 
93-15 STROKE 3

VS4
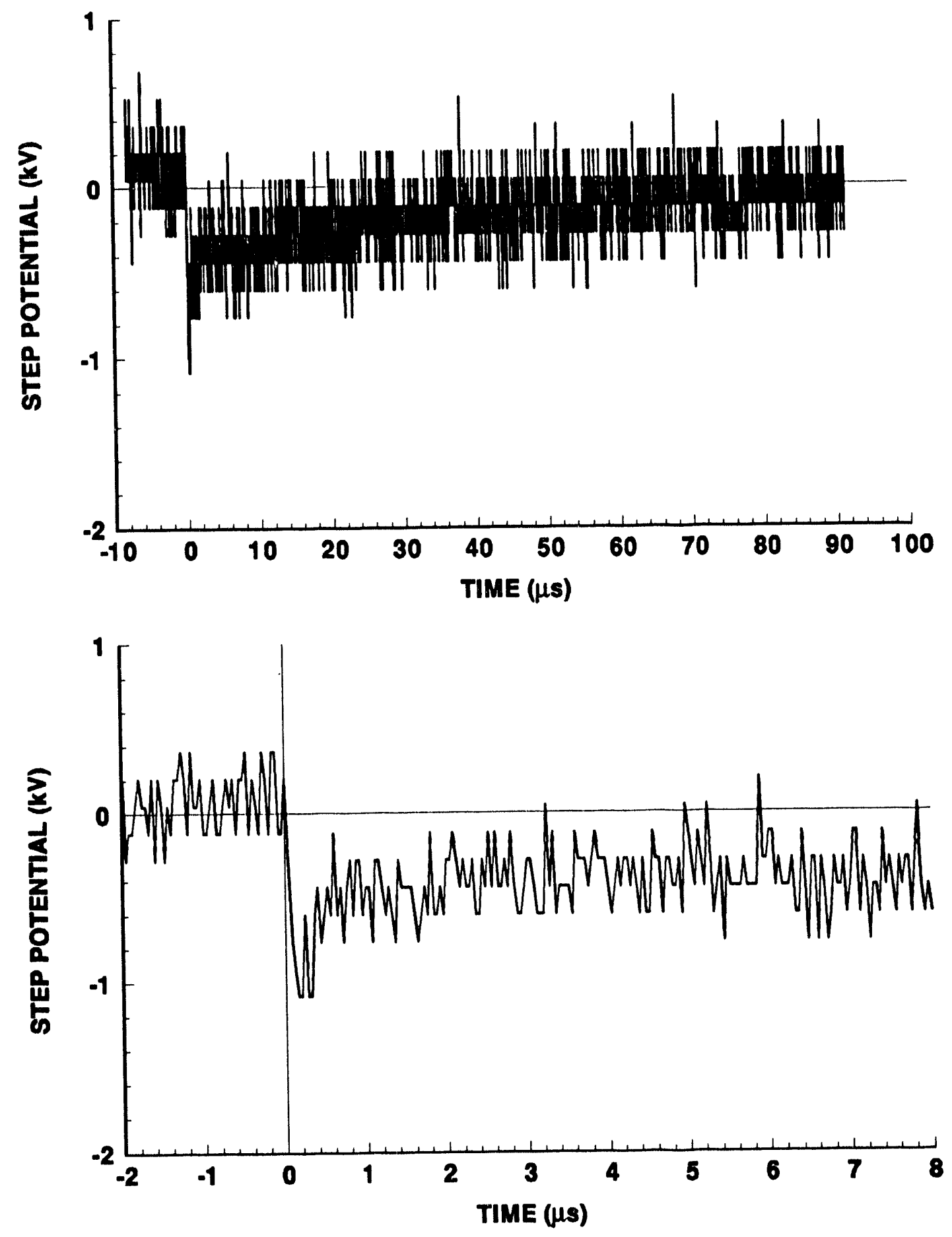


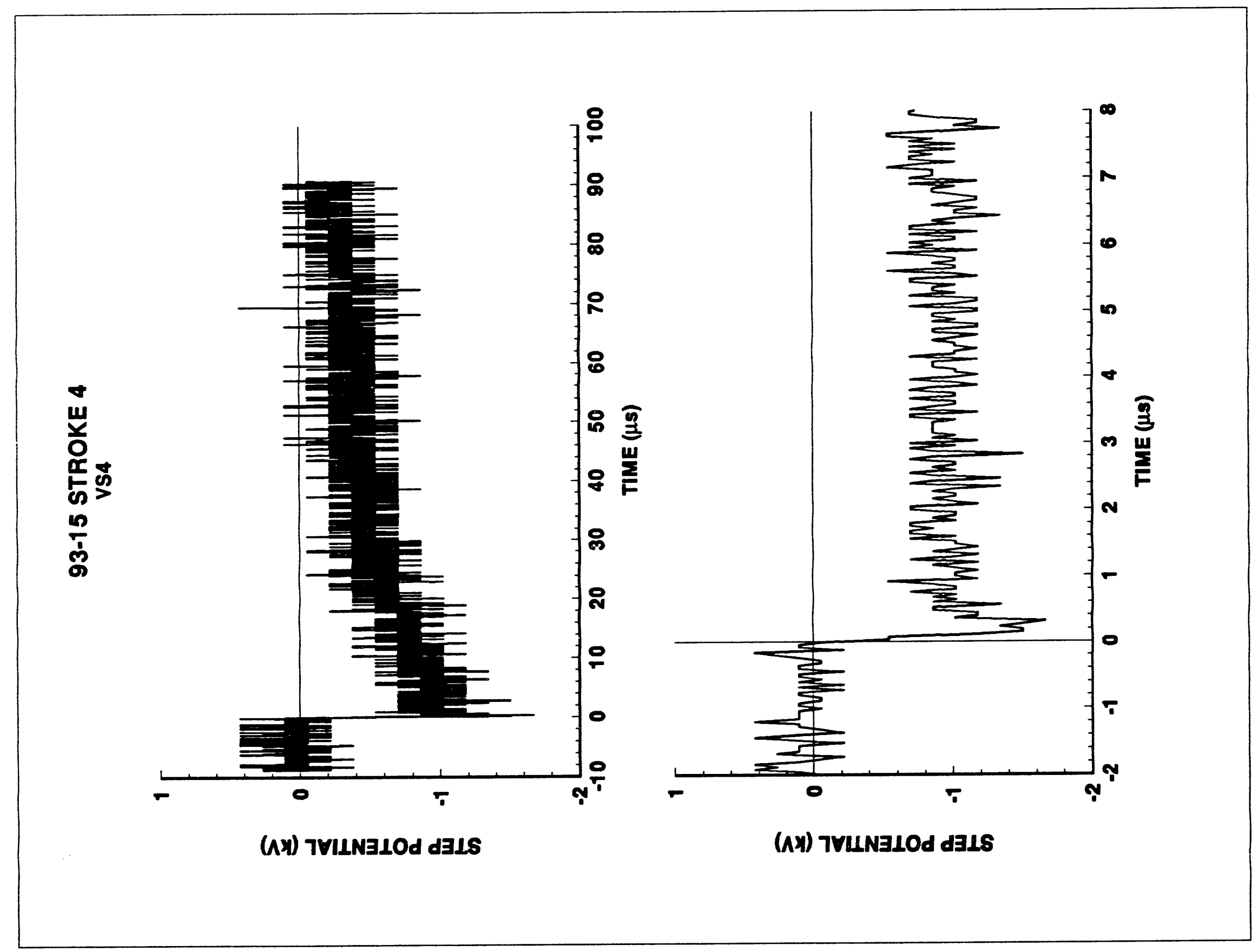




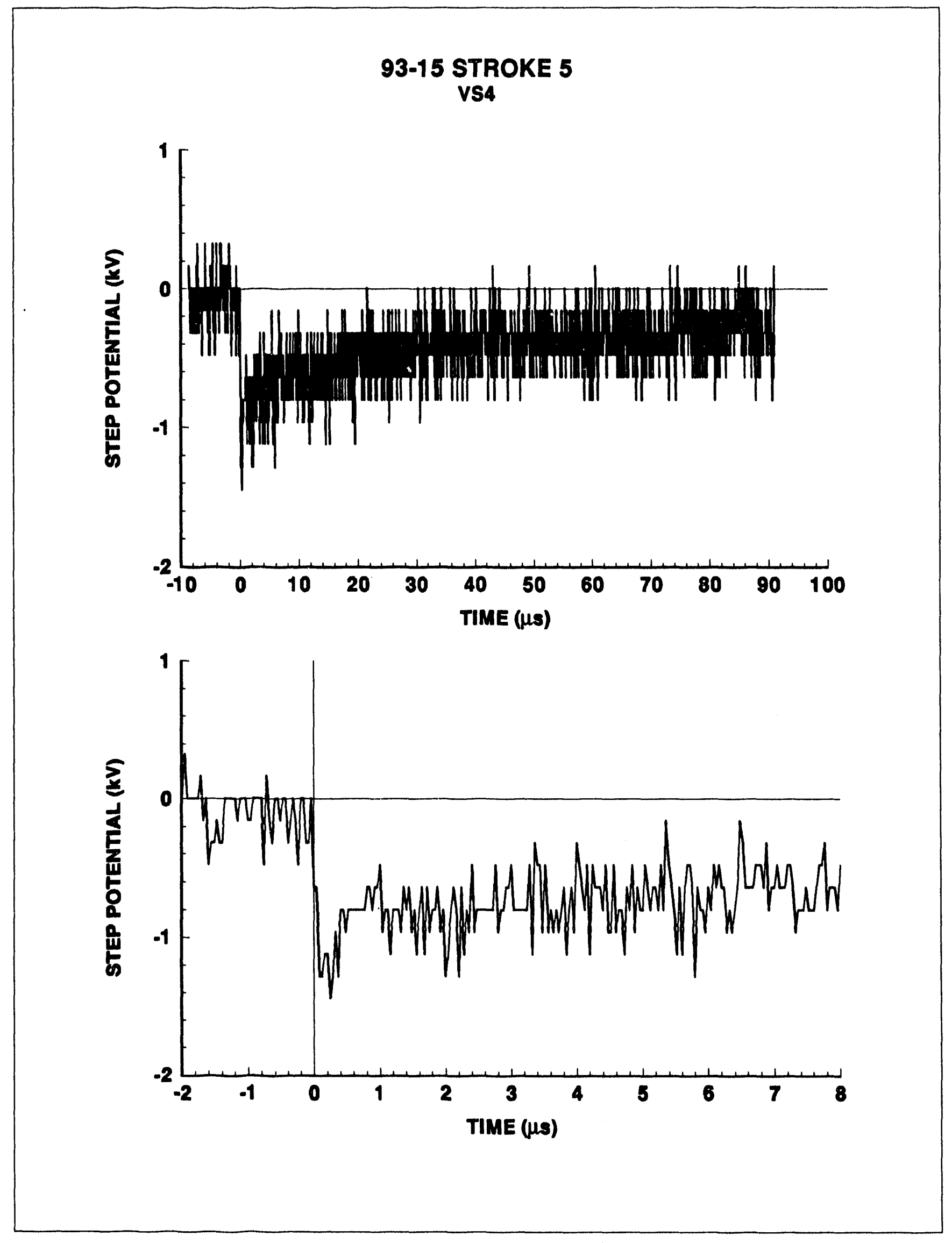




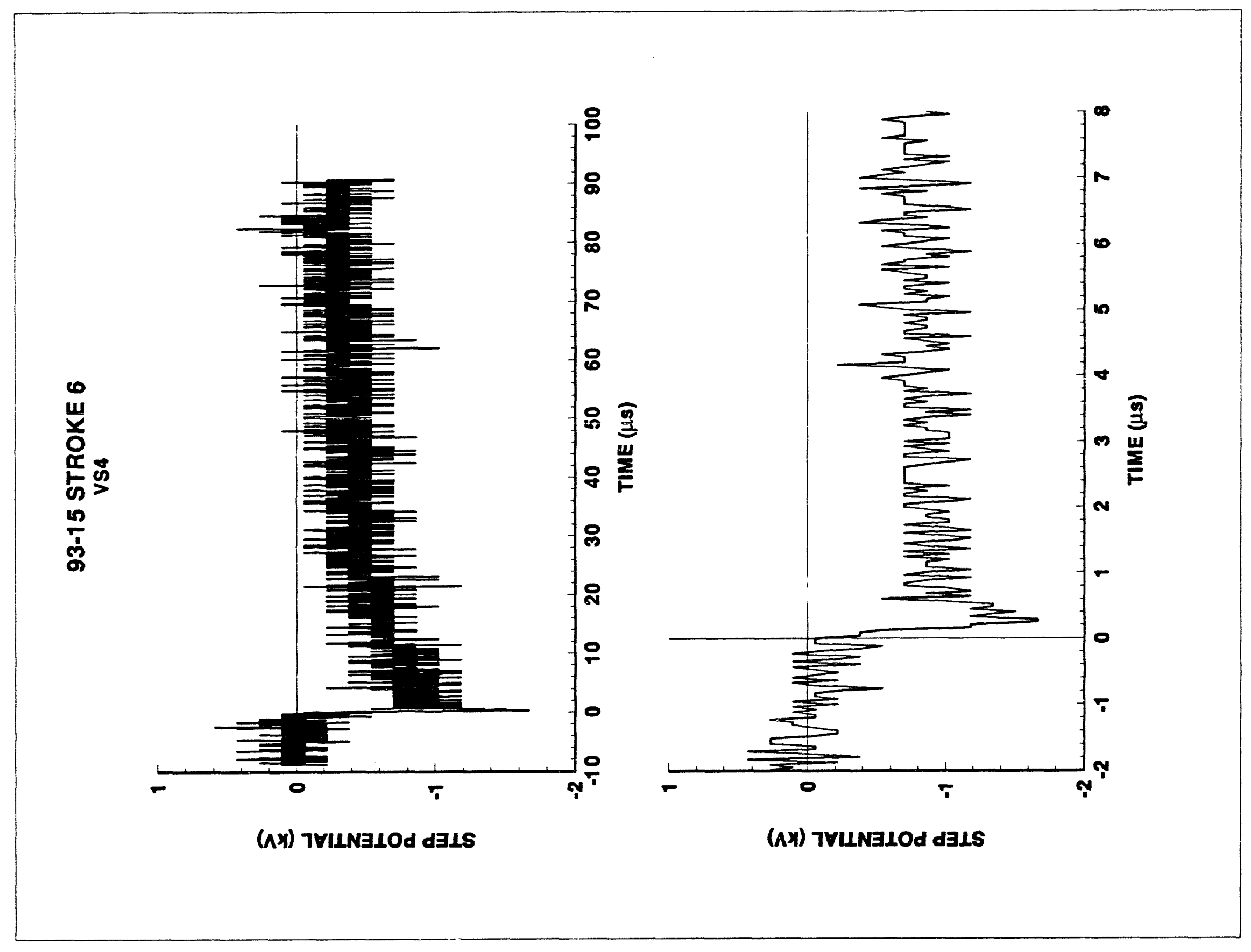




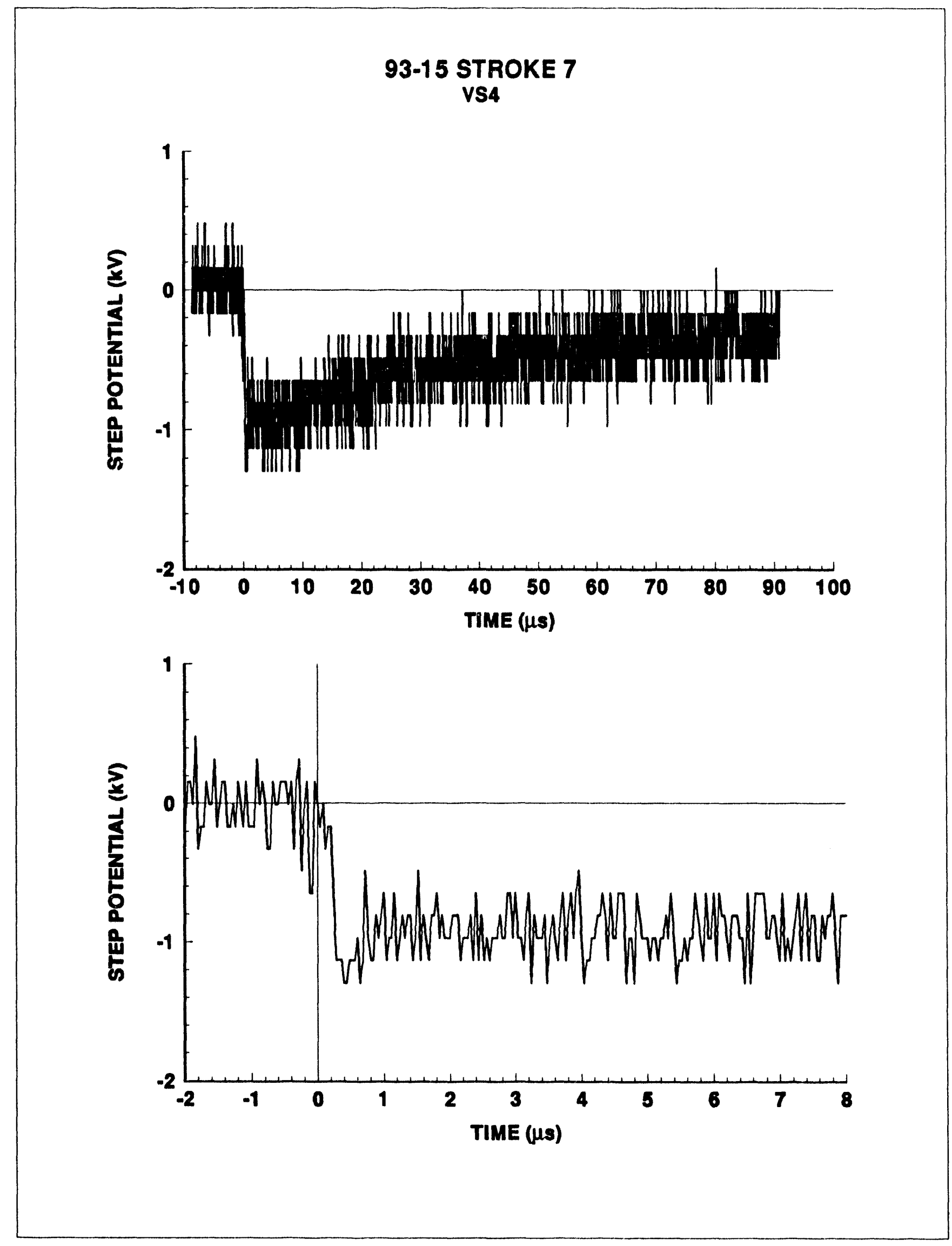




\section{3-15 STROKE 8 \\ vS4}
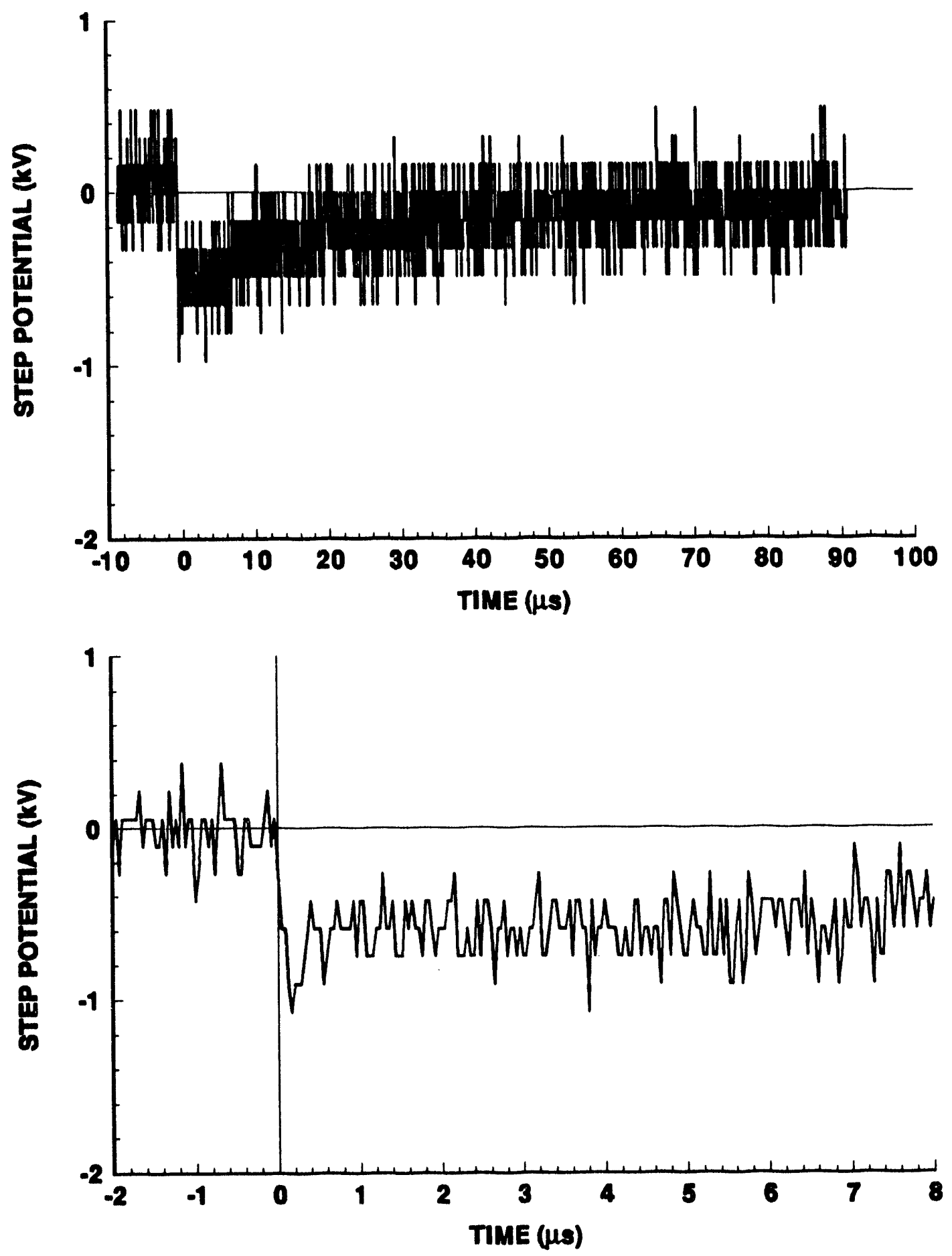


\section{3-15 STROKE 1 \\ VS5}
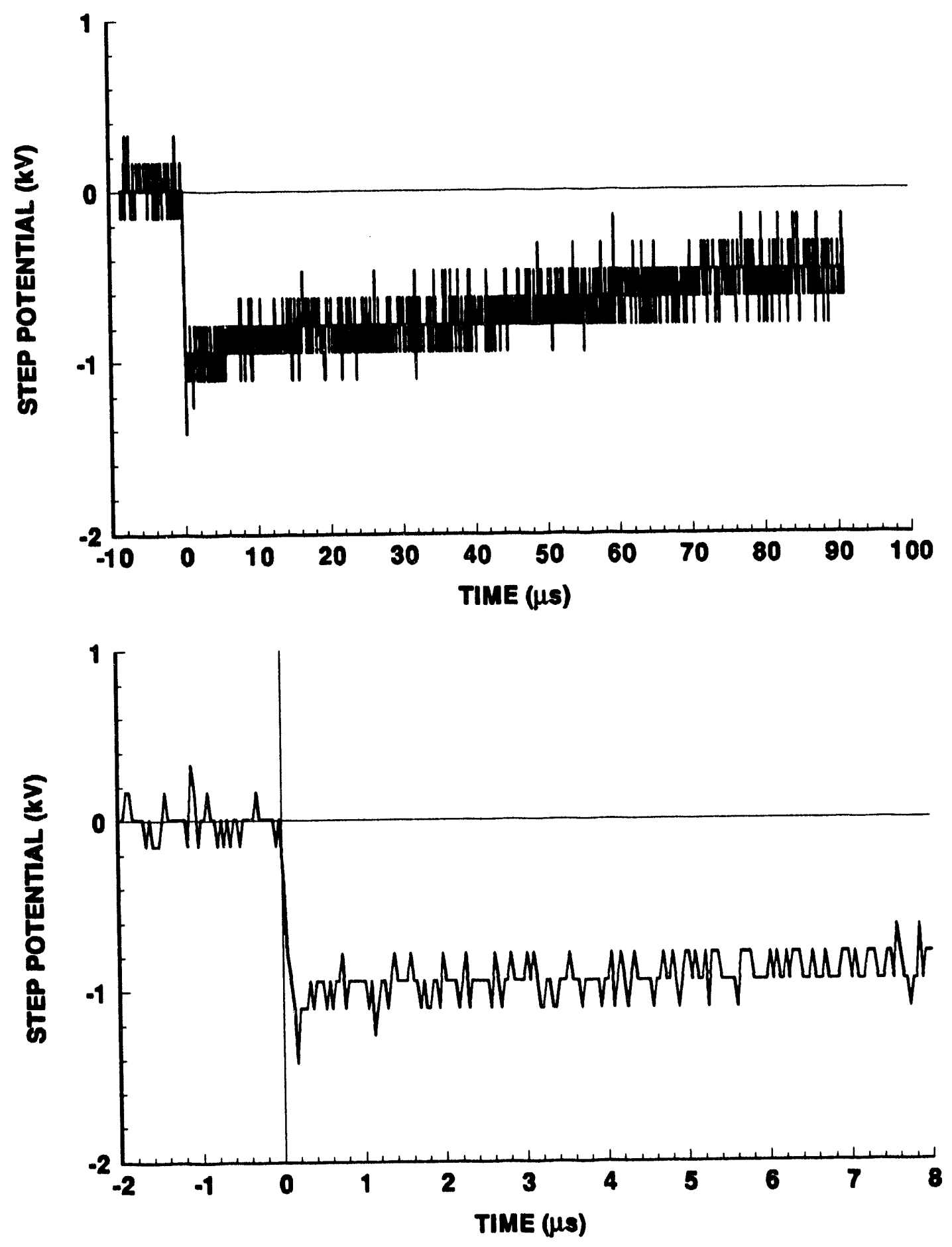


\section{3-15 STROKE 2}

VS5
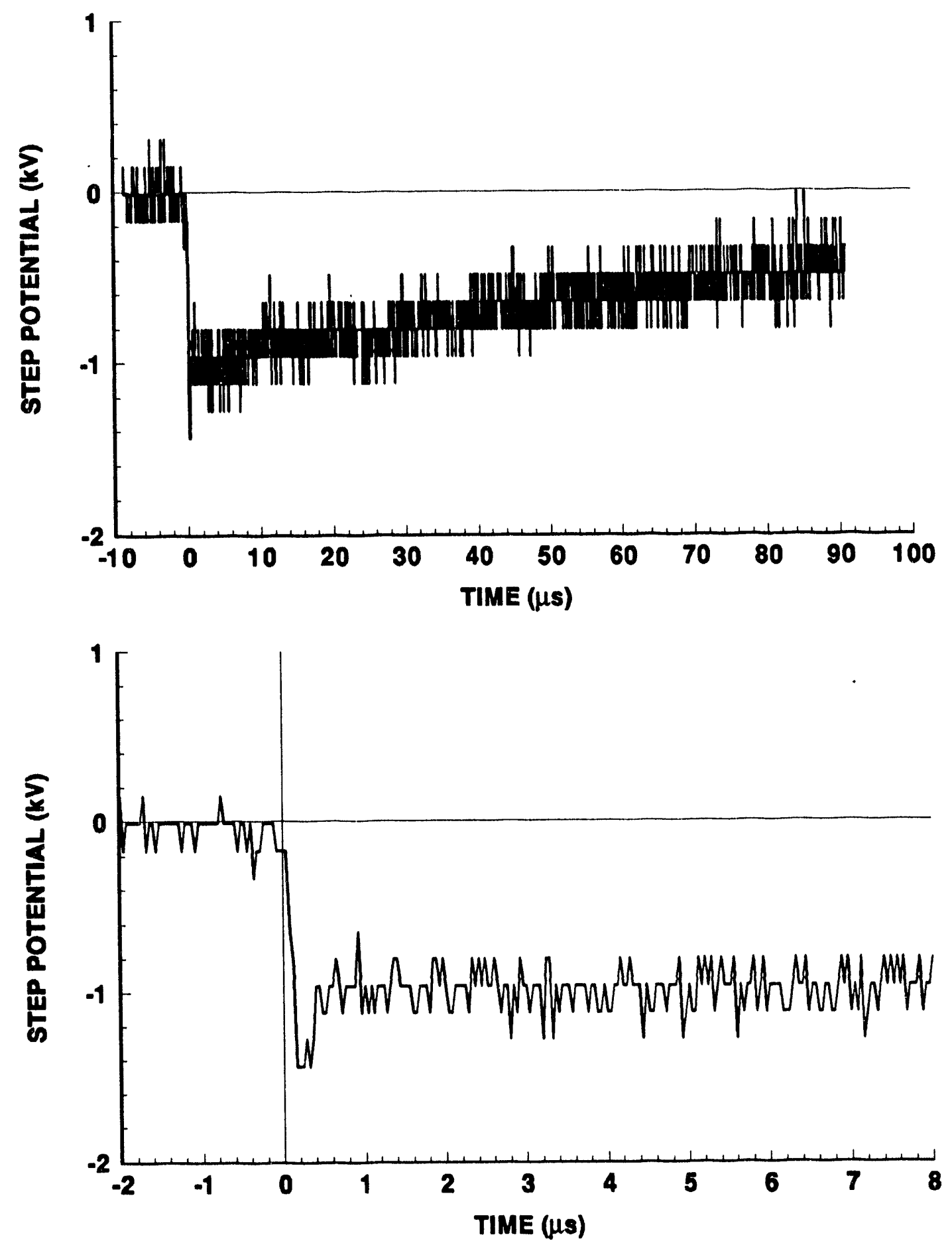


\section{3-15 STROKE 3}

v55
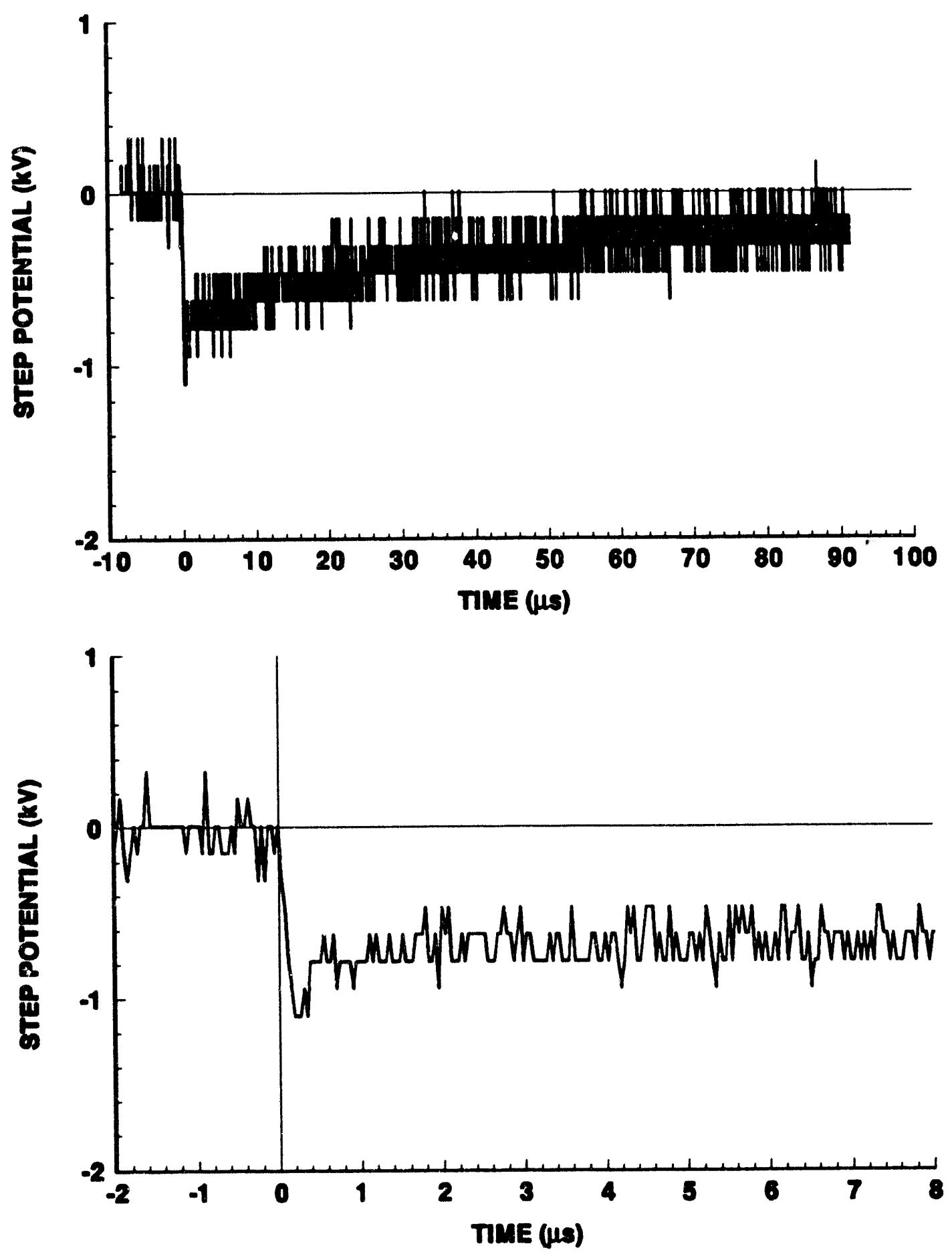


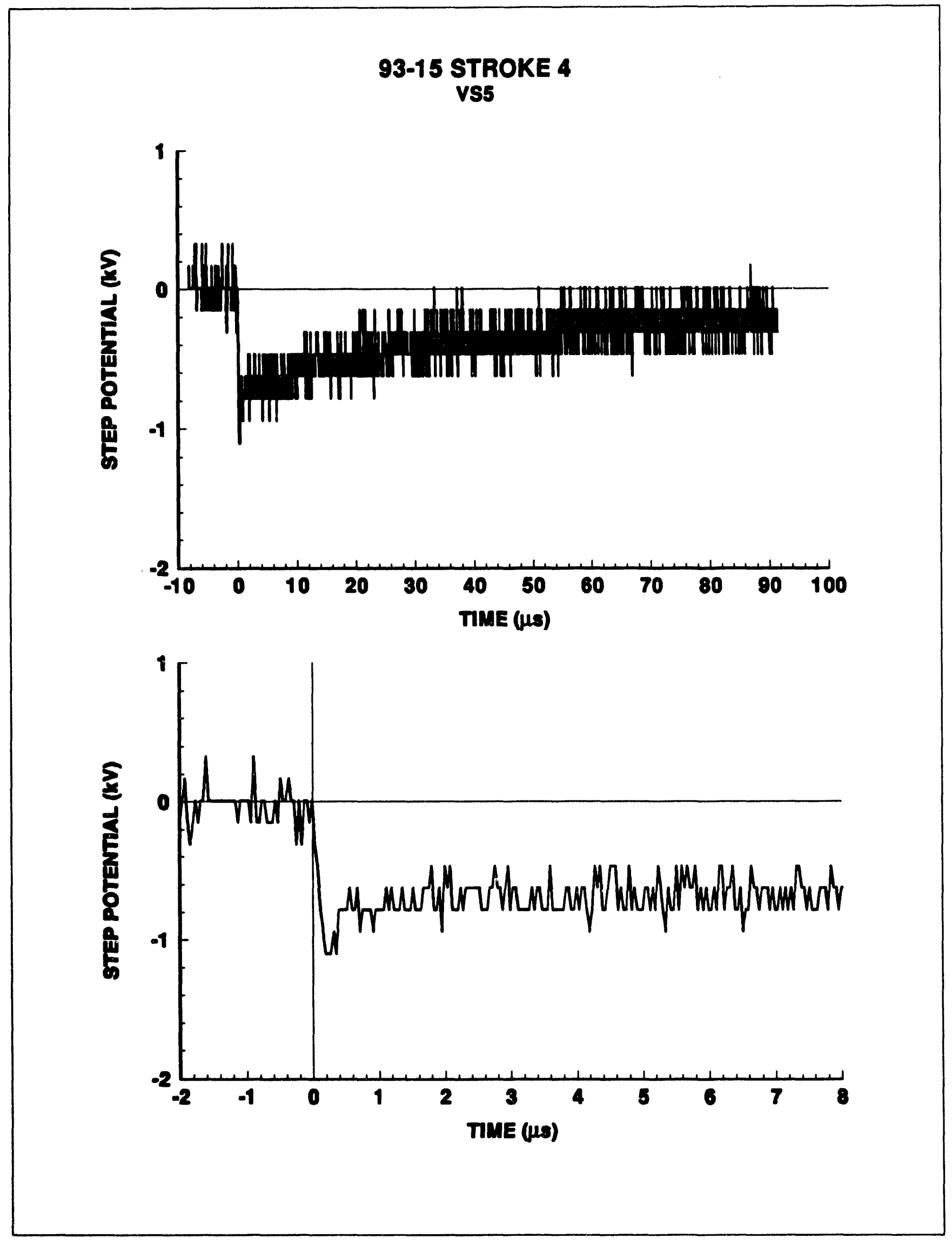




\section{3-15 STROKE 5 \\ vS5}
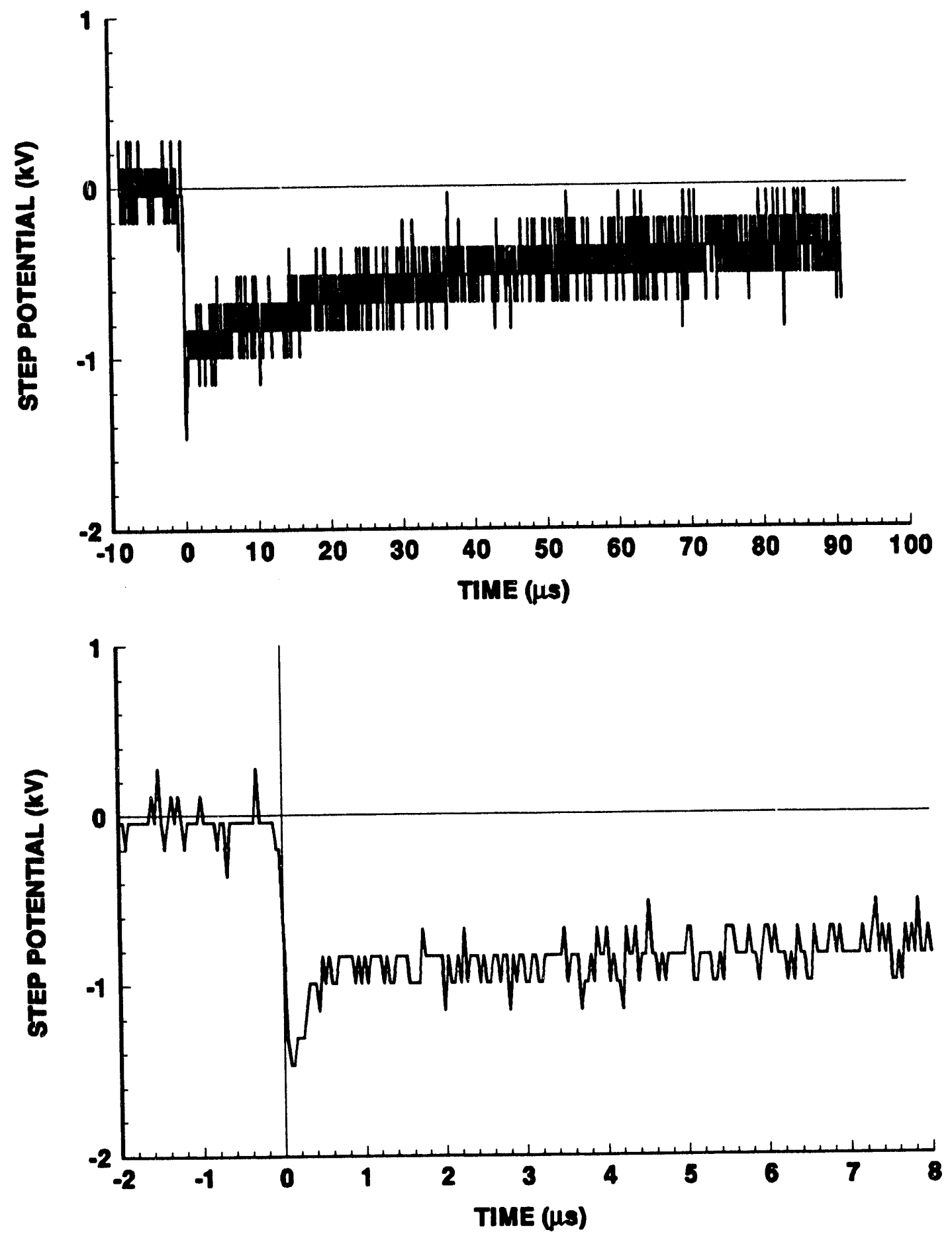


\section{3-15 STROKE 6}

vS5
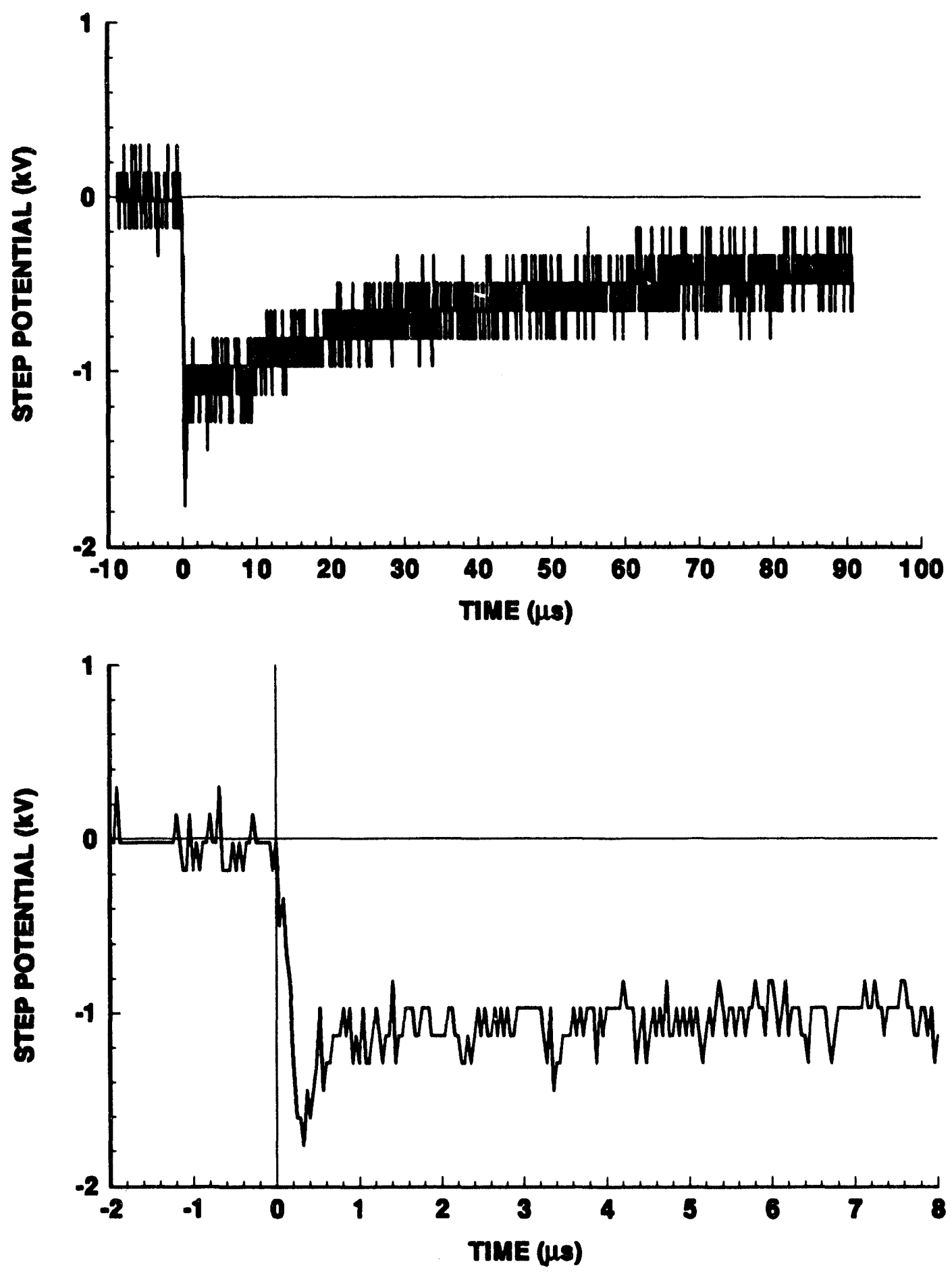


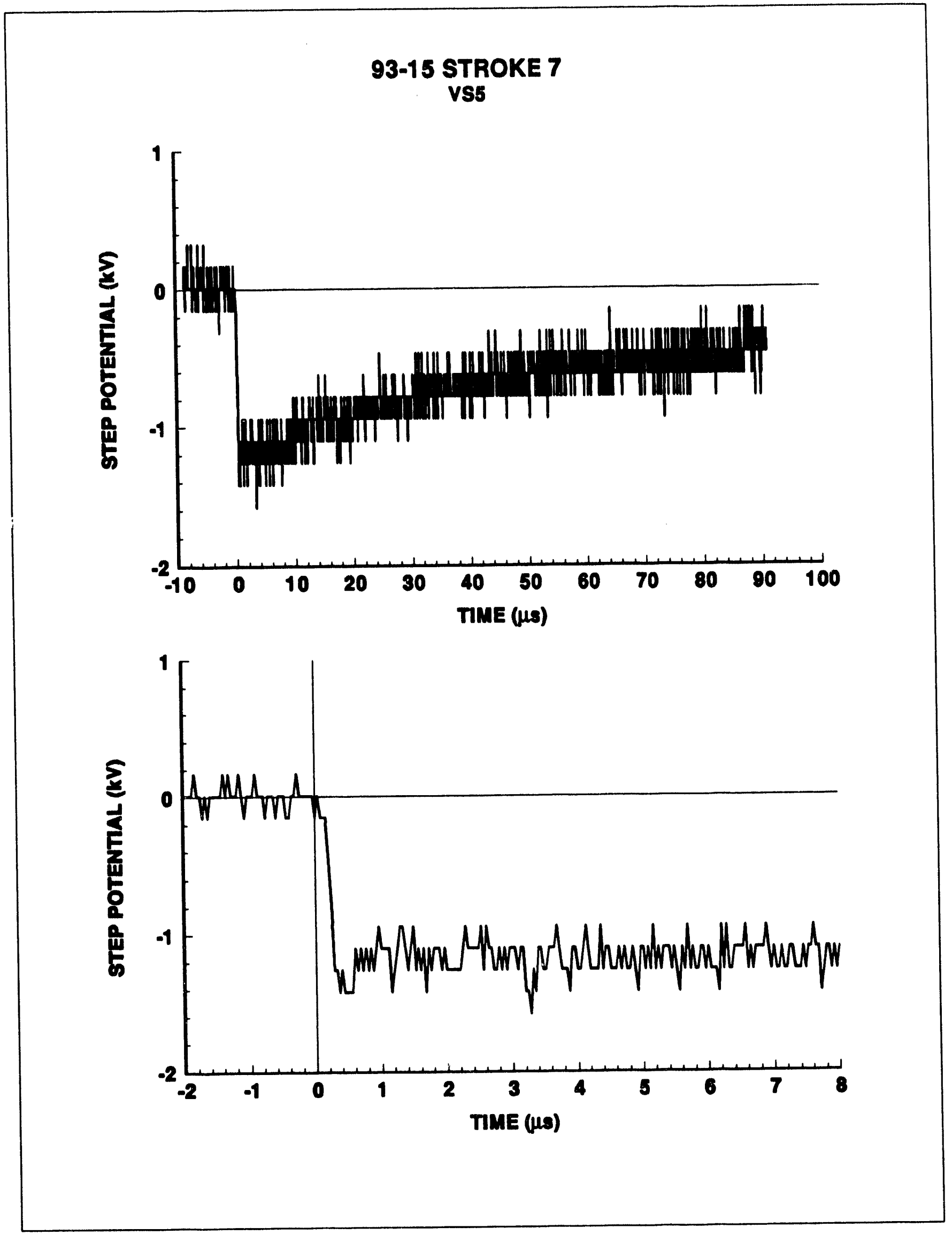




\section{3-15 STROKE 8 \\ vS5}
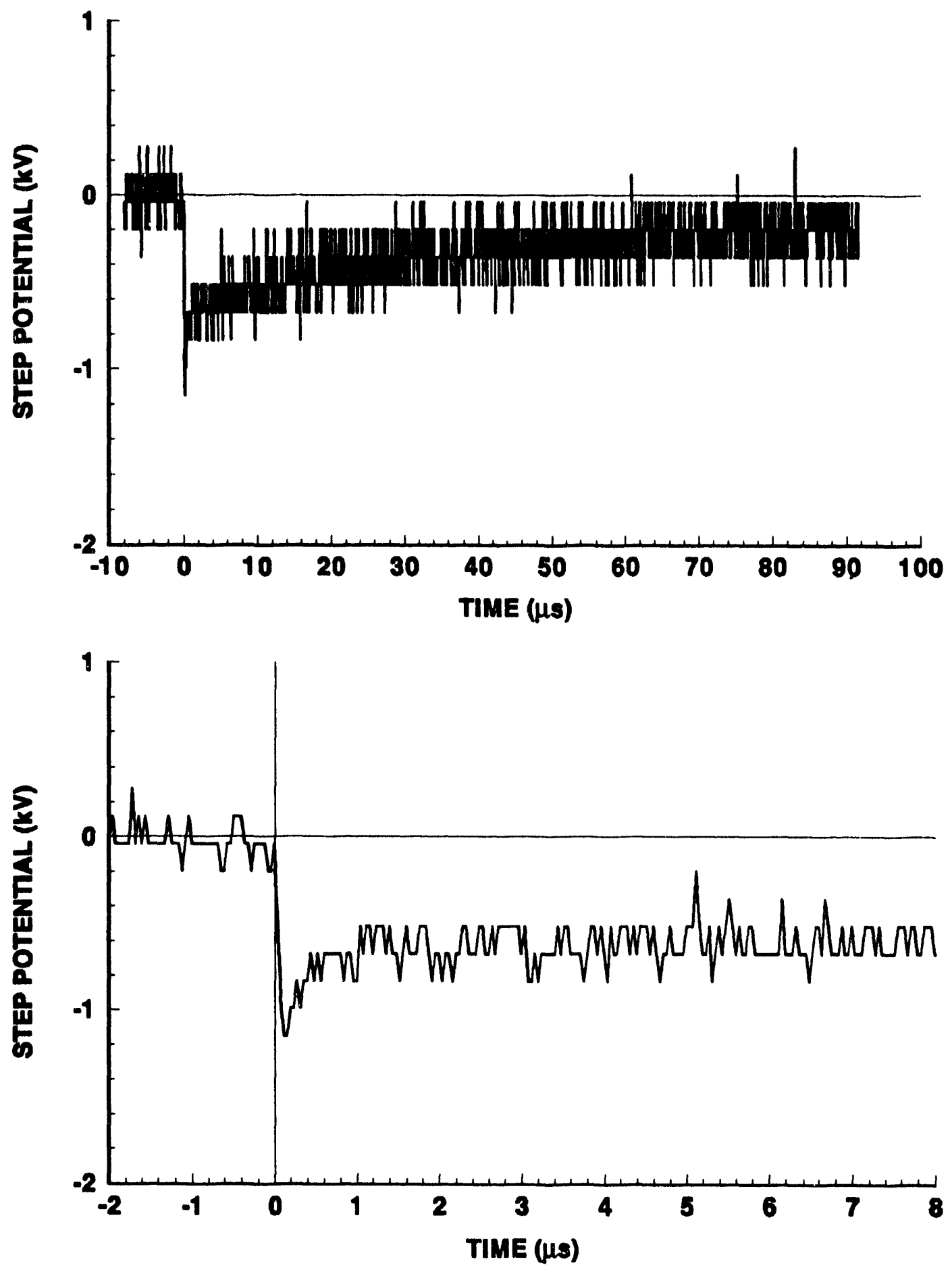


\section{Appendix B \\ Derivation of the Relationship for Obtaining \\ Earth Conductivity from a 4-Probe Measurement}

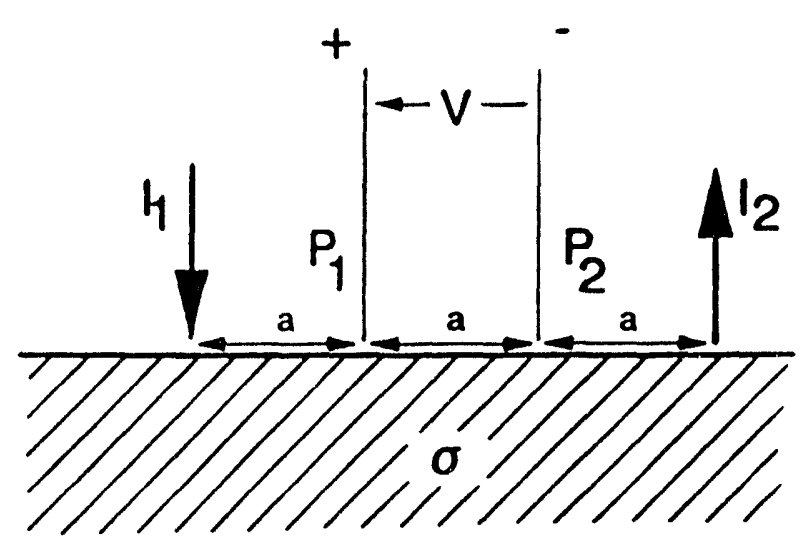

The potential difference $V$ between points $P_{1}$ and $P_{2}$ is given by

$$
V=\int E \cdot d I=\int(J / \sigma) \cdot d l
$$

in which $\mathbf{E}$ is the total electric field vector along the path of integration between points $P_{1}$ and $P_{2}, \mathbf{J}$ is the current density $\left(\mathrm{A} / \mathrm{m}^{2}\right)$ due to the current injected and extracted by the instrument at the outer probes, and $\sigma$ is the conductivity of the earth averaged over a depth comparable to the separation distance a of the probes. The conductivity is assumed to be homogenous within the vicinity of the measurement. The magnitude of the current density $\mathbf{J}$ due to a current $\mathbf{I}$ injected into the earth at a single point is

$$
\mathbf{J}=\mathbf{I} /\left(2 \pi \mathrm{r}^{2}\right)
$$

where $r$ is the radial distance outward from the point of injection.

Using (B-1) and (B-2) to determine the potential differences between $P_{1}$ and $P_{2}$ due to $I_{1}$ and $I_{2}\left(=-I_{1}\right)$ separately and then combining them by superposition yields

$$
\begin{aligned}
& V=I_{1} /(2 \pi \sigma)[1 / a-1 /(2 a)]+I_{2} /(2 \pi \sigma)[1 /(2 a)-1 / a] \\
& V=I_{1} /(2 \pi \sigma a)
\end{aligned}
$$

Finally, since $R$, as indicated by the instrument when the meter needle is properly nulled, is given by

$$
\mathrm{R}=\mathrm{V} / \mathrm{I}_{1} \text {, }
$$

it follows that

$$
\sigma=(2 \pi \mathrm{aR})^{-1}
$$


 \\ $\underset{\tilde{N}}{\sim}$}

$\underset{ \pm}{w}$

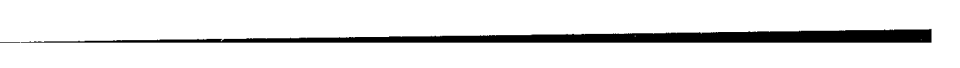


Appendix C

Potential Distribution about an Earth Lightning Strike with Conducting Surface Layer

\author{
L. K. Warne
}

Electromagnetic Analysis and Test Department

Sandia National Laboratories 


\title{
Potential Distribution About an Earth Lightning Strike with Conductive Surface Layer
}

\author{
Larry K. Warne \\ Electromagnetic Analysis and Test Department \\ Sandia National Laboratories \\ Albuquerque, NM
}

\begin{abstract}
The problem of an electric current line source terminating normally in a conductive half space, with conductive top layer, is solved. The potential distribution is determined exactly in terms of special functions. Alternatively a simple closed form approximate result is found by applying the truncation method to the integral transform.
\end{abstract}

\section{INTRODUCTION}

Figure 1 shows the geometry. Cylindrical coordinates will be used $(\rho, \varphi, z)$. Also we will assume the time variation of the current is slow enough that we can consider the problem to be static. We wish to determine the potential distribution resulting from a lightning strike to the earth. The conductive top layer is inserted to represent the water saturated region near the surface during a thunderstorm. Because the layer is considered to be thin compared to the distances at which the potential will be of interest, we have taken it to have zero thickness but described by a thin sheet boundary condition. This approximation also reduces the number of parameters in the problem, which is an advantage when the knowledge of these parameters is uncertain.

\section{FORMULATION AND SOLUTION}

The boundary condition at the surface $z=0$ is taken to be

$$
E_{\rho}=R\left(K_{\rho}^{-}-K_{\rho}^{+}\right)
$$

where $E_{\rho}$ is the radial electric field $E$ and $K_{\rho}^{ \pm}$is the total effective radial surface current density defined by

$$
K_{\rho}^{ \pm}=\int_{ \pm 0}^{\infty} J_{\rho} d z
$$

The quantity $J_{\rho}$ is the radial component of the volume electric current density

$$
L=\sigma E
$$

where $\sigma$ is the electric conductivity of the half space. We know from Ampere's law that

$$
K_{\rho}^{-}=\frac{I}{2 \pi \rho}
$$


Suppose that the earth has a conductivity function $\sigma_{E}(z) \geq \sigma$ where $\sigma_{E}(z) \rightarrow \sigma$ as $z \rightarrow \infty$, and furthermore, $\sigma_{E}$ approaches $\sigma$ for values of $z$ which are small compared to the distances $\rho$ at which the potential is of interest. We can then define the conductive layer surface resistance $R$ by means of

$$
1 / R=\int_{0}^{\infty}\left[\sigma_{E}(z)-\sigma\right] d z
$$

The special case of a top layer of thickness $\Delta$ with $\sigma_{E}(z)=\sigma_{L}+\sigma$ for $0<z<\Delta$ and $\sigma_{E}(z)=\sigma$ for $z>\Delta$ gives $R=1 /\left(\sigma_{L} \Delta\right)$.

Because the electric field satisfies $\nabla \times E=0$, it will be found from the scalar potential $\phi$ by means of

$$
E=-\nabla \phi
$$

In the lower half space $\nabla \cdot L=0$, and thus

$$
\nabla^{2} \phi=0
$$

Because the fields are independent on the azimuthal coordinate $\varphi$ this becomes

$$
\frac{1}{\rho} \frac{\partial}{\partial \rho}\left(\rho \frac{\partial \phi}{\partial \rho}\right)+\frac{\partial^{2} \phi}{\partial z^{2}}=0
$$

Separation of variables, and taking the potential to be finite at $\rho=0$, as well as vanishing as $z \rightarrow \infty$, gives the solution

$$
\phi=\int_{0}^{\infty} C(\lambda) e^{-\lambda z} J_{0}(\lambda \rho) d \lambda
$$

where $J_{0}(x)$ is the Bessel function of order zero. The radial component of the electric field in the half space is thus

$$
E_{\rho}=-\frac{\partial \phi}{\partial \rho}=\int_{0}^{\infty} \lambda C(\lambda) e^{-\lambda z} J_{1}(\lambda \rho) d \lambda
$$

Using this expression we can find the effective radial surface current

$$
K_{\rho}^{+}=\int_{+0}^{\infty} \sigma E_{\rho} d z=\int_{0}^{\infty} C(\lambda) J_{1}(\lambda \rho) d \lambda
$$

Now because $E_{\rho}$ is continuous at $z=0$ we can use (10), (11), and (4) in the boundary condition (1) to obtain

$$
\frac{I R}{2 \pi \rho}=\int_{0}^{\infty}(\lambda+\sigma R) C(\lambda) J_{1}(\lambda \rho) d \lambda
$$

The Hankel transform pair $F(\lambda)=\int_{0}^{\infty} f(x) J_{v}(\lambda x) x d x, f(x)=\int_{0}^{\infty} F(\lambda) J_{v}(\lambda x) \lambda d \lambda$, can be used to determine $C(\lambda)$ from

$$
(\lambda+\sigma R) C(\lambda)=\frac{I R}{2 \pi} \lambda \int_{0}^{\infty} J_{1}(\lambda \rho) d \rho=\frac{I R}{2 \pi}
$$

Because the potential $\phi$ is also continuous at $z=0$, the potential on the surface of the earth is

$$
\phi(\rho, 0)=\frac{I R}{2 \pi} \int_{0}^{\infty} J_{0}(\lambda \rho) \frac{d \lambda}{(\lambda+\sigma R)}
$$


The identity [1]

$$
\int_{0}^{\infty} \frac{x^{v} J_{v}(a x)}{x+k} d x=\frac{\pi k^{v}}{2 \cos (v \pi)}\left[\mathbf{H}_{-v}(a k)-Y_{-v}(a k)\right]
$$

allows (14) to be integrated as

$$
\phi(\rho, 0)=\frac{I R}{4}\left[\mathbf{H}_{0}(\sigma R \rho)-Y_{0}(\sigma R \rho)\right]
$$

where $H_{0}(x)$ is the Struve function [2] of order zero and $Y_{0}(x)$ is the Bessel function of the second kind of order zero. Asymptotic forms for the special functions [2] yield

$$
\begin{gathered}
\phi(\rho, 0) \sim \frac{I}{2 \pi \sigma \rho}\left[1-\frac{1}{(\sigma R \rho)^{2}}+\cdots\right],(\sigma R \rho) \rightarrow \infty \\
\phi(\rho, 0) \sim-\frac{I R}{2 \pi}[\ln (\sigma R \rho / 2)+\gamma-\sigma R \rho+\cdots],(\sigma R \rho) \rightarrow 0
\end{gathered}
$$

where $\gamma \approx 0.5772$ is Euler's constant.

\section{TRUNCATION APPROXIMATION TO INTEGRAL}

A simple closed form approximation to the potential can also be obtained. Let us approximate the result (14) by

$$
\phi(\rho, 0) \approx \frac{I R}{2 \pi} \int_{0}^{A} \frac{d \lambda}{\lambda+\sigma R}=\frac{I R}{2 \pi} \ln \left(1+\frac{A}{\sigma R}\right)
$$

where we take the upper limit of the "truncated" integration to be given by

$$
A=\int_{0}^{\infty} J_{0}(\lambda \rho) d \lambda=1 / \rho
$$

Thus our approximate solution is

$$
\phi(\rho, 0) \approx \frac{I R}{2 \pi} \ln \left(1+\frac{1}{\sigma R \rho}\right)
$$

If we expand this approximate solution large and small $\rho$ we obtain

$$
\begin{gathered}
\phi(\rho, 0) \approx \frac{I}{2 \pi \sigma \rho}\left[1-\frac{1}{2 \sigma R \rho}+\cdots\right],(\sigma R \rho) \rightarrow \infty \\
\phi(\rho, 0) \approx-\frac{I R}{2 \pi}[\ln (\sigma R \rho)-\sigma R \rho+\cdots],(\sigma R \rho) \rightarrow 0
\end{gathered}
$$

Noting that $\ln (2)-\gamma \approx 0.1159$ is quite small in $(18)$, we see that $(23)$ and $(22)$ are reasonably good approximations of (17) and (18). Figure 2 shows a numerical comparison of the exact solution (16) and the truncation approximation (21). (Two normalizations are given in Figure 2.) The agreement is reasonably good over the entire range of $(\sigma R \rho)$. 


\section{STEP VOLTAGE}

The quantity of primary interest is the step voltage

$$
V=\phi(\rho-s / 2,0)-\phi(\rho+s / 2,0) \approx-s \frac{\partial}{\partial \rho} \phi(\rho, 0)=s E_{\rho}(\rho, 0)
$$

where the step length $s<<\rho$. The exact radial electric field is

$$
E_{\rho}(\rho, 0)=\frac{I \sigma R^{2}}{4}\left[\mathrm{H}_{1}(\sigma R \rho)-\frac{2}{\pi}-Y_{1}(\sigma R \rho)\right]
$$

The asymptotic forms of the radial electric field are [2] $E_{\rho}(\rho, 0) \sim\left[1-3 /(\sigma R \rho)^{2}\right] I /\left(2 \pi \sigma \rho^{2}\right),(\sigma R \rho) \rightarrow$ $\infty$ and $E_{\rho}(\rho, 0) \sim(1-\sigma R \rho) I R /(2 \pi \rho),(\sigma R \rho) \rightarrow 0$. The approximated electric field from $(21)$ is

$$
E_{\rho}(\rho, 0) \approx \frac{I R}{2 \pi \rho(1+\sigma R \rho)}
$$

Figure 3 shows a comparison of the radial electric field from (25) and (26). (Two different normalizations are given.) Using the truncation approximation, we obtain

$$
V \approx \frac{s I R}{2 \pi \rho(1+\sigma R \rho)}
$$

Thus we see that if $(\sigma R \rho)>>1$ then $V \approx s I /\left(2 \pi \sigma \rho^{2}\right)$, but if $(\sigma R \rho)<<1$ then $V \approx s I R /(2 \pi \rho)$.

\section{CIRCUIT MODEL FOR APPROXIMATE SURFACE ELECTRIC FIELD}

An approximate circuit model can also be constructed which is consistent with the truncation results. Figure 4 shows the geometry with a hemisphere of radius $\rho$ superimposed. The full lightning current $I$ crosses this hemisphere. We can approximate the problem as in the circuit model of Figure 4. The lightning current divides between the resistance per unit length of the surface layer $R /(2 \pi \rho)$ and the resistance per unit length of the hemisphere of conductor $1 /\left(2 \pi \rho^{2} \sigma\right)$. This approach should certainly be valid for very small $\rho$ (where the layer dominates) and for very large $\rho$ (where the half space dominates). The result for the surface electric field is thus

$$
E_{\rho}(\rho, 0) \approx \frac{I}{\left(2 \pi \rho / R+2 \pi \rho^{2} \sigma\right)}
$$

in agreement with the truncation result (26).

\section{References}

[1] I. S. Gradshteyn and I. M. Ryzhik, Table of Integrals, Series, and Products. New York: Academic Press, 1980, p. 685.

[2] M. Abromowitz and I. A. Stegun, Handbook of Mathematical Functions, National Bureau of Standards Applied Mathematics Series, December 1972, Ch. 12. 


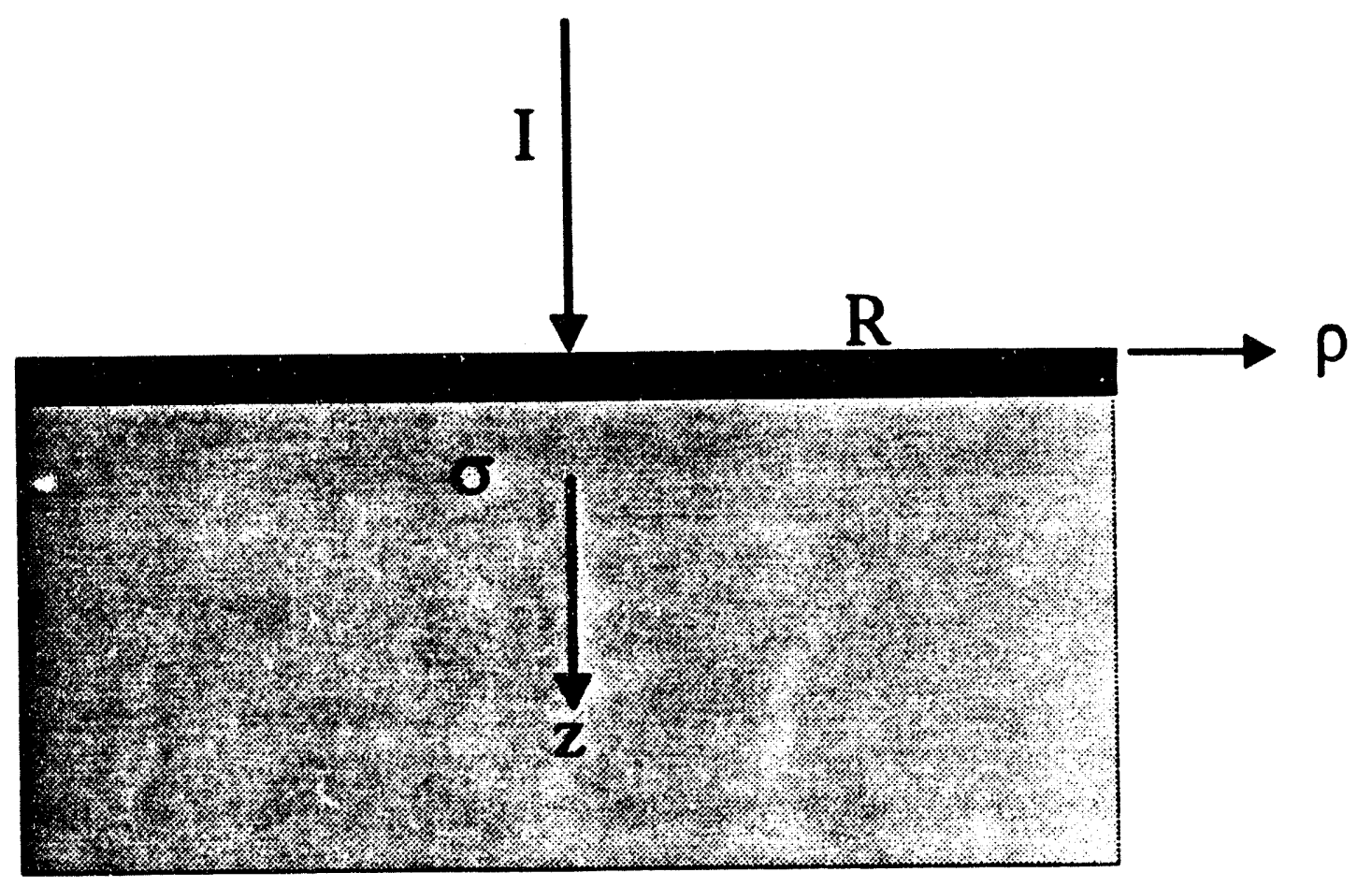

Figure 1. Filament current injected into conducting half space with conductive top layer. . 


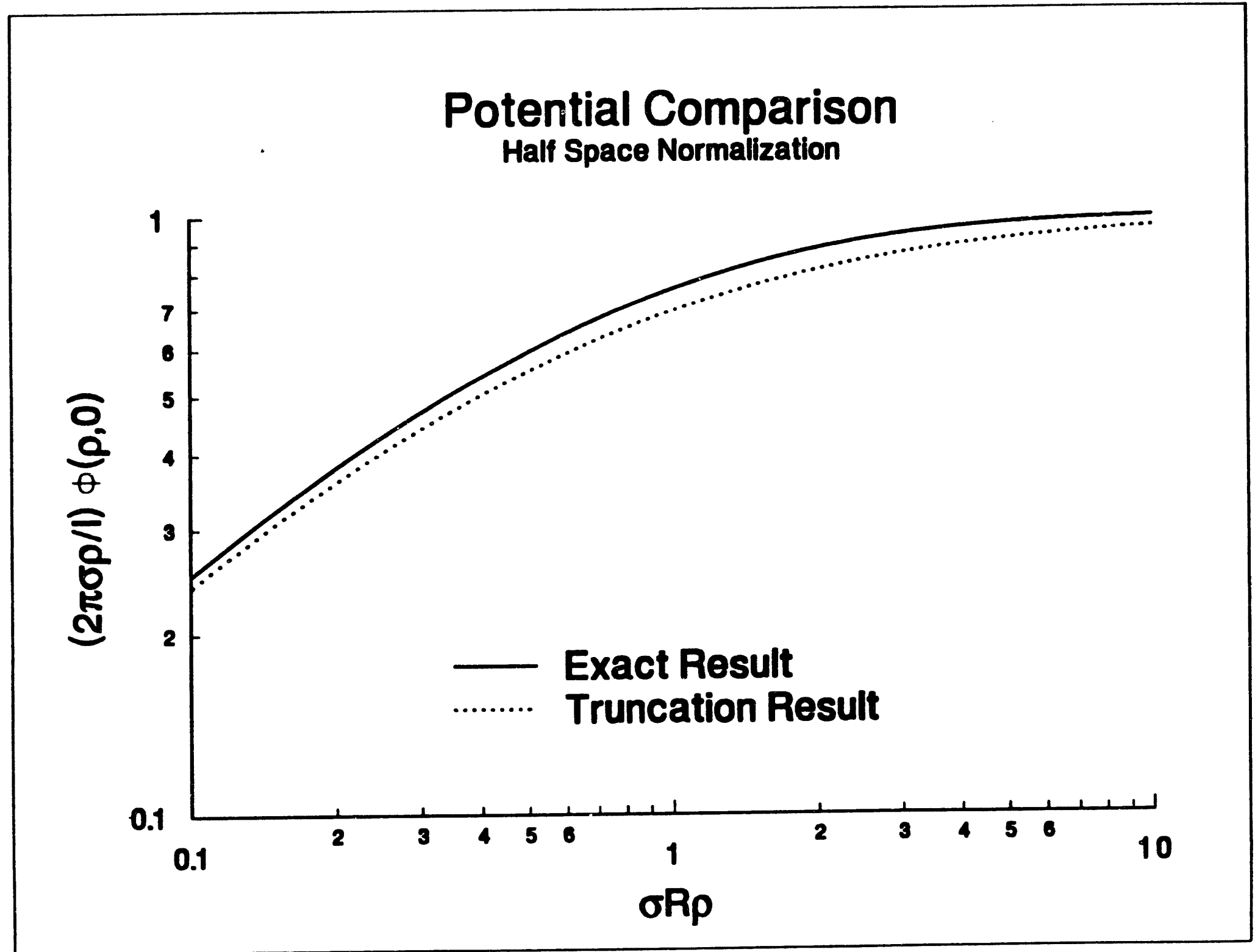

Figure 2a. Comparison of exact and approximate results for the potential with half space normalization. 


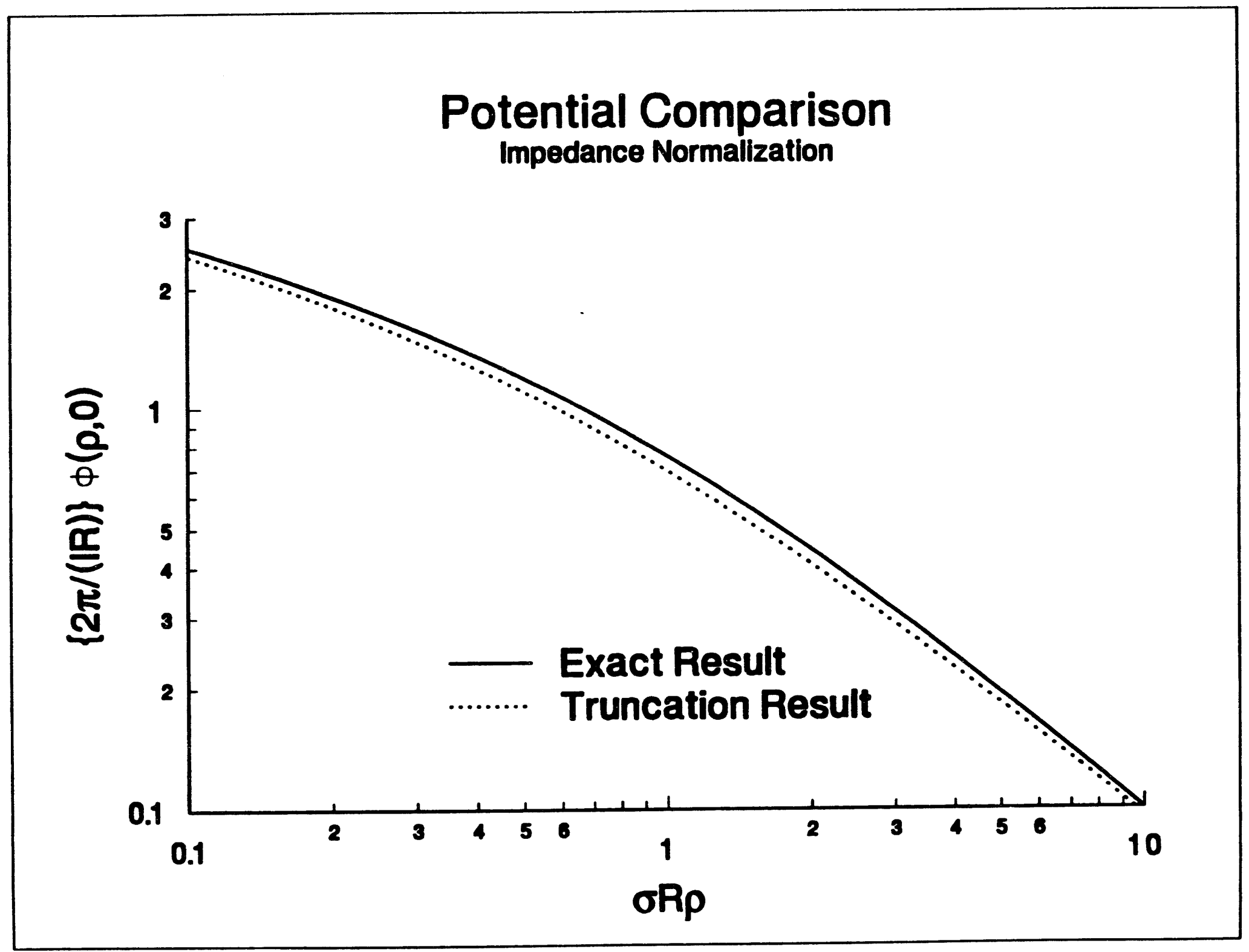




\section{Radial Electric Field Comparison}

Half Space Normalization

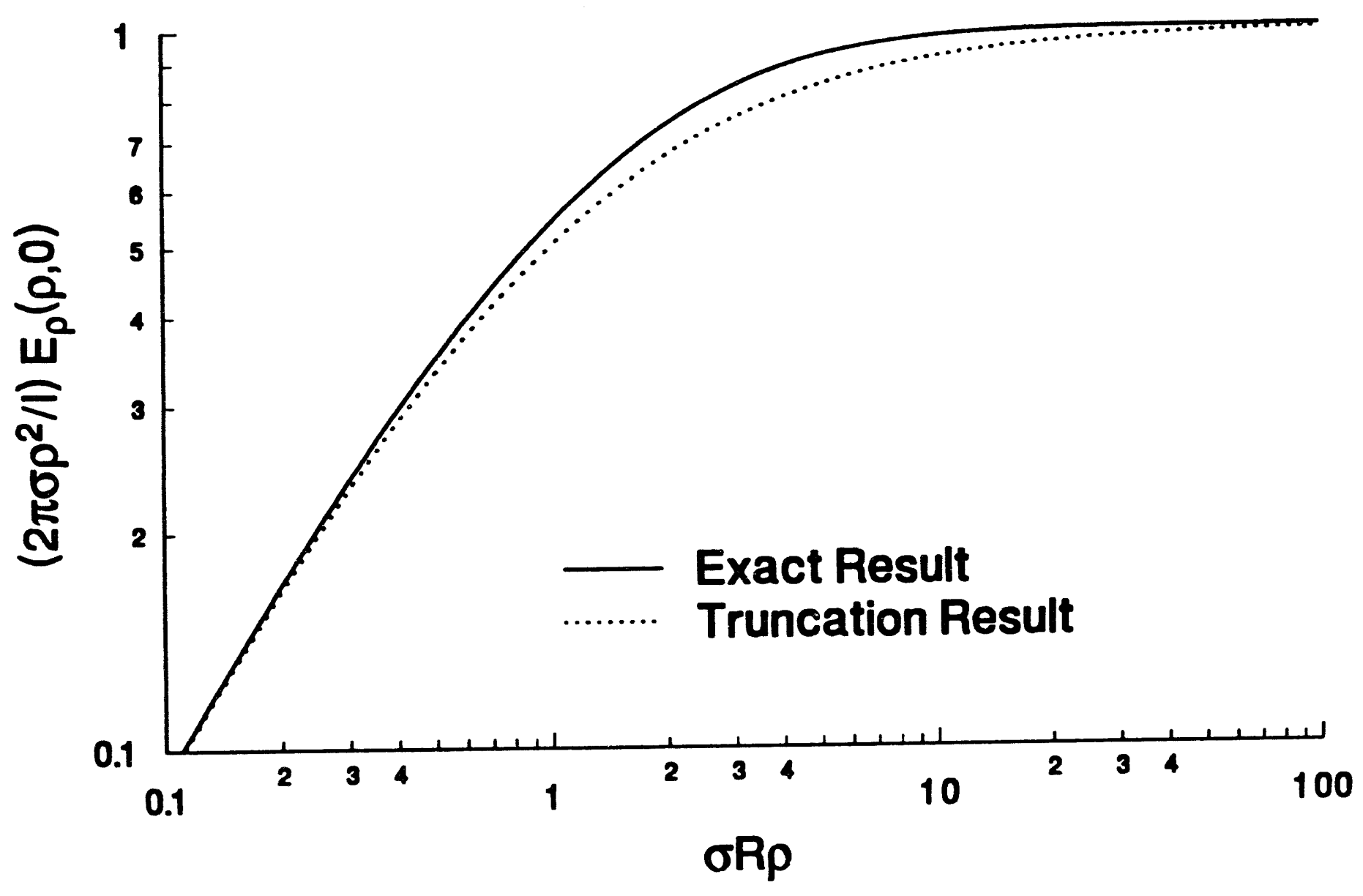

Figure 3a. Comparison of exact and approximate radial electric fields with half space normalization. 


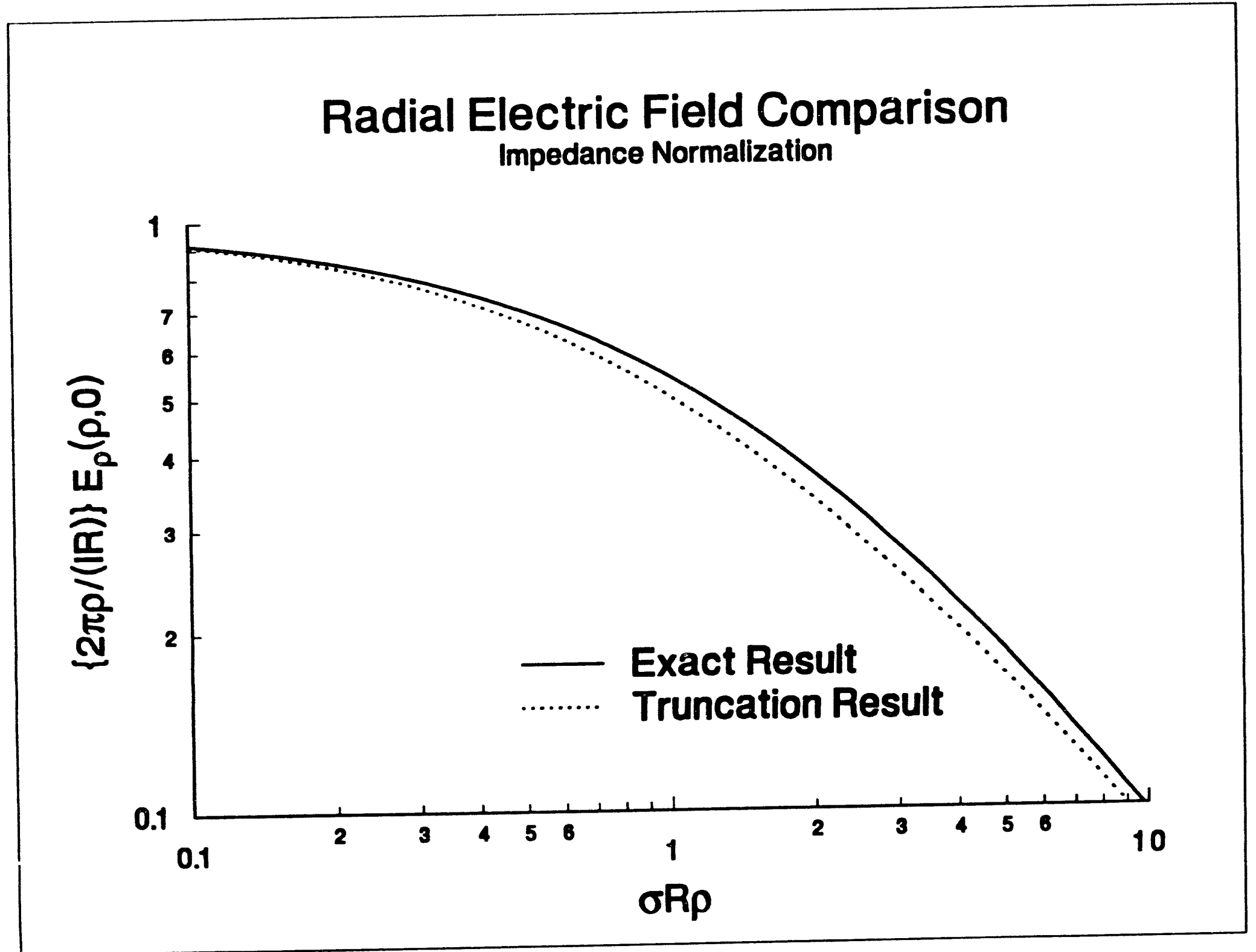



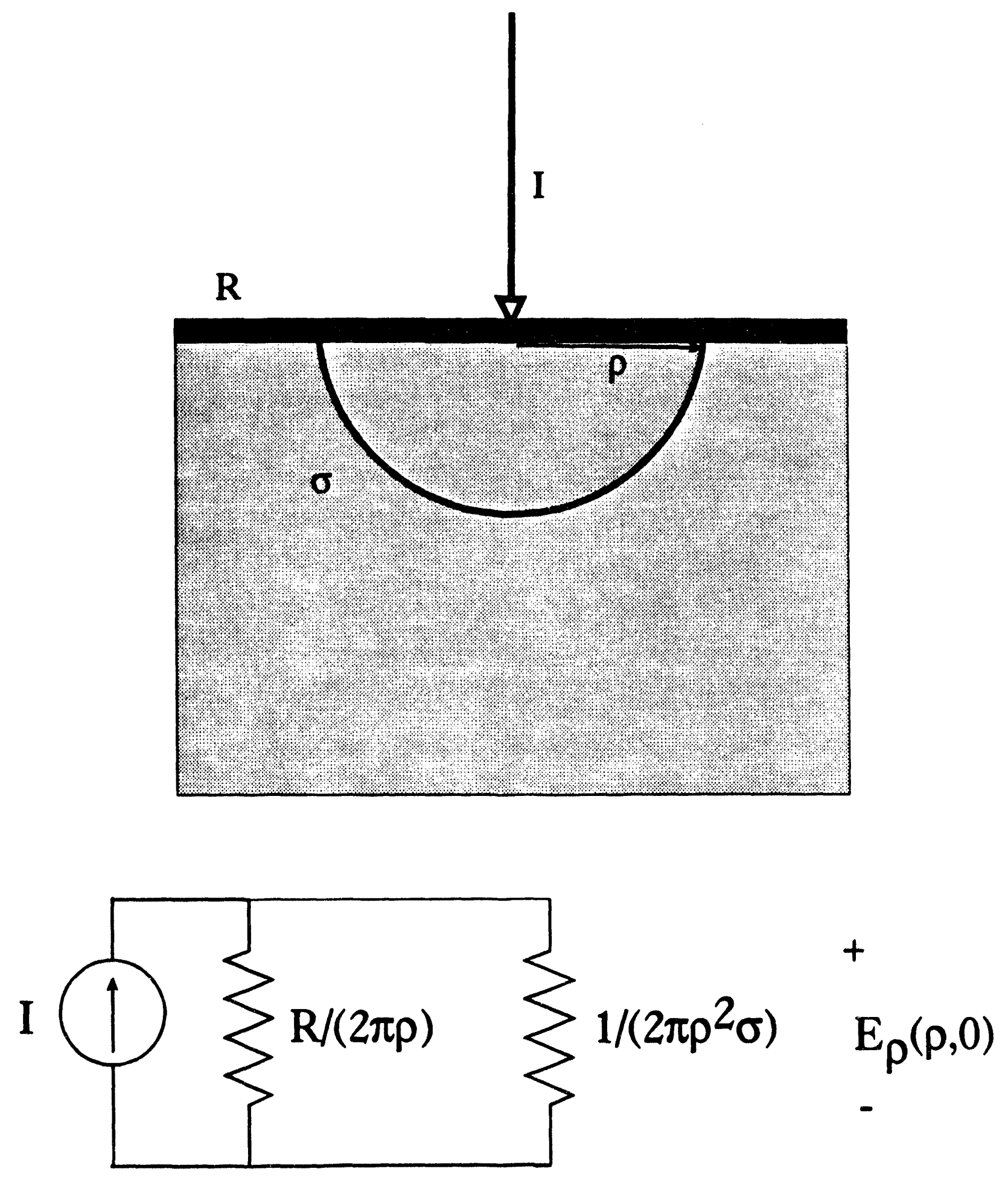

Figure 4. a) Geometry with hemisphere of radius $\rho$ superimposed. b) Approximate circuit model for surface electric field. 


\section{Appendix D \\ Annotated Schematic Diagrams \\ of the Time Histories of 1993 Triggered Flashes}

The diagrams contained in this appendix result from the frame-by-frame analysis of all available video and 16-mm records of each flash along with the various records of incident flash and stroke currents and magnetic fields. 


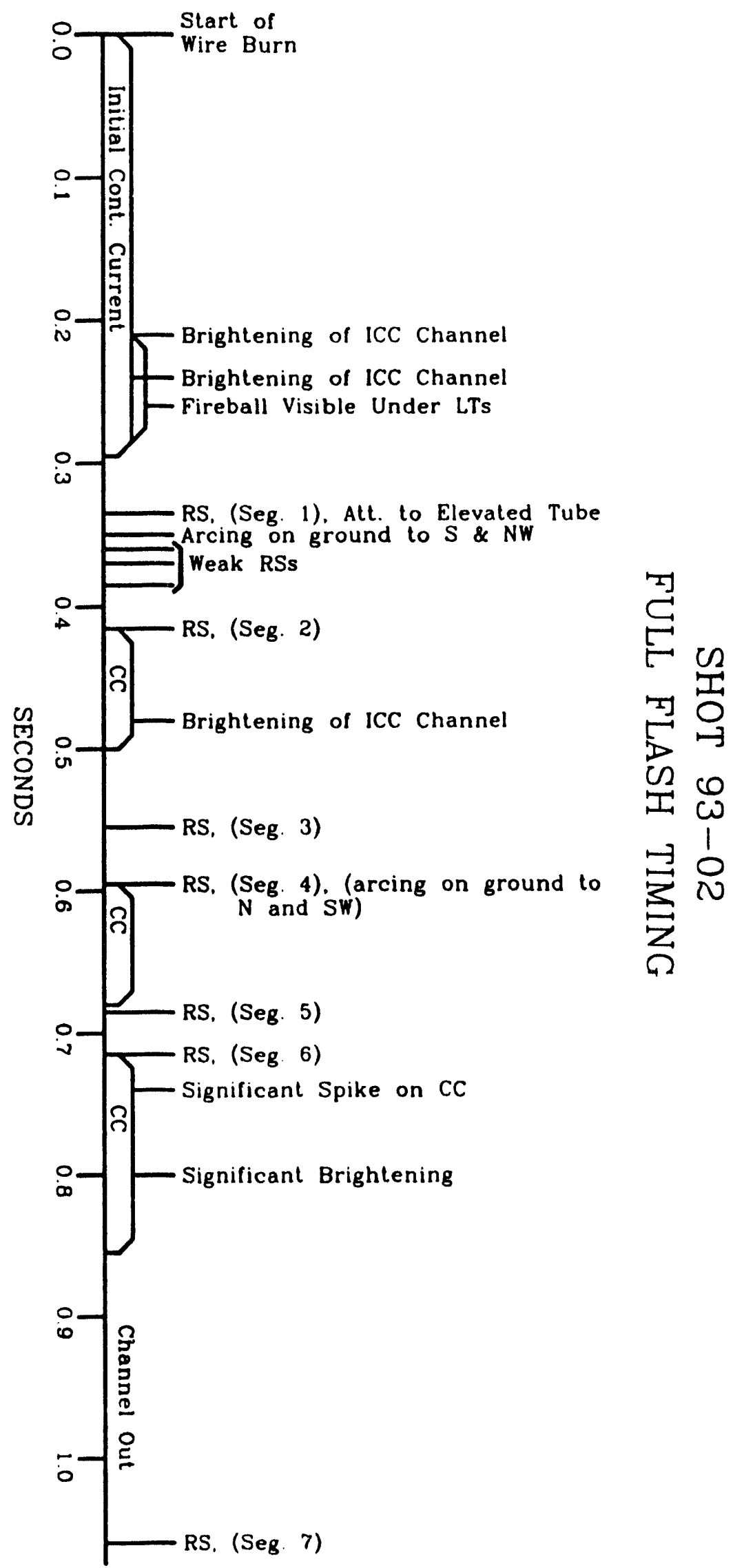




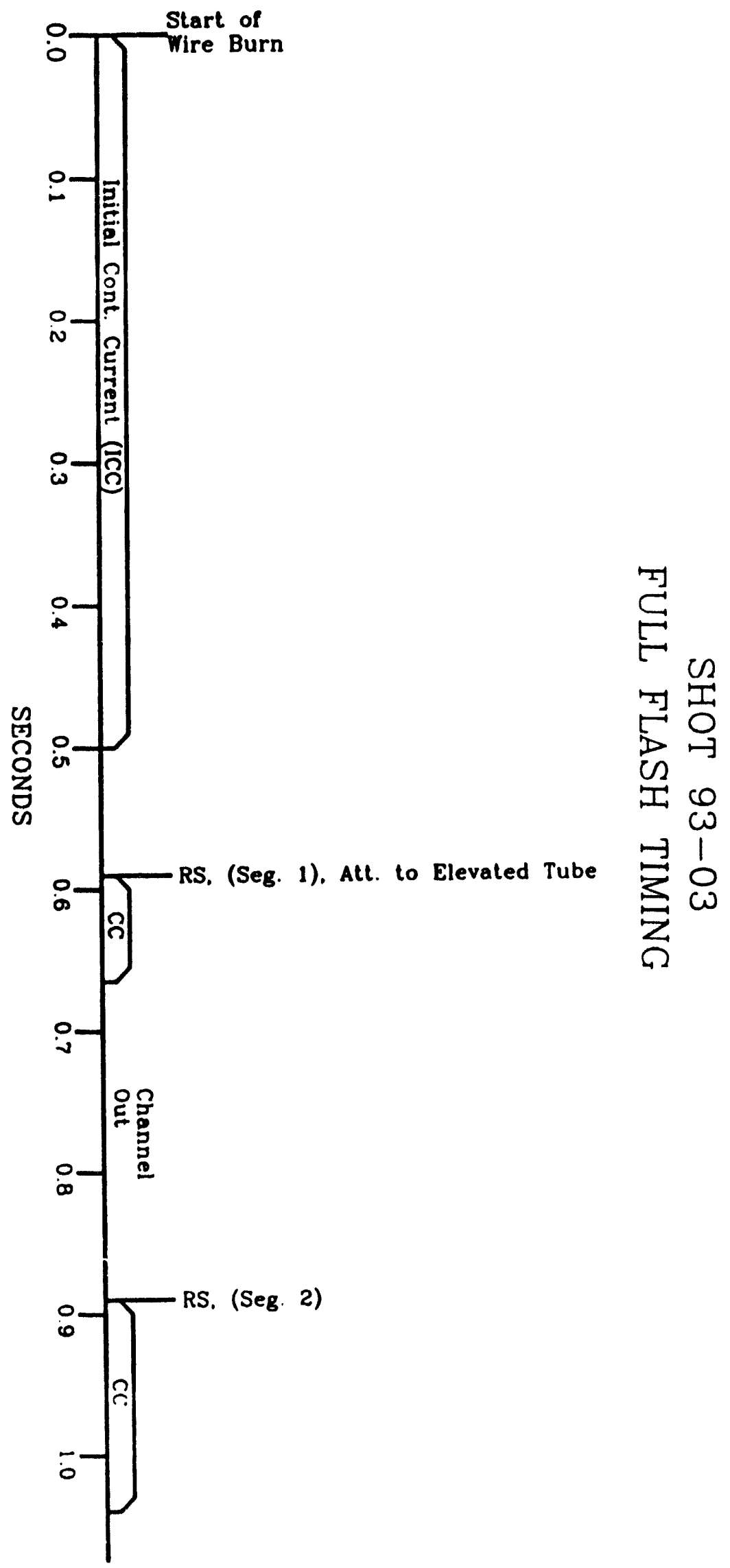




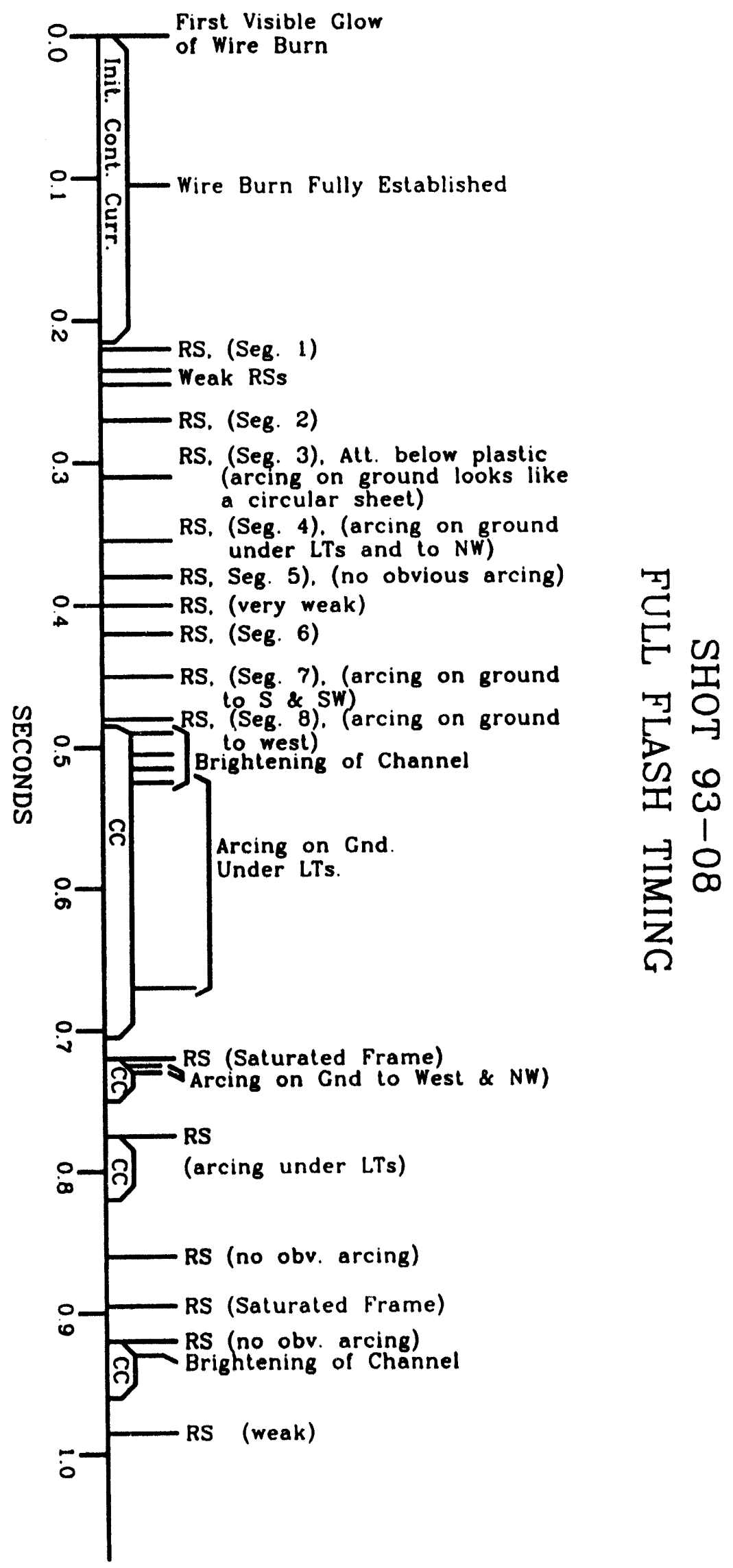




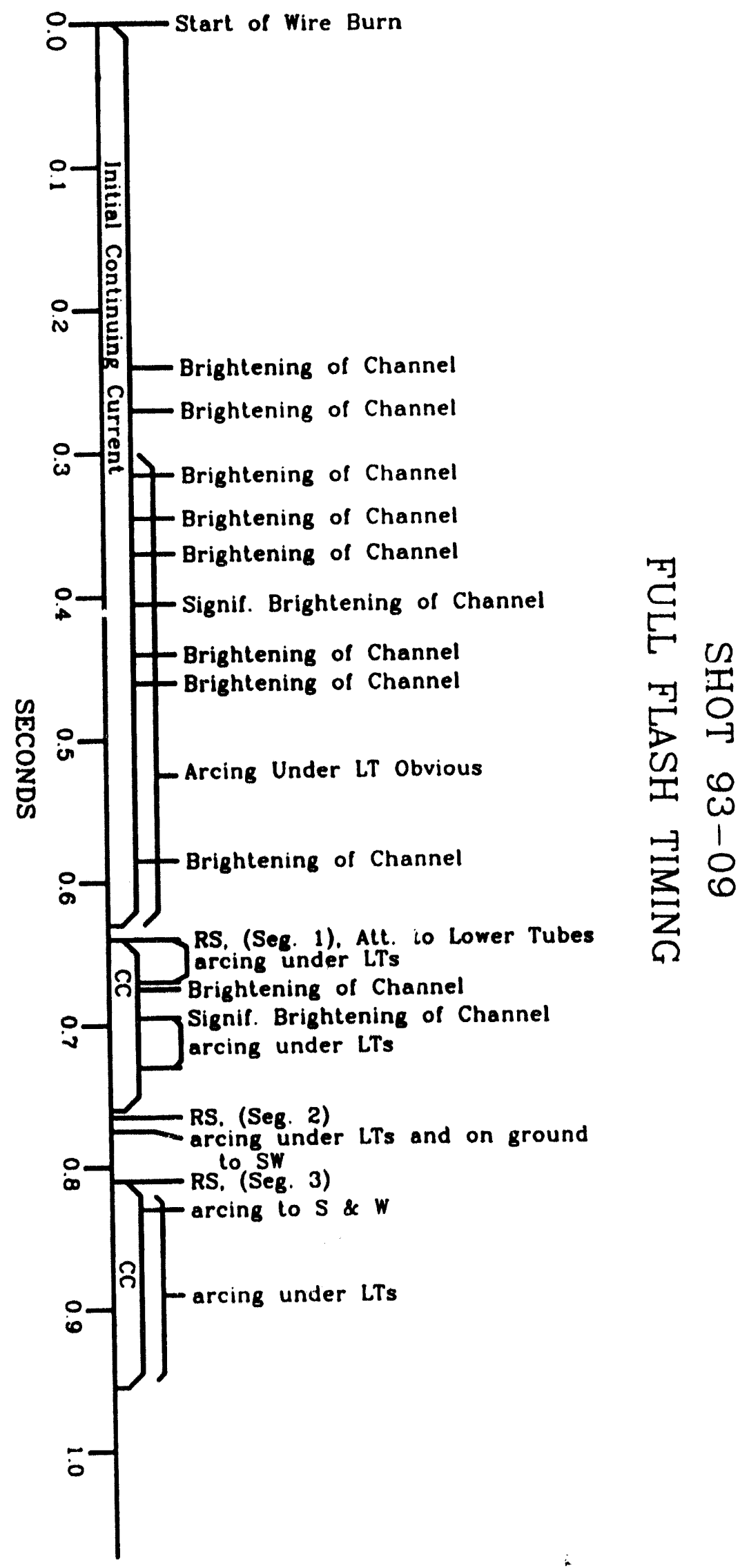




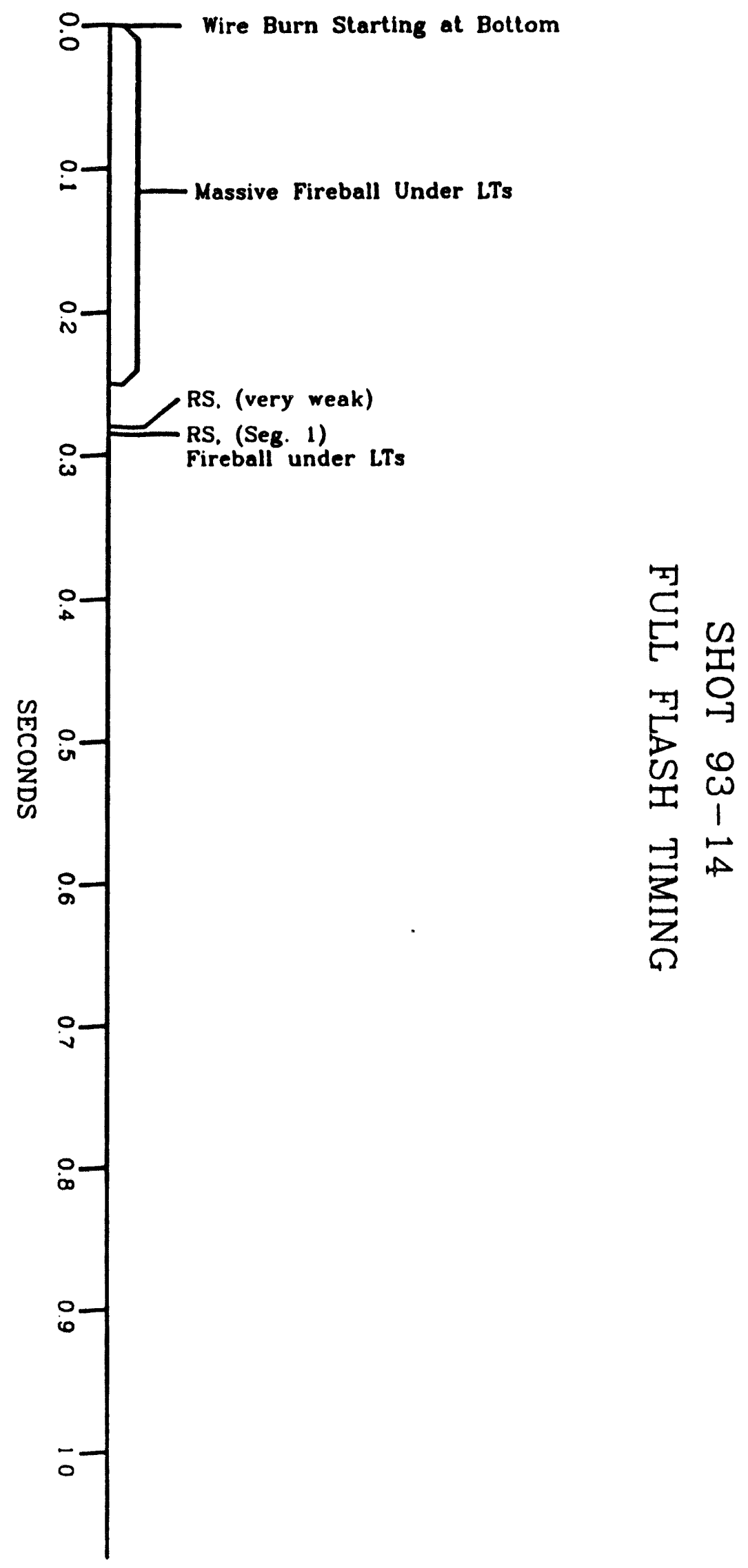




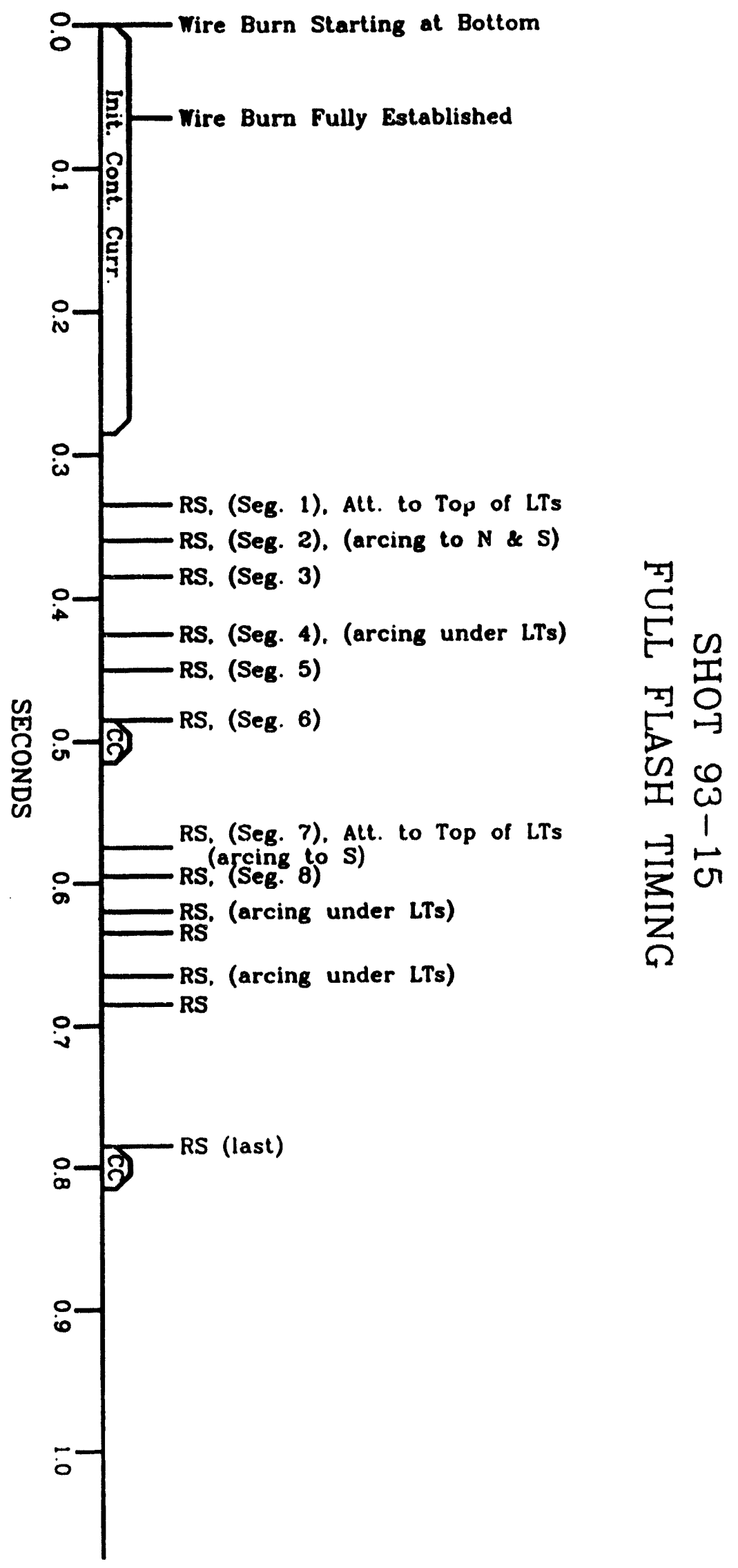




\section{Distribution}

$1 \quad$ AFGL

PL/GP/LYA

Attn: Dr. John Willet

Hanscom AFB, MA 01720

1 Philips Laboratory

GPAA

Attn: Dr. Stan Heckman

29 Randolph Rd.

Hanscom AFB, MA 01731-3010

5 Commander

USA Armament Research, Development, and Engineering Center

Attn: SMCAR-FSN

SMCAR-AEC-IM

SMCAR-FSN-T

Picatinny Arsenal, NJ 07806-5000

1 Commander, US Army Armament, Munitions and Chemical Command

Attn: AMSC-MAY-W

Picatinny Arsenal, NJ 07806-5000

1 Office of the Assistant to the Secretary

of Defense (Atomic Energy)

Room 3E1074, The Pentagon

Washington, DC 20301-3050

3 Director

Defense Nuclear Agency

Attn: R. C. Webb

LTC Wayne Andrews

J. V. Brackett

Washington, DC 20305

1 Commander, Naval Sea Systems Command

Department of the Navy

Attn: Nuclear Weapons/Munitions

Sec Div (SEA-643)

Washington, DC 20360

1 Commander, Naval Sea Systems Command

Theater Nuclear Warfare Program (PMS 423)

Attn: $\quad$ T. Rosling

Washington, DC 20362-5101 


\section{Distribution (Continued)}

4 Director, Strategic Systems Projects Department of the Navy

Attn:

B. Hannah

R. M. Jones

M. Whittaker

Ruth Ann Lee

Washington, DC 20376

$1 \quad$ US Department of Energy

Office of Military Applications

Attn: DP-22

Washington, DC 20545

2 Navy EOD Technology

Attn: Richard Burdette

Indian Head, MD 20640

2 Commander

NATC

Attn: M. Whitaker (SY84)

Patuxent River NAS, MD 20670

1 Commander, US Army Nuclear and Chemical Agency

Attn: MONA-MS (G. Long)

7500 Backlick Road

Springfield, VA 22150

1 RDA

Attn: Art Barondes

6940 S. Kings Highway

Alexandria, VA 22310

1 Dr. William Maurits

DDESB-KT

Hoffiman Bldg. 1/Rm 856-C

2461 Eisenhower Ave.

Alexandria, VA 22331-0600

1 Commander

Naval Surface Weapon Center

Attn: B. Franklin

Dahlgren, VA 22448

1 Commander

Attn: HQ AFCESA (Fowler)

Tyndall AFB, FL 32403-6001 


\section{Distribution (Continued)}

1 Commander

Attn: ASO/YQI (C. Churillo)

Eglin AFB, FL 32542

1 Commander, US Army Missile Command

Attn: Technical Library

Redstone Arsenal, AL 35898-5690

3 DoD SOTS

SMCAR-FSN-M

Attn: K. Haynes

Ft. McClellan, AL 36205-5300

1 Commander, US Army Armament, Munitions and Chemical Command

Attn: AMSMC-ASN-N

Rock Island, IL 61299-6000

1 National Severe Storms Laboratory

Storm Electricity and Cloud Physics

Attn: Dr. W. D. Rust

1313 Halley Circle

Norman, OK 73069

2 Field Command Defense Nuclear Agency

Attn: CDR F. T. Walker

Kirtland AFB, NM 87115-5000

1 US Department of Energy

Albuquerque Operations Office

Attn: NESD/WSSB

P.O. Box 5400

Albuquerque, NM 87115

4 Weapons Laboratory

Attn: D. Ulibarri/NTSW

M. Harrison/NTCA

LTCOL D. Stone/PL-LMI

Kirtland AFB, NM 87117-6006

1 Commander

NWEF

Attn: Jeff Stickney

Kirtland AFB, NM 87117 


\section{Distribution (Continued)}

1 Logicon RDA

Attn: Bill Kehrer

P. O. Box 9377

Albuquerque, NM 87119

1 Los Alamos National Laboratory

Attn: Technical Library

M. G. Wheeler, WX-1

P.O. Box 1663

Los Alamos, NM 87545

1 Commander, White Sands Missile Range

Bldg. 21225

Attn: Technical Library

White Sands Missile Range, NM 88002

1 Headquarters

Air Force Inspection and Safety Center

Attn: SEWV (Blount)

Norton AFB, CA 924097

1 Electric Power Research Institute

Project Manager/Distribution Program

Attn: Ralph Bernstein

P. O. Box 10412

Palo Alto, CA 94303

3 University of California

Lawrence Livermore National Laboratory

Attn: Technical Info. Dept.

R. T. Hasbrouck

Bill Hubbel, L-125

R. A. Woelffer, L-125

P.O. Box 808

Livermore, CA 94550 


\section{Distribution (Continued)}
MS 9006
E. E. Ives (Org. 5200)
MS 9001
C. T. Yokomizo (Org. 8007)
MS 9004
M. E. John (Org. 8100)
MS 9031
J. E. Marion (Org. 5302)
MS 9202
MS 9202
L. E. Dighton (Org. 8116)
MS 9005
C. M. Furnberg (Org. 8116)
MS 9013
J. B. Wright (Org. 5350)
MS 9014
R. G. Miller (Org. 5366)
MS 9203
D. L. Gehmlich (Org. 5371)
MS 9014
E. B. Talbot (Org 5354)
MS 9033
MS 9203
J. L. Mitchell (Org. 5371)
R. A. Pearson (Org. 5362)
MS 9006
MS 9033
S. J. Vasey (Org. 5354)
D. J. Bohrer (Org. 5202)
MS 9034
G. E. Dietel (Org. 5362)
MS 9035
D. J. Beyer (Org. 5363)
MS 9905
G. C. Story (Org. 5365)
MS 9013
C. A. Skinrood (Org. 8601)
MS 9014
K. A. Mitchell (Org. 5366)
MS 9021
J. R. Hogan (Org. 5371)
R. E. Martinell (Org. 8535)
MS 0433 R. N. Brodie (Org. 25)
MS 0465
J. F. Ney (Org. 5003)
MS 0490 S. D. Spray (Org. 12331)
MS 0492 R. E. Church (Org. 12332)
MS 0492 G. A. Sanders (Org. 12332)
MS 0490 P. E. D'Antonio (Org. 12324)
MS 0761 P. E. Rexroth (Org. 5822)
MS 0492 J. F. Wolcott (Org. 12332)
MS 0492 D. Loescher (Org. 12332)
MS 0459 T. S. Edrington (Org. 5205)
MS 1181 J. M. Hoffman (Org. 1208)
MS 1153 L. D. Bacon (Org. 1248)
MS 1188 R. G. Adams (Org. 1275)
MS 0513 H. W. Schmitt (Org. 2000)
MS 0311 R. F. Ellison, Jr. (Org. 2571)
MS 1007 J. F. Jones, Jr. (Org. 2172)
MS 0328 N. F. Siska (Org. 2574)
MS 0511 G. N. Beeler Org. 2500)
MS 1073 P. V. Dressendorfer (Org. 2277)
MS 0319 S. B. Martin (Org. 2641)
MS 0955 K. G. McCaughey (Org. 2904)
1 MS 0319 J. H. Barnette (Org. 2606) 


\section{Distribution (Continued)}
MS 0527 L. A. Andrews (Org. 2235)
MS 0523
MS 0507
R. D. Holt (Org. 2251)
MS 0865
R. A. David (Org. 2700)
MS 0865
M. E. Morris (Org. 2753)
K. C. Chen (Org. 2753)
R. J. Fisher (Org. 2753)
MS 0865
R. D. Jones (Org. 2753)
MS 0865
R. E. Jorgenson (Org. 2753)
MS 0865
K. O. Merewether (Org. 2753)
G. H. Schnetzer (Org. 2753)
MS 0865 L. K. Warne (Org. 2753)
MS 0570 C. W. Childers (Org. 5900)
MS 0471 J. P. Abbin, Jr. (Org. 5093)
MS 0459 C. C. Burks (Org. 5203)
MS 0453 R. E. Kreutzfeld (Org. 5111)
MS 0447 J. O. Harrison (Org. 5115)
MS 0447 J. S. Clabaugh (Org. 5115)
MS 0447 H. T. Lehman (Org. 5115)
MS 0436 G. L. Maxam (Org. 5147)
MS 0467 K. D. Nokes (Org. 5091)
MS $0482 \quad K$. Oishi (Org. 5161)
MS 0455 G. R. Otey (Org. 4100)
MS 1393 D. F. McVey (Org. 5609)
MS 0755 H. W. Church (Org. 6612)
MS 0971 G. H. Mauth (Org. 9203)
MS 1165 J. E. Powell (Org. 9300)
MS 1166 J. H. Renken (Org. 9352)
MS 1166 G. J. Scrivner (Org. 9306)
MS 1166 C. D. Turner (Org. 9352)
MS 1166 C. N. Vittitoe (Org. 9352)
MS 0768 J. W. Kane (Org. 5806)
1 MS 0765 D. E. McGovern (Org. 5821)
1 MS 9018 Central Technical Files (Org. 8523-2)
5 MS 0899 Technical Library (Org. 7141)
1 MS 0619 Technical Publications (Org. 7151)
10 MS 0100 Document Processing (for DOE/OSTI) (Org. 7613-2)

75 MS 0865

10 MS 0865 


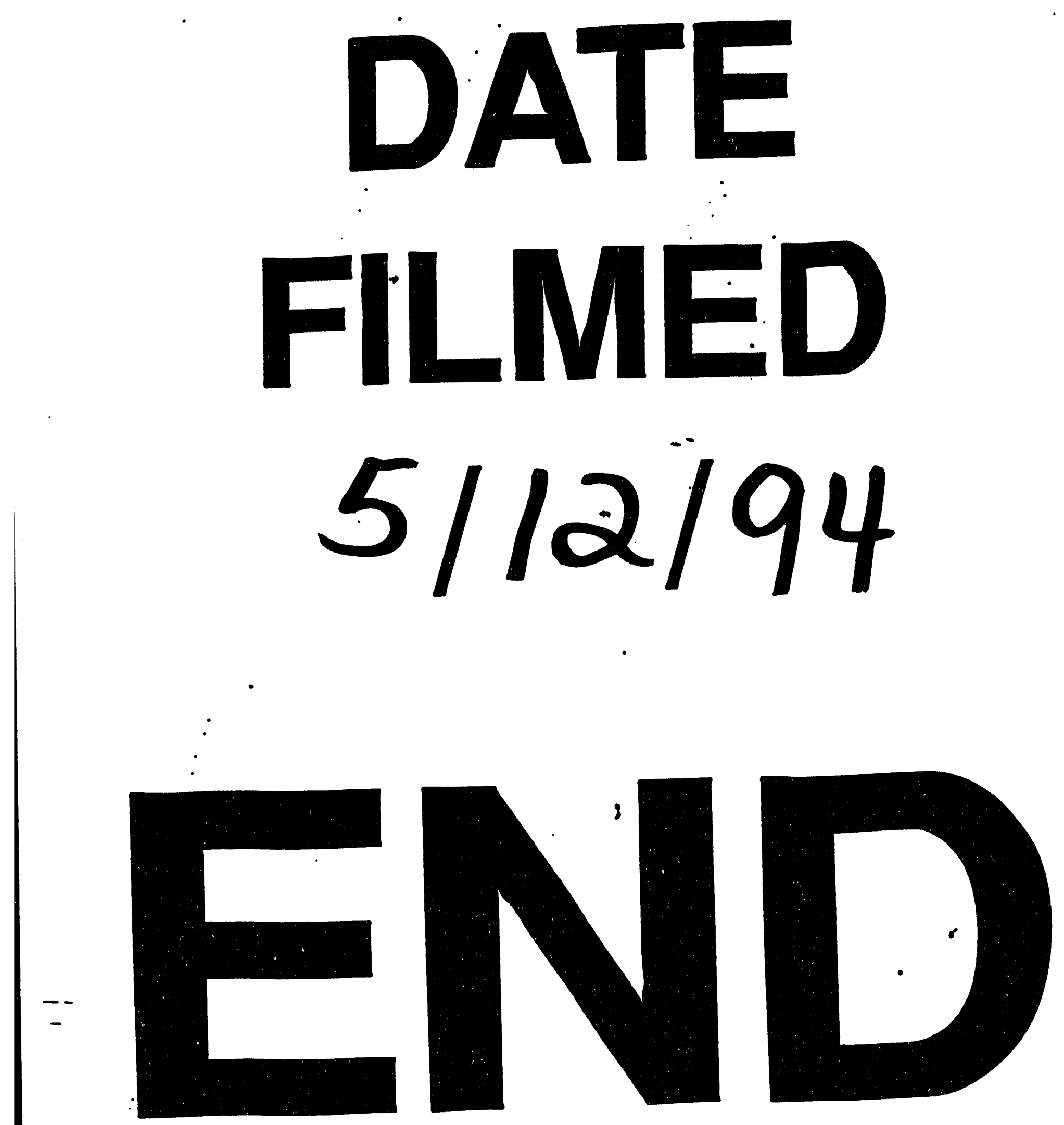




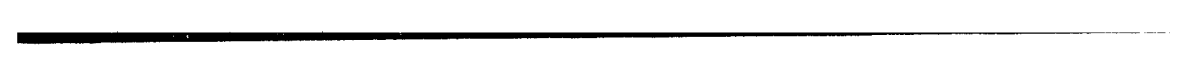

